

\section{UIBRARY}

UNIVERSTT OF

CALIFORNIA

SAN DIEGO

\section{BMA}

BIOMEDICAL LIBRARY

INNAERSITY OF CALIFORNIA, SAN DIEGO

DATE DUE

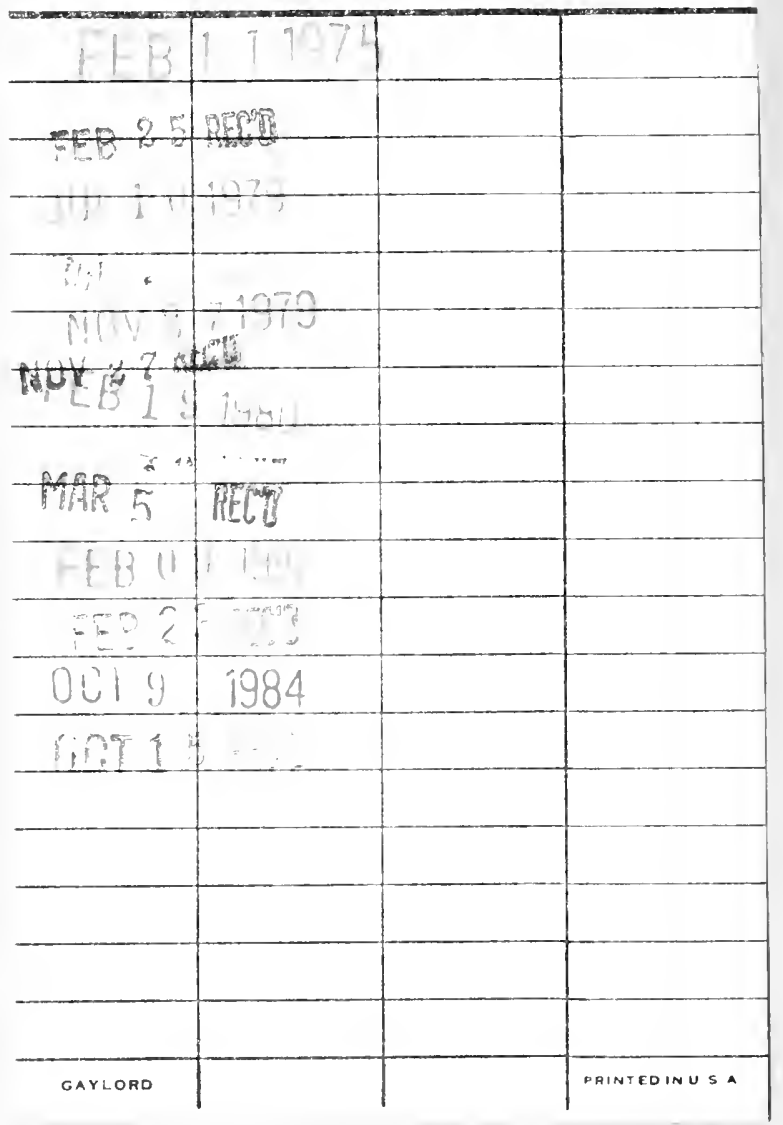



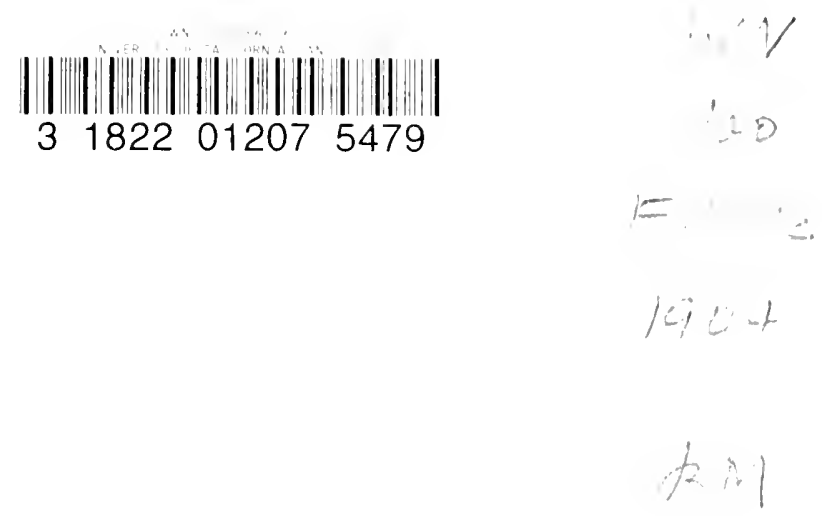




\section{Digitized by the Internet Archive in 2008 with funding from Microsoft Corporation}



Plate.
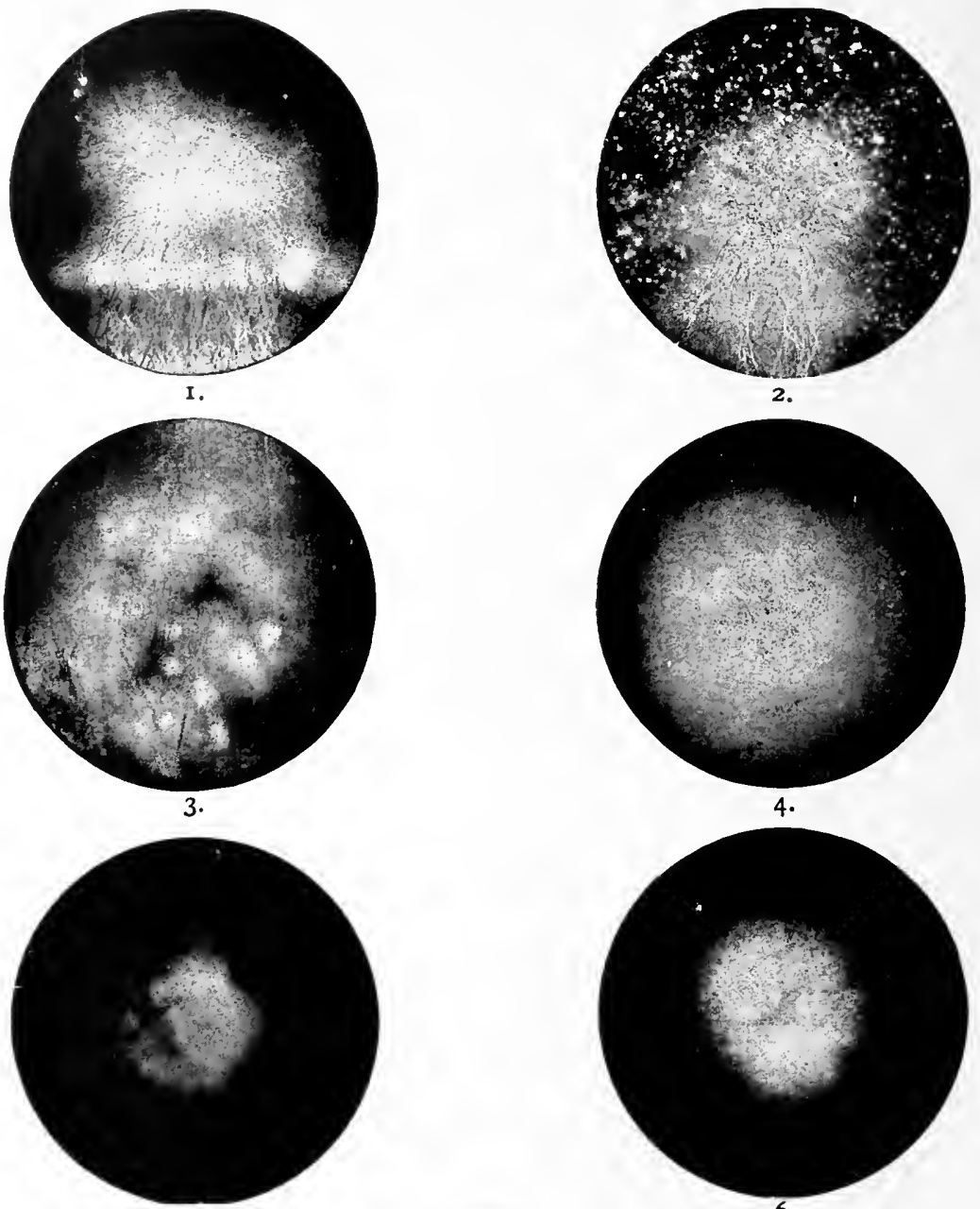

5.
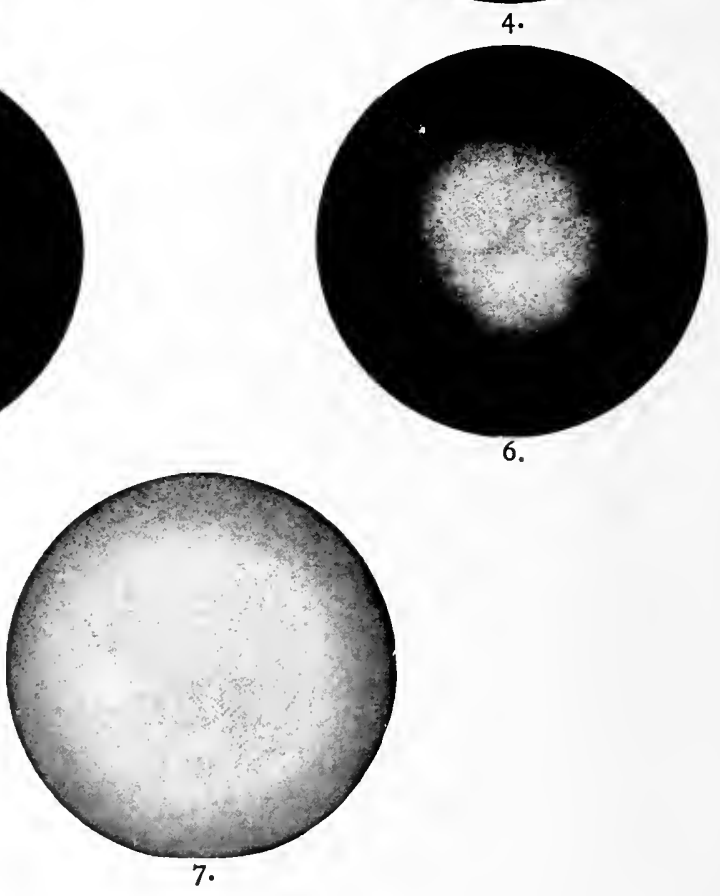

Photographs of Electric Discharges. 


\section{ELEMENTS}

$\mathrm{O} \mathrm{F}$

\section{GENERAL}

\section{RADIO-THERAPY \\ FOR}

\section{P R A C T I T I O N E R S}

BY

DR. LEOPOLD FREUND,

$\mathrm{VIENNA}$.

TRANSLATED BY

G. H. LANCASHIRE,

M.D. BrIX, M.R.C.S. ENG., L.R.C.P. LoND.

Assistant Physician to the Manchester and

Salford Hospital for Skın Diseases.

With One hundred and seten Iilustrations in the Text and one Frontispiece.

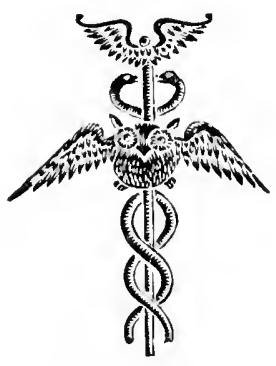

NEW YORK

REBMAN COMPANY, 10 West 23 Street, Cor. 5 Th Avenue.

LONDUN AGENTS:

REBMAN, LIMITED

129 Shaftesizury Avi, Lonino, W. C.

1904 
Entered according to Act of Congress, in the year 1904 BY

REBMAN COMPA N Y

in the Office of the Librarian of Congress, at Washington

Ali, Rigit'ts Reserved

ENTERed at Stationers' Hall.

BY REBMAN LIMITED, LONDON 


\section{TRANSLATOR'S PREFACE.}

THE translator has pleasure in introducing Dr. Freund's work to a larger circle of the English-speaking profession. For the undertaking itself he feels that no apology is needed; Dr. Freund's position in this department of medicine more than justifies the enterprise. The author is known-though the fact appears in many quarters to have been insufficiently recognised-as the man to whom, perhaps, more than any other we owe the foundation of Roentgen-therapy. The following pages, indeed, speak for his arduous labours in the whole subject of radio-therapy.

Apologies are rather needed for this English setting; these are freely extended, both to the author, whose meaning may possibly have been in places misconstrued, and to the reader. Here and there a somewhat free translation has been rendered necessary by the exigencies of the language-a necessity which all readers of German will be the first to acknowledge. In certain places omissions have been made with the author's permission.

In conclusion, the translator's grateful thanks are recorded for the kindly help afforded him by many friends.

G. H. Lancasinire.

Mancinester, January, 1904. 



\section{AUTHOR'S PREFACE.}

IN this work I have attempted to bring the essential features of a recent form of treatment before the notice of a larger circle of medical men. I had before me the task not only of describing in a comprehensible manner the technique, the indications for and results to be expected from the various radiotherapeutic methods, but also of tabulating and arranging the fundamental physical laws these nature-forces obey, thereby more fully explaining their physiological effects. Six years' arduous theoretical and practical work in all the branches of radio-therapy treated of in this volume form my excuse for a partial survey of this department of medicine, and may fairly entitle me to undertake a work of this nature.

I have not limited myself to the description merely of my own investigations and conclusions, but have quoted every author who has written on this subject, controlling wherever possible his deductions by my own experiment. Where it seemed advisable for the further comprehension and investigation of a subject, the older physical and photochemical works have been quoted. I have purposely presupposed but little knowledge on the part of the reader, as will be seen in the introductory chapters on physics. I have tried as far as possible to include the most recent ideas, drawing from the knowledge obtained in practice up to the present time, while referring the reader to the various sources which afford fuller information on the separate subjects. Above all, the present work makes no claim to have thoroughly exhausted its subject, nor must the conclusions arrived at be considered infallible. It is a truism that, however complete an observer's knowledge may seem to be, in course of time fresh facts partly contradict his earlier conclusions.

It requires but a brief glance to show the enormous amount of work which a large number of investigators have already 
accomplished, and the great progress which has been made in a few years with the question of radio-therapy. Such zeal, such intense interest as the most talented and able chemists, physicists and medical men of every country have shown in attacking these most difficult problems, remain almost without parallel. Although this branch of science can hardly be said to be more than in its infancy, gaps in our knowledge having everywhere to be filled and rubbish to be removed, we have already achieved brilliant theoretical and practical results, leading one to hope that radio-therapy will obtain an acknowledged place among our methods of treatment. One need onlyrefer to the undeniable and astonishing results achieved in the department of skin disease, which has so markedly attracted the attention of dermatologists. Nevertheless, the claims of radio-therapy as a treatment for disease are still disputed; from time to time medical men, among whom are some of repute, although none can claim personal experience, feel called upon to refer to Roentgen-therapy, d'Arsonvalisation, etc., as exploded and soon-to-be-forgotten methods. True, such croakings may deter the scientific ardour of others, but cannot really impede the progress of radio-therapy. "Science is progressive. Its development is as necessary and irresistible as the tide. It is also a stage of development of the natural forces, and as such is sure in due time to compel recognition, should not those who now try to belittle its influence and hinder its progress prefer to ally themselves with it." These words of John Tyndall, which were addressed to the opponents of natural science, might also be taken to heart by the opponents of radio-therapy. If any of my colleagues whose work lies outside this field should feel induced by my modest efforts to pay in the future more attention to this subject, or even if they merely admit the raison d'être of the methods laid down and scientifically justified in this book, my aim is accomplished. I trust that the fact of my drawing largely upon my own personal experience and investigation will not tend to prejudice the reader against my work. My heartiest and sincerest thanks are due to Hofrath Director Prof. Dr. J. M. Eder, Hofrath Prof. Dr. Anton Weichselbaum, Prof. Dr. Eduard Valenta, Prof. Dr. Anton Lampa and Docent Dr. Anton Ghon, who have light- 
ened my otherwise enormously difficult investigations and both in word and deed most kindly helped me. Special thanks are owing to my highly esteemed teacher Hofrath Prof. Dr. J. Neumann, who has always followed my work with friendly interest, and has of late given me the opportunity of furthering my scientific investigations by placing the radio-therapeutic laboratory of his clinic at my disposal.

L. FREUND. 



\section{INTRODUC'TION}

By radio-therapy we mean the employment of any form of radiation for therapeutic purposes. The name "radiation" comprises usually a series of physical manifestations which, according to the present state of our knowledge, can be divided into two groups (Lampa). In the first group fall electromagnetic radiations (radiations of electrical energy), heat rays, light and ultra-violet rays; in the second group cathode rays, Roentgen rays, the rays emitted by glow-worms (Muravka), Becquerel rays, radium and polonium rays.

All these radiations possess active powers by reason of certain inherent physical characteristics. These are known as radiant energy. Physicists have formulated various hypotheses as to the character of this radiant energy. According to one authority, radiant energy is a form of activity produced by the enormously rapid and regular vibration of an imponderable substance, ether, which permeates space and matter.

Acording to the number of vibrations which the ether-particles produce in a given second, one classifies different kinds of rays, and the effect upon our sense organs varies with the periodicity of these vibrations. We appreciate vibrations of 160 to 400 billions per second as "dark heat-rays." If, however, the rate of vibration exceeds 400 billions per second, we are conscious of them not merely as "heat-rays," but on account of their effect upon our eyes, we call them "light-rays." In this case the particular rate of vibration determines colour impression.

All ether vibrations which are under 160 and over 790 billions per second we are quite unable to appreciate. There are, however, ways and means by which these rays can be brought within our visual capacity. Thus, for instance, heat-waves of great-wave length can be demonstrated by Langley's bolome- 
ter, while short-waved ultra-violet rays can be indicated by photography or by the fluorescence of a platino-barium-cyanide screen.

Next in order to the well-known ether-vibrations of the heatrays comes a space as yet uninvestigated, which is succeeded in turn by the much slower vibrations of the more lengthy electric waves. In these latter, according to Hertz, we have only $\mathrm{I}, \mathrm{OoO}$ million vibrations to the second. The difference between lightwaves, heat-waves and electric waves is not qualitative, but merely a question of gradation. Rubens and Aschkinass showed the most lengthy heat-waves to be one six-hundredth of a millimeter in measurement, vibrating at the rate of five millions per second. On the other hand, Lampa has shown the shortest electro-magnetic waves to be four millimeters in length, vibrating at the rate of seventy-five thousand millions per second. Proceeding now along the ray-scale from the large electric waves (with wave-lengths from several meters down to a few millimeters, and vibration rates from a few millions to many milliards per second) to the smaller heat and light-waves (with wave-lengths of 0.02 millimeter to $4 \mathrm{IO}$ millionth millimeter) we come to the still quicker, shorter-waved hyper-ultra-violet rays discovered by Hertz. These latter accomplish 800 billion oscilliations per second, and are distinguished by the peculiar properties they possess.

\begin{tabular}{|c|c|c|c|c|c|c|c|c|c|c|c|}
\hline $\begin{array}{c}\text { Electric } \\
\text { waves }\end{array}$ & $\begin{array}{c}\text { Un- } \\
\text { known }\end{array}$ & $\begin{array}{l}\text { Infra- } \\
\text { red }\end{array}$ & 苟 & 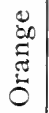 & 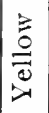 & 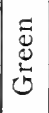 & $\stackrel{\varrho}{\Xi}$ & $\begin{array}{c}\frac{1}{0} \\
0 \\
\vdots \\
1\end{array}$ & $\begin{array}{l}\text { Ultra- } \\
\text { violet }\end{array}$ & $\begin{array}{l}\text { Hyper- } \\
\text { ultra- } \\
\text { violet }\end{array}$ & $\begin{array}{l}\text { Roent- } \\
\text { gen } \\
\text { rays }\end{array}$ \\
\hline
\end{tabular}

We might, perhaps, also include in this first group of radiations the Roentgen rays, which many physicists are inclined to regard as a species of very short-waved light-rays. The first kind of radiations belonging to the second group, the cathode rays, may be considered as consisting of small material particles ("corpuscles") which, like the ions (see p. 26), are charged with negative electricity. According to Thomson's measurements, the material amount of the corpuscles is, however, much smaller than that of the electric ions, indeed, only about one- 
thousandth of the latter, whereas, the electrical state is identical in each instance.

According to J. J. Thomson, the corpuscle is composed of a particle of solid matter bound up with a qualitatively different electric charge. A broader view, which is shared by a large number of investigators, is comprised by the conclusions Hclmholtz arrived at in ISS r. Helmholt $\approx$ observes that in the case of electrolysis (see p. 25) the ions (charged chemical atoms) are arranged in the neighbourhood of the electrodes as neutral bodies, so that a discharge or a partial exchange of charges of opposed nature takes place there. This occurrence cannot take place instantaneously; the charges must at least for a very brief time lead independent existences; one may, therefore, consider that equal charges of I "value" represent the elementary quantity of electricity; or, in other words, the electric atom. Stoney has proposed the name "electron" for these electrical atoms, a name now universally adopted. The corpuscle with its charge is identical with the electron.

According to Kauffmann, $\left.{ }^{1}\right)$ the electrical atoms are electrical particles which have the power of moving with a velocity of I-5-I-3 the velocity of light in space, but which are also able to assume independent vibrations of their own, and to transfer these to others of their kind. They can unite themselves with material particles, becoming free again, however, thereby allowing the manifestations of electrolysis. At the same time the chemical atoms which, charged with mutually neutralising electrons, appcared non-electric, discharge or cxchange their positive and negative electrons, and in doing so they must have the capacity for leading a separate existence, if only for a short period, being likewise able to freely wander in the conducting metal. Granted

') See $W$. Kauffmann, "Die Entwicklung des Elektronenbegriffes." Allg. Naturforscherzeitung. Jahrg. 1. Nr. 1.- 1. Lampa, Ueber Strahlung. Wien I902. Braumüller. 
now that you have positive and negative electrons, there is yet a remarkable difference between the two; the vibrating electron is always negatively charged, whereas the positive electron remains motionless. Hence it might happen that up to the present only negative electrons have been observed, and it is quite possible that they alone exist. On this point, however, no absolute verdict can be given. 'The electrons have been also compared with material atoms, and it has been found that the former amount to about the 2-rooo part of an atom of hydrogen. Thanks to the electron theory, an explanation is now possible of the hitherto obscurest por* tion of recent physics, including cathode rays, Roentgen rays and Becquerel rays. Hitherto physicists have endeavoured to explain electrical phenomena by mechanical laws; these attempts have not proved entirely successful. Perhaps better results would ensue from the endeavour to explain mechanical phenomena by means of electrical theories, and to assume that the supposed inertia of certain matter is only imaginary. In that case, electrons would be considered as original atoms, the various grouping of which would produce chemical elements, and it would appear reasonable to look for the known chemical elements amongst these groups which happen to be sufficiently stable, and to discover a connection between the relative distribution of the elements and their atomic weights. Finally, we may hope for a solution of the obscure problem of gravity by the aid of the electron theory.

The rays proceeding from the so-called radio-active substances (radium rays, Becquerel rays) may be classed with cathodal rays. This is evident from properties all these rays possess in common, viz.: the power of diverting the magnetic field, of causing phosphorescent effects, and of giving conductive capacity to gases. We have in the radium salts a class of bodies capable of spontaneously emitting corpuscles or electrons.

A cathodal-ray electron moving with enormous velocity must, on striking a solid body, necessarily discharge an electric 
wave in an explosive manner into space, just as a projectile produces sound-waves at the moment of impact. There are many grounds for supposing that Roentgen rays are caused in this way. ${ }^{1}$ )

This would form a second explanation of the phenomena of these mysterious rays, whose remarkable properties, as we shall see, many other theories endeavour to explain. All these rays have this property in common, wiz., the power of exciting phenomena in bodies at a great distance from the source of energy, without risible effects upon the interiening space. (In reality this is, however, an effect which is transmitted from particle to particle into space.)

The following properties may be defined as common to all the varieties of radiation at present employed in radio-therapeutics: They cause chemical changes, fluorescing bodies to glow, and alter the electrical state of bodies with which they come into contact.

The phenomena above mentioned, discovered for the most part within the last few years, have now also acquired some importance in medicine, more especially by reason of the distinct and peculiar influence they exert upon living organisms, an influence which, up to the present, we have not been able to obtain by other means. The result has been that we have been able to employ these various radiative methods as curative factors in certain diseases with absolute success, and that this branch of treatment undoubtedly signifies in many cases a distinct progress in therapeutics.

It is only of late that medicine has turned her attention to this matter, consequently, as yct, her conclusions are by no means exhaustive and the knowledge acquired by no means complete. However, it is already evident that bcsides the "distance-effect".2)

1) $W$. Kauffmann, "Die Entwicklung des Electronenbegriffes." $-A$. Lampa, "Ueber Strahlung."

2) By the expression "distance-effect" we mean in a physiological sense the effects which a stimulation produces, not at the site of irritation, but on another part of the organism by means of the circulatory or nervous system. Reflex action may be taken as an example. For our present purposes, however, we shall consider "distance-effects" in a physical sense; that is to say, those effects which the radiations produce on the part they strike. 
common to all these radiations, each of them produces appearances and changes which, despite certain differences of degree rather than of kind, can all be brought into one category.

At this juncture we must refer to the chapter on the physiological effects of radiations.

We may regard these radiations as physical irritations, which, like other ( $e . g$., chemical) irritations, in small amounts are stimulating to granulation tissue, whereas, in stronger doses, they cause destructive effects. So, as is the case with all kinds of excessive stimulation, an over-dose of radiations brings about destructive changes and cell-death. It has long been a wellknown fact that electricity employed in certain forms promotes tissue-nutrition. ${ }^{1}$ ) It is also known that Roentgen and lightrays used with lower intensity stimulate the growth of certain tissues and organisms, as well as the proliferation of hairs, colouring matter, and pigment.

Moreover, it is well known that all three agencies bring about diseased conditions when employed with greater intensity, ranging from simple inflammation to actual necrosis.

The earlier investigations of S. Exner, Kistiakowsky, Schaudinn, Joseph, Provázek, and others, have proved that light, heat radiations, electricity, and Roentgen rays influence the molecular movements of living cells, certain other elementary phenomena of life being similarly affected.

Again, according to experiment, the character of the tissue-damage and the finer molecular changes in the protoplasm appear to be in all cases similar, whatever be the particular agency employed: vacuolisation degenerations resulting from the action of light (Glebofsky), Roentgen rays (Gassman), high-frequency electricity (Freund). With regard to the visible clinical effects which may be ascribed to radiations, we may, arguing from experiment, make the following definitions :

I. The physiological effect of a radiation stands in direct relation to its intensity, in inverse proportion, however, to its wave-length; i.e., other things being equal, the short-waved rays

$\left.{ }^{2}\right)$ The reader is referred to the Preface, and also to page II2, etc., for the relations existing between d'Arsonvalisation and radio-therapy. 
cause more powerful and enduring effects than the longerwaved. $\left.{ }^{1}\right)$

2. The signs of reaction only become visible after a latent period, the length of which stands likewise in inverse proportion to the wave-length and intensity of the radiation employed.

3. The physiological effect of the radiations is enduring. As regards the penetration-effect of the radiation we can lay down no absolute rule; it would appear, however, that this also bears direct relation to the wavelength; that is to say, long-waved rays penetrate more deeply than shorter-waved (compare, for instance, blue and ultra-violet).

4. Those rays which have the property of exciting fluorescence (Roentgen rays, ultra-violet rays, blue and Becquerel rays) appear to exert more physiological effect. Very likely further experience and extension of our knowledge may tend to somewhat modify these definitions. The assumption that all the above-mentioned physical phenomena and their physiological effects may be ascribed to the same or similar elementary causes, may claim some justification. This is proved by the various change-effects which have been observed in recent years to follow from different physical phenomena (electricity, light, etc.). It is known that the latter are not independent of one another; that one manifestation may call forth another. Thus electricity may produce light phenomena, Roentgen rays, ultraviolet, light, and vice versa.

One cannot, of course, here attempt a demonstration of all those interesting matters which a number of eminent physicists have so ably argued in favour of the foregoing theorics. The reader is referred to the lucid expositions of $\left.K_{a y s e r}{ }^{2}\right)$, Janm(ann $\left.{ }^{3}\right)$, and Lecher ${ }^{4}$ ), a careful study of

$\left.{ }^{2}\right) E$. Aschkinass and $W$. Caspari suppose that the common source of the effects produced by ultra-violet. Becquerel and Roentgen rays is to be looked for in the dissociating power ("ionisation") possessed by all three. Goldstin holds the ultra-violet light alone responsible. The author, however, is inclined to ascribe an important rôle to fluorescence.

2) Lehrbuch der Plyysik. Stuttgart bei Enke 1900, pp. 56.3. .

3) Vorlesungen ucher Elektricitact und Licht. Leipzig. J. A. Barth, 190r.

•) Ueber the Entdeckung der elektrischen Wellen, etc. Ibid. Igor. 
xvi

which will amply repay the worker in radio-therapy. In the present work reference will also be made in the appropriate sections to those circumstances which justify one in explaining the different physiological changes after radiation by a reference to analogous physical occurrences. 


\section{CONTENTS}

Translator's Preface.

PAGE

PREFACE

INTRODLCTION .

Content's

\section{ELEMENTS OF ELECTRICITY}

r. Positive and Negative Electricity . . . . . . . 2

2. Conductors and Non-Conductors . . . . . . . 2

3. Transmission of Electricity . . . . . . . . . 5

4. Manifestations of Electrical Energy . . . . . . . 6

5. Electrical Distribution, Influence and Induction . . . . I2

6. Electrical Condensers . . . . . . . . . . I2

7. Electric Machines . . . . . . . . . . . . 17

8. Contact Electricity, Galvanism, Galvanic Batteries . . . 19

9. Accumulators . . . . . . . . . . . 27

ro. Ohm's Law . . . . . . . . . . . . 29

Ir. Technical Measure-Units . . . . . . . . . 32

12. Battery Connections . . . . . . . . . 34

13. Ramification of Current . . . . . . . . . 36

14. Measurements of Electrical Currents . . . . . . 38

15. Heat and Light Effects of the Galvanic Current . . . . 4I

r6. Thermo-Electricity . . . . . . . . . . 44

17. Magnetic Effects of Electric Currents . . . . . . 45

r8. Induction . . . . . . . . . . . . . 47

19. Spark Induction Apparatus . . . . . . . . 56

20. Interrupters . . . . . . . . . . . . 64

\section{TREATMENT IVITH HIGH-FREQUENCY CURRENTS}

21. High-Frequency Currents

22. Instruments for IIigh-Frequency Currents . . . . . 94

23. Technique . . . . . . . . . . . Io0

24. Physiological Effects . . . . . . . . . 104

(a) Effects on the Body Surface of the Spark I)ischarge . I21

(b) Effects on Bacteria . . . . . . . . 126

(c) Histological Experiments . . . . . . . 152

25. The Therapcutic Uses of IIigh-Frequency Currents . . . I65

Indications for the Methed:

1. The Induction of Analgesia . . . . . . If6

2. Diseases Associated with Defective Metabolism . . 167

3. Tuberculosis . . . . . . . . 170

4. Nervous Diseases . $\quad . \quad$. $\quad . \quad$. $\quad . \quad 172$

5. Diseases of the Skin and Mucous Membranc. • • 174

Résumé . . . . . . . . 180 


\section{APPENDIX}

“Permeating Electricity"

\section{TREATMENT WITH X-RAYS X-RAY METHODS}

26. Cathode and Roentgen Rays

27. Vacuum Tubes . .

28. Some Practical Hints on the Installation and Employment of X-Ray Apparatus

29. The Development of the Therapeutic Employment of Roentgen Rays

3o. Method of Treatment with X-Rays

3r. Indications

I. Diseases of the Hairs and Hairy Regions

\section{Favus}

Sycosis Vulgaris and Folliculitis Barbæ.

\section{Tricophytosis}

Blepharitis

Trichorrhexis Nodosa

Alopecia Areata

Hypertrichosis .

2. Ulcerations and Skin Diseases resulting in Ulcerations .

Lupus Vulgaris

Epithelioma, Ulcus Rodens, Mycosis Fungoides, Lepra, and Sarcoma of the Skin

3. Acute and Chronic Exudative Dermatoses and Granulation-formations

Chronic and Acute Eczema . . . . . . 295

Psoriasis

Prurigo .

Lupus Erythematosus • . . . . . $\quad$ • 298

Acne Vulgaris, Rosacea, Furunculosis . . . . 300

4. Nævus . • . • • • • • • • . $30 \mathrm{r}$

The Treatment of Internal Disorders with X-Rays . • 302

32. Physiological Effects of the X-Rays . . . . . . 305

33. The Active Agent of this Method . . . . . . . 322

34. X-Ray Dermatitis . . . . . . . . . . 337

\section{BECQUEREL-RAYS}

35. Becquerel-Rays

36. Their Physiological Effects . . . . . . . . 355

37. Some Therapeutic Experiments . . . . . . 363

\section{TREATMENT WITH HEAT AND LIGHT RAYS ELEMENTS OF LIGHT-PHYSICS}

38. Light Theories

39. Light Sources .

40. Diffusion of Light

41. Intensity of Light 
42. Photometry . . . . . . . . . . . 373

43. Katoptrics . . . . . . . . . . . 377

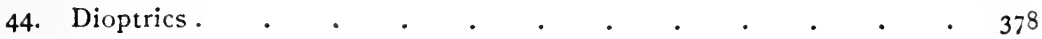

45. Aberration . . . . . . . . . . . . . . 379

46. Absorption . . . . . . . . . . . 380

47. Dispersion . . . . . . . . . . . . . $38 \mathrm{I}$

48. Ultra-Red Rays . . . . . . . . . . . . 387

49. Ultra-Violet Rays . . . . . . . . . . . . 358

50. Light Interference . . . . . . . . . . . 39I

5I. Refraction of Light . . . . . . . . . 391

52. Effects of Light . . . . . . . . . . . 392

53. Physiological Effects . . . . . . . . . 394

54. 1. The Effect of Light on Plants . . . . . . . 395

55. 2. The Effect of Light on Bacteria . . . . . . 40I

56. 3. The Effect of Light on Higher Organisms . . . . 409

57. The Therapeutic Employment of Light . . . . . . 447

58. (a) Treatment with Sunlight . . . . . . . . 450 Sunlight . . . . . . . . . . . 450

59. Sun Baths . . . . . . . . . . . . . 453

6o. Open-Air Light Baths . . . . . . . . . 454

6I. Chromotherapy . . . . . . . . . . 455

62. Concentrated Sunlight . . . . . . . . . $46 \mathrm{I}$

63. (b) Treatment with Artificial Light. . . . . . . 463

64. Treatment with Incandescent Light . . . . . . 465

65. Treatment with Arc-Lights . . . . . . . . 479

66. Therapeutic Employment of Non-Concentrated Arc-Lights . . 488

67. Employment of Concentrated Arc-Lights . . . . . 490 -Lupus Vulgaris . . . . . . . . . . . 498

Alopecia Areata $. \quad . \quad . \quad . \quad . \quad . \quad . \quad . \quad 509$

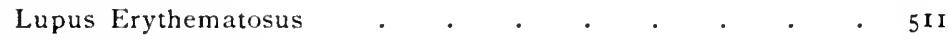

Epithelioma . . . . . . . . . . . 5 . 5 II

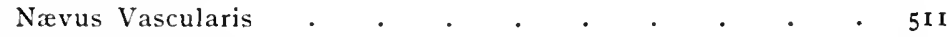

Other Skin Diseases . . . . . . . . . . 512

Venereal Diseases . . . . . . . . . . . 512

68. (c) Employment of Other Sources of Light . . . . . 513

Spark Light . . . . . . . . . . . $5 \mathrm{I} 4$

Incandescent Light . . . . . . . . . . 517

Fluorescence. . . . . . . . . . . . . 518

Phosporescence . $\quad . \quad$. $\quad . \quad$. . . . 520

Appendix to the Section on X-Ray Treatment . . . . $\quad 525$

Index to Subject-Matter . . . . . . . . . 527

Index to Authors . . . . . . . . . . 533 



\section{LIST OF ILLUSTRATIONS}

Photograph of Electric Discharges . . . Frontispiece FIG.

Tension Difference. . . . . 9

Carré's Machine. . . . . I7

Wimshurst Machine . . . I7

Lever-Rheostat (Graetz) . . . 3I

Lever-Rheostat . . . . . 3I

Lever-Rheostat (Reiniger, Schall, etc.) . . . . 3I

7 Parallel Connection . . . . 34

8 Serial Connection . : . . . 34

9 Wheatstone's Bridge . . . . 37

Io Voltmeter, outside view . . . 39

I I Ampèremeter, outside view . . 39

12, 13 Ampèremeter, inside view . . 40

I4 Crater-shaped Depression . . 43

I5 Siemens's Double T-Magnet . 49

I6 Gramme-ring . . . . . . . 50

17 Electro-Motive Force of Alternating Current. . . . 5I

18, 19 Ruhmkorff's Coil . . 57, 60

20 Spark Inductor, with Primary Coil

2I Spark Inductor Contacts

22 Spark Inductor Terminals .

23 Spark-gap · Platinim inter-

24. Dessauer's Platinum Interrupter, Platinum Break. .

25 Dessauer's Platinum Break . 66

26 Neef's Hammer . . . . . . 67

27 Foucault's Interrupter . . 68

28 Schulmeister's Interrupter . . 69

29 Boas' Turbine-Mercury-Break

30 Max Levy's Interrupter . . .

3I Mercury-break (Reiniger, Gebbert, etc.) . . . - .

32 Turbine-Mercury Interrupter for Alternating Currents .

33 Spark-Effects (Electrolytic Interrupter)

34 Max Kohl's Electrolytic Interrupter . . . . . . . .

35, 36 Hirschmann's Electric Interrupter . . . . . . 77,78

37 Platinum Interrupter. . . 79

38 Max Levy's Platinum Interrupter.

39 Tesla's Apparatus F - . -

$4 \mathrm{I}$ D'Arsonval's Apparatus..

42 Modern Type of Resonator . . 96

43,44 Rochefort's Bi-polar Resonator . . 97, 98

45 Passage of Electricity in Experiment 4 . . . . . . . 12.4

46 Experiment 22 . . . . . 135

$47,48,49$ Culture Experiment 22 . . I36

50 Culture Experiment 27 . . 138

5I Rabbit Experiment 38 . . . I46
52. 53 Culture Experiment 43 PAGE

52,53 Culture Experiment $43 \cdot \cdot$. 147

ent 43 . . 148

56 Water-Sphere Electrode . . . I49

57 Experimient 47 . . . . . $15 \mathrm{I}$

58 Cell-Infiltration . . . . . 153

59 Degeneration of Cells . . . . I54

60 Method of Local Application . 180

6I Crookes' Tube . . . . . . 20I

62 Vacuum Tube . . . . . . 203

63 Colardeau's Tube . . . . . 207

64 Ducretet's Tube . . . . . . 209

65 Mueller's (Hamburg) Tube . 2 I0

66 Dessauer's Tubé . . . . . 2II

67 Hirschman's Tube. . . . . 212

68 Adjustable Tube . . . . .214

69 Incandescent Lamps as used in charging Accumulators . 218

70 Roentgen Apparatus worked with Accumulators . . . . 219

7 Installation of Roentgen Apparatus worked from Continuous Light-Current . . . 22I

72 Apparatus with Electrolytic Break . . . . . . . . 222

73 Roentgen Apparatus by Siemens and Halske . . . . . 223

74 Auxiliaries for Roentgen Therapy . . . . . . . . 228

75 Arrangement of Tube . . 249

76 Lead Mask . . . . . . 260

77 Favus Capilitii . . . . . . 264

78,79 Folliculitis Barbæ . . . . 267

8o, 8I Lupus Vulgaris, before and after treatment... . 283

82,83 Histological changes in Lupus Tissue.... $\cdot . \cdot \cdot .287$

84,85 Plate Cultures of Staphylococcus . . . . . . 317,319

86 Hypertrichosis .333

87 Degenerated Swollen Connective-Tissue Cells . . . . 344

88 Solar Spectrum . . . . . .386

89 Curves of Thermal Action, etc. . . . . . . . 388

90, 91,92 Influence on the Skin 434, 435

93 Concentrating Apparatus for Sunlight . . . . . . $46 \mathrm{r}$

94. 95 Carbon-rods . . . . . 480

96 Iron-electrode Lamp (Bang) 485 97. 98 Iron Arc-Lamp (Reiniger) . 486

99 Iinsen's Light Apparatus . . 49 I

100 Finsen's Apparatus . . . 493

I01 Strebel's Iight Apparatus . . 495

102, 103 Trouvé's Apparatus . . 496

I04 Apparatus by Lortet and Genoud . . . . . 497

105 Giant-cell of Lupus . . . . 503

106 Lupus Nodule . . . . . 505

107 Strebel Instrument . . . . 515 

I. ELEMENTS OF ELECTRICITY. 



\section{R A D I O - TH E R A P Y}

\section{ELEMEN'TS OF ELECTRICITY. ${ }^{1}$}

\section{$\S$ I. Positive and Negative Electricity.}

As is well known, there are two electric states, which are complementary to each other. In this respect they may be compared to positive and negative quantities, or to heat and cold.

The electricity of a glass rubbed with a piece of felt or leather smeared with amalgam is not identical with that of resin rubbed with an animal skin, for one of these attracts and the other repels the clectric pendulum. Both electricities have been universally termed "frictional clectricily," the one being "glass-electricity" and the other "resin-electricity." The former is known as positive and the latter as negative electricity. Experiments have shown further that the same body may become alternately positive and negative, according to the nature of the body with which it is rubbed. For instance, if rubbed with amalgam, glass becomes positive; if rubbed with wool or fur, negative.

\section{$\S 2$. Conductors and Non-Conductors.}

There are substances which transmit electricity with extraordinary facility, and which conduct it to any distance, parting with it equally well; there are others again which conduct

$\left.{ }^{2}\right)$ Text-books: Mucller-Pouillet. Lehrh. d. Physik, Bd. 3.-H. Kayser. Lehrbuch d. Plysik. Stulgarl rooo. - F. Köner, Lelırh. d. Ply'sik. Wien und Leipzig 1897. - W'. Pscheidl, Grundriss der Naturlehre. Wien und Leip-

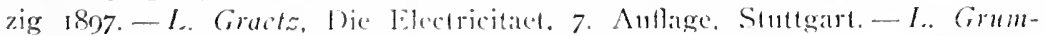
nach, die Physik. Erscheinungen und Krifte. Leipoig r8og.-J. G. Wrallon-

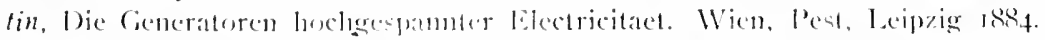

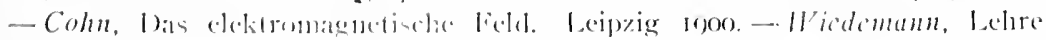

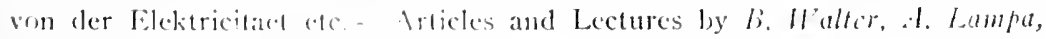
E. Lecher, ctc. quoled in the text. 
it with difficulty, and only part with it at places which happen to be touched. The first are called conductors, the second, nonconductors.

This may be otherwise expressed: All bodies offer some resistance to the transmission of electricity. In non-conductors this resistance is very marked, while in conductors it is insignificant. If a conductor is meant to retain its electricity, it is necessary that none but non-conductors be allowed to touch it.

Hence it follows that non-conductors are also insulators. A conductor can only remain electrified so long as it is insulated, that is to say, surrounded only by non-conductors. Air is also an insulator: were it otherwise, the electricity contained in charged bodies would be rapidly dissipated.

There are no absolute non-conductors of electricity, hence we should only speak of good and bad conductors. Those bodies which stand midway between these conditions are known as "half-conductors." Pfaundler gives the following table of the known conductors, half-conductors, and non-conductors, in which the various substances are arranged in their order of conductivity.

\begin{tabular}{|c|c|c|}
\hline Conductors & Half-Conductors & Non-Conductors \\
\hline $\begin{array}{l}\text { Metals } \\
\text { Coal } \\
\text { Graphite } \\
\text { Acids } \\
\text { Solutions of salt } \\
\text { Sea water } \\
\text { Riverand springwater } \\
\text { Rain water } \\
\text { Snow (wet) } \\
\text { Living vegetable mat- } \\
\quad \text { ter } \\
\text { Living animal matter } \\
\text { Soluble salts } \\
\text { Linen } \\
\text { Cotton }\end{array}$ & $\begin{array}{l}\text { Alcohol } \\
\text { Ether } \\
\text { Powdered glass } \\
\text { Flowers of sulphur } \\
\text { Dry wood } \\
\text { Marble } \\
\text { Straw } \\
\text { Ice at } \circ^{\circ}\end{array}$ & $\begin{array}{l}\text { Dry oxides } \\
\text { Fat } \\
\text { Ashes } \\
\text { Ice at } 25^{\circ} \\
\text { Phosphorus } \\
\text { Chalk } \\
\text { India-rubber } \\
\text { Etheric oils } \\
\text { Porcelain } \\
\text { Well-dried vegetable } \\
\quad \text { matter } \\
\text { Leather, parchment } \\
\text { Feathers, hairs, wool, } \\
\quad \text { silk } \\
\text { Jewels, mica } \\
\text { Glass } \\
\text { Wax, paraffin } \\
\text { Sulphur } \\
\text { Resin }\end{array}$ \\
\hline
\end{tabular}


The conductivity of a body may be modified according as the latter is soaked or corered with moisture, as well as by alterations in its temperature, or the constitution of its surface. Bodies having a rough surface conduct better than smooth. Heat operates in the first instance indirectly by its drying effect, through which bodies lose their conductivity either wholly or in part. On the other hand, other bodies become conductive by means of heat, because they soften or melt. For instance, glass, resin, and wax become more conductive when heated so as to soften. Ice also, which is a non-conductor at $-25^{\circ}$, becomes a half-conductor when heated to $0^{\circ}$.

Gases and vapours are insulators so long as they remain dry and free from dust.

\section{§3. Transmission of Electricity.}

Experiment proves that electricity which is produced by friction, or by induction (see $\$ 5$ ) has a tendency to lie only on the surface of a charged body. This phenomenon is explained by the fact that similar kinds of electricity repel one another ( \$4); consequently all the electrical particles added to the body take up a position as remote as possible from those which it already contains of a like kind. The surface-electricity is called a charge.

By bringing an electrified body into contact with a neutral body, electricity of the same kind may be transmitted to the latter.

If an insulated conductor is allowed to touch an electrified body, the electricity passing into the former does not remain at the point of contact only, but is distributed over the whole surface. By repeated contact with an electrified body an insulated conductor can be more and more charged with electricity. If the conductor be not insulated, but, for instance, held in the hand, any electricity communicated to it at once extends to the whole body and thence to the earth generally, thus becoming dissipated; the discharge has become "earlhed." 
Sunlight has the property of dissipating negative electricity, also burning magnesium and electric arclight. Polished wires, or plates of aluminium, magnesium, or zinc cannot be made to long retain negative electricity once they are exposed to sunlight.

\section{§ 4. Manifestation of Eiectrical Energy.}

With the aid of the electric pendulum, or the gold-leaf electroscope, one may demonstrate the fact that similar kinds of electricity repel, and dissimilar kinds attract one another.

The elcctrical forces which produce these results are forces capable of operating at a distance. These obey the universal law: $P=\frac{q q^{\prime}}{d^{2}}$; that is to say, they are directly proportional to the product of the electrical quantities acting upon one another, and inversely proportional to the square of their distance. (Coulomb's law.) The quantity of surface-lying electricity in a body is known as the electrical density. If a body has a surface $S$ and a quantity $Q$ of electricity, its electric density $D=\frac{Q}{S}$. This electricity, owing to the mutually repelling property of similar electrical particles, has a tendency to spread orer a body or to leave it; that is to say, it has a certain tension (potential), the amount of which depends upon the quantity or density of the accumulated electricity, or the size of the electrified body. Electricity in a given conductor can only, therefore, be considered in a state of equilibrium when the repellent effect of particles and the potential is everywhere the same. In any conductor the tension is everywhere the same; not so, however, the density. On asymmetrical bodies the density of electricity is unequally distributed, being greater where the curves of the former are small. The smaller the surface on which a given quantity of electricity is collected, the greater the electrical density in this region. The loss of electricity (point-discharge), however, increases in ratio with the thickness of the electrical density.

Electricity is constantly passing into the dust and water particles contained in the air surrounding the site of this point-discharge. 
Since like electricities repel each other, these electrically charged dust and water particles become also repellent, and so give rise to the "electric wind." Just as a charged body can unload itself at the dischargepoint, so also, if connected with the earth and placed opposite another charged body, it can exhaust the latter.

This property depends upon "influcnce." (See below.)

The proportion which the amount of clectricity contained in a conductor bears to its tension depends only upon the shape and size of the conductor. This proportion is called the capacity of the conductor.

$$
\text { Capacity }=\frac{\text { Ouantity of electricity }}{\text { Tension }} \text {. }
$$

From this formula results:

$$
\text { Tension }=\frac{\text { Quantity of electricity. }}{\text { Cirpacity }}
$$

By the electrostatic unit one signifies that quantity of electricity which exercises a force of 1 dyne at al distance of $1 \mathrm{~cm}$. upon an equal quantity of electricity.

The unit of energy (dyne) is that force which can move a mass of 1 gramme $1 \mathrm{~cm}$. in onc second.

In practice, however, one uses another unit, which is considerably larger and which rests upon other foundations. This is called the "coulomb," and is cqqual to 3,000,000,000 electrostatic units.

By the electro-static unit of tension is denoted that which produces an absolute electro-static unit of electrical quantity in a sphere of $\mathrm{I} \mathrm{cm}$. radius.

The cause of tension is called electro-motive force. Electricity contained in a body can, by reason of its tension, do work or overcome resistance when leaving that body. The amount of this capacity for work, "electric etergy," depends upon the amount of accumulated electricity, and upon its tension and quantity of electricity, and is expressed in kilogramme-metres (divided by 9.8 I, ser belowi.)

'The neighbourhood of an electrified hody is known as the "electric field;" within this zone the body is especially active in 
an electrical sense. The force brought to bear on an electrical quantity $\mathbf{I}$ is known as the electrical field-force $(H)$ in that locality. On a point containing the electric-quantity $e$ a force $H e$ will be exercised. The unit has a field in which an energy of I dyne is brought to bear on an electric quantity $(E Q)$ I. In the same way a space in which magnetic forces are acting is called the "magnetic field," and the amount of the magnetic force, acting at a given point of this field on a unit of magnetism, is called the field-energy.

In order to symbolise the influence which an electrified and magnetised body has on the surrounding space, the scientist assumes the existence of lines of force proceeding from the poles into space. These in a manner represent the energy of the poles. For instance, the expression is often used "An iron core emits lines of force," which conveys the idea that the iron is magnetised, and possesses magnetic poles. The lines of force are distributed in more or less regular curves, according to the shape of the electrified bodies brought near each other. These lines of force diverge more and more as the distance from the emitting body increases. The field-force of a given locality is indicated by the quantity of these lines of force contained therein; that is to say, their number in a sheaf whose sectional area is one square centimetre.

Two conductors in the same field have, as a rule, different tensions. If we join the two conductors by a wire, they form practically one conductor; the difference in their respective tensions becomes equalised, the electric particles move along the wire from one to the other (from the body of higher potential to the lower) until a uniform tension obtains. An "electric current" is established.

In a similar manner water, which is standing at different levels in two different vessels connected by a tube, in consequence of the law of gravity endeavours to attain one uniform level. This endeavour is called tension; it represents a force the amount of which varies with the respective altitudes of the two levels. "Tension" is synonymous with the following expressions used frequently in electricity: Potential, electric pressure, fall 
of potential, tension and pressure-difference, etc. A term which comprises all the foregoing is "electro-motive force." Employing our water simile again, this E. M. F. varies with the level difference. On the other hand, the effect of the water does not alone depend upon this difference of level, but also upon its quantity. In one case an infinitesimally small quantity of water may be acting at high pressure, in the other a very large quantity may flow from a slight altitude only. Static electricity may be compared to the former instance, where a strong, though brief, effect is produced, whereas the second instance finds a parallel in voltaic, dynamic, or galvanic electricity, where a considerable and constant energy is manifested.

Putting the matter otherwise: If an electric particle moves in an electric field from one conductor to another, the tension in these conductors is not identical; they have a "potential-difference."

The work done by the motion of an electrical quantity from one conductor to another is equal to the product of the electrical quantity and the tension-difference of the two conductors (analogous to the amount of work accomplished by a body falling from one level to another under the influence of gravity.) If this electrical quantity equals I coulomb, the work done by the forces in this motion is exactly equal to the tension-difference of the two conductors. It follow's that the tension-difference between two points $A$ and $B$ of an electric field is equal to the work required to bring a coulomb from $A$ to $B$. The unit of tension-difference at two points is found by measuring the work in kilogrammeters required to move 1 coulomb from one point to the other.

In practice, however, one never employs this unit, but another in which $\frac{\mathrm{I}}{9.8 \mathrm{I}}$ kilogramme of energy only is required to move a coulomb fron one place to another. This unit of tension-difference is called r volt.

$$
\text { I volt } \times 1 \text { couloml }=\frac{1}{9,81} \text { kgrm }
$$


The tension-difference of a place $A, B$, or $C$, as compared with that of the earth, is indicated brietly as the tension) or potential) of that place.

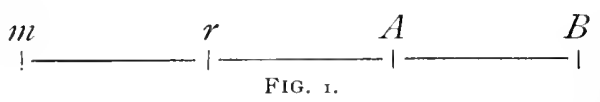

Let $m$ be the acting mass representing a point containing the electrical quantity $+m$, this point giving off energy to the surrounding space.

According to Coulomb's law, a repellent force $\frac{m}{d^{2}}$ is exercised in the direction $d$ upon a point $d$ of this space containing a positive unit of electrical quantity. The formula $\frac{m}{d}$ signifies the potential of the point $A$ subjected to the energy of $m$, or more briefly, the potential of $A$.

If this point moves in the direction of electric force toward $B$, this force accomplishes work (force $\times$ distance).

The point $A$ charged with electricity represents, therefore, a certain potential energy in the electric force-field, which may be likened to that employed in raising a weight; this energy will be exhausted in the process of removal to an infinite distance from $m$. The electric potential of a body signifies the capacity for work of its electrical tension. The potential $A$ is, therefore, the work required to bring the electrical unit under the action of the mass $m$ from $A$ to infinity; or, again, the work which must operate against the electrical field-force in order to bring the unit of positive electricity from infinity to $A$. The potential of the earth is called $O$; one can, therefore, use the phrase "from the earth" instead of "from infinity." We can only observe and measure differences of potential, or determine the potential down to a "constant" (the value of which may be disregarded), consequently, the potential of the earth may be valued as $O$.

The earth's condition is taken as a standard for all measurements, and differences of potential are compared with it. We therefore obtain the same potential-differences for different bodies, no matter what potential we assign to the earth. 
Tension, like tension-difierence, has I rolt as its unit. If, therefore, one speaks of a tension of 6 rolts as obtaining in a conductor, one means: The electric forces acting in the field must perform a work $6 \times \frac{\mathrm{r}}{9.8 \mathrm{r}} \mathrm{kgrm}$. in order to bring 1 coulomb from the conductor to the earth.

By "surface-tension" of a charge is understood the force with which electricity is discharged from a surface. Since each "quantity" here acts both as repelled and repeller, it is clear that this force must be proportional to the square of the electrical density.

With regard to this "surface tension," it must be noted that its identity with "tension" merely is not implied. It is true the word tension is frequently employed to denote "potential." It would, however, be more correct to strictly confine the expression to that force which drives off electricity from the surface of a charged conductor. In the case of a sphere the relation between "tension" of electricity on the surface and "potential" is easily indicated.

Let $\sigma$ stand for the density of electricity on the sphere; then the tension $T=2 \pi \sigma^{2}$. On the other hand, the potential being $V, E$ the whole charge of the sphere and $R$ its radius, then

$$
I^{r}=\frac{E}{R^{\prime}}
$$

Since the charge is equally distributed over the sphere, the surface-unit receives the charge $\sigma=\frac{l}{S}, S$ being the surface of the sphere; further $\sigma=\frac{E}{4 \pi K^{2}}$

Moreover, $T=2 \pi \sigma^{2}=2 \pi \frac{F^{2}}{16 \pi^{2}} R^{2}$; on the other hand, $E=$ $R V$, hence

$$
T=\frac{2 \pi}{16 \pi^{2}} ; \quad \frac{R^{2} l^{2}}{R^{2}} \text {, or } T=\frac{1}{8 \pi K^{2}} ; \quad V^{2}=\frac{1}{2 . S^{2}} I^{r^{2}}
$$

The points of an electrical field whose potentials have a constant value constitute a plane surface. No work is needful for moving an electrical guantity along a plane surface. At every point of the latter the force emanating therefrom acts at right angles to that surface. 
Electrical equilibrium can only obtain in a conductor if all points have the same potential. The unit of capacity is possessed by that conductor which receives exactly a tension of $\mathrm{I}$ volt from an electrical quantity of I coulomb. The unit of capacity is called I farad. In practice one often uses the millionth part of a farad as the unit of capacity. This is termed a microfarad.

\section{§5. Electrical Distribution: Influence, Electrostatic In- ductor.}

If an electrified body be brought near a non-electric insulated body, the latter becomes also electrified. A separation of its electricities has taken place, assuming that in every nonelectric body positive and negative electricities are already present in equal quantities at any given point. The body acted upon has become electrified by "influence," and the side turned toward the influencing body becomes the site of an opposite kind of electricity (by reason of the mutual attraction of dissimilar electric particles). This is known as "bound" electricity, being dissimilar to that of the influencing body; the similar, or "free," electricity escapes to the side farthest from the influencing body.

Experiment shows: I. That electrical quantities separated by influence are inversely proportional to the square of the distance. 2. That they are directly proportional to the influencing electrical quantity.

\section{§6. Condensers.}

It is only possible to accumulate electricity in a conductor up to a certain stage, viz.: where its potential equals that of the source of electricity. If, however, the charged conductor has sharp edges or points, its potential cannot be raised quite to this standard, since through these a certain amount of electricity is lost into the air. (See p. 5.) A "condenser" is an apparatus assisting in the storage of electricity to a higher degree. It consists of two metal plates separated by a thin insulating layer of varnish or air; one is called the collecting-plate, 
the other is the condensing-plate. If the collecting-plate be charged to its utmost capacity, it is found that on touching the condensing-plate with the finger still more electricity can be received by the collector. The explanation of this is as follows: When an "earthed" conductor is brought in the neighbourhood of a charged conductor, the tension of the latter falls considerably, in consequence of which it is enabled to retain a still further supply of electricity. The capacity of the collecting-plate (the "earthed" condenser being adjacent) is denoted usually as the capacity of the condenser. The capacity of a condenser $C$ is the proportion between an electrical quantity $E$ lying on the collecting-plate and the tension of the latter $V$, the condenser being "earthed." We now have the formula $C=\frac{E}{V}$; from his $V=\frac{E}{C}$ and $E=V C$. The capacity of a condenser depends upon the shape and size of the plates, upon their nearness to one another (the shorter the distance between the plates, the greater the capacity), and, again, upon the molecular and material constitution of the insulating medium (the "dielectric"). The condensing capacity is greater in the case of solid or liquid dielectrics than if air only is employed. The "diclectric-constant" signifies the proportion which the capacity of a condenser fitted with a solid or liquid dielectric bears to that of one which only has air as its insulating medium. Paraffin has a dielectric constant of 2.3 , india-rubber 2.9 , oil 2 to 5 , ebonite 2.6 , vulcanite 3.15 , sulphur 3.84 , glass 6 to Io, mica 8 .

If an electrified body be brought near a good conductor, the electricity of the former and the "bound" electricity of the latter accumulate on adjacent surfaces by reason of their mutual attraction. If the electrical density is in each case sufficient and the bodies are not too far apart, the two electricities combine, the effect being to produce both light and sound. In this way an "electric spark" results. Here the light is caused by glowing particles passing from one body to the other, the sound by air-condensation in the vicinity of the spark. As before mentioned, we are able to accumulate large quantitics of electricity by means of "influence" or "binding." A convenient ap- 
paratus for this purpose is to be found in Franklin's "plate." This consists of a level glass plate fitted with tin-foil on both sides, the layers of metal not being allowed to come close to the edge.

If we connect one of the tin-foils with a positively charged body, positive electricity $(+e)$ will flow from the latter until the potential becomes identical with that of the tin-foil. In the second tin-foil electricity becomes separated by "influence;" negative electricity becomes attracted, positive repelled. If this second layer of foil be "earthed," then its negative electricity becomes "bound," its positive electricity flowing away to the earth. These quantities of electricity are relatively proportional to $\mathcal{e}$, and nearly inversely proportional to the distance separating the respective layers of tin. They may, therefore, be expressed by $K e, K$ representing a fraction of which the denominator is proportional to the thickness of the glass plate. Accordingly, if the second tin-foil be on the left, the "binding" takes place through $+e$ on the right, giving $-K e$ on the left. The latter quantity, however, again binds on the right a quantity $+K e$. One can, therefore, add $K^{2} e$ to $+e$ on the right without any loss of electricity. This new quantity $+K^{2} e$ again binds on the left $-K^{3} e,+K^{3} e$ going to earth. This new quantity again binds $+K^{*} e$ on the right, and so on. One sees, theiefore, that electricity on the right-hand plate may be augmented by

$$
+c+K_{2} c+K_{4} c+K_{6} c=\frac{\mathrm{I}}{\mathrm{I}-K_{2}} c
$$

While, therefore, originally the tin-foil on the right could only receive $+c$, it is now in a position to take up $\frac{\mathrm{I}}{\mathrm{I}-K_{2}^{-}} \epsilon \cdot \frac{\mathrm{I}}{\mathrm{I}-K_{2}}=m$ is called the augmenting index. It is greater when the distance between the tin-plates is decreased.

The plate on the right, which is connected with the supply, always holds more electricity than that on the 
left, since in addition to its "bound" electricity it contains that which flows to it after the binding. If, therefore, the two plates be joined by a wire, an escape of electricity occurs from right to left, accompanied by sparking at the moment of juncture.

A convenient form of accumulating apparatus is found in the Leyden jar. This consists of a glass jar with the tin-foil pasted inside and out to 2-3 of its height. The lid is formed by an insulating cover, which is pierced by a metal rod. One end of this rod is in contact with the inner layer of tin-foil. The other end terminates in a knob. This rod transmits electricity to the inner layer. The outer layer is comnected with the earth.

The capacity of a Leyden jar depends upon its size; or, more correctly, upon the area of its tin-foils.

A considerable number of Leyden jars may be joined together, forming a battery. They may be joined by connecting all the inner and all the outer layers respectively. This gives one a connection in parallel, or in quantity; the number of jars then act like one jar of $n$-fold size. Or again, the outer foil of one jar may be joined to the inner foil of another, and so on. This gives one connection in series, or tension-connection, one then obtains between the terminal layers the $\mathrm{n}$-fold potential difference of a jar, but in the discharge the electrical quantity of a single jar only. This particular combination is called also a cascade-battery.

If a Leyden jar be discharged by a brief connection only of the layers, after a little time a second connection will produce another, though feebler, spark. In the same way, after repeated pauses, a third, fourth, or more sparks may be obtained, each progressively feebler. This is called the jar-residue.

In explanation of this phenomenon, it may be assumed that under the influence of surface-tension electricity permeates the glass from both sides; the surface-tension once remored by the discharge electricity slowly returns again to the surface.

We have seen that, dealing with a sufficient density and potential difference, an equalising of dissimilar electricities accumulated in two neighbouring conductors separated by a dielectricum can be effected in the form of a spark. The amount of 
derisity required increases with the thickness of the insulating medium. In the air, the length of the spark discharge is about proportional to the density of the electricity accumulated. If, however, the air be rarefied, the spark-length capacity increases, and in tubes filled with gases whose pressure only amounts to a few millimetres, the spark length may advance to many metres. These spark discharges produce many effects. These may be mechanical, instanced by the perforation of solid insulators, transport of solid particles from one conductor to the other, etc.; chemical, causing decompositions or combinations; thermic, igniting powder, melting iron wire, etc.; and light-effects, as shown by the visible spark, the illumination of Geissler's tubes, etc. Finally, feeble magnetic effects of the discharge can be experimentally demonstrated. In these respects the discharge simulates dynamic electricity. In the case of powerful discharges, where simultaneously large bundles of sparks are emitted, the positive pole behaves somewhat differently to the negative. From the former, trains of sparks stream off from different portions of the pole, uniting before reaching the negative pole to strike it in one focus only. Another kind of discharge takes place when there is but.little resistance. If a powerfully charged Leyden jar have its two layers of foil connected by a good conductor, more positive electricity fows to the negative layer than is required to neutralise it. This excess flows back to the positive layer, but again too freely, so that a return again takes place. In this way the current is several times reversed before equilibrium is attained. This phenomenon may be compared with the swinging to and fro of a liquid column in connection with two vessels, and is called an oscillating discharge. The number and duration of these oscillations depend upon the spark length and the capacity of the battery.

Feddersen made searching experiments upon the duration of the electric spark. He made his observations upon a spark-reflection in a rotating mirror, and saw in the first place instead of a spark-line a band of light, showing that the spark possessed a definite duration. He noticed in this band alternate light and dark 
places, a proof that the discharge stops and recommences periodically. Feddersen also calculated the period of these oscillations to be about one-millionth of a second.

\section{§7. Electrical Machines.}

Electricity may be continually formed by friction and accumulated in an insulated conductor by means of Carré's apparatus (Fig. 2). This consists in the main of two discs revolving in opposite directions. The layer of these discs $(B)$

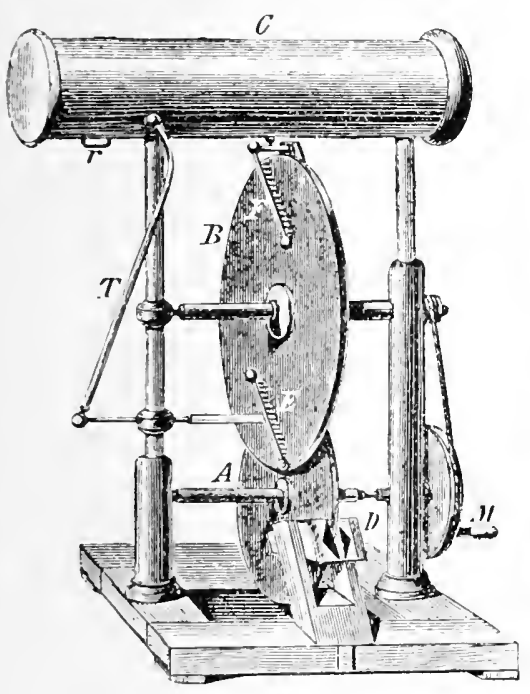

FIG. 2.

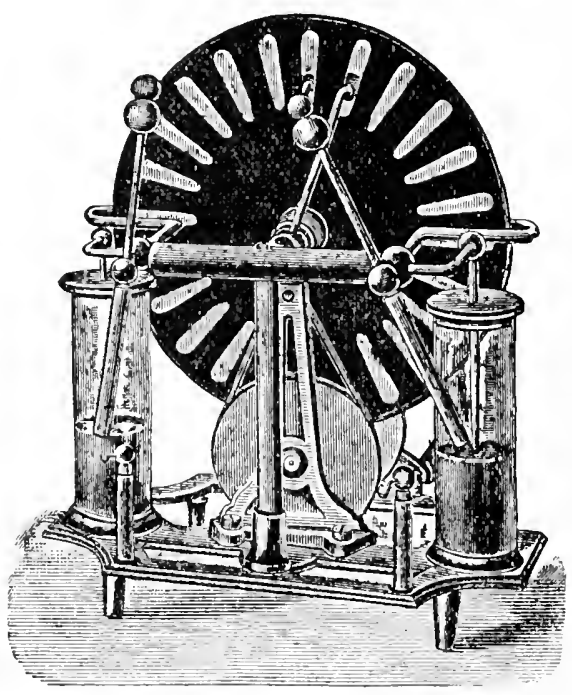

FIG. 3.

FIG. 2.-Carrés machine (from "Die Generatoren hochgespannter Electricität" von Dr. J. G. Wallentin, Wien, Pest, Leipzig, Hartleben's Verlag I884, ค. 89 ).

FIG. 3.-Wimshurst machine (from L. Gractz "Dic Electricität." Stuttgart, Engchorn, 1898, p. 34).

is made of ebonite, the smaller $(. I)$ of glass. During its rotation the latter rubs between the two friction-pads $D$ and acts as an inductor. While this disc (the glass) revolves comparatively slowly, the dise $B$ revolves very quickly. As seen from the figure, these discs partly cover each other. Opposite the 
ebonite disc $B$ stand the combs $E$ and $F$, the second of which is connected with a cylindrical metallic conductor $C$, the other with a rod $T$, terminating in a knob.

This machine acts as follows: The positive electricity of the rubbed glass disc influences, with the aid of disc $B$, the comb $E$ and draws from it negative electricity, which collects on the disc $B$; the knob of the conductor $C$ consequently becomes positive. The negative electricity of the dielectric disc draws from the comb $F$ its positive electricity, and is thereby neutralised. The conductor $C$ itself becomes negatively electric (IV allentin).

This apparatus gives good results. With but few rotations, provided the discs be large enough, one can obtain sparks up to $\mathrm{I} 5$ centimetres.

Another influence machine, which is often used for medical purposes, is the Irimsluurst (Fig. 3). This consists of two glass or vulcanite plates placed close to one another, each revolving round a common centre, but in opposite directions. Each plate bears on its outer face a number of metal sectors, and runs between a metal fork provided with spikes pointing to the plate. To each metal fork a brass rod is connected, ending in a knob, which in its turn communicates with a Leyden jar. Finally, the machine has an "equaliser" on each side. This consists of a metal arm, one end of which supports a wire brush whose bristles rub against the revolving metal sectors.

On actuating this machine electricity immediately develops, which sparks or "brushes" from knob to knob. The "equaliscrs" are not placed exactly opposite to each other, but at an angle of $60^{\circ}$ with themselves and the horizon.

The electrical output of this machine is not due directly to friction, but to the small, though constant, normal charges in the insulated sectors. These act by "influence," and as this is the case not with one sector alone, but with all of them, the terminal pole becomes more and more powerfully charged.

One cannot enter here into a more detailed explanation of the action of this apparatus. 


\section{\$8. Contact Electricity, Galvanism, Galvanic Batteries.}

If two different metals be brought into contact, one becomes positively and the other negatively electrical. This electricity in nowise differs, except in its mode of origin, from that produced by friction.

It is assumed, in explanation of the above phenomenon, that on the contact of different conductors an electro-motive force appears which develops positive electricity of a certain potential on one, and negative electricity of an equally definite potential on the other. Each conductor, therefore, possesses its own peculiar tension, a different value for each. The potential-difference of the two is constant, being dependent only upon the nature of the metals and quite independent of their size, the size of their plane of contact, or of the absolute potential value of each metal. If electricity be transmitted to both metals or to one of them, or if one of them be "earthed," the potential-difference remains the same.

Many theories have been advanced as to the origin of this separating or electro-motive force. Irolta thought that the mere contact of the metals produced their potential-difference. Others have looked for an explanation in chemical changes as a cause of voltaic electricity (F. Exner).

Volta's experiments with various metals led him to determine a tension-series. This he arranged in tabular form, the first of the couple in contact taken in the order read being positive and the second negative. This tension-series, also called conductors of the first order, reads as follows: Zinc, lead, tin, iron, copper, silver, gold, coal, platinum, manganese. If the figure 5 be taken as representing the potential-difference of zinc and lead (expressed symbolically by $Z n / P b=5$ ), according to Volta's results, we get the following values for different combinations:

$$
\begin{aligned}
& z u / / \mathrm{H}=5 \\
& C u / A g=1 \\
& I h / S n=1 \\
& Z n / \lg =12 \\
& \operatorname{Sn} / \mathrm{LiC}=3 \\
& \text { IClC } \mathrm{C} u=2 \\
& S n / C u=5 \\
& Z n \mid F_{c}=0
\end{aligned}
$$


The law relating to tensions is as follows: If a chain be formed of several metals in contact with one another, the difference in potential between the final links is equal to the sum of the potential-differences of all the links, or is equal to the potential-difference which would result from direct contact of the final links. From this it follows that the greater the distance between the individual members of the chain, the greater the electro-motive force. The electro-motive force cannot be increased by a repetition of the same combination. It can be shown by calculation that in a closed circuit of conductors of the first group the electric dissociating power equals 0 , that is to say, the tension is everywhere alike; the electricity is in equilibrium. Again, a potential-difference results when metals and liquids are brought into contact. If the potential-difference between zinc and copper be.considered as Ioo, according to Kohlrausch, the following figures are obtained in different combinations:

$$
\begin{array}{l|l}
\text { Zinc } & \text { water }=-6 \text { I.6 } \\
\text { Copper } & \text { water }=-33.0 \\
\text { Silver } & \text { water }=-17.0 \\
\text { Gold } & \text { water }=-33.7 \\
\text { Platinum } & \text { water }=-44.7
\end{array}
$$

Liquids do not obey the law of tensions. In contact with metals they become partly positive and partly negative. They are called conductors of the sccond class.

Thus, for example, every metal in contact with water becomes negative, and that in a different degree for different metals, while the water becomes positive. Water, therefore, cannot be included in the tension series.

Let us immerse a zinc and copper plate in a vessel of water. Both metals become in the first place negative, the water positive. Expressed in numbers, the tension of the zinc is the half of -61.6 , that is, -30.8 . This positive tension of the water $(+30.8)$ goes over to the copperplate. Now, however, the copper receives through the electric separating power acting on the water, the half of -33.0 , that is, - I6.5. But the positive 
tension from the water prevails over the negative of the copper (-I6.5) with the result that the copper plate receives a positive tension, $+30.8-\mathrm{I} 6.5=+\mathrm{I} 4.3$. On the other hand, the positive tension of the water, + I 6.5 (which it received by contact with the copper), is added to the negative tension $(-30.8)$ of the zinc plate. The tension of the zinc plate is, therefore, $-30 . S+\mathrm{I} 6.5=-\mathrm{I} 4.3$. The same rule applies to all other metals.

It follows that when two metals are immersed in a liquid, one of them becomes negative and the other positive. The metal which by its own separating-power becomes more powerfully negative remains always negative.

Such a combination is termed a galianic element. The ends of the two metal plates are called the poles. The copper plate contains the positive, and the zinc the negative pole. A galvanic element, the poles of which are not connected, is called an "open" one.

The law that the two metals have a definite potential-difference, which depends only upon the nature of the two metals and the liquid, and not upon their size and shape or the quantity of the liquid, applies also to the galvanic clement. This definite tension-difference is called the electro-motive force of the element.

If the poles of an element charged with a potential-difference are connected by means of a conductor, a current must result; electricity always flows from a place with higher potential to one with lower. Since, however, the source of the charge (the contacts) remains within the element, demanding the same potential-difference in the poles, fresh electricity flows at once to the latter and equalises itself through the connecting wire. One sees, therefore, that this current-flow and equalising process must be constantly going on ; the electricity never arrives at a state of equilibrium, but a continuous current results. This is called a galeanic current. The source of the current is to be found in the chemical encregy which is spent when employing conductors of the second class; these litter, as is well known, are required for a current-producing element. 
The positive electricity flows uninterruptedly from the higher tension along the wire connecting the two poles, thence through the metal and liquid. In the case of the above-mentioned element positive electricity is found at the copper pole. This positive electricity flows, therefore, from the copper pole along the connecting wire to the zinc pole, thence from the zinc plate into the water, and so to the copper plate again. We have thus a closed circuit. Or the negative electricity may be described as flowing through the wire to the copper, thence to the water and zinc. Thus the current may be followed for purposes of description in one of two ways, but the general method is to consider it as flowing from the higher potential to the lower, that is, in the direction of the positive current.

We speak of a stream of water flowing from a higher to a lower level, and in the same way an electric current is understood as passing along a conductor from a point of higher to one of lower tension. As has already been mentioned, the tension-difference between these two points constitutes the electro-motive force.

The amount of water which passes in one second a given point in the course of a stream is governed by the force of the current, and may be expressed in litres or kilogrammes. In the same way the quantity of electricity, that is, the number of coulombs passing a given sectional area of the wire in one second, is called the intensity or strength of the electric current, and is measured in units of amperes. The motive force of a stream of water, or of water falling from a height, corresponds to the tension-difference between two points in an electric current, or its electro-motive force. As regards workcapacity a comparison may also be drawn between a current of electricity and one of water.

We have seen that a current of water results from its movement along an inclined plane by reason of gravity; if there were no hindrance to its progress, the velocity of the water would become greater and greater in its course, being as great at any given point, as though it had fallen directly there from its original level. This, however, is not the case with water-streams, the current 
being found to move comparatively slowly in certain places.

This results from obstruction to its progress by friction with the bed of the strean. The work accomplished by gravity in the movement of the water over this obstruction is, of course, not lost: work, indeed, can never be lost. The energy expended receires its equivalent in a certain amount of heat, which, though hardly measurable, raises the temperature of the water and its bed.

By the aid of water-wheels, turbines, and the like, one can transform the energy of the falling water into mechanical power. The maximum of work which one can extract from a waterfall per second, is estimated by multiplying the height of the fall by the weight of water falling in that second. Just as the effect of flowing water is measured in meter-kilogrammes, the product of volt and ampère (the watt) indicates the power of an electric current between two points in its course. If between these two points there is an uninterrupted conductor, the heating of the latter constitutes the only work-effect of the electric current, and the amount of heat thus devcloped must be equivalent to the product of volt and ampère.

One can with the aid of liquids, since they do not obey the law of the tension-series, at will increase potential-difference of electro-motive force by repetitions of the same combination.

If we take a galvanic element in which the tensions of the two metals differ to the extent of I volt, and carth the zinc plate, the tension of the latter will become o; that of the copper will be I volt. Any conductor joined with this copper plate also participates in this tension; this will apply, moreover, to the zinc plate of a second element if it likewise be attached. The copper plate, however, of this second element must already possess a tension, of I rolt higher than its zinc fellow, consequently it now possesses a tension of 2 volts. Similarly, the copper plate of a third element connected in a 
like manner will possess a tension of 3 volts, and so on.

If one, therefore, in this way connects a certain number $(n)$ of galvanic elements, the tension-difference (the electro-motive force) will be greater than in any single element.

A series of galvanic elements arranged in the above manner by coupling them together, is called a chain, or battery, and this particular mode of connection is termed serial. The terminals are called battery-poles.

In addition to the galvanic elements described above, which contain only one kind of liquid, there are others in which the metals are immersed in different liquids. In this case the liquids are separated by a porous clay diaphragm. Of the two plates (of the first class of conductors), one is invariably made of zinc. In strong batteries this is always amalgamated in order to prevent corrosion while the element is at rest.

The commonest forms of galvanic elements are given as follows, together with indications of their electromotive force:

I. Daniell's cells: Amalgamated zinc in $25 \%$ sulphuric acid, copper in concentrated copper sulphate solution. Electro-motive force in volts: I.o68.

2. Meidinger's element: Zinc in solution of magnesium sulphate, copper in concentrated copper sulphate. Electro-motive force: 0.952 volt.

3. Bunsen's element: Amalgamated zinc in $8 \%$ sulphuric acid. Carbon in fuming nitric acid. Electromotive force: 1.88 volts.

4. Chromic acid element (Bunsen): Amalgamated zinc and carbon in a solution of $\mathrm{I} 2$ parts bichromate of potash, 25 parts concentrated sulphuric acid, Ioo parts water. Electro-motive force: 2.03 volts.

5. Grove: Amalgamated zinc in $25 \%$ sulphuric acid, platinum in fuming nitric acid. Electro-motive force: 1.93 volts.

6. Leclanché: Amalgamated zinc in solution of 
sal amoniac, carbon with a mixture of manganese. Electro-motive force: 1.48 volts.

The galvanic current, in addition to mechanical, magnetic, electro-dynamic, inductive-light and heat effects, causes chemical action. It if passes through a liquid, chemical decomposition always results, the process obeying well-defined laws (electrolysis).

'The immersed plate connected with the negative pole of the battery, is called the callode; the other, connected with the positive pole, is the anode. The substance underlying decomposition is known as the electrolyte; its particles separated about the plate are the ions.

If the galvanic current be conveyed through dilute sulphuric acid, hydrogen is evolved at the cathode and oxygen at the anode.

Electrolysis obeys the following laws (Faraday):

I. The amount of decomposition-products formed in a given unit of time is proportional to the intensity of the current; that is to say, to the electrical quantity passing through in that period.

2. Each unit of current at the electrodes evolves chemically equivalent quantities (that is, such quantities as are capable of chemical re-combination).

If a certain electric quantity be required to traverse an electrolyte, according to Faraday's law this can only be accomplished by the removal of a definite quantity of hydrogen from anode to cathode, thence escaping. This relation between the transmission of electricity and the ions leads one to assume that the latter are direct carriers of electricity. We see that under all conditions, whatever be the chemical combination with which we are dealing, positive electricity always accompanies the hydrogen; we may suppose, therefore, that $I I$ in the combination $\mathrm{H}_{2} \mathrm{SO}_{4}$ has a greater affinity for positive electricity than $\mathrm{SO}_{4}$, which fulfills the role of carrier of negative electricity. One may, therefore, conceive cach atom of a combination as being charged with a definite elec- 
trical quantity, base and acid, indeed, being charged with dissimilar electricity.

Every hydrogen ion bears a certain electric quantity. According to Faraday's second law of electrolysis, each current-unit produces chemically equivalent quantities at the electrodes. Therefore, the chemical value of each ion moving in the electrolyte appears joined with a definite and unchangeable quantity of positive or negative electricity. Therefore, every single-value ion conveys just as much electricity as an H-ion, while the two- and three-value ions convey double or triple electrical quantities. If one measures the current passing through, and the amount of hydrogen evolved, one can estimate exactly how much electricity falls to I grm. of hydrogen or to $I$ ion. This quantity is known as the electron (see Introduction); it amounts to about $6 \times \mathrm{IO}^{-\mathrm{xo}}=\left(\frac{6}{\mathrm{Io} \text { milliards }}\right)$ electrostatic units.

Water is decomposed by the current in the galvanic element; hydrogen and oxygen are formed, $H$ at the copper and $O$ at the zinc.

Experiments show that such plates covered with gas produce an electro-motive force and a current which proceeds in an opposite direction to the original current. This secondary current endures till the decomposition products are dissipated. Plates which are covered with decomposition-gases are described as polarised; the current which they produce is the polarisation-current.

In a galvanic element the water is decomposed, hydrogen going to the copper plate and forming a film there which breaks the current. Galvanic polarisation (electro-motive opposingforce) explains the early weakening of the original electro-motive force of the element, and its final reduction to a minimum. Herein lies the unreliability of galvanic elements. Primary elements, of whatever system, all have the disadvantage of being very dear to work compared with their capacity, either requiring very frequent and by no means easy renovation, or possessing very little current-power. 


\section{\$. Accumulators.}

Accumulators, or "secondary clements," obtain their effect by reason of the polarising action of oxygen and hydrogen arranged as ions. In Planté's accumulator two lead plates are immersed in dilute sulphuric acid. If a current be passed through, oxygen is "separated" at one plate (the positive), leading to the formation of peroxide of lead, which is seen as a reddish-brown deposit on the plate. Neanwhile, the other plate (the negative) becomes coated with sulphate of lead by the action of sulphuric acid. The accumulator is fully charged only when this latter process is complete. It has, through the influence of the electric current, received chemical energy, a chemical power of work. The saturation-point of charging is marked by the evolution of hydrogen and oxygen at cathode and anode, respectively, the electricity being no longer able to induce chemical action on the plates themselves. During the charging the sulphuric acid solution becomes more and more concentrated. If the accumulator-circuit be closed a reversed polarisation current results, reducing the peroxide to oxide on one plate, the sulphate on the other plate being likewise altered to oxide. When both plates have become chemically identical, the current ceases. By repeated charging and unloading the plates become more capable of the peroxide charge. Plantés elements had to be worked for a long time in this way before they could be made to retain any considerable charge. Faure tried to remedy this defect by pressing a layer of lead oxide onto the plates in the first instance. This layer, however, had a tendency to fall from the plate, a failing which was obviated to some extent later by grooving the plate. By constant use the chemically active portion of the plate tends to become porous and spongy, and is casily destroyed, especially when quickly charged. Moreover, the plate is then liable to get easily bent, and both plates may come into contact, thereby effecting a "short-circuit." Even without such a contingency the capacity of the accumulator is prone to become impaired through the falling of masses of the porous material to the floor of the battery, whereby the plates may become briciged across and a short circuit established. The chemical process does not penetrate 
very deeply into the lead plates; the accumulator's capacity, therefore, depends upon the size and number of these plates and their general formation. An accumulator may, however, be charged afresh any number of times, and will be capable of evolving each time a discharge current which is distinguished from that of the primary battery by its height and constant electro-motive force. The discharge current, however, never quite equals the primary current in actual quantity.

In the Tudor accumulator the positive lead plates are provided with deep horizontal grooves. The negative plates are cross-barred and contain lead-masses in the grooves, which, during the charge, become completely changed to spongy lead. The plates are cast into shapes with projections on either side fitting into the vessel holding them. A series of positive plates soldered together and a similar series of negative plates are now placed into a wooden or glass receptacle containing dilute sulphuric acid. Contact between adjacent positive and negative plates is prevented by the insertion of glass rods between them. The ressel is made deep, so that a considerable space is left between its bottom and the lower ends of the plates; here any falling substance has room to accumulate. The leaden ledges of the positive plates of one cell have grooves in which the negative plates of another cell fit, for just as one can couple up several galvanic elements, the same can be done with several accumulator-cells to form a battery. These compound-celled accumulators are arranged in wooden cases lined with lead. The separate cells are connected in series, so that at cither end a positive and negative pole is left free with which to control the battery.

Accumulators much used are those of Gottried Hagen, Guelcher, Boese, Pollak, and others.

While accumulators are of the greatest practical importance, it cannot be denied that as yet their construction is by no means perfect. Amongst the many drawbacks to their use may be mentioned their sensitiveness to shocks and over-exertion, the difficulty of transporting them, their rapid loss of power, frequent failures on account of internal derangement in the shape of bending of the plates, etc., cracking of glass cells, leakage of acid, 
their high initial cost and expensive up-keep. Many of these disadvantages are, it is claimed, obviated in the Behrend-Dessaner apparatus. This is formed of cells packed with glass powder and a hygroscopic fibre, the whole mass soaked with dilute sulphuric acid and pressing the active spongy portion of the plates in position. Thus any bending of plates, with conseciuent short circuit, is aroided, likewise falling down of the spongy lead.

Each accumulator-cell has an average tension of 2 volts. The cells should never be exhausted beyond a certain point, or the plates will be impaircd. Their limit for each size of accumulator is indicated by the maker. The electrical quantity given out during the period of discharge (expressed by multiplying the highest possible amount of discharge by the number of hours in which the current is erolved) is termed the accumulator-power or capacity in ampere-hours. Thus a battery which gives a maximum current of 6 ampères in to hours' discharge has a capacity of 60 ampèrehours. An accumulator of this capacity can also supply a I ampère current for 60 hours, a 3 ampère current for 20 hours, and so on. One cannot, however, obtain a current exceeding 6 amperes. If the ampèrehour capacity of the accumulator be divided by the product of the corresponding time of charging and the strength of the primary current, onc obtains its relative value in ampère-hours. This usually amounts to $90 \%$ or $95 \%$.

\section{$\S$ Io. Ohm's Law.}

The force or inlensity of an electric current was defined on page 24. Static electricity of the kind found in a Leyden jar is high in pressure but low in quantity, and we know that, though its discharge is violent and characterised by striking phenomena (physiological, light, etc.), these are but momentary. On the other hand, a galvanic current, though of lower tension and less electro-motive force, is comparatively rich as regards quantity. It can consequently be employed for lighting purposes, or fer mechanical or chenical purposes.

(Compare the work capacity 
of a large quantity of air or water under low pressure with a small quantity under high pressure.)

Other things being equal, the greater the electro-motive force, the greater the quantity of electricity flowing through a given sectional area of a conductor. The working value of a quantity of water depends, as we have seen, upon (I) its pressure, (2) the strength of its current. An electric current is similarly situated, but a third important factor must be added, the resistance of the conductor. A stream-current meets with obstruction from friction with its bed; moreover, caeteris paribus, a current flowing in a wide channel will be much more powerful than a narrower one. In order to obtain the same currentforce in the narrow that we have in the wider channel, it will be necessary to increase the rapidity of How or the water-pressure. With an increasing resistance the current-force decreases.

Ohm's law is as follows:

The intensity of an electric current $(I)$ is directly proportional to the electro-motive force $(E)$, but inversely proportional to the resistance $(R)$.

$$
I=\frac{E}{R}
$$

One may add that the force of the current is the same in all parts of the current-circuit when the current is continuous.

A conductor's resistance depends largely upon its composition, that is, upon its specific conducting resistance $(K)$. Again, the resistance varies directly with the conductor's length $(l)$ and its sectional area $(f)$. We may, therefore, add the following equation:

$$
R=K \frac{l}{f} .
$$

Heating increases the resisting power of metals, but decreases that of carbon.

In order to reduce a current of electricity to a required intensity it is often necessary to interpose various resistances in its path. These are known as "rheostats." Rheostats may be constructed on the decade-resisting principle, which measures re- 
sistances of I-IOO times the unit and more. When, however, the object is to quickly interpose resistances into a current-circuit, determination of this exact capacity being unnecessary, the so-called ballast-resistances are useful. The form of those usually employed for strong currents is known as a crank or leverrheostat (Figs. 4 and 5), where a series of German silver, "nickelin" or "manganin" spirals, which offer great resistance to the current, are fixed and insulated in a frame. By means

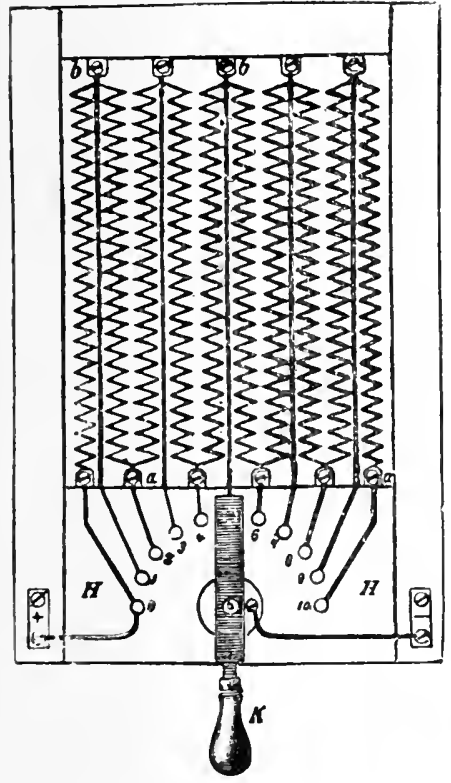

FIG. 4.

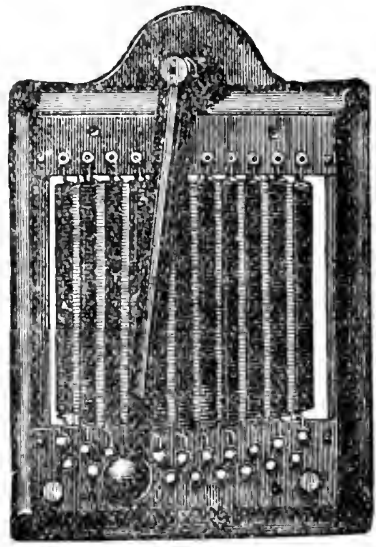

FIG. 5 .

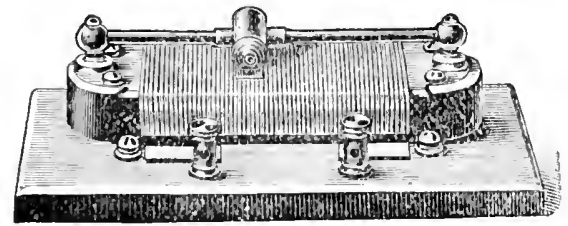

FIG. 6.

FIG. 4.-Lever-Rheostat (from Graetz L. Electricity, Stutgart, Engelhorm, p. 9 I).

Fig. 5-Lever-Rheostat of Reiniger (Gebhert and Schall, Erlangen).

of the lever a greater or less amount of these metallic spirals can be interposed in the path of the current. With the leverknob to the extreme left the current is interrupted; as it moves to the right a gradually increasing resistance is established.

Fig. 6 depicts another variety of resistance, in which a single spiral of uniform thickness, composed of German silver or nickelin, forms the resisting medium. By sliding a "contace" over 
this in either direction, the length of the resisting path can be varied.

\section{$\S$ Ir. Technical Measure-units.}

The following technical measurement-units have been agreed upon since I $8 S_{\mathrm{I}}$ as indicating current-strength, conductor-resistance and electro-motive force or potential difference:

I. The current-sirenglh unit is I ampere: This corresponds to a current-intensity capable of precipitating o.00 I I I 8 gramme of silver in one second from an aqueous solution of silver-nitrate.

Measured electro-magnetically, the unit of currentstrength is that which, traversing an arc of $\mathrm{I} \mathrm{cm}$. length and $\mathrm{I} \mathrm{cm}$. radius, acts with a force of I dyne on a magnet pole (one) situated in the middle of the circle completing the arc. The "practical" unit of the currentstrength, I ampere, is the tenth part of the above (C. G. S.) unit.

The quantity transmitted by the current, one on a time-unit through a given sectional area of conductor, is accepted theoretically as the unit of current-quantity or electrical quantity. The "practical" unit is, therefore, that electrical quantity which, with a current strength of I ampere, flows through a sectional area of conductor in one second. It is called ar ampère-second, or I coulomb. (See p. I 2) for the electro-static unit of electrical quantity.)

$$
\text { I coulomb }=\text { I ampère } X \text { I second. }
$$

From this we derive the working-unit of an "ampère-hour." The ampere-hour is that electrical quantity which traverses a conductor in one hour or 3,600 seconds with a current-intensity of I ampère. An ampère-hour, therefore, corresponds to 3,600 coulombs.

The capacity or holding-power of an accumulator is usually expressed in ampere-hours. By this one understands that electrical quantity which is evolved during the average discharge-period, and which is expressed by the product of the greatest force of current-discharge 
and the time in hours during which the current can be utilised.

2. 'The unit of resislance is possessed by that conductor in which the electro-motive force I produces the current $\mathrm{I}$. This unit is called I ohm.

The ohm is defined as the resistance of a column of mercury of I 4.452 I grammes, I square millimetre cross-section and $1.062 \mathrm{~m}$. length, measured at $\mathrm{O}^{\circ} \mathrm{C}$.

3. By the init of electro-motive force is meant that electro-motive force which produces a current of I ampère intensity in a conductor possessing I ohm resistance. This unit was termed "volt" at the electrical congress held in Paris. The unit of electro-motive force may be deduced from the phenomena of an induced current, or from the current's working capacity.

I volt is, we may mention, the electro-motive force of a zinc-copper element (Latimer Clark's normal ele$m e n t)$. It is about $10 \%$ less than the electro-motive force of Daniell's element.

The unit of current capacity (work or heat) is indicated when the work is accomplished by a current in I second, as, for example, when the heat equivalent to a unit of work is produced in a wire. (See p. 42.)

The unit is given by a current $\mathrm{I}$ and a resistance $\mathrm{I}$, or also by the electro-motive force I producing the current. The practical unit is, therefore, the volt ampère, that is to say, the capacity for the electro-motive force I volt when producing the current I ampère in I second. The unit of effect in one second is called I watt. This, multiplied by the total working period, gives the total energy involved (or accumulated). One usually expresses this in watt-hours. The consumption of electrical energy is reckoned in watt-hours. The effect of one watt $=\frac{\mathbf{I}}{9.8 \mathbf{r}}$ kilogrammeter per second. The effect of one horsepower amounts to 75 kilogrammeters or 736 watts.

The effect of one watt is, therefore, equal to $\frac{1}{73^{6}}$ horsepower. 


\section{\& I2. Battery-Connections.}

If we close the current circuit of one or more elements, two kinds of resistances are to be distinguished; the internal, or moreimportant resistance of the element, and the external, or less-important resistance. The latter $(R a)$ depends upon the crosssectional area, the length and the specific conducting-capacity of the wire joining the two dissimilar electrodes (including, of course, the apparatus to be worked). The internal resistance is offered by the liquid of the element. By connecting a fresh element, therefore, fresh resistance is unavoidably added.

As we know, Ohm's law reads:

$$
\mathrm{I}=\frac{E}{R} .
$$

Since the total resistance is made up from the external and internal resistances this formula now becomes

$$
\mathrm{r}=\frac{E}{R a+R i} ;
$$

or, substituting for the letters the measurement determinations,

$$
A=\frac{V}{\Omega(R a+R i)} .
$$

Experiments with two vessels of water provided with escapetubes show that when these are at the same altitude and the escape-tubes are in connection with one another, the escaping water is under the same pressure as if it flowed from a single vessel; it has, however, only half the resistance to overcome, since both escape-tubes are joined to form a single tube of double the sectional area. Hence, an increase in the intensity of the

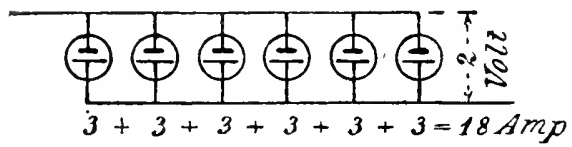

FIG. 7.

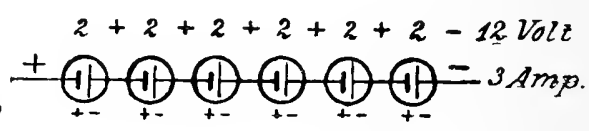

FIG. 8 .

Fig. 7.-Parallel connection (from Donath B. Die Einrichtung zur Erzeugung der Roentgenstrahlen, Berlin I890, p. 9).

FIG. 8.-Serial connection (from Donath B. Die Einrichtung zur Erzengung der Roentgenstrahlen, Berlin r899, D. 8). 
stream results. If, however, the vessels are arranged one over the other the resistance will be doubled, as also the pressure. The same thing applies in the coupling of galvanic elements. Their connection in parallel Fig. T results, therefore, in an increase of current and quantily. Connection in serics, or tension-connection (Fig. S), angments the electro-motive force.

From the above the following formula may be given for serial and parallel connections of $n$ elements:

$$
\text { г) } \left.I=\frac{n E}{n k i+k a} \quad \text { and } z\right) \text { I }=\frac{E}{\frac{k i}{n}+k a} \text {; }
$$

that is to say, in the case of serial connection the electromotive force, also the internal battery-resistance, equals the electro-motive force and the internal resistance of a single element multiplied by the number of elements joined together.

On the other hand, in the case of parallel connection one obtains a battery whose total clectro-motive force equals that of any one of the joined elements. The internal resistance of the latter, however, is correspondingly smaller, as the number of elements joined is increased.

Let us examine serial connection more closely.

If, for instance, the resistance of the connecting-wire be very small compared to the internal resistance of a single element, so that it may be disregarded, it practically follows

$$
I=\frac{\text { I. } E}{\text { I. Ki }}
$$

One therefore gains nothing in the way of current-intensity by serial connection. The total current is approximately as strong as though one were dealing with one element only. If, on the other hand, the wire-resistance be very great, far greater than the total resistance of the series, one obtains practically

$$
I=\frac{n l}{R} \frac{i}{a}
$$

Consequently the current-strength is about $n$ times as large as if - one were using one element only. 
Let us now examine conditions in the second case. If the wire-resistance be small compared with the internal resistance of the battery, so that it may be disregarded, then

$$
I=\frac{E \text { of an element }}{\mathrm{I} / n R i \text { of an element }}
$$

The current-strength is, therefore, about $n$ times greater than that of one element.

If, on the other hand, the wire-resistance be very great, it follows that

$$
I=\frac{E \text { of an element }}{R a} ;
$$

the current strength in this case is practically the same as that of one element.

One, therefore, gains nothing in the shape of currentstrength. We have, therefore, these two rules: If we wish to obtain the greatest possible current-strength when dealing with considerable external resistance connection in series is advisable; on the other hand, parallel connection should be employed when the external resistance is very weak.

\section{§ I3. Ramification of Current.}

The strength of current is not everywhere alike, when dealing with conductors of a branched form; various strengths obtain along the different branches, being inversely proportional to the resistance they offer. The two following laws given by G. Kirchhoff bear on the questions of current-intensity and the individual branch-resistances :

I. At any part of the ramification system the force of the current flowing toward that part equals the sum of the forces of current flowing away from it.

2. In the case of a ramification system forming a closed figure the sum of the current forces obtaining in each branch of the system multiplied by the resistances of the same equals the sum of the electro-motive forces existing in the current circuit.

In Whealstone's Bridge (Fig. 9) the current ramifies 
through two conductors between which a cross-communication is established, so that four branches, $a, b, c, d$, result.

A current generally flows through bridge (), whose force and direction depend upon the relations between the four branch resistances. The bridge-resistance only disappears when the following proportion exists:

$$
a: b=c: d \text {. }
$$

From Ohm's equation it is seen that if one examines the distribution of tension on the conducting wire alone, $E=I_{I} R$. Now, since the electro-motive force corresponds to the tensiondifference at the conductor's terminals, one may also say: The difference between the tensions obtaining at either end of a conductor equals the product of its resistance and the current-force. The tension, therefore, at the end of a current-conducting wire is smaller than that at the beginning, the tension-loss corresponding to the current-force mul- Fig. 9.-Whatstone's Bridge. tiplied by the resistance of the wire.

The greater the resistance of the wire, the greater is the loss of tension. Hence it follows that the tension-difference at the poles of a closed galvanic element is smaller than the element's electro-motive force. The tension-loss is here caused by the overcoming of the internal resistance of the element; it corresponds to the current-force multiplied by that internal resistance. The tension-difference at the poles of a current-producing apparatus (batter; or dynamo) is called the tension of the terminals. (This is a variable figure, being thereby distinguished from the electro-motive force of the apparatus, which remains constant.)

In the case of a branch-system in which the commencements and terminations of all the branches are sererally connected ("parallel-connection" of conductors), the resistance of an individual branch is not cqual to the sum of the resistances in all the branches, but is equal to the resistance which would be offered 
by one conductor whose sectional area corresponded to the sum of sectional areas of the individual branches.

In case of a conductor-system where the commencement of one branch is connected with the end of another the total resistance is, however, clearly increased. If the resistances of individual branches be indicated by $r_{1}, r_{2} \ldots r_{n}$ their total resistances in parallel-connection $R_{p}$ is shown by the equation

$$
\frac{\mathrm{r}}{R_{p}}={ }_{r_{1}}^{\mathrm{I}}+\cdots+\frac{\mathrm{I}}{r_{n}}
$$

their total resistance in serial connection $R H$ by the equation

$$
R_{h}=r_{1}+r_{2}+\ldots+r_{n} .
$$

\section{$\S$ I4. Measurement of an Electric Current.}

If an electric current be flowing parallel to a magnetic needle, or spirally around it, the needle becomes deflected from its usual north and south direction, the amount of deflection varying with the intensity of the current. If one imagines a person swimming in the direction of the positive current, turning his face meanwhile toward the needle, the north pole of the latter becomes deflected toward his left hand. (Ampere's rule.) This property of a magnetic needle may be employed, not only to determine the existence of an electric current, but also its direction and force. On this principle galvanometers have been constructed. In these the current passes along a few spiral turns of thick wire offering but little resistance, so that the instrument influences the current-intensity of the circuit. The amount of deflection then indicates the amount of this current-intensity. An ampèremeter is merely a galvanometer with the dial registered in Ampère's manner.

These instruments are only suitable for feeble currents. In measuring currents of greater intensity, the ampèremeter requires adaptation in the shape of "shunt-circuits." These are connected to the terminals of the amperemeter, and the resistance of each "shunt" corresponds to the resistance of the meter itself. Consequently, only a certain portion of the current 
actually flows through the instrument, the amount varying inversely with the number of shunts.

The indicated number on the dial, therefore, multiplied by the number of shunts, gives the intensity of the main current.

By its inclusion with a branch or shunt-circuit, a galvanometer may also be used to indicate tension difference.

An instrument for this purpose, however, differs from the amperemeter in that a great resistance is offered to the current, the conducting-wire being thin, and arranged in a great number of spirals. The amount of deflection caused by a current

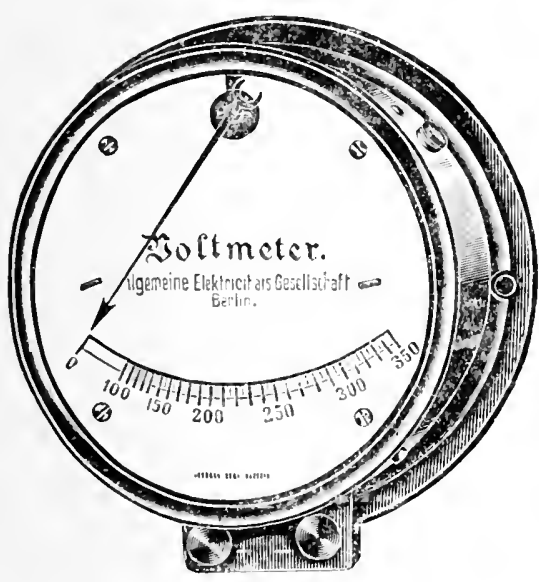

FIG. 10.

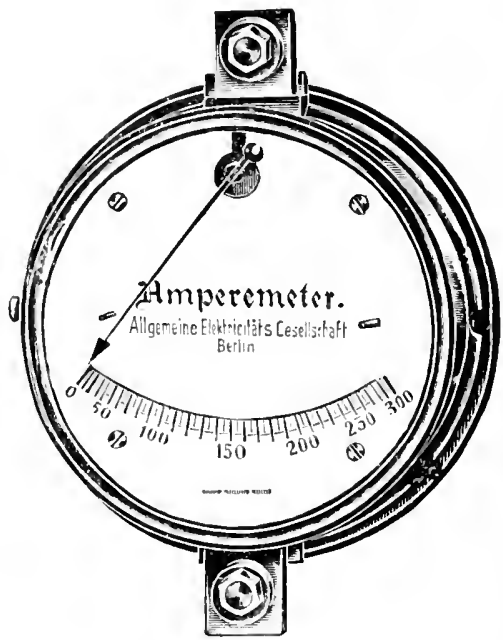

FíIG. II.

(coming from a source with small internal resistance) is proportional to its electro-motive force, or to the tension-difference of the two points in the circuit which are connected through the meter. The dial being suitably registered, one can read off the amount of this electro-motive force or tension-difference, expressed in volts. The apparatus is called a vollmeter.

Apparatus for measuring electric currents are also constructed by the aid of other magnetic properties which they possess. For example, in the amperemeter and voltmeter of the "Allgemeine Electricitätsgesellschaft" (Figs. Io and I I), the attraction of a wire spool, through which a current is flowing on 
an iron core, is utilised. A metal rod, hanging from a spiral spring, is drawn more deeply into the spool as the current increases. The force with which the metal rod is attracted varies with the square of the current-strength and the square of the number of spiral windings. By means of a lever the movement of the metal rod is transmitted to an indicator furnished with

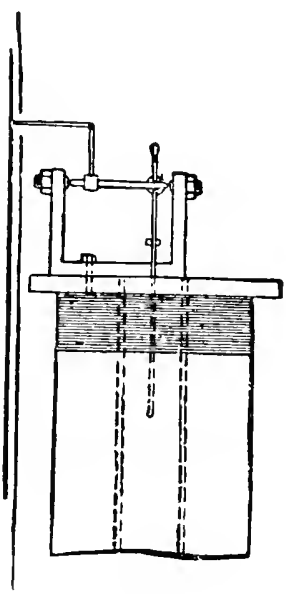

FIG. I2.

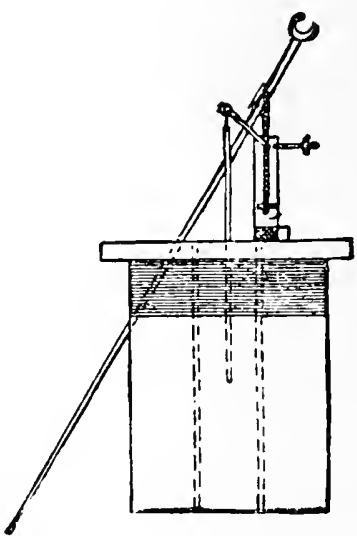

FIG. I3.

(From L. Graetz 1. c. p. 336.)

a dial. The instrument is registered in ampères of volts, according to its situation in the main-current or branch-current. Figs. I2-I3. $W$. Weber's electrodynamometer is also used for measuring continuous and alternating currents. The principle of this apparatus is founded on the electrodynamic effect which two conductors have upon one another, whereby they are constantly striving to become parallel, and the current in them to move in the same direction. The amount of this electro-dynamic force depends upon the relative situation and distance of the two current-circuits, also upon the strength of the currents. (The sine of the angle between the conductors is then proportional to the square of the current-force.) With a different arrangement in the winding of the spool the instrument can also be used for measuring tensiondifferences. 
Hummel's ampèremeter and voltmeter are often employed for alternating currents. In these instruments a piece of light iron foil is arranged so as to be capable of rotating inside a wire spool, and in such a manner that the axis of the rotation does not coincide with the axis of the spool, but is placed eccentrically. When a current passes through the spool the iron foil revolves on its axis until it reaches a plane where its weight is balanced by the electro-magnetic force. An indicator joined to the foil shows the current-force on a dial (or the tension in the case of a shunt-circuit).

It may be here noted that so-called "hot-wire instruments" are employed sometimes for the purpose of measuring the intensity and tension of alternating currents. Their principle lies in the fact that a platino-silver wire traversed by a current becomes heated, and thereby lengthened.

The wire is connected with an axis which revolves as the former alters in length, thereby actuating an indicator. The stronger the current, the greater the heating and lengthening of the wire, with corresponding greater movement of the indicator.

\section{§ I5. Heat and Light Effects of the Galvanic Current.}

A current flowing through a conductor has to overcome resistance, and, therefore, do work. This active energy is partly transformed into heat. The law relating to this heat-formation is as follows: The amount of heat developed in a given portion of a conductor in a given time increases with the square of the current-strength, and directly with the resistance of the conductor. (Jonle's law.)

$$
\lambda=R J^{2}
$$

To insure the safety of electric installations, short pieces of leaden wire (fuses) are introduced along the conductors. Their thickness is so gauged that the wire melts before the current can reach a dangerous limit, which might otherwise happen in case of short-circuiting. 
This accident of short-circuiting happens when the current finds a shorter path through contact between individual portions of the circuit, whereby any existing resistances (lamps, motors, inductors, etc.) are eliminated. A short-circuit current has naturally far greater energy, and may, therefore, be very dangerous.

This heat-effect finds an important application in electric incandescent lamps, in which carbon filaments made from cellulose are brought into a state of incandescence within vacuum glass globes. The lighting power of an incandescent lamp depends upon the current-strength and the resistance of the filament. As a rule, not merely the current-strength required for normal burning of a lamp is indicated, but also the amount of tension-difference at its terminals. Since the resistance of the lamp is known, the normal current-strength required follows directly from this tension. The resistance of an incandescent lamp is generally very high; it requires, therefore, high tension, but a comparatively weak current. Numbers are usually placed on the lamp indicating the necessary volts and ampères. Parallelconnection of lamps is the most effective.

Joule's law, as quoted above, has the same bearing upon the arc-light as upon the incandescent lamp. Whereas, however, with the latter we have an uninterrupted conductor brought into incandescence by the current; in the case of the arc-lamp two carbon points and an intervening layer of air form the conducting medium, whereby the carbons and the air laden with carbon particles are made to incandesce.

If one interrupts a powerful galvanic current, a spark is produced at the site of interruption. This phenomenon is due to change of resistance at this point, whereby high electro-motive force is induced in the circuit, and a spark flies across the air-space separating the two poles of the conductor where its continuity is broken. (For Induction, see below.)

If we send a powerful current through two pointed rods of carbons in contact with one another, the latter become incandescent at the point of contact in consequence of the high resistance offered there. If, after incandescence has been 
established in the points, the rods are slowly removed a short distance, a light violet arc-light results ("Volta's arc-light"), which is due to burning carbon particles torn off by and carried with the current from the positive to the negative pole. It is essential, however, that the electrodes (the carbons) be first in contact and afterward separated. The air then conveys the current, for if its electro-motive force and tension are sufficient, electricity can overcome the resistance of a warmed layer of air; this is accompanied by the development of such intense heat that the carbon points and the particleladen air intervening become white-hot.

A crater-shaped depression (Fig. I4) is found at the positive carbon, giving forth a dazzling radiance. The negative carbon becomes gradually pointed, and is consumed at only half the rate of the positive (their diameters being equal). In order to help in the formation of the crater, the positive carbon is made with a core of softer and better conducting material. (When employing rapidly alternating currents the condition and rate of combustion of the carbons are identical.) The arc-light is produced only when the carbon points are from 2- $\$ \mathrm{~mm}$. apart; therefore, there must be some contrivance for regulating this distance.

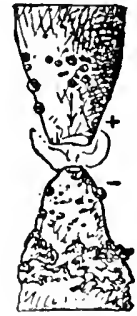

FIG. I4.

(From F. Körner, Lehrbuch der Physik, Wien und Leipzig, F. Deuticke '97, p. 389.$)$

As already mentioned, considerable electro-motive force is requisite for a l'olta's arc-light; this results more especially from the fact that in the arc-light itself an electro-motive opposing force exists of about 40 volts (perhaps resulting from destruction of the electrodes or from clectrolytic decomposition of the air), which must be overcome by the current.

Hence the terminal tension at the carbon points must always be greater than 40 volts. Moreover, the current-intensity must be very high, because, according to Joule's law. the heating of a conductor is proportional to the resistance and the square of the current-intensity. 
Naturally, the brightness of an electric arc-lamp depends not only upon the length of the arc itself, but upon the strength of the current traversing the carbons. It is customary to indicate the lighting-power of an arc-lamp by the intensity of the current required to work it.

Arc-lamps may be joined either serially (this is seldom done), in parallel, or in groups. In the latter event groups of serially arranged lamps are joined in parallel to the main current.

In the case of parallel connection of arc-lamps, resistances are placed in front of the lamp circuit.

\section{§6. Thermo-Electricity.}

We have seen (p. 23) from the laws relating to tensionseries that a current cannot be obtained from a series of metals alone. This statement, however, only holds good if the tem. perature of the whole series be constant. If at some part of the metal-chain the temperature be raised or lowered, a current results, which is known as a thermo-electric current. This current always flows from the higher-temperature locality to the lower. Thus, if at some point in the series (say, where two adjacent metals are soldered together) the temperature be lowered, the current flows towards that point. The electrical tension invoked is greater (given equal temperature differences) the farther the respective elements of the series are situated from one another. Becquerel arranged the following series for the production of a thermo-electric current: Bismuth, nickel, platinum, cobalt, silver, lead, copper, zinc, iron, antimony. The electro-motive force of a thermo-electric series also depends largely upon the temperature difference of the soldering places.

Frequent attempts have been made to produce stronger thermo-electric currents by uniting several elements in the manner of galvanic elements, to form the so-called thermo-pile. Inasmuch as the electro-motive force of even the most effectual single combination is very feeble, many separate elements require connection in series for this purpose. Guelcher's thermo- 
pile can be used for practical purposes, such as charging accumulators. In this apparatus hollow positive electrodes are cast, as in small tubes of chemically-pure nickel; the negative electrodes, likewise tube-shaped, are cast from an antimonyalloy. The tubular positive clectrodes convey gas, by which, through a small Bunsen-flame, each element receives a certain amount of heat. The small flames heat a piece of iron connecting the positive and negative electrodes. The larger-sized pile constructed thus, and consisting of 66 distinct elements, gives at average gas-pressure an absolutely constant electromotive force of 4 volts, with an internal resistance of about 0.6552 . (The internal resistance of a thermo-pile is small, the elements consisting of metals only.)

The mechanical effects of electrical currents upon one another, as shown in induction effects, effects of a current upon a magnet and vice versa, were dealt with when speaking of electro-dynamometers.

\section{$\S$ I7. The Magnetic Effects of Electric Currents.}

Iron or steel brought near a conductor transmitting an electric current become magnetised. This magnetic effect can be intensified by arranging the conductor in the form of a spiral with the several windings on the same level; or as a solenoid, which consists of rings arranged in parallel, one behind the other. A solenoid itself*behaves like a magnet. If hung up so as to be freely movable, it comes to rest in the magnetic meridian, and shows other phenomena pertaining to the magnet.

An electric current passing through an insulated wire which is arranged spirally round an iron rod, brings the latter into the magnetic state. Its poles may be determined by Ampère's law, above quoted.

The magnetising force of such an clectro-magnet is proportional to the current-intensity and number of spiral windings. With cessation of the current soft iron at once loses its magnetism, whereas steel, under the same circumstances, remains magnetic.

A current growing stronger produces less magnetism 
than a weaker-growing current. This is known as hysteresis of iron.

The "pulling effect" of a solenoid upon an iron core has been already mentioned (pp. 39, etc.).

The magnetic action of electrical currents is turned to account in the regulators of arc-lamps, in various current-interrupters, and in electric meters. An electric meter measures the quantity of electric energy consumed. An electric current does work every second which is equal to its tension (in volts) multiplied by its quantity (in ampères). The number of watts (volt-ampères), therefore, stands for the chemical energy produced each second by the current.

The period of consumption is reckoned in hours. If, therefore, we multiply the number of watts by the number of hours, we get the total consumption of energy expressed in watt-hours. Since the tension is always constant, one need but measure the number of ampère-hours (current strength multiplied by time). This number multiplied by the constant tension $V$, therefore, gives the number of watt-hours.

Aron's electrical meters, mostly used with continuous-current installations of constant tension, consists mainly of two cleck-work pendulums exactly synchronised. A metal rod hangs from the lower end of one of the pendulums, and swings with it over a solenoid traversed by the current about to be measured. The magnetic influence of the solenoid accelerates the speed of its pendulum.

This acceleration can be compared with the speed of the other pendulum, which swings free. An indicator is connected with the clock-work of both pendulums in such a way that only the difference in velocities is registered. While no current is passing through the solenoid the indicator is motionless; the moment a current passes it shows the acceleration of the magnetised pendulum. The amount of movement of the indicator is thus proportional to the current-strength, and affords exact measurement of the electrical quantity passing through the solenoid.

Where the tension is not constant the amount of energy consumed can be measured by the watt-hour-meter. This is 
similar to the preceding apparatus, but the pendulum swinging over the solenoid is not provided with a bar-magnet. In place of the latter there is a roll of fine wire, which lies in a "shuntcircuit" to the main current. The force with which the currents in both circuits act upon each other depends upon the product of their current-strengths. Since, however, the strength of the current in the shunt-circuit depends upon the tension at the terminals of this circuit (its resistance being invariable), the force depends upon the product of tension and current-strength, consequently upon the watts. The movement of the indicator by the pendulum accordingly bears direct relation to the product of the watts and the duration of the current. A dial is arranged to indicate the energy in hecto-watt-hours. This instrument can also be employed for alternating currents.

In Thompson's meter an electro-motor turns an armaturecore wound in the form of a drum. This actuates a copper disc set between magnet poles, and also a numerating apparatus. The turning of the copper disc is hindered and regulated by the magnet poles (see Foncault's currents, below). The velocity of revolution of the disc, which is affected by the current to be tested, consequently affords an indication of the watts consumed.

\section{§ 8 . Induction.}

We have already seen that an electric current produces magnetic forces. Now the reverse holds good to a certain extent, viz.: magnetism itself can, under certain conditions, produce electricity. (Faraday.)

If a magnet be brought near a conductor fitted with a galvanometer, or withdrawn from the same, the galvanometer index moves, thereby showing that the conductor is traversed by a current. This phenomenon occurs every time the magnet is approached to, or withdrawn from, a point directly opposite the conductor.

If a steel magnet be brought near the iron core of an electromagnet, the core itself becomes a temporary magnet. At the same moment an electric current appears in the closed circuit of the spool. This current is only temporary, whereas the steel- 
magnet keeps the iron core in a state of magnetism. The current is only manifested while the steel magnet is approaching the core.

The electric wave ${ }^{1}$ ) thus produced in a wire circuit while magnetism is originating is called an induced-current, being transmitted from the magnet to the distant wire-circuit (induced).

After this induced current wave is over, a fresh wave passing in the converse direction may be produced by removing the steel-magnet from the iron core and thereby dissipating the magnetism of the latter. This current is also very brief, being, like the first, merely an electric wave.

By alternately originating and dispersing magnetism an electric current may be produced in a closed wire-circuit, which continually changes its direction-an alternating current. Faraday's discovery may also be expressed as follows: If a conductor interferes with lines of force, induction takes place in it. Electrical energy is manifested therein. The electro-motive force of this induced current is greater, the greater the strength of the inducing magnet and the number of spiral windings, and again, the quicker the inducing magnet is approached to and withdrawn from the spiral. If the external resistance through which the induced current is meant to flow be increased, the electro-motive force of that current must also be increased, and therefore, other things being equal, there must be a greater number of windings. By thus augmenting the number of windings very considerable external resistance can be overcome. For this reason a secondary spiral is attached to "spark-inductors" (see below), consisting of very long and fine copper wire. The internal resistance of the spiral should probably approach as nearly as possible to that of the external resistance of the current-circuit.

The induction caused by a magnet is termed "magnetic-indiction." Just as an electric current is produced in a closed circuit by the above-mentioned method, so an induced current can be invoked in wire-spools which are made to rotate before

1) The expression "current wave" signifies the very brief duration of each induced current. 
the poles of a magnet. The same principle underlies all magneto-electro machines. Here also it follows that the induced current must constantly be changing its direction, according as a spool approaches or withdraws from a pole. These machines are, therefore, alternating-current machines, which produce electricity manifesting itself in individual electric waves, rapidly succeeding one another, and continually changing their direction.

Alternating currents can only be transforned into continuous currents by means of certain complicated apparatus (commulalors). But a continuous current may also be produced directly by one machine; the principles of this machine (which has an importance for us by reason of its use in radio-therapy) may be here brictly explained.

The earlier magneto-electric machines were very imperfect, their magnetic field being weak and not fully utilised. Siemens' double-T-mag-

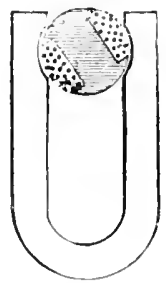

FIG. I5.

(From Köncer,

1. c. p. +15.$)$ net apparatus (Fig. I 5 ) constituted a marked improvement.

A cylinder (armature-core) rotates between two hollowed-out poles of a horseshoe magnet. Spiral wires are wound lengthwise round this cylinder, so that the windings on the axis-core lie parallet. When the armature rotates alternately north and south poles are induced in it, while alternating currents are produced in its spiral winding, which become transformed into continuous currents by a commutator.

The dynamo-electric machine of Siemens ( 1 867 ) remedied the defect of rapid loss of power by the steel magnet (see Lenz's law below). In place of permament magnets, Sicuens employed electro-magnets, producel by causing the induced current itself to flow round the iron corc. buen the softest iron core possesses traces of residual magnetism; hence, during the first revolution of the armature a current, however feeble, will result. This feeble current is first conducted round the electro-magnet, which it strengthens Thereby still stronger induced currents are produced, which strengthen the magnet anew, and so on 
until the magnet reaches its saturation-point. In this way powerful currents may be obtained in a short time from very small quantities of magnetism through the reciprocating action of magnet and spiral-winding.

By a peculiar shaping and winding-arrangement of the spool - known as the armature - a constant and continuous current can be produced in place of the wave-like currents of earlie: machines. Here we shall brietly consider the principle of the gramme-ring, a type on which many dynamos are founded. An iron ring rotates between the poles $N$ and $S$ of an electro-magnet (Fig. I 6). The ring carries a large number of wire spools, two neighbouring spools being joined with one another and a metal plate belonging to the current-collector, the whole being insulated from the axis of rotation. The collector possesses as many plates as there are spools. When the iron ring rotates, poles are formed in it in the neighbourhood of $N$ and $S$. These

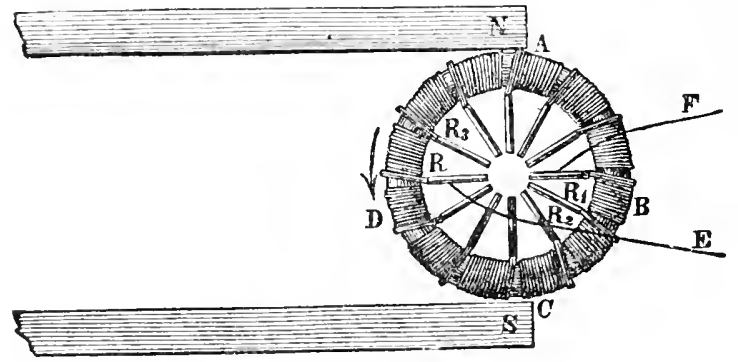

Fig. I6.

(From Lotheisen, Lehrbuch der Physik, p. 605.)

poles are apparently constantly changing their position in the ring because of its rotation, and hence moving through the spools. Consequently currents are produced in the latter, which in the case of spools above the neutral zone $B D$ are moving in one direction, while in the lower part they move contrariwise. Two wire bristles (the brushes) are in contact with those collectors which happen to be in the neutral line. One brush recerres positive and the other negative electricity, both being attached to conducting wires which convey this electricity to the apparatus requiring it. The armature has not the shape of a gramme-ring in all dynamos; other contrivances are employed 
instead, so that one therefore distinguishes ring-machines from drum-machines, and so on.

Alternating currents which, as we have seen, are produced by magneto-electric machines are not quite so useful for radiotherapeutic purposes as continuous currents. The electro-motive force of an alternating current reaches zero at a certain time $a$ (see Fig. I 7 ), increasing afterwards to the highest value $A$, and then decreasing again to zero $b$. Next the direction of the current is altered, so that electro-motive force and current-strength become negative. The electro-motive force falls to the highest negative value $B$, afterwards reaching zero again. By the "period" of the alternating current we understand that time in which a wave-movement is completed, with its

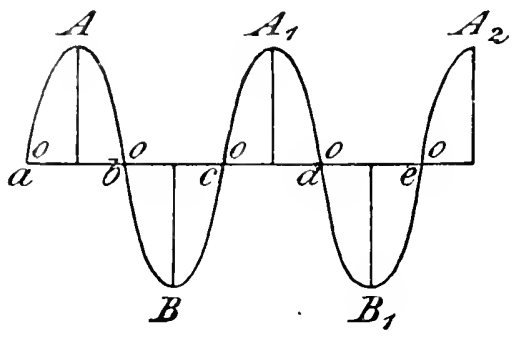

FIG. I7. concomitant position and negative electricity (the period $a c$ ). This period has a positive and a negative phase; that is to say, it corresponds to a single alternation of the current.

The greatest current-strength is known as the ampliande of the alternating current. By phase of the alternating current we understand the current-strength at a given time (reckoning from the point where this current-strength is at zero) divided by the amplitude. Two alternating currents may be distinguished from one another (1) by their average current strength, (2) by their periods (one may have a period of $\frac{1}{100}$ second, the other a period of $\frac{1}{35 \pi}$ second), (3) where their periods are equal, by the fact that they do not attain their maximum value at the same time; or, in other words, do not pass zero and change their current-direction simultaneously. A system of alternating currents possessing equal periods, but whose phases are different, has important properties dependent upon these very phase-differences. Such alternating currents acting together are called mulliple-phase currents. The rolary current is a special kind of multiple-phase current. 
In every magneto-electric and dynamo-electric machine the electro-motive force depends upon the strength of the magnetic field, the proximity of the iron-core to the magnet poles, the velocity of rotation of the anchor, and, finally, upon the number of spiral windings on the latter. If a continuous current be sent into a dynamo, the latter begins to rotate. lt is then called an clectro-motor.

In order to understand the principles of an electro-motor one needs but to call to mind the simple laws of electro-magnetism; by the flowing of the current through the anchor-windings and the ring-spools magnet poles are produced in the iron, and it is easily seen that adjacent poles in ring and anchor are of the same kind. Magnetic poles of the same kind must repel each other; the electro-motor begins to work, turning in the opposite direction to that it would have were it a generating machine.

The rotation of the motor may be transmitted and utilised in various ways by lengthening its axis: employing eccentrics, connecting-rods, etc. Alternating and rotary current motors are of a very complex nature, which cannot be described here.

(The author here describes common forms of contact apparatus.- TRANSLATOR.)

Electrical currents have also an inducing effect, known as electro- or volta-induction.

If an elcctric current be opened or closed in the inducing or primary coil of an induction-apparatus, a brief current (the induced current) is produced in the secondary coil. When the primary current is closed, the secondary current flows in an opposite direction; when, however, the primary current is opened, both flow in the same direction.

The same result is attained if one quickly strengthens or weakens the intensity of the primary current by means of a rheostat, or if one rapidly introduces the primary coil. within the secondary coil (corresponding with the closing of the main current) and withdraws it equally rapidly (corresponding with the opening of the main current). Since the free electricities which appear at the terminals of the secondary coil on opening and closing the primary current are directly opposed, the induction 
apparatus may be regarded as an alternating-current machine which, from one moment to another, is alternately producing currents of opposite direction.

With regard to the strength of the induced current, that is to say, the electro-motive force induced, the following laws apply:

The electro-motive force of the induced current is greater:

I. The greater the inducing strength, that is to say, the greater the strength of the exciting current in the primary circuit and the greater the number of windings in the primary coil; or, in the case of magneto-induction, the greater the strength of the inducing magnet. ${ }^{1}$ )

2. The greater the number of windings in the secondary coil.

3. The greater the rapidity of the interruptions, that is to say, of the changes in the main current.

4. The less the distance between the secondary coil and the inducing body (the primary coil or magnet).

The tension of the induced current depends upon the proportion obtaining between the number of windings in the two coils; the greater the number of windings possessed by the secondary coil compared with those of the primary coil, the higher is the tension of the induced current. At the same time, however, the strength becomes so much less, by reason of the great resistance offered by the many windings of thin wire in the secondary coil, whereby the current is weakened. One obtains induced currents of remarkable strength by introducing bundles of soft iron-wire within the primary coil; this results from the magnetisation of the iron core. We have then roltaic and magneto-induction acting in co-operation. IVe may also explain the mode of action of this apparatus by assuming that the current traversing the primary coil produces a magnetic field around the common axis (sce p. I 2 ), and that this magnetic field, when formed or dispersed, induces a tension in the secondary coil, the amount of which depends (apart from other factors) upon the size of the magnetic field. From this point of view the action

') See also the principle of Messaucr's apparatus, described later on. 
of the iron core may be readily understood. ${ }^{1}$ ) The discharge-current of a Leyden jar likewise produces, as Masson has shown, an induced current in a neighbouring wire. This can best be demonstrated by an apparatus constructed by Ries.

Just as a current at the moment of its make and break has an induction effect upon an adjacent conductor, it has a like effect upon its own conductor when the latter is composed of a number of spiral-windings. This is known as self-induction, and the current thereby produced is called the extra-current. When the main current is "closed," a so-called extra current of the intermption appears in the spirals of the conductor; its direction is opposed to that of the main current, and thereby the intensity of the latter is not allowed to at once attain its maximum. With "opening" of the main-current an "opening-current" is produced in the spirals, having the same direction as that of the primary current, which latter it intensifies, causing considerable augmentation of the spark at the site of interruption (see above).

On account of the high tension, the insulation of the wires may be destroyed, for which reason powerful currents must not be suddenly interrupted.

A shorter time is required for the disappearance of a current in a coil on "opening" the circuit than for its production on "closing." We know, however (see above), that a tension produced by induction is greater the quicker the successive inductions follow one another. Consequently, the induction-tension in the secondary coil is greater on "opening" than on "closing." (On closing the circuit only a small spark is formed, or even none at all, whereas it may be very considerable on "opening.")

With the help of special apparatus, which only permit either the "opening" or the "closing" waves of the induced coil to pass through a body, it can be shown that the induced current produced by the opening of the primary current possesses a greater electro-motive force than that which is produced by closing. When we come; however, to examine the chemical effects of the opening and closing currents respectively, we find that the electrical quantity traversing the induced circuit is in both cases iden-

1) B. Walter, Fortsch. auf d. Geb. d. Roentgenstrahlen. Vol. I, p. 29. 
tical. Since, however, the opening current is much briefer than the closing, it is evident that the intensity of the former must considerably exceed that of the latter.

In cases where high tensions are required only the opening tension in the secondary coil need be considered, so that the current-waves produced by the apparatus in use may be regarded as waves of ore direction. The foregoing explains, moreover, why electro-induction apparatus shows a certain polarity at the terminals of the secondary coil, in spite of the production of alternating currents, which polarity manifests itself in a striking manner when the discharge is effected in a rarefied atmosphere.

The self-induction of a conductor and the electro-motive force of its extra-current are mainly dependent (the alterations of the current-strength being equal) unon the shape of the conductor.

It follows, therefore, that the relation which the eiectro-motive force of the extra-current bears to the speed with which the current-strength is varied in the coil is merely dependent upon the shape of the coil. This relation is known as the co-efficient of self-induction, or the self-potential of the coil. Straight wires have but little self-potential. If one bends a wire in the middle, for example, so as to make it appear double, the current in each half has an opposite direction, and the self-potential in the wire is very small. If one bends it, however, in the form of a roll, its self-potential becomes more considerable, and greater still, if an iron core be placed within the spool. Each conductor, therefore, possesses a definite self-induction co-efficient or self-potential, the amount of which depends upon the shape, dimensions (length, sectional area) and winding of the conducting-wire.

Whereas the tension of the primary current may practically be regarded as a factor forcing the current into the primary coil, on the other hand, the self-induction co-efficient represents the magnetic inertia-moment of this coil, which endeavors to check the rapid development of the current.

B. Waller proved experimentally that the tension of the secondary closing current grows in direct proportion with the amount of the primary tension, and in almost inverse proportion with the amount of self-induction in the primary coil.

By increasing the amount of self-induction in the primary 
coil the closing tension in the secondary coil becomes therefore diminished, whereby, as we shall see, the "life" and scope of regulation of a "soft" Roentgen tube are considerably augmented. If one diminish the self-induction, for instance, by sending the primary current through fewer windings of the wire, a greater current-strength must be employed if the magnetic and inductive effects are not to be impaired. Lenz lays down the following law relating to the direction of induced currents: In all cases of electro-magnetic induction the induced currents have such a direction that their counter-effect strives to hinder the process of their development.

Induced currents are met with not only in linear conductors but in massive metallic bodies; these are called Foncault's or whirl-currents. According to Lens's law, the direction of these induced-currents is such that they counteract the activity of the conductor-mass. The opposing currents produced in this way in the massive core of an electro-magnet on closing the current thereby hinder the development of the magnetism; in like manner, on opening the current, induced currents are produced in the iron mass of the core, which have the same direction as the vanishing current, and delay the disappearance of the magnetism. Follcault's currents, consequently, account for a considerable and useless consumption of energy; they are, moreover, troublesome by being transformed into injurious heat-effects (see Jonle's law). One tries to remedy this defect as far as possible by suitable distribution and arrangement of the metal-masses. Thus the iron-cores of electro-magnets are not arranged as one solid mass, but in bundles of thin insulated iron-wires or plates. In these no very pronounced induced-currents can arise, since they are confined to interrupted conductors. For the same reasons the coverings of the iron-wires are not made of metal, but of an insulating material, such as ebonite.

\section{$\S$ I9. Spark Induction Apparatus.}

For present purposes, perhaps the most interesting application of clectro-magnetic induction is to be found in spark-inducing apparatus. By their means we may produce all those phenomena for which high-tension electricity is required. The main 
object of the apparatus is, therefore, to transform currents of low tension to others of high tension. Ruhmkorfl's coil (Fig. I 8 ) consists of a primary thick-wire coil $P$, the core of which is filled with a bundle of thin iron wires $M$ insulated from one another, and of a secondary coil of thin wire $S$ arranged outside the primary coil, and having a very great number of windings.

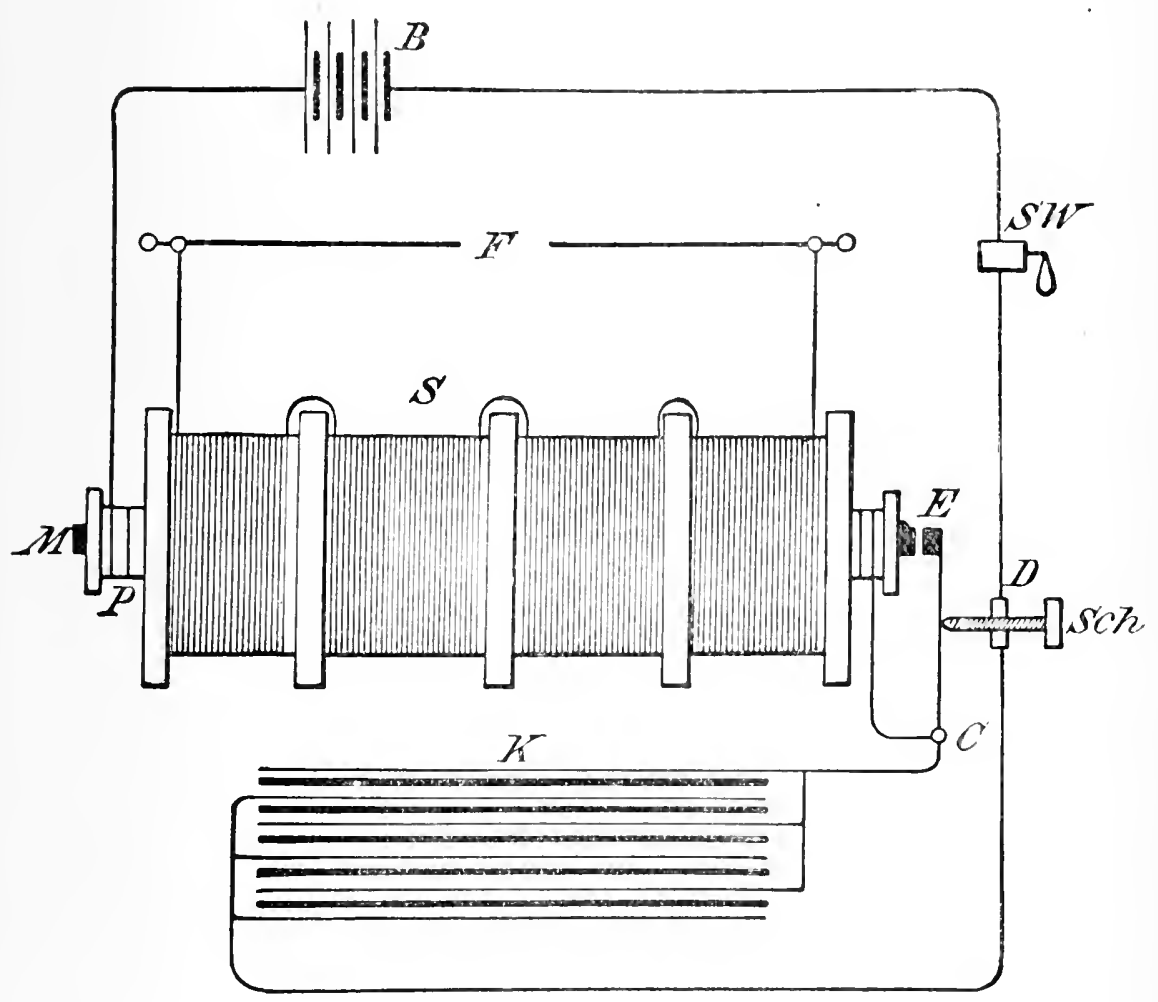

FIG. I8.

In order to produce rapidly-succeeding induced currents in the secondary coil the primary coil current has to be opened and closed with corresponding rapidity; this is done by means of an interrupter ("rheolome"). Interrupters are of various types, and will be referred to later. The efficacy of the whole apparatus is greatly increased by means of the condenser K (Fizean's condenser), which is connected with the primary coil. This con- 
sists of a series of supcrimposed sheets of tin-foil, insulated from each other by sheets of paper which are larger than the tin-foils and are impregnated with resin. Sheets of oiled silk are often used instead of paper. The tin-foils are so arranged that the first, third and fifth sheets, and so on, are in connection with one another and overlap the paper sheets on one side, the second, fourth, sixth, etc., overlapping on the other, and being likewise in connection. In the case of large induction coils the layers of the condenser sometimes reach the size of $20 \mathrm{qm}$. These layers are in connection with those parts of the interrupter where the make-and-break occurs. The effect of this is to make the spark at the site of interruption (created by the extra current in the primary coil) smaller. "Extra-current" electricity passes into the condenser, positive towards one layer and negative towards another. These opposite electricities immediately reunite through the medium of the primary coil, the battery, and the current-circuit which connects the two layers. Hence a current arises which is in opposition to that of the battery, whereby a momentary "demagnetising" of the iron core results and the induced current is made of shorter duration. Thus the condenser allows the interruptions to take place with greater rapidity, with the effect of increasing the tension in the secondary circuit and the spark-length. Whereas without a condenser the sparking is very pronounced at the interrupter, by introducing this appliance into the primary circuit the interruption-spark is made very much weaker. A condenser's value does not alone depend upon its size: it requires to be "tuned," as it were, to the primary coil, to which it must bear a definite proportion.

Experiments by T. Mizuno ${ }^{1}$ ) ('Tokio) have shown that the capacity of the condenser has a great influence on the spark-length, and that by exceeding a certain capacity the spark becomes shorter again. Mizuno mentions a series of experiments showing that the sparklength increases with the strength of the primary current, and that a definite condenser-capacity goes best with a given current-strength.

1) Phil. Magazine. 
It is, therefore, advisable to have an apparatus whose capacity can be regulated by means of certain contrivances. Such an apparatus has been constructed by Radiguet.

According to $/ H^{\prime}$ erlheim Salamonsen (Fortschr., Vol. IV., No. 3), the introduction of a small resistance in the condenser's circuit has a powerful effect in diminishing the "vibrations" which arise at the moment of interruption, by leaving the so-called lime-conslant $l^{1}$ of the inducing current. These vibrations cause a considerable potential-difference at the two ends of the current-circuit at the interrupter, which is equalised by the spark. By using Salamonsen's apparatus one is consequently able to avoid those disturbing explosions which are apt to occur when using mercury interrupters.

Coils fitted with electrolytic interrupters (IV ehnelt's interrupter, see below) are not worked with a condenser. The selfinduction of the primary coil (which is negatived by a condenser) is an important adjunct in interrupters of this type.

A Ruhmkorff's coil possesses yet another contrivance (Fig. I $S, S W^{\prime}$ ) by means of which it is not only possible to rapidly interrupe the current passing through the primary coil, but also to change its direction at any time and, therefore, alter the poles of the induced circuit. The apparatus consists of a cylinder of ivory, vulcanite, or wood (Fig. I 9 ) resting on a couple of pivots insulated from each other. The cylinder can be turned by means of a handle between two copper beds. To the latter screws are connected, which receive the ends of the primary (a) coil-wire $(b)$. A couple of small metal-plates are screwed fast to the cylinder-one screw joins the right-hand plate with the upper pirot, another the left-hand plate with the lower pirot. Springs slide over both plates, and each spring is in connection with a pole of the electrical source. The drawing

1) Time-constant $=\frac{2 L}{R} \quad(L=$ self-induction co-efficient $; R=$ resistance $)$. It determines the amplitude of the viluations: $\left(i=A c-\frac{R}{2 L} \sin B+\right)$ in single periods. 
shows the course of the current for one position of the commutator. On rotating the cylinder through $\mathrm{I}^{\circ} \mathrm{O}^{\circ}$ the current is

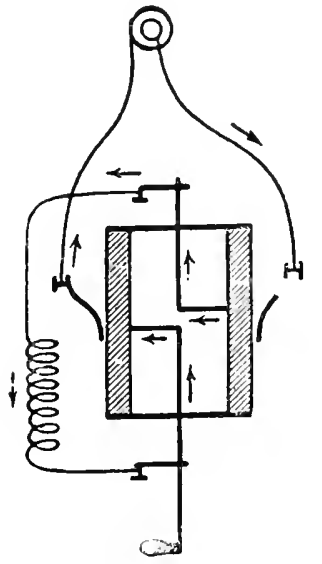

FIG. I9. reversed; at $90^{\circ}$ the springs no longer slide on the metal plates of the cylinder, but on its wooden non-conducting portion, whereby the current is interrupted.

Another frequently used form of disconnector consists of a flat piece of metal fastened to a wing-shaped horizontally rotating handle. As the handle turns the wings come into connection with two flat springs which are in connection with the conductors. Thus the circuit can be made or broken.

The primary coil consists, as before mentioned, of thick wire of shorter length than that of the secondary coil. This wire is wound on a hollow cylinder of wood or papier-maché. The cylinder is filled with a bundle of thin soft-iron wires, each wire being separately varnished. The cores of modern coils (those, for instance, of the Allgemeine Elektricitäts-Gesellschaft, Berlin) are made of layers of iron plates. Besides great magnetic conducting capacity an almost perfect freedom of "whirl-current" is thereby obtained, so that losses of energy in the iron are practically obviated.

We have already seen (p. 58 ) that self-induction plays an important part in the working of a coil with $/ V$ ehnelt's interrupter, and that this applies more especially when working the apparatus in conjunction with a Roentgen-ray tube. It follows that in order to be able to properly regulate and increase the life of a vacuum tube we require some method for increasing the self-induction of the primary coil. For these cases in which it is required to weaken the effect of an inductor worked with $W$ ehnelt's interrupter $B$. $I V$ alter recommends the following proceeding ${ }^{1}$ ): On a thick roll of cardboard $3 \mathrm{~cm}$. in diameter about 150 turns of copper wire $\frac{2}{3} \mathrm{~mm}$. thick, and

1) Fortschr. a. d. G. d. Roentgenstr., Vol. II, p. 225. 
Wehnelt's interrupter-two conditions upon which the interrupter of the primary current largely depends. In order to weaken the induction-effect, we may, however, augment the selfinduction in the current-circuit. This is accomplished by the simple method of $W^{\prime}$ alter already mentioned.

$W$ alter constructed for the same purpose primary coils with

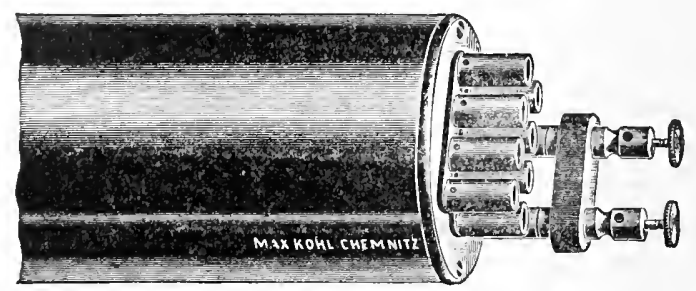

FIG. 2I.

variable self-induction, which adapt the capacity of the inductor to the vacuum of the Roentgen tube. This he effected by arranging the windings of the primary coil in several divisions, allowing the current to flow through one, two, or more of these divisions by means of a suitable switch (Fig. 20). The individual coil-windings can be connected serially, in two groups, or in parallel. The ends of the wire windings terminate in "contacts" at one side of the primary coil (Fig. 2 I). At these "contacts" pins are arranged on which plugs fit, according to the connection desired between the terminals of the several

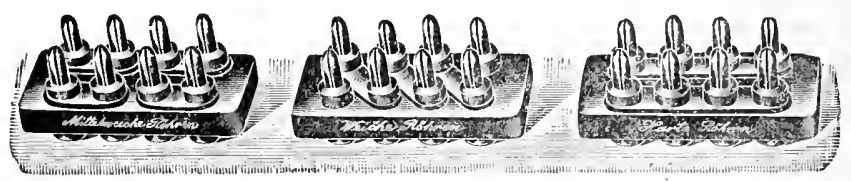

FIG. 22.

coil-windings (Fig. 22). By connecting the windings serially (for soft tubes) the self-induction of the primary coil is considerably increased; by connection in two groups (for tubes of medium density), or in parallel (for hard tubes), the selfinduction becomes, on the other hand, diminished.

The primary coil is covered with an insulating layer of 
glass, ebonite, gutta-percha or paraffin. Many makers construct the primary coil in such a manner that it forms a separate and independent part of the apparatus, so that it is introduced when required within the secondary coil, and may be exchanged for another at any time. In other kinds of apparatus the secondary coil is wound directly on the primary. The former consists of a very thin and long copper wire, which is everywhere uniformly insulated. The diameter of the wire varies in different apparatus from $\frac{1}{5}$ to $\frac{1}{20} \mathrm{~mm}$. Its length is considerable, reaching many kilometers in the larger models. By increasing the length of wire one grets a greater potentialdifference; by increasing its diameter one gains in electrical quantity.

One of the greatest difficulties met with in the construction of coils lies in the proper insulation of the secondary circuit, the least defect in this respect leading to discharges within the apparatus which in a short time fuse the wire and render the apparatus worthless. The several layers of wire, which is already of itself well insulated, are, therefore, insulated from one another. This is done by embedding them in paraffin, or each layer may be varnished or covered with wax or silk; or again, a sheet of waxed paper or gutta-percha may be arranged between the layers. Even with these precautions it may be very difficult to induce a current with safety through so long a wire, so that with the larger-sized coils the plan recommended by Poggendorff is adopted. In this method the secondary coil is built up of several short coils, separated from one another by insulating plates, but with their wire-ends in contact (see Fig. I 8 ). The insulation is thereby much improved, and in case of short-circuiting repairs are much more readily executed.

M. L.evy has constructed coils in which the gutta-percha insulation can be readily remored and replaced when showing signs of deterioration, which this kind of material is apt after a time to do.

F. Dessaner's apparatus gives a sccondary current which is very strong, but of relatively low tension. This is arrived at by diminishing the resistance in the secondary circuit. According to Dessauer, the large coils used for Roentgen-ray work 
(whose secondary coils are made up of an enormous number of windings of exceedingly thin wire) supply a current of very high tension; but this on the other hand has to overcome a tremendous resistance in the coil. This is not an ordinary resistance, but a so-called "impeding-resistance," for the current traversing the secondary coil is not a regular but rather a rapidly pulsating current, whose intensity increases to a maximum and then decreases with each pulsation. The resistance increases with the length of the wire in far greater ratio than the tension with the number of windings. By shortening the wire in the secondary coil, therefore, Dessauer obtains a lessening of the resistance, and consequently an increase in the current-intensity of the secondary circuit.

The ends of the wire in the secondary coil are in connection with two perforated terminal screws. One of the latter bears a rod with a metal point, the other a rod with a metal disc.

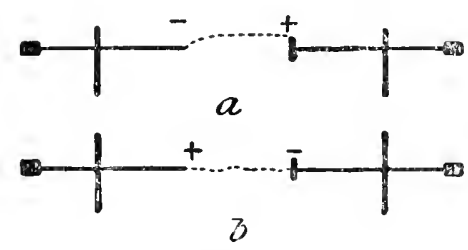

FIG. 23. The tension-difference of the terminals determines the distance which the disc shall be placed from the point in order that a spark may jump from one to the other. The sparking-distance of a coil is the greatest distance which a spark will jump from one terminal to another. The sparking-distance is often, though not quite correctly, taken as a measure of the capacity of the coil.

If one examines the course of a spark in the spark-gap between the terminals (see Fig. 23), one sees that with the current flowing in one direction the spark jumps between the point and the edge of the disc $a$; with the current reversed the jumping is from the point to the centre of the disc. In the first instance the point is known as the cathode, the disc as the anode; in the second vice versa.

\section{$\S 20$. Current Interrupters.}

The capacity of an induction-apparatus depends very largely upon the manner in which the interruptions occur in the pri- 
mary current. The interrupter must be capable of giving a great number of interruptions, and the latter must take place suddenly, completely and equally. Interruptions can be effected in a primitive manner by hand with a file, or a toothed wheel the points of which are made to close the current.

Generally, however, automatic interrupters are used, by which the process can be more effectively carriced on. The simplest appliance of this kind is to be found in Neef's hammer, which can be readily understood from lig. IS. lt consists of a spring provided with an iron piece $E$ (the hammer) placed opposite the iron core of the coil. 'This spring is connected with one of the battery poles through the wire of the primary coil. The wire from the other battery pole is connected with the platinum screw $S c h$, which can come into contact with the spring supporting $E$. When the current circulates through the primary coil it makes the iron core magnetic, so that the hammer $E$ becomes attracted. Is a result of this the current becomes broken, the iron core is demagnetised, the hammer springs back into contact with the platinum screw, and a fresh current is established. The whole process is then repeated, and in this way makings and breakings of the current rapidly succeed each other. The number of interruptions in a time-unit depends upon the length and tension of the spring and the size of the armature-core. The same principle underlies Depres's, Ernecke's, the Allgemeine Elektriciläls-Ge'sellschafl's, Max Lacry's and other interrupters.

F. Dessancr ${ }^{1}$ ) arranges contact plates (Fig. 2+) on both sides of the middle of the spring, and opposite these two contact screws $K^{1} K^{-2}$ are placed. With $K^{1}$ touching $P^{1}$ the current is closed; the magnetised core then attracts the spring, $K^{1}$ is released and the current broken. By swinging forward the contact-plate $P^{\prime 2}$ strikes the screw $K^{2}$ and the current is again established. Now the magnetised core endeavours to keep the spring in this position, but this force is met by the inherent elasticity of the spring. Since the magnetism can only act upon the length of the spring (from p to the armature core),

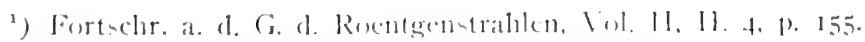


whereas elasticity obtains throughout its whole length, the latter prevails, and the spring moves away from the core. By this arrangement Dessaner's break gives double the number of in-

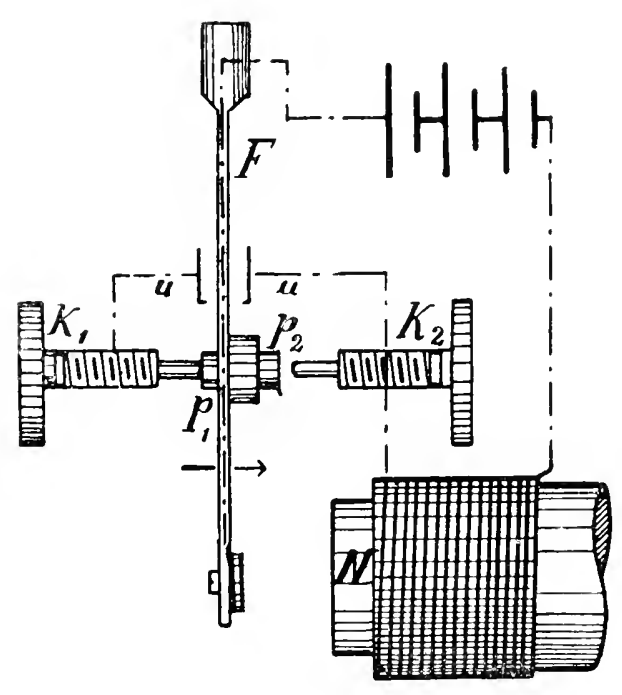

FIG. 24. terruptions compared with Neef's apparatus. It gives, moreover, a much longer period of contact, and, consequently, more powerful induction effects (Fig. 25).

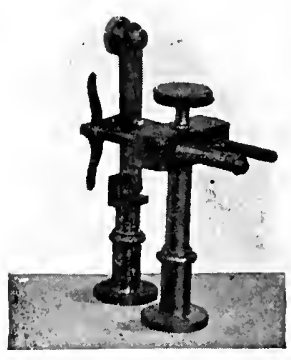

FIG. 25 .

FIG. 24. $-F$. Dissaucr's platinum-interrupter.

FIG. 25-F. Iessaucr's platinum-break, Aschaffenburg.

All platinum-interrupters have one defect in common: they are liable to be soon destroyed, the surfaces in contact being burned away. Morcover, the spring often "sticks." The rapidity of interruptions again is small in proportion to what is attained with more modern apparatus. This rapidity, nevertheless, must not be too great, otherwise the extra current formed at each closing of the inducing curreni cannot properly escape, and the core of the coil cannot be fully magnetised, in consequence of which the induced current does not reach its maximum of intensity. Spring-interrupters have the advantage of cheapness and simplicity, and may be useful in dealing with less powerful primary currents and smaller coils.

Foucault invented a mercury-intermpter for working large coils. The main principle of this apparatus consists in closing 
the current by means of a metal rod dipping into a ressel of mercury. In order to effect a sudden interruption, aroiding at the same time the formation of an extra-current spark in the air and the production of mercury-fumes, the mercury is corered with a layer of water, or a mixture of water and alcohol. By allowing the interruption to occur in liquids of low conducting power, instead of in the air, we are able to obtain a more rapid series of interuptions in the primary current, also a more rapid escape of the "opening" induced current, whereby the latter gains in intensity. Again, with the air-spark at the site of interruption we have connection maintained for several moments between the separated portions of the interrupter; this favours the strengthening of the opening-spark by the extracurrent. When employing a badly conducting liquid as the medium the formation of the opening-spark is prevented, whereby the circuit is more rapidly broken.

Fig. 26 shows on the right the simplest form of mercuryhammer-interrupter (on the left is a Nef's hammer). The illustration clearly shows its mode of action. In smaller coils

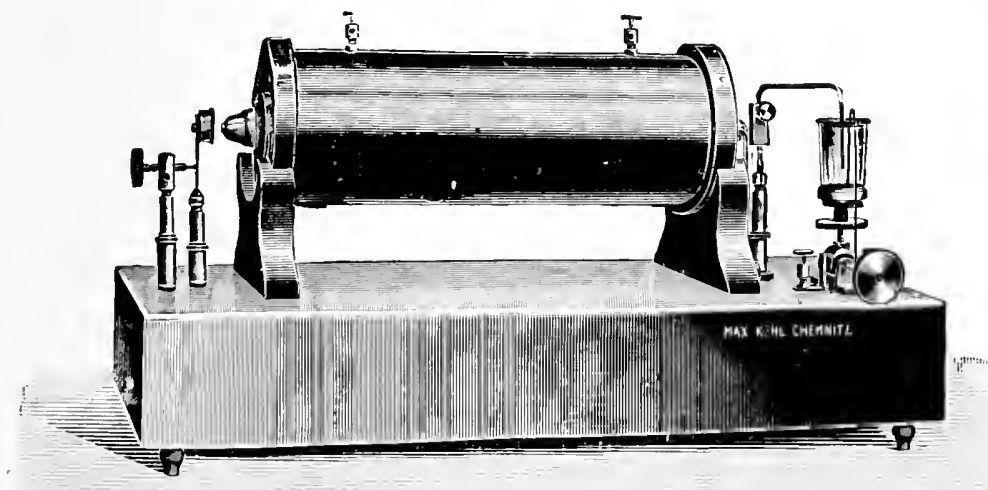

Fis. 26.-Siark-coil with Necf's hammer and mereury-interrupter, by . Hax Kohl. Chemnit\%.

the contact-pin is attracted directly by the iron core. In the case of larger apparatus, however, the interrupter acts independently, having its own electro-magnet and special electrical 
supply. In order to be able to regulate the rapidity of interruption, the lever, which carries on one hand the contact-pin, and on the other the anchor for the magnet, is provided with a vertical rod along which a movable weight glides. By raising or lowering this weight the movement of the lever can be varied.

Interrupters of this kind have two mercury vessels with ascending and descending contact-pins. At one of these the

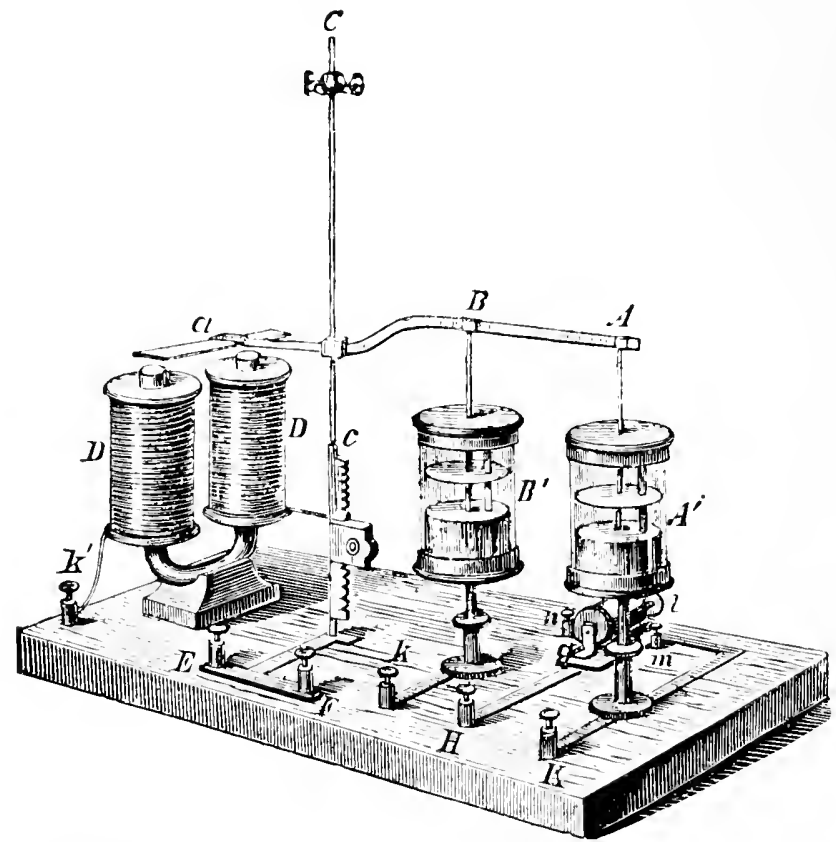

FIG. 27.-Foluculte's interrupter. (From J. H'allentin, Die Generationen der Spannungselcktricität, Wien, Leipzig, Budapest bei Hartleben, p. 223.)

interruption occurs of the special current intended for the electro-magnet of the interrupter. It the other, at the same time, the curient traversing the primary coil is alternately opened and closed (Fig. 27).

Foucaull's interrupter cannot work above a certain speed. It is, however, desirable for many purposes to have a greater range of current-alterations within the time-unit at one's disposal. This requirement is met by the recently-constructed mercury-motor, turbine and electrolytic interrupters. 
In the first, small rapidly-moring motors are employed, worked by a special current. A rertical movement is obtained from the rotation of the motor (by means of a small eccentric

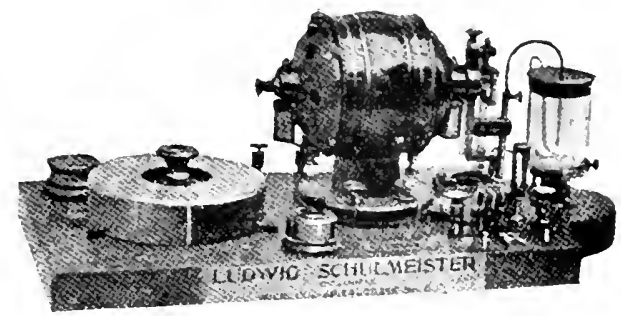

FIG. 28.-Ludaig Schulmcister's interrupter, Vienna.

or a crank-pirot and connecting-rod) and transmitted to a silvered rod dipping into a ressel of mercury which can be raised or lowered. With the rod immersed in the mercury the current through the primary coil is closed; with the rod raised from the ressel the current is opened. By means of a regulating resistance the rapidity of the interruptions may be varied

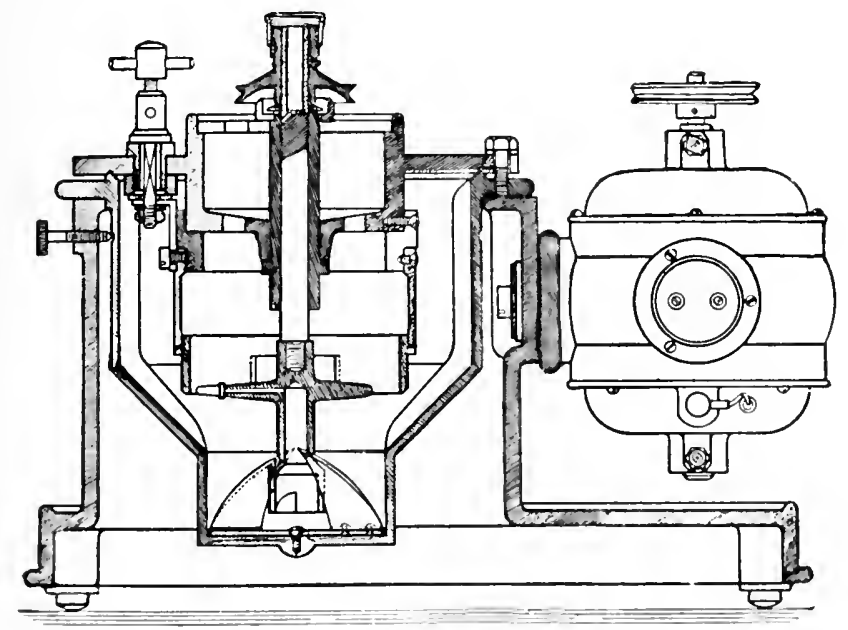

Fis, 29-Turbine-mercury-break by Boas (Allg. Elek. Gesell., Berlin).

through a wide range. The mercury is covered with a layer of alcohol, petroleum, or pure water. Fig. 28 shows the design of an interrupter mate by $I$. Sithlmeisher, of Vicnna. 
Like the coil to which it belongs, this is worked by a branch from the main (street-current, when this is continuous), consuming but little electricity, is comparatively noiseless, and is of very simple construction. Consequently it can readily be taken to pieces and put together again, and the mercury vessel can be easily cleansed. The resistance-dial indicates approximately the number of interrupters per time-unit. An interrupter of another kind, which gives a still higher rate of interruptions, is the turbine-mercury interrupter of the Allgemeine Elektricitäts-Gesellschaft, Berlin (Fig. 29). A metal tube, bent at right angles, has one arm vertically immersed in mercury. By rapid rotation of the tube, with this vertical arm as axis, mercury is sucked up and emitted from the horizontal arm in the form of a jet, owing to centrifugal action. The jet strikes a metal ring perforated at intervals with apertures. With the jet striking the metal ring the current is

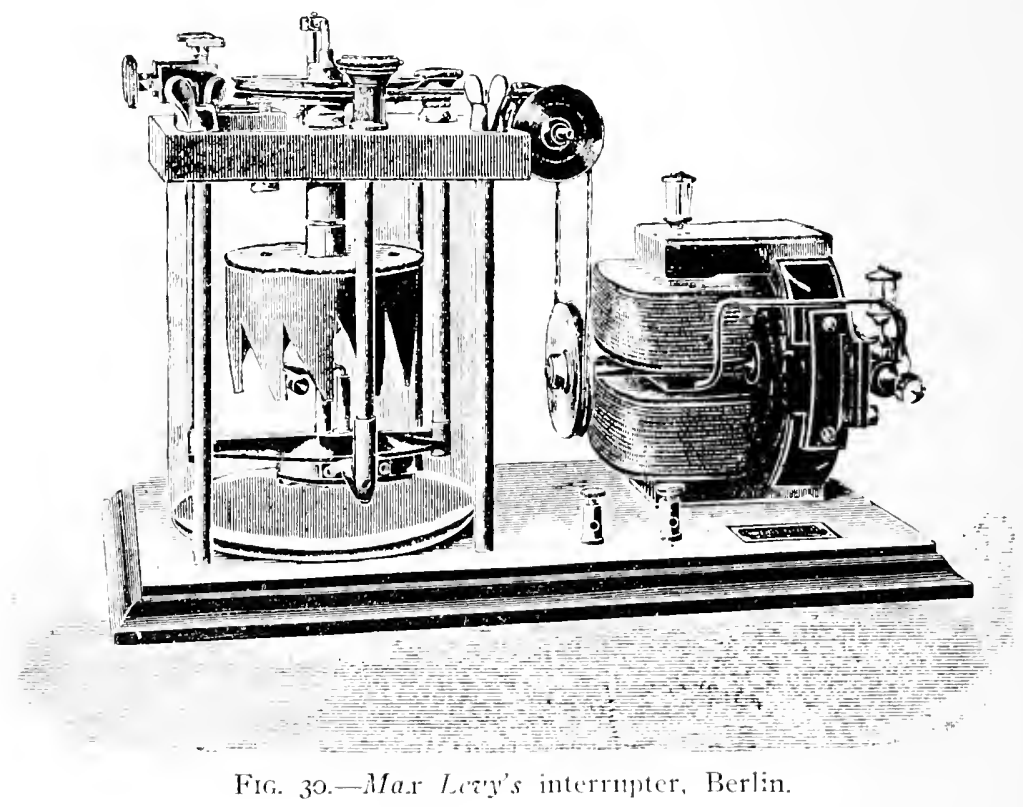

closed; each time, however, it coincides with an aperture the current is opened. Consequently the number of interruptions in a given time can be increased by employing rings with a 
greater number of apertures. The rate of interruption, of course, also depends upon the speed of revolution of the tube (which is actuated by an electro-motor whose speed can be varied by resistances). "The mercury is covered with a layer of

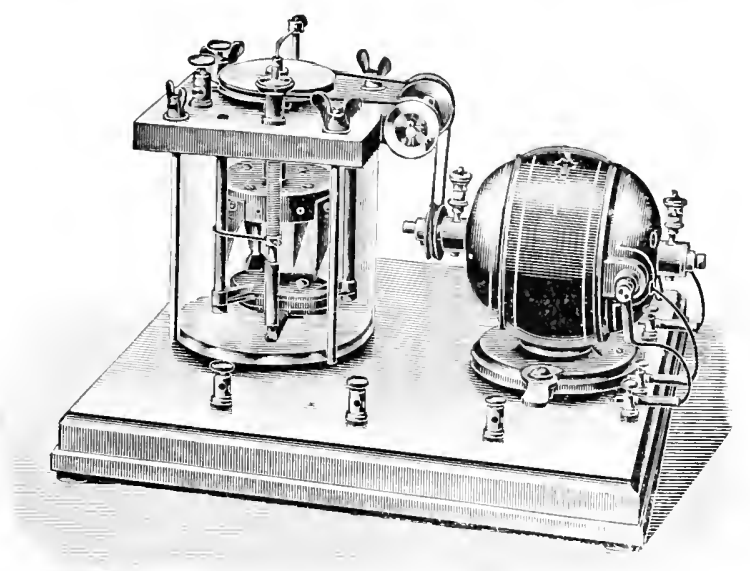

Fig. 3I.-Mercury-break by Rimiger, Gibbert and Sihall.

alcohol. About $\mathrm{I} 50 \mathrm{c.cm}$. mercury and $1400 \mathrm{c.cm}$. absolute alcohol are required to fill the interrupter.

Max Levy, of Berlin, has devised a modification of the apparatus (Fig. 30). The chief difference lies in the fact that the mercury jet rotates, and not the "contact-ring." By means of a suitable pump, which has an axis in common with the ring, mercury is driven up the rectangular tube and squirted in the form of a jet against the contact ring and its apertures alternately. The contact-pieces being triangular, with the apices below, by adjusting the height of the mercury tube it is possible to vary the duration of the individual current-periods. The pump works even when the motor is going very slowly, so that this type of interrupter can be used when a very slow rate of interruption is required. In the original apparatus a glass covering encloses all the working-parts; the condition of the mercury, etc., can thus be kept under observation and control. In Reiniger, Gellert and Siluall's apparatus the mercury (Fig. 3I) is squirted from a fixed tube $I$ ) a gainst a fixed contact plate $C$, the interruption being effected by rotating wing-shaped 
insulating pieces $F$, which cut the jet at intervals and prevent it from striking $C$. The mercury is pumped from $D$ by a centrifugal-pump placed in the case $C$, whose axis also carries the interrupting wings. The length of the mercury jet can be varied by means of a screw placed on the cover of the apparatus which raises or lowers the contact plate. The rapidity of the interruptions depends upon the rate of rotation of the main axis, the number of wings and their size, and upon the distance of the contact plate from $C$.

The turbine-mereury-inlerrupters for alternating currents of the Allgemeine Elektricitäts-Gesellschaft, Berlin, are constructed on the same principle as those for continuous currents. Their rate of interruption, however, cannot be changed at will; it depends always upon the number of periods of the alternating current which is used, and this in most cases amounts to $5^{\circ}$ per second. The apparatus is constructed exactly on the same lines as the turbine-continuous-current interrupter. The motor, however, is not fitted at the side, and is not joined to the turbine axle by an endless cord, but lies over the interrupter and is directly coupled with the axle. The speed of the motor exactly conforms with the alternation-rate of the current, so that the current-closings and interruptions always occur at the same periods of the alternating current-phase. 'The arrangement is of such a kind that the current is only closed in the same half of the current-phase, so that only current waves of one direction enter, their effect being, therefore, identical with that of an interrupted continuous current. In order that the motor may run with a certain velocity, corresponding with the rate of alternations of current, it requires some regulating. This is effected by means of a hand-wheel which is pressed against the axle of the interrupter by an eccentric. A Roentgen-ray tube introduced within the secondary circuit of the coil shows by its regular end even fluorescence when synchronism has been attained in the interrupter (Fig. 32 ).

A. I.onde and L. Leroy, also $I V$. A. Hirschmann, constructed rotary interrupters with sliding contacts working without mercury jets.

In these the interruption is brought about by a 
contact brush sliding over a round metal disc provided with insulating sectors. These appliances give a considerable rate of interruption (up to 2,000 per minute). Quite recently an interrupter has been brought out which works on an entirely different principle and is a simple and effective apparatus. 'This is $A$. W'elunelt's ${ }^{1}$ ) electrolylic merrupler, in which all the parts are fixed, and whose effect lepends entirely on electrolytic action. It can give an enormous rate of interruption (from sereral hundreds to 2,000 per second), and this with regularity.

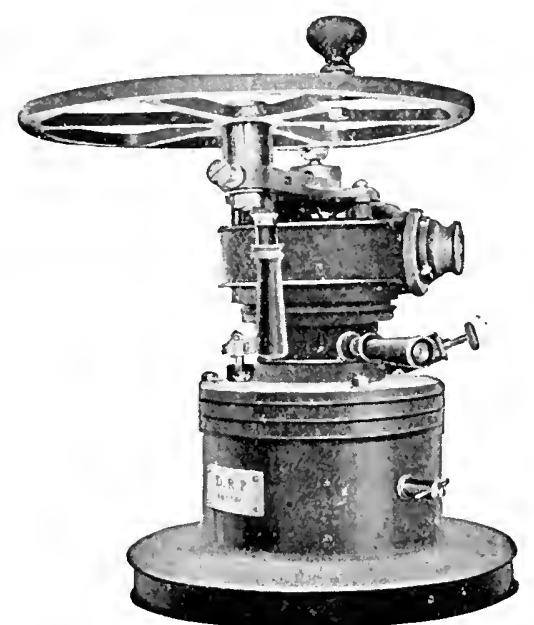

FIG. 32--Turbine-mercury-interrupter for alternating-currents. (Allg. Elek. Gesellsch., Berlin.)

Many physicists have studied the peculiar light- and heat-effects which are produced by passing powerful currents from small electrodes through a conducting liquid. While experimenting on the question of electrolysis $/ /$ elunelt was struck by the humming sound elicited when the smaller of the two electrodes (using thin platinum wire and a large lead plate in dilute sulphuric acid solution) was connected with the negative pole of a powerful hattery. Richars (1890-1892) had already shown that this humming sound corresponded with irregularities in the current traversing the fluid.

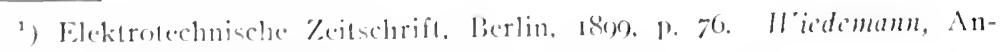
nalen, 1890. Bir. I.Xlill, 1, 23.3. 
Whehnell now examined this inconstancy of the current more closely and found that it consisted of a series of total interruptions. It is calculated from the position of the humming sound in the scale that there are some 1,700 interruptions per second.

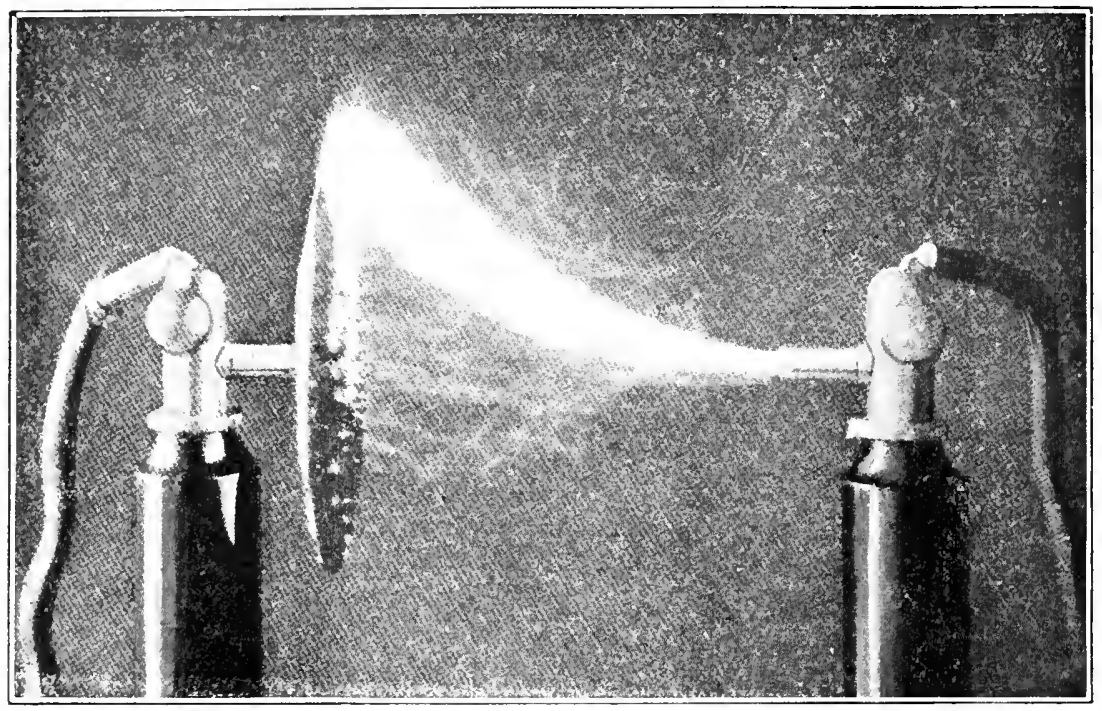

FIG. 33.- Spark effects with an instrument (electrolytic-interrupter) by Man Lea'y, Berlin.

Acting on these observations, the idea occurred to $V^{\prime}$ elhnelt of placing an electrolytic apparatus in the primary circuit of a coil; he thus obtained a most effective interrupter. IVith higher tensions ( r o volts) the smaller (negative) electrode soon became red-hot and melted away, the spark-stream of the secondary circuit becoming feeble and irregular. Much better results were obtained when the smaller (the "active") electrode was made positive (anodal) and the larger one negative (cathodal). "The result was surprising. On passing the currents (about ron volts) a powerful arc-light was produced between point and plate of the secondary coil terminals. When point and plate were separated for about $25 \mathrm{~cm}$. this arc-light was broken up into a sheaf about the thickness of a lead pencil, composed of innumerable sparks interwoven one with another." Fig. 33 gives an illustration of this phenomenon. 
If the Ruhmkorff's coil be excluded from the current-circuit no interruption occurs, but the active electrode immediately begins to glow; on introducing the coil this glowing ceases and a distinct sound is heard, the note of which corresponds with the interruption-rate. A reddish-yellow veil of light now surrounds the active electrode, but when the latter is also negative this light becomes of a bluish-white tint. Spectrum analysis shows in the former instance the spectrum of hydrogen, in the latter that of platinum.

The foregoing observations show that the active electrode must be connected with the positive and the other electrode with the negative pole of the main current in order to obtain good and constant action of the electrolytic interrupter. The most common form of this interrupter consists of a large square glass ressel covered by a perforated rulcanite lid. On the lat-

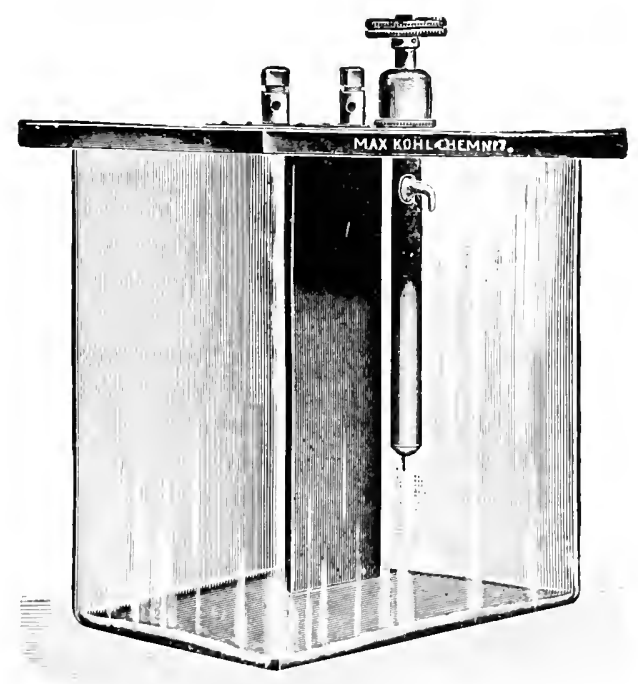

FIs. 34-Max Kohls clectrolytic interrupter, Chemnitz.

ter lies a terminal in comnection with the negative lead plate. A metal rod pierces the lid, bearing at its lower end a platinum pin, while a porcelain tube encloses the rod itself. Some acid always rises between the anode-pin and the porcelan tube; this, 
if allowed to rise as far as the ressel lid, would penetrate the fittings of the tube and rod, and so reach the surface, thereby causing a side-circuit. It is, therefore, necessary to allow a vent for this unavoidable rising of the acid (which is partly an effect of the explosion), and this is done by fixing a small glass tube on one side of the upper part of the porcelain tube. The upper end of the metal rod (anode) is connected with the positive pole of the main supply by means of a terminal screw. Here, also, is a regulating screw by which the platinum pin may be made to emerge more and more from the porcelain tube; thus the active surface of the anode (i. e., that part of it through which the current enters the fluid) may be increased or diminished at will.

Other things being equal, the rate of interruptions depends upon the area of this active surface; the greater the surface, the lower the interruption-rate. Thus by increasing the active surface of the platinum pin, by pushing it further out of its insulating cover, the rate of interruptions is lowered; on the other hand, the current-strength is increased. If one increases tension by switching off resistances at the current-regulator, without at the same time increasing the platinum anode-surface, the rate of interruption and the current-strength are both augmented. This is at once made evident by the louder humming sound, the pitch of which is raised.

Other factors on which the interruption-rate depends are the resistance and the self-induction of the circuit made and broken by the interrupter. Now the part both these factors play bears relation to the fact that the interruption takes place at a certain current-strength, penetrating through the surfaceunit of the active surface. This current-strength must be attained before an interruption can possibly occur. The time required for this purpose depends to a great extent upon the resistance and the self-induction of the current-circuit, as we have learned from the theories relating to current-closing. The smaller the self-induction, the greater the number of interruptions per second. In the case of a circuit without self-induction the apparatus does not work, but gives one interruption only. Without self-induction in the closing-circuit the tension 
required for the production of the above phenomena is generally a higher one. The current-intermptions of an electrolytic interrupter are of such a kind that no condenser is required for the coil. Self-induction, which in other cases is most carefully aroided, or at least minimised as far as possible, is not only absolutely harmless when using interrupters of the I' ehnelt type, but actually farours the production of the Roentgen-tube phenomena and exact interruptions.

Ioller and $I V$ alter explain this peculiarity by the fact that the primary opening tension (which with other interrupters produces disturbing secondary effects with the opening-sparking, having, therefore, to be diverted into the condenser) is actually a farourable factor with electrolytic interrupters by reason of its decomposition-effect on the water-gases, whereby the continuity of the current can be restored.

In some recent Roentgen-ray apparatus the electrolytic interrupter is so arranged that in order to work tubes of varying degrees of hardness several ( 3 to 6 ) different sizes of active electrodes are employed. These may be switched on at the connecting-board without having the troublesome arrangement of the platinum-pin to go through. ( $/{ }^{\prime}$ alter and AlbersSchönberg's compound Wehnett.)

$I^{\prime}$. A. Hirschmann's electric interrupter with surface contacts (Figs. 35 and 36 ) consists of a platinum plate lying be-

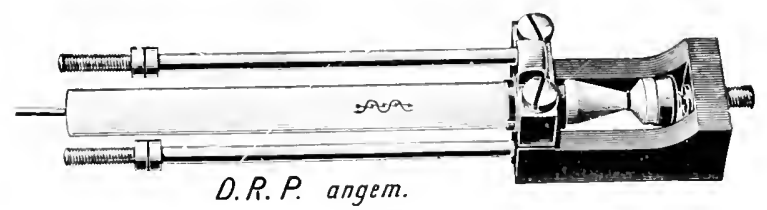

FIG. 35.

tween two porcelain surfaces, and in comnection with the positive wire, and also a rod-shaped leaden electrode, the size of which is varied according to the current-strength. The platinum electrode may be converted at any time into another of different thickness. By this arrangement not only is a smaller current consumption secured, but another practical advantage is gained. For any defect in the porcelain tube arising from 
wear and tear can be repaired by simply grinding off the end of the tube. With other electrolytic interrupters the porcelain tube after a time becomes hollowed out by the flame proceeding from the platinum pin, whereby it is rendered useless

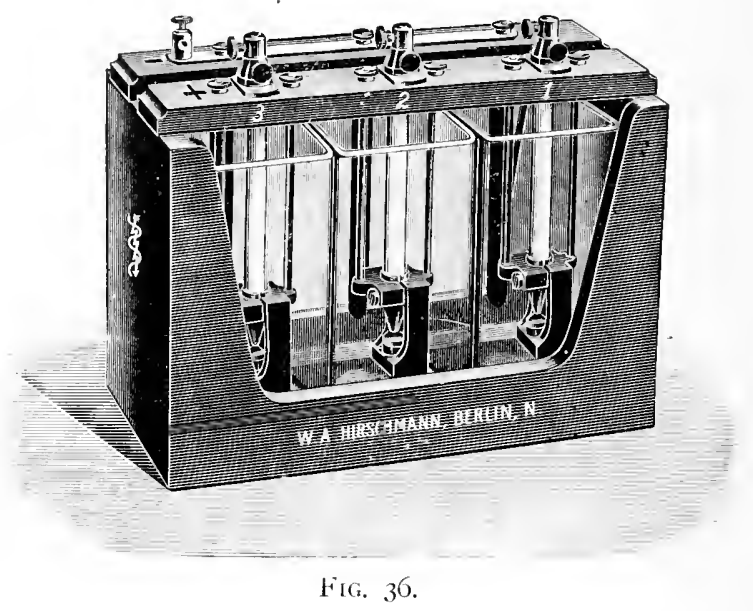

and must be replaced. Interrupters of this type are also constructed with several pairs of electrodes.

In order to gain a proper conception of the events taking place in an electrolytic interrupter, it is necessary, as Voller and II alter have shown, to examine the gases appearing at the electrodes, both in regard to quantity and kind. We find the gas evolved at the active electrode consists of not only oxygen, which must appear at the anode, but also of hydrogen, which with normal electrolysis appears at the cathode.

This apparent abnormality might be explained by assuming that the great heat attained in the locality of the active electrode (resulting from the narrow sectional area of the conductor here) causes an evaporation and decomposition of the water, so that in addition to the oxygen which is electrolytically separated, both hydrogen and oxygen appear from the steam. The cause of the interruption may, therefore, be found in the gaseous envelopment of the active electrode. As soon, however, as the current (and with it the heat-supply) is cut off, condensation of the steam occurs by the surrounding cold liquid, where- 
by the current circuit is restored. It may be mentioned in proof of this theory that as soon as the water has reatched a temperature of $90^{-}-C_{\text {. }}$ whereby condensation can no longer take place, the apparatus ceases to work. The action of the apparatus is helped by the opening-spark, which Hies through the zone of steam and gas round the anode (hence the luminosity in this region), thereby farouring the union of hydrogen and oxygen in case these have not already escaped.

The foregoing gives an outline of the theories which If ellnelt formed on the mode of action of his interrupter. Moreover Simon ${ }^{1}$ ) considers that the heat developed so energetically in certain parts of the current-circuit, as described above, may be regarded as the essential cause of the interruption. On this hypothesis he has formulated a theory of I'ellmelt's interrupter which is certainly in harmony with experiment, so far as the interruption-rate is concerned. A double cell (Fig. $37, d t$ ) with two large lead plates $F F$ as electrodes, divided by a porcelain partition $D$, which is furnished with one or more small apertures, should act as an interrupter (CaldwellSimon interrupter). This is found to be the case. The interruption takes place at the small aperture through which the cur-

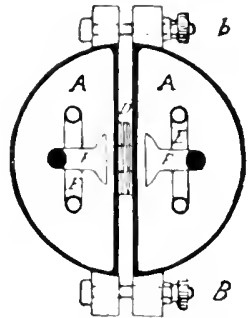

Fiti. 37. - Platinuminterrmpler. (After Ruhmer. ly Matr Live. Berlin.) rent is made to pass. Here watery rapour is periodically evolved, followed each time by condensation, and thus the current is alternately broken and made. According to Simon, this interrupter differs from /l'huell's in being independent of the current direction, working equally well with continuous or alternating currents. The apparatus, moreover, does not stop working when the acid has become heated after long working. "l'hmell's apparatus, as has been mentioned, is useless when once a temperature of $90^{\circ} \mathrm{C}$. has been attained. I'Arsoneal found that /'ehme't's interrupter works well with an alternating current of 110 volts, and is as

1) Elekirotechn. Zeitschr., 18(x). 1. 4.40. 
good for Roentgen work under these conditions as a continuous current. This proves that the current interruption only takes place during one phase. The effect on the interrupter during that phase when the active electrode is negative is quite insignificant, and may be disregarded. When using an alternating

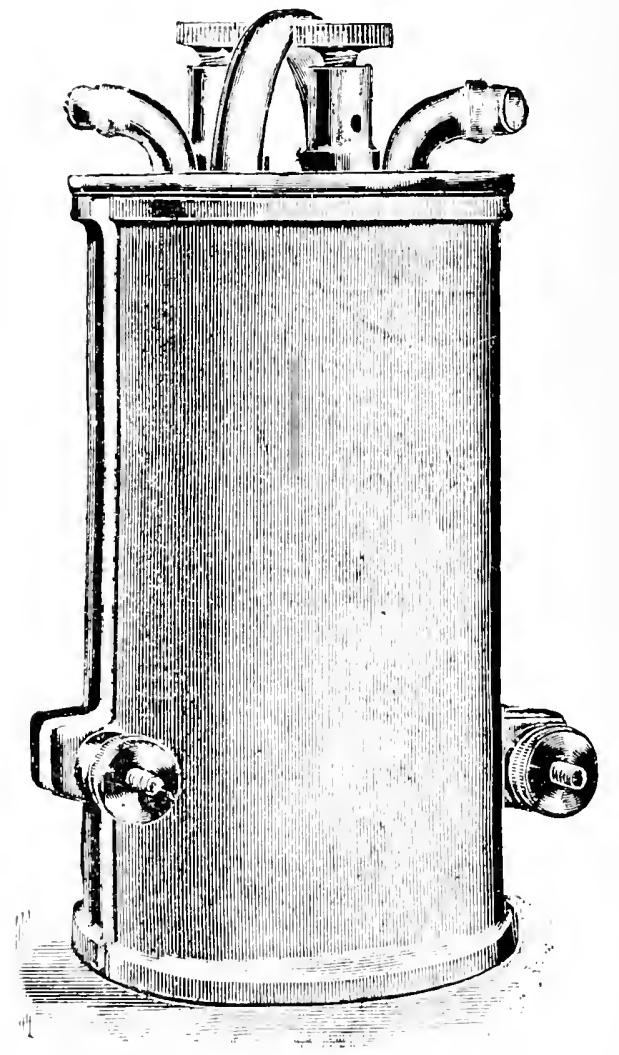

Fig. 38.-Mar Ler'y's platinum-interrupter, Berlin.

current the wear and tear of the platinum pin is considerable; it requires, therefore, to be made of corresponding strength and size.

Simon's interrupter acts, however, with each phase of an alternating current, being independent of the current-direction, and is consequently not suitable for X-ray work in its original form. By utilising a property of aluminium discovered by 
Pollak ${ }^{1}$ ) and Graels-), however, this interrupter may be so modified that by its use an alternating current may be made continuous. These observers found that by employing this metal as anode a great resistance is offered to the current's passage in certain electiolytic liquids (especially potash and soda), and that if the tension does not exceed a certain limit the current-flow is completely barred. If, then, one of the lead electrodes $F$ (Fig. 37) be replaced by an aluminium plate, the current only flows through to the primary coil when the aluminium is acting as cathode.

The platc-intermpter is a modification of Simon's apparatus. In order to alter the size of the plate-apertures, and thereby the rate of interruption, the apparatus is provided with porcelain plates $D$ having holes of different sizes. The plates are interchangeable. Over-heating from high current-strengths or prolonged use is prevented by means of a system of cold-water circulation through a porcelain tube $E$ attached to the lid of the apparatus:

The use of electro-magnetic induction apparatus in radiotherapy will be discussed in later chapters.

1) Elektrotechn. Zeitschr., I897, p. 359 .

?) Sitzungslor, d. k. bayr. Akad. d. Wissensch, I Mai, 1897. 

II.

TREATMENT WITH HIGH-FREQUENCY CURRENTS. 



\section{TREATMENT WITH HIGH-FREQUENCY CURRENTS. ${ }^{1}$}

\section{\$2I. High-Frequency Currents.}

In the year I $8 S_{1}$ Morton ${ }^{2}$ ) described a method of using electricity for the local treatment of muscle and nerve disorders. He brought the terminals of the discharger of an electrical machine so near together that sparks passed between them. He then put his patient in the circuit connecting the tin-foils of the condensers. Morton was the first to produce by means of this arrangement and to therapeutically apply high-frequency currents, for he had used the oscillating character of the condenser-discharge to increase the frequency of an alternating current. The condensers were charged in this case through an induction apparatus. Morton's high-tension oscillating currents have been used by Leduc ${ }^{3}$ ) and F. I'inkler $^{4}$ ) in similar affections, and, of course, with results similar to those produced by other forms of high-frequency apparatus.

In the year i 893 Tesla described an arrangement by which he produced currents of rapid alternations and high-tension. He passed them through glass tubes containing a partial vacuum, thereby obtaining brilliant effects of illumination. These currents of Tesla are of great medical interest, since they produce powerful physiological effects, and may be applied

$\left.{ }^{3}\right)$ Literature referred to: Noumcr, Annales d'électrobiologie, électrodiagnostique et électrotherapic, Vol. I-IV. - Dr. H. Kurella, Zeitschrift für Elektrolherapic und ärztl. Elektrotechnik. Vool. I-IV. - Fouréu de Courmclles. L'annéc électrique, I900, 1901.-F. Winkler. Festschrift für Hofrath Prof. Noumann, igoo. - L. Bouchacourt and 1. Rómond. Annales d'électrobiologie, Inoo. Vol. III, D. 334. - Reiniger (Gebbert \& Schall's Catalogue, 1902 )

2) Qunted by Doumer \& Oudin. Ann. d'électrobiolngie, Inoo, p. 507.

3) Compt. rend. du XII Cong. int. de Médicines. Vol. II, Sec. IVa, p. 70.

-) Wiener med. Presse, Iyoo, No. 4I. 
successfully in the treatment of various diseases. In order to understand Tesla's discovery, one must have some knowledge both of the laws of electrical induction and of the phenomena accompanying the discharge of a Leyden jar. We know that electricity at the moment of its appearance and disappearance induces electricity in a neiglibouring conductor, and we know that one can produce alternating currents of high tension by means of induction apparatus. We have already learned (p. I 8 ) that when a Leyden jar is discharged the whole positive and negative quantities of electricity are not dissipated, but that many discharges of gradually decreasing intensity occur at very short intervals afterwards.

These facts have been known for many years. They were first theoretically deduced by Kirchioff and Thomson, who applied to electricity the laws already known dealing with the motion of liquids. Under like conditions the behaviour of electricity may be compared with that of water which is accumulated in two vessels in communication with each other at the bottom by means of a wide pipe. If the water level be lowered in one ressel so that it rises in the other, on leaving the liquid to itself repeated alterations in the level will occur whereby the water repeatedly flows to and fro within the pipe; the two columns of water will oscillate until equilibrium is finally established. Now the same thing occurs when the positively and negatively charged tin-foils of a Leyden jar are brought into contact. The electricities counterbalance themselves in the same way as do the differences of water level in the communicating vessels. The process of discharging is not completed in one transition of electricity from the inner to the outer tinfoil, but a repeated passage of electricity to and fro occurs.

It would seem as though electricity possessed inertia, though this is not the case. Self-induction takes the place of "vis inertix." When the excess of electricity begins to flow from the interior of the Leyden jar (which we will suppose to be charged at the beginning with positive electricity) an excessive amount goes to the outer tin-foil, leaving the interior of the jar lacking in positive electricity; the inner foil is thereby negatively charged. The superfluous positive electricity collected on the 
outside flows back again to the inside, but again in excess, so that a new current of electricity is produced in the opposite direction.

When the conducting wires of the two tin-foils are brought near together a spark results. The electricities in this spark are in reality flashing to and fro until equilibrium becomes established. This phenomenon is called electrical oscillation.

Clark Maxwell showed that a kind of magnetic transverse undulation occurs in the space surrounding a conductor where this electrical oscillation is going on. This undulation he believed to possess all the qualities of waves of light, and that while it may be transmitted with any wave length it must always have the velocity of light, i. e., $300,000 \mathrm{~km}$. per second (the electro-magnetic theory of light).

Hertz proved experimentally that an electro-magnetic disturbance is as an effect of induction transmitted with the velocity of light through space, and that these electric waves possess the same qualities as light. It is indisputable that electro-magnetic induction, electric and magnetic manifestations are transmitted through the air not instantaneously, but with the velocity of light. An electrical wave of this kind would, therefore, be identical with a light ware. As far as we know, the source of electrical oscillation is to be found in the discharging of larger or smaller quantities of electricity.

Kirchlooff has proved that electrical discharges are transmitted not only in the air but also along wires with the velocity of light. Lecher showed that the transwerse ether waves are transmitted between parallel wires in a direction parallel to the latter, whereas the direction of the wares of the main current, of the electrical force, is perpendicular to the surface of the wire.

Hertz proved that from such electric vibration ether waves proceed which are transmitted through space and non-conducting bodies (diclectrics) with a velocity which in the air is almost equal to that of light. These waves of electric power are subject to the same lawsof reflection, refraction and polarisation as ordinary light. Hertz reflected the wares from a perpendicular screen of metal; 
"fixed waves" were formed by interference between direct and reflected waves, between the primary conductor and the screen. In other words, at certain points there was no ether vibration. Hertz's electric waves penetrate insulating bodies, such as glass, paraffin, sulphur, wood, but never metals.

The current flowing through the wires in this experiment is repeatedly changing its direction; it is an alternating current. The current-reversals produced by discharging Leyden jars are extraordinarily rapid (alternaling high-frequency currents); it has been proved that they may take place ıoo,ooo times, even I, ooo, ooo times per second. (Alternating currents as supplied for ordinary purposes do not usually change their direction more than So times per second.) This alternating current soon loses intensity. But when an immediate re-charging of the Leyden jar is provided for by suitable apparatus a permanent alternating current can be supplied.

Tesla produced a series of remarkable phenomena by means of the electric oscillations caused by the discharging of Leyden jars. When the current of an ordinary alternating-current machine is conveyed through the inner winding of an induction coil the latter shows very powerful induction effects; the alternating-currents produced by the secondary coil are strong enough to light an incandescent lamp placed in their circuit. The inducing power becomes greater when the rate of alternation is increased.

Tesla alternatively charged and discharged Leyden jars by means of alternating-currents, by rapidly succeeding currents from a Ruhmkorff's coil, thereby intensifying the frequency of the currents. He converted these comparatively strong highfrequency currents into currents of extraordinarily high tension by conveying them through a primary coil of slight resistance (and self-potential); over this primary coil he placed a secondary one, formed by numerous windings of thin wire. He thus obtained induction-currents of remarkably high-tension and high-frequency. Primary and secondary coils together are called Tesla's iransformers.

In Fig. 39 we have a sketch of Tesla's apparatus. The 
inner tin-foils of the Leyden jars $C_{1}$ and $C_{2}$ are positively and negatively charged from the secondary terminals of the Ruhmkorff's coil $A$. The outer tin-foils are in connection through the primary winding $P P$ of Tesla's transformer and through the spark-gap $B$. Alternating-currents are produced in the primary coil of the transformer; they are synchronous with the oscillations of the discharge in the spark-gap. These high-frequency alternating currents induce alternating currents in the secondary coil $S S$; these currents combine high-frequency with high-tension. On account of the enormous ten-

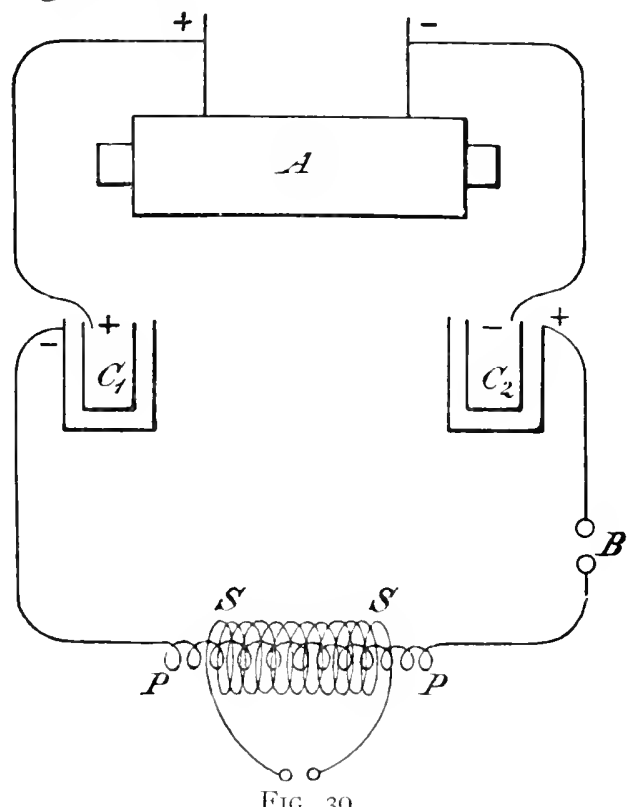

sion obtaining in the windings of the secondary coil of the transformer, the latter requires to be immersed in oil, which forms the only satisfactory insulating medium; otherwise sparks would inevitably pass between the separate windings of the wire and soon destroy the apparatus.

These high-frequency currents (T'esla's currents) possess some extraordinary physical properties. They do not require a closed conductor for their transmission; they are transmitted as electric waves through the air. They illuminate Geissler's tubes even from a considerable distance. They also possess a 
quality which is of a special medical interest: they are devoid of danger despite their enormous tension. When we consider that ordinary alternating currents of about 2,00o volts' tension are very dangerous to life, it is an extraordinary fact that these alternating currents, though possessing a tension of a hundred times greater, are absolutely harmless. An electrode of a Tesla transformer may be touched with a metallic rod held in the hand, without danger. An incandescent lamp, the poles of which are held by the experimenter and an assistant, will be illuminated the moment the current of sparks passes into the metallic rod. The experimenter's body forms part of the conductor for the current which is illuminating the lamp; the current must, therefore, be of considerable intensity; nevertheless it does no harm and causes no disagreeable sensation.

'This fact was known to the French physiologist $A$. d'Arsonval, even before Tesla. D'Arsonval proved that a solenoid traversed by high-tension-currents induces powerful currents in an organism which it encloses. A man's body placed within a large solenoid forms an electric conductor in which currents are induced of the same high-frequency as those in the solenoid. When the man within the solenoid forms a circle with his arms which is closed by a small incandescent lamp, the latter becomes lighted by the induced currents circulating through his arms. According to d'Arsonval, this experiment is more successful if the subject of the experiment first moistens his hands with a saturated solution of sal ammoniac. By so doing the resistance between the two hands is lessened by about 600 ohms. The experiment can also be performed with other animals, $e$. $g$, eels. Although this procedure acts very vigorously on the organism, no sensation is produced. D'Arsonval published the results of his experiments in $\mathrm{I} S 9 \mathrm{I},{ }^{1}$ ) but he employed a rather different apparatus to that of Tesla.

There are two Franklin plates (condensers) C (Fig. 40); the tin-foils of one side of these plates are in circuit with the poles of the secondary coil of the induction apparatus $A$ and spark-gap B. The tin-foils of the other sides are connected by

') Société de Biologic, Fel, 24 and April 24. IS9I. 
a thick copper wire $D$, which forms a little solenoid having from I 5 to 20 windings. Besides this smaller solenoid a larger one $E$ can be placed in the circuit of the discharge-current at $H$; the patient is placed within the larger solenoid. Synchronously with every discharge through the sparkgap a corresponding discharge takes place on the tin-foils of the condensers in connection with the solcnoid. These discharges pass through the solenoid, where in consequence high-frequency currents arise which are synchronous with the oscillations of the dischargespark at the condenser, and possess about the same hightension as currents from an electro-static $\mathrm{machinc}$ (about 2 o, o o o volts). D'Arsonial originally arranged the experiment so that the terminals of a "shunt" were in connection with two windings of the

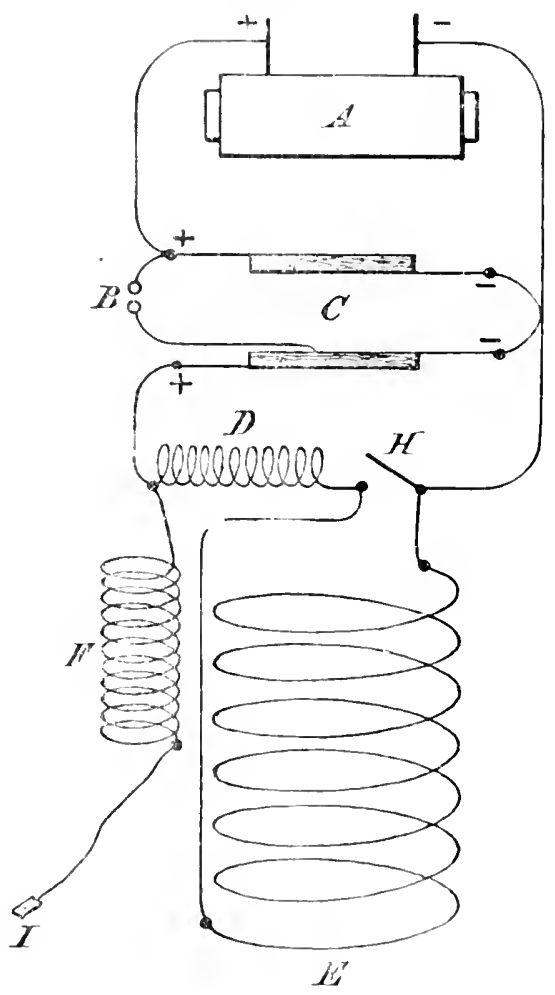

FIG. 40., solenoid $D$. This formed a conductor of weak resistance and helped in the production of self induction ${ }^{1}$.

The effect of the high-freguency currents in the shunt was more intense when its points of contact were far apart. The maximum effect was gained when the contact was connected with the first and last winding.

The high-frequency currents passing through the solenoids create an electric field in their vicinity. This can be demonstrated by the fact that an incandescent limp placed in a metallic

1) A mowalle core of soff irom whin the sulemerel alow helps in this way. 
circle which is suspended within the solenoid $E$ without contact with the same is illuminated as soon as the solenoid is traversed by the high-frequency currents. If the bulb of a thermometer be wound round with several turns of thick copper wire, through which high-frequency currents are passing, the mercury in the bulb becomes heated and may even be brought to boiling point. The electric field existing in the vicinity of high-frequency currents has a marked effect in the cure of several diseases. $D^{\prime} \mathrm{Ar}$ sonval utilised this physiological effect on the human body by exposing the whole body of the patient to the influence of the electric field ("general treatment").

Faraday, Maxwell and Hertz found that conductors had the effect of localising the electric oscillations on their surface, so preventing them from becoming diffused; acting on this idea Oudin arranged an apparatus by means of which high-frequency currents could be localised and made to act on circumscribed parts of the body. He constructed an apparatus like a tuning fork, having a certain rate of vibrations; through this the highfrequency currents were passed, while a second tuning fork was brought near the first (both forks being in tune with each other). When the first fork vibrated the second also vibrated in sympathy with it, producing the same note. Hertz proved by means of this instrument, which he called a resonator, that within the compass of the waves of a primary electric conductor vibrations are induced in another (secondary) conductor; these are strongest near the elevations of the waves and weakest near the nodal points. The intensity of electrical resonance depends upon the shape and size of the secondary conductor. Hertz found that a resonator of a definite capacity is necessary for a given primary apparatus in order to obtain the best effect. The resonator must be tuned to the primary conductor.

Oudin proved that the electrical vibration in the resonator depends directly upon the capacity and self-induction of the circuit formed by the resonator.

The vibrations which occur in a d'Arsonval's solenoid may be used as "primary" oscillations; differences of electrostatic tension will arise in every conducting body of a certain capacity placed near the solenoid, and these differences will produce cur- 
rents in the body itself. When the conductor is formed of several windings of copper wire, currents will be formed by self-induction in the separate windings strong enough to enable sparks to be drawn from the end of the conductor; here, too, the maximum of efficiency will be obtained with bodies of a definite electric capacity. This capacity must be rariable according to the construction of the high-frequency apparatus which is being used. Metallic bodies placed in the neighbourhood of the solenoid emit a very long spark or none at all, according as the rate of vibration produced in them is increased or decreased; in other words, the capacity of the body has to be tuned to that of the solenoid. These differences become still more marked when the body is connected to the solenoid through a metal wire; but they are only then shown when the body possesses a certain self-induction. Thus such effective vibrations can be produced in proportion to the self-induction and capacity of the body as to give very long sparks. If one of these factors, either the self-induction or capacity, be altered, the oscillations rapidly diminish, and may even disappear entirely when only a slight difference obtains between the two factors.

Oudin's resonator considerably increases the tension of the high-frequency current; it is easily adjustable, so that it can be adapted to all phases of d'Arsonval's apparatus, i. e., it can be tuned to another electrical pitch. In effect the resonator consists simply of a solenoid of copper wire having a low resistance, which is wound round an insulating cylinder. When one end of d'Arsonval's solenoid $I$ ) is connected by wire with a certain point of this resonator a powerful and continuous aigrette of sparks becomes visible at the end of the resonator. In this attached resonator the electrical oscillation takes place synchronously with that in the connecting wire (solenoid).

By a suitable arrangement an aigrette of sparks 9 to $10 \mathrm{~cm}$. in length may be emitted from the resonator, while that from the primary solenoid is only is to $20 \mathrm{~mm}$. in length. When the point of contact of the wire is movel some centimetres in one direction or the other, the length of the sparks becomes rapidly diminished. The secondary solenoid must, therefore, be tuned to the primary in order that its vibrations may be synchronous. 
When the capacity of this resonator is increased by connecting its free end with a wire terminating in an electrode, the rate of oscillation is modified; in this case the point where the current enters the resonator must be altered in order to obtain at the electrode the same aigrette and the same spark as were emitted by the resonator without the electrode. It is only the last spiral of the resonator or a conductor joined to it which emits sparks. The effect of the remaining windings upon each other prevents them from giving discharges into the air. When a larger capacity is added to the resonator, $e$. g., larger Leyden jars, the sparks grow more rigorous and may cause a painful sensation. (Oudin consequently only used small condensers.) A resonator which is made of thin wire emits a thin, long, sinuous and comparatively painless spark, and the aigrettes (or quiet discharges) are less vigorous. By using the thicker wire both discharge and spark grow more powerful.

\section{\$22. Apparatus.}

Direct currents from the main, accumulators, or bi-chromate batteries of about 6 ampère intensity are usually employed as generators of high-frequency currents for medical purposes. These currents are first transformed from low to high tension by means of a Rullmkorff's coil, the sparking capacity of which need not exceed $25 \mathrm{~cm}$. The primary current is interrupted by means of a motor-mercury break, a turbine interrupter, a I'chnclt's apparatus, which latter is especially suitable. The alternating current which is produced by the coil, and which cannot be used directly, is transformed by the condenser into a highfrequency current. The condenser consists of two Franklin's plates, arranged as in Fig. 40 and placed within a fat box (Fig. 4 I ), outside which may he seen the small solenoid and the sparkgap with the connecting screws. Or the apparatus may be constructed as shown in Fig. $60 e$. Here two Leyden jars are placed behind the spark-gap (which is put under a bell-jar to deaden the sound of the discharge; within the bell-jar a small ressel containing soda-lime is placed to absorb the nitrous fumes. From the outer tin-foils of the jars two conductors issue which end in two terminals, between which a third is placed. Where 
general d'arsonvalisation is required the small and the large solenoid are joined to this terminal.

The author made his large solenoid as follows: A basket wound round with twelve turnings of thick copper wire is placed on a long table. The windings of copper wire are continued through two terminals on either sicie of the table into other windings below the table top; the two terminals of these windings are in connection with the terminals of the condenser

Ondin's earlier resonators consisted of 40 to $50 \mathrm{~m}$. of copper wire one-half to $3 \mathrm{~mm}$. in thickness, wound round an insulating cylinder 40 to $50 \mathrm{~cm}$. in height and $30 \mathrm{~cm}$. in diameter,

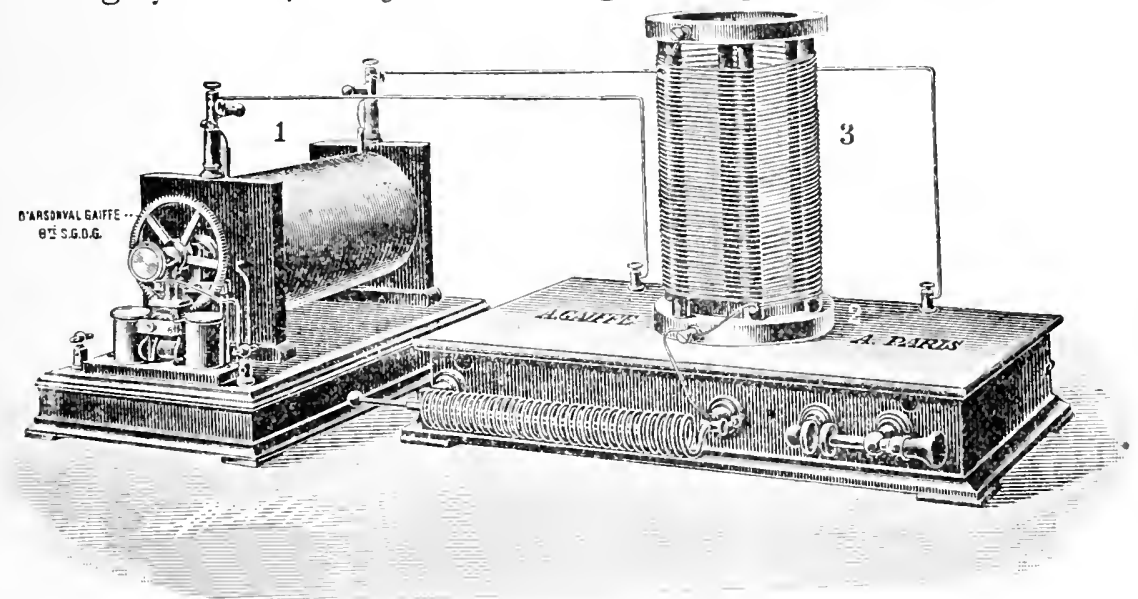

FIG. 41.-D'drsonzal's apparatus.

the distance between the several spiral windings being $1 \mathrm{~cm}$. By means of a terminal the conducting wire from one end of a d'Arsonval's solenoid was fixed to a suitable part of the resonator. The other end of the solenoid remained free or was earthed. (Later on Ondin comnected this with the lower end of the resonator.) 'The modern type of resonator, $c . g$., that by Radignel, Dncretet, Bonelli, has the following construction (Fig. 42 ) : the inner tin-foils of the Leyden jars are connected with two spherical terminals, which can he approached to or removed from each other. The resonator proper consists of a vertical cylinder covered with paraffin wax and having a spiral groove in which the wire is laid. The wire is $2 ! \mathrm{mm}$. in thick- 
ness and has 50 turnings, with a distance of $8 \mathrm{~mm}$. between each turning; the whole length of the wire amounts to $45 \mathrm{~m}$.

The electrode is attached to the upper end of the wire; the lower end is connected with the outer tin-foil of one of the Leyden jars, while the outer tin-foil of the other jar is in connection with one of the lowest windings of the resonator, its wire being attached to a metallic ending which can be moved up and down the resonator along a groove. Thus the resonator is divided into two solenoids.

We have now to produce electrical oscillations in both systems. The rate of oscillation depends on the product of the

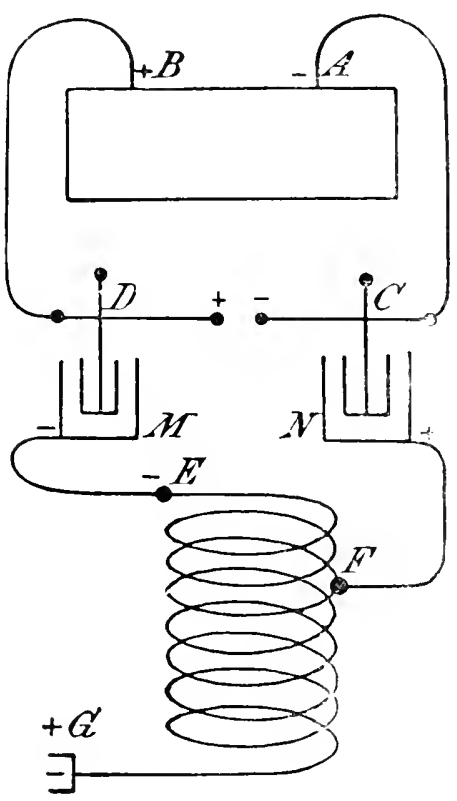

FIG. 42. capacities and self-inductions of these systems, and the arrangement is most effective if both systems have an equal rate of oscillation. The first system consists of the Leyden jars $N M$ (of constant capacity), the outer tin-foil of which is discharged through the spiral winding $E F$ (of variable self-induction). The second system includes a conductor of variable capacity, the body which is to be electrified, and a wire coil of constant self-induction. The second system must allow of being "tuned" in order to make the product of its capacity and self-induction harmonious with that of the first (resonance). This is accomplished by altering the number of windings, $i . c$., adding or taking away whole windings or portions of them (Oudin). Kurclla ${ }^{1}$ ) "earths" the end of the remainder of the resonance coil which is not included in the circuit so that this portion may not disturb the resonance.

O. Rochefort devised a bi-polar resonator (Fig. 43). He

1) VII Congr. d. deutsch. dermatolog. Gesellsch. Breslau, 1901. Sitzungsber., p. 488 . 
divided each of the outer tin-foils of his two condensers into two parts by connecting two of the inner tin-foils of 4 Leyden jars with each other, and attaching to each of these two connecting parts a metal rod ending in a knob $(. \perp B)$. These knobs placed opposite each other formed the discharger; the 4 outer tin-foils are placed in the circuit at four points $F I I M L$ of two resonators, as shown in Fig. 43. Under these conditions the two resonators, although acting upon each other, are, electrically speaking, separate and act like two resonators. They are worked by the same interrupter and each gets an equal cur-

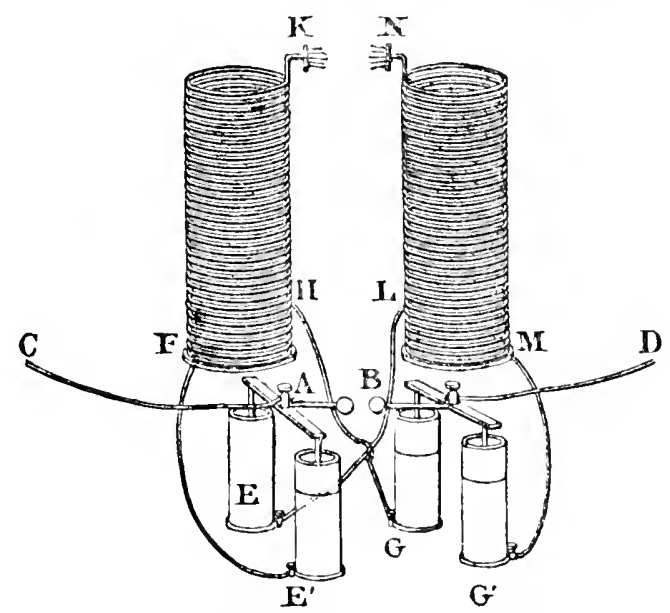

FIG. 43.-Rochefort's Bi-polar Resonator.

rent, though these run in contrary directions through the primary spiral. According to Oudin, the capacity of the primary spirai $F H$ must correspond with that of the secondary one, in order that the maximum of effluvium may be obtained. The secondary capacity consists of the capacity of the secondary spiral $H K$, and that of the whole conductor in connection with the point $K$. In order to make these capacitics agree the point $H$ must be moved elsewhere; both resonators require to be regulated in this way.

The direction of both effluria is toward one another, not because they are of contrary electricitics, but because the capacity of one resonator attracts the efflurium of the other. When equal effluvia are produced in both resonators by connecting the 
wire which leads to $H$ with $F$, and that which leads to $F$ with $H$, the mutual repulsion of both elfluvia can be easily seen in a dark room; this is shown still more clearly in a photograph. By arranging the apparatus in the way just mentioned, Rochefort obtained bi-polar effluvia $50 \mathrm{~cm}$. in length.

Rcchefort made a bi-polar resonator with two simple condensers by connecting the lowest spirals of both resonators with each other (Fig. 44, $M F$ ) and also the external armatures with two points near the middle $H L$. This arrangement, however, makes each resonator too dependent on the other; moreover, it

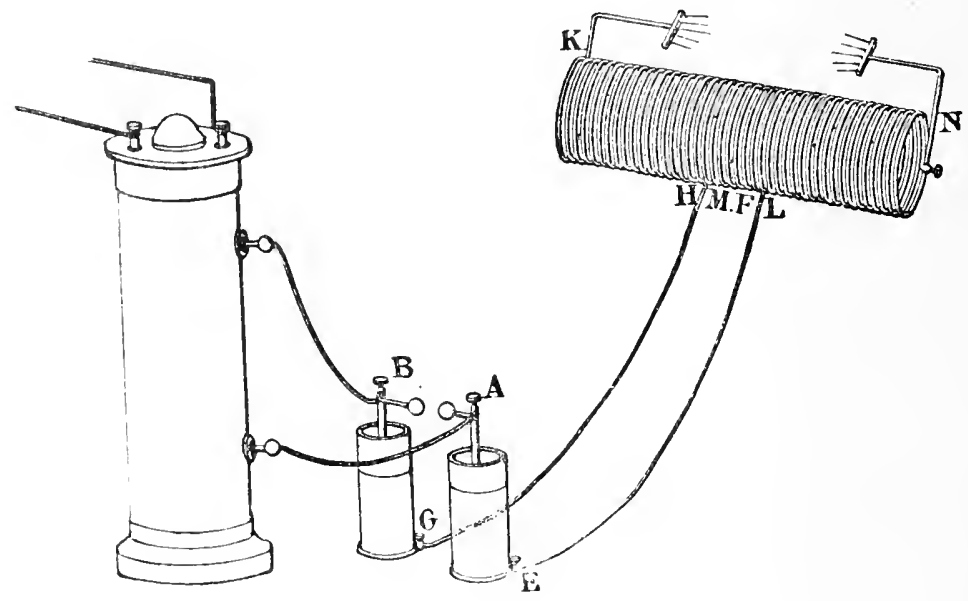

FIG. 44 .

is difficult to regulate them when the capacities of their secondary coils are very different.

The electrodes are the same as those employed in galvanisation and faradisation, $i$. e., plate-electrodes, knob-electrodes, brush-electrodes, etc. Oudin's electrode is often used for local treatment. This consists of a long ebonite handle at the end of which a thick metal rod is fixed, covered by a small glass dome. The conducting wire is conveyed through a side hole in the handle to the metal rod.

Oudin used a very pliable material woven from metal wire, such as is used for theatrical dresses, as electrodes, covering the material with moistened chamois leather. By this means he was able to spread the current over a fairly large and well-con- 
ducting surface, thereby preventing the patient from receiving disturbing sparking effects.

M. Kohl constructed internal electrodes for use in the body cavities. A conducting wire is passed through small ebonite sticks of various length, thickness and shape, according to the cavity to which they are to be introduced. The part of the body which is to be treated does not come into direct contact with the conducting wire, but serves in a manner as a condenser's foil.

When the bi-polar condenser is used the patient holds in his hand a brass cylinder connected with one of the resonators, whilst the end of the other resonator is joined to the other electrode. In every case the length of the resonator must be adapted to the capacity of the electrode and that of the patient's body if perfect resonance is to be obtained.

The author has obtained considerable augmentation of power by connecting the condenser in the following way: The conductors from the poles of the secondary coil of the induction apparatus are led to the inner tin-foils of the two Leyden jars; the outer tin-foils of the jars are connected with each other as well as with one knob of the spark-gap; the other knob being connected with one of the two rods which lead to the inner tinfoils of the jars. In this way the spark is made much brighter in the spark-gap; it is a fatter and noisier spark than that produced by the ordinary mode of connecting the two jars; moreover the discharges from the resonator, if this be suitably arranged, are more vigorous. Nercrtheless, by the ordinary arrangement a longer spark-gap can be traversed.

When both jars are connected in parallel the sparking becomes still more vivid. The two jars now act like a single jar of double the size; hence the quantity of the discharge is increased, whilst with the ordinary arrangement one obtains double the difference of potential but only the electrical quantity of a single jar; but the discharge from the resonator, other things being equal, is weaker in the case of parallel connection of the jars. Parallel connection is, therefore, not suitable for medical purposes.

The cffluvium from the resonator can be modified, firstly, by 
regulating the primary current of the induction-coil (by taking resistances out of the circuit of the rheostat or adding more cells to the accumulator; when I' elnelt's interrupter is used the same object is attained by altering the length of the anode-pin); secondly, by altering the distance between the knobs of the sparkgap, the farther these knobs are apart the stronger the effect; thirdly, by regulating the resonator; fourthly, by the use of different electrodes.

Oudin has experimentally proved that the resonator produces greater electrical energy than electrostatic apparatus. The intensity of high-frequency currents was accurately measured by d'Arsonial.

\section{$\S 23$. Technique of the Application of High-Frequency Currents.}

D'Arsonval distinguishes between an indirect and a direct application of the currents.

In the former (general d'arsonvalisation) either the method of anto-conduction or of condensation may be employed. In the anto-conduction methot a large spiral, running round the patient's couch, is placed in the circuit from the outer tinfoils of the condenser; the patient, fully dressed, sits within the spiral without being in actual contact with the conductor ${ }^{1}$ ); the patient is thus placed within the electrical field and totally charged with electricity, so that sparks can be drawn from any part of his body. In the condensation method, the patient is laid on a couch made of some badly-conducting material; the bottom of the couch is made of metal and is connected by a wire with one end of the solenoid $D$ (Fig. 40), while the patient takes hold of the second part of the condenser (represented by the couch), i. e., an electrode leading to the other end of the solenoid.

$\left.{ }^{1}\right)$ A solenoid of this description can easily be arranged in a corner of the operating-room by making a door so as to shut off a triangular space here, two sides of which are made by the walls of the room and the third by the floor. In this space the solenoid is placed. The conducting wire runs ronud the three sides of the enclosure, suitable contacts being made so that the circuit is closed when the door is shut. 
Direct application of high-frequency currents is performed as follows: The patient is connected with one end of the solenoid $D$ by two metal plates on which he places his feet, or by a foot-bath, while the current is closed by an electrode which is connected with the other end of the solenoid and placed in the patient's hand-bi-polar applicalion. Local bi-polar application of the currents is arranged by placing in the patient's hand a metallic electrode which is in connection with one of the terminal spirals of the small solenoid, the other teminal spiral being connected with a second electrode which is brought near the portion of skin to be treated; or Ondin's resonator may be attached to one end of the solenoid and a Oudin's electrode connected with the other end, and applied to the skin as desired.

When the bi-polar resonator is used its discharges are applied by connecting the end of one coil with a metallic electrode placed in the patient's hand, the effluvium from the end of the other coil being directed toward the part of the body under treatment. The above directions are quite sufficient for auto-conduction and for condensation; the sittings may take place twice or three times a week or daily for ten to thirty-five minutes. The patient must be warned of the noise made in the spark-gap, lest he be alarmed; he must also be very careful not to touch any part of the apparatus which is not given into his hands by the operator; otherwise he runs the risk of receiving shocks.

One proceeds differently with the application of high-frequency currents according to the condition of the discase under treatment. When, for example, the electrolytic effect of the current is especially called for, the proper electrode should be rapidly approached to the skin and pressed tightly upon it so as to spare the patient any disagreeable sensation ( see Fig. 60). Then according to requirements the electrode is left for a longer time in situ ( $e . g$. , at the tender spots in the case of sciatica) till a lively sensation of warmth is felt; or the electrode may be slowly and with some pressure passed along the course of the nerve trunks; the latter may be accomplished either with the point of the electrode or with the latter so held that it covers a greater surface. When, howerer, the mechanical effect of the discharges is required the electrode is kept at some slight dis- 
tance from the skin; a bright brush of sparks then plays upon the skin from the electrode followed by a distinct sensation of warmth and pricking. The explanation of this phenomenon is as follows: When the electrode is in close contact with the skin, the resistance to the passage of the current is very weak; with the electrode at some distance from the skin, however, the resistance is much increased, for the current has to traverse the air, which is a bad conductor. ${ }^{1}$ ) According to the formula $J^{2} R$ (p. 4I), the degree of heating depends upon the resistance. Much of the effect depends upon the kind of electrode employed. (The reader is referred to the different ways in which the discharge can be increased by altering the modes of connection, p. 98.) When using Oudin's electrode there is an appearance of violet light radiating toward the skin from the most adjacent point of the electrode. This luminous aigrette is seen on careful inspection to be composed of an infinity of fine, shining sparks; part of these would seem to penetrate the glass wall, part are absorbed by it. Small sparks issue in much smaller number from the outside of the glass covering of the electrode when the latter is kept at not too great a distance from the skin; the effect caused by these sparks is quite insignificant.

The fact that the sparks actually penetrate the glass covering, especially if the latter be thin, is proved by the presence of minute fissures in the glass after some considerable wear. These fissures are found to lie in the line of passage of the sparks. To some extent a condensing power exists in the glass case which may be conceived as acting like that of a Leyden jar.

The spark discharge from the metallic terminal of the electrode to its glass covering becomes more power-

1) According to Lord Kclain-

A spark-gap of $0.5 \mathrm{~mm}$. corresponds to a tension-difference of 2.9 ro volts.

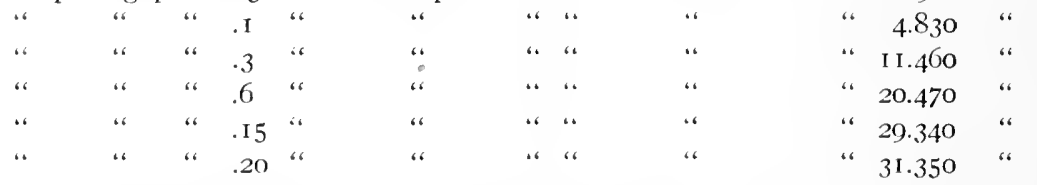

The above figures indicate the high resistance of the air, which can only be overcome by high tensions. 
ful the nearer the apparatus is placed to the skin and the moister the state of the latter. Since the treatment usually causes a slight perspiration at the parts treated, the apparatus works more effectively toward the end of the sitting.

With the glass covering removed there is no diminution in the intensity of the discharges; the sparks now strike the skin with undiminished rigour. Used in this way, the apparatus is more powerful and decidedly more effective, and it may be recommended in this form for the treatment of skin disease.

Still more vigorous effects will be obtained by arming the ends of the wire from the resonator with a faradic brush. This gives one an apparatus which is very useful for local stimulating purposes.

When the electrodes are kept at some distance from the skin, a spark-brushing is produced which may be compared to that obtained from monopolar discharges from induction coils (sce below), though the effect is weaker.

The patient's body may be charged with high-tension electricity by placing a metallic electrode in his hand; the operator then massages the diseased part with his bare hand, drawing sparks from the patient's skin in the process; but the electric current is much more effective when locally applied than when used in this manner because of the strong resistance offered by the body. Another variation consists in the operator holding the electrode in one hand while he passes the other hand over the affected region of the paticnt.

Local d'arsonvalisation is best applied daily from 10 to 15 minutes; the appearance of a red-brown colour on the skin is an indication for stopping the treatment until the part resumes its normal appearance. For the local treatment of the head or face the patient is best seated in a revolving chair having an adjustable head-rest. Care must be taken that the wire leading from the apparatus to the electrode loes not come in contact with the patient; care must also be taken that sparks are not too long applied to a small area of skin, otherwise blisters and sores may develop. 


\section{$\S 24$. Physiological Effects of High-Frequency Currents.}

High-frequency currents are not injurious to the human organism; they traverse it with an intensity and tension sufficient to light an incandescent lamp, without causing the least sensation. D'Arsonval ${ }^{1}$ ) showed that neither muscles nor the peripheral ends of sensory nerves are impressed by these extraordinarily rapid oscillations; on the contrary tissues, especially epidermis and denuded nerve endings through which high-frequency currents have passed, are said to become less sensitive to normal stimuli. Thus an anæsthesia lasting from 5 to 20 minutes may result, though it does not penetrate very deeply. In the author's experience the anæsthesia produced by high-frequency currents is very insignificant, though he would by no means call into question d'Arsonval's opinion on this point. Probably, as before suggested, the different results obtained by these most able French physiologists are to be explained by differences in the arrangement of their apparatus. Doumer and Oudin believed that the anæsthesia described by d'Arsonval and recommended by him for surgical operations is the first stage toward celldeath, which is quite analogous to the anæsthesia caused by freezing. ${ }^{2}$ )

Baedaeker, on the other hand, states that hyperæsthesia is induced by high-frequency currents.

D'Arsonval propounds two theories with the view of explaining the remarkable phenomenon that sensory nerves are not affected by high-frequency currents.

Ist. The currents do not act on the organism, because they only spread throughout its surface, or,

2nd. The peripheral nerves are only sensitive to electric vibrations of low-frequency; just as the terminals of the acoustic and optic nerves will remain unaffected by ether vibrations which are above or below certain limits.

Against the first theory we may urge the fact that sinusoidal currents $^{3}$ ), without causing any marked sensation, have some in-

1) Ann. d'électrobiologie, Vol. I, No. I.

2) Ibicl. 1900, Vol. III, p. 5із.

$\left.{ }^{3}\right)$ These are alternating currents, consisting of gradually increasing and decreasing waves. They are produced by means of suitable transformers 
fluence on tissue-nutrition; they must, therefore, be penetrative to some degree.

Vittorio Maragliano ${ }^{1}$ ) states that the heat effects which are produced by high-frequency currents, after they have traversed a considerable depth of living or dcal tissue, prove that $d^{\prime} \mathrm{Ar}$ sonial's currents take not only a superficial course, but also penetrate more deeply.

L. Hoorwe $\left.g^{2}\right)$ is also of opinion that d'Aroncal's high-frequency currents do not remain on the surface of the human body, but penetrate it completely; this is also shown by the results of Einthofen's and Leyden's experiments.

In the author's opinion even d'Arsonval's second theory is unnecessary. When a primary current in a Faraday's induction-apparatus is very rapidly interrupted by means of a motorbreak violent muscular contraction can be eroked without the least sensation of electricity on the part of the patient (d'Arson$v a l)$. In this case a current of definite intensity and tension induces a certain number of alternating currents of likewise definite qualities. When we diminish the rate of interruption without interfering with the primary current, the tension at the poles of the secondary coil becomes raised, i. $e$., the induced current has (the resistance not having been interfered with) a greater intensity than before. The author's bacteriological experiments have proved (see later) that with at higher current-intensity more powerful effects are obtained. We are, therefore, entithed to expect that a reduction in the rate of interruption of the primary current is followed by an increased intensity of the secondary current, and in consequence of that more marked physiological effects. $e$. g., sensation. This, indecd, is the case. We may reasonably suppose the same to ohtain in the case of high-frequency currents; by reason of the rapid alternations the intensity of any single shock may conceivably be very insignificant. On the other hand, we maly expect that by raising the intensity of high-frequency currents (by suitable windings of the

which regulate and reduce the intensity and tension of currents from the main (alternating or rotary).

1) Clinica medica, 1901. No, 7.

2) P'Aliger's Archix., Vol. I.XXX!1, 8. 
coils and construction of the condensers) the physiological effects will become much more marked.

In the low intensity of these currents may lie, therefore, the explanation of the remarkable fact that they produce so trifling a sensation. We must, however, distinguish between the effects of the electric current and that of the electric discharges on the sensory nerves. The latter produce the same sensations in the case of high-frequency currents as do the discharges of any other kind of static electricity. Again, we must not associate the two facts that on the one hand high-frequency currents cause no sensation, while on the other hand they are said to produce analgesia.

H. Kurella $a^{1}$ noted that when the resonator to which the electrode is attached is only connected in a uni-polar manner with the solenoid $D, d$ 'Arsonval's current evokes a marked sensation of formication and a tetanising effect in the muscles; the latter does not take place in the case of bi-polar connection.

D'Arsonval infers from his experiments that high-frequency currents have a great influence on metabolism and cell-production. By their action respiratory combustion is intensified, the quantity of oxygen consumed in the unit of time as well as that of carbonic acid eliminated is heightened. In one instance an increase took place in the latter of from 17 to 37 litres. This augmentation of the process of combustion is also indicated by the increase in the amount of urea excreted, while that of uric acid is diminished (Lazat and Gautier). At the same time the body parts with more heat; nevertheless, despite this augmentation in the process of combustion the body temperature is scarcely raised, but a further proof of heightened combustion was furnished by the loss of weight of the animals used in these experiments. A little guinea-pig, for instance, lost under ordinary conditions 6 grammes in weight in sixteen hours; when exposed to the action of high-frequency currents it lost 30 grammes in the same time. After that when left to itself it recovered the lost weight in 2 hours.

Berlio $\left.{ }^{2}\right)$ tested the urine of 280 patients who were under

$\left.{ }^{1}\right)$ Zeitschr. f. Elcktrotherapie und ärztl. Elektrotech., Igoo, p. 59.

2) Compt. rend de l'Académic des Sc., March I8, I895. 
treatment by auto-conduction, examining in all $76 \mathrm{I}$ specimens. He found, first, increased diuresis and better elimination of the excreta; secondly, increased organic combustion; thirdly, the proportion between uric acid and urea approached nearer the normal ( $\mathrm{I}: 40)$. Apostoli observed with his patients increased diuresis and excretion of urea, increase in the percentage of oxyhæmoglobin in the blood, better capacity for work and walking, etc. Hæmospectroscopic analysis by Henocque's method shows the vigorous effects of these currents on nutrition change. The process of nutrition is both stimulated and regulated.

Tripet ${ }^{1}$ ) made researches on the influence of high-frequency currents on the reducing power of oxyhxmoglobin. He found this power increased in 37 cases of patients suffering from defective metabolism ("rheumatism").

In 10 cases of diabetes where the reducing power was much augmented before treatment it became lowered by high-frequency currents. In 6 cases marked by general organic decay the treatment was unsuccessful. Guillanme ${ }^{2}$ ) also pursued investigations on the lines of Tripel. His patients were affected with arthrodynia, sciatica, chlorosis; in them the quantity of oxyhrmoglobin was increased together with its reducing power; often, too, the general health became improved. This improvement, however, wats partly due to the modified nutrition, as was shown by the analysis of the blood and urine. In any case the good effects cannot be ascribed to the high-frequency currents alone. The inhalation of orone acts in the same way on hæmoglobin (Labbé), and it is well known that high-frequency currents are always accompanied by the production of ozone.

Lacaille") found that in his cases the quantity of urea excreted while the patients were under the influence of auto-conduction increased from I I grammes to 43 , and even 60 grammes.

Denoyés, Marre and Ronvive ") found that under the influence of high-frequency currents the daily quantity of urine excreted increased in the same proportion as did the urea, uric acid,

\footnotetext{
1) Acad. d. Sc., June 25, 1900 .

2) Gazetle des Hopitaux, Feloruary 7. I901.

3) Bullet offic. d. I. Socicté fr. d'électrothérapic, March, I900.

") Compit. rend de l'Acad. d. Sc, July I, igor.
} 
nitrogen, phosphates, sulphates and chlorides. The increase varies with different people, and is maintained to some extent for 3 days after suspending treatment.

G. S. Yinai and G. I ictti $^{1}$ ) also found in two cases that d'arsonvalisation increases the total amount of nitrogen and phosphates excreted.

These statements of d'Arsonval and his pupils have, however, not been generally confirmed.

Guilloz enquired into the tissue change in muscles, and could not observe any increase in the assimilation of oxygen brought about by auto-conduction. He does not, however, doubt the results of d'Arsonval's enquiries, which indicated an increased assimilation of oxygen in the body, but maintains that this increase cannot be due to increased oxygenation in the protoplasm.

L. Querton ${ }^{2}$ ) found not the slightest increase in the output of carbonic acid by the use of alternating currents of high-frequency and tension.

Reale, Renzi and $V^{\prime i n a i}{ }^{1}$ ) were of opinion that Tesla's currents considerably increased the oxygenation of the body by promoting the elimination of uric and phosphoric acid.

Querton ${ }^{3}$ ) observes that d'Arsonzal does not mention if the guinea-pigs employed in his experiments were fed during the process, or if the temperature remained normal.

J. E. Baedeker ${ }^{4}$ ) reports that rabbits which were subjected to the auto-conduction method showed an increase in the rate and depth of respiration, and along with that an increase in respiration-quantity (from 6, I 40 to I I, OOO c.cm.). This effect was maintained for five minutes after the operation, but gave way after fifteen minutes to normal conditions. In the case of other animals the respiration was in no way affected.

A. Locwy and T. Cohn ${ }^{5}$ ) made experiments on the respiration of eight persons. They found "in one case an increased consumption of oxygen, which exceeded the normal physiological

\footnotetext{
1) Giorn. d. elettr. medic., I, 2, p. 6I.

$\Rightarrow$ Ann. d'électrobiologic, V'ol. III, p. I4.

$\left.{ }^{3}\right)$ Institut Soláay, 1899.

$\left.{ }^{4}\right)$ Wiener Klinik, Vol. XXV'II. Nos. 10 and I I.

$\left.{ }^{5}\right)$ Berlin. klin. Woch., 19co, No. 34.
} 
limits of 6 per cent. by 12.6 per cent., in another case by 19 per cent."

N. Spaski ${ }^{1}$ ), on the contrary, found a decreased assimilation of oxygen and of carbonic acid and a decrease in the exhalation of aqueous vapour in animals which underwent general d'arsonvalisation; he therefore denics any effect on the interchange of gases by this proceceling.

The third important property ascribed by d'Arsonial to high-frequency currents is their influence on the vaso-motor system. When a rabbit is subjected to the currents it will be observed that the ressels on the animal's ear rapidly dilate, just as they do after section of the sympathetic nerve. This phenomenon is succeeded by marked contraction of the ressels. The blood pressure of a person undergoing general d'arsonvalisation, as shown by the sphygnograph or sphygmomanometer is seen first to fall and to rapidly rise. The same thing is shown by a mercury pressure gauge brought into contact with an artery. D'A sonval observed that the blood issuing from an incision in a rabbit's foot flowed more freely after the action of high-frequency currents.

But these statements of d'Arsoneal are also not generally confirmed.

Carvalho found no change in the blood pressure after autoconduction, neither could he ohserve any motor or sensory reaction. But when a very strong current was directly applied to the skin the sensibility of the latter became affected, and at the same time the blood pressure decreased. He obtained the same motor and sensory reactions with muscle-nerve preparations from frogs as are to be obtained from other kinds of currents.

Moulier ${ }^{2}$ ) states that he decreased arterial pressure by means of auto-conduction, which is precisely the opposite of d'Arsonzal's finding ${ }^{3}$ ). The same author, ${ }^{3}$ ) however, produced an increased arterial pressure of 2 to $3 \mathrm{~cm}$. by applying the efluvium from the resonator along the spinal column.

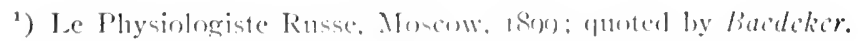

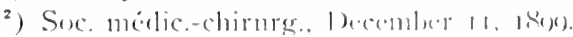

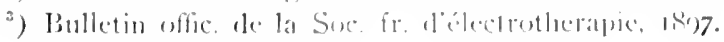


Leduc ${ }^{1}$ ) found that high-frequency currents induce cutis anserina and superficial anxmia, which is necessarily followed by an increase in the general'arterial pressure.

Ondin $^{2}$ ) raised the arterial pressure of anæmic patients from 9 to $14 \mathrm{~cm}$. in one sitting under the effluvium of the resonator; he examined the capillary pulse by Laulanie's sphygmometer, and found that whenever the efluvium of the resonator played on the region of the body the capillary vessels at once spasmodically contracted, thereby effecting a fall in the curve; after suspending operations the pulse resumed its previous character, but it was some time before it recovered its previous amplitude.

Doumer and Oudin, therefore, recommended this treatment for affections due to deficient tissue change and for local inflammations accompanied by arterial or venous stasis.

Baedeker ${ }^{3}$ ) re-examined all d'Arsonval's statements as to the effect of high-frequency currents on the blood pressure. He found that general d'arsonvalisation effected an increase of the "extinction-pressure" amounting to $6 \mathrm{~cm}$. and more; this could be observed on the human body by means of $V$. Basch's sphygmomanometer: with rabbits, however, he could not confirm the appearance of the phenomenon of the ear-vessels, or of the blood-How from an incised foot, or any increase in the bloodpressure of the carotids.

Experiments by Locwy and T. Cohn yielded quite negative results.

According to Cafferena, ${ }^{4}$ ) high-frequency currents have a distinct effect on gastric peristalsis, especially in cases of atony of that organ.

Another property ascribed to high-frequency currents is their effect on microbes and their toxins. D'Arsonval and Charrin $^{5}$ ) tested their bactericidal effects on the organisms found in the dregs of beer and on bacillus pyocyaneus. D'Arsonval

1) Quoted by Doumer-Oudin.

2) Ann. d'électrobiologie, I900, Vol. III, p. 3 I4.

$\left.{ }^{3}\right)$ Wiener Klinik, Vol. XXVII, Nos. Io and I I.

4) Clinica medica, I9or, No. 7.

${ }^{5}$ ) Académie d. Sc., Feb. Io, I8g6. 
also found that toxins can be powerfully modified in their virulence and converted into anti-toxins. ${ }^{1}$ )

D'Arsonical's arrangement for the experiment was as follows: 'The liquid to be dealt with was placed in a U-tube, in both arms of which platinum pins were immersed, which were in connection with the ends of the solenoid. The U-tube was surrounded by a cooling apparatus.

D'Arsonval and Phisalix ${ }^{2}$ ) found also that the venom of serpents (viper, cobra) also loses its poisonous properties after exposure to high-frequency currents. Bonome and Viola ${ }^{2}$ ) achieved still greater success in this direction, and state that their method proves an effective antidote against even diphtheria.

Haller ${ }^{2}$ ) observed a similar destructive effect on alga, fungi, and bacteria when he allowed the currents to traverse a liquid in which these organisms were suspended. Dubois, of Rheims, ${ }^{2}$ ) confirmed d'Arsonval's statements as to the lessening of the virulence of toxins; he was not, however, able to produce anti-toxins by the process.

The literature of the subject is marked by the most diverse opinion on this point. Oudin and Doumer,;) for instance, could find not the least effect from the resonator discharge on the arresting of organic development.

Discharges from the resonator cause, when applied to the surface of the skin, first, marked anæmia and cutis anserina. This condition continues for one or two minutes, and is combined with a considerable sensation of burning and smarting. The anxmia afterwards gives way to a fairly marked erythema; this erythema may last according to the intensity and duration of the operation, also to the patient's capacity for reaction, for several hours or even for two days. Oudin observed the appearance of blisters after a protracted local application of the currents.

1) Sociélie de liologice, 1806 .

$\left.{ }^{2}\right)$ Quoted by Doumer-Oudin, Annales d'électrobiologic, Vol. III, p. 5 I7.

$\left.{ }^{3}\right)$ Ibid. 
The author observed a case of this kind in a lady who had a very sensitive and tender skin. Powerful discharges from the bare metal electrode were thrown onto the knuckles (where the cuticle is highly stretched and the bones are lying near the surface). Small bullæ, half the size of a pea, made their appearance, surrounded by normal skin. On the following day the bulla had dried up and the skin soon became quite normal. M. Bisseric told the author that excessive application of high-frequency currents may induce actual destruction of tissue, as do the Roentgen rays.

Very often small beads of perspiration make their appearance on the part treated, accompanied by some considerable wedema. After the repeated application of spark-discharges a brownish discolouration often persists in the skin; this is a hemoglobin stain.

Arguing from the circumstance that in d'Arsonval's apparatus electrical vibrations are produced which can be focussed on definite parts of the body by means of Ondin's resonator, we might fairly look upon the treatment of skin diseases by means of d'Arsonval-Oudin's apparatus as a branch of radiotherapeutics; but from the author's experiments he cannot look upon the physiological, biological and therapeutic effects of the method, so far as they concern the skin, as being due to electric vibrations. He believes, on the contrary, that the sparking which accompanies these vibrations is the most active factor, and he believes that the effects of spark-discharges from $d^{\prime} A r$ sonval-Ondin's apparatus only differ in degree from those which are produced by other generators of high-tension electricity. When an experiment was arranged in such a way as to prevent the impact of the spark-discharge on the exposed object without hindering the progress of the electric vibrations (e. g., by a thick wooden board), results were entirely negative.

Negative results, moreover, were obtained from experiments as to the final effect of the dynamic electricity emitted by Oudin's apparatus.

The phenomena which d'Arsonval observed in the case of 
a living being enclosed within his solenoid and described by him, may be looked upon as the effects of "influence," or of electrical vibrations produced by the apparatus. These phenomena bear some relation to the biological effects of other radiations, and we may fairly characterise this method as a branch of radio-therapy.

We must make the following distinctions in the biological effects of the discharge from d'Arsoncal-Ondin's apparatus:

First, the direct effects of the electrical (i. c., of the dynamic electricity) and those of the electrical vibrations on the internal organs.

Second, the effects which are partly the direct and partly the indirect consequence of the changes brought about on the body-surface by the static discharge; the latter effects are shown by modifications in the functions of the internal organs.

There is a wide province for future work on the first-mentioned group, but little being at present definitely known on the subject. Some experiments, howerer, which the author undertook in the year 1900 under the direction of Prof. Anton $\mathrm{H}^{\prime}$ eicltselbaum in the Patho-Anatomical Institute of Vienna, throw some light on the manner of the local action of discharges from high-tension and rapidly-alternating currents.

At the same time the effects of the discharges from a $R u h m$ korff's coil were also carefully studied, the author believing, as he did, that in the treatment by every kind of high-tension electricity (faradisation, franklinisation, high-frequency currents) we are dealing with the effects of one and the same physical phenomenon, i. e., with spark-discharges.

The sparking is more intense according to the height of the tension, more brilliant according to the quantity of electricity. Sparks are cmitted from the poles of an electro-static machine or from those of Faraday's, Ruhmkorfl's or d'Arsonval's apparatus when the machine is brought near the human body; these sparks only differ from each other in their shape, intensity, length and the rate at which they succeed each other. By means of suitable contrivances the sparks from all these apparatus can be changed into quiet discharges, in which the high-tension electricity flows off as "electrical wind." 
Faradic discharges onto the skin produce clinically slight pain, hyperæmia and erythema, the latter varying with the intensity of the discharge; also some degree of cutis anserina. The sensitiveness of the skin is much increased and the feeling of pain diminished. The circulation in remoter organs is markedly influenced. Thus, for instance, it is possible to produce cerebral anemia even to the stage of syncope by faradisation of the abdomen $(V$. Basch $)$. By reflex action, moreover, vaso-motor, sensory and motor nerves, glands and other organs containing involuntary muscle fibre can be excited. Organs which are deeply situated can be relieved of congestion by producing hyperæmia of the cuticle. According to Beard and Rockwell, this method of electrisation acts not only as a stimulant, but also as a very effective tonic alterative and sedative (Lewandowsky), especially in various conditions of asthenia and disturbances due to malnutrition (anæmia, chlorosis, rheumatism). Spilker and Goldstein stated that induced electricity was also capable of destroying bacteria. This statement has since been disputed.

Local faradisation gives similar results, especially as regards local effects. Discharges of static electricity produce first anæmia, the duration of which depends upon the intensity of the discharge; this anæmia is afterwards replaced by a correspondingly enduring hyperæmia. The effects of the spark discharge can, moreover, be considerably increased, being then associated with marked feeling of pain, the appearance of papules, vesicles, and even large blisters. When acting on the whole organism this method increases the pulse rate, ${ }^{1}$ ) the excretion of sweat, saliva, and urine, $\left.{ }^{2}\right)$ causes even an increase in the body temperature, ${ }^{3}$ ) facial erythema, and an exciting or sedative effect on the nerves. I'igourou ${ }^{4}$ ) maintained that franklinisation powerfully stimulates the process of nutrition; he therefore urged its employment in those diseases associated

1) Cavalho and Wilkinson, Traité complet. d'électricité, I777-I785.

") Mauduyt. Article "Electricity" in the 18 th Century Encyclopedia.

$\left.{ }^{3}\right)$ Sigaud de la Fond.-De l'électricité médicale 177I, and Journal de médicine de Vandermond, Vole. IXX and LXXII.

4) Tigouroux, Gazette médicale, i\$78. 
with defective metabolism. Further defects are said to be regulation of the catamenia, improrement of the appetite, soporific, anti-spasmodic, and anodyne effects. Destot and Dubard maintained that this kind of electricity possessed bactericidal properties.

Before proceeding to a description of the author's own experiments, some remarks may not be out of place here concerning the different kinds of electric sparks. The following observations are in part quoted from the classical work of Bouchacocrt and Rédmond ${ }^{1}$ ) on this subject.

Experience teaches that by the use of the same electrostatic machine or the same static mduction apparatus, which is actuated by the same motor, we may produce sparks of very different shape.

In the case of the electrostatic machine the shape and area of the surfaces between which the sparks strike are of great importance in this respect.

When the hand is brought near a pole of the electrostatic machine a series of branched sparks are produced, which become ordinary sparks as the hand is approached still nearer. The ramifications in the first instance are to be explained by the atmospheric resistance; the discharge always follows the line of least resistance through the air, and these lines are determined by the presence of aqueous vapour and particles of dust, which increase the atmospheric conducting power. When the apparatus is worked very vigorously these ramified sparks are changed more and more into a so-called "aigrette"; the latter can only be seen in the dark, but still consists of a series of finely-ramified sparks. The electric discharges become more uniform when the machine is acting vigorously or when the terminal knob is small; the thinner the end of the conductor, the more continuous becomes the discharge, $e . g$, the less can one observe single ramifications from the aigrette; the latter becomes smaller and smaller, devolving finally into a small point of light.

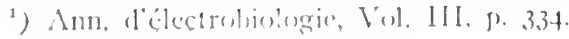


Faraday described four kinds of electrostatic discharge:

I. The spark proper.

2. The brush.

3. The aureola.

4. The invisible discharge.

I. The Spark. When an earthed conductor is brought near another conductor charged with electricity, a shaft of light is seen between the two, indicating the intermittent discharge between them. When the distance between the conductors is small, the path of the spark is marked by a straight luminous beam of equal thickness throughout; the thickness and brightness of the spark depends solely upon the quantity of electricity; when the spark-gap is made longer the spark maintains its brilliancy and thickness at both ends, but becomes thinner in the middle. When the distance exceeds a certain limit and the discharged electrical quantity increases, the spark takes the form of a series of zig-zag lines having purplecoloured ends; when the sparking distance is still more increased, ramifications make their appearance and streaks of light are seen flowing off from the corners of the zig-zag. B. $\mathrm{F}$ alter ${ }^{1}$ ) found that the electric spark is preceded by the appearance of several intermittent and successively longer brush discharges; each of the latter follows the path of its predecessor, extending it a little on each occasion. Thus we finally obtain either a series of ramification discharges into the air, or, with a more powerful current, a spark traverses the spark-gap.

2. The Brush. When a static apparatus is put into action in the dark a series of shining rays or brushes are seen flowing off from all prominent parts of the conductor with a noise resembling that of a steam-jet. As a rule, these brushes consist of a straight and brilliant stem ramifying into branches which again ramify in their turn. These phenomena are better seen when the second

1) H'iedcmann's Ann., Pd. LXVI, p. 636. 
(earthed) conducto: has a considerable surface, as, $c . g$., that of a sphere or a large disc. The electric brush is only produced on the positive points of the static machine; it varies in appearance in these places according to the shape, distance and arrangement of the conductors inducing the discharge. The negative points are only discernible as shining dots like stars. Both the brush and the spark itself are intermittent discharges; this can be proved by means of rotary mirrors. The brush is formed by the rapid succession of weak discharges, which only represent a fraction of the total electricity collected on the conductor; the spark proper, on the other hand, discharges the conductor completely. Both kinds of discharge, however, according to Faraday and Gangain, are seen where the same potential-difference obtains; other conditions may, therefore, transform a brush into a spark or vice versa. For instance, sparks were produced by an influence apparatus working with a definite sparking distance, which only gave brushes when the rotation of the apparatus was accelerated, the capacity of the conductor increased, a condenser connected, or an auxiliary spark cmployed.

When a thin metal wire is suspended from a conductor which is drawing sparks from a powerful electric machine the wire becomes luminous in the dark, short rays being seen to dart from it in all directions. The same phenomenon is observed in the wires connecting the secondary poles of a powerful Ruhmkorfl's coil with a conductor offering considerable resistance, such as a very hard Roentgen tube. I ligh-frequency currents, too, produce brush discharges on conducting wires through which they pass.

3. The Shimmering-lighl (aureola) and Invisible i)ischare. When the terminals of the condenser of a static apparatus end in very small metallic spheres, often a light of varying brilliance and size is to be observed playing about the spheres.

According to faraday, in order to convert the brush 
into an aureola the dimensions of the conductor must be diminished, the output of electricity from the machine must be increased, and the conductor must be strictly isolated. In the air the negative aureola is much less than the positive. This peculiarity is particularly well shown when the discharge takes place in rarefied gases. Examined spectroscopically, the auroelia shows appearances which depend upon the nature of the conductor, upon the character of the gaseous medium, and upon the pressure of the latter, the strength of the discharge, etc. Lines are found in the spectrum of the sparks which are characteristic of the metal of which the electrode is composed. The spectrum of the brush-discharge shows the same qualities as the aureola and the spark proper.

Gaugain (quoted by Mascart) showed that by using equal-sized spheres as conductors the sparking distance and the tension are almost proportional; according to Riess, however, this only holds good for small distances up to 4 or $5 \mathrm{~mm}$. Discharge occurs most readily between a small positive and a large negative sphere.

According to Harris, the quantity of electricity necessary to produce a spark is directly proportiona! to the atmospheric pressure in the spark-gap.

Döbereiner and Cailletet observed that the length of the spark decreases when the pressure is raised beyond one atmosphere.

Numerous investigations have proved that a spark passes through the air much more freely when the pressure decreases; under these conditions the spark is also "fatter." This, however, only holds good up to certain limits; heyond these the resistance of the gaseous medium becomes progressively more effective; when, indeed, the vacuum is absolute the resistance becomes invincible.

According to Harris, the sparking distance between two spheres in connection with both terminals of the condenser is proportional to their electric charge. Thomson found that the potential difference required to pro- 
duce a spark in the air between two parallel plates does not increase in the same proportion as the sparking distance when the plates are further removed from each other. The relation between these two quantities (potential difference and sparking distance) is only constant at distances of more than $\mathrm{I} \mathrm{mm}$.

W'icdemann's and Rïhlmann's experiments showed that the potential difference necessary for the discharge increases more slowly at low atmospheric pressures as the sparking distance is increased. This applies to sparking distances between 2 and $30 \mathrm{~mm}$. Gangain assumes that the resistance of the medium is overcome by electrical pressure; he thinks it of greater inportance to know the electrical density at which spark discharges occur than to define potential difference. He showed by means of two charged cylinders placed one within the other that the production of sparks depends upon the size of the surface of the inner cylinder, i. $c$., to the density of the electricity accumulated on its surface.

Riess found that the heat dereloped by an electrical discharge depends upon the same law that Joule enunciated in the case of electric currents--the caloric quantity produced in a conductor which is traversed by an electric discharge increases in duplicate proportion with the intensity of the current, and in direct proportion with the resistance in the conductor.

Franklin, Arago and Ridolfi demonstrated the magnetising effect of static discharges. Masson proved also that induction phenomena followed the static discharge. The character of the sparks produced by a static conductor depends mainly upon the intensity of the currests, the rate of interruption, the sparking distance and the quality of the conductor surfaces. The action of positive and regative discharges will be discussed later. With a comparatively slow rate of interruption ( $c$. ... by Verf's hammer) the spark makes its appearance as a simple flash of light; with a quicker interruption rate (e. $x^{x}$, by a turbine interrupter), as a sheaf of sparks; with an extremely rapicl interruption rate (such as is 
produced by an electrolytic interrupter), as a thick stream of light showing sometimes dark spaces (the "caterpillar" discharge); with a very quick rate of interruption, noiseless discharges, aigrettes, and bundles of sparks may be produced, resembling very closely the spindle-shaped bundle of violet sparks evolved from a l'imshurst apparatus.

The spark from an apparatus worked with a $W$ ehnelt's interrupter strikes horizontally between two points which are placed opposite each other. When the terminals are placed vertically and parallel to each other the discharge takes the shape of a Gothic arch. E. Lecher experimentally demonstrated the mobility of $U^{\prime}$ elnclt's sparks in a magnetic field ${ }^{1}$ ).

F. Himsted $t^{2}$ ) fixed a fine-pointed terminal on one pole of a Tesla transformer. At some distance (so great that no spark could traverse it) an insulated disc was placed. He found that the disc became always positively charged in the atmosphere or in oxygen, but always negatively charged in any other gaseous medium. This occurred quite irrespective of the direction in which the primary current was sent through the apparatus, or whichever pole of Tesla's coil was used. As the disc is removed further from the point the positive charges become weaker and weaker, disappearing finally to be replaced by negative ones. From this we may infer that more positive electricity than negative is radiated from the point into the air, but that negative electricity, nevertheless, is more capable of being transmitted far into space than is the case with positive electricity.

In working out his experiments the author thought it advisable to investigate, firstly, whether direct sparks discharged on the living skin bring about changes which correspond to those caused by Roentgen rays; secondly, the histological changes in a skin subjected to the sparking process; and, thirdly, the effect of direct sparks on micro-organisms.

The following apparatus was used to produce the sparkdischarges:

$\left.{ }^{1}\right)$ Sitzungsbericht d. Kais. Akad. d. Wiss. Part IIa, Vol. CVIII June 8, 1899.

") Iricdemann's Ann., Vol. LXVIII, I899, p. 294. 
I. A Roentgen outfit, consisting of a to cm. coil, manufactured by M. Kohl, Chemuit\%. On the primary coil are 220 windings of $3 \mathrm{~mm}$. thick copper wire; the secondary coil, however, has 58,000 to 60,000 windings of $075 \mathrm{~mm}$. wire, with a resistance of 20,000 ohms. The primary current in one series of experiments was provided by a battery of accumulators ( 12 volts); in another series by direct current from the main (I ro volts). The primary current was interupted by a mercury-break. The rate of interruption varied between 5,8 and I 6 per second, according as larger or smaller resistances were inserted into the motor circuit.

2. A Roentgen apparatus manufactured by Sicmens $\&$ Halske, consisting of a $30 \mathrm{~cm}$. coil and with windings as follows :

Primary coil, tos. Windings. 0.25 olm, 2. mm. thickness.

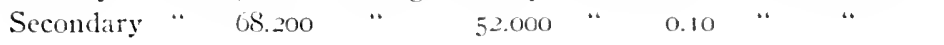

This apparatus was always worked from the main. A mercury turbine break was used in conjunction with it, giving a constant rate of interruption of 100 per second.

3. A d'Arsoncal apparatus manufactured by Gaiffe, of Paris.

Each experiment was repeated two or three times, and was only regarded as conclusire when the results were in agreement on each occasion.

(a) Investigations on the iction of the Spark-Discharge on the Body Sinfaie.

Exp. I. February 23rd, 1900 . A healthy guineapig was placed in al suitable wooden case haring a $2 \mathrm{~cm}$. square aperture cut away corresponding to the right flank of the animal. The end of a wire coming from one pole of the coil $I$ was fixed opposite this aperture at a distance of $3 \mathrm{~cm}$. from the animal's skin. The aperture in the case was closed by a thin cardloard covered with aluminim foil; the foil wis connected by a wire with the floor, the lower end of the wire being inserted 
in a crevice between the wall and the floor. The primary coil was connected with the main; the voltmeter constantly indicated I ro volts, while the hand of the amperemeter indicated 4 or 5 ampères during the experiment. There were 16 interruptions to the second; time of exposure, 45 minutes. When the sparks were first applied the animal squeaked a little, afterwards it became quiet and showed only a quicker rate of respiration. During the whole experiment sparks were incessantly striking from the end of the conducting wire into the floor.

The same procedure was adopted on February $24^{\text {th }}$ and 25 th. On the 26 th the hair of the treated skin yielded to slight traction; by the 28 th there was a bald spot the size of a finger nail. The animal remained for a time quite lively and well and took nourishment properly, but on the night of March Ist it died. Prof. W eichselbaum made a post-mortem examination and found but slight hyperæmia on the lungs, the liver and the kidneys. There was no ecchymosis on the surface of the heart nor on the endocardium. It is an open question whether the animal died from the effects of electricity or from exposure to cold, since on the night it expired the weather was very chilly and the animal had been kept in a room without fire. Small apertures were visible both in the aluminium foil and in the cardboard, showing that thin sheets of this kind afford no protection against spark-discharge.

The result of the experiment seemed to indicate a depilatory effect on the direct spark-discharges.

Exp. 2. March $7_{\text {th, }}$ 1900. A robust hare, which had recently changed its fur so that its present hair-coating was firmly fixed, was placed in a case constructed similarly to that in Exp. I. The aperture, however, was a little larger; the bare end of the conducting wire was not arranged as before, but at a distance of $\mathrm{I} \mathrm{cm}$; a contrivance was placed of the following description:

A wooden frame was used as the electrode, covered 
by metal points piercing a tin-foil covering to the wood. The metallic points were arranged in the figure of a cross, and were in circuit with the secondary current. The object was to produce a cross-shaped polar discharge of sparks, and thus, if possible, a cross-shaped patch of baldness on the hare's skin. At the same time it was hoped that the larger quantity of electricity would be collected on the electrode by giving the latter a larger surface, and that thereby the physiological effects would be promoted. This expectation, however, was not realised, for the discharges did not take place simultaneously from all points, but generally from one point only to a portion of the skin which probably offered the least resistance. This single spark was sometimes seen wandering along the arms of the cross, though it always returned to its starting point. The distance of the electrode from the skin was I cm.; the primary current was of 2 to 3 ampères, 12 volts, and 16 interruptions per second; the time of exposure was 30 minutes. The animal, though at first evidently disturbed, behaved quite quietly on the whole. The same procedure was repeated on March 8 th and 9 th.

On March Ioth numerous hairs fell off from the parts treated, the remaining hairs being very much loosened. The wool among the hairs was still adherent and had hecome singularly clogged and twisted. On the rest of the body the hair was not affected.

Exp. 3. I Titherto only one pole of a spark-coil had been employed in connection with the electrode; now the second pole was earthed: the discharges then from the electrode to the skin appeared much more vigorous and traversed a greater sparking distance than in Exp. 2.

Exp. \%. 'The case with the animal in it was connected with the floor in the following way: The receptacle was placed on a metallic sheet which earthed by a wire. By these means we ohtained the same result as in Exp. 3; the sparks were more vigorous and traversed a greater distance. 
Clearly the resistance offered to the passage of electricity from one pole to the other was lessened by the above contrivances. The entire resistance offered in the passage of the discharge from one pole to the wire, the electrode, the air, the animal, the earthing wire, and the floor back to the second pole was apparently less than the resistance offered by the air between the two secondary poles themselves. (See diagram 45.)

The animal was treated daily in this manner until March I \& th. On this day several bald spots were visible on the right flank within the zone of the radiation. Everywhere else the hair was quite firm, except at the root of the left ear and on

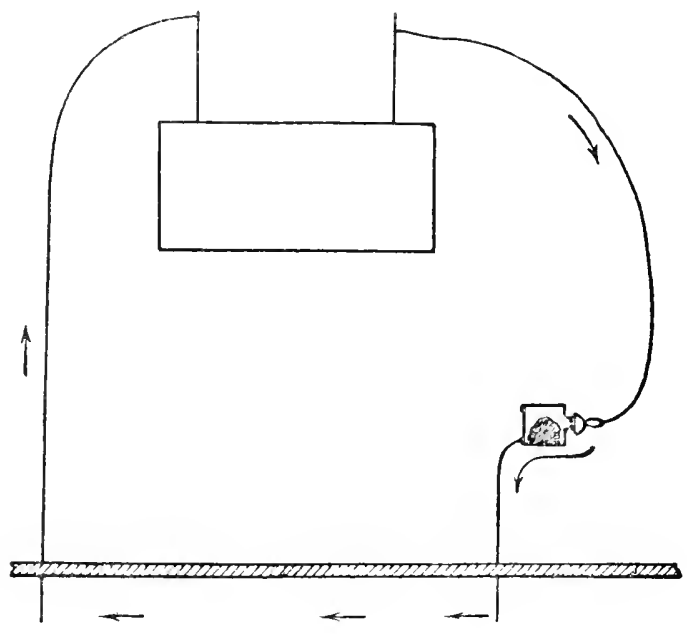

FIG. 45 .

the outer side of the left fore-paw; here also there were bald places. It was found that the latter places had been in contact with the metallic fastening of the case during the experiment, for from time to time sparks had been observed striking between these parts of the body and the metal.

On March igth a completely bald white patch 3-4 mm. in width was observed on the skin corresponding to the horizontal arm of the cross of metal points. There were, moreover, three bald spots the size of a lentil, arranged in a straight line and corresponding to the vertical arm of the cross. Be- 
sides these spots the rest of the skin coinciding with the aperture appeared but sparsely covered, and there were numerous places the size of a kroner which were quite destitute of hair. The skin itself appeared quite normal, with the exception of some few excoriations the size of a pinheal; the latter were probably due to very strong sparks.

Fresh hair began to grow again in the course of a week on all the places except those corresponding to the cross; in the latter situation about four weeks elapsed before there was any recovery of hair.

Exp. 5. March 2 Ist, I goo. The animal was now so placed that its left fank coincided with the aperture in the case; in front of this case the same electrode was fixed, at a distance of $1 \mathrm{~cm}$. The electrode was connected with the coil terminal of an Oudin's resonator; the latter again was in connection with a d'Arsonval's condenser and the spark-coil $I$. The primary current of the coil was of $\mathrm{I} 2$ volts and 2 to 3 amperres, the rate of interruption 16 per second; time of exposure, 10 minutes. By this arrangement much brighter sparks were induced than in the case of the previous experiment.

On the following day a scab of dry blood the size of a lentil was visible on the exposed area of skin; the operation was repeated under the same conditions on March 22 nd and 23 rd.

On March $24^{\text {th }}$ the animal's coat showed the same changes as in the case of direct polar discharges from the coil, $i$. $e$., loosening and falling of the hair and clogging together of the woolly undergrowth. The experiments were continued on March $24^{\text {th }}, 25$ th and 26 th. By the latter day the area of skin corresponding to the electrode appeared almost bald; the rest of the hair, moreover, coinciding with the aperture of the case had become very thin and loose. The skin itself appeared quite normal during the whole experiment, except for the above-mentioned excoriation, which soon healed. In this case, also, fresh hair did not appear until the expiration of some weeks. 
The above experiments conchnsively proved that in animals the hair can be made 10 fall by applying spark-discharges, whether in the form of a direct polar discharge or as the effluvium from a d'Arsonval-Oudin's apparatus.

\section{(b) Experiments on the Action of Spark-Discharges on Bacteria.}

The organism chosen for these experiments was staphylococcus pyogenes aureus, this possessing an average capacity for resisting deleterious influences.

Exp. 6. April 3rd. A small portion from a fresh culture of staphylococcus pyogenes aureus was taken on a strip of platinum, and with this the nutrient agar of 2 Petri's dishes $A$ and $B$ were inoculated. Culture $A$ was deposited for 24 hours in the incubator, and served for control purposes; culture $B$ was uncovered and exposed to the electrode employed in Exp. 2 at a distance of $\mathrm{I} \mathrm{cm}$., the electrode being connected with one pole of the secondary coil of a Ruhmkorff's apparatus. The primary current was of 2 ampères and $\mathrm{I} 2$ volts; there were I6 interruptions per second. The culture was treated to the sparks for I 5 minutes, a metal dish being afterwards placed beneath it and earthed by means of a wire; the culture was then exposed to the discharge for I 5 minutes more, and after that placed in the incubator for i 8 hours.

April 4th. Culture $A$ shows a well-developed growth; culture $B$ shows very slight signs of growth. In the middle of it, indeed, there is an absolutely sterile patch the size of a kreutzer. This area corresponds exactly with the middle portion of the metallic electrode, from whence most of the sparks had been seen to strike.

The experiment was repeated several times, and the conclusion appeared justified that the effect of direct sparking is to hinder the development of cultures of staphylococcus pyogenes aurcus.

The object later was to disperse the spark-discharge over 
a large surface, making it everywhere of equal intensity. The cross-shaped electrode was, therefore, dispensed with, and a metallic brush $4 \frac{1}{2} \mathrm{~cm}$. by $2 \mathrm{~cm}$., the construction of which was like that of the brush electrodes used in general faradisation, was employed (electrode II) ; or a faradic brush having a diameter of $\mathrm{I} \mathrm{cm}$. was used (electrode III).

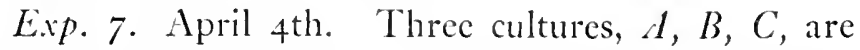
made on nutrient agar from plate $A$ of the last experiment, a streak $2 \mathrm{~cm}$. in width being inoculated in each instance. Culture $A$ is piaced in the incubator; electrode II is placed over the middle of cultures $B$ and $C$ so that its longest diameter coincides with that of the streak of bacteria. Culture $C$ is electrically earthed; distance of the electrode, I cm.; primary current, 2 ampères, I 2 volts; interruptions, 16 per second; time of exposure, 20 minutes each. Culture $C$ is seen to receive stronger sparks; after the experiment each culture is deposited in the incubator for is hours.

April 5th. Culture $A$ has developed well, also culture $B$, the latter showing a uniform growth. Culture $C$, on the other hand, shows eren to the naked eye a bare strip $8 \mathrm{~mm}$. in length, corresponding to the middle of the electrode.

This experiment shows that by earthing the current the development of cultures of this or anism is materially arrested. Exp. 8. Three streak-shaped cultures of the same organism are made on three agar plates, as in the last experiment $(A, B, C) . \quad A$, the control culture, is placed in the incubator; a fourth culture $I$ ) is made in a crossshaped manner. Culture $B$ is earthed by a wire, electrode II being placed opposite the middte of the streak at a distance of $3 \mathrm{~cm}$. An ordinary brush electrode (III) is placed opposite the middle of culture $C$, at a distance of $\frac{1}{2} \mathrm{~cm}$, but $C$ is not carthed. $\left.I\right)$ is arranged in such a way that the centre of the cross is situated immediately under electrode $I$, at a distance of $1 \mathrm{~cm}$. $I$ ) is clectrically earthed.

$B$ and $I$ are exposed for 20 minutes each; $C$ for 30 
minutes; primary current, 2 ampères, 12 volts; interruptions, I 6 per second; after the experiment all the cultures are placed in the incubator for i 8 hours.

The object of this experiment was to discover whether the distance of the electrode counts as a material factor, also whether the absence of proper electrical earthing may be compensated for by longer time of exposure and shorter distance of the electrode, and, finally, whether the area of the sterile zone is proportional to that of the active surface of the electrode.

April 6th. The result of experiment 8 is as follows: $A$ seemed to be well developed; in $B$ also no arrest of development could be noticed; $C$ shows a spot of deficient derelopment in the centre; $D$ an absolutely sterile spot the size of a kreutzer in the centre.

These results clearly show that the bactericidal action of spark-discharges rapidly decreases as the distance of the electrode from the cultures increases; further, that absence of proper electrical earthing may be to some degree compensated for by more prolonged exposure and shorter distance from the electrode.

In the case of culture $D$ the experiment shows that electrode II was not adapted for equal dispersion of the spark-discharge.

The next experiment was undertaken with the object of finding whether stronger currents and longer exposures can compensate for greater distance between the electrode and culture.

Exp. 9. April 6th. In 3 Petri dishes, $A, B$, and $C$, ribbon-shaped cultures of the same organism were made on agar as before; $A$, the control culture, is placed in the incubator; $B$ is exposed to electrode II, at a distance of $3 \mathrm{~cm}$., being duly earthed. Primary current (from the main), 4-6 ampères, I Io volts; rate of interruption, i 6 per second; time of exposure, 20 minutes.

$C$ is also earthed and placed so that the middle of its culture streak is at a distance of $3 \mathrm{~cm}$. from electrode II, and exposed for 45 minutes. Primary current, 2 ampères, I 2 volts; interruptions, 16 per second. Each 
culture is placed in the incubator for is hours after the cxperiment.

April 7 th. $f$ is well-developed; $B$ and $C$ show in their centres a sterile patch, that of $B$ being the more conspicuous.

This expcriment shows that, in spite of greater distance of the electrode, the desired inhibitory effect on the organism can still be attained by prolonging the expositre and increasing the strength of the primary current.

Exp. Io. In order to investigate the manner in which the conduction of electricity to the Hoor takes place, capsule $B$ of the last experiment was placed, not directly on a metal plate as before, but a sheet of black paper was inserted between them. During the experiment powerful sparks could be seen Hashing up and down along the walls of the Petri dish. After the experiment the paper, when held up to the light, showed a circular line, consisting of numerous tiny holes, the arrangement of which exactly corresponded with the outline of the base of the glass dish. Thus the sparks had eridently not struck through the bottom of the dish, but had taken their way from the surface of the agar over the edge of the disin, so transfixing the paper.

The above experiments clearly prove that spark-discharges have the effect of hindering the derelopment of cultures. It now remained to be seen whether they were also able to destroy cultures already grown.

Exp. II. April gth. Two P'tri dishes, $A$ and $B$, were each inoculated in a cross-shaped manner with the same organism; $I$ serres for control purposes; $B$ is corered with a sheet of paper and placel in electrical contact with the Hoor. l'lectrode $H$ is fixed above the paper at a point coinciliner with the centre of the cross. Primary current, 46 anpires, is rolts, 60 interruptions per second.

An isclated spot of a well-dereloped two-days old culture (I:xp). ()) $I:$ is placed opposite the bare end of the wire, at a distance of $1 ! \mathrm{cm}$., and exposed for 20 
minutes; primary current, 2 ampères, I I 2 volts; interruptions, 16 per second. Afterwards a small part of this irradiated portion is removed on a strip of platinum and an I-shaped figure drawn with it on a fresh nutrient agar $E$; from a non-exposed portion a II-shaped figure is drawn on the same agar $E$; all the plates are then placed in the incubator for 18 hours.

April 1 oth. $\alpha$ is well developed; $B$ is also well developed, but shows in its centre an absolutely sterile spot the size of a pfennig; the $I$ and the $I$ of capsule $E$ are also well developed; no difference can be discerned between these respective growths.

From the first part of this experiment we may conclude that the discharges have a deleterious action on organisms, even through a paper diaphragu. The sheet of paper showed numerous punctiform holes in the centre.

In the second part of the experiment any destructive effect on grown-up cultures had failed to show itself.

Exp. J2. April I ith. A colony of a culture of the same organism two days old, the size of a pinhead $a$ is placed half a centimetre below electrode II; this brush electrode is connected with apparatus II ; 20 minutes' exposure is given. The same is done with a second colony (b) for ro minutes. Then "I" from $(a)$, "II" from (b), and "III" from a non-exposed portion of the colony are grafted on fresh agars, and these three plates (I, II, and III) are deposited in the incubator for is hours.

During the sparking, which was extremely vivid, the colony and its immediate surroundings assumed a bleached appearance; this vanished after an hour or so and was replaced by a brownish hue; similar changes, but less marked, were observable with colony $b$, which had not been so long exposed.

April i 2th. Only the last of the plates which had been deposited in the incubator shows a III formed by colonies; the others are absolutely sterile, showing only 
the furrows of the $I$ and 11 in the agar. The spots $a$ and $b$ on the exposed plate appear more sterile than their surroundings.

Exp. I3. April I 2 th. Lxp. 12 is repeated, but at a distance of $I_{2}^{1} \mathrm{~cm}$. from the electrode.

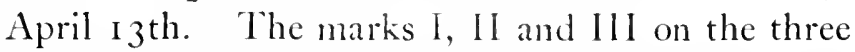
plates show a uniform development of bacteria; thus the greater distance of the electrode had counteracted the effect of the discharge.

Exp. If. April I 3 th. The general arrangement of Exp. 12 is adopted, but a larger colony of culture $A$, in the shape of a streak $\mathrm{I} \mathrm{cm}$. in length and $2 \mathrm{~mm}$. in breadth, is exposed for 20 minutes. From this an I-shaped inoculation is made on plate $B$; also a IIshaped inoculation from a non-exposed portion is made on the same plate.

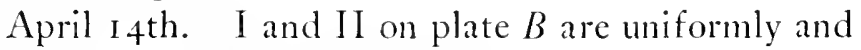
thickly covered with bacteria.

Frequent and careful repetitions of Exp. I 2 showed, however, that small colonies the size of a pinhead may be destroyed by a Io-minutes' exposure under the above-described conditions. The failure of Exp. I 4 may, therefore, be ascribed to a too short exposure of so extensive a colony. It follows from this that by employing sufficientiy strong high-tension primary currents and a sufficiently quick rate of interruption, spark-discharges can be produced which, suith the electrode at a moderate distance and a comparatively long exposure, can destroy bacterial culuures which are several days old and fully developed.

In the later experiments the current in the primary coil was so directed that the disc of the spark-gap was always cathode (-) and the point anode $(+)$. (Sce p. 64.) The object of enquiry now was to discover whether positive and negative discharges have the same physiological effects.

Exp. I5. April i 3 th. On a plate of agar $(A)$ two colonies of the organism are deposited in the shape of two parallel streaks, each about $1 \mathrm{~cm}$. wide $(1,2)$. The plate is earthed and the middle of each streak exposed to the brush electrode for 15 minutes, at a distance of 
I cm.; the electrode is in connection with the anode (point) in the case of streak $\mathrm{I}$, with the cathode (disc) with streak 2 ; primary current, 2 ampères, I 2 volts, 16 interruptions per second.

During the experiment a marked difference can be discerned in the character of the two discharges. The sparks from the negative pole are thrown in a shaft directly and vertically on the portion of the agar nearest to the electrode. They always occupy the same site; the discharges from the positive pole, however, are seen jumping from one metal point of the electrode to the other, and show a tendency to reach the circumference of the electrode.

The same procedure is repeated with a second plate $B$, which is exposed for only 8 minutes. After both experiments both plates are deposited in the incubator for is hours.

April i 4th. Both streaks on plate $A$ are well developed, but show a sterile region in their centre. This in the case of streak $\mathrm{I}$ is seen in the shape of a clear circular spot the size of a lentil; streak 2 shows in its centre more irregular sterile streaks and foci.

On plate $B$ only streak I shows a small circular ster. ile patch; streak 2 is nowhere interrupted, but uniformly covered with the growth.

This experiment shows that the bactericidal effect of positive spark-discharges is not so posverful as that of negative; also that the action of the latter is limited to a smaller area.

Further repetitions and control experiments confirmed these conclusions.

Exp. 16. April 25th, I900. A streak inoculation is made on two agar plates $(A$ and $B)$. A brush electrode in connection with d'Arsonval-Ondin's apparatus is fixed opposite the middle of streak $A$ at a distance of I cm. Primary coil current, 2 ampères, I 2 volts; interruptions, 16 per second.

The brush electrode is also fixed at the same distance opposite the middle of streak $B$, but connected with 
the cathode of the coil; both plates are earthed, and exposed for 5 minutes each.

April 26th. The growth on $B$ is well developed, but shows a sterile spot in its centre; streak $A$ is uniformly developed.

Exp. 17. April 26th. A streak-shaped colony of the organism in a P'etri's dish is treated in the middle with discharges from a d'Arsonval-Oudin's apparatus for 25 minutes.

April 27 th. The streak shows a sterile patch in the middle.

These last experiments show that spark-discharges from d'Arsonval-Oudin's apparatus act in the same way on bacteria as direct polar discharges from the coil, but that the intensity of action of the latter is greater.

Exp. 18. April 27th. Two streak-shaped cultures of the organism are made on agar; the middle one, plate $A$, is exposed at the distance of $\mathrm{I} \mathrm{cm}$. to a brush electrode conveying the discharges of a secondary current, which is induced by a primary current of 3 to 4 ampères, I 2 volts, and 16 interruptions per second. The middle of the second streak $B$ is exposed to the sparks from a primary current of 3 to 4 ampères, I I O volts, and $\mathrm{r} 6$ interruptions per second. Time of exposure in each case 3 minutes, followed by incubation for i 8 hours.

April 2Sth. Both cultures show uniformly welldeveloped uninterrupted streaks of organisms.

Exp. I9. The last experiment was repeated, this time with a longer exposure, viz., Io minutes; both streaks now show equal-sized sterile patches.

When the time of exposure was varied in still other ways no difference was found in the action of primary currents of rarying tension, but of equal intensily and rate of interuption.

These findings are quite analogous to the results of a series of experiments made by $B$. I'aller.') The latter found that

1) Fortechr. auf dem Gehiete der Röntgenstrahlen. Vol. II. p. 3I. 
the amount of tension obtaining in the circuit of a primary current has no direct effect on the length of spark evolved. ${ }^{1}$ )

Exp. 20. April 27th. 'Two streak cultivations of the organism are made on agar plates; the middle part of one (plate $A$ ) is exposed to the spark-discharge from a brush electrode at a distance of $\mathrm{I} \mathrm{cm}$. The electrode is in connection with a secondary circuit induced by primary currents of 2 ampères, I 2 volts, and I 6 interruptions per second. Plate $B$ is subjected to the same treatment, with the exception that in this case the primary current is only interrupted 8 times per second. The plates are earthed in both instances. Time of exposure 4 minutes, followed by incubation as before.

April 28th. Plate $A$ shows a well-developed colony, with a sterile patch in the centre; the growth in the case of plate $B$ is absolutely uniform.

We may conclude that the rate of interruption of the primary current is an important factor concerning the effect of the spark-discharge.

Exp. 21 . This is a modification of the last experiment. The rate of interruption is again 16 per second, but the intensity of the primary current is altered from I to 4 ampères by varying the resistances and raising the mercury vessel of the interrupter.

The result showed that a colony of bacteria exposed to the discharges from a current of I ampère for 8 minutes was not hindered in its development; whereas, the latter event occurred when another colony was exposed to a current of 4 ampères under precisely the same conditions.

Exp. 22. April 3oth. A square piece of annealed copper sheeting $\mathrm{I}$ sq. $\mathrm{cm}$. in size is placed on the floor of a sterile Petri dish (Fig. $46 K$ ) ; from the edge of this piece of metal a copper wire $B$ leads to the side of the dish, and eventually to the floor. An ordinary nutrient agar medium is placed on the copper sheet, and

$\left.{ }^{1}\right)$ Fortschr. auf dem Gebiete der Röntgenstrahlen, Vol. II, p. 3 I. 
after coagulation is uniformly covered with a small quantity of the organism. Over the colony the following arrangement is placed: $A$ disc of glass $6 \mathrm{~cm}$. in diameter and $2 \frac{1}{2} \mathrm{~cm}$. thick, with a central hole $2 \mathrm{~cm}$. in diameter, is supported by three glass pedestals; on the upper surface of the disc a sheet of paper $(P)$ is attached with sealing-wax so as to cover the central hole; on this paper a circular piece of fresh human skin $(I)$

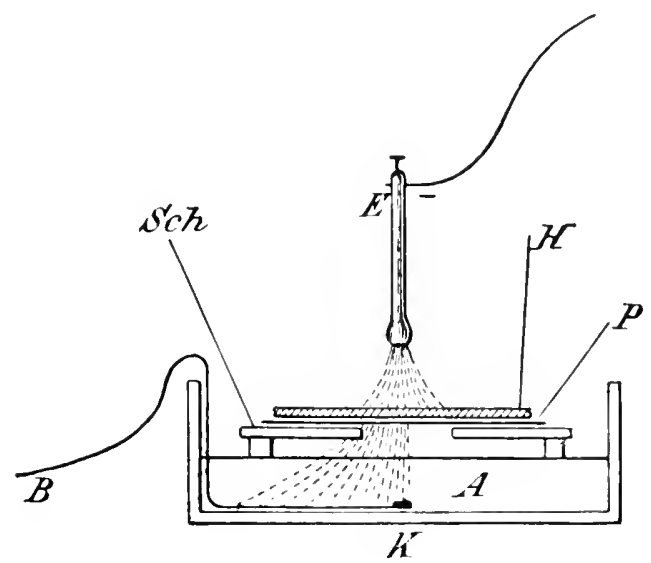

FIG. 46.

is placed. The edge of the skin does not extend quite to that of the glass disc; the hole in the latter is arranged just above the copper sheet. Above the whole a brush electrode is fixed at a distance of $1 . \mathrm{cm}$., and connected with the negative pole of the secondary circuit. Primary current, 4 to 5 amperes, 1 ro volts; roo interruptions per second; time of exposure, 20 minutes. During the whole experiment a copious discharge of sparks took place between the perforation in the glass disc and the copper sheet. After the experiment the surface of the skin appeared slightly singed within an area coinciding with the aperture in the glass disc; the paper showed a multitude of small holes with seorched border. The glass dise was then removed; the surface of the nutrient agar showed no evident changes; the 
Petri dish was then closed and placed in the incubator for two days.

On May and the culture presented the appearance shown in the photograph (Fig. 47). The agar is seen to be completely sterile in the region corresponding to the piece of copper sheeting and its wire; everywhere else the nutrient medium is seen to be uniformly covered with a well-developed growth.

This experiment proves that, ezen by interposing a piece of human skin, living bacteria can be absolntely prevented from developing by treating them with negative polar discharges of hish-tension induced currents.

The experiments hitherto described were intended to shed light on the particular circumstances under which the bactericidal effect of the electric discharge takes place.

We now proceeded to test the action of polar discharges on various other kinds of bacteria, dealing especially with those micro-organisms which bear a causal relationship to certain diseases of superficial parts of the body.

Exp. 23. April 2oth. Two streak-shaped colonies $(a, b)$ of a culture of typhus bacillus are made on nutrient agar in a Petri dish $A$, the dish is placed in the incubator for 36 hours. After this time a uniformly

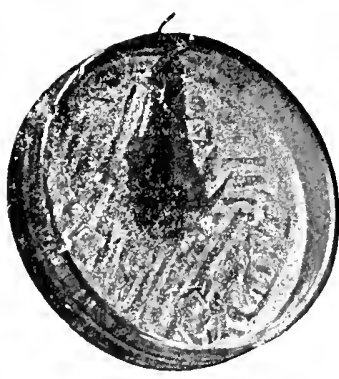

FIG. 47 .

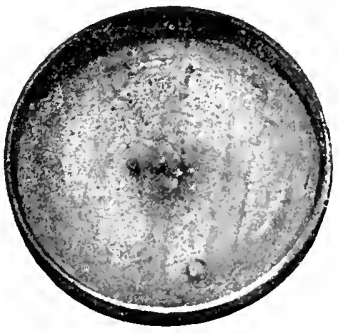

FIG. 48 .

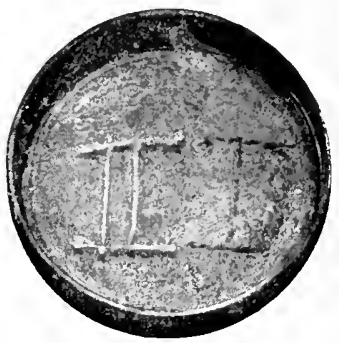

FIG. 49.

well-developed growth is seen in the sites of inoculation; streak $a$ is now exposed in the central part to the negative discharges of a secondary current, which latter is induced by a primary current of 2 ampères, I I o volts, and $\mathrm{I}$ oo interruptions per second. Discharges are pro- 
jected from a brush electrode at a distance of $\stackrel{1}{2} \mathrm{~cm}$. The Petri dish is electrically earthed; time of exposure, io minutes. After the experiment a small quantity is taken from the exposed portion (which appears rather dry) and an I-shaped figure is inoculated with it on sterile agar $B$. A portion is also taken from the non-exposed streak $b$ and a II-shaped figure inoculated on $B$; both dishes are then placed in the incubator.

April 2 Ist. Streak $a$ in dish $A$ shows a small hollowed spot in its midst; otherwise it is uniformly corered with growth. On the agar of dish $B$ the furrows made by the "I" are completely sterile; on the other hand, the "II" is everywhere corered with thicklygrown colonies.

Exp. 24. April 25th. The middle ( I) of three cultures of diphtheria bacilli sevcral days old is exposed to the negative discharge of a secondary current through a brush electrode; primary current, 2 ampères, I IO rolts; Ioo interruptions per second. The culture ressel is electrically earthed. From the exposed portion "I," as in experiment 22, is drawn on a second agar $B$; also on the same agar a "ll" is drawn with a non-exposed portion of culture 2. Both plates are then placed in the incubator.

April 26th. Streak I in plate. 1 shows a slightly brownish colour in its centre; the $I$ in plate $B$ is completely sterile, the 11 thickly corered with colonies (Fig. 49).

Exp. 25. April 25th. Exp. 23 is repeated with the same arrangement, but this time cultures of aphthe two days old are used; the results are quite similar to those in the case of the diphtheria hacilli.

Exp. 26. April 25th. The same procedure is adopted with cultures of anthrax two days old, which are treated by direct negatire polar discharges from a brush electrode. The first experiment gives negrative results; acting on Prof. I'richsellonum's adrice, the colony is moistened with sterile bouillon. V'ivid sparks ensued, 
with the evolution of moist heat, in which the organisms perished more easily. By these means their destruction is achieved.

Exp. 27. May I 2th. A culture of tubercle bacillus four months old, cultivated on glycerine agar in a test tube $(A)$ and well developed, is treated as follows:

A wire in connection with the negative pole of a secondary coil perforates the woollen plug in the test tube so that its end is placed vertically and at a distance of

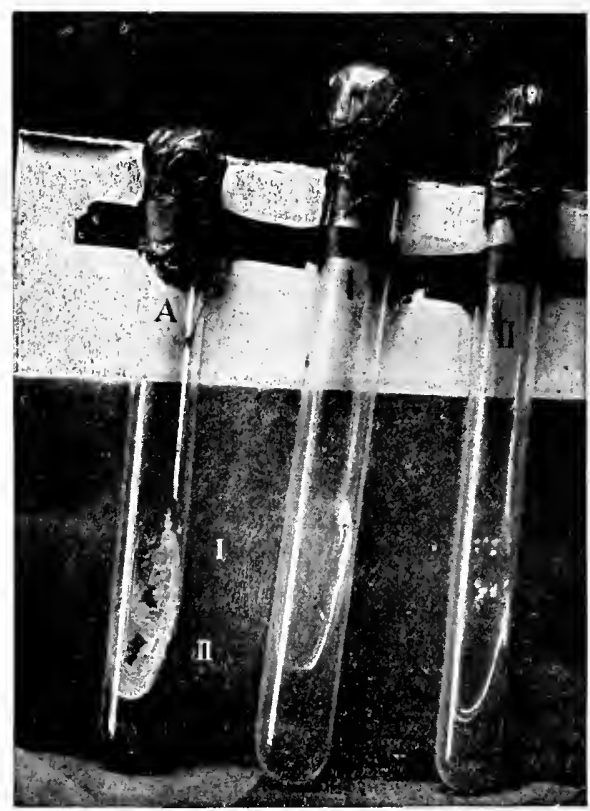

FIG. 50 .

$4 \mathrm{~mm}$. abore the culture. Outside the test tube and opposite the wire a small sheet of metal is fixed; a wire connects the latter with the floor; primary current, 2 ampères, I Io volts; I oo interruptions per second. Fxposure is continued for Io minutes, during which time sparks are seen freely striking through the tube in the direction of the metal strip outside. (The surface of the nutrient medium was placed in a slanting direction within the tube so as to be traversed by the sparks in 
their passage.) After the experiment the exposed portions of culture are apparent as two dark-brown spots the size of a lentil. (Fig. 50.) From these spots a small quantity is taken on a platinum spatula, mixed with a few drops of sterile bouillon, and a sterile sloping surface of nutrient glycerine agar in test tube $I$ inoculated with it; in the same way a portion of culture $A$ which had not been exposed to the discharge is inoculated on a third agar (test tube $/ I$ ). These three test tubes are then closed with plugs of cotton wool covered with gutta-percha tissue and placed in the incubator.

After three weeks (June 2nd) they were inspected. Test tube $A$ shows four sterile places exactly like each other; test tube $I$, on which the irradiated portion had been ingrafted, shows the surface of the agar to be completely sterile; test tube $I I$, however, in which non-exposed portions had been inoculated, shows several welldeveloped colonies (see Fig. 50).

Exp. 28. A culture of farus, in three well-developed streaks on agar and a fortnight old $(\mathcal{A})$, is exposed in the centre of its middle streak to negative discharges from the brush electrode at a distance of $\frac{1}{2} \mathrm{~cm}$. for $\mathrm{I} 5$ minutes; primary current, 2 ampères, I I o volts, 100 interruptions per second; earthing of the current. The exposed portion is then moistened and mixed with sterile bouillon and "I" of this is inoculated on a second agar B. Also a "II" of one of the other non-exposed streaks is inoculated on $B$. Both vessels are then closed and placed in the incubator.

Three days later the "I" on vesse] $B$ appears sterile; the "II," however, is thickly grown over with colonies.

In addition to the preceding experiments, experiments 23 to 28 show that it is possible by direct polar discharges striking from metal (end of the conducting wire, or brush electrodes) to prevent the development of bacterial cultures; also, under suitable experimental conditions, to absolutely destroy ezen acell-developed colonies, sereral days old, of staphylococcus 
pyog. aur., typhus bacillus, diphtheria bacillus, anthrax bacillits, aphthe, tubercle bacillus, and achorion Schönleinii.

We must not, of course, assume too much from the results of the last two experiments, since it is quite possible that these results were merely accidental in view of the difficulty presented in the cultivation of these kinds of bacilli (tubercle bacillus, achorion Schönleinii). The results of the other experiments, however, may fairly be regarded as conclusive, since in their case no difficulty of this kind exists.

Some reports on the action of electricity on bacteria already exist. Mendelsohin ${ }^{1}$ ) had shown that the galvanic current is capable of destroying micro-organisms. Apostoli, ${ }^{1}$ ) Laguerrière, $\left.{ }^{2}\right)$ Prochownik and Späth ${ }^{3}$ ) ascribed this effect to the electrolytic action of the current. Some authors ascribed similar effects to the inducing properties of electricity; especially Spilcker and Gottstein, ${ }^{4}$ ) Burci and Froscani. ${ }^{5}$ ) The statements of Spilcker and Gotistein were disputed by Friedenthal ${ }^{6}$ ) and Krïger. ${ }^{7}$ )

Klemperer, ${ }^{8}$ ) Kriiger, ${ }^{9}$ ) and Smirnow ${ }^{10}$ ) made anti-toxines from bacterial toxins by treatment with constant currents of 80 to Ioo m. a.; d'Arsonval and Charrin $^{11}$ ) succeeded in converting the toxins of pyocyaneus and diphtheria bacilli by the electrolytic action of high-frequency currents. Bonome and Vial $^{12}$ ) Meade Bolton and H. D. Pease ${ }^{13}$ ) confirmed d'Arson-

$\left.{ }^{1}\right)$ Coln's Beiträge zur Pflanzenphysiologie, I879; quoted by Gottstcin and Labarsch-Ostertag, I897, P. S2.

2) Sem. méd., isgo.

$\left.{ }^{3}\right)$ D. med. Wochenschr., ISoo.

4) Centralbl. f. Bakt., Vol. IX.

") Quoted by Labarsch-Ostertog. 1897 .

$\left.{ }^{6}\right)$ Centralbl. f. Bakt., Vols. XIX and XX.

${ }^{7}$ Zeitschr. f. kin. Med., Vol. XXII.

$\left.{ }^{8}\right)$ thid. Vol. XX, p. I65.

i) $1 . \mathrm{c}$

1") D. Med. Wochenschr., I894, No. 30.

11) 1. c.

$\left.{ }^{12}\right)$ Centrallil. f. Bakt., Vol. XIX, p. 849.

$\left.{ }^{13}\right)$ Journal of Experimental Medicine, I896, Vol. I, No. 3. 
val and Charrin's experiments on the conversion of toxins into anti-toxins, but stated that bacteria themselves were not in the least influenced. Marmicr ${ }^{1}$ ) repeated the experiments and came to quite other conclusions. He believed that by the electrolytic decomposition of the sodium chloride contained in the culture fluid hypochlorites are produced which destroy the toxins. He found that by employing d'Arsonial and Charrin's method the diphtheria toxins were heated in I 2 minutes to $\delta_{I^{\circ}} \mathrm{C}$.

Tolome $i^{2}$ ) enquired into the effects of direct sparks on acetic fermentation. When sparks from a $R u h m$ korff's apparatus strike the surface of the fermenting liquid, some arrest in the development of the organisms occurs in the case of fairly strong discharges; the liquid, however, is not sterilised, for after stopping the discharge the fermentation proceeds again, though to a less extent. According to the report of Destot's and Dubard's papers, read before the Congress on Tuberculosis, Paris, I $\$ 9 \$$, bactcria can be destroyed by static electricity.

How are these negative discharges of high-tension induced currents, which have proved to be so efficacious, to be used in practice? The use of the apparatus in the manner described for the foregoing experiments is obviously out of the question; for one thing the pain would be considerable, and the surface of the body, if irradiated in this manner, would be almost certainly damaged. Moreover, the discharges would be applied over too small an area.

Many experiments had been made with the object of dispersing the spark-discharge over a larger surface, with negative results. In no case could a simultaneous discharge be induced from all points of the surface of the electrode. The author made a series of photographs of discharges from the electrode in the following way:

1) Annales de l'institut Pastenr, 1805, 1), 53.3: 1896, p. 468.

${ }^{2}$ L'Orosi, Vol. Xill, p. 40I-409. Ref. Centrablbl. f. Bakt., Vol. IX, p. 540. 
A bromide of silver collotype film was placed in a dark room on a metal plate, the latter being earthed. An electrode was fixed at a distance of $\mathrm{I} \mathrm{cm}$. above the middle of the collotype and a current passed from the coils. The time of exposure amounted to only a few seconds; with a longer exposure the whole collotype would have been diffusely blackened. In this way a graphic picture was obtained of the positive discharges which were seen to flow off in all directions towards the metal plate (see plate, Fig. I). Another picture showed the more constant course of negative polar discharges, which always keep the same situation and follow the shortest course to the metal plate (see plate, Fig. 2). Pictures of the discharges from brush electrodes (plate, Fig. 3), also those from pencil electrodes (Fig. 4 ), show very distinctly a series of round spots where the sparks successively struck the film. From these photographs, as well as from inspection of the process of sparking itself, it became quite clear that none of these electrodes would quite meet the case. In consideration of the fact that in all these experiments good conductors had been employed, the author now determined to try bad conductors.

Exp. 29. A piece of wood was placed within the lumen of an ordinary wooden bobbin and connected with the conducting wire of the negative pole. The bobbin was fixed above the collotype in the same way as before.

The negative, on being developed, showed a bright circle corresponding to the base of the bobbin, with a completely blackened surrounding. This blackening was evidently caused by the quict (invisible) discharges from the bobbin. This experiment suggested the following:

Exp. 3o. By Prof. Valenta's advice the wooden electrode was shaped like a cylinder, the thickness of a thumb, with its ends rounded; this was placed in a glass receptacle which left only that side free which faced the collotype; the conducting wire was conveyed through the glass to the electrode.

The photograph obtained by the use of this apparatus appeared distinctly more promising. In addition to a number of 
spots corresponding to the rarying position of the spark-shaft, there was a rather diffuse and more uniform action, as shown in the plate (Fig. 5).

Instead of an imperiect conductor, such as wood, a thoroughly bad conductor, glass, was now chosen. Experiments with glass alone failed. An electrode was now contrived (Exp. 31 ) by filling a test tube with water, closing it with a cork, and conveying the conducting wire through this cork to the surface of the water. It was soon seen that one's object had been more nearly attained; discharges proceeded from the lower end of the test tube in a regular and uniform manner, in the form of a number of thin blue rays streaming off simultaneously. The photograph, too (see plate, Fig. 6), save satisfactory resultsthe discharges had produced a diffuse circular blackening figure, which showed only an insignificant bright spot in the centre. (The plate, of course, shows the photographic process reversed, $i$. e., the black spots are white, and vice versa.)

. The last defect was remored when, instead of the test tube, a small glass flask of about $8 \mathrm{~mm}$. in diameter was employed (Exp. 32), and arranged as the test tube had been. Discharges from this instrument gare a perfectly uniform and simultaneous effect. Their photograph appeared as a circular uniformly blackened dise ( see plate, Fig. 7), the diameter of which amounted to about $8 \mathrm{~cm}$, that is, about 10 times the diameter of the electrode.

These diffusely radiating discharges, which cause but fecble light effects, have heen known for a long time; the peculiar light effects which are seen in the dark on mast-tops, the ends of lightning rods, tree-tops, etc., which were named after Castor and Pollux by the ancients, and called St. Elmo's fire in our times, belong in the same class of phenomena. Points which are approached to a conductor charged with electricity collect this electricity in a high state of tension and part with it to the surrounding air. The electricity thus imparted to the air is repelled by that of like kind still remaining in the points, and leads away the electricity accumulated on the conductor; thus the result is the same as though the electricity streamed directly from the points. When the electricity streming off points in 
this manner is conducted to the ground, the appearance of a bluish vapour is produced; when, however, the space between the point and the earthing wire is increased, the electricity streams away almost invisibiy: it is now more readily discernible by the sense of touch than that of sight.

Exp. 33. An investigation now took place of the action of negative polar discharges from the last-mentioned (Alask) electrode when directed towards an earthed metal plate; also as to their behariour when a glass plate is inserted between the electrode and the metal plate. The following table gives the results:

\begin{tabular}{c|c}
$\begin{array}{c}\text { Flask-Electrode } \\
\text { Glas-Piate } \\
\text { Earth-Conduction }\end{array}$ & $\begin{array}{c}\text { Flask-Electrode } \\
\text { I }\end{array}$ \\
\hline
\end{tabular}

1. Contact of the electrode with the glass plate gives a powerful diffused blue light, from which no other visible rays stream off.

2. When the eleetrode is gradually removed the light becomes concentrated into numerous blue rays, with one central brighter ray.

3. With still greater distince of the electrode, the central ray becomes brighter and more pronounced, while the other rays become correspondingly feeble.

4. With still greater distance the central ray also disappears: a diffused bluish halo is now seen round the electrode.
I. Contact of the electrode with the metal plate gives numerous but weaker luminous rays.

2. With the electrode a short distance from the plate, one thick luminous ray appears.

3. With the distance inereased numerous weak, but still luminous, side-rays appear surrounding the central ray.

4. With the distance still increased, the central ray disappears, and

5. 'The electrude appears surrounded by a bluish luminous halo.

Exp. 3t. Similar phenomena were observed when the electrode was only half filled with water, in which the end of the conducting wire was immersed.

Exp. 35. The experiment was repeated with the electrode empty. With the electrode in contact with the glass plate a feeble light was seen; this disappeared as the electrode was remored. Precisely the same effect was produced when the metal plate was substituted for the glass plate.

Exp. 36. In order to decide whether the size of the electrode has anything to do with the quality of the dis- 
charge, a spherical electrode filled with water was used, $4 \mathrm{~cm}$. in diameter. With this apparatus no such dis. persion of the discharges could be effected as with the smaller electrode. When the electrode was placed $\mathrm{r}$ cm. from the glass plate, about a dozen thick bluish rays were seen striking from its base. As the clectrode was approached still nearer to the plate the rays became thimner and gradually brighter.

E.xp. 37. In order to decide whether these discharges caused any sensation of pain or touch, the back of one's hand was approached to the electrode in action (electrode VI). At a considerable distance eren the hand had the sensation of being breathed upon; this sensation increased as the hand was brought still nearer to the electrode, and at a distance of $1 \frac{1}{2} \mathrm{~cm}$. a spark struck the skin. There was absolutely no feeling of pain. Repeating the experinent with the larger watersphere electrode (electrode (11), a faint sensation as though the skin were being stroked with hair was felt even at a distance of half a meter. This phenomenon occurred when the hand was moved in any direction within this radius.

The effects of uni-polar discharges of high-tension induced currents differ slightly from those of high-frequency currents. In the form of a simple spark-discharge they produce a small wheal on the skin, which shows as a white spot well defined against hyperæmic surroundings. Sparking of this kind causes a smarting, burning sensation; afterwards the sensibility of the skin for further shocks rapidly diminishes. When the discharge is converted into a "quiet" one, the skin becomes first red and then bluish, slightly swollen and ordenatous. Sensibility is mostly altered in the direction of analgesia; sometimes, however, the reverse occurs.

Exp. 3S. A rabbit, whose coat was perfectly fast, was placed in a wooden receptacle as before, and the left side of its chest exposed to diffused necrative polar discharges from the witer-sphere electrode VI at a distance of + cull. Primary current, 3 amperes, 110 volts, 
I oo interruptions per second. No earthing of the current. Time of exposure, 30 minutes. No visible sparks were evident during the whole experiment, and the animal showed no signs of discomfort.

The treatment was continued in this way for 8 days (June 1st-Sth). 'The animal, which for want of proper

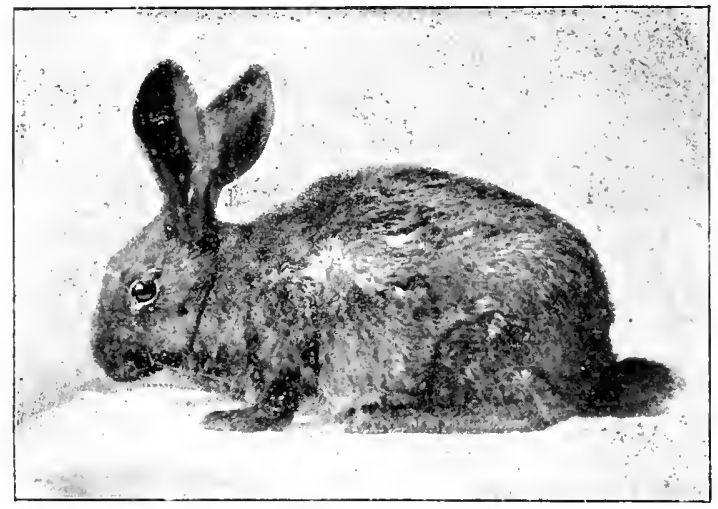

FIG. 5I.

accommodation had to be kept in a very warm room, failed gradually in health and died on June Sth. By this time, however; changes were seen in the fur exposed to the discharges; these changes exactly corresponded to those produced by direct spark-discharges: thinning of the hair, bald spots in places and clogging of the woolly undergrowth in others. (Fig. 5 I.)

Exp. 39. April 26th. A Petri dish containing nutrient agar is uniformly covered with a culture of staphylococcus pyog. aur. The test-tube electrode is fixed at a distance of $\mathrm{I} \mathrm{cm}$. above it. The apparatus is earthed. Primary current, 2 ampères, I I o volts; I oo interruptions per second. No direct sparks are visible; a bluish halo of "quiet" discharges surrounds the electrode. Time of exposure, 25 minutes. The Petri dish is afterwards placed in the incubator. April 27 th: The centre of the mutrient agar shows a sterile circular patch the size of a 
kreutzer; elsewhere a thick uniform growth is seen (Fig. 52).

Exp. fo. April 26th. Exp. 39 repeated without the earthing of the current; exposure, 35 minutes. The result was the same as in Exp. 39.

Exp. fr. May 5th. Two agar plates, $A$ and $B$, are uniformly inoculated with anthrax bacillus. $A$, the control-plate, is placed in the incubator; $B$ has its central portion exposed to electrode $\mathrm{VI}$ and is earthed. Primary current, 24 ampères, I io volts, roo interruptions per second. Exposure, 20 minutes, followed by incubation.

May 6th. A shows well-developed colonies; $B$ shows a central sterile patch and colonies only round its border.

Exp. 42. May 9th. Plate $A$ of the preceding experiment, now a culture + days old, is exposed in its central portion to the water-sphere electrode for one hour; the discharge is invisible. From the centre of $A$ an "I" is marked on a second nutrient agar in dish $B$, while from the periphery of $A$ a " 11 " is marked also on $B$.

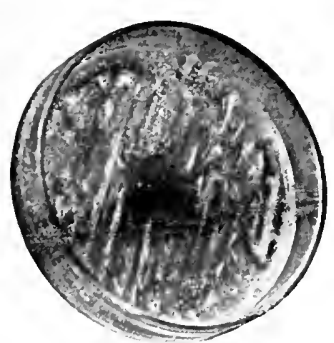

FIG. 52.

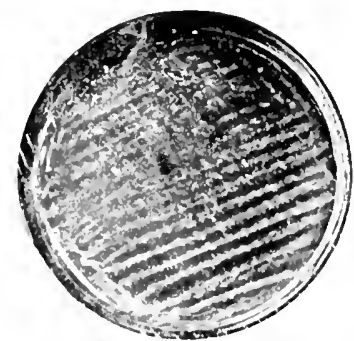

FIG. 53-Control-plate A (Exp. 43).

May 1oth. Both marks, "I" and "Il," on dish B are completely and uniformly covered with growth. The dischare had, therefore, been unable to destroy the inoculation from 1 .

Exp. 13. May oth. Two agar plates, $A$ and $B$, are covered with anthrax bacilli and placed in the incu- 
bator. After $S$ hours $B$ is removed; it shows a slimy, delicate film throughout its surface. A cover-glass preparation is made from this and stained with Moeller's carbol fuchsin-methylin blue, to show the spores. These and the bacilli are seen under the microscope to be very abundant. The central portion of $B$ is now exposed for $\mathrm{I}_{1}^{1}$ hours to electrode V'I (the water-sphere), conveying quiet negative polar discharges. Primary current, 2 ampères, I I O volts, I oO interruptions per second. Afterwards an "I" from this exposed portion is marked on a third dish $C$, while a "II" from its periphery (unexposed) is marked also on $C$. $B$ and $C$ are then placed in the incubator.

On the following day $d$ is seen to be copiously covered with colonies. $B$ is seen to be sterile, with the exception of some scanty colonies round its border. The "I" on plate $C$ is only seen by its furrow; it is quite sterile. The "II," on the other hand, is well marked with bacterial growth (see Figs. 53, 54, 55 ).

Summing up the results of the last experiments, we see that by suitable appliances we can transform direct spark-discharges

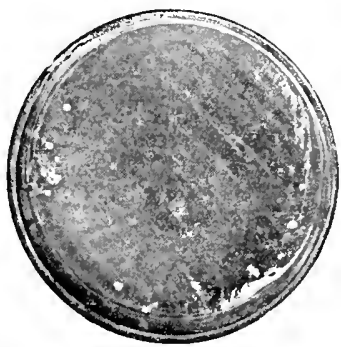

FIG. 54 .

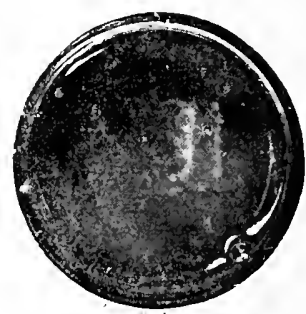

FIG. 55 .

Fig. 54.-Plate $B$ (Exp. 43).

Fig. 55.-Plate $C$ (Exp. 43).

into quiet discharges, whereby they lose somechat in their effective power; that in order to make the effect of these quiet discharges equal that of direct sparking the exposture must be prolonged; that the quiet discharges from the water-sphere electrode have a larger spliere of action.

The physiological effects of these quiet discharges exactly 
corresponded with those of direct spark-discharges: they calsed hair to fall off, they prevented the deselopment of bacteria, and they destroyed the already-developed colonies of anthrax bacilli. Electrical conduction to the ground was advantageous in this instance as before.

Direct observation of the phenomena themselves and comparison of the effects of quiet discharges and direct sparking suggests the idea that, just as the thick shafts of sparks become divided into several feebler rays, so also the physiological effect becomes weakened, but on the other hand spread over a wider area. The painlessness of quiet discharges is certainly a practical point in their favour. In using the water-sphere electrode it must be very gradually approached towards the object. The

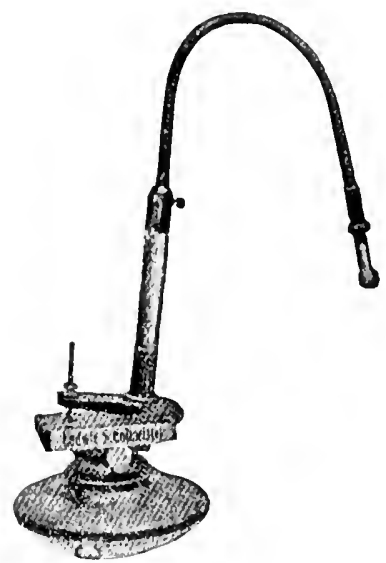

FIG. 56. author had a special apparatus made for him by Messrs. Schulmeister \& Ott, Vienna (see Fig. 56), which enables this to be attained very efficiently.

Exp. 4f. May 17 th. A cover-glass preparation of anthrax bacilli is made, and the spores shown by suitable staining. The microscope reveals an abundant culture of spore-bearing bacteria. A small quantity is, therefore, taken from the culture and mixed with I c.cm. of sterile bouillon. 'The test tube containing this mixture is placed for one day in the incubator; typical anthrax-colonies are seen to develop. Three drops, therefore, are taken of the culture and placed in the centre of a sterile and empty Petri dish. This latter is then exposed to direct sparking for 15 minutes. Brushelectrode I cm. distance; earth-conduction; primary current, 2 ampères, IIo volts, 100 interruptions per second. After the sparking a trace of the culture so treated is taken on a sterile platinum point and an "I" drawn with this on a sterile agar $B$. On the same agar 
a "II" is drawn from the non-irradiated bouillon-culture in the test tube. $B$ is then placed in the incubator.

May I 8 th. The "I" in $B$ is sterile; the "II" shows well-developed bacteria.

From this experiment it follows that micro-organisms, even when suspended in liquids, are susceptible to the destructive effects of spark-discharges.

When the conducting wire of a d'Arsonval-Oudin's apparatus in action is observed in the dark, bluish rays (brush-discharges) are seen to strike from it at right angles in its entire length. The rays are very similar to those of our water-sphere electrode, and they evoke similar sensations in the skin. In order to discover if these rays were the cause of the well-known phenomenon-the illumination of an incandescent lamp placed within (but not in contact with) a metallic spiral traversed by high-frequency currents-the following experiment was made:

Exp. 45. Within such a spiral a large pasteboard cylinder was placed, covered above and below with glass plates. Within this cylinder two spirals of wire with an incandescent lamp between them were suspended. As soon as high-frequency currents were sent through the solenoid the lamp began to shine. Since thick pasteboard, such as was used in this instance, prevents the passage of brush-discharges (as the author had proved by other experiments), we had clearly to deal here with an induction-effect within the cylinder.

The question now arose, had this inductive action any rôle in the destruction of germs by high-frequency currents?

Exp. 46. Diffused cultures of staphylococcus pyog. aur. are made on two nutrient agars (with sloping surfaces) contained in test tubes. Culture $A$ is for control purposes. Tube $B$ is wound round, opposite the agar, with four turns of thick insulated copper wire. The ends of this wire are attached to the first and last winding respectively of a d'Arsonval's solenoid $D$.

D'Arsonzal states that within a spiral of this de- 
scription sufficient heat is developed to boil mercury in the bulb of a thermometer. Consequently the spiral and test tube are placed in a ressel of ice-water. The apparatus is then set working and the culture in the test tube exposed for 45 minutes to the action of highfrequency currents.

A mercury-thermometer immersed in the tube registered $10^{\circ} \mathrm{C}$. throughout the experiment. After the exposure of the tube in this way both $A$ and $B$ were placed in the incubator.

On the following day both agars were found thickly and uniformly covered with colonies.

The experiment showed that high-frequency currents have no bactericidal effect achich can be referred to induction.

Exp. 47. In order to examine the action of spark discharges on the blood-ressels a frog was curarised. A fold of mesentery was then arranged over a perforated cork-as is done in examinations of the blood-circulation-and exposed to the direct discharges from apparatus 1 . Under the microscope a ressel was seen, from which the diagrammatic sketch (Fig. 57) is taken. A fissure $\left(S_{c h l}\right.$.) is seen in the front and hinder walls of the vessel; the direction of this fissure is perpendicular to that of the path

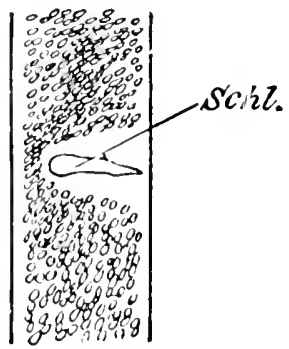

Fit. 57. of the discharge. The walls of the vessel must in some way have become fused together round the fissure, for no signs of extravasation were to be seen. The direrted course of the blood-stream is shown in the sketch. This condition obtained for nearly 48 hours.

In order to examine the histological changes which take place in living skin exposed to spark-discharges, Exp. 48 was undertaken. A rabbit was anxsthetised and a portion of its skin treated for 3 minutes to the powerful sparks from 
apparatus $I$, using the negative polar current. A second portion of the skin was exposed to sparks from apparatus $I I$, and a third, for the same time, to the effluvia of a d'Arsonval-Oudin's apparatus. Although the animal was deeply anæsthetised, breathing but superficially and showing no conjunctival reflex, it responded instantly to the first spark-discharge and made efforts to escape. The prompt appearance of these stimulating effects of high-tension induced current discharges is a point of practical significance.

After the experiment small extravasations of blood were seen on both portions of the skin which had been exposed to spark-discharges. The animal soon recovered from the effects of the chloroform and appeared quite healthy. A day later it was killed, and the exposed portions of its skin deeply excised and prepared for microscopic examination.

\section{(c) Histological Investigations.}

The specimens of skin were hardened in Mueller formol, embedded in celloidin, and cut at right angles to the surface. Horizontal sections were made of the piece which had been treated by the discharges from apparatus $I I$. The sections were stained with hæmalum-eosin, hæmotoxylin-eosin, polychrome methylene-blue, and also after the methods of TänzerUnna, Weigert and van Giesson. Specimens of skin which had not been subjected to the experiments were similarly prepared for purposes of comparison.

Examination even by low power showed that changes had occurred. These changes were localised in the upper layers of the skin in the form of a focus; with increasing depth they were spread out over a gradually increasing area.

In the stratum mucosum a cell-infiltration appeared, which contrasted well with its surroundings (see Fig. 58). This small cell infiltration separated the cellular tissue of the rete malphigii, partly taking the place of the latter in both its upper and lower layers. 'The infiltration consisted entirely of polynuclear leucocytes, thickly clustered in the upper layers and here hardly distinguishable from each other, while in the lower lay- 
ers of the infiltration the cells could be readily defined, and were seen to contain numerous eosinophile granules in their protoplasm.

Further important changes were found, at varying depths, in the pars papillaris and pars reticularis. Here, even with the

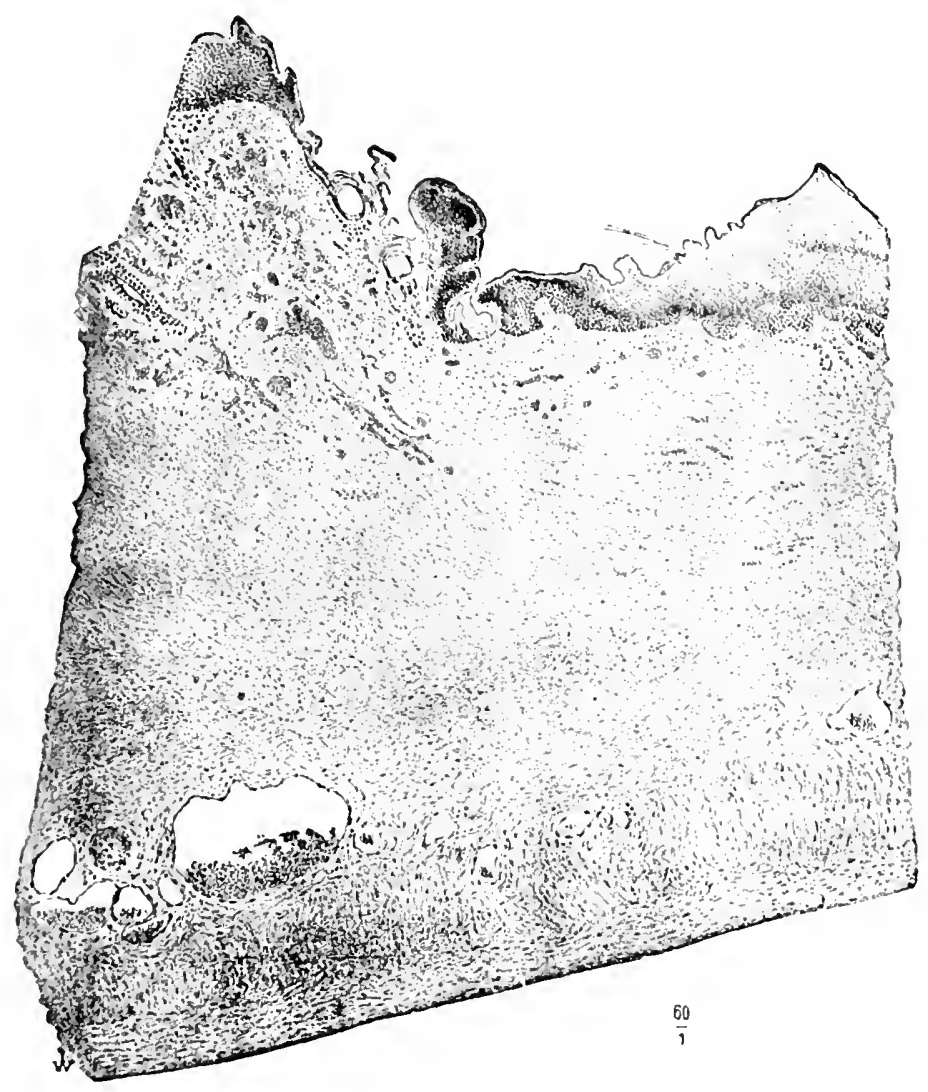

FIG. 58.

low power, an infiltration with red blood-corpuscles was evident; this filled the interstices of the tissue in the form of streaks, and surrounded the hair-follicles like a web. This was plainly shown in the horizontal sections of specimen $I I$. The infiltration was evidently a hxmorrhage into the cuticular tissues. 
Here and there on the surface of the epidermis were crusts of necrotic material; cell elements were visible here also, which proved to be red corpuscles.

Still more striking were the conditions obtaining in the bloodvessels. A casual inspection even showed marked vascular dilatation, the veins especially being widely dilated and gorged with blood. These vascular changes were more marked in the deeper layers of the corium.

The vessel walls showed especial changes in the case of the arteries (Fig. 59). In some of these the intima appeared so thickened that it occupied the greater part of the lumen. In

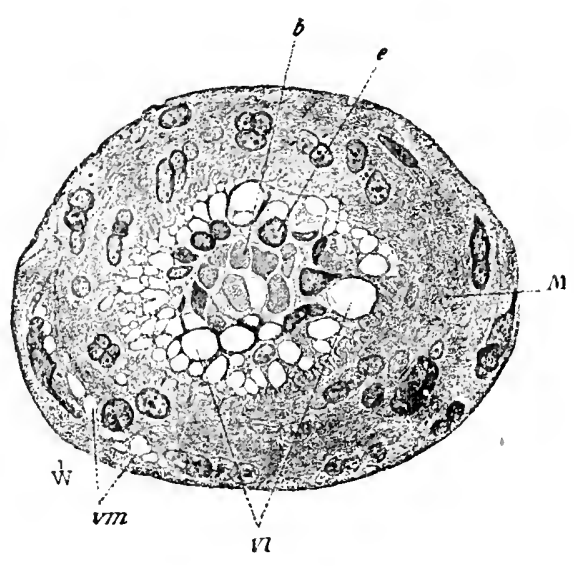

$\frac{050}{1}$

FIG. 59. the intima and media peculiar round spaces (vi) the size of a cellnucleus were visible; these spaces were packed together ( 4 or more being seen in some sections), partly raising the intima from its underlying layer, so that it was only connected by septa with the rest of the vessel-wall. Examination by the high power showed that the spaces were not really formed by the raising of the intima, from the elastica, but by vacuole degeneration of the cells. They are, moreover, not arranged purely concentrically, but quite irregularly, being here adjacent to and there remote from the vessel lumen. The spaces (vacuoles) were, as has been said, also visible in the media ( $\mathrm{vm}$ ), but to a less extent. The formation of vacuoles to the extent just described was only seen in a few sections; most sections showed only two or three vacuoles.

The nuclei of some of the cells within the vicinity of the vacuoles possessed a peculiar property. Whereas the nuclei of other cells took the stain readily and appeared finely granulated 
and dark, these nuclei remained light-coloured and homogeneous, showing no traces of granules, but rather having the appearance of drops of oil or water. Where nuclear granulation was seen this was coarser and more marked than that of the cell-protoplasm. The granules were mostly arranged at the periphery, leaving the centre clear, with perhaps several vacuoles there situated.

Many cell-nuclei in the hair root-sheaths showed similar peculiarities. Many of the hair-sheaths were empty; a few still contained hair, and in these no abnormality could be observed.

Pigment was formed in clumps in parts of the upper cuticular layers; it did not appear to be increased in quantity. In the sections stained with orcein, according to Tänzer-Unna's method, elastic fibres were visible amongst the connective tissue, both of the pars papillaris and pars reticularis. Where the hæmorrhages obtained, these fibres were sometimes pressed apart from one another and destroyed. Mast-cells, plasmacells, and giant-cells were not seen in any specimen.

Summed up, microscopical examination revealed mainly (a) small-celled infiltration in the deeper layers of the epidermis, $(b)$ extensive extravasation of blood, $(c)$ vacuolisation in the intima of the arterioles.

Comparing these facts with what Gassmann observed in a case of Roentgen-dermatitis, we are first struck by the similarity in the condition of the vessel walls in both instances. Ultraviolet light, moreover, as Glebowsky proved, produces similar changes in the cell-protoplasm. There can be no doubt that in our case these changes may be ascribed to the electric discharges; this was proved by comparison with sections of skin which had not been so treated. Moreover, only those vessels which were within the range of the discharges were affected; other vessels in the same preparation were free from change.

The author cannot decide whether the vacuolisation must be considered as due to the direct mechanical action of the sparks, or whether it is a degeneration caused by electricity itself; the latter, however, appears to him to be more probable.

A second important change in the exposed skin is repre- 
sented ty the hæmorrhage. It is quite possible that this phenomenon may bear some relation to the marked pigmentation following Roentgen-radiation; that is to say, the electric discharges from the Roentgen-tube may cause hæmorrhage, followed by the deposit of hæmosiderin.

The above-described transformations, sometimes more marked in one respect than in the others, were seen in all the sections made from the three portions of skin which had been treated by discharges from different apparatus. We may assume, therefore, that high-tension discharges, of whatever ori, gin, produce physiological effects which are identical as regards their quality and differ only in their intensity.

Many authors state that not only the cells of vessel walls, but, under certain circumstances, other structures are transformed in like manner.

Thus, on reviewing the literature of the subject we find that several botanists speak of the disorganisation of protoplasm under the influence of electricity. Kle $\mathrm{mm}^{1}$ ) mentions swelling of the protoplasmic layers and fibres and plentiful production of vacuoles of an indefinite size. Pfeffer ${ }^{2}$ ) states that the nuclei are more sensitive to electrical influence than the unformed protoplasm. In the case of tradescantia the nucleus swells almost to twice its original size, becomes circular, and then sud. denly collapses and becomes irregular in its outline; it often takes a stain before the body of the cell colours at all.

Kuehne ${ }^{3}$ ) and Vercorn ${ }^{4}$ ) observed that the galvanic current produces granular decay in the protoplasm of actinospharium, mycomycelis, polystonella and pelomyxa. Verworn traces the opacity and shrinking of the protoplasm, which is "typical of contractor-excitement," to a very fine vacuolisation (becoming froth-like and honeycombed) of the homogeneous hyaline condition which previously obtained in the protoplasm.

$\left.{ }^{1}\right)$ Jahrb. f. wissensch. Bot.. I895, Vol. XXVIIT. p. 627.

$\left.{ }^{2}\right)$ Abh. d. Saechs. Ges. d. Wiss., Nw.-math.-ph. Cf., Vol. XVI, p. I85.

$\left.{ }^{3}\right)$ Arch. f. Anat. und Phys., I859, pp. 564 and 748.-Unters, ueber das Protoplasma, Leipzig. Engelmann.

4) Arch. f. d. ges. Physiol., Vol. XLV., p. I.267: Vol. LXV, p. 47, and Sitzs. der Berl. phys. Gesellsch., Dub. Arch., I894, p. 538. 
Le also observed granular destruction of the protoplasm resulting from alternatung currents.

Roux $x^{1}$ ) found that alternating currents caused migration of pigment in frog-spawn, and contractions so powerful that the ora became broken and the yolk escaped.

C. Hess") explains the opacity of the lens which occurs after a lightning-stroke by the destruction of ephithelium in its capsule.

K. Kiribuhi") exposed rabbits' eyes to powerful spark-discharges. He found both in the protoplasm and the nuclei of the capsular epithelium a formation of racuoles, which he ascribed to electrolytic processes.

All these reports go to show that the damage caused to protoplasm by electricity consists of a loss of cohesion and a solution of its ingredients, which is probably brought about by chemical transformation (vacuolisation).

Several authors describe changes in the nerre-cell from electrical stimulation. These changes are visible under the microscope, or show themselves in the form of "fatigue."

Forybut-Daskicitis ${ }^{4}$ ) found an enlargement of the nuclei in the cells of the medulla which are in connection with the electrically-excited nerres.

Hodge ${ }^{5}$ ) found reduction in the size of the nucleus, vacuolisation of the protoplasm, and lessened staining power.

Magini") found disappearance of the nucleoli and a better staining capacity in certain cells of the anterior cornua.

$\left.I^{Y} a s^{\top}\right)$ observed swelling of the nucleus and the cell-body, also dispersion of the nucleus and the chromatic substance towards the periphery of the cell. I.ambert's") observations

1) Sitzungsb. d. K. Akad. d. Wiss., Math. Nat. Classe. Abth. III, I89r, Vol. CI p. 27, and Pflueger's Arch., 1896. Vol. LXIII.

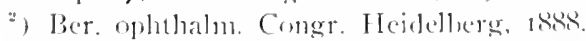

3) i'. Gracte's Arch. f. Ophlhalm., 1. I.. rooo.

1) Arch. f. mikirok. Anat., I, s, p. 51.

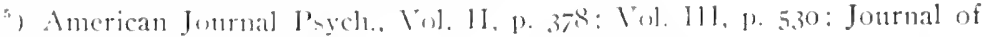
Morphology, Inl. IX, 1). H't.

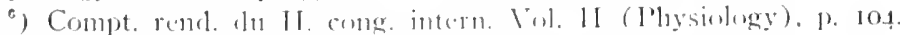

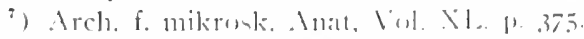

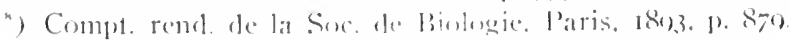


agree with those of $\operatorname{Vas} . \operatorname{Mann}^{1}$ ) found that the size of the cell, the nucleus, and the nucleolus increases during the stage of irritation, and that the chromatic matter is destroyed. In the stage of fatigue the size of the nucleus diminishes and its staining becomes more diffuse. Lugaro's ${ }^{2}$ ) observations agree with this. Ialenza $\left.{ }^{3}\right)$ found that after prolonged irritation of the electric organ of the torpedo-fish by high-tension and high-frequency currents important retrogressive changes occurred in the nerve-cells. These were shown especially in the nuclei as "chromatolysis caryorixe" and as hyperchromatosis. When the cells were adjacent to the electrode the nuclei contracted with irregular outlines and hyperchromatosis of its contents; the nucleoli, moreover, became absent. Parts more remote from the electrode, however, showed an increase in the volume of the nucleus, hyperchromatosis of the nuclear wall alone, while the nucleolus still remained in evidence. By the application of less powerful currents he found various changes in the nervecells.

G. Corrado $\left.{ }^{4}\right)$ killed dogs by the direct application of highpotential currents (400-920 volts, I O-23.5 ampères). The current was applied through broad metallic electrodes, one of which was attached to the forehead and the other to the back, near the animal's tail. With the highest tension he observed spark-shocks at the site of the electrodes, but there was always also a certain amount of burning without the formation of sparks. The autopsy revealed congestion of the meninges, the sinuses of the dura mater being engorged with blood. Often effusions of blood would be seen on the surface of the cerebrum, and on one occasion there was hamorrhagic effusion into the lateral ventricles. On the surface of the medulla oblongata numerous ecchymoses were found. The heart in most cases was in a state of systole, especially the left ventricle. In one case bubbles of gas were found in the blood, in another

1) Journ. of Anatomy and Physiology, Vol. XXIX, p. 100.

2) Lo Sperimentale a 49, sect. hiolog., Florence. 1895. 1. I59.

$\left.{ }^{3}\right)$ Atti della R. Accad. d. Sc. fisiche e nat. di Napoli, Vol. VIII. ser. 2.

4) Ann. d'électrobiol., Vol. II, 1899. p. 245. The experiments made by this anthor are very similar to those printed later by $S$. Jollinck. 
they were in the meninges of the medulla, while in a third case they occupied the cerebral fissures.

Corrado described in the first place marked alterations in the body of the nerve-cells. Many nerve-cells could be recognised through Golgi's method of staining, or by convergence of nerve fibres towards them. Most of them appeared globular, irregular, or torn. By other methods of staining (e.g., Nissel's) the fragments of the lacerated cells were also discernible; often the protoplasm near the well-preserved nucleus appeared granular. The condition was, therefore, one of cytorrhexia. The cells of the spinal marrow were less affected than those of the cerebrum. In certain of the microscopic fields cells could be seen which were absolutely normal, in addition to those which were badly damaged. Often a cell had the appearance as though parts of the protoplasm had been expelled from onc side.

In the cell-body Corrado found a removal of the chromatic substance, a homogeneous powder-like condition; in a word, marked plasmolysis. Often distinct vacuolisation was observed. The chromatic substance showed a tendency to separate itself from the remainder of the cell-body and to congregate at one side of the cell, the other side remaining uncoloured. (Corrado believes that the situation of the decolourised portion indicates the direction of the current.) In most cases the nucleus appeared changed only in its general aspect and in its rolumeits outlines were angular and irregular. The chromatic substance of the nuclei was either completely destroyed or left a few thready remnants near the periphery. The situation of the nucleus in the cell-body varied, but was generally somewhere near the circumference of the cell, especially at that side where the chromatic substance had collected. The nuclear membrane was sometimes torn. The nucleolus was generally uninjured, and stained well. Sometimes, however, it was destroyed; it changed its situation, tratersed the nuclear membrane, and arrived at the periphery of the cell.

The processes of the nerve-cells often showed a condition of "varicose atrophy." They were throughout fragmentary and disarranged. Not infrequently a spiral and highly char- 
acteristic condition was to be found in the protoplasmic processes of the pyramid cells in the cortex. Corrado believes that there is not only a chemical but also a mechanical action on the nervecells; moreover, the bubbles of gas are evolved in the interior of the cells, as in the meninges and the blood.

S. Jellinck $k^{1}$ ) observed that ganglion-cells in the spinal-malrow burst and ecchymosis also resulted after the use of powerful alternating current-discharges.

Light has been shed on the mechanics of the effects of sparkdischarges partly by direct observation of irradiated cultures, partly by microscopical investigation of the condition of the exposed skin. Short exposures produced an immediate drying effect on the inoculated nutrient medium; after longer exposure heal-effects were noticed-the nutrient medium became browncoloured, swollen, and fluid. With sparking at a rapid rate, a small flame flashed up from time to time, vapours arose, and the sound of the boiling agar was to be heard.

It is quite comprehensible that these two factors alone (desiccation and heat) would be sufficient to cause destruction of bacteria and perchance therapeutic effects also. Possibly marked desquamation - the result of desiccation, and perhaps also of the electrolytic destruction of the superficial layers of cells in the skin-may explain any good results achieved in the case of acne, seborrhoe, and similar affections. Both desiccation and rise of temperature are inimical to the regetative forms of bacteria. Even the spores which longer resist such deleterious influences may be destroyed, as the author has proved, by spark-discharges from suitable apparatus.

Tesla explained the heat-effects of high-frequency current as follows: A body, properly insulated in the air, becomes heated simply by connecting it with a source of rapidly-alternating high-tension electricity. The body becomes heated by its electrical bombardment, through the air or through any medium of a molecular or atomic constitution. The body itself may be a good or a bad conductor; this does not materially affect the heat phenomenon. The human body happens to be a good

1) Wiener klin. Wochenschr., I902, 1. 450. 
conductor; when a person standing insulated in a room comes into contact with a generator of high-frequency currents his skin becomes heated by the electrical bombardment. The extent of the calorific effect depends upon the dimensions and general arrangement of the apparatus.

The physiological effects ( $c$. g., lessened sensibility) may also reasonably be ascribed in part to "mechanical concussion," the mechanical action of the rapid bonbardment on the tissues.

Besides these factors we have, howerer, to consider still others; this was shown by the author's histologrical examination of skin treated by spark-discharges. Where the discharges had only operated for a short time, and had been produced by weak currents, merely superficial inflammation and small-celled infiltration was discernible in the upper layer of the cutis. When, however, powerful and rapidly-succeeding sparks had been in operation for a considerable time, very marked alterations were produced in the tissues.

According to Engelmann, Klemein, Davenport and others, these changes consisted in an impairment of cohesion and a solution of the cell-ingredients (vacuolisation); the latter effect is probably brought about by chemical change, the former more indirectly. Electrolytic processes can be readily understood to be going forward in the cells, and these, acting in concert with the local increase of temperature, may conceivably hinder the development of micro-organisms.

Physiological investigation thus shows, Isl, that spark-discharges of achatever origin may induce desiccation; 2nd, heateffects; zrd, that by these means the dezelopment of bacteria may be hindered; fith, that in all probability an electrolytic, and 5th, a mechanical action are to he ascribed to them. We must also bear in mind the possible action of the actinic light-rays (blue, violet, ultra-riolet) which accompany the sparking phenomena, the freely-developed o\%one, and the remote effects of the electric wares themselies.

It is theoretically possible that by means of the local action on the skin indirect effects maly be attained on the whole organism. The passive hyperamia induced on the body surface might, were a sufficiently large area treated at once, result in the 
relief of congestion in deep-lying organs. Thus in the case of d'Arsonval's method of auto-conduction, could it be proved that really efficacious high-tension discharges take place between the solenoid and the body of the patient (and the author himself has never been able to verify this), one would be the better able to understand the beneficial effects claimed for this method.

The same physiological effects on the skin can be obtained by the use of strong discharges from a static machine, or from a Roentgen apparatus. If these act on a large surface of the body for a considerable time one may also, for the above-stated reasons, expect some effect upon the general organism. In this sense the action of these electrical methods of treatment is probably comparable with that of hydro-therapeutics and light-treatment. Each aims at a local irritation of the skin. The therapeutic effects of faradic currents are probably also to be explained in this way; here also we are dealing with the discharges of induced currents, albeit very weak ones, applied to the skin.

As regards the remote (electrolytic) effects of high-frequency discharges, these are probably, on account of the low intensity of the currents employed, inferior to those produced by the employment of direct spark of brush-discharges at the exposed surface.

Arguing from the results of his own experimental and histological enquiries, and comparing these with the findings of other investigators, the author came to the following conclusions regarding the mode of action of electricity:

There is no hard and fast line to be drawn between the biological effects of statical, faradic, and high-frequency electricity. Any differences which may exist depend simply upon the strength of current employed in any particular instance. The effects are mechanical, electrolytic, and thermic; the most important action of electricity on the tissues is to be found in its disorganisation of living protoplasm (vacuolisation degeneration). When the electrical discharges are of high intensity the effect will be manifested not only at the site of their application, but also in remoter regions of the body to which the current has been conveyed. Thus in addition to local cell 
destruction at the site of impact we may get disorganisation in the cell-tissue of more remote vital parts (as in death from currents from the main or from lightning, where we may get hxmorrhages into the tissues of the central nerrous system in addition to local effects at the part struck). When, howerer, the intensity of the current is low, the effects are merely local, producing anatomical changes in the skin and nerve fibres, though sometimes functional effects (conrulsions) may also be produced. Further histological investigations are required before it can be decided whether the good results claimed for the treatment of internal diseases by the application of electricity which possesses no great intensity are to be ascribed to the direct influence of that electricity on deep-lying organs, or to the local irritation of superficial tissues (the skin, the sensory nerres) whereby secondary changes (depletion, nervous reflexes, etc.) are eroked in deeper parts. The author himself inclines to the latter view.

The author believes that the local irritation of the nerveterminals by electrical discharges plays a considerable part in ordinary electro-therapy. It appears to him rery unlikely that ordinary faradic induced currents of low intensity can have any influence on remote organs, even if it be presumed that they are conveyed to those organs along the nerve trunks. But the strong local action of alternating-current discharges as applicd by the brush-electrode must be regarded as therapeutically important; this action may be compared with that of chemical irritants, though it is possibly more powerful than that of the latter. The application of constant galvanic currents, however, if these be of fair intensity, must have some derivative effect; at the site of the electrodes considerable electrolytic action and signs of irritation in the superficial tissues are manifest. The latter show themselves by more or less marked ery. thema, often followed by pigmentation. If a powerful galvanic current be passed through the tissucs it may produce marked destructive changes. Conceivably by these diseased tissues fibres or cells might be destroyed, and so prepared for absorption.

The above considerations may serve to explain the good 
effects of faradic brush-applications in cases of neuralgia, etc., of galvanic treatment in cases of painful or tender regions in the spine.

The shocks produced by current-interruptions are certainly of importance in a therapeutic sense. Any sudden disturbance of the electrical equilibrium of the body probably acts physiologically. In this way also the body may be possibly affected by being placed in any field of electric vibrations. Undoubtedly the sudden opening and closing of currents acts as a powerful stimulus on nerve terminals; this may be transmitted along nerve trunks to the muscles, and so excite contraction. It would appear quite reasonable to apply this property of electrical, and especially faradic, currents to medical purposes.

Many authors have proved that electric vibrations produce certain effects on living tissues. B.J. Danilcwsky ${ }^{1}$ ) showed by a series of experiments that many tissues and organs (nerves, muscles, the brain, the organs of sense, protoplasm in general) are affected when placed within an electric field, even when the electric source is some distance from the organism and not in direct connection therewith. This inductive action undoubtedly exists also in the case of an organism placed in a field of atmospheric electricity. Electrical waves or rays cause physiological effects eren at considerable distances, and partition walls of wood or even stone cannot prevent these manifestations. The physiological effect shown by increased irritability, restlessness, or a feeling of oppression, according to the intensity of the electrical action and the circumstances under which it is produced. In all these instances the essential condition of electric stimulation is the oscillatory character of the electric field.

Radzikowsky, $\left.{ }^{2}\right)$ Chatzky,") Kurella, ${ }^{4}$ ) and others have made similar observations. Capriati") obtained in this way (through the agency of the electric field) an influence on the

$\left.{ }^{1}\right)$ Centralbl. f. Physiologie, XI, No. I9, 20. See also Compt. rend. du XII Congr. intern., Moscow, Vol. II, Section II, p. 59.

2) Quoted by S. Jellinck, Wr. klin. Wochenschr., 1902, p. 450.

3) Zeitschr. f. Elektrotherapie u. aerztliche-Elektrotechnik, June, Igoo.

4) Ibid.

$\left.{ }^{5}\right)$ Ibid. 
development of tadpoles; while Selim Le mstroem ${ }^{2}$ ) and $\mathrm{Bcr}$ thelot ${ }^{2}$ ) observed an effect on the growth of plants.

The detailed account given of the foregoing experiments shows that d'Arsonial-Ondin's apparatus owes its efficiency mainly to the action of spark-discharges on the skin. At the same time the possibility camnot be ignored that the electric waves sent forth by the apparatus may have some conceivable effect on other organs. This latter effect might then be applied for medical purposes, as d'Arsmeal intended. Could the existence of such an action be definitely proved, the method of d'arsonvalisation might fairly be ranked amongst other radiotherapeutic procedures.

\section{$\S 25$. The Therapeutical Application of High-Frequency Currents.}

Soon after the publication of d'Asonval's researches, his method became exploited by many writers as the best for all sorts of diseases, both internal and external. Of late, however, there has been a marked change of opinion, and the earlier enthusiasm has given way to scepticism. The reason for this is probably to be found in the fact that in many cases repetitions of d'Arsonial's experiments gave negative results. Clinically, too, results fell far short of what had been expected; consequently in some quarters the whole method received unqualified condemnation.

This attitude is by no means justifiahle, nor is it fair to stigmatise all d'Arsomal's deductions as incorrect. The author is bound to confess that he obtained no more success than many others who experimented on "auto-conduction": nevertheless he is of opinion that his failures were probably due to certain details lacking in his technicue, and more especially to deficiencies in the currents he employed. The statements of the man to whom we owe so many important discoveries are not to be disregarded in so off-hand a manner. It would, however, be very desirable if d'Arsonval would give us minuter details of the

1) Elcktrotechnische \%(cit., 18(y), Nis. 4.

") Quroted by S. Jillinck. 
arrangement of his experiments so that they could be repeated under exactly the same conditions.

As regards the utility of these currents in medicine, there can be no doubt that they are successful in dealing with certain affections where other methods have failed. Apart from their value in asthenic conditions of the nervous system, their beneficial effects in certain local and superficial affections is beyond dispute. These good effects are quite on a level with those produced by faradic and static apparatus.

\section{Indications.}

D'Arsonval infers from his bacteriological experiments that d'arsonvalisation produces a direct stimulant effect on the vital processes in the cell-protoplasm while exerting no influence whatever on the nervous system. Previous methods of electrification acted directly on the nervous system and only indirectly on metabolism by the medium of sensory, vaso-motor, and nutrition-nerves. Apostoli says d'arsonvalisation "is a medicament for the cell and a powerful modifier of the general nutrition, which it can at once promote and regulate."

D'Arsonval and his pupils, M. Benedict and others, acting on the results of their physiological experiments, laid down the following indications for the employment of high-frequency currents :

I. The production of analgesia in minor operations and the relief of superficial neuralgia.

2. Those diseases which are due to defective tissue-change (Bouchard) : Diabetes, mellitus, gout, rheumatism, obesity.

3. Parasitic diseases (tuberculosis).

4. Diseases of the nervous system.

5. Various local diseases of the skin and mucous membrane.

\section{Analgesia.}

The high-frequency method can scarcely be recommended for ansesthetic purposes in the case of surgical operations. Indeed, its analgesic effect would appear to be but trifling at the best. What there is may be accounted for partly by the 
mechanical concussion-effect on the tissues partly by the local anæmia caused by the discharges, by which the sensory nerveendings suffer in nutrition. Bateker ${ }^{1}$ states that in his case d'arsonvalisation caused impaired sensitiveness lasting for five minutes; this was succeeded by hyperasthesia and increased sensibility for temperature. Cruet and Ondin, $\left.{ }^{2}\right)$ Requier and Didsbury ${ }^{3}$ ) recommend the process as an anxsthetic for dental surgery. They stipulate that the current be of 150 to 300 ma. intensity. Dr. Bum has recently experimented in the author's establishment with Oudin's efflurium for tooth extraction. Sometimes the patients said that they felt less pain, but results were often negative: it is, however, interesting to note Bum's observations that bleeding was diminished.

\section{Diseases Associated W'ith Defective Metabolism.}

D'Arsonial reported to the Académic des Sciences (July 6 th, 1896$)$ that he had been successful with two cases of diabetes and one of obesity. The patients stood in a foot-bath in which one pole was immersed, the other-a forked electrodewas held in the hand.

In the first case of diabetes the daily amount of urine fell in 42 days from I I litres to 7 ; the sugar from 620 grm. to $\mathrm{I} 80 \mathrm{grm}$; arterial pressure, the pulse-rate, and the temperature were raised. The body-weight at first rapidly decreased, but soon advanced again. Treatment: daily sittings of $\mathrm{I} \cap \mathrm{m}$ mutes.

In the second case also there was a diminution in the sugar-secretion; here, however, the treatment was not so well borne, but left a fecling of lassitude.

In the third case (a fat cabman, weighing I,30 $\mathrm{kgrm}$. and having irregular action of the heart) daily sittings of Io minutes were given; later, as the patient complained of dyspnoxa, the sittings were reduced to 3

1) I,oc. cit.

2) Soc franc, délectrothérapie, 1808 .

$\left.{ }^{3}\right)$ Le progres mér. Vol. XIV. N⿴囗1 3. 
minutes. In this case an increase was noted in the amount of urea secreted.

Apostoli ${ }^{1}$ ) asserts that he has obtained splendid results by means of general d'arsonvalisation in cases of this class. He treated 5 I 8 patients, giving altogether 12,728 sittings and noted invariably:

Gradual improvement in the general health, increase of energy, return of the appetite, better sleep, improved digestion, an improvement in the patient's temper, better working and walking capacity. Arthritis and chronic rheumatism improved rapidly after the "auto-conduction" in from 4 to 30 sittings; movement became easier and painless.

According to Apostoli, high-frequency currents are injurious in acute rheumatism, not very efficacious in subacute rheumatism, but excellent for chronic rheumatism; they are good, moreover, for obesity and asthma (which is said often to accompany arthritis), anæmia, chloræmia, and diabetes. He states that in the case of the latter sugar is "often diminished"; in many cases where the sugar was not lessened in amount, the general health was nevertheless improved. Similar opinions are offered by $T h$. Guillos. Moutier ${ }^{2}$ ) states that he has cured two patients with urinary calculus in 25 sittings held 2-3 times a week (auto-conductions followed by "static bath" and gentle discharge-currents of 20-30 minutes). After the first few sittings stones are said to have been voided in masses (a doubtful testimony in favor of high-frequency currents). Moutier also reports the cure of two cases of biliary calculus.

Laquerriere ") speaks well of the method in the treatment of diseases associated with defective tissue-change.

On the other hand, T. Cohn and Loevoy, also Doumer ${ }^{3}$ ) state that they have observed no improvement in the case of diabetes. Bacdeker treated three diabetics in the large solenoid. The sugar was not altered in amount thereby, but some troublesome symptoms (pruritus, dryness in the throat) were relieved.

') Compt. rend. du XII Congr. internat, de médicine, Vol. II, Sect. 4a. 1). 69 .

2) Annales d'électrobiologie, Vol. II, P. 47.

3) Ibirl. Vol. III. 
Kindler ${ }^{1}$ ) also only obtained negative results with these affections.

Th. Guilloz $\left.{ }^{2}\right)$ reports that a case of obesity which had much improved under the direct continuous current again put on flesh when high-frequency currents were applied by auto-conduction.

Moutier $\left.{ }^{2}\right)$ remarks that d'arsonvalisation effects no real loss of weight in obesity, but a general decrease in corporeal bulk, the tissues increase in density.

Fovean ${ }^{*}$ ) found d'arsonvalisation ineffective in obesity but states that thyroid treatment is better borne in conjunction with it. Doumer ") stated at the Congress for Radiology, 1900, that there existed unanimity of opinion as to the ineffectireness of d'arsonvalisation in diseases associated with defective tissuechange. The author's own experience in this direction has not been very extensive. He has treated some cases, which would be classified among the "arthritis" group by the French school, by means of auto-conduction, but has seen no very definite results. Now and then it is true that patients declared themselves feeling better, but these were generally neurotic individuals who were probally under the influence of suggestion. Nevertheless he thinks it quite possible that d'arsonvalisation, as well as any other form of high-tension electricity, may exert a favorable influence on this kind of disease. Benclicial effects in the case of internal disease might reasonably be looked for, bearing in mind the counter-irritation produced by the currents on the skin. Where the skin surface treated is fairly large, one might assume that by making it hyperiemic the hlood-supply of internal organs would be somewhat modified. The local irritation of the skin plays a considerable part in the often successful treatment of these diseases by water or light. This local irritation is, of course, quite insignificant in the treatment by aluto-

1) Annales d'électrolinlogie. Vol. Ill

${ }^{2}$ ) Congr. de Radiologrie, l'aris. boo.

$\left.{ }^{3}\right)$ Acarl. d. Sc., May 1, 1899.

4) Fortschr, d. Medicin, trgor, No. 13.

s) I Congr. d. Ratlinlogie, I'aris, Comple rend. 
conduction, but more marked when the currents are applied locally.

Ondin states that by means of the local application of highfrequency currents he obtained results quite similar to those produced by d'Arsonzal's auto-conduction method. He claims that by local applications along the spinal column he obtained an increase in blood-pressure, improvement in arthritis and old pleuritic effusions, and in pulmonary tuberculosis.

\section{Tuberculosis.}

In the year i 899 the newspapers published a sensational report that Tesla had succeeded in curing pulmonary tuberculosis by high-frequency currents.

The report was not confirmed. Tesla had evidently only suggested the possibility of this cure, arguing from the bactericidal effects of his currents, and this possibility is, of course, not precluded. But the history of medicine teaches that the therapeutical efficacy of a method may be quite independent of its physiological value; what succeeds in the laboratory often fails entirely in clinical practice. Consequently we must not take it as a matter of course that a method which proves efficacious in dealing with pure cultures of pathogenic micro-organisms will be equally successful in dealing with the disease itself.

In the second part of the Annales d'électrobiologie, etc. ( I 900$)$, E. Doumer mentions a series of I 7 cases of advanced tuberculosis which he had treated 4 years previously with highfrequency currents. He exposed those parts of the chest-wall corresponding to the lung trouble, the supra-clavicular region, and the supra- and infra-spinal regions to the effluvium from an Ondin's resonator. In another series of cases he used the effluvium from the secondary coil of Tesla's transformer. The sittings took place daily as a rule for 5-I 2 minutes. He did not try to avoid direct spark-shocks, but on the contrary endeavored to produce them.

The first effects (after 5- $\delta$ sittings) included easier breathing and disappearance of the evening rise of temperature. After 2 months the patient began to put on flesh, while the cough and 
expectoration diminished. The stethoscopical signs persisted longer. During the course of treatment, bacilli in the sputum gradually decreased in numbers, sometimes disappearing altogether for a time, to return later. Since improrement in the generai health always precedes diminution in the number of bacilli, Donmer is inclined to ascribe the beneficial effects of high-frequency currents in this disease to their power of so improving the general nutrition that the patient's resisting powers against the micro-organisms are likewise strengthened.

Oudin also reports similar improrements in pulmonary tuberculosis. ${ }^{1}$ ) He used the strongest possible discharges from his resonator. He applied the current to the thorax for 10 or I 5 minutes, laying his fingers on an adjacent part of the chest wall "so as to direct the currents as far as possible to the diseased portion of the lung." Towards the end of each sitting he stroked the skin with a brush, thereby causing it to be bombarded with numerous sparks. Gandil") states that he obtained rapid cures with Ondin's resonator even in advanced stages of the disease and under bad hygienic conditions. $R i$. viere reports similar results. $R$. Sudnik.") states that he cured a tubercular abscess of the bone and several glandular abscesses by means of high-frequency currents. (His account of the case of bone-abscesses is, howerer, by no means convincing; it might have healed spontaneously in much the same way he describes.)

The above reports are no doubt very interesting, and deserve careful analysis. The author, in conjunction with Dr. Kahane, has undertaken a series of like investigations, and results will be published in due course. He thinks it better to wait until lengthy and careful ohservation of many cases, with detailed examination of all the physical symptoms from time to time, entitle one to form conclusions.

It may here be mentioned that pulmonary tuberculosis is said to have been cured also by other varieties of electrical dis-

1) Annales d’édectrobiologice. V'ol. [1, p. 382.

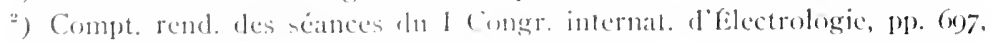
755.

3) Hhid. page 312 . 
charges, $e . g$. , those from static apparatus ( $/$ assilief $\left.^{1}\right), \mathrm{Ni}$ kolsky $y^{2}$.

\section{Affections of the Nervons System.}

Influenced, no doubt, by their teacher d'Arsonval, who says that high-frequency currents have no effect on the peripheral nerves, either sensory or motor, Apostoli, Berlioz and others, deny their beneficial action in any case of nervous disease. Apostoli, indeed, says that they only aggrarate cases of ncurasthenia, hysteria and neuritis; he regards these diseases, which are influenced so farourably by static electricity, as contra-indications for the employment of high-frequency currents.

In the author's opinion one cannot distinguish between the physiological effects of a static apparatus and those of an Oudin's resonator. Both kinds of apparatus are, as stated above, producers of spark-discharges, and if one acts beneficially in certain cases, the other should also be of value. The author has repeatedly found that certain nervous affections for which franklinisation is recommended as the remedy "par excellence" have improved under uni-polar discharges from induction apparaus, or discharges from Ondials resonator; on the other hand, there are unfortunately rery many cases where neither franklinsation nor high-frequency currents are the least use. Nerertheless, Apostoli's statement would appear to be far too sweeping and by no means proved. The author, indeed, holds that in certain nerrous diseases good effects are sooner obtained (by means of high-frequency currents) than in other diseases of the general organism. We must, however, carefully distinguish the effects of auto-conduction from those of direct local application.

Auto-conduction would appear to have a strong "suggestive" effect upon the patient. He is likely to be impressed, and his imagination stimulated, by the sight of the apparatus with its accompanying phenomena; it seems to him that such a powerful treatment must necessarily be successful.

1) Klin.-therap. Wochenschr., 1898, No. 22.

2) 11 ratsch, rgoo, No. 15. 
The whole procedure may be regarded as a "suggestive" remedy, therefore, which is justifiable only in severe cases, and especially those of the depressed type. Cases of functional impotence, for example, have done remarkably well under this method after other methods ( $c$. .., hydrotheriphy) which were known to the patient had failed. On the other hand, cases associated with nerrous excitement mostly do badly, their restlessness, insommia, etc, being algeralrated by the treatment.

The effect of a resonator-discharge is of a different and more material order. The results are often sumprisingly good, and may be explained by the counter-irritant effect upon the skin and the direct action upon the nerves themselves. Sciatica, the shooting pains of Tabes corsalis, neuritis, neuralgia, and arthritic pains have been relieved in this way.

A patient, aged 30 , who had been incessantly tortured by neuralgic pains along the right sciatic and anterior crural nerves, so that walking and standing were difficult and his sleep much disturbed, was vastly improved by this method. The painful regions were lightly stroked daily for 10 minutes with an uncovered electrode. The first few sittings relieved the patient, and after 7 sittings he was permanently cured.

A female patient was recently under treatment, in the author's institute, for aural neuralgia with insomnia. This case D)r. Kalane speedily cured by local application of high-frequency currents.

Bacdeker ${ }^{1}$ ) speaks well of the effect of auto-conduction on the insomnia of nerrous and hysterical patients. I lis opinion is corroborated by $T$. Cohn") and Botsean de Rocher"). Bacdacker also obtained good results in local d'arsonvalisation for neuralgia (sciatica, cervical, interestal and occipital neuralgia). He found that neuralgia of the fifth nerve was ageralrated by the resonator-discharge; myalcria, however, improved. Arthralgia and erythromelaçia are said to have improved after a few sittings. I Ieadache of nervous, animic, or uremic origin was

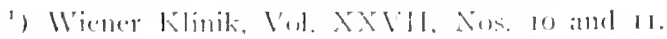

$\Rightarrow$ Berlin. Klin. Mindr. 1000.

3) Compl. rend., 1. 351. 
cured by d'arsonvalisation. E. Kindler $r^{1}$ ) found it improved the general debility, pariesthesia, hyperæsthesia and insomnia of neurasthenia; Apostoli obtained success with migraine, arthritic neuralgia, lithiasis, varix, hæmorrhoids, constipation and dyspepsia.

In cases where the spark-discharge from a resonator has been indicated, the author has applied this daily, from 6 to 20 minutes in the first stages, afterwards every second or third day only. The spark-application should be continued until the skin shows distinct signs of reaction; the time of the appearance of this, of course, depends upon the intensity of the discharge.

\section{Affections of the Skin and Mucous Membranes.}

It can hardly be questioned that definite results are obtained by discharges from an Oudin's resonator upon certain superficial diseases. These results may be ascribed to the local effects of the spark-shocks, and also to the more quiet discharges, whereby mechanical, chemical, or thermal effects ensue. Possibly in some cases, $c . g$., pruritus, dynamic electricity may play a lesser part; in the main, however, the mechanical effect of the sparking must be considered as the chief factor. Proof of this may be found in the fact that when the electrode is kept tightly pressed against the skin no alteration takes place in the lesion; results are only obtained when the electrode is kept at some little distance away, so that sparks bombard the skin. In the first case, dynamic electricity alone is operating, while in the second we have the addition of static discharges.

Already this method of applying high-frequency currents has found a large field in dermatology. Oudin, Bollaan and others have used it successfully for molluscum contagiosum, psoriasis, eczema, pruritus, impetigo, herpes zoster, furunculosis, acne, acne rosacea, seborrhoa, sycosis, tuberculosis of the skin and venereal warts; Ondin and Barthélemy for keloid; Bisserié, Bordier, Gaston, Chabry and Rieder for alopecia areata; Pearsons and Rivièe for epithelioma; Bisserié for lupus erythematosus, and Brocq for atrophia cutis, etc.

1) Fortschr. d, Medicin, 1901, No. 13. 
Thus we may see that many writers claim to have achieved good results upon ulcers and parasitic affections of the skin by this method, which combines high-tension clectricity with sparkdischarges. Lupoid, trophic, varicose and venereal ulcers, impetigo, acne, furunculosis, gonorrhoa, and alopecia areata (which most of these authors regard as a parasitic discase) are said to have been cured in this way.

We may therefore regard parasitic skin diseases as suitable for this particular mode of employing high-tension electricity. The author's own experience is as follows: By the use of sparkdischarges, however produced (whether from high-frequency apparatus of static machines, etc.), he has obtained a distinctly good effect upon ulcers. The earliest sign has been a drying and cleansing of the ulcer base. With lupus ulcerations he observed after a few days a tendency towards healing, even in obstinate cases. But, while acknowledging this improvement, he fecls bound to state that up to the present he has not succeeded in absolutely curing an ulcer of infective origin by this process. Together with $S$ chiff, he treated a soft chancre in this way, with the view of testing the parasiticidal action of sparkdischarges. The same effects were observed as with lupus ulcerations - a cleansing and drying of the ulcer-base. Nevertheless the ulcer showed no tendency to heal quicker than usual, and after 6 sittings the treatment was abandoned in farour of the iodoform method. The impression gained was that at the best the high-frequency method in this case was no improvement on older methods. Broc $\left.q^{1}\right)$, Bisserié $e^{2}$ ) and Gaston obtained a temporary and slight improvement with lupus vulgaris; even this, however, was not lasting ${ }^{3}$ ).

Probably the morbid infiltration in these cases is too deepseated for the spark-discharge, which loses its effect at the surface. The author's microscopical investigations lend support to this theory.

$\left.{ }^{1}\right)$ Traitement des dermatoses, Paris, 1808 .

2) Journ. des malard. cutan., I80, 1). 372

$\left.{ }^{3}\right)$ Chisholm W'illians (meeting of Pritish Merlical Association, Manchester, igo2) reports that he cureel a cane of facial lupus by general d'arsonvalisation. This experience appears to be mique. 
IVilh a suitable arrangement of the apparatus (sufficiently powerful currents, elc.) the discharges may be made to penetrate very derply (see the experiments quoted on p. Iog and I29; according to Flemming's reports (quoted by Strebel), alternating currents of lesser frequency, $\iota . g$., Ioo per second, penctrate about $26 \mathrm{~mm}$. into copper, while those of $\mathrm{I}, 000,000$ per second penetrate only $\frac{1}{15} \mathrm{~mm}$. Those of slower rate can penetrate ciarious media, including human skin, and exert vigorous bactericidal effecis of some depth. It has, however, not proved possible as yet to use the apparatus necessary for this penetration in practice.

The author's experience of high-frequency currents in the treatment of alopecia areata is not quite in accordance with that of other writers. Many of them claim to have cured this disease by faradic and static electricity and by d'Arsonval's highfrequency currents. The writer treated a boy suffering from alopecia areata by uni-polar discharges on a bald plaque. After some time lanugo appeared, but on the whole the result was quite inconclusive. Schilf afterwards treated the same patient by Elrmann's method of faradisation, but without success. Since that time the author has often repeated the experiment, but with no better results. Assuming that the treatment has been successful in some cases of alopecia areata-and the bona fides of other workers is not to be questioned-the question arises, is the good effect due to the destruction of micro-organisms, or to the counter-irritations and consequent increased blood-supply to the definitely-acting hair papilla? Before leaving the subject of the treatment of alopecia areata by high-frequency currents, we may refer to an observation by Neumann, who aptly states that decided proof of the efficacy of the method can only be looked for in a universal alopecia of several years' standing, in which the possibility of spontaneous healing is practically excluded. It would appear desirable that experiments should continue in this field. Processes which are undoubtedly infective in origin (such as trachoma) should be subjected to this treatment.

A second group of skin-diseases which are benefited by high-tension electricity is to be found in those disorders which 
are characterised by hypertrophy of the fibrous tissues and chronic cell-infiltration of the cutis. From many reliable sources come reports of success with keloid, cicatrices, sclerema circumscriptum, and elephantiasis by these means. Bisseric, whose authority is beyond question, gives an account in a notable work ${ }^{1}$ ) of the treatment of 62 cases of lupus erythematosus, 33 of which were completely and permanently cured by high-frequency currents. Jaquot, too, cured 39 cases out of 56 with this disease.

These results are probably to be explained by the electrolytic effect on the tissues by the high-tension electricity; again we must count as favourable factors the heat-effects, the mechanical effects ("electrical massage"), the mechanical destruction of important parts of the diseased tissue, and the decreased nutrition of the morbid growth which results from alterations in its vascular supply.

The author himself had a case of lupus erythematosus of the face under treatment, in which his experience agreed on the whole with that of Bisseric. A dark-red erythema first appeared, not only in the lesion itself, but forming a border $\mathrm{cm}$. in breadth around it. The scales and crusts gradually left the diseased site. Then the erythema began to diminish, finally disappearing altogether. Some pigmentation was left hehind. The treatment could not be continued, unfortunately, as the patient failed to attend.

We may note the fact that Roentgen-treatment with hard tubes, in which high-tension electricity probably plays an important part, succeeds also with this class of discase (elephantiasis, lupus erythematosus).

The third important indication for the application of hightension electricity is to be found in prurilus and pruriginous affections generally.

The author, in company with others (Broce, Bisserie, Leredde, and even Stranss, who believes this to be the only lecritimate use of high-frequency currents) has observed a favourable

1) Journ. de Médicine et de Chirurgie protiques, February 25. Ig0r, 
effect in most cases of essential and symptomatic pruritus (e.g., in jaundice and eczema) by means of high-frequency currents, faradic electricity, and uni-polar discharges from a Ruhmkorff's coil. The distressing itching is markedly relieved, sometimes in a comparatively short time (after 5 sittings). The author has not been able to histologically examine a portion of the treated skin and so determine the cause of this success, but clinical observation and theoretical reasoning would support the idea that the production of heat and the mechanical impact of the sparks are of importance. Possibly, too, the stimulation of the local circulation, the counter-irritant effects of the spark-discharge and its mechanical or electro-chemical influence on the peripheric nerveterminations are to be considered. The relief of the pruritus of impetigo, eczema, etc., may be explained by the destruction of the denuded nerve-endings. But the whole question requires further and more exact investigation for its elucidation.

Polar discharges from high-tension induced currents employed in this way have a distinct depilatory effect. This was confirmed by $S$ chiff $^{1}$ ) by experiment on his own cuticle, and also by Kaiser $^{2}$ ). Disturbances of the circulation in the skin are successfully treated by the discharges from an Oudin's resonator. We have already seen that the effluvium causes some local anæmia from vascular spasms; this property may be utilised in the treatment of paretic conditions of the skin capillaries. Thus the high-frequency currents may be used with advantage in the treatment of chilblain and certain other angioneuroses. The author, in company with Thielée, Baudet, and others, has seen very good results with perniones, especially as regards the relief of itching. It must be noted that the same success can be obtained by using electrical discharges which are otherwise produced; for instance, faradic currents have the same effect. This was first proved by Gautier. We have already mentioned ( $p$. I60) the use of spark-discharges in producing desquamation of the epidermis (for cases of acne, seborrhea, ephelides, chloasma). The author has seen definite though not very extraordi. nary results achieved in this way.

$\left.{ }^{1}\right)$ K. k. Gesellschaft der Aerzte WVien, Dec. I4, 1900.

") Wiener klin. Woch., I90I, No. 3I. 
Some writers speak well of the use of high-frequency currents in affections of the mucous membranes. Thus $E$.

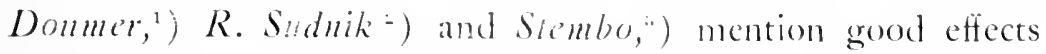
in cases of hamorrhoids, which they describe as antiphlogistic and "resolving." The former effect-the antiphlogistic-is said to relieve the congestive symptoms after a few sittings, while the "resolving" appears later, but is seen in even old and inveterate cases. Doumer, moreorer, mentions extremely good results with fissura ani, which he states is cured in from 2 to 6 sittings of $3-6$ minutes each.

Tschdanow ${ }^{+}$) made extensive trials of the method in 85 cases of fissura ani with very good results. He used bi-polar discharges, without resonator, curing his cases after $15-30$ sittings applied every other day. The pain was first relieved, then the straining, later the sphincter-spasm, the difficult defiecation, and the bleeding. The fissures healed perfectly in every calse.

$R$. Sudnil ") reports that he has used high-frequency currents successfully in conjunctivitis granulosa and in blenorrhora urethre with paraphimosis. He treated gonorrhera by wrapping the penis in cotton-wool covered by a zinc-plate, to which the currents were conducted. Or the penis was placed in a glass tube with a metallic bottom, and containing boracic lotion; the metallic bottom was then conducted with one end of the solenoid, the other end being joined to an electrode placed over the perineum. He treated the urethro-vaginitis of women by introducing a moist plug of cotton-wool into the vagina and connecting it with one end of the solenoid, the other end being connected with an electrode applied to the hypogastrium. By this proceeding the micro-organisms are said to be killed; nevertheless, the discharge is not completely remored. Application of the currents to the perineum is said to suppress inflammation of the back part of the urethra in recent cases. From other experiments Sudnik infers, moreover, that the antiphlogistic action of high-

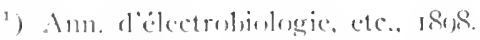

2) 1hirl. 18roy.

") Dentecle merl. Wuehentelir. Ironz. No. S.

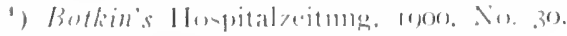

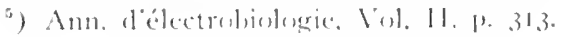


frequency currents is useful in boils, buboes, orchitis, and acute articular rheumatism. Doume'r ${ }^{1}$ ) confirmed Sudnik's opinion. He found that in gonorrhoea the inflammation was rapidly suppressed, while the discharge and pains soon disappeared.

The reader is referred to an earlier description for the technique of the method of local application (see Fig. 60). It

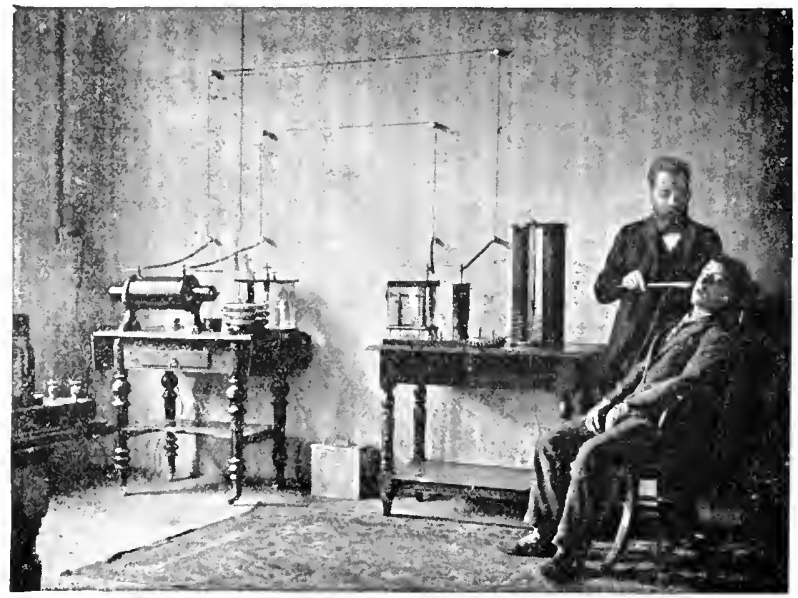

FIG. 60.

is sometimes advisable to apply the currents daily; at others twice or thrice a week will suffice. The sittings may vary in time from 2 to 10 minutes. The operator must be guided always by the reaction seen on the skin. As a rule, several weeks are required for a cure, if this be attainable. Bisserié gives 70 sittings as the utmost required for the treatment of lupus erythematosus. For other diseases, however, such as pruritus and eczema, even from 3 to 5 sittings may suffice.

\section{Summary.}

In the d'Arsonval-Oudin method of treatment we have clearly one which utilises both electrical waves and static discharges. IVe have seen that while the high-frequency currents

1) Congr. intern. d'électrobiologie. August I, 1900. 
produced by the present-day apparatus give us physiological and therapeutic results which are somewhat indefinite, the results obtained from the static discharges of Ondin's resonator may be more exactly demonstrated.

IVe have thus to deal mainly with effects which are quite similar to those produced by faradisation and franklinisation. The only difference is one of degree, corresponding to the difference in current intensity of the several apparatus.

From a survey of the physiological and clinical effects of high-tension electricity (including the discharges from induction-, influence-, and d'Arsonval-Ondin's apparatus) we may say: That this method possesses many adrantages in common with Roentgen-therapy; thus it dispenses with dressings and causes little pain, the sittings last but a few minutes each, so that no great sacrifice is demanded from the patient, and finally that the cosmetic results are very good.

We have seen that the method possesses certain valuable properties (bactericidal, desiccating, anti-pruritic) which are of advantage in several skin-diseases; we have found, however, that its virtues are not so pronounced that older and more approved methods may be disregarded. Nevertheless, due attention must be paid to a new department which has undoubtedly enriched our therapeutical resources, trusting that in time it may receive the improvement and scientific development which it now so greatly lacks.

The treatment by monopolar vollaic currents should probably he included in radio-therapy. NarkiewiezJodko and Colombo utilise the cathodal waves from a Ruhmkorff's coil for curative purposes. The operator holds in his hand a test-tube containing liquid in which the anodal terminal (a copper plate) is immersed. The operator then strokes the paticnt with the other hand. The patient thereby receives electrical waves from the cathode, and becomes chared with necrative electricity. It is said that by this method the same results are achieved as by d'. Irsonzal's auto-conduction process ${ }^{1}$ ).

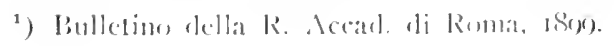




\section{Appendix.}

$\$ 25$. In the treatment of the so-called "permeating electricity" method of E.K. Mueller (Zürich) the patient is exposed to the influence of a magnetic field of high intensity produced by a powerful wave-forming current.

The apparatus consists in the main of a wire spiral of over 200 windings traversed by a current of high intensity (20-60 ampères) but low tension and frequency. By these means a magnetic field of low frequency, but high intensity, is produced; the lines of force in this magnetic field penetrate the body which is to be treated.

The wire spiral surrounds a core composed of layers of paramagnetic material (soft iron, nickel, etc.), and containing a central space for system of cold-water circulation. The coil itself, moreover, is provided with a cooling arrangement.

The coil may be supplied with a continuous or an alternating current. The current merely needs to vary periodically in intensity, its direction being immaterial. This undulating supplycurrent, whether continuous or alternating, will always produce alternating currents in the windings of the coil by self-induction, and these in turn will create an undulatory magnetic field. The para-magnetic core is not absolutely essential, but it enhances the magnetic effect.

According to Rodari, ${ }^{1}$ ) the treatment has the reverse of the stimulating action on the organism produced by d'Arsonval's method. The effect is said to be mainly on the nervous system.

Rodari gives the following indications for its employment: r. Peripheric nerve diseases (neuroses) : neuralgia of the trigeminal, occipital and temporal nerves; diffuse headache; migrain; neuralgia of the brachial plexus, of the intercostal nerves; sciatica; conditions of a neuralgic nature, especially writers' cramp, cramp in the calf; also lumbago and muscular rheumatism. 2. Central neurosis: irritative forms of acquired neurasthenia with insomnia. 3. Sensory disorders of internal organs: angina pectoris, hyperæsthesia of the gastro-intestinal tract. 4. Local

$\left.{ }^{1}\right)$ Berlin. klin. Woch., I90I, Nos. 23 and 24. 
acute gout. 5. Ataxia and the shooling pains of tabes dorsalis. Since the publication of these indications another has been added to the list, viz.. 6. Subacute arlicular rhenmatism.

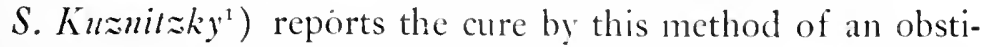
nate lumbago, which disappeared after 5 sittings of 20 minutes each. A valuable St. Bernard (log suffering from palsy of the hindlegs is said to have been cured in 3 sittings. P. Isherosk $y^{2}$ ) states that his investigations show that an electro-magnetic field produced by alternating currents of high-frequency and high tension causes considerable changes in the organism and in the skin. He noted especially increase of blood-pressure, the pulse becoming slower and fuller, and the respiration less frequent and deeper, while the sensibility of the skin for perception of space and faradic irritation became heightened. Moreover, he found a striking increase of general liveliness on the part of the patient, and rapid recovery from the effects of hard mental work. We may conclude from this that an undulating magnetic field does not act soothingly in Rodari's sense, but rather as a stimulant, like d'Asoncal's alternating currents. Here, too, the disturbing effects on the electrical equilibrium act as physiological irritants (see p. I64).

That magnetism is under certain circumstances capable of exciting similar physical phenomena to those produced by the other kinds of "radiation" treated of in this work may be proved by Ph. Braham's ${ }^{3}$ ) experiment. In this a sensitised photographic plate is placed between the poles of a powerful clectromagnet having an interrupted current; a similar impression is made on the plate as by ordinary light, a black portion appearing on development corresponding to the part situated directly between the poles.

Grence ${ }^{4}$ ) has made similar observations; Ch. Graf,") on the other hand, was unable to confirm these experiments.

\footnotetext{
1) Aerztliche Rundschau, Munich, I901, No, 50.

$\left.{ }^{2}\right)$ Nachr. 1. kais. Militaes. med. Akad. J'eterslurg. 1901. No. 3.

3) Phot. News, 1889, p. 6.20

4) 11,id. 1889, P. 751

5) Phot. Chronik., r890, p. 82.
} 
The following experiments are more reliable:

D'Arsonval (Compt. rend. Acad. d. sc., Vol. I 26, p. 919) states that the eye is affected as by light when it is brought into an electro-magnetic field. Dr. Beer has recently confirmed this observation. Even in the year I 857 the well-known discoverer of the "Od," Baron Reichenbach, declared that "sensitive" subjects have a sensation of bluish phosphorescence under the influence of the north pole of a powerful electro-magnet, combined with the sensation of a refreshing breath of wind; under the influence of the south pole there is the sensation of yellowish phosphorescence and of a tepid enervating current of air.

According to Hermann's ${ }^{1}$ ) researches, animal substances are diamagnetic.

Pluecker ${ }^{2}$ ) found that when blood was brought into a magnetic field, there was a repellent effect amongst the corpuscles. Milk showed the same phenomena with its fat globules.

Ch. Feré : $^{\text {) }}$ tried to prove by experiment that a magnet is capable of increasing muscular power; at first the power is said to diminish, then to increase. North and south poles act alike.

Many other physiological effects have been ascribed to magnetism; the statements are mostly, however, from unreliable sources, and will not bear investigation.

1) Pfueger's Arch., Vol. XLIII, pp. 217, 218.

$\left.{ }^{2}\right)$ Pogg. Ann., 1848, Vol. LXXIII, p. 549.

3). Soc. d. biologie, I902. 
III.

TREATMENT WITH X-RAYS. 



\title{
TREATMENT WITH X-RAYS.
}

\author{
The X-Ray Method ${ }^{1}$ ).

\section{S 26. Cathode and Roentgen-Rays.}

We have already seen that air ofters great resistance to the passage of electricity, which can only be overcome by very high tensions.

If one discharges an electric machine or a Ruhmkorff's coil through a medium of rarefied gas, one obtains a greater sparklength with the same potential ").

By lessening the density of the air, therefore, the resistance opposed to the passage of electricity is also lessened.

The pressure of a gas, as is well known, is measured by the height in millimetres of a column of mercury which it counterbalances. Normal atmospheric pressure —called "atmosphere"-is the pressure equivalent to

$\left.{ }^{1}\right)$ Literature: E. P. Thompson and $\Pi$. A. Anthony. Roentgen-rays and phenomena of the Anode and Cathode. New York, $1896 .-$ Fortschritte auf dem Gebiete der Roentgenstrablen, I Iamburg, Jahrg. I-V. - J. M. Eder, Jahrbïcher für Photographie, etc. Jahrg. X-XVI. Halle bei Knapp.-Puscy. Roentgen-rays in the treatment of skin disease. etc. Jonrnal of cutaneous and genito-urinary discases, July, 1900 . - Anmales délectrobiologie, Jahrg. I, II, III, IV. - Kalischer, Streifzuege durch dats Gebet der X-Stralilen. Elektrotechnische Zcitschrift, I8g8, I1. 24 H. - Magnus Moller, Der Einfluss des Lichtes auf die IJant, Suttgart. - Bucthor and Muller, Technik und Verwendung der Roentgenstralilen, I and 11, Aull, Halle bei Knapp.-

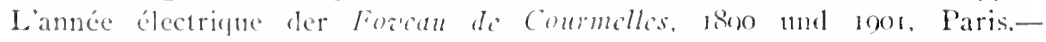

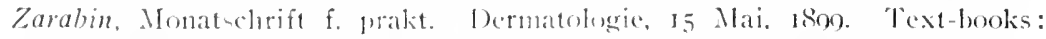

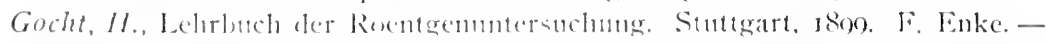

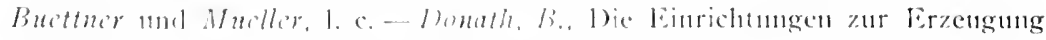

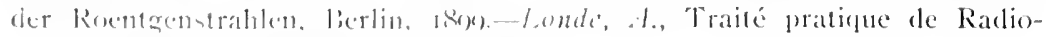

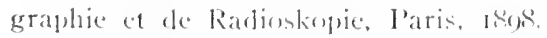

") Electricity traverses a "lonricellian" vacumu with the production of light-phenomena. 
$760 \mathrm{~mm}$. of mercury. In a vessel whose atmospheric contents have been reduced by means of a pump to $\mathrm{I} \mathrm{mm}$. pressure, there remains but $\frac{1}{60}$ part of the original quantity of air.

In order to utilize highly-rarefied gases and vapours for these purposes, Gassiot, Pluecker, and Goissler sealed them in tubes and globes of glass. The glass vessels were first provided with platinum or aluminium wires arranged in suitable places as electrodes, and these latter connected with the secondary terminals of a Ruhmkorff coil. The electrode conveying positive electricity is known as the cinode; that which conveys negative electricity is the cathodc. The electrodes are not invariably simple wires; they are fashioned according to requirement in the form of plates or rings.

A high-tension current can easily traverse a tube exhausted to an atmospheric pressure of $3 \mathrm{~mm}$., causing its contained gas to glow ${ }^{1}$ ). Entirely different light-phenomena, however, are seen at the electrodes; the negative terminal is covered by a thin light layer, then follows a darker space-the dark cathode-space - which is again succeeded by a light band-the lighl calhodeband. From this glowing light rays extend with diminishing brightness towards the anode. Then follows a dark space, behind which the positive light begins. This consists of a great number of alternately dark and light bands.

The electric glow-light in vacuum tubes is now generally looked upon as a kind of fluorescence-or phosphorescence-phenomenon of the gas, which glows under the influence of the negative electrical particles, the cathode-rays which are thrown off by the cathode. $H$. Ebert ${ }^{2}$ ) shewed that the properties causing the visible phenomena of light continue after cessation of the light, yet remain so effective that they considerably influence the succeding discharge and its characteristic appear-

$\left.{ }^{1}\right)$ H'iedemann proved (H'icdemann's Ann., V, p. 500, I878; VI, p. 278, 1879) that the gas legins to glow at a temperature below $100^{\circ}$. We must, therefore assume that the electric discharges act directly on the "ether." (See also the Electron-theory.)

") Ilicdemann's Annalen, 1899, d. LXIX, p. 372 . 
ances. These after-effects appear mainly to be due to the continuance of certain charges, which, during the process of discharge, are communicated to the gas. It is principally in the zone of gas around the cathode that these after-effects are manifested.

If the current is passed for a long time in the same direction through the glass ressel, the wall of the latter, which faces the negative electrode, becomes corered with a metallic deposit from the electrode.

If the air be gradually exhausted from a Geisster's tube, the light first hegins to disappear from the cathode, where soon a luminous point only remains, then from the anode, and finally altogether. On the other hand, the ressel wall begins to fluoresce, between $\frac{1}{1} \overline{0}$ and ind mun. pressure, the light being green or thlue, according to the nature of the glass. This furescence is caused by a certain kind of rays proceeding from the cathode. They were discorered by $/{ }^{\prime}$. IItlorf in I $869^{1}$ ). These rays, whose study was continued with such success by Goldstein, Herts, lenard, Crookes, Iother, I'iedemann and others, proceed from the cathode. Goldstin called them cathode-rays, Crookes "radiating maller." Their cathodal origin may be assumed from the fact that only that part of the tube-wall fluoresces which is directly opposite to the cathode. They have not the power of penetrating glass, but are transformed inlo heal. In other words, they become completely absorbed as soon as they strike the wall of the tube. These cathode-rays proced in a perpendicular dircetion from the surface of the calhode, auite independently of the anode's position. If, therefore, the cathode he shaped as a concare mirror, the rays become focussed in some spot, and here the temperature may reach such a height that any body encountered at this focus begins to glow. Again, the tube-wall may be so softened by the heat as to become broken through by the external atmospheric pressure. Substances which fluoresce well in ordinary light show the phenomenon in a still higher degree under the influence of cathode-rays. The effect of ratys proceeding simultaneously

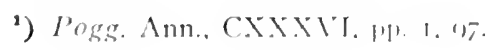


from several cathodes is very remarkable. They mutually divert each other, as can be seen from the fluorescence on the tubewalls. Crookes introduced a metal cross in the path of the rays. He found this arrested all the rays striking it, giving a shadow of the cross on the wall of the tube, and thus incidentally furnishing proof positive that these rays proceed in straight lines. A powerful magnet has also the property of deflecting cathoderays, as can be easily demonstrated by first bringing a shadowcasting boly in their path. E. I'iedemann and Ebert observed that this deflection is fan-shaped '). The shadow always takes another position and shape ${ }^{2}$ ), according to the position of the magnet. Cathode-rays seen also to possess a motive power. If they impinge on a lightly-hung $\mathrm{Hy}$-wheel, the latter begins to move. Not only the glass of the tube, but also nearly every (non-metallic) body with which they come into contact is made to Huoresce by cathode-rays. The colour of this fluorescence depends upon the nature of the body. Moreover, these rays have an active effect upon photographic plates. Becquerel and Goldstein found also that the rays have the power of imparting rivid colour to certain colourless salts. This property E. IVicdemann and G. B. Schmidt believe to be due to a reducing action. Goldstein found that not only "alkalihaloid" salts, but also sulphates, phosphates, and carbonates were coloured if first powerfully heated.

Radium-rays, ultra-violet light, and Roentgen-rays (IIolzknecht) are capable of producing the same colour effects.

\footnotetext{
$\left.{ }^{1}\right)$ Sitzungsher. der phys-med. Soc, zul Erlangen, December, I89o.

2) It is interesting to note that Prof Goldstcin, of Berlin, described two kinds of cathorle-rays in I866-those which can be deflected by a magnet and those which cammot. The latter, as we shall see, are the Roentgen-rays. Linard (Vortrag v. d. Gesellseh. Deutsch. Naturforschr., Frankfurt, 1897) found that this penetrating power of cathode-rays decreases the more they are diverted by a magnet. He could not deflect the external cathode-rays (Lenard-rays) magnetically. Lenard also demonstrated the fluorescence within the tube caused by the rays, their power of penetrating opaque bodies, lheir increase of the electric conducting-power of air or gases which they traverse, and their photographic action (Goldstin). The greater the electric force employed in the production of these rays, the less are they capable of being deflected from their course.
} 
Most bodies, especially metals, are opaque to cathode-rays. Aluminium, however, as Hertz has shown, is an exception. Lenard ${ }^{1}$ ) made tubes of this metal so that the cathode-rays could penetrate the tube-wall, and thereby be more carefully examined.

The so-called chamel-rays radiated backwards by a perforated cathode, which were discovered by Goldstein, do not, according to $\mathrm{W}$ clenelt, originate at the cathode, but are merely positive ions which have traversed the cathodal perforations. Ollo Ber $\left.\mathrm{g}^{2}\right)$ satisfactorily proved that these channel-rays are in reality anodirays.

In the year i 895 Willelm Konrad Roentgen ${ }^{3}$ ) found that from the site of the vacuum-tube which is struck by the cathoderays, a new kind of rays emerges which are not visible to the human eye, but which possess the same properties which Lenard found in his "external cathode-rays." If one deflect the cathoderays within the tube by means of a magnet, then the new part of the vessel-wall struck by them becomes also the site from which these new rays emanate. Rocntgen showed that these rays, which he called $X$-rays, but which are also known as Roentgen-rays in honour of their discoverer, penetrate the glass-tube, proceed in straight lines, and have an actinic effect upon photographic plates. He also showed their remarkable power of exciting fluorescence (as, for instance, in a screen coated with bariumplatino-cyanide).

The bulk of the fluorescence in a Roentgen-tube is caused not by the cathode-rays themselves, but by the $\mathrm{X}$-rays to which they give rise. If one brings a piece of glass near an active Roentgen-tube covered with black cloth in a dark room the piece of glass begins to fluoresce ( $\mathrm{W}$ alter).

A fact of great practical importance established by Eder and

1) Wicdenann's Annalen, 1894. 51, 225, and 1897. 6.3. 253.

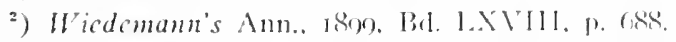

$\left.{ }^{3}\right)$ Eine neue Art von Strahlen. Stahel'sche Hof- und Univ-Buchhandlung. Würzhurg, 1895 . 
$V$ alcnt $\left.a^{1}\right)$ is this: one can only obtain good photographic results with these rays by using gelatine plates. A collodionplate is comparatively insensitive. The effect of X-rays, as is the case with ordinary light, decreases with the square of the distance. X-rays penetrate objects which for ordinary lightrays are opaque with even greater facility than cathode-rays ${ }^{2}$ ). Thus, for instance, a thick book, a double set of playing cards, thick blocks of rood, etc., are easily penetrated by X-rays, as is glass by ordinary light.

Since, as has been before mentioned, phctographic plates proved sensitive to X-rays, Roentgen found that he could take photographs in a lighted room with a plate hidden in a wooden case or paper bag. Speaking broadly, one may say that the lighter the specific gravity of a body, the more transparent is it to X-rays. On the other hand, a body's opacity for the rays increases with its density, though not in the same proportion. Variations in the density of a given body give, therefore, variations in its capacity for penetration.

$I^{\prime}$. Novik and $O$. Sule, ${ }^{3}$ ) also $V$ oller and $/ V$ alter $\left.{ }^{4}\right)$ proved that the transparency of a body for X-rays depended less upon its density than its atomic weight.

Eder and I'clenta found that almost all organic bodies are transparent to X-rays when they do not contain any heavy metals, but consist only of carbon, hydrogen, nitrogen and oxygen.

From the important transparency-data published by the above-mentioned investigators it is seen that magnesium-ribbon $\left(\frac{1}{10}\right.$ and $\frac{1}{2} \mathrm{~mm}$.) is easily penetrated, also tin-foil; with strips of the latter of $\mathrm{O}$. I to I $\mathrm{mm}$. thickness they succeeded in making graduated transparency-meters, recalling some of the photo-

1) Eder tund l'alenta. Versuche neber Photographie mittels der Roentgenstrablen. Wien und Halle, 1896.

2) It lunst be noted that other kinds of rays are known which penetrate opaque bodies. For instance, a dark indine-bisulphide of carbon solution allows radiant heat to pass through it without loss. Again, electric Wates, ats we have seen, penetrate solid bodies. The penetrating power of catherle-rays with cortain media las already been mentioned.

$\left.{ }^{3}\right)$ Zeitschr. f. plyss. Chemie, I8,66, 19, 489.

${ }^{4}$ ) Zeitschr. f. angewandte Chemic, I897, Heft 15. 
meters used in photography. Copper and silver in layers of 0.1 to $0.2 \mathrm{~mm}$. thickness are not quite opaque. On the other hand, lead, platinum, and gold are highly opaque, as are also zinc (as thin plate), nickel, iron, and especially mercury.

It is interesting to note that rock-crystal is not much more transparent than crown-glass, and that Hint-glass stops X-rays even more than crown-glass.

Glass with a thickness of I mm. weakens the rays (even if one uses the purest crown-glass or "solinglass"), and much more than an aluminium plate of the same thickness. On the other hand an aluminium plate $\mathrm{I} \mathrm{cm}$. in thickness affords a very considerable resistance. Hence, it follows that in estimating the transparency of a body for X-rays one must take into account not only its atomic weight, but also its thickness. A body, therefore, which in thin layers may be comparatively transparent to the rays, becomes in thicker layers quite opaque. This was well shown in the photograph of a camco taken with $\mathrm{X}$-rays by Eder and Valenta, the different gradations of thickness being well marked. Layers I mm. thick, of bone or mother-of-pearl, absorb the rays about as much as glass. A layer of flesh $\mathrm{I} \mathrm{cm}$. thick is as transparent as a layer of bone I mm. in thickness. Horn is similar to Hesh in this respect. Wood, even in thick pieces, is very transparent; soft wood is more so than hard. Caoutchouc, wax, strong leather, woollen cloth, linen, dressings for wounds, mica, and celluloid are all very transparent. Carbon, in the form of coal, charcoal, soot, black-lead, or diamonds, is very transparent. Of phosphorus, selenium and sulphur, the first has the greatest penctrability, and the last the least. According to Marangoni, ${ }^{1}$ ) lithium is the most transparent of all metals for X-rays. According to Chaband ${ }^{2}$ ) and Sehricald, the addition of halogene to the organic molecule greatly increases its opacity in this respect. This effect is particularly well seen in the case of chloroform, bromoform and iodoform. Very dark shadows are cast by clear solutions of bromoform, also by liquid tetrachloride of carbon. Since these combinations

2) Atti R. Ace dei Lincei (5) 5. 2. Sem., 1896, page 403,

8) C. R. 122, 1896, p. 237. 
are mostly penetrable by ordinary light-rays, they afford, as Schricald observes, a very good filter for $\mathrm{X}$-rays ${ }^{1}$ ). As regards liquids, water and bi-sulphide of carbon are very transparent to the rays. Others again are somewhat more opaque, c. g., blood and, as the author has himself observed, solutions of sulphurated hydrogen.

If one wraps the vacuum-tube in black cloth in order to exclude all visible light-effects, and places near the cathodal-ray region a screen coated with some fluorescent body (the best is Darrum-platino-cyanide), the latter at once begins to shine with a light-green radiance when the tube is in a state of activity. If one then places between the tube and screen a body whose density varies in parts (e.g., a boot with iron nails, a box of weights, a human hand), the dense portion will be found to cast deeper shadows than the more transparent.

It is to this property, their power of easily penetrating softer parts, while others, such as bones, are more opaque, and metals (foreign bodies) absolutely so, even varying gradations of shadows being corresponding to tissues which are air-containing or otherwise, rich in blood or the reverse, that the $\mathrm{X}$-rays owe their enormous value in medical diagnosis.

As regards the chemical effect of $\mathrm{X}$-rays per se opinions are much divided; while many investigators (for instance, Hemp. tinne) are inclined to deny the existence of such an action, others arguing from certain facts take an opposite view. P. Villard ${ }^{2}$ ) is to be included among the latter. He noted that glass is coloured violet by X-rays, whereas cathodal rays blacken it.

Roentgen thought it probable that his rays are capable of exerting a heat-effect, since they certainly possess the power of producing light-phenomena (fluorescence). He found also that they are not refrangible--they are not deflected by mica, rubber or aluminium prisms or lenses. Moreover, he could not succeed in finding any very definitely marked reflection of the rays; on the other hand, he found that a photographic dry-plate exposed with its glass side towards the vacuum-tube and bearing

1) Naturwissensch. Rundichan, II, 1896, 1). 50.3.

") I'oggenderf's Beiblätter zu den Annalen der Physik, I90o, p. I35. 
bright strips of aluminium, iron, platinum, tin and zinc, on the film-side, gare on development darker patches corresponding to the last three metals. The actinic effect was particularly marked under the zinc-strip, while the aluminium had absolutely no effect whatever. It would seem, therefore, that lead, platinum, and zinc do to some extent reflect the rays. Later on, Carmichacl of Lille succeeded in reflecting Rocntgen-rays by means of steel-mirrors. Rocnlgen showed that X-rays are entirely different from cathode-rays, and cannot, like the latter, be deflected by a magnet.

It has been found no more possible to bring X-rays to a condition of "interference" than to deflect, refract, or polarise them. On the other hand, they are found to have a discharging effect on electrified bodies. The leares of a charged electroscope rapidly fall together if the instrument be brought within the field of an X-ray tube. According to H. Starke, ${ }^{1}$ ) this action of $\mathrm{X}$-rays is to be compared with the similar effects produced by ultra-violet and Becquerel-rays. The action of ultra-riolet light is confined to the cathode; Roentgen-rays, however, have the same effect whether they strike the positive or negative electrode. The effect on both poles on the part of X-rays is perhaps only an apparent one. According to $S a g n a c$, solid bodies which are struck by $\mathrm{X}$-rays emit new rays having similar properties. These secondary rays likewise farour an electrical discharge. It is therefore possible that the anodal effect is to be attributed to the secondary rays emitted by the irradiated anode towards the cathode.

Sella and Majorana ${ }^{2}$ ) found from a series of experiments that the discharge depended largely upon the nature of the irradiated body. The discharging effect of X-rays increases, acaccording to J.J. Thomson,") Righi ${ }^{4}$ ) and Roenlgen, with the atmospheric pressure on the chared body. Roenlgen maintains that it is the air itself which, excited by the $X$-rays, brings about the discharge.

1) Hiedemann's Amm. IRos. B., I.XVI, p. 1000.

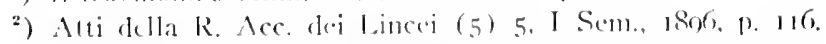

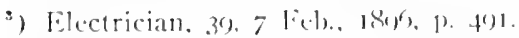

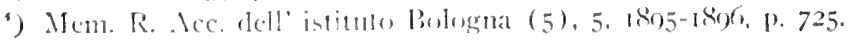


Perrin, $\left.{ }^{1}\right)$ Villari ${ }^{2}$ ) and Winkelmann ${ }^{3}$ ) believe that this is due to a so-called "ionisation" of the gas, $i$. $e$., the gaseous molecules are arranged in oppositely-charged atoms-the "ions"-_of which some are repelled by the charged body, while others are attracted, and thereby neutralise a portion of its charge.

Roentgen-rays, like ordinary light, increase the conducting power of selenium.

The intensity and penetrating capacity of X-rays depend very largely upon the nature of the body struck by the cathoderays, or, in other words, upon the nature of the emitting surface. Kanfmann's ${ }^{4}$ ) and Roiti's ${ }^{5}$ ) investigations seem to prove that the metals have a greater radiating power the higher their atomic weight. Kaufmann lays down the following series, in which each individual metal has a greater radiating capacity than its predecessor.

$$
\mathrm{Ag}, \mathrm{Fe}(\mathrm{Ni}, \mathrm{Cu}, \mathrm{Sn}, \mathrm{Zn}) \mathrm{Ag}(\mathrm{Cd}, \mathrm{Pt}, \mathrm{Pb}, \mathrm{U}) \text {. }
$$

In the above those metals included within the brackets have practically the same radiating capacity.

If one imagines the radiating part of the tube (the anti-cathode-see below) to be a luminous flame and the exposed body a shadow-casting object, one can readily understand what will be the geometrical configuration of the shadow; for since the X-rays proceed in straight lines, the same laws of shadow-formation obtain as in optics. The picture seen on the fluorescent screen or photographic plate entirely bears out this view ${ }^{6}$ ).

Every imaginable hypothesis has been brought forward to explain the nature of X-rays. Roentgen's earlier view was that they were longitudinal waves, progressive condensations and rarefications of the light-ether. Other distinguished scientists,

1) C. R., I23, 35I, I806, and I24, 455, I897.

2) Atti della R. Acc. dei Lincei (5), 6, I897, 343 .

$\left.{ }^{3}\right)$ Jenaische Zeitschrift f. Naturw., Bd. XXXI, N. F., I897, I74.

4) Verhandl. d. phys. Gesellsch. zu Berlin, 30, 4, I897.

3) Atti R. Ace. dei Lincei (5) 6, II Sem., Pp, I23, I29, 1897.

6) In a work published with Schiff. whose main contents are given later on, I have referred to the circumstances that the geometrical shape of the changes in the skin brought about by X-rays depends upon the distance of the skin from the tube. 
including Boltzmann, Lord Kelizin and Lodge, seem inclined to agree with him. After referring to the analogy which obtains between light-ether and certain elastic (particularly gelatinous) bodies as regards the capacity of both for longitudinal and transverse vibrations, Boltamann") observes: "In all elastic bodies, especially gelatine, longitudinal waves are transmitted with far greater rapidity than the transierse ones. If one assume this also of light-ether, Roentgen-wares, in spite of the very brief duration of their vibrations, may still have fairly large wave-lengths. The short vibrating period would explain the phenomenon of Huorescence, which probably is manifested as soon as vibrations occur which are approximately as rapid as those of the molecule. The comparatively large wave-lengths would explain the power these waves have of penetrating most bodies, in which respect they resemble Hertzian waves. Do I not observe every night that from the music in the next-door house the low deep notes, which have the greatest wave-length, penetrate the wall far more easily than high notes. . . . Cathodal rays, on the other hand, are probably longitudinal, but of very short wave-length; in the first respect they resemble Roentgenrays, in the latter (their short wave-length and therefore greater capacity for being absorbed) they are similar to ultra-violet light."

Many physicists are of the opinion that X-rays are ultraviolet rays (that is to say, transerese vibrations of very short wave-length), with which they have in common the property of extending in straight lines, of exciting fluorescence, and most likely of producing chemical action. They cxplain the impossibility of breaking or polarising $X$-rays by the shortness of their wave-lengths, and again by the imperfections in our apparatus.

Many others again believe X-rays to be a kind of cathode. rays. Thus Roentgen himself in a later work") thinks this idea by no means an impossible one. We have already seen that there are many transitions from one kind of ray to the other, and that they are at all erents closely related. In his third

3) \%eitschr, für Elektrotechnik, 1806, 15, I.

2) Sitzungsber. 1. Berliner Akad., 1897, 20, 589 . 
treatise on the subject, Roentgen is inclined to hold that Roentgen-rays and cathodal-rays are phenomena of the same nature.

Now Crookes has formulated the following theory on the nature of cathode-rays: One must imagine that every gaseous body is composed of individual molecules; these are quite elastic. The so-called kinetic theory of gases teaches that the molecules of any gas are charged with energy and move with infinite velocity through space. In doing this they must impinge upon one another eventually. They are then mutually repelled by reason of their elasticity, bounding apart like billiard balls which have collided, thereby changing their direction and hurrying forward again in straight lines. The more molecules there are in an enclosed space, the oftener will they bound against the wall enclosing that space. The total amount of these impacts corresponds to the pressure under which the gas lies. The fewer molecules there are (i.e., the lower the pressure of the gas), the greater is the energy with which they can travel on in straight lines with a correspondingly larger course before they collide with other molecules, and so become diverted. Crookes concludes that the very low pressure in his tubes makes it possible for the few remaining gas-molecules to travel with enormous force and thereby develop the remarkable properties seen in cathodal-rays. He holds, therefore, that these rays are constituted by the gas molecules, and that they are minute particles of gas (proceeding from the cathode) to which a tremendous motive power has been imparted by the electric current. The comparatively small number of these particles prevent their being hindered in their career by one another; they thus come into violent contact with any body which lies in their path, causing it to become heated by the molecular bombardment and so perhaps to glow or melt.

Gimll and Puluj held a theory that cathode-rays were due to material particles of the cathode hurled forward in straight lines, heat being developed as the result of their impact against the tube-wall.

Crookes' gas-molecule theory, as described above, would seem to explain fairly well most of the phenomena observed in connection with cathodal-rays, such as their deflection 
in a magnetic field, their heat-effects, and supposed mechanical effects.

Closer investigation, however, proved that Crooke's hypoth. esis was untenable. Quite recently this question has again come to the fore in connection with a theory first propounded by

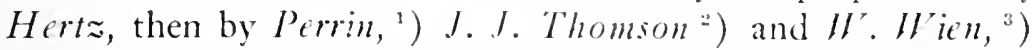
that cathode-rays carry a negative electric charge with them, which is essential to their very nature-that they are themselves negative electricity in motion. The researches of $E$. Wiechert,") $W$. Kanfmann,") E. Aschkinass,") Ph.Lenard,") Th. de's Coudres ") all showed that Crookes' theory required modification only in order to satisfactorily explain practically all the phenomena. The cathode-rays shoukd, according to these physicists, be regarded as charged particles, but the ordinary material particles of gas must not be held to constitute the carriers of the charge. These are to be looked for in much smaller elements, the "ions," "component parts of the ether" (Lenard) or "electrones" (Stoney), which are considerably less in size than ordinary material atoms. By examining a whole series of the properties of cathode-rays it became possible to determine the amount of the charge in the case of these particles. These data corresponded very closely with those which Ze'man had already obtained for the particles associated with the optical phenomenon which bears his name. Hence, "Vichert's") hypothesis, that in both cases one is dealing with the same particles, $i$. $i$., electrones, would appear reasonable. Wichert estimates the velocity of an electron in its flight to be $\frac{1}{3-1}$ that of light. In consequence of this enormous velocity the impact of an elec-

2) C. R., I895, I2I, P. II 30.

2) Phil. Mag. (5) 4.4. I897. p. 293.

3) Verhandlungen der phys. Gesellsch., Berlin, Nov. 19, 1897, i6, 165.

4) Sitzungsher, der phys. -̈̈kon., Gesellsch.. Königsherg, 1897, 1. 1. Naturw. Rund., Nlay. 1897 .

") I'icdmann's Amn. 61, 544 (I.897); 62, 506 (1897); 65. 431 (1898); $66,649(1899)$.

") Wiedcmann's Ann., 62. 589 (1897)

7) Hiedemann's Ann., 64. 279 ( I898): 65. 504 (1898).

") Vehr. 1. phys. Ges. Berlin. 17. 17 (18, 89$)$.

") Goettinger ged. Nachrichten, Irogn. 1) 1, 260. 
tron against a solid body sends an explosion-like electric wave into space, just as a projectile at the moment of impact emits a sound-wave. Wiechert thinks it not unlikely that Roentgen-rays may be electro-dynamic wave-movements, manifested as a series of short, rapidly-succeeding waves. The real carrier of these wave-movements is the all-parading ether.

Walter, ${ }^{1}$ ) Batelli and Garbaso, $\left.{ }^{2}\right)$ Michelson, $\left.{ }^{3}\right)$ Muraoka, ${ }^{4}$ ). $I^{\prime}$ osmaer, Ortt ${ }^{5}$ ) and other scientists regard Roentgen-rays as cathode-rays which have given off their charge at the tube-wall or at the anti-cathode from whence they spring, thereby gaining in power of penetration. They are therefore cathode-rays sifted, as it were, by the media through which they pass. Assuming that Roentgen-rays are themselves non-electrical, we can the more readily understand that on the one hand they tend to discharge an electrified body, and on the other hand, like all non-electric or non-magnetic bodies, they are not deflected by a magnet. Welnelt's ") observation again, that the production of X-rays depends on no factor so much as the presence of rapidly-suppressed discharges, that is to say, a disturbance of electrical equilibrium giving rise to powerful electric waves, induces $B$. Donath ${ }^{7}$ ) to assume that there is some connection between Roentgen-rays and electrical waves, and that the former are electrical waves of the most diminutive wavelength.

It is very likely that, just as white light is composed of various colours, Roentgen-rays may also be of various kinds, which differ from each other in their penetrative power as well as in their physical and physiological effects. We shall examine this question more closely when speaking of vacuum-tubes.

1) Fortschr. a. d. G. d. Roentgenstr., Bd. II, H. 4.

2) Nnov. Cim. (4) 3, 1896, p. 299.

3) American Journal of Science (4) 1. 1896, p. 3I4.

$\left.{ }^{4}\right)$ I'iedemann's Ann., 59, 1896, p. 773.

5) Nature, 56, 1897 , p. 316 .

") I'iedemann's Ann., 65.511, I898.

) B. Donath, Die Einrichtungen zur Erzengung der Roentgenstrahlen, Berlin, 1899. 


\section{$\$$ 27. Vacuum Tubes.}

A vacuum-tube is an apparatus in which electrical energy is transformed into Roentgen-rays. The quality of the rays depends upon: I, the kind of electrical energy employed; 2, the condition of the tube.

The intensity of the rays increases with the quantity of electrical energy consumed. Consequently not only higier tension, but also greater current-intensity, causes a more powerful emission of X-rays.

The condition of the tube is always a factor of prime importance for the production of an abundant, strong, and constant supply of X-rays. It was the caprices of tubes and their slight resisting capacity which accounted for so many failures in the earlier history of X-ray work.

The earlier forms of Crookes' tubes were pear-shaped or cylindrical. The cathode was button-shaped, or later on consisted of an aluminium disc or concave mirror (Fig 6r). A pin or ring of aluminium was first used for the anode; later on the latter was shaped in the form of a plate fitting in a sidechamber of the tube.

Now in these earlier forms of tube the most effective rays proceeded from a comparatively large and curved surface. Consequently they were lacking in power, and gave only ill-defined shadows of an object. The portion of the glass bombarded by the cathode-rays again was very liable

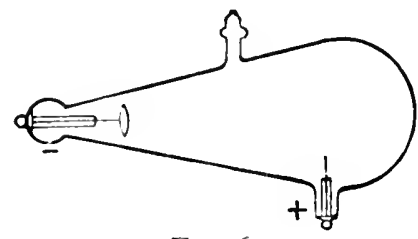

FIG. 6I.

(From A. Londe, Traité pratique de Radiographie. Paris. I8ys, p. Gr.) to become over-heated, whereby it softened and gave way under the external atmospheric pressure. Fortunately the production of $\mathrm{X}$-rays is not solely dependent upon the impact of cathodal-rays on glass-on the contrary, other materials are just as suitable, or even more so. Roentgen soon found that his rays could be emitted from a platinum surface, and here he was dealing with a material which withstood high degrees of heat and was therefore all the more suitable. In order that clear shadow-pictures 
may be given, the source of the rays must be in the form of a point, or at least very small, and this requirement was met by employing an aluminium concave mirror as cathode.

On these principles Roentgen, Jackson and others constructed the so-called focus-tubes; these marked an era in X-ray. work. Focus-tubes are so arranged that the rays produced in them proceed from one point only, whereby considerably greater intensity is attained. For this purpose, as mentioned above, an aluminium concave mirror is used as cathode. A small platinum plate-the anti-cathode-is now fixed between the disc-shaped anode and the cathode, opposite the centre of the latter. This anti-cathode is so placed that it coincides with the apex of the cone of cathodal-rays from the concave mirror, and these rays, moreover, strike it at an angle of $45^{\circ}$. Since every solid body struck by the cathode-rays becomes the source of Roentgen-rays, these latter are emitted at once from the focus-point on the anticathode. This point emits X-rays in all directions, though most powerfully in the half of the tube-space corresponding with the cathode. (Emission of X-rays, however, takes place in the opposite direction as well, through the platinum-plate of the anticathode. The fluorescent screen at once proves that the rays are evolved also from the dark half of the tube. The glass walls of the tube and the secondary anode also emit X-rays.) By this arrangement excessive heating within the tube is obviated. The anti-cathode in these tubes is generally so arranged that by means of a connection with the anode it may be turned into a secondary anode. Thereby the tendency towards destruction of the platinum is minimised.

In many tubes (those, for instance, of the Allg. ElektricitätsGesellschaft, Berlin), while the cathode is made as above described, the anode itself is made to form the anti-cathode by making it carry a platinum mirror set at an angle of $45^{\circ}$, from which the X-rays emanate. If, however, the anti-cathode is made to work in this way as anode also, the tube soon becomes blackened by the dispersion and precipitation of platinum-particles on the vessel-wall; this does not occur where the anticathode is insulated. The coating of platinum-particles has an affinity for absorbing gases, and so seizes on the traces of air 
left within the tube. According to $/$ 'ild, the cause of this dispersion of platinum-particles from the anti-cathode is explained by the fact that the latter is always surcharged with negative electricity, and becomes, indeed, a secondary cathode, which develops its own rays. These, according to the "bombardmenttheory," are a current of material particles flung off by the cathode. The "penctrator" of Messrs. W'atson $\&$ Sons is a globe with two long tube-shaped processes which contain the electrodes. The cathode is shaped like a concave mirror, and the anode-wre, which terminates in a ring, is placed in a separate glass tube. A prolongation from the latter supports a platinum mirror (the anti-cathode), which is thus insulated from the anode-wire. The cone of cathode-rays has to pass

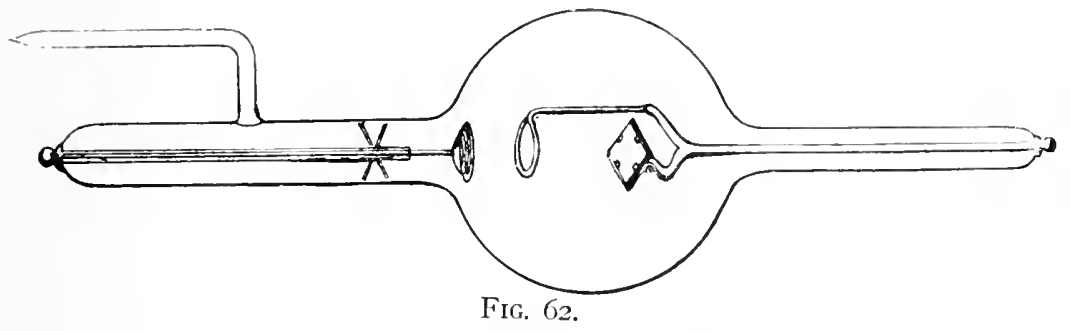

(From A. Londe, i c., p. 64 .

through the anode-ring before it strikes the anti-cathode (Fig. 62 ).

Richard Mueller-Uri of Brunswick constructed a Roentgen tube giving its effect within a small and strictly limited area and with small current-quantities. The cathode, supported on a long stem, is contained within the cylindrical part of the tube. The anode-an aluminium ring-is placed in the middle or wider part of the tube. Opposite to it the secondary anode (reflector) is fixed; this is contained within the globular end of the tube, which is cone-shaped. The peculiarity of the tube consists in this situation of the reflector at one side, with its finger-like encasement. The rays from it act only upon an area the size of a thumb-nail. The efficiency of an X-ray tube is limited by the fact that when the cathode-rays have attained a certain intensity, the anti-cathode gets red-hot. As a result of this the glowing 
platinum parts with a certain amount of absorbed gas, whereby the degree of vacuum in the tube becomes altered. Again the dispersion of the platinum in the form of fine particles, as mentioned above, is considerably augmented. By these two means the tube is gradually rendered useless; and it is not always safe, therefore, to work the coil at its full capacity and so.supply to the tube more energy than it can properly deal with. In order to prolong the "life" of a tube, F. Kurlbaum" ${ }^{1}$ ) covers the anticathode with platinum-black. This material parts with heat much more freely than sheet platinum, and therefore is much less liable to become red-hot.

Many makers, with the idea of obviating this excessive heating of the anti-cathode, construct the latter of comparatively large blocks of copper (voltohm tubes), or provide it with a layer at the back consisting of some substance which has a greater heat-capacity and a larger radiating-surface (Max Levy, Dessauer). This black layer of insulating material (porcelain) is intended on the one hand to retain the heat, so that the platinum-mirror gets into the glowing state, in which it evolves the greatest quantity of highly-penetrative rays. At the same time this kind of anti-cathode, even when heated, only parts with very small quantities of gas, so that the vacuum is not altered very much. Max Levy believes that a second function of the insulating material is to be found in its power of storing a large portion of the energy developed on the anti-cathode.

In order to cope with powerful current-supplies having rapidly-succeeding interruptions-such as, for instance, obtain when using the electrolytic interrupter-tubes have been made with cooling-chambers adjoining the anti-cathode. These chambers contain cold water, which absorbs the surplus heat, and moreover tends considerably to diminish the dispersion of the platinum. The water in tubes of certain kinds is kept circulating through a system of tubing, whereby a stream of cold water is constantly in relation with the anti-cathode. In another and still better system the anti-cathode is formed by a platinum-mirror fixed at the bottom of a glass vessel containing water. The water does

$\left.{ }^{1}\right)$ Elektrotech. Zeitschr., I900, p. 237. 
not require renewing, since the tube works efficiently even with the water at boiling-point ( Dr. I'aller, Fig. 65).

These appliances prevent in a great measure any increase in the vacuum of a tube, which would, other things being equal, be inevitably accompanied by some loss of efficiency. For if the platinum be allowed to glow very much, it has a well-known tendency to absorb gases on cooling, and so heighten the vacuum ${ }^{1}$ ). Now the quality of the X-rays depends very largely upon the degree of the tube's vacuum. With the heightening of the latter the tube is said to become "hardened"; it offers a greater resistance to the passage of the discharge, and while the radiation gains thereby in intensity, it also gains in penctrative power. If, for instance, working at a certain vacuum, the tube gives a sharp silhouette of the hand (since the X-rays only penetrate the fleshy parts, but are absorbed by the bones, as is the case with soft tubes), it will be found on increasing the vacuum that one obtains rays which penetrate all the tissues almost equally well, whereby constant effects become lost (hard tubes). We see, therefore-a fact which was soon discovered in X-ray work-that by varying the degree of vacuum, we can also vary the penetrative capacity of the rays.

Eder and $V^{\prime}$ alent ${ }^{2}{ }^{2}$ ) observed in 1896 that the effectiveness of tubes varied with their degree of vacuum; Porter ${ }^{3}$ ) classified X-rays as follows: Rays which penetrate the flesh easily but bones with difficulty ( $X_{1}$-rays); those which are largely absorbed by flesh ( $X_{\text {-rays}}$ ); and those which penctrate both bones and flesh equally well ( $X_{\text {-rays }}$ ).

Albers-Schocnberg ${ }^{4}$ ) recognised four degrees of vacuum: I, hard (grey); 2, medium-soft (grey-black); 3, soft (deepblack); 4 , very soft $\left.{ }^{5}\right)$. Here the intensity of the shadow of the metacarpal bones on the fluorescent screen is taken as an index.

1) With a high degree of racumm lhe lube somelimes explodes under the atmospheric pressure.

${ }^{2}$ ) Versuche ueber die Photographie millet der Roentgen Stralnen. Wien und Halle. isgo, p. 5. Amm.

${ }^{3}$ ) Cit. bei l: l'alenta, Oest. Chemikierztg.. I, Nr. I, $180 \mathrm{~s}$.

") Fortschr. a. 1. Gel, (1. Roentgensir., Bid. 111, 11. 4. p. I43.

") Kienbock dictinguishes yet another, a fifth grade, the "over-hard" tube. (Wr. Klin. Woch., I900, No. 50.) 
Contrasts are more with "soft" tubes, as mentioned above, than with hard ones. According to Gassmann and Schenkel, ${ }^{1}$ ) the degree of hardness of a tube is best determined by means of the skiameter. This, however, only measures the penetrative power of the rays, and not their intensity. Two tubes having an identical penetration capacity may act very differently upon a photographic plate; moreover, one and the same tube, given a fixed degree of hardness, produces very different radio-chemical effects when worked with different strengths of currents. In order to estimate their intensity, one is obliged to examine the effect of the rays on the fluorescent screen. Gassmann and Schenkel propose gauging the skiameter by having the tin-foils of a determined thickness and counting the number of foils required to just block out the rays. Of course, the apparatus would have to be worked at a definite distance, say $30 \mathrm{~cm}$., from the anticathode. Benoist (see p. 245) constructed his radio-chromometer very much on these lines.

Acting upon the hypothesis that the degree of penetration of X-rays for different bodies depends not only upon their thickness, but upon their atomic weight, the author has for some time past been engaged in the construction of an instrument consisting of a graduated series of substances of different atomic weights for this purpose.

$U$. Kaufmann ${ }^{2}$ ) found that the velocity of the cathoderay particles is proportional to the square-root of the tensiondifference between anode and cathode.

Since in order to work hard tubes higher tensions are required, it may be presumed that in these tubes the cathode-ray particles move with considerable higher velocity and consequently bound with greater force against the anti-cathode than is the case with soft tubes.

It must be here noted that the degree of hardness of many tubes alters perceptibly during their use; moreover, the extent and time of the appearance of this change varies with different

1) Fortschritte auf dem Gebiete der Roentgenstrahlen, Bd. II, H. 4, p. I3I.

$\left.{ }^{2}\right)$ Wicdemann's Ann., 6I, 544, 1897, and 62, 596, I898. 
tubes and apparatus, so that no hard and fast rule can be laid down as to the degree of hardness permissible for radio-therapy.

The glass wall of a tube absorbs a considerable amount of the rays, thereby diminishing their effect. In order to do away with this loss, Colardean made a tube in which the site where the rays are emitted is only $\frac{1}{10}$ millimeter thick. The life of the tube is further prolonged by a communicating side-chamber of great capacity (Fig. 63).

The Allgemein. Elektricita'sgessellschaft also construct a tube having a very thin wall where the rays appear and have

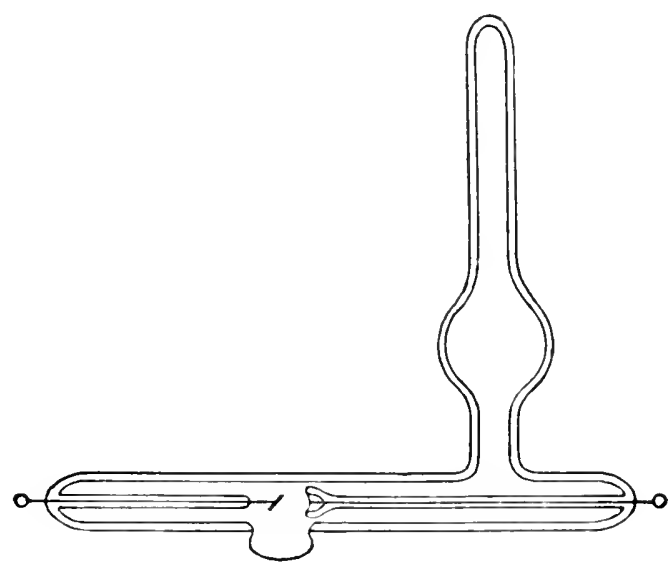

FIG. 63.

Colardian's tube.

their greatest intensity. This region lies at right angles to the axis of the tube.

At Stuetzerbach in Thüringen, Messrs. Groiner E Friedrichs make tubes of borax-glass, which are very transparent to X-rays and fluoresce with a blue colour; while Segny and Gundelach make theirs of a glass containing powdered potter's earth and "chlordidymium." This glass Huoresces red, and allows double the ordinary amount of rays to pass.

After prolonged use a Roentgen-tube begins to lose in effectiveness. This is partly due to the electro-static charging of the tube-wall, a fact which is readily proved. It has been supposed that the gas-molecules within the tube are driven towards the 
wall as a result of electro-static attraction, whereby the central part of the tube becomes of higher vacuum. Efforts have therefore been made to obviate this change of vacuum by removing the electro-static charge.

Porter $\left.{ }^{1}\right)$ with this object in view, placed a wire ring around the tube in the plane of the cathode-mirror. This ring was brought as near as possible to the outer surface of the tube, without actually touching it, and was "earthed" by means of a connecting wire.

By this arrangement constant discharge from the tube-wall is effected. The same result is attained by covering the cathodal part of the tube with tin-foil and fitting an "earthed" wire ring over the latter, or again by covering the cathodal region with a wooden cylinder, whose inner wall is kept moist.

Other means are also available for prolonging the life of the tube. Thus the air-molecules attracted to the metallic coating of the tube-wall may be dispersed by warming the latter. This method, however, proves effective only for a few times and for short periods.

Again the so-called adjustable tubes have been constructed, which are founded on two systems. In the first of these, substances are placed within the tube, or side-chamber, which give off gases on being heated.

Crookes varied the tube vacuum by means of a piece of caustic potash in a side-chamber. This absorbs the last traces of moisture left within the tube, thus raising the vacuum. On heating the potash, however, a certain amount of vapour becomes liberated, whereby the vacuum can be lowered. In the latest tubes of this class, the heating of the potash is effected automatically through the agency of the discharge-spark, which, when the vacuum is too high, jumps across and outside the tube.

In the "Queen self-regulating-tube" made by Queen E Co. of Philadelphia, also in those of C. F. Mueller of Hamburg, and E. Ducretet of Paris (Fig. 64), there is some caustic potash placed within a secondary tube $R$, which is attached to the main tube. When the vacuum in the latter becomes too

2) Nature, 54, 149, I896. 
high, there is too much resistance offered here to the passage of the current from the poles $I$ and $K$. Consequently the current passes through $f$ to the side-tube, which is also a vacuum-tube in which $m$ represents the cathode. The apex of the cathodal-ray cone from $m$ coincides with $R$, whereby the caustic potash hecomes heated and emits vapour; this rapour passes into the main tube and so lowers its racum and resistance. In adjustable lever $E$ is in connection with the side-circuit, which permits the introduction of a larger or smaller spark-gap. With the lever at some distance ( 5 to $7 \mathrm{~cm}$.) from the cathode of the main tube, the latter works "harder," at a less distance $(1-3 \mathrm{~cm}$.) we have a constantly lower racum, and the tube works "softer." At any time when the resistance of the main tube becomes greater than

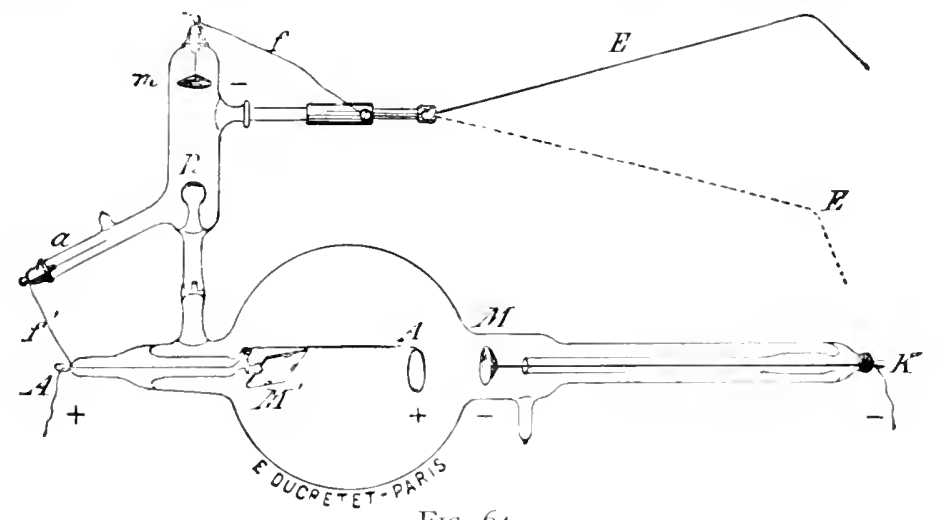

Fig. 64 .

that of the spark-gap the current passes through the side-circuit and heats the potash. The presence of sparks, therefore, in the spark-gap shows that the automatic-regulating process is procecding.

In the latest types of tube made by C. II. F. Mueller of Hamburg (Fig. 65) a loop is attached to the lever C. To this, if it be required to quickly lower the vacum, the negative pole of the coil is directly attached, so that the current can be sent in its full forec through the sccondary tube. It is generally aldisable, however, to begin with weaker currents.

The last-mentioned tube has also a derice for "hardening" it. 'This is useful when the "softening" has by accident been 
carried too far. In order to harden the tube, the positive wire from the coil is not connected with the secondary anode $G$ of

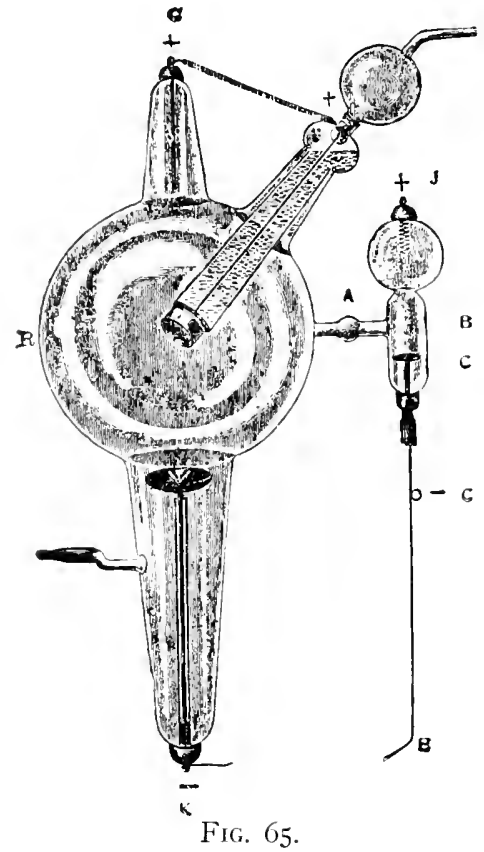
the main tube, but with the spiral-shaped electrode $J$ of the side-tube, care being taken that the brass lever $E$ is not in contact with the cathode $K$. If now the current be turned on (in the same direction as for softening), metallic particles from the spiral $J$ are freely driven off against the wall of the side-tube, whereby some of the gaseous contents of both tubes become also bound to the vessel-wall and the vacuum of the main tube is raised.

Another method of controlling the vacuum tubes is founded on the principle of osmosis.

Here one utilises the property which heated platinum possesses of being penetrable by hydrogen. For this purpose the main tube carries a side-chamber terminating in a platinum tube closed at the end (Fig. 66). If the vacuum be too high, one heats this platinum over a Bunsen- or spirit-flame till it beconies red-hot. Hydrogen gas from the flame now permeates the platinum and so gains access to the interior of the main tube, the vacuum of which is thereby lowered. 'This process may be carried out while the tube is working, so that the degree of vacuum can be gauged by the colour of the fluorescence. When the latter begins to show a bluish tint the heating operation should be suspended, for now the vacuum is evidently very low. According to B. Walter $^{-1}$ ) and Lester Leonard, ${ }^{2}$ ) the

1) Fortichritte auf dem (Gehiete der Roentgenstralılen, Bd. I, p. 84 .

2) Ann. d'électrobiolog., Bd. III, p. 48I. 
degree of vacuum of a Roentgen tube may be at once gauged from the spark-length of the electrical tension necessary for its excitation, so that one may speak of the "spark-length" of such a tube. Since the electrical resistance of a tube depends upon its vacuum, while the same factor controls the penetrating power of the rays, one will in the measure of the resistance possess also a relative measure of the quality of the Xrays. The sparklength of the coil supplies this measure, since the spark jumps across the secondary current-circuit and is parallel to the discharge-path within the tube. (See Spintemeter, page 245.)

The metallic lever on the automatically-regulated tube (Figs. 64, 65) affords a means of varying the vacum of the tube by modifying the resistance of the secondary current-circuit, which again influences the resistance and the vacuum of the tube. As the lever approaches or recedes from the cathode,

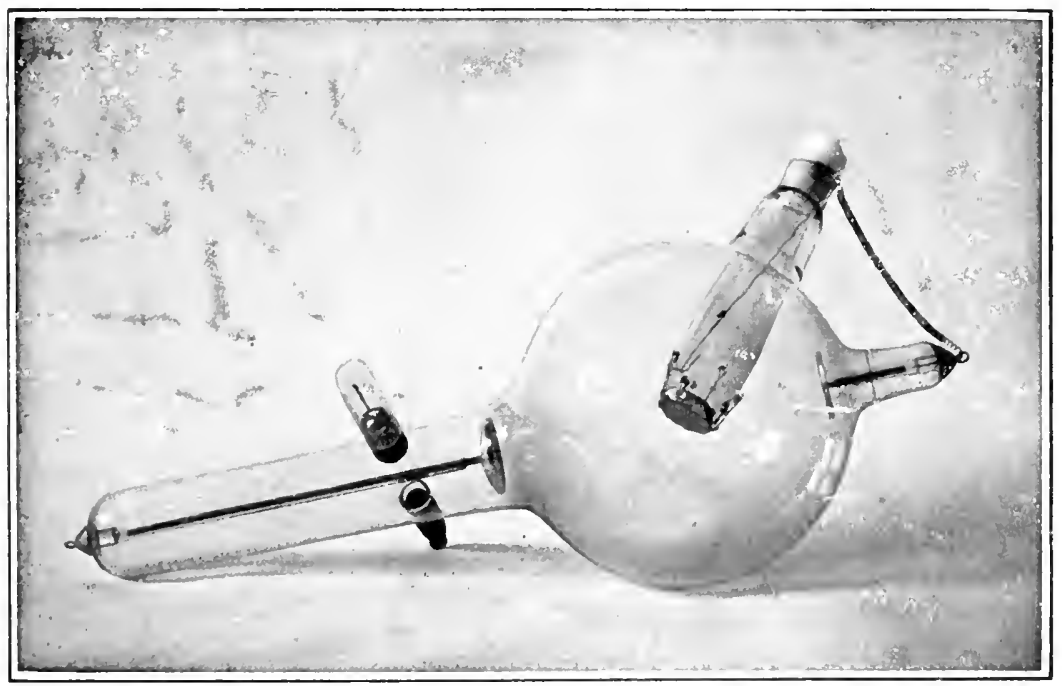

Fig. for.-Fricdrich llessumer's adjustable tube.

the resistance in the secondary current-circuit is accordingly increased or diminished.

By using a self-regulating tube one can work, throughout the expose, with X-rays which are of even quality. According 
to Leonard, a "soft" tube corresponds to a spark-length of 2-5 $\mathrm{cm}$. at the coil; it allows a current of great energy to traverse it. The same authority defines a "hard" tube as one requiring a spark-length of $5 \mathrm{~cm}$. and more at the coil. It sometimes happens that on reducing the vacuum of an over-hard tube the process is carried too far and the tube made too soft. Such a tube is useless for work with a I'ellnelt's interrupter. On first closing the circuit, a temporary yellowish-green illumination of the tube is seen, with violet circles and shadows, but after that there is no further fluorescence. The author has remedied this defect by lengthening the platinum-pin of the interrupter; or should this prove ineffective, by placing another tube (a hard one) in front of or behind the over-soft one. On sending a strong current through both tubes, at first a slight violet glimmer is seen in the soft one. I ater on a violet line appears between cathode and anti-cathode, and this is succeeded by greenish fluorescence which gradually gains in intensity, the riolet being lost; when

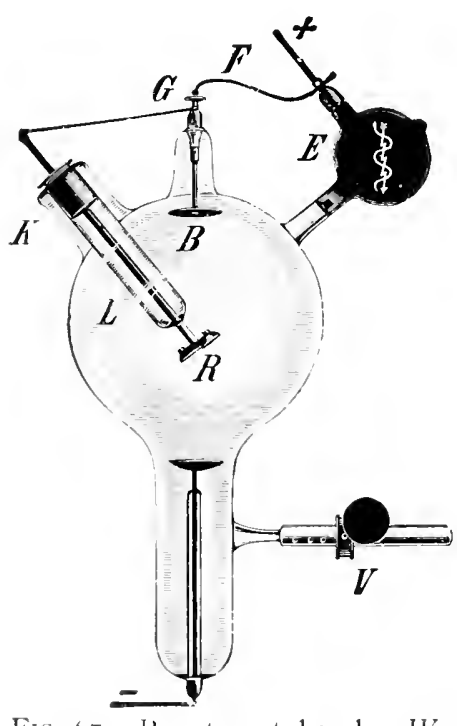

FIG. 07.-Roentgen-tube, by $W^{\prime}$. 1. Hirschmann, Berlin. this happens sparks appear in the spark-gap of the coil. By serial connection of hard and soft tubes in this way the author has moreover at times succeeded in restoring over-hard tubes. Very hard tubes can also be got to work for a time by removing the wire connecting anode and anti-cathode.

The adjustable tubes made by $I^{\circ}$. A. Hirschman and Max Lery of Berlin are founded on another principle. In these there is a contrivance whereby minute quantities of air are allowed to enter the tube when it has become over-hard. Fig. 67 represents such a tube. By turning the valve-screw $r$, which is kept tightly closed at other times by atmospheric pressure, a minute quantity of 
air gains access to the tube and so softens it. These tubes also possess an appliance $E$ for letting air escape from them, whereby it is possible to harden soft tubes while they are working. This is managed simply by lifting the spring $F$ with a glass rod away from $G$ til! no sparks jump across. The globe $E$ is, it should be mentioned, coated with a layer of finely-divided platinum, which absorbs air while the current is passing.

The anti-cathode is in this tube provided with a water cooling chamber $L$. If the character of the $\mathrm{X}$-rays changes, the situation of the focus-point of the cathode-rays will also change. The ideal is to have one point on the anti-cathode, which is at the same time both the focussing-point of the rays coming from the concave mirror and the point of origin of the X-rays. In most tubes, however, the $\mathrm{X}$-rays do not emanate from a point on the anti-cathode, but from a surface of rariable size. This surface is at its smallest when the tube possesses a certain degree of vacuum. The need, therefore, existed for some arrangement whereby the cathode-rays could be accurately focussed at one point in the anti-cathode. Moreover, the X-rays must proceed from one point only, and this point must remain fixed, cren though the penetrating capacity of the rays may alter. For exact work again those rays which exist apart from the main body of rays - the so-called "wandering rays"--must be eliminated, and one should be able to regulate the quality and the penetrating capacity of the rays during the expose as desired, and that without admitting or expelling air.

With these ideals in view, a tube has been constructed by Gundelach and Dessaucr. Gundelach utilises an observation made by Hillorf and P'uluj to the effect that the static charge of a glass tube hinders the free passage of the cathode-rays. When cathode-rays traverse a tube the latter becomes statically charged, whereby the progress of further cathode-rays is impeded. If the tube be narrow, the cathode-rays may be altogether suppressed. If, howerer, it he somewhat wider the rays pass through, kecping as far alwy as possible from the sides of the tube and becoming concentrated into one single ray which runs in the line of the tube's axis. 'This concentrated ray will strike the anti-cathode somewhere, and X-rays will be evolved 
with almost mathematical precision from this point. Gundelach placed a metallic covering over his tube, which considerably heightens the effect. This metallic mantle has another function. The site where the $\mathrm{X}$-rays strike it has an area about the size of a pfennig-piece. This area allows all the regular rectilineal rays to pass, but completely obliterates all "wandering" rays.

$F$. Dessauer had, however, noticed that the quality of the rays changes in certain tubes according to whether one uses the anti-cathode and secondary anode conjointly or not. In the former case some tubes were considerably softer.

Acting on this observation, tubes were made (Fig. 68) with a variable spark-resistance between the external poles of the anti-

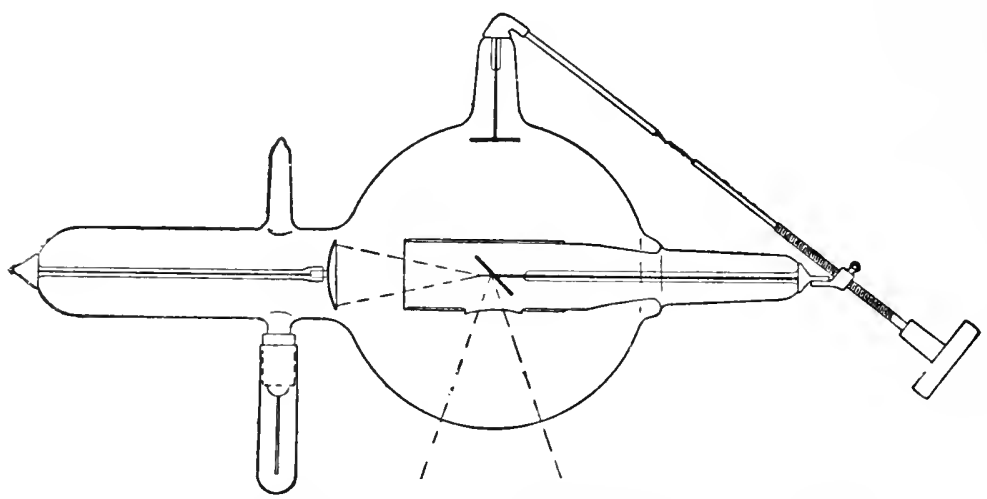

FIG. 68.-Adjustable tube by Gundelach and Dessauer.

cathode and anode. By means of a vulcanite handle the sparkgap could be increased or diminished, so that any desired current-quantity could be supplied to the secondary anode and the penetrating capacity of the rays controlled.

$W^{\prime}$ ild and ${ }^{\prime}$ (alter ${ }^{1}$ ) have shown that Roentgen-tubes are adversely affected by the closing currents of the coil, which give wrong current direction. The presence of this reversed closingcurrent is shown by the interspersion of spots, rings, and shadows in the green fluorescence of the tube.

Experience soon showed that in order to be able to properly regulate soft tubes, as well as to free them from closing-current

$\left.{ }^{1}\right)$ Fortschritte auf dem Gebiete der Roentgenstrahlen, Bd. II, p. 60. 
effects, the primary coil should be constructed with variable selfinduction. The latter can then be readily increased for these soft tubes ${ }^{1}$ ).

For this purpose coils are made as directed by $B$. II aller, in which the primary winding is composed of several sections. By means of a special cross-connecting arrangement the current may be made to flow through one, or to increase the self-induction, through two or more of the sections.

Another method of banishing the closing-currents from the tube consists in introducing the so-called Drossel tubes to the secondary current-circuit of the coil. The cathode-rays, according to Puluj, that is to say, the electric discharges in a vacuumtube possessing a free and an enclosed electrode, go only from the latter to the former, since a greater resistance is opposed to the passage of a discharge in the reversed direction. The Drossel-tube takes advantage of this fact. It offers no special resistance to the "opening" induced currents, but, on the other hand, "closing" currents, which pass in the opposite direction, are practically suppressed.

The Drossel-tube is comnected directly to the Roentgren-tube, being itself in connection with the positive wire from the coil. As a rule, the Drossel-tube itself is provided with an appliance for regulating its vacuum. ( $) r$. Max l,ery's, $F$. Dessaner's and Chabaud's tubes.)

The author's own experience with Roentgen-tubes leads him to prefer those which are regulated on the "osmosis" principle. These tubes he has found practically indestructible, and has indeed had two of them in constant use for fire months, working daily for several hours, without finding them suffer any deterioration. He has found the ordinary non-adjustable short-lived tubes so very troublesome that he has discontinued using them.

\section{$\S 28$. Some Practical Hints on the Installation and Use of X-Ray Apparatus.}

When buying an X-ray outfit, the current source must be the first consideration. Primary currents are no longer used. Most

1) See page $\sigma_{2} \mathrm{ff}$. 
tubes are now worked by accumulators, or by currents, continuous or alternating, from the town supply. In rare instances static machines (see p. I 7 ) are used to supply the current, and here the installation is perhaps the simplest. These generators, however, cannot as a rule be recommended on account of their comparatively feeble capacity.

Presuming some system of accumulators to be selected, the first thing to ascertain is what current-strength and what tension are required by the coil. Supposing $\mathrm{I} 2$ volts to be necessary, at least 6 cells must be provided. It is, however, better to use an accumulator somewhat larger than this, as small accumulators suffer if too great a demand be made upon them. It must be remembered also that the charge of the cells falls after use below 2 volts, and, moreover, various auxiliary apparatus has to be connected with the current-circuit. The following table of the Allgemeine Elektricitaets-Gesellschaft, Berlin, shows the lowest tension necessary for a coil in order to maintain the maximum spark-length. A mercury-interrupter is supposed to be used which interrupts the current i 8 times per second, the duration of current-opening and current-closing being identical.

\begin{tabular}{|c|c|c|c|c|}
\hline \multirow{2}{*}{$\begin{array}{l}\text { Spark-Length } \\
\text { of Coill in Cm. }\end{array}$} & \multirow{2}{*}{$\begin{array}{l}\text { Mrinimum } \\
\text { Tension in Volts }\end{array}$} & \multirow{2}{*}{$\begin{array}{l}\text { Minimum of } \\
\text { Celis required } \\
\text { in Battery }\end{array}$} & \multicolumn{2}{|c|}{ For Roentgen-Ray Work } \\
\hline & & & $\underset{\substack{\text { Minimum } \\
\text { Tension }}}{\text { Then }}$ & Number of \\
\hline 18 & 12 & 6 & I6 & 8 \\
\hline 23 & 14 & 7 & I6 & 8 \\
\hline 28 & I6 & 8 & 20 & IO \\
\hline 33 & 20 & IO & 24 & I 2 \\
\hline 43 & 24 & 12 & 28 & 14 \\
\hline 54 & 28 & If & 32 & 16 \\
\hline 65 & 32 & 16 & 40 & 20 \\
\hline 75 & 40 & 20 & 48 & 24 \\
\hline
\end{tabular}

With a quicker rate of interruption more cells are required, or the coil will not be worked at its full capacity.

Charging the Accumulators. This must be done at a certain current-strength only, which is defined by the maker of the cells. Primary elements, or a thermo-pile, or a dynamo-supply, may be used for this purpose, but the first two should only be used when the last-mentioned method is not available. Generally 2 Bun- 
sen-elements are required to charge a single cell, so that an accumulator of 6 cells requires 12 elements for charging.

When using a thermo-pile, the battery requires first connecting with the gas-supply, and it is important to see that all the lights are burning. Since a thermo-pile only furnishes low-tensions, the accumulator must be provided with an arrangement whereby all the cells may be connected in parallel; in this manner, as is well known, the tension of the battery is made no greater than that of an individual element. After the thermopile has become heated, its + terminal is connected with the + terminal of the accumulator; the - terminals are similarly joined. Charging an accumulator in this way takes a considerably longer time than in the manner next described.

The best method of all is to use the continuous current from a dynamo. The poles on the conducting-wires from the latter must be first determined. The following chemical methods may be adopted for pole-finding: (a) By dipping the ends of the two wires into dilute sulphuric acid, say the contents of a $W$ chnclt's interrupter. The negative wire shows a free development of gas, whereas the positive wire remains practically free from gas, but is coated with a black deposit of copper oxide. (b) By bringing both wires into contact with a piece of paper moistened with potassium iodide solution. The positive pole is indicated by a black stain. W'ilke's "pole-finding paper," which is treated in the same way, gives a red stain at the site of the negative pole. 'This paper may be purchased from the trade, bound in booklets. (c) A small apparatus can be obtained which consists of a small tube filled with a liquid into which platinum wires project from either side. If these be connected with the conducting wires the liquid becomes dyed red at the negative pole. The colour disappears on shaking the tube.

When the pole signs are not marked on the accumulator, the positive electrode may he known by its brown, and the negative by its grey colour. Care must be taken when charging, that the positive and negative electrodes are in connection with the corresponding terminals of the supply current.

In order to protect the accumulator from damage by too strong a supply-current, a rheostat and ampèremeter must be 
first introduced into the circuit. Or a series of incandescent lamps of definite size, connected in parallel, may be used as resistance. In this case no ampèremeter will be required. The current-consumption of a lamp is at the same time a measure of the current-strength which it allows to pass through. If, for instance, a lamp of the series works at 98 volts and 25 candlepower and uses I ampère of current, it will, where there is par-

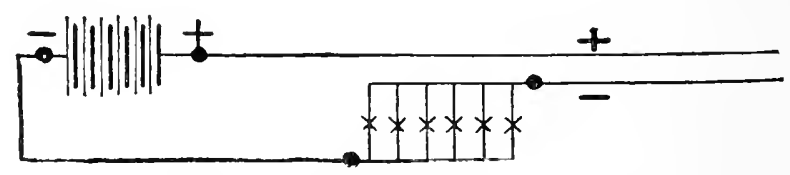

FIG. 69.-Scheme for the arrangement of incandescent lamps as front-resistances in the charging ố accumulators. (After Max Kohl, Chemnitz.)

allel-connection (Fig. 69), allow just as much to reach the accumulator. A second lamp passes a second ampère, and so on.

When the accumulator is not properly connected, the lamps burn more brightly than usual. A lead-fuse placed in the current circuit and calculated for the maximum current-strength protects the lamps from destruction.

As a rule the current-strength should be maintained at an even level during the process of charging. In no case should too great a current-strength be used, or the cells will be damaged, and towards the end of the charging the strength of the supply should be allowed to fall $30-50 \%$. If too feeble a current be used, the time required to charge the cells is unnecessarily prolonged. The charging of the accumulator must be allowed to proceed until a moderate amount of gas is developed in the cells. Notice should be taken if the formation of gas is equal in all the cells; should one of them be deficient in this respect, it will probably be found that short-circuiting has occurred, owing to the presence of conducting media between the electrodes. These should be removed by means of a glass-rod, and if the trouble cannot be removed in this way the cell should be withdrawn. Hermetically sealed vessels which may enclose the electrodes should be opened during the process of charging, $e$. g., by removing plugs.

When discharging an accumulator the current-strength used 
must not be allowed to exceed certain limits, which are defined by the manufacturer. At the beginning of the discharge the tension of each cell averages 2 volts; later on it suffers a gradual decrease, while as the cell approaches the stage of exhaustion this loss of tension becomes more rapid. The discharge limit is reached when the terminal tension of each cell drops to $\mathrm{I}_{3} \mathrm{~s}_{3}$ volts. A cell should never be worked down to 1.8 volts or its durability will be impaired.

The following points should also be borne in mind when working Roentgen apparatus from accumulators:

A fully charged accumulator standing idle discharges itself in the course of 2-4 weeks; consequently it requires periodical charging whether in use or not. The liquid in the cells requires renewal from time to time, either with distilled water, or fresh acid and water of the original strength.

Accumulators must be moved with great care and guarded

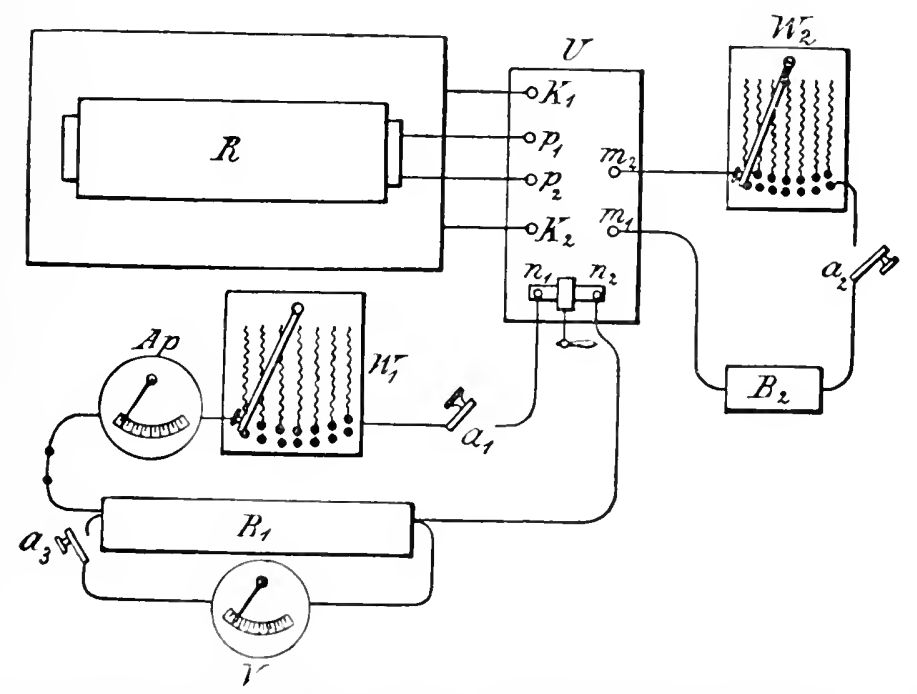

FIG. 70.-Scheme for Roentgen apparatus workel with accumulators. (After B. Donath, c. p. 9I.)

from shocks. They must never be used too long at a time, and must be frequently recharged.

When connecting the accumulator with the coil (Fig. 70), one of the poles of the battery is joined by wire with one of the 
terminals $\left(n_{2}\right)$ of the primary coil. The second battery-pole should first be connected with a lead-fuse, the latter with an amperemeter $(A p)$, a rheostat $\left(H_{1}{ }_{1}\right)$, and a current-interrupter $\left(d_{1}\right)$ which is in connection with the other terminal $\left(n_{1}\right)$ of the primary coil. A volt-meter $(V)$ is arranged in a side-circuit (parallel), the function of which is to indicate the tension in the accumulator. For use with a Nerf's hammer, the above arrangement is quite suflicient, but in most cases quicker interrupters are employed for therapeutic purposes, so that the second battery $\left(B_{2}\right)$ will be required for working the interrupter. One of the poles of this battery is connected with a terminal $\left(m_{1}\right)$ of the motor working the interrupter. Between the other battery-pole and the second motor-terminal $\left(m_{2}\right)$ a current-interrupter $\left(A_{2}\right)$ and a rheostat $\left(V_{2}\right)$ are introduced, by means of which the rapidity of the interruptions may be regulated. In addition to the terminals $m_{1}$ and $m_{2}$, there are three further pairs of terminals at the interrupter board. Of these, the first pair, $n_{1}$ and $n_{2}$, receive the main current for the primary coil, the second pair, $p_{1}$ and $p_{2}$, are joined to the poles of the primary coil, and the third, $K_{1}, K_{2}$, are connected with the terminals of the con. denser.

The condenser is in connection with both sides of the site of interruption, its function being (p. 57) to receive the electrical quantities which are produced here by the extra-current.

The switch between $n_{1}$ and $n_{0}$ enables one to change the direction of the current at any time. The wires con. necting these parts of the apparatus must be well-insulated thick copper cables.

The apparatus should be worked with a continuous current supply of 1 I o volts wherever this is to be obtained. Apart from the fact that a cheaper form of apparatus may be used with a lighting-circuit, no restriction is placed upon the choice of an interrupter, and the whole plant can be worked more efficiently. Fig. 7 I shows a scheme for the arrangement of a Roentgen apparatus worked from the lighting-circuit. From the main cable, shown at the top and left-hand side of the sketch, two wires branch off - a thin wire $L_{1}$ for working the interrupter, and 
a thicker wire $L_{\text {.: }}$ for the coil. Resistances $\left(A I^{\prime}\right.$ and $\left.R I^{\prime}\right)$ and switches $(A)$ are situated in the course of both wires: $l, .2$ moreover possesses an amperemeter $(A p)$ and a volt-meter $\left(I^{\prime}\right)$ in a side-circuit.

Where a I'ehnelt's interrupter is used, the main lightingcircuit is in direct comnection with the primary coil (Fig. 72),

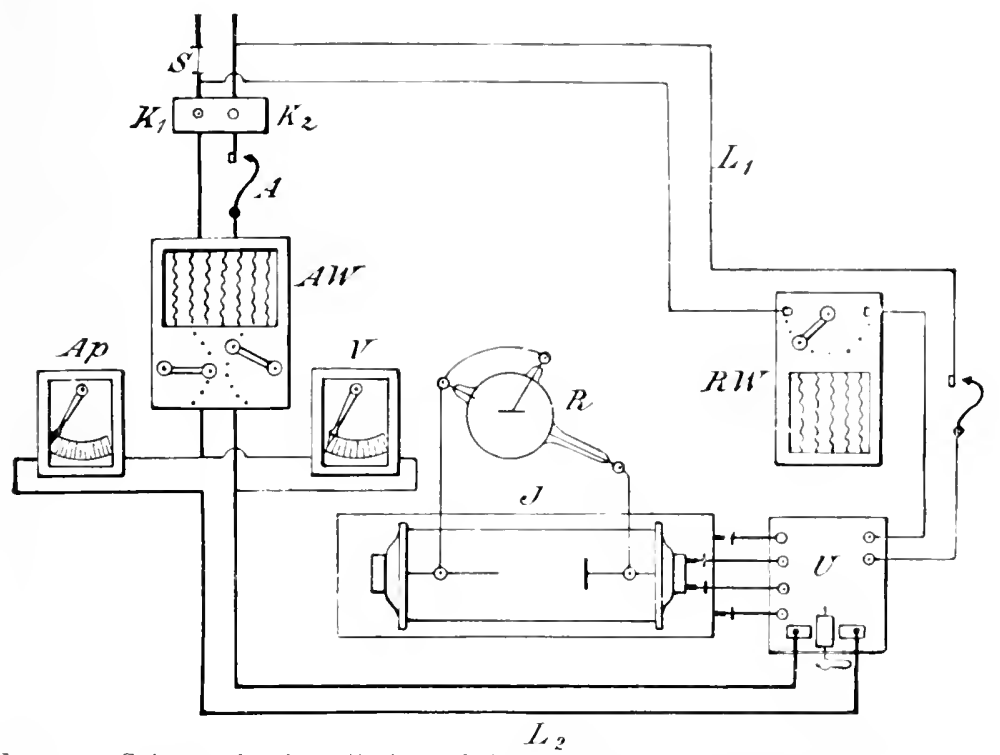

Fig. 7I.-Scheme for installation of Roentgen appatatus worked from a continuous lighting-current. (After B. I) onath, l. c., p. of.)

and provision is only made for a lead-fuse (5), a rheostat $\left(I I^{\prime}\right)$, an amperemeter $(A p)$, and a current-break $(d)$. Care must be taken that the positive pole of the main current is in connection with the platinum pin of the electrolytic interrupter, the negative pole being in connection with the lead plate of the same.

When working the Roentgen apparatus with a mercury break the full current may be used for the motor; the tension of the main circuit is, however, too high for the coil itself.

High tensions camot be employed because of the excessive amount of sparking which would occur at the interrupter: whereby the latter would be soon destroyed. Moreover, with 
high tensions the current-strength in the primary coil would reach such a dangerous height with each current-closure that there would be injurious heating of the coil and damage to its

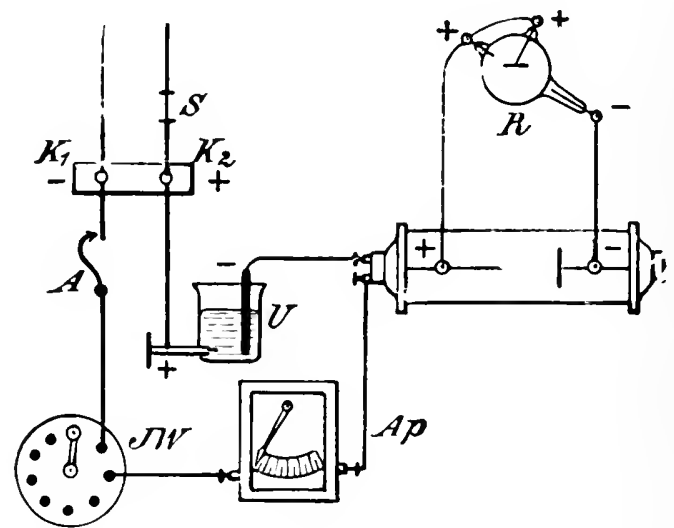

FIG. 72.-Scheme for an apparatus with electrolytic break. (After B. Donath, c., p. 96.)

insulation (B. Donath). Resistances have therefore to be provided which are capable of absorbing the greater part of the energy supplied.

To regulate the current-supply, resistances are usually employed which reduce the tension to $35-5$ volts and the current strength to about 3 ampères. As has been before mentioned, the higher tension of the main current can be used direct for the motor of the mercury interrupter. The motor is situated in a branch-circuit from the main (See Fig. 7I). In order to facilitate their manipulation, all the connecting, regulating, and measuring apparatus belonging to the Roentgen outfit are generally mounted on a wooden or marble slab fixed to the wall at a height convenient for the operator. In the latest types of Roentgen apparatus, with electrolytic interrupter and variable self-induction for the primary coil, the controlling mechanism is arranged on a small table, fixed on wheels so that it can be moved to any place desired (Fig. 73). The apparatus, moreover, possesses resistances which are capable of the most delicate regulation, and on the table every combination can be produced between the various electrodes of the interrupter. 
and the different divisions of the primary coil (apparatus by Siemons E Halske, Richard Seifort $\mathbb{E}$ Co., Max Kohl, etc.).

The interrupter may be placed at some distance if its noise inconveniences the patient. When working with a turbine-interrupter the conducting wires from the coil may be passed through two holes in the wall to the racuum-tube in an adjoining room, where the patient sits. Thus all noise is obviated, and a nerrous patient is spared the sight of formidable-looking apparatus. A switch in the operating room controls the whole apparatus.

In case a d'Arsonial-Ondin's apparatus is intended also to be worked with the X-ray coil, the arrangement should be as

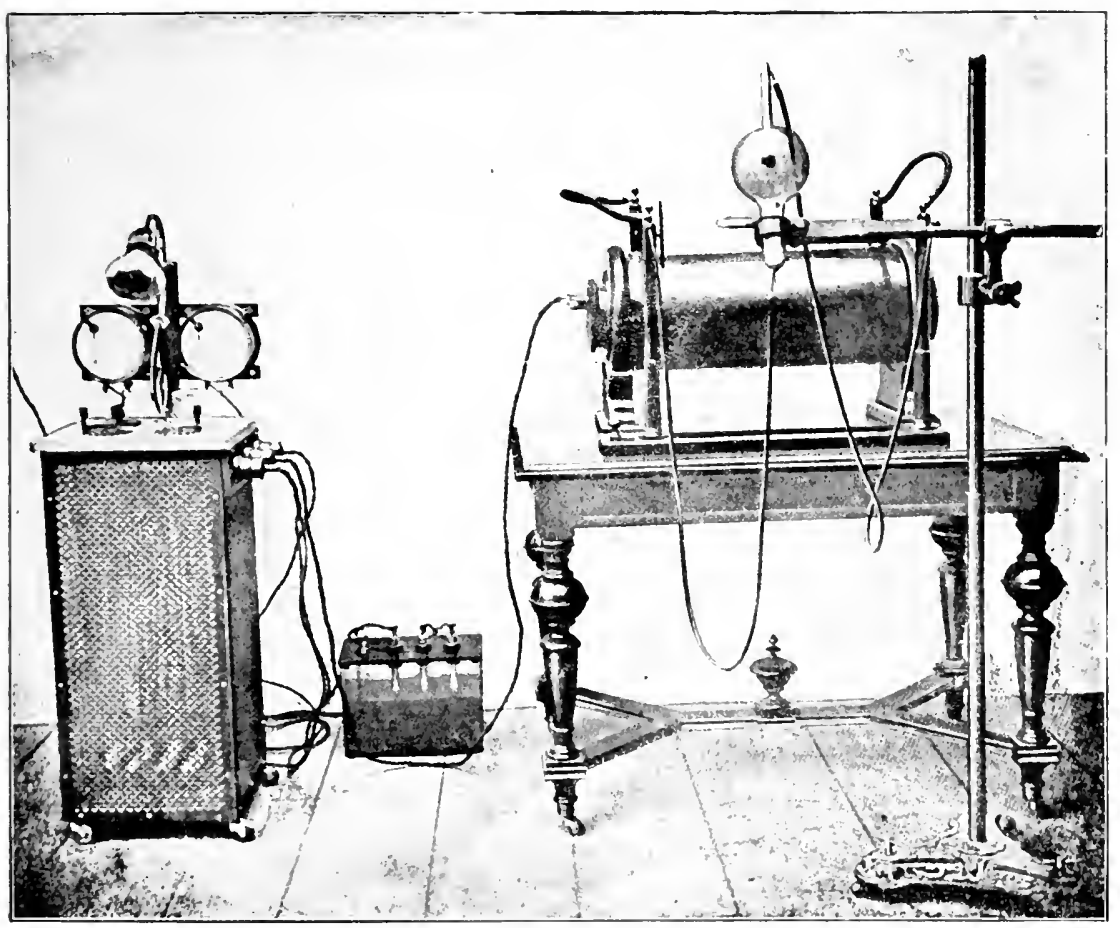

Fig. 73--Roentgen apparatus by Siemens of Malske.

in Fig. 6o. An earth-wire can be laid hetween the two wires going from the secondary coil to the inner layers of the leyden jar. This earth-comnection should go not to the gas, but the 
water-pipes, since the former are often in the vicinity of the electric-light cables.

The apparatus for the production of uni-polar discharges with Ruhmkorff's coil will not work with W' chnelt's interrupter. In cases where a continuous current-supply is not to be had, but where an alternating or rotary current alone offer, three courses are open for Roentgen work: $\mathrm{r}$. An electrolytic interrupter may be used. In this case the platinum-pin is made a little thicker, to cope with the increased wear and tear. Siemens \& Halske make a I'ehnelt's interrupter which may be used in direct connection with an alternating current-supply. A spark-gap is introduced into the secondary circuit, which only allows the interruptions arising from one phase of the alternating current to inHuence the tube, those of the other phase being equalised in the spark-gap. 2. By means of a turbine-mercury-interrupter. With this apparatus the current is always closed in one and the same half of the current-phase, so that only waves of the same direction enter the primary coil; consequently their effect is identical with that of an interrupted continuous current. As already mentioned, the turbine-interrupter must be first regulated by hand by means of the fly-wheel. The number of interruptions exactly equals the number of periods of the alternating current. 3. The most adrantageous plan is to transform the alternating or rotary current into a continuous current. This is done by means of a motor, which is coupled to a continuous-current dynamo; the latter supplies the current for the Roentgen apparatus. (A transforme: must also be used in case the continuous current supply has a very high tension, say 440-500 volts.)

The commutator must always be properly arranged, so that in the spark-gap of the coil sparks jump from the point (anode + ) to the centre of the disc (cathode -). Where the commutator is wrongly placed the sparks jump from the edge of the disc (see Fig. 23) to the point.

The tube must be arranged so that its anode is in connection with the positive pole of the coil, while its cathode (the concave mirror) is joined to the negative pole.

The wires connecting the tube with the coil may be thinner than those joining other parts of the apparatus. Many kinds 
of insulated wires are on sale with the object of preventing the patient or operator from electric shocks. The author has found none of these really effective; he now uses plain copper wire. The only thing necessary is to aroid coming too close to the wires.

What should be the position of the tube with regard to the patient? The first thing is to ascertain where the most effective Roentgen-rays are evolved from the tube. This a Huorescent screen or photographic plate soon shows. By bringing either of these close to an active tube, not only the contour of the irradiated field, but its most intensive area can be seen. The latter, in the case of most tubes, lies round a line proceeding from the point on the anti-cathode (coinciding with the apex of the cathode-cone) at right angles to the tube-axis. In some tubes, however, the most active zone lies around a line at right angles to the anti-cathode itself. In the first case the tube should be placed parallel to the object to be exposed, and then mored until the middle of the sphere is exactly opposite that object. In the second instance, the tube must be turned until the anti-cathode is parallel with and exactly opposite the object.

An apparatus with the electrolytic break is easily placed in action. The resistances at the rheostat are first disconnected, and the length of the platinum-pin is regulated by means of the screw according to requirement. Should the interrupter fail to act there may be various causes, of which the following are amongst the commonest.

a) The lead-fuse may have melted, requiring renewal. The fuse must not be replaced by a stronger one, or a copper wire, as by so doing the wires would become red-hot with too powerful a current. The insulation of the supply-cable must be looked to at the first opportunity.

b) A gas-bubble may be fixed at the platinum point, in which case it should be dislodged by tapping or shaking the lid.

c) The tube may be too soft, or, where no tube has been connected, the spark-galp in the secondary circuit may be too small. To obriate this difficulty a harder tube may be cmployed, or the original tube may he hardened by reversing its position for a few seconds (see also p. 2 12). 
d) The acid in the interrupter may be too hot, requiring the addition of colder acid. The liquid again may be too concentrated. The best proportion of acid is $\mathrm{I}$ in 20 ( $\mathrm{I} .06$ specific gravity, $=8^{\circ}$ Beaumé) .

e) The porcelain may become too hollowed around the platinum-pin after prolonged use, needing replacement.

The current must be turned off at the main switch, not at the rheostat.

When using interrupters which are not actuated by the primary current itself they must first be set going before sending the primary current through the coil. When disconnecting the apparatus the procedure must be reversed. Should it be found that the current is passing in the wrong direction through the tube (which is at once seen from the character of the fluorescence), the current-reverser must be used immediately.

The interrupter may be regulated by (I) altering the position of the contacts in the rheostat in the case of turbine- and mercury-jet-interrupters. (2) The make and break may be modified in mercury-motor-interrupters by raising or lowering the mercury vessel. (3) The intensity of the primary current may be regulated by means of its own special resistance. The following practical points are important: No wires should be left lying about on the floors or tables in the operating room; all wires which are not in use should be put tidily away, and the operator must not indulge in gesticulations with wires in his hands.

Wires from the coil to the tube must not be allowed to lie against the former. The coil should stand somewhere away from the window, otherwise in the summer there will be a risk of the sun's heat melting the paraffin insulation of the windings. If the temperature be very high, the coil should be cooled with damp cloths, or by using an electric fan.

Great care should be taken that the apparatus be not set working while anybody is busy with the wires; moreover, no bystander must approach too near the conductors from the secondary coil while the latter is in action.

The spark-gap of the coil must never be extended beyond its 
limit of capacity, even with the tube in position. Otherwise, if the resistance between the terminals be too great, there is a risk of short-circuiting through the insulation of the coil. It is much better to have the spark-gap somewhat shorter than absolutely necessary, for in this way a kind of safety-ralve is provided; in case the resistance of the tube becomes too high, the discharge does not take its way round the tube or through the coil-insulation, but finds rent in the spark-gap.

Where the platinum contact of a platinum break becomes fused, it may be necessary to use a file to the place, or a fresh "hammer" may have to be provided. The motor working a mercury-interrupter requires cleaning and oiling daily. When not in use, the copper rod dipping into the mercury must be removed from the latter-otherwise an amalgam will form. Plugs of cotton-wool should be placed in the oil-holes to prevent them leaking. The motors should be seen to from time to time, lest they get overheated. This may happen if they are worked too long at a stretch, especially if too strong a current be used; the result may be some damage to the insulation. Again, if a layer of spirit be used over the mercury in a mercury-break, there is some danger of it becoming ignited. The author has found water better to use for this purpose. Not only is it free from risk of firing as compared with spirit or petrol, but the apparatus is more easily cleaned afterwards. This is readily done by placing the mercury-vessel under a strong jet of water, which washes the muddy accumulation away and leaves the mercury clean. This process should be repeated daily.

A turbine-interrupter does not require cleaning quite so often; once a fortnight should suffice. The pure alcohol should first be poured off and placed aside in a vessel. What is left in the interrupter should be poured into an iron bowl, and the residue of alcohol ignited. When the alcohol has burnt away clean mercury is left lying on a layer of muldy debris. The mercury should be poured off and the mud stirred with a stick, whereby the rest of the mercury separates and is ready for collecting. The whole process should be completed in the open air on account of the poisonous mercury fumes evolvel. Do not neglect after this operation, or after much handling of the leaden 
masks (see later), to always wash the hands with soap and water so as to avoid lead or mercury poisoning.

It is adrisable to remove the electrodes from the acid when an electrolytic interrupter is not in use.

Vacuum-tubes must be kept in a dry place, free from shocks

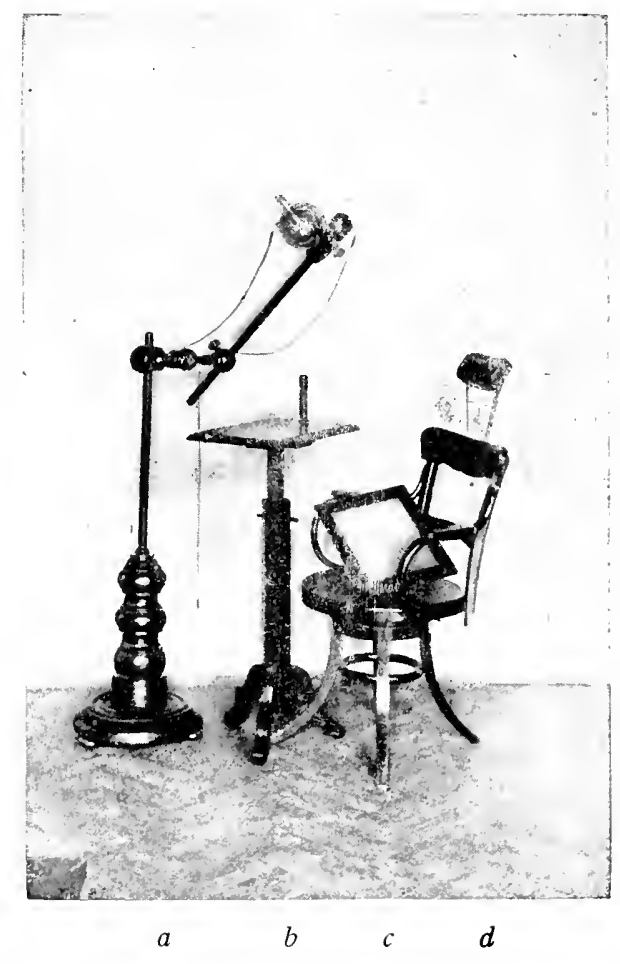

FIG. 74 .

or risk of violent shaking. The best plan is to line the box in which they are kept with wood-wool or cotton-wool.

The following articles are necessary auxiliaries for Roentgen-therapy :

I. A tube-stand, either fixed to the wall or movable, and preferably with two arms, so that two tubes may be connected if desired (Fig 74a).

2. An operating-chair with adjustable head-rest $(d)$. 
3. A small table with an adjustable top $(b)$.

4. A clock for timing the sittings.

5. A couch.

6. A fluorescent screen, for examining the quality of the tubes (c., Fig. 74). If the room cannot be darkened, this screen must be fixed in a darkened box.

7. Several leaden plates tapes, pasteboard masks, lead-fuses, and a variety of mechanical tools, such as pliers, files, screw-drivers, ctc.

\section{$\S 29$. The Development of the Therapeutic Employment of Roentgen-rays.')}

Roentgen-therapy has already undergone considerable development and has received definite indications for its employment and method, whereas the theoretical investigations of the detailed physiological effects of the process has only recently been commenced, and this with comparatively small success. Consideration of this circumstance induces the author to place the chapter on Roentgen-therapy before that on Roentgen-physiology. Many facts, moreover, to be found in this section are of significance in a physiological sense, and will not require repetition later, but merely a reference to the present chapter.

The origin and development of the Rocntgen-therapy of skin diseases dates from an experiment made by the author in 1896 with the object of removing the hair from a large navus pigmentosus pilosus in a girl. This experiment was prompted by a newspaper article in Junc, 1896 , in which it was reported that a man working with $X$-rays became aflicted with a dermatitis accomparied by extensive loss of hair from the head; further by an article appearing soor after that in No. 30 of the "Deutsch. Med. Wochenschr.," from the pen of Dr. W'. Marcuse of Berlin, who noticed the same effects after a fortnight's radiation on the head of a young man.

1) A Jucid accomt of Renentern therapy is given in the excellent article by Herr Prof. Kotp in He llambluch der phy ik. Therapie Th. II, Bd. I. 
The author made the following arrangements for this experiment ${ }^{1}$ ) :

A Ruhmkorff's coil by Keiser and Schmidt of Berlin, having a $25 \mathrm{~cm}$. spark-length, was worked from accumulators, and vacuum-tubes which had been proved to be rich in X-ray powers were used. The tubes were capable of giving a Roentgen photograph of a man's hand at a distance of $15 \mathrm{~cm}$. with one minute's exposure, and the fluorescent screen was used from time to time to prove their proper working. These tubes possessed platinum anti-cathodes and aluminium electrodes, and the most serviceable of all were found to be those of Messrs. Frister of Berlin. Ordinary Hittorf's tubes were only used in those experiments where it was desired to exclude the cathode-rays as far as possible. The child was made to sit with its back bared, and the tube was placed at a distance of $\mathrm{IO} \mathrm{cm}$., so that the zone richest in X-rays coincided with the nape of the neck. In this way a large part of the nævus from the scalp down to about the middle of the dorsal spine was irradiated. At first a thick leaden mask with an aperture corresponding to the area of the nævus was used; this, however, was soon dispensed with, the result of the experiment being apparently so doubtful. The exposure was two hours daily.

The author soon convinced himself that no perceptible heat was evolved from the tube. The child, though a sensitive one, endured the sittings extremely well and remained well and lively throughout. For the first ten days no change whatever occurred; not a single hair fell. On the eleventh day (December $3 \mathrm{rd}, \mathrm{I} 896)$ the mother removed several bundles of loose hairs from the inter-scapular region, and the

\footnotetext{
$\left.{ }^{1}\right)$ Wiener med. Wochenschr., I897, No. Io. The assertion made repeatedly in the latter by Prof. Dr. E. Schiff to the effect that the author made these experiments under his (Schiff's) control is not borne out by facts. They were carried out independently by the author in the K. K. graphisch. Lehrund Verstehsanstalt in Vienna; Herr Hofrath Director Dr. J. M. Eder alone assisted him with his advice in the purely physical questions.
} 
author did likewise. With gentle pulling, bundles of 5-Io hairs came out each time in one's fingers, with absolutely no sensation of pain on the part of the child. The individual hairs were thin, showing no bulbar enlargement at the root to the maked eye. A microscopic examination by Dr. Ad. Zemomn showed atrophy at the roots. The skin in this region was quite smooth and showed neither reddening nor any other change. The shedding of hair continued steadily in the nape of the neck (although for reasons which will appear later the irradiation was discontinued in this region), and by the Sth of December there was an actual alopecia involving the lower part of the back of the head. From the roth December the shedding of hair decreased in amount somewhat, though persisting for some time in lesser degree. On the I Ith December, that is, eight days after the commencement of the hair-shedding, a dermatitis developed from two small excoriations which had already been noted on the nape. This consisted of a diffuse reddening, with here and there sereral small moist excoriations varying from the size of a lentil to that of a sixpence. This dermatitis disappeared after a few days' treatment with ichthyol ointment, and with it went the few remaining hairs at the nape. The back of the head and the neck and the upper inter-scapular region was then quite denuded of hair, and only a slight reddening left by the dermatitis was to be seen at the nape of the neck. It must be noted that a large portion of the bald surface had nerer shown the least signs of dermatitis.

On the tenth day the commutator of the coil was, through an orersight, so arranged that for 15 -20 minutes no cathode-rays were evolved from the tube. When on the eleventh day the hairs began to fall it was thought possible that this result was due to "anode-rays." ") To verify this the experiment was changed. The navus on

1) Three years later $O$. Berg experimentally proved the existence of incole-rays. (Sec page 19r.) 
the right arm, which was at some distance from the previously exposed region, was exposed for twelve days to a "reversed" tube. The results were negative.

The task still remained to exclude any possible effects from electric currents in the shape of spark discharges from the tube to the skin. With this object in view, the lowest part of the nævus was exposed to the $X$-rays, but between the tube and the skin a sheet of thin cardboard covered with aluminium-foil was interposed. The aluminium, which is very transparent to the rays, was suitably "earthed." This screen, therefore, while allowing X-rays to penetrate freely, acted as a discharger for the electricity. The experiment was begun on December I 8 th, I 896 , and eight days later the hair on the irradiated area began to fall. Till the 2 nd January this shedding of hair was only small in amount, being about 5 or 6 hairs daily.

After that it increased to 5-ro hairs daily. On January $5^{\text {th }}$ slight erythema was noticeable, and by then the hair had almost disappeared from the middle of the field. Altogether this region was exposed for 42 hours.

These three experiments clearly showed that the cause of the phonomena above-mentioned was to be found in the Roentgen-rays. Neither the anode-rays nor the high-tension electric waves could have produced the effects. Moreover, in the second series of experiments, sparks were often seen to jump from the tube to the arm to which it was adjacent, and again the hairs bristled up towards the tube (both events showing that electricity was passing from tube to skin), but no loss of hair resulted.

Another point brought out by these cxperiments was that the comparatively thin and long hair of the scalp fell out more readily than the strong short hairs of the mens? in the latter simation again it was found that the fine lanugo-hairs fell more freely, and after a shorter exposure than the stouter hairs. 
Finally, it became erident that the X-rays only produce their effect after a long exposme ( 11 to 2.2 hours), but that this effect is maintained for some time ( $S$ to I2 days) afterieards; the rays have therefore a cumblative action.

Was the falling of the hair due to the dermatitis alone, being analogous to the well-known alopecia symptomatica seen after erysipelas and eczema? Probably not, bearing in mind the late appearance of the dermatitis (seven days after the first falling of hair) and its slight character. The author published the facts concerning the later condition of the child's skin, and the conclusions to be drawn from the whole history of the case, in the Wiener Med. Wochenschrift, I $\$ 97$, No. 19. From this article it may be learned that the nape of the neck showed no change till February 2 2nd, when a little fine down was to be seen on the occiput. These new hairs were confined to the scalp region at first, the nape of the neck remaining quite bare till March I 4th, when fresh hair grew here also. In the dorsal region the changes were very surprising. This part had been last irradiated on January isth, I 897 , the total exposure having been about 42 hours. On January i 8 th the erythema here became more intense and all the hair fell out at the same time. On January 2oth and the succeeding days the erythematous portion of the skin showed numerous small resicles, which soon burst. Next the epithelium peeled off, learing a large raw surface, which was bright red, hot and oozing frecly. After a few days the inflammation became less acute. By February 6th a whiter patch the size of a child's hand was to be seen within the bright-red granulating excoriation in the region of the gth dorsal rertebra, and from this similar streaks radiated on to the neighbouring surface. On this part the contral rays of the light-cone had fallen. The base of the excoriation appeared deepened, and at the lower border onc could see two round epithelial islands. It secms remarkable 
that in spite of the appearances above mentioned the child had no special pain. The chief thing complained of was itching round the borders of the excoriation, where some eczema had resulted from the irritating discharge. The temperature and urine were normal! Instead of the applications of liquor burowii cooled in ice, which had hitherto been used, dressings of ointment were now prescribed (Ung. paraffin, ung. boracic, etc.), and later on baths, but none of these influenced the dermatitis in any way. Bad nursing may also have contributed to the want of success, since various household nostrums were used without the author's knowledge at that time.

Towards the end of February the child's general health began to suffer. She was obliged to stay in bed altogether, became pale, lost her appetite and slept badly. There was now much pain in the raw places. This pain came on often in paroxysms, especially during the night, and the attacks, lasting some fifteen minutes, were localised to the sides of the chest and abdomen. From what the child said, the pains appeared to shoot obliquely downwards towards the bladder. There was no sensitiveness on pressure anywhere. The attacks of pain, which were so severe that they always left the child bathed in perspiration, always came to an abrupt termination and were followed usually by micturition. Examination of the urine showed an acid reaction, some albumen, and deposits of urates, but otherwise nothing abnormal. By the Ioth of March the white patch above mentioned became more prominent, while the surrounding excoriation became duller and of a pale reddish-yellow colour. The base of the ulceration was considerably deeper and a delicate red flush could be seen on its odematous borders. The ulceration proceded apace; soon the entire inflamed region was transformed into a large ulcer, whose uneven base was covered with dark-brown putrid shreds of tissue. The child had now a high fever and was quite prostrate, 
while the urine showed an abundance of albumen. With sublimate-gauze dressings and baths the patient rallied somewhat and was taken to the dermatological clinic. Here the improvement became marked in a few days and the ulcers showed signs of healing. The parents unfortunately withdrew the child from the hospital on March 29th. Further progress of the case was marked by granulation and closing in of the wound. By May, I90I, the wound was closed by a somewhat hypertrophic scar with the exception of a patch the size of a florin, which also was slowly healing. The scar next unfortunately gave way as the result of injury, necessitating further surgical aid. The following conclusion was drawn from the progress of the case as above described. In the first place, the unexpected appearance of the ulceration gave signal warning that caution was to be observed in experimenting with X-rays. It suas evident that irradiation too long contimued might produce disagreable and reen dangerous results. It appeared that not only the total length of exposure, but the time of the individual exposures had to be considered. On the other hand, shorter total exposures (20 hours or less) suffice for the therapeutic effect of epilation without cansing real damage to the tissues or the organism. The cumulative effect of the $X$-rays constitutes the chief difficulty to be contended. with in determining the proper period of exposure.

It will be noted that in the case just described, the really harmful effects took two months to develop. In other cases (when the X-rays were used with a different object in view) the author has seen the hair fall after a much shorter exposure. Other factors beside the time of exposure have to be considered, viz: the intensity of the irradiation and the distance of the tube from the skin.

Since, as the first series of experiments showed, it was possible to obtain complete epilation effects lasting two months and a half, while avoiding any disagreeable 
after-effects, it appcared that Roentgen-rays might reasonably be used experimentally for the removal of hairs provided the warnings above mentioned were obeyed; apart from the fact that the effect was maintained for a considerable period, the brevity and painlessness of the treatment as compared with the other methods of dealing with hypertrichosis were a further inducement. in this direction. Moreover, it seemed likely that results might be made permanent, if, acting upon Kaposi's theory, one endeavoured to maintain the paretic condition of the papillary vessels. This might be accomplished by giving short exposures throughout a long period. Inasmuch as a nerve which is paralysed for some time often never recovers its function, it might be possible to so influence the raso-motor nerves in this way that they would lose their power of providing for the nutrition of the hair follicles; in this way the existence of the hair itself would become impossible. Moreover, the epilatory powers of the X-rays might be turned to acconnt in other skin affections, such as sycosis and farns, where a temporary epilation is of great advaniage. Experiment only was wanted to verify these hypotheses.

The data obtained from the above-mentioned experiments, giving promise of a rational therapeutic employment of X-rays, induced the author to pursue his investigations in spite of warnings from an authoritative source (Kaposi). A definite action of the rays on the skin had been experimentally proved, and this was sufficient for its judicious employment in the treatment of skin-disease.

It seemed somewhat tempting to experiment with an agent which evidently had such an extraordinary influence on the cuticle and which, according to later reports, had a favourable effect on the inoculation-tuberculosis of animals, as a means of dealing with local tuberculosis of the skin (lupus vulgaris). As a matter of fact X-ray therapy soon proved that in this affection very good results could be obtained, both as regards radical cure and cosmetic effect. The first report bearing on 
this question was made by Kïmmel at the Congress of the Deutsche chirurgische Gesellschaft, Easter, i 897 . At the same time, and independently of Kiimmel, the author and $E$. Sihiff (to whom the publication of these experiments was left ${ }^{1}$ )) treated and cured two cases of lupus rulgaris by means of $\mathrm{X}$-rays. Soon after this, reports followed in quick succession of favourable results in various kinds of skin disease treated in this way.

Thus $\operatorname{Hahn}^{2}$ ) first employed the method in dealing with chronic eczema, $S_{\text {chiff }}$ ) used it for lupus erythematosus, the author ${ }^{4}$ ) for sycosis and farus, Ehrmann" for (dermatitis papillaris, Ziemssen ") for psoriasis, Sorel') for elephantiasis, Pokitonoff") for acne rulgaris, Julass") for navus flam. meus, Kienböck and Holsknechl") for alopecia areata, Scholt ${ }^{11}$ ) for lepra and mycosis fungoides, Sjïgren and Stenbeck ${ }^{12}$ ) for epithelioma.

At the present time all those diseases are regarded as suitable for this treatment in which a temporary or permanent removal of hair is indicated (such as hypertrichosis, sycosis, farus); most writers, too, speak favourably of its employment in lupus rulgaris and epithelioma. In the case of other skin affections opinions are more divided, which is only to be expected considering the comparatively recent introduction of X-ray therapy. In many quarters the riew was and still is held that good therapeutic effects are only to be obtained by first exciting powerful reactions. Many operators, indeed, make it

\footnotetext{
1) Archis. f. Dermatologie und Syphilis, Bel. Xl.ll, I. Heft.

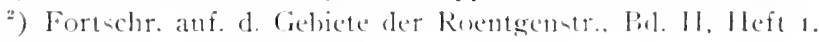

3) Hidl. Bal. [I, I left 4 .

4) Wiener dermatolog. Gesellichafi, May 10, temo.

s) Mr. Med. Mochenschr., Mo1, Nr. 3\%, 31

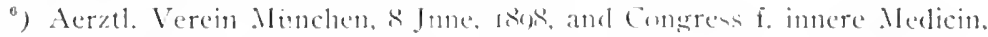
Wiesbaden, isgs.

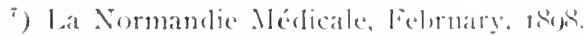

") Xll Intern. med. Congr. Moncow, Ison. Dematulogical Section.

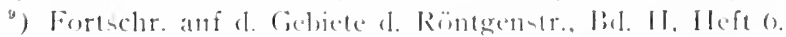

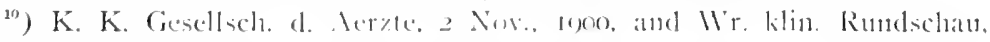
Ig0I, No. 41 .

") Arch. f. Dermatologie and Syphilis, lid. I.IX, Iteft 3.

12) Xlll. internat. med. Congrest. Partis, lyoo. 
their first aim to arouse violent inflammations. Under these circumstances it is not to be wondered at that more prudent men (Richl, for example), who saw some of the results of this heroic school, looked askance at the whole method.

A report, however, submitted by the author to the XIIth International Congress held in Moscow, showed conclusively that under proper conditions the treatment could give the best results and yet be free from these untoward accompaniments.

In riew of some of the facts noted in the table below it can be seen that epilation is quite possible without disastrous effects accompanying the process. The first experiment undertaken by the author proved conclusively that the cause of the Roentgendermatitis lay in too intense an irradiation.

\begin{tabular}{|c|c|c|c|c|c|}
\hline $\begin{array}{l}\text { Observer } \\
\text { and } \\
\text { Publication }\end{array}$ & $\begin{array}{l}\text { Dis- } \\
\text { tance of } \\
\text { 'Tubes }\end{array}$ & $\begin{array}{l}\text { Duration of } \\
\text { Exposure }\end{array}$ & $\begin{array}{c}\text { Part } \\
\text { Exposed }\end{array}$ & Physiological Effect & Remarks \\
\hline $\begin{array}{l}\text { Sihradeld, } \\
\text { beutsche nued. } \\
\text { Wochensehr., } \\
1896 . \text { No. } 42\end{array}$ & $14 \mathrm{~cm}$. & $\begin{array}{l}45 \text { minutes: } \\
\text { one exposure }\end{array}$ & $\begin{array}{l}\text { Abdomen: } \\
\text { boy, I } 3: 2 \\
\text { years old }\end{array}$ & $\begin{array}{l}\text { After it days slight } \\
\text { clermatitis and pig- } \\
\text { mentation. Falling } \\
\text { of lanugo-hair, lit- } \\
\text { tle sweat secretion }\end{array}$ & - \\
\hline $\begin{array}{l}\text { Mariuse, } \\
\text { Deutsche med. } \\
\text { Wachenschr., } \\
\text { 1806, No. } 30\end{array}$ & $25 \mathrm{~cm}$. & $\begin{array}{l}\text { Once or twice } \\
\text { daily (5-Io } \\
\text { min.) for } 4 \\
\text { weeks }\end{array}$ & $\begin{array}{l}\text { Face a nd } \\
\text { scalp }\end{array}$ & $\begin{array}{l}\text { In one place derna- } \\
\text { titis, in another } \\
\text { alopecia; the latter } \\
\text { not inflamed }\end{array}$ & $\begin{array}{l}\text { Hair grew } \\
\text { again } \\
\text { after } \\
3 \text { months }\end{array}$ \\
\hline $\begin{array}{l}\text { condex Medic., } \\
\text { Aus., Isgo }\end{array}$ & - & $\begin{array}{l}\text { A repeated long } \\
\text { exposure for } \\
\text { photographic } \\
\text { purposes }\end{array}$ & - & $\begin{array}{l}\text { The mons tache } \\
\text { fell, and changed } \\
\text { its colour }\end{array}$ & - \\
\hline $\begin{array}{l}\text { Nacintye, } \\
\text { Nature, } 1896, \\
\text { No. } 1412, \\
\text { Vol. } 55, \\
\text { roth Nov. }\end{array}$ & 一 & $\begin{array}{l}\text { Worked for } \\
\text { months with } \\
\text { X-rays }\end{array}$ & Hand & Loss of hair & - \\
\hline $\begin{array}{c}\text { E. E. Kins, } \\
\text { Canalian lirac- } \\
\text { titioner. } \\
\text { Nov., Ingo, }\end{array}$ & - & $\begin{array}{l}\text { Worked for } \\
21 \mathrm{months} \\
\text { daily }(2-6 \text { hrs. }) \\
\text { with X-rays }\end{array}$ & 一 & $\begin{array}{l}\text { After } 5 \text { weeks loss } \\
\text { of hair (eyebrows, } \\
\text { heard, hand) and } \\
\text { nails }\end{array}$ & - \\
\hline $\begin{array}{c}\text { Kelle, } \\
\text { Brooklyn Ied. } \\
\text { Journal, Ingo, } \\
\text { X, i2 }\end{array}$ & - & Once to min. & Head & $\begin{array}{c}\text { Alopecia after i } 6 \\
\text { days; no dermatitis }\end{array}$ & $\begin{array}{c}\text { Hair grew } \\
\text { again } \\
\text { later }\end{array}$ \\
\hline $\begin{array}{l}\text { I. Manid, } \\
\text { Med. Record, } \\
\text { Isogo, } \\
\text { Vol. }+9,17\end{array}$ & $\begin{array}{c}1 \frac{1}{3} \\
\text { inches }\end{array}$ & Once I hour & Head & $\begin{array}{l}\text { Alopecia after } 21 \\
\text { days; nodlermatitis }\end{array}$ & - \\
\hline
\end{tabular}




\begin{tabular}{|c|c|c|c|c|c|}
\hline $\begin{array}{l}\text { Observer } \\
\text { and } \\
\text { Publication }\end{array}$ & $\begin{array}{l}\text { Dis- } \\
\text { tance of } \\
\text { T'ubes }\end{array}$ & $\begin{array}{l}\text { Duration of } \\
\text { Exposure }\end{array}$ & $\begin{array}{c}\text { Part } \\
\text { Exposed }\end{array}$ & Physiological Effect & Remarks \\
\hline $\begin{array}{l}\text { University of } \\
\text { Minnesota, } \\
\text { Med. Record, } \\
\text { 1896, Vol. 50, } \\
\text { No. } 25\end{array}$ & - & $\begin{array}{c}\text { For a consider- } \\
\text { able period }\end{array}$ & $\underset{\text { Ear a n d }}{\text { sealp }}$ & $\begin{array}{l}\text { Skin as if frozen; } \\
\text { the hair had fallen } \\
\text { out; no subjective } \\
\text { symptoms }\end{array}$ & - \\
\hline $\begin{array}{c}\text { W. B. } \\
\text { Banister, } \\
\text { Med. Record, } \\
\text { I897. Vol. } 52 \text {, } \\
\text { No. } 4\end{array}$ & - & $\begin{array}{l}\text { Numerous cx- } \\
\text { posures }\end{array}$ & $\begin{array}{l}\text { Abdomen, } \\
\text { chest and } \\
\text { neck }\end{array}$ & $\begin{array}{l}\text { Well-marked der- } \\
\text { matitis; the beard } \\
\text { became grey and } \\
\text { fell out in parts }\end{array}$ & - \\
\hline $\begin{array}{c}\text { Broca, } \\
\text { Séances de Soc. } \\
\text { fr. d. Phys., } \\
\text { I } 8 \text { th Dec., } 1896\end{array}$ & - & - & - & $\begin{array}{l}\text { Long after the ir- } \\
\text { radiation the skin } \\
\text { and hair fell off }\end{array}$ & - \\
\hline $\begin{array}{l}\text { Paul Kicher } \\
\text { and } \\
\text { Albert Londe }\end{array}$ & - & $\begin{array}{l}\text { Worked for } \\
2-3 \text { mont hs } \\
\text { daily for sev- } \\
\text { eral hours } \\
\text { with X-rays }\end{array}$ & Hand & $\begin{array}{l}\text { Dermatitis, falling } \\
\text { of the fine hairs, } \\
\text { alteration of the } \\
\text { nails }\end{array}$ & - \\
\hline $\begin{array}{c}\text { Forster, } \\
\text { Deutsche med. } \\
\text { Wochenschr., } \\
\text { I } 897, \text { No. } 5\end{array}$ & $10 \mathrm{~cm}$. & $\begin{array}{l}\text { Twice } 25 \mathrm{~min} . \\
\text { each time }\end{array}$ & $\left|\begin{array}{r}\text { Head of a } \\
\text { man at } 30\end{array}\right|$ & $\begin{array}{l}\text { After } 6 \text { days alo- } \\
\text { pecia, pigmenta- } \\
\text { tion of the hair- } \\
\text { roots, disturbed } \\
\text { sensation of the } \\
\text { temperature; no } \\
\text { dermatitis }\end{array}$ & - \\
\hline Idem. & $\begin{array}{l}8-10 \\
\mathrm{~cm} .\end{array}$ & $\begin{array}{l}\text { Once } 15 \text { min., } \\
\text { after } 7 \text { days } \\
30 \text { min. again }\end{array}$ & $\left|\begin{array}{c}\text { Head of a } \\
\text { man at to }\end{array}\right|$ & $\begin{array}{l}\text { After } 3 \text { days alo- } \\
\text { pecia, no dermatitis }\end{array}$ & - \\
\hline $\begin{array}{c}\text { J. Mies, } \\
\text { Deutsche med. } \\
\text { Wochenschr., } \\
1897 . \text { No. } 26\end{array}$ & $1 \mathrm{~cm}$. & $\begin{array}{l}\text { For I6 days } \\
\text { twice } 10 \text { min. } \\
\text { daily }\end{array}$ & $\begin{array}{c}\text { Check of a } \\
\text { girl of } 23\end{array}$ & $\begin{array}{l}\text { On the ioth day der- } \\
\text { mititis, a nd a lo- } \\
\text { pecia; at the bare } \\
\text { place no dermati- } \\
\text { tis ; disturbed sen- } \\
\text { sibility }\end{array}$ & - \\
\hline $\begin{array}{c}\text { J. Jutassy, } \\
\text { Orvosi Hetilap, } \\
\text { I897, XLI., } \\
24-25\end{array}$ & $\begin{array}{c}\text { About } \\
10-40 \\
\mathrm{~cm}\end{array}$ & $\begin{array}{l}\text { Seven sittings } \\
\text { of } 2 \text { hours } \\
\text { each }\end{array}$ & $\begin{array}{l}\text { Face, neek, } \\
\text { chest, of a } \\
\text { girl }\end{array}$ & $\begin{array}{l}\text { At the places near } \\
\text { the tube derma- } \\
\text { titis; at those very } \\
\text { far from it alopecia, } \\
\text { a'bout dermatitis }\end{array}$ & $\begin{array}{l}\text { For more } \\
\text { than } 3 \text { mos. } \\
\text { no after- } \\
\text { growth of } \\
\text { laair }\end{array}$ \\
\hline $\begin{array}{c}\text { Foneau de } \\
\text { Courmelle's, } \\
\text { Soc. de Biolog., } \\
\text { i th July, } 1897\end{array}$ & - & L & $10 \quad f \quad f$ & $\begin{array}{l}\text { Alopecia, aithout } \\
\text { inflammation }\end{array}$ & Aftor $g_{1}$ \\
\hline $\begin{array}{l}\text { Frewnd, } \\
\text { Wr. Nedic. } \\
\text { Wochenschr., } \\
1897, \text { No. } 10\end{array}$ & $15 \mathrm{~cm}$ & $\begin{array}{l}12 \text { days for } 2 \\
\text { hours each }\end{array}$ & $\mid \begin{array}{ccc}\text { Back } & \text { of } \\
\text { the lnead } \\
\text { of a } \\
\text { of } 5\end{array}$ & $\begin{array}{l}\text { Alopecia: at the } \\
\text { nape of the neck } \\
\text { slight dromatitis: } \\
\text { at the oceiput mo } \\
\text { dermatitis }\end{array}$ & $\begin{array}{l}\text { After } s_{1} \\
\text { days the } \\
\text { hairstart- } \\
\text { colgrowing } \\
\text { again }\end{array}$ \\
\hline ldem. & $15 \mathrm{~cm}$ & 42 hours & 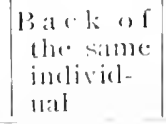 & $\begin{array}{l}\text { Alopeciat well- } \\
\text { mated dermatitis }\end{array}$ & \\
\hline
\end{tabular}


In a later communication ${ }^{1}$ ) the author, in conjunction with Schiff, explained how satisfactory results had been safely obtained in a series of cases.

The treatment is controlled by:

I. Increasing or diminishing the output of X-rays.

2. The mode of administration of the same.

As factors of importance in (I) we may note: tube.

(a) The quality of the coil, interrupter and vacuum.

(b) The electro-motive force and current strength employed.

With regard to (2) the following are of importance:

(a) The distance of the tube from the object; (b) the duration of the individual exposures.

In order to ascertain in the first place whether the patient possesses an idiosyncrasy in the shape of special susceptibility to X-rays (which happens now and then, according to some writers) trial exposures are given, as suggested by the author. These consist of one or two sittings of $\mathrm{I} 5$ minutes cach at a distance of $20 \mathrm{~cm}$., the rays being of medium strength; other authorities state this to be the smallest "dose" capable of causing dermatitis. The patient is then watched for three weeks. In the event of any evident reaction being noticeable in that time (though the authors never saw this happen) some modification in the exposure is called for. Patients who are undergoing treatment for hypertrichosis are warned against pulling out the hair (a prevailing habit with these people) so that the extent to which the exposure should be pushed may be gauged.

The intensity and quality of the rays emitted from a given tube appear to depend only upon its state of vacuum and the character of the primary current, etc., but also upon certain inherent properties of the tube which up to the present time are imperfectly understood.

') Wiener med. Wochenschr., I898, Nos. 22-24. 
The fluorescent-screen must consequently be employed as a means of estimating the intensity of the rays in a given instance.

The course of erents in the two lupus cases mentioned above was as follows ${ }^{1}$ ):

After a time the lupus nodules became dark-red and turgid; moreover, small foci of disease became visible in parts which hitherto had appeared healthy. Later on the nodules disappeared, leaving sharp-cut ulcers the size of a pin's head. The final result was as follows: The site of the nodules became occupied by reddish depressed scars, the surrounding skin being smooth and white.

Where the method was employed for the removal of hairs the best therapeutic effect was obtained by exposing for $17-30$ short sittings. Beyond an occasional slight erythema no inconvenience was felt by the patients. In several cases pigmentation was noted one or two days before the hair began to fall. This pigmentation increased for the next day or two, afterwards disappearing rapidly within three or four days. A remark. able effect was seen in the case of several brunettes who were under treatment, the hair before it fell out becoming snow-white. In one case the pigmentary change was repeated on exposing the second and third crop of hair after the initial removal. Another noteworthy effect was seen in skins disfigured by folliculitis and scars (often the result of electrolysis). These became comparatively smooth after the treatment.

In all these cases the cumulative effects of $\mathrm{X}$-rays to which Freumd and later Forster and others had called attention, were well seen.

In the same communication arguments were given in favour of Fremend's assumption that the physiological effects of irradiation are due rather to $\mathrm{X}$-rays than to electrical discharges. It was noted, for instance, that

1) This was outlined by Schiff in the publication already mentioned. 
the epilatcd surface was always circular, the circle being larger when the tube was farther from the skin. This could be at once understood, bearing in mind the coneshaped field of the $\mathrm{X}$-rays as emitted from the anticathode. The leaden masks again completely confined the physiological effects to the area of skin exposed to X-rays, while they did not altogether protect the skin underlying them from spark discharges. If, indeed, the electric discharge were the causal factor, why should physiological effects be first manifested in the deeper layers of the skin, to be followed later by surface phenomena? For in most cases the falling of the hair preceded the erythema. Finally tubes of high vacuum requiring powerful currents for their working produced. slighter physiological effects than softer tubes working with much feebler currents.

The writings of Kienböck, ${ }^{1}$ ) Strätter ${ }^{2}$ ) and $S$ cholt ${ }^{3}$ ) are of importance in the history of Roentgen-therapy. These authors insisted on the necessity of giving due consideration to the state of vacuum of the tubes employed, pointing out that "soft" tubes were more effective than "hard ones." This fact had already been referred to by the author in I 897 , at the International Medical Congress in Moscow; also in the work with Schiff above mentioned. Those works of Kienböck and the rest proved, moreover, that the author's original view, ascribing therapeutic results to X-rays rather than to electrical discharges, was correct. For the author had been in the meantime led somewhat too far by experiments with spark-discharges alone, which gave results very similar to those of X-rays and led him to somewhat underestimate the value of the latter.

It will be noted that a knowledge of the fact that different tubes possess different actinic effects, according to their state of vacuum, is of the greatest importance also in avoiding excessive reaction.

In the carlier stages of the history of Roentgen-therapy

\footnotetext{
1) Wicner Klin. Wochenschr., I000, No. 50.

2) Deutsche med. Wochenschr., igoo.

$\left.{ }^{3}\right)$ Archiv. f. Dermat. u. Syph., Bd. LIX, H. 3.
} 
efforts were chiefly made to widen the field for its employment and to perfect its technique rather than to investigate its mode of action. The last two years, howerer, have seen much work of moment in the latter direction. Not only has the question as to which is the most effective agent in radio-therapy been dealt with and brought nearer to a solution, but writings are to be recorded on histological investigation into healthy and diseased tissues which have been under treatment, on bacteriological investigations, and experiments with protozoa and other low forms of life, all helping to a better understanding of the biological effect of Roentgen-radiation. In this connection reference must be made to the important works of Gassman, ${ }^{1}$ ) Grouren, Ehrmann,"2) Scholtz,") Joseph and Provazck, ${ }^{4}$ ) and others.

\section{§ 30. Method of Treatment with X-Rays.}

The main factors which have to be considered in X-ray therapy are:

I. The strength of the primary current.

2. The capacity of the coil.

3. The intensity of the rays and the state of vacuum of the tubes.

4. The duration and frequency of the sittings.

5. The distance of the tube from the exposed region.

6. The susceptibility of the tissue exposed.

In the first place it is essential that the primary current be not of too high intensity. It must be remembered that Roentgen-rays represent a condition of transformed electrical energy. The greater the amount of electrical energy supplied, therefore, the more powerful will be the output of the rays; moreover, the electrical discharges from the tube will be correspondingly intensified.

The beginner should never work with currents above $1:-3$ ampères, and eren the expert should employ the greatest caution

\footnotetext{
1) Fortsclir. auf d. (ichiete d. Roentgenstr., Bd. II, II. 4.

z) Wiener meel. Woch., roor, Nos. 30, 31.

3) Arch. f. I) Imal. 11. Syph., Bil. I.I. . II. 3, 1. 424.

4) Zeit-chr. f. allg. I'hysiologic, Bd. 1, 11. 2, 1902.
} 
if compelled to work with more intense currents, say up to 6 ampères.

The second condition-the capacity of the coil-must be considered in connection with the preceding one. Currents only should be chosen which give but short spark-lengths at the coil. If only a large coil is at one's disposal it should never be worked at its full capacity; if necessary, resistances should be used with the primary current, bringing down the spark-length at the coil to $\mathrm{I} 5 \mathrm{~cm}$. Coils whose maximum spark-length capacity is 30 $\mathrm{cm}$. are the most suitable for X-ray therapy.

As has been mentioned before, soft tubes are therapeutically more effective than hard ones, other things being equal. If, however, a hard tube be worked with a more powerful current, it may give as rich a supply of $\mathrm{X}$-rays as a soft tube worked with a weaker current.

The author arrived at this opinion as follows:

An elderly neurotic woman had been treated for hypertrichosis intermittently for about a year, using hard tubes and a Kohl's coil $(30 \mathrm{~cm}$.) without a trace of dermatitis, the hair falling regularly after 2 I to 30 sittings. The patient insisted upon the treatment being accelerated, and irradiation was then combined with applications of uni-polar discharges from the coil. 'After I I sittings of I 5 to 20 minutes each, a somewhat intense dermatitis (without ulceration) arose, which it required some weeks to subdue. Some months later treatment was resumed of the skin, which still remained hairy. Two or three brief courses of treatment with hard tubes, a course consisting of Io or I 2 sittings of I 5 minutes each, again produced temporarily a perfectly smooth skin without any ill effects. The patient, nevertheless, clamoured for a stronger reaction, having noticed that the skin remained permanently smooth at the site of the former inflammation. Only one other apparatus was at the disposal of the operator, and this had clearly to be employed with great caution on account of the high intensity of its secondary currents. The hardest tube was therefore used with this appa- 
ratus, a brilliant fluorescence being the result. Nine sittings were given, each lasting 7 to Io minutes, and the result was a violent dermatitis which produced an ulcer the size of a kroner piece.

From the above ${ }^{1}$ ) it may be deduced that a hard tube which, working with weaker currents, may safely produce useful results, gives altogether different effects if worked with a powerful coil and stronger currents.

Nevertheless, where only one apparatus is available, soft tubes will be found more generally useful (worked with due precaution) than hard ones.

The tubes by Gundelach and F. Dessaner, and Iirschmann, in which the vacuum can be regulated, have already been described (p. 212-214).

By employing 3 sets of apparatus, Béclire") professes to be able to produce rays which penetrate the tissues to any required depth. The instruments used are: (1) the "amponle a osmo-régulatem" of rillard, which is said to prolong the life of the tube indefinitely, and to allow one to control its penetrating capacity; (2) Béclire's "Spintemeter." This apparatus affords indirect evidence of the penetrating capacity of the rays through the character of the spark-discharge at the terminals of the coil. It has already been mentioned that this sparking bears direct relation to the resistance offered in the sccondary circuit. With hard tubes the spark is long, being short with soft tubes. The apparatus is nothing more than the graduated spark-gap which has been supplied for many years by certain German manufacturers, and its value would appear to be only relative. (3) The radiochromometer of b'moist. This indicates the strength of the $\mathrm{X}$-rays. It consists of a thin silver plate having a broad aluminium border. The latter is divided into I 2

1) Apropos of hard tubes the following case may be cited: Bernard and Ruotte (Fozeau de Conmelles), L'anme dectrique, Revme de roor, p. 350, radiated a young man for 35 uninules will a sery hatrd tule placed $15 \mathrm{~cm}$. from the skin. The prinary currens was one of 15 volts and 4 amperes, and a $25 \mathrm{~cm}$. coil was used. An aluminum plate atrthed through the gas pipes was fixed between lhe tube and llo slin. Afler fiftern days a dermatilis developer, which was not properly healed after len montlis.

$\left.{ }^{2}\right)$ Journal des malarl. cut, el sypll, rooz, lleft 3. 
sections, of graduated thickness. By means of the fluorescent screen the particular aluminium section is noted, whose shadow equals the density of shadow cast by the silver plate, and thus some measure of the intensity of the rays is arrived at. The apparatus, however, is by no means infallible, since the penetrating power of X-rays depends not only upon the thickness, but upon the density of the body traversed. The first and third of the author's experiments quoted above show plainly that the effects of irradiation depend very largely upon its duration. While comparatively brief exposures give good results, those which are too prolonged must be regarded as highly dangerous.

What is understood by too long a duration of treatment?

An absolute duration of exposure must be clearly distinguished from a relative one-that is, the duration of the individual sittings. The effect of one sitting of a definite duration is not the same as the effect of a series of sittings whose total sum covers the same length of time. A single exposure below a certain limit has no effect. The total length of exposure necessary to produce a certain effect varies accordingly as one gives a single or several sittings.

If, for example, a patient be irradiated for 50 minutes without interruption, the effect on the skin is much more marked than if the exposure be made for Io minutes on five succeeding days. 'The fact must be especially borne in mind when working with powerful apparatus, that is to say, strong currents and tubes of high capacity. If the treatment is to be completed in one or very few sittings, powerful currents and tubes being to hand, it must be remembered that a long exposure is a most powerful agent, and that an "over-exposure" of but a few minutes only will have an important effect on the final result.

In such cases, therefore, the utmost caution is necessary, and the need for this is intensified by the fact that the full effects are not seen in different individuals in precisely the same time, although the irradiation has been identical in each case. The energy of Roentgen-radiation varies inversely, as is the case with light, with the square of the distance of the ray-source. A photographic plate, whose sensitive surface is weakly acted upon at a certain distance shows at half that distance an effect which is 
four times that of the former. This fact must be carefully borne in mind when arranging the position of the tube, for a difference of 1 or 2 centimetres only, when considered in connestion with other factors (current strength, state of racum of the tube, relative time of exposure, etc.), maly be of some importance.

A proper estimation of the inlensily of the X-rays employed is of the highest importance. Difterent methods hare been advised to this end, but mostly they leare something to be desired in the way of precision. The author relies most on the character of the fluorescence as seen in the tube. In soft tubes the fluorescence is almost yellow and very copious, while in hard tubes it is greenish, transparent, and watery-looking. The amount of fluorescence atiords a fairly good means of estimating the ray-intensity. The rate of interruption of the primary current is a factor not to be overlooked, since it plays an important part in the production of physiological effects. One school of writers believes that greater effect may be had from rapid interruptions, another pins its faith oin slower. L pholders of the first doctrine argue that by doubling the rate of interruption in a given time the number of X-ray "waves" striking the skin is likewise doubled. Whereas the others hold that the higher current-tension obtained by interrupting more slowly is of adrantage ${ }^{1}$ )

The truth probably lies half-way. ITp to a certain limit (40-50 interruptions per second) acceleration of the rate of interruption is attended by an increase of physiological effect. Beyond this, however, $i$. ', with still more rapid interruptions, the effect is not proportionately increased. Indeed, the author has found that with a turbine apparatus working at roo interruptions per second, he could obtain a certain effect with fewer sittings than when using a $/ V^{\prime}$ 'hmell's apparatus giving 1700 interruptions per second.

It is of the greatest importance to bear in mind the fact that the tissues of different individuals do not possess the same sensitiveness to X-rays. Many authors, it is true, deny this, though

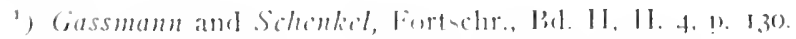


it would appear that even they have met with differences in the mode of reaction of different persons ${ }^{1}$ ).

Other writers attribute a special susceptibility to reaction to certain parts of the body, moreover, stating that the colour of the skin and hair indicates susceptibility in some people. Thus Jutassy says that blondes are especially sensitive. Scholtz has observed that dermatitis is more apt to arise without warning on the scalp and beard than in other regions. Both Bisserie's and the author's cxperience is in accord with this, and the latter has further observed that reaction is more readily induced in tissues immediately overlying bone.

Jutassy, Benedikt, and Kicuböck noticed that children and persons who are ill-nourished are more sensitive to the rays, ${ }^{2}$ ) also that mucous membranes, the face and the back of the hands, react more readily than the face and the trunk. The author has not been able to verify the statement made by $\mathrm{Hal}_{\mathrm{n}} \mathrm{n}$ and Albers-Schönberg to the effect that women who wear veils have tenderer skins and are consequently more susceptible to X-rays. He has observed, ${ }^{3}$ ) however, that skins affected with sycosis or farus, being already somewhat inflamed, are soon influenced by the rays. This has been confirmed by others (Schiff, Kicuböck, Schollz). Kienlöck denies the possibility of some people possessing an idiosyncrasy which renders them prone to excessive reaction. He maintains that in every case the effect depends simply upon the amount of irradiation.

The whole subject of Roentgen-therapy is still a vexed one. There are those who would deny its raison d'étre, doubting if it is really capable of producing physiological effects ${ }^{4}$ ); others who while admitting these effects are of opinion that they sig-

1) These writers do not say: "The reaction appears after irradiations," lust rather. "To ohtain a certain effect n-m sittings must be given." If the morle of reaction were identical in all eases the effects procinced by a given number of exposures of the same intensity would always arise at the same time. This is loy mo means the case.

$\left.{ }^{2}\right)$ Mathu and Albers-Schönbers, on the other hand, belicve children to be more resistant ( Wïnchen. med. Woch. I901, 9-11).

$\left.{ }^{3}\right)$ Wiener ned. Presse, isog. No. 31.

4) Borgmann, Vers. der Naturforscher und Aerzte in München, i899. 
nify dangerous damage to the body ${ }^{1}$ ). On the other hand, those who are agreed as to the value of the method are by no means unanimous with regard to the details of its technique. Much controversy still obtains on the question of the right kind of racuum-tube.

In consideration of the fact that soft tubes have a more powerful effect than hard ones Kienböck adrises the following procedure $^{2}$ ): An adjustable Roentgen-tube whose vacuum has

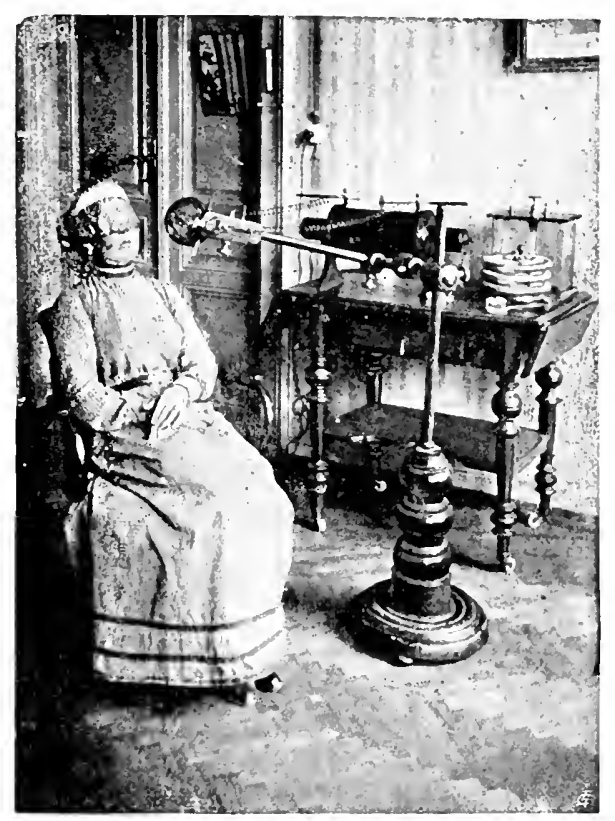

FIG. 75.

been brought to the "soft" or "medium-soft" stage is worked by means of a primary current of $3-6$ amperes, the interruptions being secured through a turbine apparatus at the rate of 15-20 per second. The tube must give rays capable of penetrating the thorax of an adult at a distance of $1-2 \mathrm{~m}$. This tube is fixed about $20 \mathrm{~cm}$. from the skin, and from 3 to 5 sittings of 10 to 15 minutes each are given on succeeling days. The sittings are

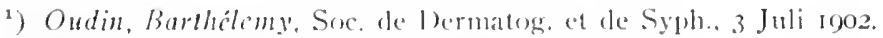

2) Wiener kilin. Hoch., 1900. p. 1103. 
then suspended for two or three weeks so that the reaction may develop. After all reaction has subsided the sittings are resumed as before.

The author does not approve of this method, since by its adoption one hardly knows if the exposures have been pushed too far or too little. Now Kaposi ${ }^{1}$ ) has rightly remarked that one of the chief drawbacks to Roentgen-therapy lies in this, that one is working more or less in the dark and is ignorant of what has been really done.

Amongst a series of cases which were for purposes of comparison treated exactly on Kienböck's lines the author obtained good results in two cases of sycosis; in a case of hypertrichosis again results were negative - the exposure had been insufficient; while in a case of favus a somewhat violent inflammation set in which required several weeks to heal.

The method indicated in I $\$ 9 \$$ by Schiff and the author, which the latter has since employed with a few modifications, ${ }^{2}$ ) is as follows: A Ruhmkorff's coil $\left(30 \mathrm{~cm} .{ }^{*}\right)$ ) is worked either from the main or from a 6-cell accumulator, the current being between $I \stackrel{1}{2}$ and 2 ampères, and the interruption averaging $\mathrm{I} 6$ per second. The tube is hard, yet one giving a good greenish flare.

The tube must be so placed that the most intense rays proceeding from the anti-cathode strike the centre of the region to be exposed (Fig. 75). It has been shown elsewhere (p. 224) how the region of most intense radiation may be discovered.

The distance of the tube from the skin is at first $\mathrm{I} 5 \mathrm{~cm}$., but this is afterwards gradually decreased to $5 \mathrm{~cm}$. In like manner the sittings are gradually prolonged from 5 to 10 minutes, and the part is irradiated until signs of reaction occur, when operations are suspended. The author has found the following early

1) K. k. Gesellsch. d. Aerzte, 27 Oct. 1899.

${ }^{2}$ ) Nany other workers have found this method successful, c. g., Goorg J. Miller, Grouz'cn, Mazas, Pus'y, Zcchmeister, Merk, Török, Schein, Gastou, l'irira, Hall-Eda'ards, Boczar, etc.

${ }^{3}$ ) Coils by Kiser \& Schmid, Kohl, Sicmens \& Halske, Riniger, Gebbert \&o Schall and Dessaucr were used. 
signs of reaction in by far the greater number of his cases treated as above described ${ }^{1}$ ) :

I. Intumescence of the skin.

2. Pigmentary changes.

3. Erythema.

4. Loosening of the hair.

5. Subjective symptoms.

The intumescence is an early sign which is nearly always to be observed. A cheek, for example, which is being treated for hypertrichosis, appears after about three weeks fuller and rounder than the other; the skin appears tenser, a little oilier and perhaps more shining. At this stage no alteration in the colour of the skin can usually be observed. Indeed, at first the changes are not very striking and they may readily escape the notice of an inexperienced operator. They are, however, noticed by the patients themselves, who usually scrutinize their skins most assiduously; they come, therefore, to the practitioner saying that the treatment does them a lot of good, and that they "look much better than before." In cases where the sittings are prolonged for some time the intumescence is, of course, longer maintained, whereby scars and irregularities in the skinsurface are temporarily levelled, and the general appearance of the integument improved. The intumescence disappears after the sittings are suspended, but often a more lasting cosmetic effect is gained through the resulting desquamation. It must be noted that the intumescence is never sharply circumscribed, but rather diffuse,-its border cannot be exactly defined. Finally, the sense of touch is not so useful in discovering its presence as that of sight. The alterations noticcable by palpitation of an irradiated skin are almost imperceptible.

The question naturally arises, To what anatomical changes is this clinical effect due?

Behrend ${ }^{2}$ ) assumed that the tissues become permeated with a serous exudation which not only affects the parts containing blood-vessels, but also the decper layers of the epidermis, which

$\left.{ }^{2}\right)$ Wiener med. Presse, 18 on, No. 3r k. k. Ges. d. Aerzte in IVien, 27 Oct. 1899. Congr. d. deutsche dermalohg. Gesellsch., Breshau, 1001.

2) Berliner klin. Wochenschr., isos, No. 23. 
becomes so soaked that the lymph-spaces between the elements of the stratum spinosum are distended. As a result the individual cell-elements are forced apart, and in the case of a violent exudation even larger spaces may be formed, filled with serous fluid and overarched by the less yielding horny layer of the epidermis, thus giving rise clinically to a vesicle.

The author is not aware how far this theory of Behrend's depends upon histological investigation. It will be seen later, however, that Scholiz has verificd the existence of this serous exudation into the tissues by experimenting with animals.

Roentgen-radiation often causes singular pigment changes in the skin, and these may even be the first sign of commencing reaction. They may be characterised either by the appearance of freckle-like spots on the skin, or the whole exposed area may assume a diffuse, slightly yellow, brown, or greyish tint which is in most cases ephemeral. Again, pre-existing freckles or other pigmentary abnormalities may become darker, or they may in some cases become somewhat lighter in hue. It must be noted that these pigment changes very often make their appearance without erythema having preceded them; they are evidently not always dependent on the latter phenomenon.

A discolouration of the hair, especially in the case of brunettes, has been repeatedly observed as the first sign of reaction. Sehrwald, ${ }^{1}$ ) Gocht, ${ }^{2}$ ) Gassmann and Schenkel ${ }^{3}$ ) have seen depigmentation of the exposed surface with increased pigmentation around its border. The author has himself seen this as a later effect of Roentgen-radiation, notably in a case of lupus erythematosus treated by $S_{c h i f f}{ }^{4}$ ).

In this case the pigment scemed as though it had been displaced from the white area marking the site of the disease into its vicinity (Schiff and Freund, Beiträge zur Radiotheraphie, Festschr. f. Hofrath, Prof. Neumann, page So6. The author again saw a case

\footnotetext{
$\left.{ }^{1}\right)$ Deutsche med. Woch., I901, No. 30.

$\left.{ }^{2}\right)$ Fortschr., Bd. I, H. I.

$\left.{ }^{3}\right)$ Ibid. Bd. II, H. 4 .

4) Ibid. Bd. II, H. 4 .
} 
recently, that of a young lady, a brunette, who had been treated for hypertrichosis. Dermatitis here had been followed by the appearance of pale patches in the skin surrounded by dark brown rings of pigment. One recalls the fact that freckles are particularly apt to appear on blondes, and melanoses on white animals. Ph. J. Pick, who calls attention to this fact in an able article (Vierteljahrschr. f. Derm. u. Syph., I $\delta_{4}$, page 24), is of opinion that the pigment defect in the hair of animals with melanosis accounts for its excessive deposit in other parts.

The author concluded from his researches on the physiological effects of electric discharges on the skin that pigmentary accumulations arise from hæmosiderin, which in its turn is the result of hæmorrhage into the tissues.

S. Ehrmann ${ }^{1}$ ) gave the following explanation of the pigment-phenomena, based on microscopic examination: By the direct influence of irradiation on the capillaries, the walls of which become adapted for diapedesis (not hæmorrhage), hyperæmia is produced. At the same time there is a copious exudation of blood-plasma in which hemoglobin is dissolved. This explains the yellowish tinge of the hyperæmic skin. Very soon gold-coloured hæmosiderin is deposited from the colouring matter of the blood in the tissue interstices. The hæmosiderin is absorbed after a few weeks, until which time the skin retains its yellowish-brown tint. When, however, the sun's rays, or the $\mathrm{X}$-rays, encounter a skin possessing melanoblasts (cells producing melanotic pigment, which is not formed within the cell-spaces, and is chemically different, moreover, from hæmosiderin) melanin is more copiously secreted as the result of the irritation of these cells. Hence the sepia-brown discoloration, which may persist a twelve-month.

The skin of fair-haired people as a rule contains fewer melanoblasts; consequently they usually escape this excessive brown pigmentation.

1) Wiener med. Woch., 1901, Nos. 30, 31. 
L. Török and M. Schein ${ }^{1}$ ) have observed an abundant development of closely packed comedones after irradiation of the face.

The erythema which is so often seen as an early sign of Roentgen-reaction differs in character from most erythemata. It differs, for instance, from the erythema resulting from the internal or external use of certain medicaments (erythema venenatum); it has rather the character of an erythema solare. The skin looks as though it had been much exposed to the sun, or as though the patient had been amongst the glaciers. The erythema is at first of a delicate light colour, later on a reddishbrown tint with a slight bluish cast develops. This darkening of colour is probably due on one hand to the combination of erythema with pigmentation, on the other to the fact that the hyperæmia is "passive." This erythema of the skin may be either diffuse or patchy.

In a great number of cases loosening of the hair-shafts is the very first sign of reaction. It is quite wrong to suppose, as many have done, that this effect can only follow after other inflammatory phenomena have been established. A good method to discover this loosening of the hair is to seize two or three shafts between the finger-tips close to the skin, then, exerting slight pressure, to slide the fingers towards the free ends of the hairs. Normal hairs are not affected by this manœuvre, whereas loosened hairs readily fall out. To pull forcibly at one part of the hair, either with the fingers or with a forceps, is a crude and much less reliable method; it is, moreover, one which is quite useless for determining the earlier and slighter alterations in the fixity of the hair.

In order that a reaction may be recognised early it is most important that any patient who is undergoing treatment for a hair affection does not shave or otherwise cut the hair short; in cases where the hair has been recently cut operations should be deferred until it has reached a certain length. Difficulties in this direction will often be encountered in the case of women who are being treated for hypertrichosis, these patients being

1) Wiener med. Woch., 1902, No. I8 ff. 
accustomed to preserve appearances by the use of depilatories or the epilation-forceps. Nevertheless, the rule must be strictly enforced, for it is impossible to tell when closely cropped hairs are really loose, the hair-roots, lacking friction on their shafts, will otherwise remain for a long time in situ, and may be only removed in the end by the lifting force of fresh-growing hairs. Under the latter circumstances the falling of the hair could give no indication whatever for the suspension of treatment.

Subjective symptoms are often the forerunners of a reaction. They include itching, burning, and a feeling of tension, and are apt to be more marked by night. It is, however, in but rare instances that patients will on their own initiative mention these sensations; they regard them usually as insignificant. It is important, therefore, that they be informed of the great importance of mentioning these symptoms as soon as they occur. Sometimes neurotic patients who are under treatment, especially in the later stages, will suffer from an attack of intense pruritus of the exposed region often accompanied by urticaria. Should this happen, operations must be suspended. The above-mentioned early signs of reaction are far from intense if the treatment is properly conducted, indeed, they often escape notice by begimners. They afford, however, a most valuable index to the more expert to the progress of erents, and enable him in time to complete the treatment with safety to his patient. Refcrence may here be made to the fact that suspension of the sittings at this comparatively carly stage almost always meets with opposition from the patient. I le often begins treatment with somewhat prejudiced feclings, inasmuch as he sees nothing of what is going on, on account of his mask. Consequently he objects to resting at a time when he "hardly feels anything," and protests that he has been treated insufficiently. Ilysterical women who are being treated for hypertrichosis are particularly troublesome in this respect, especially if dering an earlier treatment they have been relieved from their hairy troubles. On no account, however, will the operator who has a proper conception of his responsibilitics give way to these importunities.

Both the methods which have been explained in full above have this in common: The object is to produce a certain effect 
by means of a "normal" intensity of irradiation and period of exposure. It is true that this "normal" has been empirically determined, but it cannot be exceeded without danger. One can obtain the same final result with hard or soft tubes, weak or powerful rays, varying distance of the tube, etc., provided the "dose" be regulated accordingly. In the first method the "normal" is attained in a few sittings compared with the second; the final effect is the same in both. Whereas, however, the effect upon the skin in a single irradiation by the second method is very feeble, in the first method it is fairly strong, owing to the employment of more powerful tubes. Later experience has confirmed the author's earlier view, that by employing the first method the operator is using a strong remedy, the effects of which he may be unable to control; on the other hand, taking into consideration the different reacting power of individuals, there is the possibility of him administering a "normal" irradiation which the subsequen four-weeks resting time may prove to have been insufficient.

An experiment by Oudin and Barthélemy ${ }^{\mathbf{1}}$ ) furnished an interesting proof of this assertion. They exposed successively 12 patients under identical conditions of exposure-period, etc., to a tube whose vacuum could not be regulated. At the beginning of the experiment the tube was very soft, towards the end it became very hard. The first patient, who had been exposed to the "oversoft" tube showed no reaction whatever. Succeeding patients, as the tube became altered in vacuum, showed erythema, phlyctenulæ, bullæ, loss of hair, and finally severe dermatitis. The eleventh patient (the tube being now very hard) showed complete loss of hair without other signs. In the twelfth patient there were no signs whatever.

The author has already expressed the opinion ${ }^{2}$ ) that the Roentgen treatment will only become popular amongst medical

1) II Congr. intern. d'électrologie et de radiologie, Sept. I, I902.

2) The same opinion was expressed by the author at the VII Congress of the Deutsche dermatolog. Gesellschaft. Breslau, I90I, before a large company of experts. 
men when it is perfected to such an extent that by definite "doses" it produces definite effects whose presence informs the operator when he has treated the patient sufficiently and within the limits of safety. It is probably for this reason that upholders of the "first method" of treatment have endeavoured to so modify it that it may meet the requirements, thereby bringing both methods more into line.

Thus Kienböck,") who at first called our method "complicated, confusing, unnecessary, and tedious," has recently recommended, in addition to his original method, two more methods in which the total exposure is distributed over several sittings of less intensity. By these Kieniö̈k"2) means daily exposures of 5 minutes ("irradiation of lesser efficiency"), or longer sittings twice a week ("medium strength irradiations," which produce their effect in about a fortnight).

Scholt $\approx^{3}$ ) begins with a comparatively strong exposure in order to soon bring about reaction. At the first sign of this he promptly reduces the intensity of the irradiations; these are continued in mild doses until the desired effect begins to show itself. This plan is also adopted by Török and Schein, and the author has frequently used it with marked success.

$V$. Lion ${ }^{4}$ ) uses medium-soft tubes, a coil with spark-length of $30-40 \mathrm{~cm}$., current strength of 3-4 amperres, 30 volts tension, and I $500-1800$ interruptions per minute. In the carlier sittings the tube distance is $30-50 \mathrm{~cm}$, and the time of exposure 5-10 minutes. Later on the distance is reduced to $5-10 \mathrm{~cm}$., and the exposure increased to 30 minutes.

Gastou, Vicira and Nicolon ") use hard tubes corresponding to a $15 \mathrm{~cm}$. spark-length and to No. 6 of Bennoit's radiochrometer. The primary current has an intensity of 5 amperes and a 25 volt tension. Tube-distance, $15-30 \mathrm{~cm}$. Duration of exposures, I m mutes every other day. Under this condition the

1) 73. Versamml. d. Naturf. u. Aerzte in Iamburg, Forticlur. a. d. Geb. d. R., Vol. V. Part I, p. 34.

2) Fortsclir. a. d. G(d). d. Roentgenutr., Vol. V', Part I, p. 34.

3) Archir. f. Derm, and Syph., Vol. LIX, Part III, 1. 424.

*) Vil Cong. d. deutsche derm. Geellech. in Preslau, I901,

b) Soc. de dermatologic et de Syphilis, July 3, 1902. 
hair begins to fall after 6-1 I sittings. "Signs of inflammation were not observed."

Oudin $^{1}$ ) gives very brief exposures, using a soft tube which is brought within $5 \mathrm{~cm}$. of the skin. He exposes at first for $\frac{1}{2}$ minute only, gradually increasing the time to 5 minutes. He continues this treatment until signs of reddening show themselies and itching is felt.

Before closing this chapter the author must once more state his opinion that in the patient's interests a plan which, while involving more time, is yet harmless, and achieves definite results by gentler means, is far preferable to one which steers straight for its goal with great energy. The latter plan is undeniakly briefer, but may prove either too vigorous, in which case painful complications are in store for the patient, or insufficient, whereby much time is lost. The first plan, though more tedious, is far safer, albeit much practice and experience are required for its successful adoption. In fact, this younger branch of medical science may fairly be considered as an art.

For the rest, especially in view of the varying capacities of different apparatus, the beginner would do well to proceed with extra caution. He should first test his apparatus, finding out the correct exposure by first commencing with very short sittings (say 4 minutes). Should results prove negative after three weeks, the patient should be given 3 weeks' rest. After that the sittings can be resumed, the exposure being gradually increased to $5,6,7,9$ minutes and so on until the desired limit is reached. The current strength and tension, the tube distance, etc., must be as already indicated; hard tubes may be chosen if they show evident fluorescence (see p. 247). When soft tubes are used the total exposures must not exceed 40 minutes. When once the time of exposure necessary to loosen the hairs with each series of sittings has been ascertained, one has gained valuable data for the treatment of various affections, which may now be undertaken with greater confidence. Hence, one may proceed a little more boldly, and, by modifyng the treatment in accordance with the instructions already given, hasten the cure. The best and

1) Soc, de dermatologie et de Sypliilis, July 3, 1902. 
safest plan is for the beginner to start with the treatment of a disease like sycosis. It is only after considerable experience he should undertake cases of hypertrichosis, the after appearance of the skin being here especially a matter of the greatest moment.

Since it occasionally happens that a single irradiation, even one which is by no means intense, may cause dermatitis, a brief trial-exposure should be given in every fresh case, ${ }^{1}$ ) so that due allowance may be made for the special susceptibility of any particular case. Bearing in mind the law that the intensity of an irradiation varies inversely as the square of the distance between the ray-source and the object, while the size of the area irradiaated increases with the square of the distance, it will be necessary to increase the distance between the tube and the patient where large surfaces are under treatment; at the same time the exposure must be correspondingly lengthened. One must remember that at the skin-surface which is nearest to the tube-wall (the part corresponding with the most brightly illuminated portion of the fluorescence screen) the effects are most pronounced. When the whole face is under treatment the cheeks should be exposed in turn and then the chin; one should not forget, however, that by so doing, some regions may be twice cxposed.

The question of screening the healthy skin from the action of the rays must now be considered.

$U n n a^{2}$ ) recommended a zinc paste with the admixture of a body arresting the passage of $\mathrm{X}$-rays, such as bismuth; Scholtz ${ }^{3}$ ) tried a mercury plaster; Kaiscr ${ }^{*}$ ) tried a funnelshaped piece of lead sheeting, intending by this means to concentrate the rays on the skin (forgetting, however, that they are not capable of refraction or reffection). Schïrmayer ${ }^{5}$ ) recommended leaden screens which were "carthed" through a conducting wire. The author has found leaden masks the most effective. In place of the stiff, heavy and comparatively ex-

1) Albers-Schönbere (Fortschr. II, 4. 181) and Scholls (1. c.) advise this.

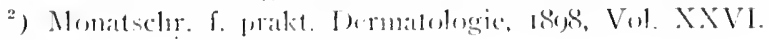

3) 1. c.

1) Wiener kin. Woell. inol, No, 31.

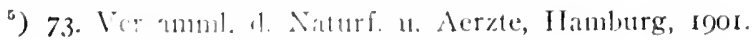


pensive masks made by the plumber for this purpose, he now uses those which are made as follows:

A piece is cut from a leaden sheet $\frac{1}{2} \mathrm{~mm}$. thick (Fig. 76). The measurements are $a d=42 \mathrm{~cm} ., a e=8 \mathrm{~cm}$., $\mathrm{kg}=16 \mathrm{~cm}$., $a b=e d=9 \mathrm{~cm} ., b f=c h=I 2 \mathrm{~cm}$. The sheet has "billrothbattist" sewn to one side; at $m$ and $u$ two slits perforate it; through each of these a ribbon passes which is sewn to the lining. The mask is now ready for the upper part of the face. In case this mask has to be worn by several patients $(e . g$. , in outpatient practice) they should be instructed to each bring a handkerchief to be placed beneath the mask. The mask may be roughly shaped to fit the face. Apertures should be cut in the mask corresponding to the diseased area. All parts of the body coming within the X-ray field which not do require exposure

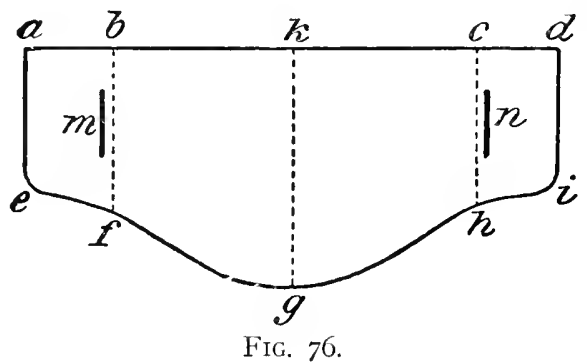

must be carefully protected from the rays by means of masks. Thus, if the chin is under treatment, not only the upper part of the face, but also the chest, requires protection, and where the angle of the jaw is the site of operations, the shoulder must be screened.

In many cases it is a good plan to place the vacuum-tube within a lead-lined box, having an aperture at one side corresponding to the diseased area.

As a special protective measure, Kaiser ${ }^{1}$ ) recommends the use of vacuum tubes made of red glass; Jutassy ${ }^{2}$ ) again says he has never seen any bad effects accompanying the use of blue lead-oxide tubes.

1) Wiener klin. Woch., I90I, No. 3I.

${ }^{2}$ ) Fortschr., Vol. II, Part V, p. 195. 
The operator's own safety must not be forgotten. One has known zealous radiographers who have, after some hours' use of the Huorescent screen, dereloped severe dermatitis. It is probably cases like this which have led to excessive precautions being taken by some of those who busy themselves with X-ray therapy. The author has known a conscientious operator who only worked with an armour of lead plate, a leaden helmet on his head; his appearance was scarcely calculated to soothe the patient.

Undoubtedly the wamings and precautions have been exaggerated. The operator should remember that the tube only aftects changes on a skin which is for some time exposed to its field of action. He will aroid placing his own skin under these conditions. A brief manipulation of the apparatus, a brief examination of the mask, a change in the patient's position, etc., can be made repeatedly each day without affecting onc's health in the least. For the last 6 years the author has been working daily from 6 to 7 hours in his Roentgen laboratory without ill effects; nor has he worn protective appliances of any kind to hinder him.

\section{\$ 3I. Indications.}

Ve now come to the indications for the employment of Roentgen-therapy. Physiological experiments shew that Roentgen-radiation, like that with light and electricity, has a stimulating effect when administered in moderate amount, but a destructive action on certain tissue-clements (cells) when employed with greater intensity. We may assume that when acting beneficially the rays modify the process of nutrition in badly-healing ulcerative conditions and in paretic states of certain tissues, thereby promoting the healing of the former and the resumption of function in the latter ( $e . . .$, the hair papilla in alopecia areata). It would appear by no means unlikely that the irritant effect of the irradiation, like that of certain chemical agents (silver nitrate, copper sulphate, camphor, etc.), stimu- 
lates the function of the connective-tissue cells, thereby promoting scar-formation. Since this stimulating effect penetrates more deeply, it would appear applicable in cases where chemical agents, by reason of their more superficial action, are useless.

The X-ray method is especially applicable for certain pathological conditions in which the lesions are widely spread; for others which are more circumscribed surgical treatment, treatment by drugs, or the "light-method," are preferable.

In by far the greater number of cases the destructive action of X-rays is what is called for; this is especially so in affections which have their seat in cellular structures, such as the epidermis, the glands, and the hair follicles, or in which the essential feature is an orer-production of cellular elements, such as psoriasis and epithelioma.

Clearly, however, both the destructive and restorative properties of Roentgen-radiation can often find their metier in one and the same disease; thus the healing of a lupus may be due as much to the stimulating effects of the rays, whereby hyperæmia and improved nutrition are afforded and scarformation assisted, as to the destructive action upon the diseased tissue.

The following groups of diseases suitable for X-ray treatment may be classified from a purely empirical and histological standpoint :

I. The so-called diseases of the hairs (including those of the hairy skin).

In this group we may include all those abnormal conditions for the cure of which epilation is the first desideratum, whether or not other effects of irradiation are called for (favus, sycosis, folliculitis barbæ, blepharitis, dermatitis papillaris capillitii, trichotylosis, hypertrichosis, alopecia areata).

2. Ulceration processes in the skin.

In this group we include not only simple, non-infected ulcerations, in which irradiation acts favourably by reason of its stimulating effects, but also those skin affections due to direct infection which so often end in ulceration. Here we utilise the destructive action of the rays upon the morbid tissues and so prevent further infection of surrounding tissues (lupus vulgaris, 
scrofulodermia, tuberculosis cutis, epithelioma, ulcus rodens, lepra, mycosis fungoides, atonic and varicose ulcers, etc.).

3. Acute and chronic exudative dermatitis (inflammations) and granuation-formations. The explanation of the good effects obtained in discases of this class is still obscure; perhaps it is to be found in the destructive action of the rays upon inflammatory products. Grone'en and Scholls hare shewn that this is so in the case of psoriasis and lupus.

It would seem not unlikely that the high-tension electricity already referred to (p. I62), as streaming from the tube, plays a considerable part.

This group includes eczema, psoriasis, prurigo, lichen ruber, pemphigus, lupus crythematosus, acne rulgaris and rosacea, furunculosis.

4. Diseases which owe their origin to morbid changes in the blood-ressels.

The histological changes in the blood-ressels which can be proved to follow irradiation explain the good results obtained with this class of discase (nærus flammeus).

5. Progressive disturbances of nutrition in the skin (rerrucæ, næri, spili, elephantiasis).

1. Diseases of the Mair and Mairy Regions.

\section{Favus capillitii.}

Complete epilation of the whole scalp is in essential feature in the Roentegen treatment of this affection. If the scalp be only partially denuded of hair, recrudescence of the disease is practically certain, the fungus growing again from the hairy foci where it has been left. In order that the whole scalp may be exposed the tube should be first placed orer the middle of the frontal region, next opposite the sides of the head (with the anti-cathode opposite the middle point of a line between the ear and vertex), and finally opposite the centre of the occiput. The tube should be plated at some distance $(25-30 \mathrm{~cm}$.$) , so$ that as larege a surface as possible may be irradiated, and each exposure should be from 6 to 10 minutes. 
As a rule, a somewhat intense irradiation is necessary for the cure of favus; this is probably due to the thick hairy coating of the diseased parts. (Hair does not materially prevent the penetration of X-rays; it is, however, a very bad conductor of electricity.) Before commencing operations, the hair should be cut to about the length of $2 \mathrm{~cm}$.

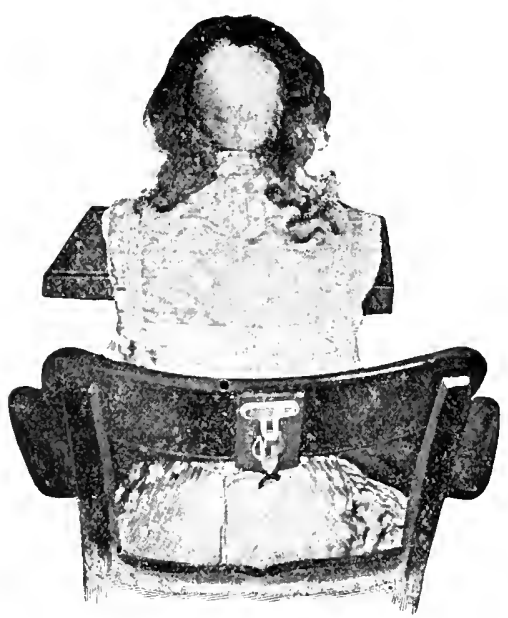

Fig. 77.-Favus capillitii. Occiput, treated with X-Rays.

The usual course of events is, that after about 3 weeks (when hard tubes and weak currents have been used), the hair is found to be loosened on grasping it, or, on parting the hair, the skin is seen to be slightly reddened. Within the next 2 weeks the hair falls out in a circular patch. Any scutula in this region exfoliate, leaving superficial, pale-red, oozing excoriations behind, which very soon (in about 8 days) become covered with skin. The part then presents a smooth, shining appearance (see Fig. 77), and about 6 or 8 weeks after the termination of irradiation becomes covered with lanugo; later on the scalp fully recovers its hair. In many cases the scalp remains permanently cured, and this result may be confidently expected if a relapse does not show itself within so weeks. Sometimes, however, a slight relapse necessitates a short supplementary treatment.

Török and Schein believe that relapses are not due to fungus being left behind in the hair follicles, but to fresh infection from without. Hence the advisability of treating the whole of the scalp as above recommended.

As an extra safeguard against relapse, the following ointment may be used for the bare places on the scalp after reaction has subsided: 
Glycerin acid. carbolic.

Lanolin

2.5

50. 0

This is to be thoroughly rubbed night and morning into the scalp by the patient, the object being to get the carbolic acid (which, according to Calderone, ${ }^{1}$ ) is the best destroyer of the fungus) well into the hair follicles. With this method very obstinate cases of farus may be cured in a few weeks' time.

The author knows of sereral cases which have remained well since this treatment, which took place from I to 3 years ago.

Should the carbolic treatment fail to prevent relapse, the Roentgen method must be repeated once or twice. Care must be taken, however, to aroid strong reactions; if the latter are frequently repeated the scalp will remain pemanently bald.

It will very often happen that old cases of favus which have been correctly and carefully treated by X-rays shew bald patches after healing. These are not due to irradiation, but are the effects of the disease itself.

The beneficial effects of this treatment in the case of farus do not depend upon any parasiticidal properties of the rays themselves. We shall see later that the rays possess practically no qualities of this kind, and the fungus on the fallen hairs is not found to have in any way sulfered in vitality. The good apparently consists in keeping large areas of the scalp bald for a considerable time; in this way much rantage ground for the fungus is eliminated with a great part of the fungus itself, and diseased hairs are remored which would otherwise retard the healing process. Then there is the stimulative effect of the irradiation to be considered, which improses the nutrition of the hair follicles; also a peculiar action of the rays in causing degeneration of the cell elements among which the fungus proliferates, and so inclirectly acting inimically on the latter. Up to the present time no minute histological investigation in this line have been published.

The favourable results obtained by this method of treating farus, which was first introluced by the

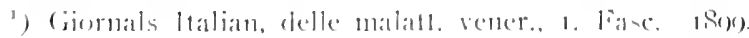


author ${ }^{1}$ ) (and published later in connection with Schiff), have been confirmed by Ziemssen," $)$ Neumamn,") Albcrs-Schönberg, $\left.{ }^{+}\right)$Hahn,") Grouven, ${ }^{6}$ ) Lion, $\left.{ }^{,}\right)$Norman I'alker,") Scholtz,") and Spiegler,,$^{10}$ ) Gaston and Nicolon, ${ }^{11}$ ) Boc zar and Bukofsky. Spiegler states emphatically that the method radically cures favus in a shorter time than any other known method, and is, in fact, the best possible means for treating the disease.

\section{Sycosis vulgaris and Folliculitis barbae.}

The mode of application of the Roentgen treatment for this class of affection must be guided by circumstances, such as the character of the sycosis and other conditions to be considered later. If we are dealing with an acutely suppurating process, tending to excoriation, it is better to give but few sittings (4 to 6) of $8-10$ mintues' duration each, using a hard tube at a distance of $15 \mathrm{~cm}$. In such cases even this brief treatment suffices to determine very evident results, the skin becoming drier, the pustules drying up without fresh pustular formation, the infiltration absorbing, and the itching, pain, and tension gradually lessening; all this happens without the loss of hair. The appearance of these signs and symptoms, together with a darker reddening of the skin, should be an indication for suspending the treatment. Should there be a relapse after a few weeks, the process may have to be repeated once or even several times. Nore powerful, but more radical, effects are obtained

${ }^{1}$ Freund, Wiener med. Woch., I897, No. 19, Wiener dermatol. Gesellsch., May 10. IR9o. Fortschr.. Vol. IIJ. 1. Io9.

2) Aerztl. Verein in München. June S. I898.

3) K. K. Gesellsch. d. Aerzte, 19 Oct. 1900.

$\left.{ }^{4}\right)$ Aerztl. Verein in Hamburg. IS. Oct. I898, and Fortschr. III, Part IV, 1. 160.

$\left.{ }^{5}\right)$ Aerztl. Verein in Hamburg. 19 Nox. 1900.

6) Fortcher. IV, Part V, p. 18.3.

7) V'll dermatol. Gesellsch.. Preslau, rgor.

") Lancet, 1900. January, p. 27.

9) Arch. f. Derm. u. Syph. Vol. LIX, Part IJI.

1") Wiener Klin. Woch. 1900. P. ro94.

${ }^{11}$ ) Soc. de dermatolog. et de Syph., July 3, 1902. 
by epilation, which is produced by stronger irradiations (see below, also Figs. 78 and 79 ).

In the treatment of this kind of skin-affection (folliculitis barbe) by means of $\mathbb{X}$-rays, it sometimes happens that the skin reacts riolently after comparatively few ( 3 to 5 ) and weak irradiations; the reaction partakes rather of the nature of an exacerbation of the sycotic process than of an ordinary Roentgen-dermatitis. Numerous fresh pustules appear, which often run together; the whole skin becomes tense and exceedingly painful. Often deep-seated abscesses are formed, where

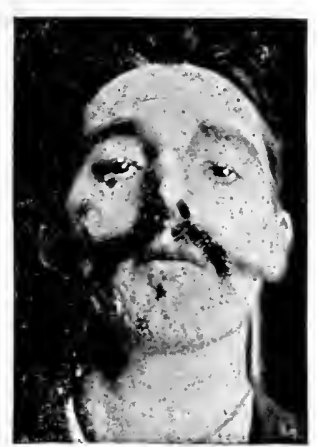

FIG, 78 .

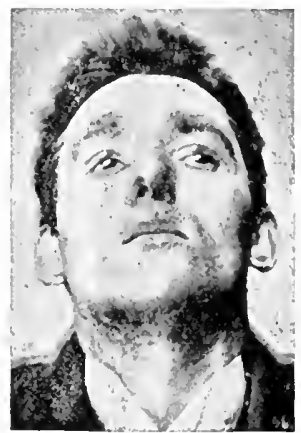

FIG. 79 .

the pus is collected sub-cutaneously in considerable quantities. Since the skin is already highly intumescent, so that the addition of Roentgen irradiation with its effects might even induce sloughing, the author in such cases suspends the treatment, applying appropriate dressings until the acute inflammation has subsided, after which the sittings may be cautiously recommencel.

The sycosis again may manifest itself in the shape of small, red, infiltrated, superficially scaling nodules. These occupy the sitc of the follicles, being pierced by the hairs. In this variety of the disease (acne pilaris. Besnier, ulerythema sycosiforme, folliculitis barba $)$, it is advisable, if the patient can conveniently remain beardless for some time, to continue the treatment to the stage of epilation. Since a reaction is soon obtained in these cases, on account of the pre-cxisting inflammation, the skin 
should not be exposed for more than 14 days with hard tubes. After this period, as a rule, the commencement of reaction is indicated by increased tension in the skin, with smoothening of its surface and a decp-red appearance. Loosening of the hairs soon follows.

In order that we may be in a position to recognise the important sign of loosening of the hair, the patient must, at the outset, be told not to shave or otherwise interfere with the beard; nor should the treatment be undertaken before the hair has reached such a length that it can be grasped by the finger-tips.

It often happens, however, that the treatment cannot be pushed to the stage of epilation, many patients being obliged for social reasons to retain the beard. In such cases it is advisable (as with cases of the first kind) to proceed more gently, exposing the skin only until the pustular and nodular formation ceases, repeating the process after three weeks' interval until no further lesions manifest themselves.

It is by no means clear to what we are to attribute the favourable action of Roentgen-radiation in this disease.

The observation that epilation of the hairs in the affected region is a better procedure than shaving permits of the assumption that good results are due not so much to the better penetration of medicaments after epilation, as to the actual removal by this procedure of an agent which maintains the follicular inflammation; just as gauze-strips introduced within an abscesscavity prevent the latter from healing, although freely granulating, so the presence of a dead hair may conceivably interfere with the healing of a follicle whose walls are in a state of inflammation. The author assumed, therefore, that keeping the skin hairless for some time would have a beneficial effect on the process, and on this ground recommended the use of $\mathrm{X}$-rays in the treatment of sycosis and farus. ${ }^{1}$ )

Kaposi ${ }^{2}$ ) was of the opinion, however, on being shewn a case of this kind by $S$ chiff and the author, that sometimes a

1) Wiener med. Woch., I\&97, No. I9-“Light Treatment," etc.--Compte. rend. du XII Cong. internat, de médicine, Moscow, I897, Dermatolog. Section, P. 4 I 4 .

2) Wiener dermatolog. Gesellsch., May ro, 1899. 
sycosis is dependent on the irritation resulting from certain anomalies in the hair-growth (such as the presence of scveral hairs in one follicle, or a want of proportion between the diameter of the hair and the follicular lumen), and that rcappearance of the hairs would be associated with relapse of the disease. It must, of course, be admitted that relapse is common in cases of sycosis. Still Kaposi's view is hardly justified, for if in these cases sycosis were actually provoked by the anomalies referred to above (follicles with several hairs, or hairs of too large a size), the first visible signs of new hairs would inevitably be accompanied by traces of the disease. This is, however, not the case. In all these cases the hairs rapidly grow again, and in normal fashion. The skin appears healthy, and remains so for some time. Then, after the patient has apparently had a healthy beard for a while, there comes a recurrence one day of his sycosis. This can be speedily removed in 4 or 5 sittings, without having to push the treatment to the stage of epilation.

According to Kaposi, a radical cure of sycosis associated with anomalous growths of the hair is not possible by means of Roentgen treatment. This view is not correct, however.

Of 17 cases of sycosis treated by the author up to November, I90 I, 5 cases were thoroughly cured by the first course of sittings; in 5 cases, I; in three cases, 2 , and in I case 3 supplementary sittings were required for a permanent cure. Of the remaining 3 cases, some are still under treatment, the rest not having put in an appearance since the first sitting. These results shew that sycosis is certainly amenable to treatment by irradiation. Gassmann and Schenkel ${ }^{1}$ ) report a case so treated which had remained well a year later. AlbersSchönberg and Haln, ${ }^{2}$ ) Grouve'n,", Spiegler, $\left.{ }^{4}\right)$ Lion, ${ }^{5}$ ) and Scholtz, ${ }^{6}$ ) G. H. Lancaslite, ${ }^{7}$ ) Gaston and

1) Fortschritte auf dem Geh. d. Roentgenstr., Vol. II, p. 1.

2) Ibid. Vol. III, p. I60; Acrztl. Verein in Hamburg, ") Nov. 1900.

$\left.{ }^{3}\right)$ Niederrh. Gesellsch. f. Natur und Heilkunde z.11 Bonn, Feb. 2, 1900.

-) Wiener derm. Gesellsch. Nov. 14, 1000.

s) VII Cong. d. deutsch dermatolug. Gesellsch., Breslau, I90I.

e) Archiv. f. Dermatolog. u. Syph., Vol. LIX, Part III.

7) Brit. Med. Journal, May 31, 1902. 
Nicolou ${ }^{1}$ ) also report the radical cure of obstinate cases.

From these reports we may conclude that a radical cure of sycosis may be effected in a comparatively short time, very often in a single series of sittings. Moreover, in the majority of cases, there is no need for bringing about Roentgen-dermatitis or permanent loss of hair, as has been suggested by two writers on the subject.

The prospect of cure by this method, which was first recommended and used by the author himself, is therefore quite good. The treatment may be combined, if desired, with the use of drugs. In that case a 15-30\% sulphur-oil may be used, after all inflammatory signs of reaction have subsided.

The relapses which undoubtedly occur from time to time after the employment of radio-therapy in this disease are urged sometimes as an argument against the method. But, after all, the repetition of the sittings after six weeks or so, followed perhaps by yet another course of sittings later on, is no great hardship to the patient. The inconvenience is one that is amply compensated for by the patient's feeling that he is no longer a conspicuous object for pity amongst his fellows. One must bear in mind what the ordinary treatment of the disease commonly entails, viz.: constant treatment for a very long period, daily shavings and epilation of the tender skin, unsightly dressings which may bar the patient from society. The fact that he may, after a painless treatment by $\mathrm{X}$-rays which inconveniences him but little and lasts perhaps three weeks, be made once more presentable is surely an argument in favour of radio-therapy.

Recently Ehrmann ${ }^{2}$ ) has shewn that the disease called by him "sycosis scleroticans," but which is more generally known by Kaposi's title of "dermatitis papillaris capillitii," can only be cured by Roentgen-treatment or electrolysis.

The author treated such a case in which the disease was accompanied by nodular glandular infiltrations the size of a walnut. After 7 sittings the hair became

1) L. c.

2) Wiener med. Woch., I90I, Nos. 30, 31 . 
loosened, and the nodules decreased in size. A second course of treatment acted still more energetically. The technique is the same as for ordinary sycosis.

\section{Hyphogenic Sycosis (Trichophytosis).}

Growen, $\left.{ }^{1}\right)$ H. Zechneister" ${ }^{2}$ and Lion ${ }^{1}$ ) report good results with this disease. Zechmeisler obtained a radical cure in onc case after a single course of 2 I sittings, the exposures lasting from 5 to 15 minutes each and the tube-distance being from 25 to $15 \mathrm{~cm}$. He used hard tubes, and noted the spontaneous disappearance of a fluctuating tumour, the size of a hazel-nut, which had existed within the zone of disease.

Brmo Chaves ${ }^{3}$ ) recommended the treatment for herpes tonsurans. J. Pollizer informed the writer that he obtained good results also with this affection.

\section{Blepharitis.}

The author had repeatedly observed that patients who were under the Roentgen-treatment for sycosis barbx, eczema faciei, acne rosacea, lupus vulgaris of the face, were incidentally soon cured of a co-existing blepharitis. He published the first case of this kind in conjunction with E. Schiff; this was a case complicating a chronic eczema of the beard. Since that time he has treated 3 cases of uncomplicated blepharitis.

As a rulc the diseased region reacted after 4-9 weak irradiations of the closed eye, using hard tubes. Response to the treatment was ajuickly obtained, whether the disease was of the ulcerating or scaly type. Crusts became detached, the colour of the part became lighter, excoriations skimned over, and the skin became once more healthy in appearance. All this happened without the eyclashes falling, and no after-treatment by drugs was necessary. The accompanying conjunctival catarrh improved rapidly after the cure of the blepharitis.

1) L. C.

2) Monatich. f. prakt. Dermatolugie. Vol. XXXll. No. 7.

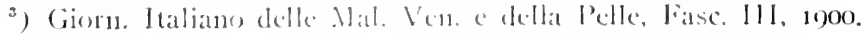




\section{Trichotylosis (Trichorrhexis nodosa).}

The author has used Roentgen-treatment in a case of this peculiar disease, the origin of which is as yet unknown. This was a remarkably obstinate instance of the affection, which had for years withstood other methods of treatment.

The patient, whose moustache presented the characteristic appearance of this disease, had twelve sittings in September, I9OI. The apparatus comprised a $25 \mathrm{~cm}$. coil, worked from a supply-current of 6-8 ampères and I I 0 volts; hard tubes were used, the sittings occupied 7-IO minutes each, and the tube-distance was $10 \mathrm{~cm}$. Slight erythema and partial falling of the hair ensued. Unfortunately, about this time the treatment had to be interrupted. After 6 weeks' time the hairs 'began to grow again in a normal manner. At the present time (April, I902), the patient has a normal moustache, the hairs of which average $2-2 \frac{1}{2} \mathrm{~cm}$. in length and shew no abnormality when examined microscopically. (Later note: Some trifling relapse is noted at the places from whence the hair had not previously fallen under exposure. But the new growth of hair appears quite healthy six months after the treatment. The patient is recommended further and more energetic treatment with the rays.)

\section{Alopecia Areata.}

R. Kienböck ${ }^{1}$ ) treated a man, æt. 26, who had for over 3 years suffered from severe alopecia areata of the whole head, by the Roentgen method, giving six sittings of 20 minutes each with a tube-distance of $20 \mathrm{~cm}$. He exposed the frontal region only.

The small lanugo hairs soon fell out over the irradiated area, and were succeeded two months later by a strong growth of dark and normal hair. The rest of the scalp, which had not been treated, retained its diseased condition. Kienböck con-

$\left.{ }^{2}\right)$ K. k. Gesellsch. d. Aerzte in Wien, Nov. 2, I900. 
cluded that the irradiation, with its consequent epilation, had determined the fresh growth of normal hair.

G. Holzkncht ${ }^{1}$ ) soon after this reported another case in which, after 5 sittings of 10 minutes each with a $20 \mathrm{~cm}$. tubedistance, the same result was obtained. In this case, however, the epilation was not complete, a ring of thick black hairs being left surrounding each plaque. The plaques themselses became reddened 3 weeks after the irradiation, whilst the healthy portion of the scalp (in which reaction had shewn itself after 8 days) remained pale. New hair first grew on the plaques, which soon became covered with soft, thick black hairs; this occurred before the healthy portion of the scalp (which had been denuded by the rays) recovered its hair.

In these cases fresh hair grew, as is usual after Roentgenepilation, two months after the last exposure. In two more cases similar results were achicred. ${ }^{2}$ )

Holsknecht recommends two methods of treating alopecia areata: ( I) in which only the plaques themselves are irradiated, together with a ring $\mathrm{I}$ cm. wide surrounding them where the disease is presumably latent (the rest of the skin is protected by lead masks; (2) in which the whole scalp is expesed, including both healthy and diseased areas.

The above-mentioned two cases of alopecia areata shewed unmistakably that Roentgen-radiation caused the growth of fresh hair. Do we possess in this method a specific for the affection?

The author treated in this manner a boy, at. I 2 , who had alopecia areata capitis. After 8 sittings of 10 minutes each, with a hard tube, lanugo appeared in all the bare places, one plaque indeed shewing a fresh growth of black hair.") In this case there was no preliminary falling of the hair. Improvement, however, was not maintained when the treatment was continued.

$\left.{ }^{2}\right)$ K. k. Gesellech. 1. Aerzle in Wi(n. 7 l)ec. Inoo. Wiener klin. Rund-

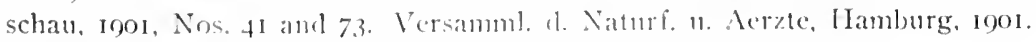
Fortschr. a. d. Cich, d. R.. Vol. Y., No, 1, 1. (12.

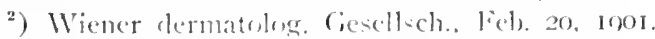

${ }^{3}$ ) K. K. Giencil ch. (1. Acrzte, 1)ec, 7, 1000. 
$J$. Nenmann ${ }^{1}$ ) rightly insists that in these cases we must bear in mind the possibility of a spontaneous cure. In connection with this point we may recall the fact that alopecia areata which has involved the entire scalp, in the case of elderly people, is generally incurable, whereas healing is the rule with younger people. The discreet type of the affection again usually heals of itself in due time.

Few data are to hand of this particular branch of X-raytherapy, nevertheless it is clear that it has been by no means universally successful. Holzknecht ${ }^{2}$ ) found some of his cases to be absolutely refractory, and Kienböck ${ }^{3}$ ) himself reports to the same effect. The author has had the same experience in two instances, and has learned from private communications received from several Viennese dermatologists that they also failed in several cases.

We shall probably, therefore, have to abandon the hope of curing universal alopecia by the $\mathrm{X}$-ray method, since it often fails to improve even the less severe forms of this malady.

Kicnböck attempts to explain the beneficial effects of Roentgen-radiation in alopecia areata, when these do occur, by ascribing some parasiticidal qualities to the rays. According to this writer, moreover, it is equally reasonable to expect good results if the disease be regarded as atrophoneurosis. With regard to the first point, it has not yet been demonstrated that all cases of alopecia areata are of microbic origin. Then again, $\mathrm{X}$-rays cannot be accepted as possessing any microbicidal properties at all. Even supposing them to be so endowed, the irradiation would have to be so vigorously maintained for a long time, in order to destroy the organisms, that serious injury to the skin could hardly be avoided. Finally, no experimental proof has as yet been forthcoming of the destruction of Sabourand's microorganisms in this manner.

$\left.{ }^{1}\right)$ K. k. Gesellsch. d. Aerzte in Wien, Nov. 2, I90o.

$\left.{ }^{2}\right)$ Wiener klin. Rundschan, Igor, No. 4I.

${ }^{3}$ ) Verhandlingen d. deutschen dermatolog. Gesellsch., VII Congress, p. 447 . 
The theory advanced by several writers, including Silhif, ') Ehrmann,") Holsknechl") and the author," much more tenable, viz.: that we have here mainly an effect of mechanical irritation.

It has often been observed that a few short exposures do not cause any falling of the hair, but rather some slight irritation which may lead to thicker growth. The effect is quite analogous to that produced by certain chemical agrents (tr. aconite, reratria, cantharides), which are also cmployed as stimulants to the scalp. Probably, howerer, the effect of Roentgen-radiation (and also, according to Finsen, phototherapy) is more penetrative, and therefore more likely to reach the structures concerned with hair-growth than is the case with these chemical agents, whose effect is more confined to the surface of the skin. We know that under certain circumstances alopecia areata may be cured also by other physical irritants. Thus Ehrmann shewed this with faradisation. If this view be correct, the X-ray method should on theoretical grounds possess a certain therapeutic value in this affection. Undoubtedly it does, under certain conditions, stimulate the process of hair-growth, though it is an open question if the origin of this stimulus is to be found in the $\mathrm{X}$-rays themsclves or in the high-tension electricity flowing off the tube. In any event, to obtain this effect it is not necessary (as the author's experiments prove) to push the irradiation in the first instance to the stage of epilation.

The property which Roentgen-radiation possesses of stimulating hair-growth is by no means so reliable as its action as a depilatory. It is interesting, however, as Kienböck remarks, to note that identically the same remedy may be employed to produce diametrically opposite results.

Holzknech tried the method in the treatment of alopecia pityroides, but without any definite success.

1) IV Intern, clematoleg. Cong. P'aris, Inoo.

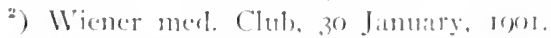

$\left.{ }^{3}\right)$ 1..

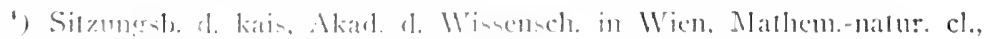

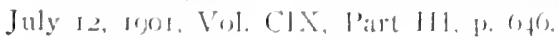




\section{Hypertrichosis.}

The methods of treating this skin affection are in the main those already indicated in general Roentgen-therapy. Almost every operator has his own technical-modifications, which he believes to be the best. The first essential is to become thoroughly acquainted with the capacity of one's apparatus; it is only after that that one may safely introduce niceties of detail.

The author himself uses hard tubes showing greenish fluorescence in a non-darkened room. He employs a $30 \mathrm{~cm}$. coil and currents of. 2 or 3 ampères and I Io volts, and a tubedistance (skin to glass) of I $5 \mathrm{~cm}$. If the cheeks and chin are to be exposed, a mask is used which covers the upper part of the face and extends to the edge of the lower lip. The chin is irradiated with the patient's head stretched far back, and at the same time care must be taken to protect the chest with a leaden mask. If the upper lip is to be exposed, a mask should be used covering the entire face, having an aperture coinciding with the part treated.

Treatment should not be commenced before the hairs are long enough to be grasped, and the patient must be told never to pull out the hairs herself. The first signs of skin-reaction are usually seen after 20-2 5 sittings; these signs have already been considered at length (p. 255). The other methods of irradiation were also described in detail (pp. 250, 257, 258) and need not here be repeated. The author has not observed any differences with regard to the reaction of blondes and brunettes. Occasionally fine lanugo falls more readily than the thicker medullated hairs; the reverse is, however, far more frequently the case.

The later course of the reaction is as follows: After a few days, during which the earlier signs of reaction increase in intensity, the hair begins to fall out in thick tufts when lightly grasped, or it is seen on the towel after the patient's toilet. The hair-roots are thin and atrophied (see the "Physiology of Roentgen-radiation"), the skin is smooth and white, and the patient often feels a slight burning and sensation of tension. These signs disappear in a few days ( 5 to 8 ), including the sometimes marked pigmentation (which is especially seen in the 
case of brunettes), and the skin again appears quite normal. If no further treatment be adopted fine hairs again make their appearance in the course of $6-8$ weeks; these grow gradually to their former size. ${ }^{1}$ ) If, however, after + -6 weeks (even before there are the slightest signs of relapse) a brief supplementary treatment be undertaken, the skin will preserve its good appearance for another 4 or 6 weeks, after which period the process must be repeated. In this way the treatment must he kept up for I 2-I 8 months, after which time a permanent cure may be expected.

The author has had already a large experience, which warrants him in saying that the series of supplementary courses of treatment as indicated above turns the depilatory effect from a transient to a permanent one. He bases this opinion on the repeated examination of a number of patients since their final exposures to the tube, in whom not the slightest signs of relapse were erident.

At the termination of the sittings the skin looks plump and well nourished, as described earlier (p. 25I). After a few months, however, it alters somewhat in appearance, the change depending solely upon the extent to which the treatment was pushed.

The final effect is most perfect in those cases in which, during the whole series of operations, the intensity of the irradiation was regulated to such a nicety that no very marked changes were visibly produced in the skin. In this event the skin appears quite normal, and more or less smooth according to the age of the patient. In the site of the follicles one finds either very tine whitish points, which disappear after a time, or (in places which have been less exposed to the tube), excedingly small white lanugo-like hairs; the latter do not increase in size and are practically unnoticeable.

Jutassy and $K$. Minich found on microscopic examination the following conditions in a rabbit's skin which

2) If the initial cource of trealment was more energetic relagse will take place much later - after three or four momblis. 
had been epilated by X-rays. ${ }^{1}$ ) The stratum malpighii presents a straight line bordering the cutis; only here and there can a hair-follicle be seen. These follicles penetrate to various depths; the major part are found in the superficial layer of the cutis. They possess no lumen; in place of the hairs we find atrophied epidermic cells or their remains. Some of the follicles penetrate to a deeper layer of the cutis; in these thin, black hair-rests are to be seen, which are shrunken from the papilla. The papilla pili looks atrophied, and consists of small, light-coloured degenerated cells. The hair-groups which characterise the normal skin are absent, nor can their follicles be discovered. In place of the latter one finds richly-cellular connective tissue. Pigment granules are found here and there in the deeper layers of the cutis; in this region, too, the blood-ressels are found widely dilated and filled with blood-cells; there are, however, no thrombi. No abnormalities can be found in the larger nerves. The main feature is the atrophy of the follicles and the hair-papillæ.

If during the course of treatment the signs have been more severe-marked intumescence and erythema, or perhaps slight excoriation-one finds changes in other parts besides the follicles. The sites of the latter are marked by small scar-like depressions, and the whole of the irradiated skin appears thinner and more wrinkled than is natural. The wrinkles may not be noticeable while the facial muscles are at rest, but they become quite evident when the patient talks or laughs. ${ }^{2}$ )

In other cases, particularly with brunettes, the skin remains darker than usual for some months; in course of time this excessive pigmentation disappears. In one case, that of a fairhaired girl who had been under treatment intermittently for I 5 months for hypertrichosis of the forearms, and in whom somewhat marked erythema (without excoriation) had thrice arisen, a reddish-brown spotted discolouration remained after

1) Orvosi Hetilap, 1898, 2I-2,3.

$\Rightarrow$ According to Ehrmam, the wrinkling results from atrophy of small bundles of connective tissue in the skin. 
treatment which disappeared rery slowly. The elephantiasisor sclerodermia-like thickening of the skin, described as an aftereffect of Roentgen-radiation by many writers (Balzer and $\mathrm{M} \mathrm{CH}$ seaux, Salomon, Barthélemy', Billerend, Mallopian and Gadean), has not as yet been seen by the author. He has, howerer, seen cases treated by others which during their course had dereloped excoriations and uleers. The scars were marked by extensive telangiectases which considerably impaired their appearance, the scars themselves being smooth and otherwise not very disfiguring.

Another result of too intense and too frequent cxposure was described by $/$. C. Johnston. ${ }^{1}$ ) A medical man, who for four or five years had been working with X-rays, observed the back of both his hands to be groing red, dry, slightly scaly and somewhat itching. About 20 small tumours developed, varying in size from a pin-head to half an inch in diameter, and forming hard, shining, horny elevations which were depressed in the centre and surrounded by a reddish areola. On microscopic examination, these proved to be epithelial accumulations. The epidermis was considerably thickened throughout; moreover there were large masses of epithelial cells lying in trabecula, and small clusters between the connectire-tissue fibres. Iolnsson regarded the condition as one of those keratoses which, like leucoplakia oris or verruca seborthoxica, are considered to be often the forerimners of carcinoma.

Reviewing, the adrantages and disadrantages of this treatment for hypertrichosis, we find we have here one of the most reliable remedies within the reach of the medical man, inasmuch as its depilatory effect is certain. We must note that it causes all the hairs-the lanugo-hairs as well as the thicker ones-to fall; that large areas of the skin can be treated at a time; that though a relatively long time is repuired for permanent cure the actual sittings occupy but a brief period, so that the patience and endurance of paticnt and operator are not too severely

1) Jhitarletphia Merlical Journal, Fed, 1, 1902. 
tried. It must be borne in mind, moreover, that by proceeding on the right lines in the first instance and giving supplementary sittings as they are required, the epilation which is first produced may be made permanent. We possess in the Roentgentreatment an absolutely painless method of epilation, a method which enables us to dispense with drugs, dressings, manipulations on the part of the patient. It is, morever, one which does not attract attention or compel the patient to interrupt her usual avocations or social intercourse.

We find also that the treatment has a definitely limited duration; it matters not whether a small area of skin or the whole face has to be treated. At the same time it must be remembered that under certain circumstances changes in the skin occur which cannot be considered as advantageous to the complexion. It is possible that, with increased experience and ability, we may be able to avoid this contingency with certainty; nevertheless at the present stage of our knowledge the possibility of this contingency is one which must be put to the patient before commencing treatment: she must be asked whether she prefers her hirsute appearance to some facial wrinkles which may develop after treatment.

Where the hairy places are small and circumscribed, the author, in common with Ehrmann and Schiff, would unhesitatingly advise electrolysis. Under any circumstances it would seem advisable to avoid risible reaction as far as possible.

Considering the numerous undoubtedly successful results which not only the author and Schiff, but many Austrian, German, English, American, Hungarian and Swedish writers report (Grumnach, ${ }^{1}$ ) Levy-Dorn, ${ }^{2}$ ) Gronecn, $\left.{ }^{3}\right)$ Hahn, ${ }^{4}$ ) Benedikt, $\left.{ }^{5}\right)$ Sjögren and Sederholm, Sharpe, Dumsirey,,$^{\circ}$ ) Neville Wood, ${ }^{\circ}$ )

2) Deutsche med. Wochenschr., 1899, 37.

2) Eulcuburg's "Real-Encyclopädie," Arlicle "Roentgenstrahlen."

3) Niederrh. Gesellsch. f. Natur-11. Heilk, zu Bonn, Feb. I2, I900.

4) Aerztl. Verein in Hamburg, Nov. 19, 1900.

$\left.{ }^{5}\right)$ Wiener med. Wochenschr., Nov. I9, I900.

$\left.{ }^{6}\right)$ Fortschr., Vol. IV, p. 163 .

${ }^{7}$ ) Archives of the Roentgen-rays, Jan. Igor.

$\left.{ }^{8}\right)$ Schmidt's Jahrbücher, Vol. CCLVI. ') The Lancet, Jan. 27, I90o. 
James Startin, $\left.{ }^{1}\right)$ Kicuböck," ) J!ntassy, $\left.{ }^{3}\right)$ Havas, ${ }^{4}$ ) Ullmann,") Pusey,") G. H. Lancashire, $\left.\left.{ }^{7}\right)\right)$-results some of which have been demonstrated and acknowledged at many congresses of leading dermatologiststhe objections raised by (Oudin and Barlhélemy s) against the Roentgen-treatment of hypertrichosis seem hardly justified. Thesc writers are apparently of the opinion that internal organs may be injured by the process. Probably the "visceral accidents" they speak of, and which they ascribe to some influence on the sympathetic system, are to be explained by the hysterical character of so large a proportion of the class of women who would resort for this treatment to the Paris hospitals.

Under certain circumstances the depilatory effect of the Roentgen-rays may be used for other purposes. Its application in this respect for sycosis, etc., has already been mentioned. Gocht ${ }^{9}$ ) reports the case of a patient who had been operated upon for lymphadenoma of the neck. The edges of the wound became folded over, and owing to the irritation caused by the hairs healing was impossible. Epilation by Roentgen-rays resulted in perfect healing of the wound.

2. Ulcerations and skin-aflections leading to ulceration.

\section{Lupus vulgaris.}

The radio-therapy of lupus has received the greatest attention and study by dermatologists. A really certain and reliable remedy for this most serious and obstinate disease has long been

2) The Lancet, March 3, Igoo, and Nov, I6, I901.

2) Wiener med. Club, Feb, 6, 1900.

$\left.{ }^{3}\right)$ L. C.

1) Festchr. f. Prof. Kaposi.

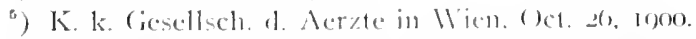

") The Journal of the Americal lledical Association, Sept. 28, rgor.

7) Brit. Merl. Journal, May z1, roses.

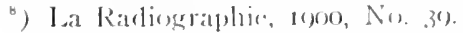

๑) Forlschir., Iol. 1, l'art 1, 1. 17. 
a desideratum; the affection is one which has been treated for decades past with no altogether satisfactory results. It must still be left an open question whether in Roentgen-radiation we have attained what has been so long wished for-a specific for lupus-and if the treatment will supplant all other methods.

That lupus vulgaris can be cured by $X$-rays is beyond dispute; it still remains, however, to be proved that the process is easier of adoption and quicker to achieve success than other methods. The author is of opinion that the advantage of the Roentgen method is to be found: ( I) in its being a blookless process: (2) in its excellent cosmetic results (see Figs. So and $\left.S_{I}\right)$. As regards the time required for the treatment, this is much the same as that required for the older methods or for Finseil's light-treatment.

Just as in the Roentgen-treatment of hypertrichosis we have the choice of two schools. The one deprecates the production of dermatitis, regarding this as not only superfluous but as actually injurious and retarding the cure (Schiff, Freund, Albers-Schünberg, Haln, Groncen, Kïmmel). On the other hand, the opposite school recommends the deliberate excitation of powerful reactions, believing that in this way a scar may be obtained of grood appearance and free from relapse for a considerable period (Lion, Schollz).

The technique of the rival methods has already been described in this work. Suffice it to note here that by one method (with hard tubes and considerable tube-distance) a comparatively large number of sittings is required, whereas with the other method (soft tubes and shorter tube-distance) a smaller number of sittings has the effect of producing very strong reactions.

The usual course of events where the first method is adopted is as follows: the first signs of reaction in the lesions shew themselves in the form of an alteration in their colour. They become hyperæmic, and visible nodules become dark-red; at the same time the morbid tissue swells. Later on the visible nodules break down and often necrose entirely away, leaving small, sharp-cut ulcers in their site. The whole site of the disease may be deeply indurated, and neighboring glands enlarged. The 
ulcers soon heal, leaving small scars. These scars for the first few weeks may present a perfectly sound and healthy appearance, the general intumescence and tension of the parts having not as yet subsided. The tuberculin-test applied at this stage, however, proves cure to be incomplete (Teisser, Lion, Scholla), and after a time, when the swelling has subsided and the tissues have again become lax, more deeply-seated nodules become evi-

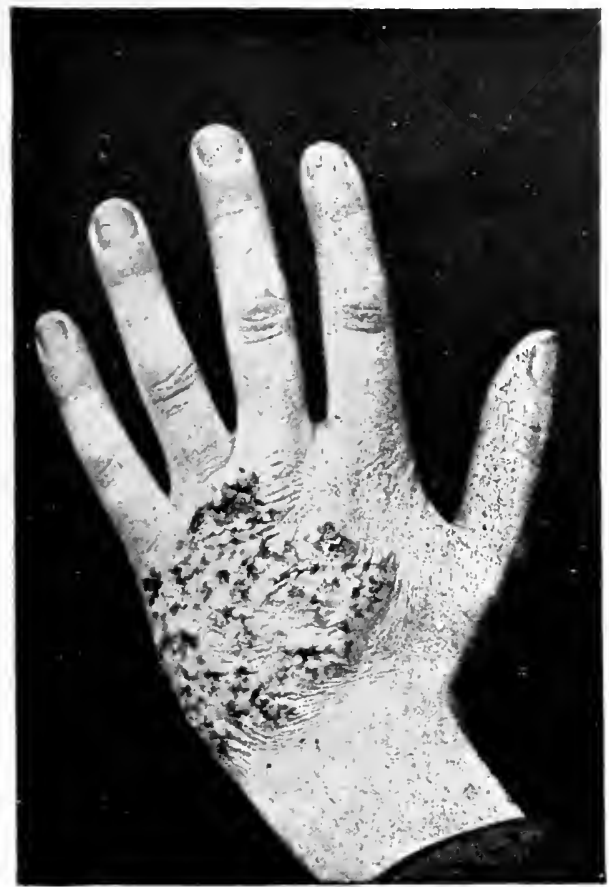

FIG. 80.

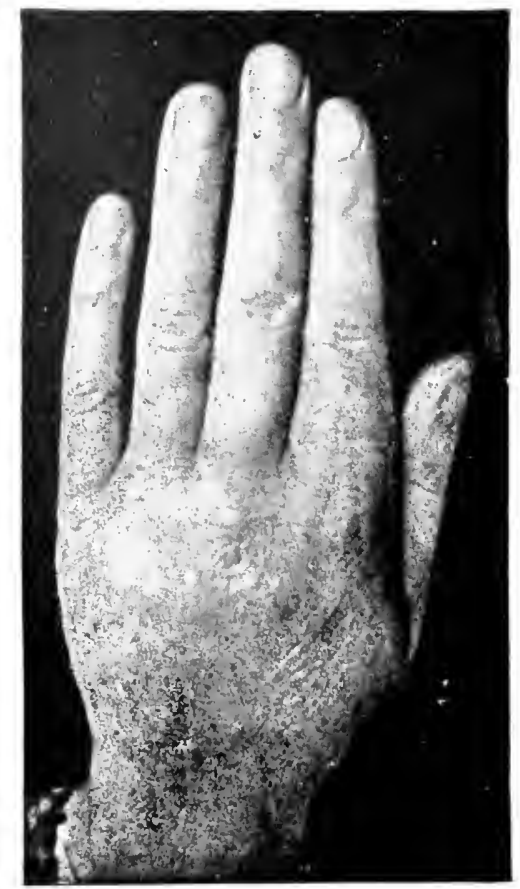

Fig. $8 \mathbf{I}$.

FIG. 8o-Defore treatment.

FIG. 8I.-After one year's intermittent treatment with X-rays.

dent. After a few wecks, therefore, the treatment requires to be repeated again and again, until all traces of disease have finally disappeared. 'This in a case receiving no auxiliary treatment may take a year or more.

It is worth noting that after a fairly prolonged treatment with $\mathrm{X}$-rays lupus nodules often become visible 
in regions which had hitherto been considered healthy. The rays would appear to have an elective action on the lesions-a peculiarity which was demonstrated by the physicist E.P. Thompson to exist in the case of many substances exposed to $\mathrm{X}$-rays.

The other method has for its object the destruction of the disease "en masse," it may be regarded, though not altogether correctly, as a cauterisation of the whole lesion by means of Roentgen-radiation. The method is certainly more radical, and the duration of the actual treatment is very short. Nevertheless, it is very questionable if the patient gains any advantage even in this direction. In place of his disease, he is now provided with an ulcer which is exceedingly slow in healing and is, moreover, excessively painful; thus he is deprived of what should be the chief advantage of radio-therapy, viz.: its painlessness. Scholt $\mathrm{z}$ has shown the histological changes to be similar whicherer method be employed-molecular destruction of the morbid cell-growth, reactive inflammation and replacement of the lupus tissue by connective tissue. But the whole course of reaction is much milder with the first method, the patient is spared pain, and the proceeding is more conservative of healthy tissue; whereas with the more drastic method the whole tissue, healthy and diseased, within the zone of irradiation becomes necrosed and has to be replaced by scar-tissue. As has been pointed out earlier, the milder method requires more skill in its practice.

Comparing the X-ray treatment of lupus with Finsen's method, it must be noted that according to reports published up to the present time the latter shews a larger percentage of radical cures.

In connection with this point it may be observed that up to now Finsen's method has had the advantage of being carried out in well-appointed public institutions, on a properly regulated system. Thanks to the courtesy of Hofrath Prof. I. Nemmann, the author has now the opportunity of treating hospital cases also; he hopes in due course to be able to supply reliable data therefrom.

Nevertheless the author must again remark that by the aid 
of the Roentgen-treatment he has succeded in a great number of cases (sometimes, it is true, with the additional help of chemical applications) in obtaining radical cures of lupus. The scars have been of excellent appcarance, moreorer. He would advise those who contemplate the practice of radio-therapy, but whose resources are limited, to purchase an X-ray apparatus. Public institutions and private individuals who can afford the outlay should certainly possess Finsen's apparatus as well.

In case the Roentgen-treatment of lupus be decided upon, one should undoubtedly employ the milder method of its application where large areas of disease are concerned. Smaller and more circumscribed lesions may be destroyed, too, in this way, due care being taken to protect the surrounding healthy parts with leaden masks. In these cases, however, the author's own predilection is in favour of radical excision and suture or Thiersch's method of transplantation.

G. H. Lancashive ${ }^{1}$ ) recommends the X-ray treatment of lupus where (I) the disease is too widespread for Finsen's treatment; (2) where there is much ulceration; (3) where unsightly scars add to the existing disfigurement; (4) where the mucous membranes are involved.

$J$. Nenmann") believes it to be the treatment "par excellence" for "lupus tumidus et exfoliativus" of the face, also for the hypertrophic forms involving the skin and sometimes the fascia and muscles, which have hitherto been considered incurable.

Lupus ulcerations of a sluggish character assume quite another appearance after a careful series of irradiations; healthy granulations spring up and cicatrisation begins. I upus of the mucous membranes is also farourably influenced. The first sign of reaction in these cases is increased secretion; thus a patient with nasal lesions presents the symptoms of nasal catarrh. Cavities of this kind may be irradiated through a leaden tube or speculum, the skin round the orifice being carefully protected by a leaden mask.

$\left.{ }^{1}\right)$ British Med. Journal, May 31, Ino2.

$\left.{ }^{2}\right)$ K. k. Gesellsch. d. Aerzte in Wien, Oct. 26, 1900. 
Himmel ${ }^{1}$ ) found the following histological appearances in a section of a lupus nodule which had been subjected to the rays :

The epithelium, which formed a thin narrow border to an apparently homogeneous mass, was perforated by small apertures, the size of which did not exceed the diameter of a cellnucleus. Nowhere could the exact outlines of the individual epithelial cells be made out. The corium appeared shrunken. The individual bundles of connective tissue were packed closely together, thickened, and translucent. Elastic fibres could not be recognised. In stained specimens the cells of the connective tissue appeared shrunken; they took the stain badly. The lupus growth itself appeared greatly changed. The giant-cells were small; they were seen as small, shrunken homogeneous bodies in which the nuclei were but faintly indicated; the epithelioid cells were four or five times smaller than usual, had lost their normal shape, were homogeneous, and their nuclei did not stain. The lymphoid cells had become transformed also into small shapeless bodies. The outlines of individual cells could often not be distinguished, they being so closely pressed together.

The general microscopic appearance of the sections suggested a slow necrotic process going on in the lupus nodule, the epithelium, and part of the corium. Hucter ${ }^{2}$ ) found that the tubercular tissue gradually underwent a fibrous transformation. A remarkable feature was to be found in the large number of giant-cells present. These were often situated eccentrically or close to the periphery of the nodule; many nodules seemed to consist almost entirely of giant-cells. The zone of leucocytes bordering the tubercular foci was very narrow or entirely absent. The nodules were closely encircled by bands of thick connective tissue, rich in nuclei. The line of demarcation between the morbid growth and this connective tissue was sharply pronounced. No tubercle bacilli were seen.

$\left.{ }^{1}\right)$ Archiv. f. Dermatologie und Syph., Vol. L. p. 3,35 .

2) Quoted by Hahn and Albers-Schönbcrg. Nünchener med. Wochenschr, I900, Nos. 9-II. 
Dr. Growen, whose investigations in this field are most valuable, reports as follows ${ }^{1}$ ) :

"When examining with low powers, we are struck by the abundance of connective tissue which encapsules the several tubercular foci in the form of thick bundles of fibre, penetrating more or less freely the interior of the foci themselves (Fig. 82 ), and thus forming a closely woren network enveloping the residuum of the morbid growth. The spindle-cells, which are

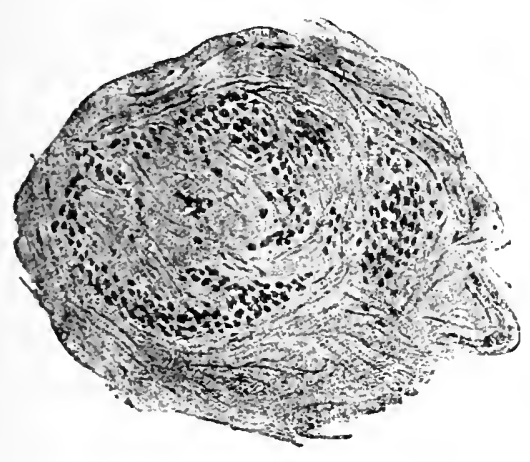

FIG. 82.

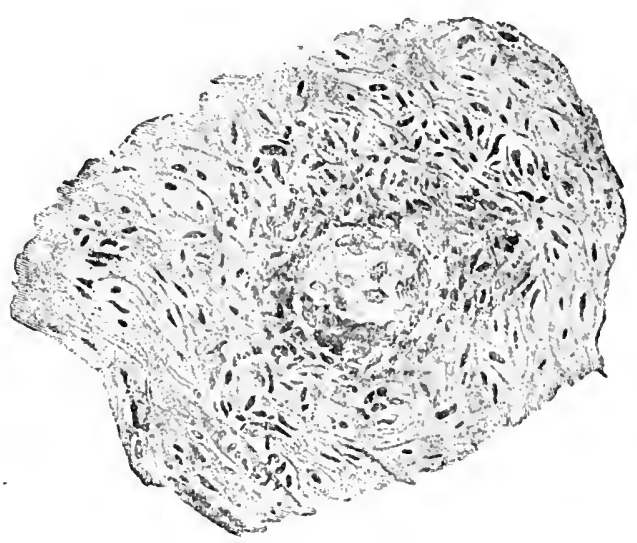

FiG. 83.

FIG. 82.-From C. Grouich, "Histological changes in lupus tissue after Roentgen treatment." Fortschr. a. d. Geb. d. Roentgenstr., Vol V. Part 3. p. 186 .

Fig. 83.-A giant-cell enclosed by new-formed connective tisstre, highly magnified. From C. Gronern, 1. c., p. 187.

present in great numbers (Fig. $8_{3}$ ), suggest the active proliferation of connective tissue. Under higher powers the epithelioid and lymphoid cells are seen to be greatly degenerated; this degeneration is shewn by the poor staining-properties of the nuclei, disorganisation of the nuclei, and vacuolisation changes in the cell-protoplasm. The general changes are analogous to those described by Gassmann as occurring in the ressel-walls of Roentgen-ulcers."

1) Niederrh. Gies, f. Nat, 11. Hcilw., Bomm, June 17, roor, Fortschr. a. d G. d. Roentg. Vol. V. Part I. 
Doutrelepont describes the process of healing of lupus under Roentgen-treatment as follows:

"The first effect of irradiation is the production of hyperæmia; this leads to increased migration of leucocytes from the ressels. Just as in the tuberculin-reaction the infiltration of leucocytes begins at the border of the diseased focus; it, however, penetrates the latter also, there to transform itself probably into spindle-cells and fibrous connective tissue. The morbid cells themselves degenerate; thus we get a gradual absorption of the diseased tissue and its replacement by cicatricial tissue."

According to $S$ cholt $z,{ }^{1}$ ) the primary effect is not hyperæmia, leucocyte infiltration, and fresh formation of connective tissue, the secondary one being destruction of lupus nodules. He believes that the reverse holds good: under the action of Roentgen-radiation we have, in the case of lupus, first a degenerative process in the cell-elcments, especially in the giant- and epithelioid-cells of the morbid lesions themselves; this is followed by inflammatory reaction. Scholtz believes the healing of lupus is mainly brought about by this inflammatory process, that in consequence of the degeneration in the morbid cells the inflammatory reaction is directly concentrated upon the diseased foci, and that we have, therefore, to deal with a selective effect of irradiation in this instance.

$N^{\prime}$ eisser ${ }^{2}$ ) compares the effect of Roentgen-radiation with the tuberculin-reaction. In the former, however, the process is a slower one, and is attended by passive hyperæmia. By this the cicatricial encapsulation, and, under certain conditions, the absorption and destruction of the tubercular foci is effected. "Especially in the case of Roentgen-dermatitis one can see," says this author, ${ }^{3}$ ) "that the old view with regard to inflammatory irritation is wrong, and that $/$ eigerl was correct in advancing the doctrine that in all these processes we have always to deal with primary tissue-lesions, which are followed by inflammatory reaction." What we see in the most marked forms of

\footnotetext{
1) Archiv, f. Dermatol, u. Syph., Vol. LIX, Part 3.

") Ehstcin-Schacalbe's Handbuch der prakt. Med., Vol. III (Skin Diseases).

$\left.{ }^{3}\right)$ Quoted by Scholtz. p. 242.
} 
Roentgen-necrosis takes place most probably also in those milder alterations in which actual destruction docs not become visible under the microscope.

Many writers speak of good results with this treatment. The first report in this field came from kïmmel (26th Congr. d. deutsch. chirurg. Gesellschaft, 1897). The author, in conjunction with Schiff, treated two cases on which he reported at the 12 th Internat. Med. Congress, held in Moscow, and which Schiff mentioned at the Naturforscherversammlung, Braunschweig, i\$97. Further reports came from Gocht ${ }^{1}$ ) and Albers-Schönbers,") followed by Sonncuburg.:") Neisser, ${ }^{4}$ ) o. Ziemssen,") Gassmann and Schenkel,") Stenbeck, $\left.{ }^{7}\right)$ Ch. Schmid,") Grimmach,", Sharpe, $\left.{ }^{1 "}\right)$ Neriman, ${ }^{11}$ ) Hall-Edwards, ${ }^{12}$ ) Sholeficld, $\left.{ }^{12}\right)$ Rona, ${ }^{14}$ ) Jutassy,, ${ }^{15}$ ) Gron-

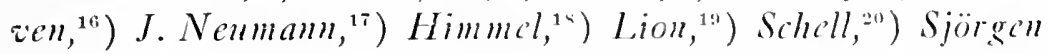
Eo Sederholm, ${ }^{21}$ ) Clark and Smith, ${ }^{22}$ ) Pusey,,${ }^{2: 3}$ ) Geyser, ${ }^{24}$ )

1) Fortschr. I, I, I5.

${ }^{2}$ ) Ibid. I, 2 and 3; II, 1, 23; Münchener Med. Woch., 1900, 9-II.

$\left.{ }^{3}\right)$ Freie Vereinig. d. Chirurg., Berlins, Jan. I0, $1 \$ 98$.

*) Schles. Gesellsch. f. vaterl. Cultur, Nay 20, I898.

$\left.{ }^{5}\right)$ Aerztl. Ver. München. Junc \&, I 898 .

${ }^{\circ}$ Fortschr., II, 4, I.

${ }^{7)}$ Hygiea, Vol. LXI, p. 568 .

$\left.{ }^{8}\right)$ Fortschr., III, I, I.

$\left.{ }^{9}\right)$ Deutsclie med. Woch., I899. No. 37.

$\left.{ }^{10}\right)$ The Roentgen Society of London, Nov. 6, 1899 . Archives of the Roentgen Rays, Jan. Igoi.

11) The Roentgen Soc., London, Nov. 6, I899.

$\left.{ }^{12}\right)$ Edinburgh Medical Journal, March, Igoo.

${ }^{13}$ ) Lrit. Med. Journal, May 5, 1900.

") Königl. Gesellsch. d. Aerzte, Budapest, Nov. 9, I899.

$\left.{ }^{15}\right)$ 1. c.

16) I. C.

${ }^{37}$ ) K. k. Gescllsch. d. Acrztc, Oct. 26, 1900.

$\left.{ }^{14}\right)$ Archiv. f. Dermatol. u. Syph., Vol. L, p. 3.35.

$\left.{ }^{19}\right)$ L. c.

20) Archiv. délectr. médic, experiment ct clinic, I900, No. 96.

$\left.{ }^{21}\right)$ Fortschr., IV, 5, I49.

22) Buffalo Med. Journal, Jan., 1901.

${ }^{23}$ ) The Jonrmal of the Anerican Medical Association. Dec. 8, 1900, and Sept. 28, I001.

"2) Aerztegencllsch., New York, January, igor. 
Jones, $\left.{ }^{1}\right)$ Knox, ${ }^{2}$ ) Lapinski, ${ }^{3}$ ) Kirmisson, ${ }^{4}$ ) Norman Walker, ${ }^{5}$ ) Jadassohn,") Van Dort, ${ }^{\top}$ ) Thurnwald, ${ }^{\circ}$ ) Jeney, ${ }^{9}$ ) Du Castel and Fovean de Courmelles, ${ }^{10}$ ) J. de Nobele, ${ }^{11}$ ) Stenbeck, ${ }^{12}$ ) Holland, $\left.{ }^{13}\right)$ P. R. Eganl, $\left.{ }^{14}\right)$ G. H. Lancashire, $\left.{ }^{15}\right)$ G. H. Radman, $\left.{ }^{16}\right)$ J.C. Squance, $\left.{ }^{15}\right)$ P. A. Morrowe, ${ }^{18}$ ) and others.

Most of these writers agree that Roentgen-treatment acts beneficially in the case of lupus, but that the duration of the treatment is a very long one, and that it is advisable to combine this method with others, such as cauterisation, scraping, etc.

Gronven and Aronstam ${ }^{19}$ ) reported similar good results in the case of scrofulodermia.

Ivar Bagge ${ }^{20}$ ) cured tuberculous ulcerations which had appeared on the chest in the site of old cauterisation-scars in an elderly man by means of X-rays. The rays were only directed on the chest, but this writer reports that at the same time some ulcerations existing on the back of the patient also healed.

Sjögren and Sederholm treated 5 cases which they considered to be of the nature of tuberculides with very successful results. (According to their reports, these cases seemed to partake somewhat of the nature of perniones, or to suggest lupus erythematosus.) After I5-58 sittings the swelling and infiltra-

$\left.{ }^{1}\right)$ Philadelphia Med. Journal, Jan. 6, I9oo.

2) Journal of the American Med. Association, Nov. Io, I90o.

$\left.{ }^{3}\right)$ Gazette lekarska, Vol. XIX.

${ }^{t}$ Soc. de Chirurgie, Paris, I898, 2, in.

${ }^{5}$ ) Lancet, January, 1900.

") Encyclop. d. Haut- und Geschlechtskrankheiten, 1900.

i) Tijdschr. v. Geneesk, I900, No. I8.

") K. k. Gescllsch. d. Aerzte, Oct. 26, 1900.

$\left.{ }^{9}\right)$ Wissensch. Verein d. Militäraerzte der Garnison, Wien, Jan, 26, I90I.

10) Annales d'électrobiologie, I898, Nov. I5.

$\left.{ }^{11}\right)$ Ibid. Vol. III, p. 236.

$\left.{ }^{12}\right)$ Quoted by Mocller, Der Einfluss des Lichtes, etc., Bibliothek. med., p. 12I.

${ }^{13}$ ) Archives of the Roentgen Rays, May, I90I.

1*) Anterican Medicine, Nov. I6, I900.

1., Brit. Med. Journal, May 3I, I902, p. I329.

$\left.{ }^{10}\right)$ Lancet, Nov, 16, I90I.

$\left.{ }^{17}\right)$ Ilidid.

14) New York Dermatological Society, March 25, 1902.

$\left.{ }^{10}\right)$ Journal of Tuberculosis, Oct. 190 r.

$\left.{ }^{20}\right)$ Fortschr. auf. d. Geb. d. Roentgenstr., III, 6, p. 218. 
tion receded and the irritation disappeared. In some cases there was slight atrophy of the skin left afterwards.

\section{Epithelioma, Ulcus rodens.}

In his able monograph "On the Influence of Light upon the Skin," with which we shall deal more closely in the next section, Magnus Moeller mentions a case of epithelioma of the face, treated by Sjögren, in which Roentgen-radiation had a marvellously good effect. The illustration bears out this impression. Irradiation with hard tubes was employed; the current-strength was 2.5-3 amperres, and the tube-distance I $5 \mathrm{~cm}$., the duration of each exposure being 10 minutes. After I 5 sittings the scabs became detached and the ulceration commenced to heal. In the course of two months the ulceration had entirely healed; there was at no time any marked reaction, and the skin retained an almost normal appearance. Later on, however, an acute dermatitis set in on the cyclid which led to ectropion of the lower lid. Altogether 100 exposures were given.

Sequeir ${ }^{1}$ ) reported four cases of ulcus rodens, the diagnosis of which had been microscopically confirmed. In these Roentgen-radiation had given excellent results. In a later publication ${ }^{2}$ ) the same writer reported 12 cases of the same disease and perforating ulcer treated by X-rays. Five of these cases were cured, but of their permanency the writer could not at that time spcak. In a third report (British Medical Associ:tion, Section of Dermatology, Manchester, 1902). Sequeira stated that he had indeed obscrved relapses after X-ray treatment, but that these were in turn entirely removed by further treatment. Out of So cases of rodent ulcer $3+$ were cured. He believed that those cases were unsuitable for the treatment in which bone or cartilage are involved; he found the hardgrowing border of the disease resisted the treatment for a long time.

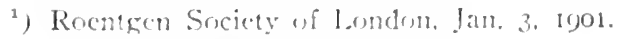

${ }^{2}$ Treatment, March, 1001, Nis. I. 
Th. Stenbeck ${ }^{1}$ ) treated 2 cases of epithelioma by X-rays, exposures being giren daily for several months. Reaction set in after 8 days. On pressure fairly long yellowish plugs could be squeezed from the diseased area. The cases healed completely and without relapse. In Stenbeck's opinion the irradiation acts electively.

In 4 later cases $S j o ̈ \mathrm{gren}^{2}$ ) observed after 25-33 sittings absorption of incluration, removal of scabs, healing of ulcers, and disappearance of subjective phenomena, such as prickingsensations and tenderness. The skin afterwards looked soft and smooth and a shade darker in all these cases. The treatment was carefully carried out, reaction being avoided as far as possible.

Stenberk and Bollean published in the July number of the Archives d'électricité médicale 5 other cases of malignant disease treated in this way.

In a report published in the $73 \mathrm{rd}$ Naturforscherversammlung, ${ }^{3}$ ) Sjögren advised the deliberate induction of energetic reaction so that necrosis and sloughing of the morbid tissue might be effected. He states that the result of this procedure is to leave a clean open wound which heals with a clear atrophic scar. Of the cases thus treated by him several had remained free from relapse up to the time of writing ( $\frac{1}{2}$ to I year).

Johnson and Merill $^{4}$ ) observed in 5 cases of epithelioma that Roentgen-radiation diminished suppuration and, after about 50 sittings, brought about healing almost without any visible scar. Beth operators used soft tubes, and endeavoured to produce "Roentgen-burns" (sic). Chamberlain") obtained very favourable results in 13 cases of carcinoma of the skin by means of hard tubes at 4-6 inches distance, 6 minutes exposures, and sittings held daily, or later twice a week. This author

1) Congr. internat. d'Electrologie et de Radiologie médicales, Paris, I9oo. Annales d'électrob., Sept., Oct., 1900.

2 Fortschr., IV. 4. 1. I 66.

3) Fortschr., V, No. 1, p. 38 .

4) Philardelphia Medical Jomrnal, noo, No. S.

5) Jourmal of Electro-therapentios, New York, May, igor. 
recommends for X-ray treatment those cases in which there are as yet no signs of glandular involvement. Other good results in cases of carcinoma were reported by $S$ mith, $\left.{ }^{1}\right)$ /Villiams," Pusey,") G. B. Ferguson, ${ }^{4}$ ) I. B. Leciack,") I. F. Rinchart,"

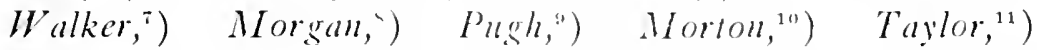
Williams, ${ }^{12}$ ) and $S$ (hilf ${ }^{1: 3}$ ). Puscy observes that he employed the method recommended by the author and Schiff (hard tubes). G. H. Lancashire describes the course of events in a case of ulcus rodens treated by Roentgen-rays as follows: In the first place the hard border of the growth becomes softened, the ncighbourhood becomes crythematous; the wax-like appearance of the ulcer-base gives way to a darker red, its smoothness disappears and granulations shew on the surface, which grow apace. In course of time the base of the ulcer becomes level with the surrounding skin, and may even reach a higher level. Graclually new epithelium spreads orer the ulcer from its borders.

On the whole, from the comparatively little experience so far gained, it may be said that Roentgen-radiation is an effectire remedy in malignant affections of the skin which have not already involved the lymphatic glands, a remedy which remores the disease at any rate for a considerable time, and gives excellent cosmetic results. As regards the question of the intensity of irradiation which should be applied, the remarks already made on this subject when dealing with hupus will apply here with equal force. Several writers (Sjögren, Chamberlain,

3) Philadelphia Medical Journal, Dec. I. Inoo.

${ }^{2}$ ) Boston X. \& S. Journal, Jan. i7 and April 4. Ioor.

3) The Journal of the Ancrican Nedical Asseciation, Sept. 28, 1901.

3) Brit. Med. Journal, licb. I, 1902.

") Scot. Journal, Fel., 1902.

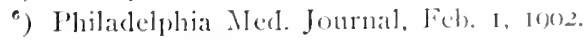

7) I.iverpoes Med. Inst., April 17, 1002.

") Ibid. F(b). 13, 1902.

") Brit. Med. Jomrnal, April t2, 1902.

1") Nerl. News, April 5, 1902.

11) Brit. Ned. Journal, May 3, roz, and Brit. Ned. Assoc, Nanclester, I9O2.

12) $11, i 11$.

13) K. K. Ciesellschaft der Aerze in 11 ien, Fels, 2I, 1902. 
Pusey, Schiff) have recorded that they have obtained good results with the milder method-the use of hard tubes.

For several weeks preceding the publication of his work the author, in conjunction with Prof. Ehrmann, had been treating a case of carcinomatous ulceration of the soft palate. This was in a patient 60 years of age, and the diagnosis had been confirmed by means of the microscope. The ulcer was the size of a thaler and $\mathrm{I} \mathrm{cm}$. in depth. Its borders were hard, the base coarsely granulated and readily bleeding. There was considerable pain. The method of treatment was as follows: The patient held in his mouth a tube lined with lead-foil, one end of which was placed over the ulcer. The face was protected by a leaden mask. A hard tube was placed opposite the external aperture of the tube, and exposures given for ten minutes daily. After the seventh exposure the borders of the ulcer felt considerably softer, and its size had decreased centripetally to that of a kreuzer; there was no visible reaction. Moreover the pains had been relieved and the swelling of the lymphatic glands had been strikingly diminished.

Scholt ${ }^{1}$ ) found by microscopic examination of Roentgenised-carcinomata that the malignant cells degenerated and were finally destroyed just as in the case of normal epithelium. One specimen shewed abundant signs of commencing mitosis.

\section{Mycosis fungoides, Lepra, Sarcoma of the Skin.}

Scholtz describes 3 cases of mycosis fungoides in which an energetic treatment by Roentgen-rays was followed by superficial necrotic changes leading to complete disappearance of the morbid lesions, including both fully developed tumours and pre-mycotic foci. Norman $/$ ' alker and $H$. G. Brooke have also reported good results in the case of the same affection ${ }^{2}$ ). One case of sarcoma of the skin was also favourably influenced. In two cases of lepra, however, Scholta achieved no success; de la Camp's experience was similar ${ }^{3}$ ).

1) L. C.

$\left.{ }^{2}\right)$ Brit. Med. Assoc., Manchester. July 30, I902.

$\left.{ }^{3}\right)$ Fortschritte, Vol. IV. 
Scholtz examined histologically the effect on the lepra lesions by Roentgen-radiation. He found the morbid infiltration rather less in the regions treated, while the bacilli, which were abundantly present, were slightly more granular in appearance than elsewhere. They were, however, readily stained, and had certainly not diminished in numbers. Irradiation had therefore produced practically no effect on the pathogenic micro-organisms.

Chronic ulcerations of various kinds.

Many reports have been published which shew that chronic ulcers of various kinds which had proved partially or completely refractory to other methods of treatment healed readily under X-rays. Thus Sjögren and Sederholm ${ }^{1}$ ) described four cases of ulceration (their exact nature was not indicated) in which 17 to 38 exposures of Io minutes each brought about healing. Weak currents were employed.

Colleville ${ }^{2}$ ) treated varicose ulcers in this way, and Sequeira ${ }^{3}$ ) some cases of perforating ulcer. Both obtained successful results. Further experience in this branch of Roentgen-therapy is much to be desired.

3. Acute and chronic exudative Dermatilis, and granulationformations.

Halm and Albers-Schönberg ${ }^{4}$ ) foumd that Roentgenradiation in the case of chronic and acute ecsema resulted in diminution of the discharge, drying of the skin, relief of itching, and removal of crusts and scales after comparatively few exposures $\left.{ }^{2}\right)$. Nany other writers confirmed these observations $\left(\right.$ Grummach $\left.{ }^{5}\right)$ r. Ziemsien,") Julassy,") Sharpe,", Sjögren

1) Fortschritte, Tol. TV. No. 4. 1\% I(12.

2) L'Union med. rlu Nord-Est, Aug. 30, 189y.

3) Merlical Recurd, inol.

4) Fortschritte. Vol. II, No. 1, IP. B(i-2.4.

s) Deutsche mel. Wochenschr., IRon. No. 37.

*) Congr. f. Innere Merlicin, Wienbatlen, Ingrit.

7) Fortschritte, Vol. III, No. 3, p. IIS.

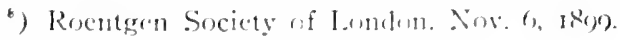


and Sedcrholm, ${ }^{1}$ ) Payne, $\left.{ }^{2}\right)$ Scholt,$\left.^{3}\right)$ Schiff ${ }^{4}$ ) and others). All these writers stated that a course of 6-20 sittings considerably relieved the trouble. Sjögren and Sederholm noted especially good results in cases associated with lichenification and exudation.

The author's own experience in this field is not very large. Up to the present time he has seen no reason to abandon the well-tried treatment of eczema, as taught by the Viennese school, in favour of Roentgen-therapy. He believes that no new form of treatment deserves recognition unless it proves effective when other methods have failed, or unless it does better than what has been done before. Nevertheless, he has tried the Roentgen-treatment in some cases of eczema by way of experiment, and can quite confirm the opinions of Hahn and Albers-Schönberg. In these experiments the author adopted his usual technique, using hard tubes at a good tube-distance, and suspending operations at the first signs of reaction. The effect was at first to produce diffuse reddening, slight intumescence and signs of desquamation. In two cases the results were not permanent, the disease afterwards appearing again. In a case of eczema, with tylosis of the palm, the treatment proved ineffective.

Roentgen-treatment for

\section{Psoriasis}

was adopted with more or less success by Albers-Schönberg, ${ }^{5}$ ) Grnnmach, ${ }^{6}$ ) v. Ziemssen, $\left.{ }^{7}\right)$ Rubinstein, ${ }^{8}$ ) Sharpe, ${ }^{9}$ ) Payne, ${ }^{10}$ )

1) Fortschritte, Vol. IV. No. 5, p. I75.

${ }^{2}$ ) Roentgen Society of London, Nov. 6, I899.

$\left.{ }^{3}\right)$ L. C.

3) Dermatologen-Congress, Breslau, I90I.

b) Fortschritte, Vol. II, No. 4. p. IfI.

๑) L. C.

$\left.{ }^{7}\right)$ Aerzte Verein München, June 8, i898.

$\left.{ }^{8}\right)$ Berliner med. Gesellsch., Nov. 8, 1899.

๑) L. C.

$\left.{ }^{10}\right)$ L. C. 
Grouven, $\left.{ }^{1}\right)$ Hahn, $\left.{ }^{2}\right)$ Sjögren and Sederholm, ${ }^{3}$ ) and Scholt $\left.{ }^{4}\right)$. They found that after the first 4 or 6 exposures the scaly plaques fell off without learing the bright-red points characteristic of psoriasis. (Hahn, Albers-Schönberg). Large plaques must be exposed at a considerable tube-distance. Exposures are given daily for the first two or three times, then every alternate day, and finally every third day (Schollz). The author himself recommends treating the disease, when it involves a large surface of the body, with the tube at $30 \mathrm{~cm}$. distance, giving exposures of IO-I 2 minutes each; small plaques can be treated at a shorter tube-distance and with correspondingly short exposures. The usual course of events is as follows:

Should there be any very red plaques present they become lighter in colour after three or four sittings, yellow-brown pigmentation collects round their borders; this gradually becomes darker, reaching finally a brown-black shade. The scales fall, hyperxmia disappears, and the pigmentation spreads still further. The latter, however, soon disappears. Scholtz's experience agreed with the foregoing in most cases. Sometimes the results were lasting: in other cases relapses occurred. Other observers, on the other hand, report relapse to be the rule and state that it often actually occurs during the treatment.

Scholtz recommends an auxiliary treatment by the ordinary methods on the termination of reaction and desquamation.

Microscopical examination of psoriasis-lesions after exposure to X-rays were made by Scholtz. He found scarcely more than the ordinary appearances seen in the disease itself. The prickle-cell and granular layers were deeper than normal, and slight infiltrations were still to be noted on the papillary body and round the subpapillary vessels. The epithelial cells shewed the same changes which are found in normal skin exposed to X-rays. Peculiar pigmentations were visible in the papillary body and in the rete. According to Scholtz,

\footnotetext{
1) I.. c.

2) Fortschritte, Vol. IV, No. 5, p. go; Vil. I, Nu. I, p. 39

8) L. C.

•) L. c.
} 
the situation of this pigment is not intercellular, but rather in the cell-walls and their protoplasmic substances.

\section{Prurigo.}

Scholt $\approx$ treated this affection also to X-rays by way of experiment, but without conspicuous success. On the other hand this observer, also Sjögren and Sederholm, obtained decidedly beneficial results in cases of pruritus ani and vulve after very few exposures ( 6 Sjögren and Sederholm). From the few accounts to hand, unfortunately, the benefit does not appear to be lasting.

Scholtz obtained a temporary improvement in cases of pemphigus foliaceus. In a case of lichen ruber planus he obtained striking success; the papules were absorbed, the process being accompanied by desquamation and pigmentation.

The relief of pruritus in general by means of Roentgentreatment is quite comparable to that obtained by d'arsonvalisation. It should be borne in mind that the presence of the hightension electricity accumulated on the surface of a Roentgen-tube in action is quite sufficient in itself to explain the relief afforded in the cases of pruritus.

\section{Lupus erythematosus.}

In I 898 E. Schiff treated a female suffering from this affection by means of X-rays. The disease took the common form of "butterfly-patch" on the face, and for purposes of comparison the left side only of the lesion was irradiated. Schiff observed ${ }^{1}$ ) that the exposed region became of a darkred colour; scales became detached, and the border of the patch became levelled. Here and there spots varying in size from a pin-head to a linseed became conspicuous by reason of their intense red colouring.

In course of time the infiltration within the exposed area entirely disappeared, the skin became perfectly smooth, white, and level. The sight of the original plaque was surrounded by a thin ring of pigment. The further course of events in

1) Wiener med. Presse. I890, No. 2. 
this case was somewhat remarkable, as described in a report published a year later by the author in conjunction with Schiff ${ }^{1}$ ).

Sone little time after apparent healing fresh foci of disease appeared round the original site in the shape of prominent reddish lesions, scaling, and with plugged follicles. The hair fell from the left temporal region, this part having come within the X-ray cone. In the course of the next few weeks the secondary lesions disappeared, leaving the skin white and smooth. The alopecia, however, persisted. The appearance of permanent alopecia after a single course of irradiation without any marked reaction is quite unique. The author can only explain this circumstance by the supposition that an outbreak of lupus erythematosus must have been determined in the temple itself, since this disease, as is well known, usually leads to permanent loss of hair when affecting the scalp. There were distinct evidences, as has been said, of fresh disease in the immediate neighbourhood of the site of the original disease. These were probably determined by the irradiations. We know that other forms of irritants, such as caustics (Kaposi), will often provoke fresh eruptions of lupus erythematosus.

In the treatment of this case extreme caution was employed; any marked reaction was avoided by the use of hard tubes.

Sjögren") procecds on different lines in his Roentgen-treatment of lupus erythematosus. Il object is to provoke a strong reaction (redness, swelling, exudation, and scab formation). He states that after this reaction has subsided the crusts fall, leaving an atrophied light-red skin which shows no traces of follicular apertures. Schols a alopts the same procedure.

The author, in company with Silhif and many others

1) Beiträge zur Dermatologgin mul Syblilic. Jestschrift für IIofratl Neumann, igros.

2) I. . . 
$\left(\right.$ Jutassy, $\left.{ }^{1}\right)$ Haln,,$\left."\right)$ I. Neumann,", Grouven, $\left.{ }^{4}\right)$ Llaberia, $\left.{ }^{5}\right)$ Lion, ${ }^{6}$ ) Scholt,${ }^{7}$ ) Woods, ${ }^{8}$ ) Taylor,")), has found that though often enough satisfactory results have been obtained in the first instance, treatment has constantly to be resumed on account of ever-recurring relapses. By very prolonged treatment in many cases a permanent cure may be attained. The scars are then smooth, very white, and level with the surrounding skin. It must be admitted, however, that many cases are made worse by the treatment (Nelmann, P.S. Abraham).

\section{Acne vulgaris, rosacea Furunculosis.}

Gautier and Pokitonoff ${ }^{10}$ ) reported in I 892 upon I 7 cases of acne vulgaris and rosacea, which they had cured after daily exposures of 5-6 minutes each. They used currents of 4 ampères and $\mathrm{I} 8-20$ volts, and a tube-distance of $30 \mathrm{~cm}$. After the sixth sitting the disease began to diminish, the skin became paler, dilated vessels became less evident, and healthy skin appeared amongst the pustules and papules.

K. Ullmann's ${ }^{11}$ ) experience in a case of obstinate acne of the back was as follows: After IO-I 5 sittings the skin became diffusely reddened and the papules swollen; the latter, however, did not suppurate, but became absorbed, whilst the overlying skin exfoliated. The disease disappeared completely after 50 sittings, leaving well-marked pigmentation behind.

$H a h n^{12}$ ) and Jutassy ${ }^{13}$ ) also obtained successful results in the case of both of these affections.

1) Fortschritte, Vol. III, No. 3, p. 1 I9.

$\left.{ }^{2}\right)$ Ibid. IV, 2,86 .

$\left.{ }^{3}\right)$ K. k. Gesellsch. d. Aerztc, Nov. I4, I900.

1) L. c.

s) Fortschr., V, I, 56

$\left.{ }^{8}\right)$ Dermatolog. Congr., Breslau, igor.

$\left.{ }^{7}\right)$ L. C.

$\left.{ }^{8}\right)$ The American Journal of Med. Sc, Dec., I90r.

$\left.{ }^{9}\right)$ L. c.

1") Compt. rend. du XII. Congr. internat. de Medécine Moscow, Vol. IV, pp. $382-386$.

ii) Wiener dermatol. Gesellsch., Nov. 28, 1900.

12) Fortschr., Vol. IV, No. 2, p. 96; Vol. V, No. 1, p. 39.

$\left.{ }^{13}\right)$ L. C. 
The author's own experience includes I case of rosacea and 2 cases of acne vulgaris. In these distinct improvement was brought about after a rather long period of treatment with weak irradiations. Small red spots persisted for some time in place of the papules; these spots afterwards became pigmented. In one case of acne a relapse occured a few months later. The other cases were only recently discharged, so that nothing can be said as to the permanency of their cure.

In a case of chronic furunculosis of the neck the patient was relievel of the trouble for a considerable time by irradiation pushed to the stage of epilation. Török and Schein explain the successful action of Roentgen-rays in affections of this class, on one hand, by their parasiticidal qualities (?), and on the other hand, by their influence on the sebaceous glands, in which, as in the sweat glands, the secretion is diminished ${ }^{1}$ ). In the author's opinion the desquamation which so often results from irradiation is the most important factor.

\section{Nowils flammens.}

Julassy ${ }^{2}$ ) claims to have cured a case of this kind after inducing an X-ray dermatitis. He says that irradiation produces contraction, thrombosis, and obliteration of the vessels. In the Breslau-clinic, however, the results were less successful (Lion, Scholtz).

In conclusion we may mention that Sorel and Soret claim to have cured a case of elephantiasis-like induration of the hand, of rapid onset, after 3 sittings of $10-25$ ininutes each $\left.{ }^{3}\right)$. Sjögren and Sederholm cured a case of verrucas and finally, other operators (Gocht and Schollz) cured nevi spili, and Török and Schein urticaria pigmentosa by means of Roentgenradiation.

1) I. . C.

2) Fortschr., Vol. II, No. 5. p. Int.

3) Lat Normandie Mialicale, Fill., ISes. 


\section{The Treatment of Internal Diseases by X-Rays.}

No account of the therapeutic uses of X-rays would be complete without some reference to the facts which warrant the employment of this method in the treatment of certain internal disorders.

Several able and impartial writers have already furnished us with noteworthy data upon this subject. Thus Voigt ${ }^{1}$ ) mentions a case of pharyngeal carcinoma in a man, xt. 89 , in which the pain was relieved. Gocht ${ }^{2}$ ) describes 2 cases of inoperable mammary carcinoma in which daily irradiation soon relieved the excruciating neuralgic pains, without, however, affecting the appearance of the growth in any way. Johnson and Merill $^{3}$ ) described similar results.

The author himself learned from a private communication which he received from an eminent surgeon in Vienna that a woman suffering from metastic carcinomatous growths in the peritoneum was relieved of intense pain by the X-ray method; the malignant process itself was, however, uninfluenced. Clark states that in a case of ulcerated carcinoma of the breast he has seen distinct diminution in the size of the tumour and of the auxiliary glands after about 30 sittings of 15 minutes each ${ }^{*}$ ). Despeignes ${ }^{5}$ ) relates the case of an old man who suffered from cancer of the stomach, in whom "considerable improvement" took place after 80 sittings held twice daily for $\frac{1}{2}-\frac{1}{4}$ hour. P. H. Ei kman ${ }^{6}$ ) claims that he has cured a case of carcinoma of the breast, and we learn from Dr. Weldor of New York ${ }^{\top}$ ) that John G. Gilman of Chicago has cured 50 cases of cancer by means of X-rays. Quenisset and Seguy report that they obtained improvement in a case of recurring and metastatic sarcoma ${ }^{\mathrm{s}}$ ). C. Beck treated a recurring sarcoma of the lower:

1) Acrztl. Verein, Hamburg, Feb. 3, 1896.

$\left.{ }^{2}\right)$ Fortschr., Vol. I, No. I.

$\left.{ }^{3}\right)$ Philadelphia Med. Journal, No. 8, 1900.

${ }^{4}$ ) Brit. Med. Journal, No. 8, I90I.

5) Lyon Méd., Dec. 20, 1896.

") Krebs und Roentgenstr., Harlem. Ino2.

7) Revue des Revues cit. "Wissen f. Alle," I902, No. I, p. I4.

${ }^{\circ}$ ) Quoted by Fov'cau de Courmellos, Ann. de médecine et Chirurgie infantile, Vol. V, No. 5, p. I64. 
part of the thigh in a man, aet. 36 , by exposure given 2-3 times a week for $10-45$ minutes. Ife accomplished a "cure" which lasted for 9 weeks (sic) ${ }^{1}$ ).

The writer was induced by a Vicnnese practitioner to treat an inoperable chondro-sarcoma of the left parotid gland in a man aet. 70 . Treatment was griven daily for 20 days, using hard tubes at a distance of I $5 \mathrm{~cm}$., for 6-S minutes. Results were entirely negiltive.

A similar case treated by a colleague proved equally unsuccessful.

Another property of Roentgen-radiation which has already been referred to, viz., its analgesic effect, suggested its cmployment in painful affections generally. Stembo") succeeded in relieving 21 out of 28 cases of neuralgia, giving from 3 to 10 sittings of about 5 minutes each. The author has himself proved experimentally that analgesic properties of X-rays are not to be ascribed to suggestion-effects, since with the current reversed no improvement can be obtained. The true explanation is to be found, he believes, in the electrical stimulation of the peripheral sensory nerves, which inhibits the functions of deeper-lying nerves and so relieves the neuralgia.

The author succeeded in the case of an old man who suffered from a trigeminal neuralgia, which was scarcely relieved even with morphia, in bringing about marked improvement after I I sittings; there was no visible reaction in this case. In a second case irradiation proved unsuccessful.

Grunmach ${ }^{3}$ ) employed Roentgen-therapy with varying success in cases of neuralgia of the face, occiput, and intercostal nerves, and in articular and muscular rheumatism. Southgale Leigh ${ }^{4}$ ) reported before the Seabord Medical Society the case of a patient who had been shot in the upper part of the thigh, who complained of pain in the knee-joint. The pain disappeared

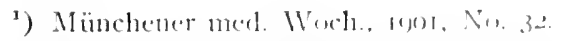

2) D. Therapic 1]. Giegenw., 1400, No. (1.

s) Deulsche med. Woch., trome. No. 37.

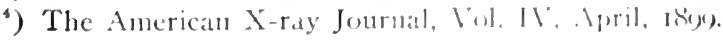


after an exposure lasting 4 hours. In another case irradiation is said to have removed colic due to biliary calculi.

Rheumatic and tuberculous joint-affections are said to have been improved by Roentgen-radiation. Sokolow treated acute and chronic rheumatism in children's joints by covering the diseased parts with woollen blankets and exposing them for I O-20 minutes at a tube-distance of $50-60 \mathrm{~cm}$. He states that after $\mathrm{I}-4$ sittings the pains disappeared, the swelling perceptibly diminished, and the mobility of the limb improved ${ }^{1}$ ).

According to Southgate Leigh, a tubercular elbow-joint was completely cured by 2 -hour exposures, administered two or three times a week.

Kirmisson states $^{2}$ ) that he much improved a tubercular wrist-joint affection, in a girl of 14 , in 65 sittings lasting I o minutes each. Similar reports come from $B a z y$, Lancaster, Sainton ${ }^{3}$ ) and Escherich ${ }^{4}$ ).

Even pulmonary tuberculosis and acute inflammation within the chest are stated to have been cured by some authors (according to Rendu and $d u$ Castel, ${ }^{5}$ ) by Roentgen-treatment; Bergonié and Mongour, ${ }^{6}$ ) Sinapius, ${ }^{\top}$ ) Chanteloube, Descamps and Rouilliés, ${ }^{8}$ ) Destot and Dubard ${ }^{9}$ ). Alusset and Bédard ${ }^{10}$ ) report on a case of chronic tubercular peritonitis. After 50 sittings given daily for $\frac{1}{2}$ hour, employing powerful tubes at a distance of $20-23 \mathrm{~cm}$., the ascites disappeared, likewise the hard irregular masses which had previously been felt through the abdominal walls, and the general condition of the patient improved. Two years later these writers reported a similar case. In conclusion we may mention that Paulin Méry and

$\left.{ }^{1}\right)$ Wratsch, 1897, No. 46.

2) Soc. de Chirurgie, Feb. 2, i 898 .

3) Quoted by Bergonié, La sem. méd., ז898, p. 349.

${ }^{4}$ ) Revue mensuelle des maladies de l'enfance, May, i898.

") Soc. méd. des Hôpitatix, Jan. I,5, I897.

") Acad. de médecine, July, I897.

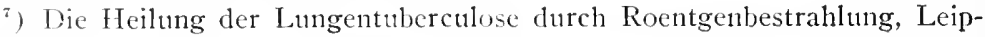
zig, 1897 .

") Arch. d'électric. méd., May I5, I\$97.

5) Gazette des Hopitaux. Aug. I3, 1898 .

$\left.{ }^{10}\right)$ Echo méd. dı Nurd, I898, No. 46 I. 
Campenon ${ }^{1}$ ) are said to have hastened the union of a fractured bone, and $A$. de Lancastre" ) to have cured a case of suppurating osteo-periostitis by means of Roentgen-radiation.

The author has but little to contribute with regard to this particular department of Roentgen-therapy; he has seen practically no physiological or therapeutic effect which could be ascribed to the influence of the rays upon internal organs. Nevertheless he is not prepared to deny the possibility of such an effect existing; he believes that Kienbock") goes too far when he says that X-rays can only produce deep-lying changes when an ulcer is first formed. There may be rays of another kind from the tube which influence tissues other than the skin.

\section{$\S$ 32. Physiological Effects of X-Rays.}

Soon after the publication of Roentgen's discovery it became known that his rays have a definite influence upon the protoplasm of living tissues. Investigations were duly made, with the following results:

The chief objects of enquiry were as follows:

I. The influence of Roentgen-rays upon the higher organisms, upon certain regions and their functions.

2. The histological changes in the cells.

3. The influence of the rays upon bacteria.

4. Their action upon the plasmodial activity of various low forms of life.

As regards I, there is little to be added to what has been already stated in the previous section. Capranica ${ }^{4}$ ) observed that moles exposed to Roentgen-rays are excited for several hours afterwards, but that no alteration could be noted in the amount of carbonic acid they excreted.

1) Quoted by Foa'can de Courmilles, Amm. We meid. el Chirurg., Igor, No. 5.11 .164$.

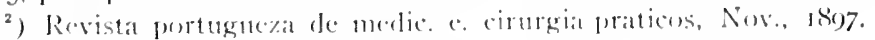

3) 11 r. med. Presse, 1001, No. 1r).

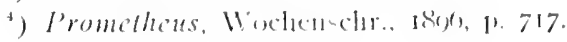


On the other hand, Tarkhanoff ${ }^{1}$ ) states that frogs whose cerebra have been exposed to the rays sustain a loss of reflexsensibility. After replacing the creatures in water their skin remains very dark-coloured, and only regains its lighter hue after several hours.

Gaston Seguy and F. Quenisset") noted that after they themselves had been exposed to the rays for a long time they suffered from violent and irregular cardiac palpitation. A female patient also who had been under treatment complained of the same trouble, coupled with a feeling of oppression.

Sabrazis and Rivicre ${ }^{3}$ ) carefully examined the heart's action in cold-blooded animals (frogs) after Roentgen-radiation. They could observe no change in cardiac rhythm even after prolonged exposures.

Destot ${ }^{4}$ ) watched the pulse while the hand was being irradiated by a vacuum-tube worked from a static apparatus, and afterwards from a coil. The exposure on each occasion lasted one hour, the tube-distance being $5 \mathrm{~cm}$. A pulse-tracing was taken by a Marey sphygmograph. The static machine caused the disappearance of the dicrotic wave after Io minutes; the whole curve became higher, but the rate of pulsation was not affected. When the coil was used to work the tube the pulse shewed at first a higher tension. This, however, soon fell, and was succeeded by arhythmia and intermittent pulsation. In both cases the radiation was of like intenstiy. Destot concludes that the cause of the physiological disturbances observed in Roentgen-radiation is to be found in the effect upon the sensory nerves; these affect the spinal cord, and, in a reflex manner, the vascular system.

L. Leccrcle") irradiated the shaven posterior regions of rabbits, and observed the temperature of the skin and rectum both before and after the experiment. ('The time of exposure is not stated.) The first effect was a fall in the temperature;

1) Gaz. Butkin, 32.

$\left.{ }^{2}\right)$ Compt, rend. de l'Acad. de Sc., Vol. CXXIV, p. zyo.

3) Ibid. 1). 981.

4) Compt. rend., Val. CXXIV, p. 1115.

") Compt. rent. Acad., Vol. CXXV, p. 234. 
this was soon followed by a rise to above normal. The same observer found ${ }^{1}$ ) that the anmal's skin parted with more heat after irradiation, and this phenomenon was maintained for a considerable time. In a later publication lecercle ${ }^{2}$ ) states that the function of perspiration in rabbits becomes temporarily impaired by exposure to the rays. In yet another series of experiments it was shewn that exposure on 3 successive days caused increased excretion of phosphates in the urine, which change was maintained for a couple of days.

Several writers argued from certain clinical phenomena (headache, insomnia, dificulty in micturition, menstruation, etc.), which appeared occasionally after irradiation, that the rays had some influence upon internal organs. Thus Oudin, Barthélemy and Daricr ${ }^{3}$ ) observed that irradiation with too powerful a current, or with certain other kinds of defective technique, resulted in gastro-intestinal disturbances, palpitatation, etc.

In a later publication ${ }^{4}$ ) these authors described "visceral complications" as occurring after radio-therapy in cases where there were no actual signs of gastric-disorder. They refer these phenomena to a disturbance of the sympathetic system. ('They would seem to overlook the fact that in emotional people these occurrences not uncommonly result from any kind of psychic disturbance.) Amongst the many thousand Roentgen operations which the author has conducted for the past six years he has, however, met with no such case of "visceral complicatıon." The trembling and disturbances of sensation which Oudin mentions as occurring on the hands of those who work with X-rays are not to be explained in this way. The trembling may, of course, be due to very different causes; the sensory disorders may result from the dermatitis which careless operators of ten contract.

IV alsh") also describes complication similar to those men-

1) Compt. rend. Acarl., Vol. CXXY. 1. 5i.3.

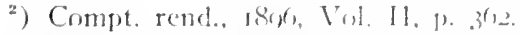

3) Monatsch. f. prakl. Dermatrilogie. Vul. 25. No. 29.

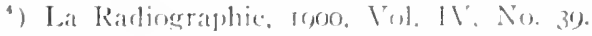

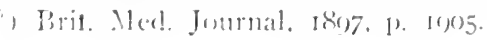


tioned by Oudin and Barthélemy. He has seen effects like those of sunstroke, also gastric disturbances.

Rodet and Berthin ${ }^{1}$ ) after exposing animals to intense irradiation produced dermatitis, paralysis, and convulsions followed by death. They found at the autopsy meningomyelitis (thickening and adhesions of the meninges, congestion of the spinal cord, cell-hyperplasia and small hæmorrhages), which was evidently not due to sepsis; bacteriological examination of the blood, peritoneal fluid, and spinal cord giving negative results.

Scholtz found on making enquiries into the effect of irradiation upon internal organs that even intense exposure, such as leads to necrosis of the tissues, causes no evident signs of mischief along the gastro-intestinal tract. Young animals, whose anterior fontanelles had not yet closed, died sometimes with paralytic symptoms, and sometimes without any definite symptoms at all, after their crania had been exposed to the rays. Several other observers state that small animals, such as guineapigs, suffer from paralysis after irradiation of the occipital region; sometimes sudden death ensues. Considering the thinness of the skin and bones in these cases, one can readily understand the possibility of the rays penetrating to the central nervous organs and so producing dangerous or fatal lesions. It is known that high-tension electricity has an unfavourable influence upon small animals. (See the author's experiments, pp. I 2 I, I 45, I 74. )

Intense irradiation of a rabbit's eye has produced necrosis in the neighbourhood of the eyeball, but no important changes in the eye itself.

Investigators have for some time been concerned with the question as to whether Roentgen-rays are visible, or whether they exert any effect upon the retina or other structures of the eye. Several observers have assumed that the retina is sensitive to Roentgen-rays. Thus 1 xenfeld ${ }^{2}$ ) came to this conclusion after some experiments he made upon arthropedes. He placed the animals in a box made of half wood and half lead, and

1) Gazette des Húpitaux, May 7, 1898 .

3) Centralbl. f. Physiologic, X. No. 6, 1. 147 and No. 15, p. 436. 
exposed this box to the rays; the animals all moved to the wooden portion. Blinded animals did not act in this manner. Brandes ${ }^{1}$ ) made an experiment to see if aphakic persons are sensitive to the rays. A girl, both of whose lenses had been removed, received an impression of light from a Crookes tube which had been previously covered with a dark cloth.

Brandes also believed he received a risual impression from Roentgen-rays after covering both his cyes with leaden masks; he believed that the rays gained entrance round the masks (which were shaped like spectacles), though they did not appear to traverse a central aperture which had becn made in the masks themselves ${ }^{2}$ ).

This observation was disputed later by Radiguet and Guichand, ${ }^{3}$ ) also by Daricix $\left.{ }^{4}\right)$. Chalpuccky found that only apertures above $4 \mathrm{~mm}$. allowed the passage of perceptible rays. The rays, moreover, were only effective in the periphery of the visual field; the central part of the lens would appear to be opaque to them, and this probably explains why radiograms of the lens give shadows almost as dense as those of the whole eyeball. G. Bardet, ${ }^{5}$ ) to some extent, confirms Brandes' experience; he belieres that any one within the zone of action of a vacuumtube receives an impression of light. For this, howerer, it is essential that the room be absolutely dark and that there be no colours on the walls, since many colours fluoresce on exposure to X-rays. Bardet finds that the eye placed a few centimetres in front of a covered vacuum-tube in action receives just such a feeble impression of light as is produced by moving a lighted candle before the closed lids. When the apparatus stops working this impression disappears, and the same happens if a screen of iron, copper, or lead be interposed. A glass screen, however, diminishes the effect but little, an aluminium screen not at all. If the tube be turned on its axis through $I 80^{\circ}$, so that the cathode-rays strike the side of the tube which is furthest from

1) Sitzungsh. d. Preuss. Akarl. d. Wics. ISg6. p. 547.

2) Quoted by Gebhardt. Die Iteilkralft des Lichles, Leiprig, I898, p. 278.

-) Acad. de Mérlec. Nov. iro, isog.

-) Qunterl by G. Bardit.

-) Compt. rend. de l'Acard de Sc., Vul. Cxxiv, p. Izs8. 
the eye, no light impression is received, showing that the "electric field" can have no share in this phenomenon.

Foreau de Courmelles ${ }^{1}$ ) examined 204 blind children. He found that only 9 of these received any impression of light from $\mathrm{X}$-rays.

It has long been known that electric currents and discharges applied to the eye cause a sensation of light. Purkinje ${ }^{2}$ ) produced this sensation by the electric stimulation of the retina and optic nerves.

G. E. Miller ${ }^{3}$ ) found that a constant electric current sent through the retina in an afferent direction produces on "closing" a light blue-violet sensation; applied in the reverse direction, however, there is a sensation of dark yellowish green. On opening the circuit, the reverse takes place. Darier ${ }^{4}$ ) found that in cases of amblyopia, without nerve-lesion, a sensation of light is induced by very feeble currents; where, however, there is atrophy of the optic nerve far stronger currents are required.

D'Arsonval ${ }^{5}$ ) shewed that the eye receives a quite similar impression of light when brought into an electromagnetic field produced by an alternating current of 42 periods. (Recently Berthold Beer ${ }^{6}$ ) has published observations to the same effect.) D'Arsonval emphasises the fact that a magnetic field acts like other physiological irritants, not by virtue of its intensity alone but by its alternations.

In variance with the above testimony, which speaks for some influence upon the eye by Roentgen-radiation, the experiments of Fuchs, Kreidls ${ }^{\top}$ ) and Gatti $^{\mathrm{s}}$ ) would seem to indicate

$\left.{ }^{1}\right)$ Compt. rend. de 1'Acad. de Sc., Vol. CXXVI, p. 919.

2) Briicke, Vorlesungen über Plyssiologie, Vol. II, p. 910.

$\left.{ }^{3}\right)$ Zeitschr. f. Psychologie und Physiologie der Sinnesorgane, Vol. XIV. p. 329 .

4) Bull. de la soc. franç., d'ophtha., 83, quoted by $\mathrm{Nagcl}$, Jahresübersicht. I884. P. 242 .

$\left.{ }^{8}\right)$ Compt. rend. de l'Acad. de Sc., Vol. CXXIV, p. I389.

") K. k. Ges. d. Aerzte, Wien, Jan. I7, I90z.

$\left.{ }^{7}\right)$ Centrall,l. f. Physiolngie, X, No. 9, p. 249.

") Annal. di Ottalm., XXVI, p. 344. 
no sensibility for the rays on the part of the retina. These observers found that no change takes place in the appearance of the risual purple on exposure to the rays; the latter, moreover, have no effect upon the pigment-epithelium. On the other hand, changes do occur in the anterior parts of the eyeball; these, however, are quite analogous to the changes in irradiated skin. The author called attention some time ago to the mild attacks of conjunctivitis which are liable to occur in patients whose eyes are not suitably protected. Himmel ${ }^{1}$ ) mentions the case of a patient who developed symptoms of photophobia after Roentgen-treatment for a patch of lupus on the forehead; this Himmel refers to irritation of the optic nerve. The author has pointed out in a previous communication ${ }^{2}$ ) that troubles of this kind can generally be aroided merely by the patient closing his eyes during the sitting. It would seem, therefore that the Roentgen-rays themselves cannot be held altogether responsible for the conjunctivitis, etc., since they frecly penetrate the thin tissue of the eyelids. On the other hand, were high-tension electricity the cause of the trouble, it can readily be understood that by closing the eyelids this electricity would be conveyed to other parts of the body and so easily earthed. Again, the author has frequently seen conjunctivitis make its appearance, although the eyes have been covered by leaden masks. Oudin looks upon accidents of this kind as effects of the X-rays upon the general organism ("accidents generaux").

Chalupecky's experiments ${ }^{3}$ ) shewed that inflammation of the conjunctiva, when the rays are directed straight upon the eyeball, may involve, by extension, the anterior chamber and its vicinity. This observer irradiated a rabbit's eye for $2+$ hours and found the following progressive changes: Inflammation of the eyelids, falling of the eyelashes, conjunctivitis, and irregularities in the cornea; the latter becane greyish-white, and, like the conjunctiva, covered with false membrane. $R$. B. / / ild ${ }^{*}$ ) saw a case in which panophthalmitis supervened upon excessive

\footnotetext{
1) I.. C.

2) Wiener mal. Presse, I899. Niı.31.

2) Centralbl. f. prakt. Angenheilk. Ang., Sept., p. 267.

-) British Med. Assoc. Manchecter, July 30, 1902.
} 
exposure to X-rays, necessitating enucleation. Chalupecky believes that these effects upon the eyeball are common both to X-rays and ultra-violet rays. As a matter of fact, we find in the literature of this subject morbid processes quite similar to the above which are ascribed to the action of intense white light.

The effect of Roentgen-radiation upon living skin, and the clinical and histological changes produced therein, will be more fully dealt with later; we have already discussed some of these changes when describing the method of treatment. Here we will only refer in a general way to the physiological peculiarities which characterise the Roentgen-reaction. These, which must always be borne in mind during the practice of Roentgentherapy, are:

I. The cumulative effect of irradiation. Although the immediate effect of each exposure may be but insignificant, the sum of these effects goes on accumulating, so that in the end we may get a sudden and unexpected reaction. The more intense the exposures, the sooner do the naked-eye changes become evident. The intensity and time of appearance of the reaction, therefore, afford a criterion as to the character of the irradiation (see Introduction, p. 5) ; they also give some indication of the course the reaction itself will probably run.

The author believes he was the first to point out the cumulative action of X-rays (Wiener med. Wochenschr., I 897 , No. Io), though most writers give Forster the credit. Kienböck and Scholt $\approx$ first indicated the important part played by the intensity of the irradiation in determining the time of appearance of the reaction.

2. The peculiar character and course of the changes brought about by irradiation (reaction, dermatitis).

3. The changes first affect the cells of the skin; from this the peculiar effect on the hairs arises.

4. A skin treated by Roentgen-radiation is for a long, time afterwards prone to again react after comparatively weak exposures.

Reports upon the influence of Roentgen-rays on bacteria are somewhat conflicting. Whereas many observers deny the rays 
any bactericidal action whaterer (Beck and Schultz, ${ }^{1}$ ) Beanregard and Guichard,") Berton,") S. Brunlon Blaikic, ${ }^{4}$ ) Blaise and Sambuc,") Grunmach,") Minck,") Polt,") Sabrazes and Rivière, $\left.{ }^{\prime}\right)$ Sormani, $\left.{ }^{11}\right)$ Wittlin, $\left.{ }^{11}\right)$ M. Wolff $^{12}$ ) and others), others again found them to possess distinct bactericidal properties (Bonomo and Gros, ${ }^{1 .}$ ) Fiorentini and Luraschi, ${ }^{14}$ ) Frantzins, $\left.{ }^{15}\right)$ Lortet and Genond, $\left.{ }^{1 \mathrm{1i}}\right)$ Miillsam, ${ }^{17}$ ) Rieder, ${ }^{1 \mathrm{~s}}$ ) Holzknecht and Spieler ${ }^{19}$ )).

We can only quote a few of the experiments dealing with the question of the influence of Roentgen-irradiation upon bacteria.

F. Berlioz (Compt. rend. Acad. d. Sc., I $\$ 96$, Vol. II, p. I09) irradiated bouillon-cultures of diphtheria bacilli for 16,32 , and 64 hours. Guinea-pigs were innoculated afterwards with these cultures, and it was found that the bacilli had not in the least lost in virulence. This result agrees with $/ V^{\prime}$ ade's (Brit. Med. Journal, February, I 896$)$ and Minck's experience.

Pott ${ }^{20}$ ) inoculated 25 test-tubes containing glycerinagar-pepton with tubercle bacilli. The tubes were kept for I month at a temperature of $37^{\circ} \mathrm{C}$. By this time

1) Zeitschr. f. Hyg., I896, p. 490.

2) Soc. de biolog., July 27, 1897 .

$\left.{ }^{3}\right)$ La sem. méd., I896, p. 283 .

${ }^{4}$ The Lancet, 1898 , II, p. 1425.

") Soc. de biolog., July Io, 1897 .

") Quoted by Bergmann.

7) Münchener med. Woch., r896, No. 5 ; rS98, No. 9.

") The Lancet, Nov. 20, 1897.

") Acad. de Science, May 3. 1897.

1") Quoted by Möller.

$\left.{ }^{11}\right)$ Centralbl. f. Baht., 2, p. 676.

12) Berlin med. Gesellsch., March 2, 1898 .

13) Giorn. med., June, I 897 .

11) Revue internat. d'électrothérapie, F(h., March, 1897. 1. 223.

$\left.{ }^{15}\right)$ Centralbl. f. Bakt., March 5, 1897.

$\left.{ }^{16}\right)$ Compt. rend., March 30, 1\&igo.

${ }^{17}$ ) Chirurgenverem, Perlin, Jan. 10, 1898.

$\left.{ }^{14}\right)$ Münchener merl. WVoch., 18,8, No. 4, 25.

1") Wiener merl. Club, Jan. 30, 1901.

") The Lancer, Nov. 20, I897. 
characteristic and pure colonies had developed. Of these 25 tubes 8 were retained for control purposes and I 7 were exposed to the rays. (It had already been determined that the glass-tubes themselves offered but slight resistance to the passage of X-rays.) Means were taken to secure an equal exposure for all the tubes by placing them on a revolving stand, above which the Crookes tube was fixed. Photographs were taken from time to time to make quite sure that the Roentgen tube was emitting $\mathrm{X}$-rays. The 17 tubes were exposed daily for 3 months, and in such a way that always the same 2 tubes were placed out of reach of the rays after $\frac{1}{2}, \mathrm{I}, 2,4,6,8$, and Io hours respectively, while the last three tubes were exposed for II hours each day. At the end of the experiment it was found that the cultures had been entirely unaffected by this treatment; they differed in no way from each other, or from the cultures in the $S$ control-tubes.

This experiment conclusively proves that Roentgen-rays possess no influence upon tubercle bacilli, and that a Roentgencure of tuberculosis (if, indeed, such has been actually effected) must be due to other causes.

J. Sabrazès and P. Riviere ${ }^{1}$ ) exposed cultures of the bacillus prodigiosus in a case covered over with black paper for 20 days, giving daily irradiations of I hour. Absolutely no effect was produced on the organisms. In a second experiment a small opening was made in the abdominal wall of a frog by means of a cautery. Into this aperture a capillary tube, which had been previously drawn through a culture of bacilli, was inserted. After an irradiation lasting several hours, the tube was withdrawn and its contents examined. The results were entircly negative; no increase in the number of white blood-corpuscles or change in the process of phagocytosis, as compared with non-exposed control animals, was discoverable.

1) Compt. rend. de l'Acad. de Sc., Vol. CXXIV, p. 979. 
H. Rieder ${ }^{1}$ ) exposed cultures of the micro-organisms of cholera, anthrax, typhus, diphtheria, also puscocci and bacteria coli. The cultures were covered by leaden plates, in the centre of which a large square aperture had been made. He found that after 48 minutes the colonies coinciding with the aperture perished, whereas the covered colonies continued to thrive. In these experiments Rieder used a $30 \mathrm{~cm}$. coil, the primary current being interruped 3 oo times per minute; distance of anti-cathode from the object, $10 \mathrm{~cm}$., time of exposure, 1-3 hours. In some cases the aperture in the leaden plate was covered with black paper. In order to ensure a sufficiently prolonged action of the most intense rays, a second interrupter in the form of a metronome was introduced into the circuit; this was selfacting and adjustable. It seemed unlikely that the bactericidal effect was due in any way to a chemical change in the nutritive medium brought about by heat-rays or fluorescent light from the vacuum-tube; there still remained, however, the possibility of effects caused by the electrical discharges from the tube. In order to eliminate this possible factor, Rieder employed a tin-foil screen to carry away the electricity. It was found that this procedure scarcely affected the bactericidal process. Moreover, on suitable arrangements being made to eliminate the X-rays themselves no bactericidal action could be secured. Finally, any action on the part of ozone was proved impossible.

Rieder also claims to have proved by experiment that the production of Roentgen-dermatitis also is solely due to $\mathrm{X}$-rays themselves ${ }^{2}$ ). By still more recent experiments, Rieder") claims to have confirmed his earlier deductions.

L. I.ortet and Genoud ${ }^{4}$ ) also cnpuired into the

1) Nünchener med. Woch., ISgR. No. 4. 11). so1-104.

") Mënchener merl. Woch., Jume 21, 18ise.

3) Il,icl. Matrch 11, 1002.

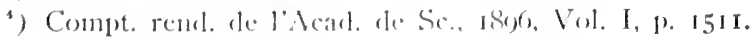


influence of Roentgen-radiation upon micro-organisms. They were of the opinion that even thin-walled glass test-tubes offer considerable resistance to the passage of X-rays; consequently they irradiated animals inoculated with micro-organisms (tubercle bacilli). These animals were exposed daily to the tube for at least one hour

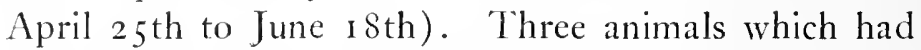
been treated in this way shewed no signs of tuberculosis, whereas other animals employed for control purposes (inoculated, but not irradiated) shewed abscesses and rapid emaciation. As the result of these experiments, followed by microscopic investigation, Lortet and Genoud concluded that Roentgen-radiation had modified the effects of inoculation. Fiorentini and Luraschi ${ }^{1}$ ) confirmed this opinion.

R. Mïhsam ${ }^{2}$ ) inoculated guinea-pigs with tuberculosis and irradiated 12 of them for one hour daily, another I 2 being kept for control purposes. Results shewed that general tuberculosis was not prevented by irradiation; there was merely some weakening effect on local tuberculosis.

Scholt 3 $^{3}$ ) exposed well-developed plate cultures of typhus, cholera, pyocyaneus and trichophyton germs to soft tubes for I to 4 hours with entirely negative results. He believes that $X$-rays have no serious bactericidal effect. He was unable to obtain positive results moreover in the case of animals inoculated with tuberculosis.

The author made the following experiments bearing upon this question: A Gundelach's vacuum-tube was employed, having a leaden screen fixed on it at the junction of the tube-neck with the globe. By this arrangement (Fig. 84 ) X-rays were excluded from the region of the tube-neck.

Two plate cultures of staphylococcus pyogenes aureus on agar were placed, one at a distance of $\mathrm{I} \mathbf{c m}$.

1) L. C.

") Dentsche med. Woch., 1898, No. 45 .

3) L. C. 
below the globe $K(A)$, and one $(B)$ at a distance of $\frac{1}{2} \mathrm{~cm}$. below the narrow end $(H)$ of the Roentgen-tube. Below $d$ a photographic plate $P_{1}$ was placed, wrapped in black paper, and with its film uppermost. Between $A$ and $P_{1}$ a leaden letter $A_{1}$ was placed. A leaden letter $B_{1}$ was also placed between $B$ and a second photographic plate $P_{2}$. Plate $A_{1}$ was corered in addition by a thick aluminium screen $A l$, which was connected with the earth. After 5 minutes' working of the tube the photographic plates were developed. On plate $P_{1}$ was to be seen a well-marked white image of the letter $A_{\mathrm{e}}$; the other plate $P_{2}$ shewed a very faint image of the letter $B_{1}$ and the plate as a whole was almost entirely white.

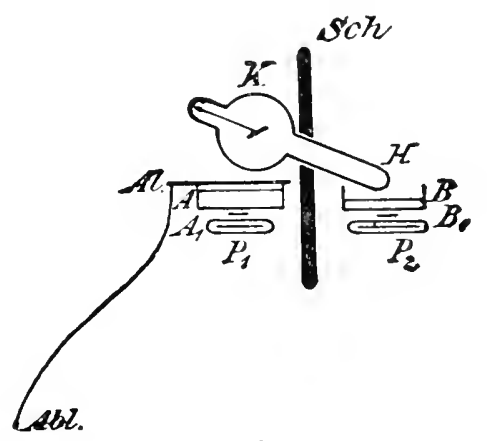

FIG. 84 .

Fresh photographic plates were treated in preciscly the same way and developed after 5 minutes; the results were the same as before. Fresh photographs were taken at intervals during the half hour the plate cultures were exposed. The negatives always proved that X-rays were freely passing through the culture $I$ and the aluminium screen covering it, whereas through $B$ only rays in small amount were penetrating. On the other hand, $B$ was throughout accessible to the silent discharges of hightension electricity from the tube-surface, while $d$ was protected from electrical discharges by means of the aluminium screen. The "earthing" wires $(A b l)$ from the latter ran to a cleft between the ceiling and the wall of 
the room. The Roentgen-tube became very hot during the experiment. After half an hour both plates were covered and placed in the incubator. If now the electrical discharges and not the X-rays formed the bactericidal factor in Roentgen-radiation, it was expected that culture $B$ would appear destroyed and culture $A$ unharmed. The reverse, however, proved to be the case. $A$ had a sterile patch in the centre, whereas $B$ was uniformly grown over with colonies.

This experiment seemed to indicate that the Roentgen-rays themselves have bactericidal properties.

On carefully considering the conditions of this experiment, however, the author began to doubt if $A$ had after all been efficiently protected from electrical discharges; the earthing of the aluminium plate was possibly defective. Again, it seemed very likely that the electric discharges striking $B$ were less powerful than those proceeding from the curve of the sphere towards $A$, so that $B$ might have been insufficiently exposed.

In order to eliminate these objections the next vacuum-tube experimented with was one made by the Voltohmgesellschaft of Munich. This possessed two spheres of almost equal size; the cathode-rays were produced in one sphere and projected from the anticathode in an opposite direction from the other splrere. In this experiment earthing was secured by connection with the water-pipes. The leaden screen was fixed on the narrow neck of the tube between the spheres.

Two diffused cultures of staphylococcus pyogenes aureus on agar in Pétri-dishes were used. Dish $A$ was covered by a lid filled with parafin wax and placed beneath the sphere $K_{1}$. Dish $B$ was left uncovered and placed beneath $K_{2}$. Each dish was I $\mathrm{cm}$. distant from the tube; the earthing in connection with both dishes is shewn in the diagram Abl. Photographic plates and leaden letters were placed below the dishes as in the preceding experiment, and over $A$ an earthed aluminium screen $d l$ was arranged as before (Fig. 85). 
The above arrangements precluded any possibility of dish $A$ being struck by electric discharges; on the other hand X-rays were allowed to penetrate freely, as the photographs shewed. Dish $B$ received, however, no X-rays at all, the photographic plate $P_{2}$ shewing no darkening on development. This dish, however, received practically the same amount of electric discharge from the sphere $K_{2}$ as was evolved from sphere $K_{1}$. Exposures of one hour were given. Dish $B$ was then covered and both dishes were placed in the incubator.

On the following day it was seen that dish $A$ shewed an abundant and uniform growth of colonics. Dish $B$

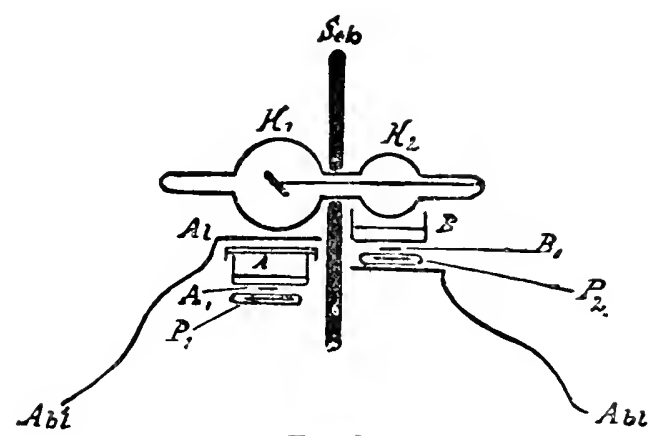

Fig. 85.

shewed a sterile patch in the centre the size of a kreutzer. The dishes were left in the incubator for 2 days more and then photographed.

The experiment was repeated five times; each time the same result was obtained, even when longer exposures were given ${ }^{1}$ ).

1) Kicuböck and Holaknecht urged igainst this experiment that the exposure was too brief. Quite recent experiments by (roumach (l. c.), Scholiz (1. c.). Zcit (Boston Merl. and Surg. Journal, Nov. 1yor), Aschkinass and Caspari (1. c.), however, funte contirm the author's experience.

Even assuming that longer expostre with soft tules would have demonstrated the bactericidal effect of $X$-rays it must be borne in mind that no such exposures could le taken advantage of in actual practice, since lley would ineviably produce severe $\mathrm{X}$-raty dermatitis. 
From these experiments it becomes evident that the bactericidal action of X-rays-presuming such to exist-is very slight compared with that of the electrical discharges given off by the vacuum-tube. It may, indeed, be regarded as practically a negligible quantity.

For the purpose of studying the more delicate intracellular processes which result from the irradiation of living matter, causing visible changes and the impairment of protoplasmic function, one turns from complicated higher organisms to the phenomena of plant-life, to germinating seeds and plant-cells, where one can readily observe the protoplasmic circulation. Or one may employ the cells of protozoa, whose usual course of existence is comparatively well understood (so that any departure from the normal can be at once recognised) as the objects of this elementary physiological enquiry.

According to Maldincy and Thouvenin, ${ }^{1}$ ) seeds of convolvulus arvensis, lepidium sativum, and panicium miliaceum, after one hour's exposure to Roentgen-rays, germinate sooner than others kept unexposed for control purposes.

According to Lopriore, ${ }^{2}$ ) Roentgen-rays have an accelerating effect upon the protoplasmic circulation in vallisneria spiralis. If after $\frac{1}{2}$ hour's exposure the rays be withdrawn normal conditions again obtain amongst the cells. After I hour's exposure harmful effects can be noticed: the protoplasm assumes a yellowish colour, becomes granular and vacuolised. After 2 hours' exposure the protoplasm ceases to circulate, and the chlorophyll bodies begin to lose their colour. Lopriore also examined the germination of the pollen of genista and darlingtonia coronillæfolia under the influence of X-rays. He found the process became suspended. After the experiment germination began again, the pollen germs having absorbed water freely during the exposure.

F. Schandinn ${ }^{3}$ ) examined the effect of irradiation upon

1) Compt. rend. de l'Acad. de Sc., Feb. I4, 1898.

$\left.{ }^{2}\right)$ Azione dei raggi sul protoplasma della cellula vegetale vivente in: Nuova Rassegna. Catania, 1897.

3) Ueber den Einfluss der Roentgenstrahlen auf Protozoen Pflïger's Archiv. f. d. ges. Physiologie, p. 29. 
separate animal cells, using cultures of protozoa in open glass receptacles for this purpose. One culture was always reserved for control purposes, being covered with a lead plate. The vacuum-tube, covered with a black cloth, was placed $20 \mathrm{~cm}$. above the cultures, and the exposure occupied I 4 hours.

Schaudinn found that under these conditions amebia princeps Ehrberg at first shewed signs of increased activity. After 5 or 6 hours this, however, generally diminished; after io hours the cells became clumped together in globules and remained stationary. After the experiment many of the cells dicd. 'The rays had an unfavourable influence after 4 hours upon "amœba lucida Gruber" and "pelomyxa palustris Greef."

Trichospharium sieboldi Schneider did not react at all to the rays. On the flagellates (chilomonas paramecium Ehrberg, etc.) irradiation had a harmful effect.

Spirostomum ambiguum Ehrberg (infusoria) was also exposed to the rays; after 4 or 5 hours activity was diminished, after 6 hours the infusoria died.

Schaudinn argued from his experiments that Protozoa varied much in their powers of resisting Roentgen-rays. Many kinds did not appear to respond to the irritation in the least, others reacted slightly, and others again very markedly. The condition of the plasma seemed to bear a certain relationship to the capacity for reaction. Those which reacted quickly possessed a loosely-lying plasma, and contained more fluid than those which were only affected slightly or not at all.

Recently $H$. Joseph and $S$. Prowazek ${ }^{1}$ ) have published a series of interesting investigations on the influence of Roentgenradiation upon living protoplasm.

They found in the case of bryopsis plumosa (algx) that a I 5 minutes' exposure distinctly slowed the protoplasmic circulation; also that the plasma did not appear so freely at the sites of artificially produced lesions. The chlorophyll granules became spindle-shaped and arranged in rows, or sometimes in star-shaped conglomerations. Some time after the irradiation conditions became normal again. These results are somewhat

$\left.{ }^{1}\right)$ Zcitschr, f. allg. Physiologie, Vol. I, Part II, I902, 
remarkable, in view of the fact that Lopriore found in the cells of vallisneria spiralis an acceleration of the protoplasmic circulation after a half-hour's exposure.

When infusoria (paramæcium caudatum and volvox) or daphnix were placed in a tube filled with water, one half of which was covered with lead-sheeting, the animalculæ collected after IO-I 5 minutes in the portion where they were protected from the X-rays. In order to exclude the possibility of white light having some share in this phenomenon the half of the tube not covered with lead was enveloped in a cloth. The result was, however, the same.

Joseph and Prowasek describe many other experiments upon low forms of animal life. Hard or soft tubes gave like results.

All these experiments go to show that when administered to a certain degree Roentgen-rays have a stimulating and vivifying action, while beyond this limit they begin to be injurious. These deductions agree entirely with clinical experience. It is known that weak irradiations stimulate the granulation of atonic ulcers, the proliferation of pigment, and the function of hair-papillæ. The latter fact explains why patients undergoing Roentgenradiation for hypertrichosis are left with a stronger growth of hair if for any reason they are obliged to suspend treatment at an early stage. These facts (including the circumstance that irradiated tissues have for some time afterwards a tendency to react after comparatively mild exposures) are similar to those observed after irradiation by light. Axenfeld, Joseph, and Prowazek's observations have shown that Roentgen-radiation stimulates cell-movement. We can, therefore, assume that the effect of Roentgen-rays, like other irritants, is to (I) stimulate, (2) act injuriously, (3) promote activity (movement).

\section{$\S 33$. The Active Agent in Roentgen-therapy.}

A vacuum-tube in action emits various kinds of rays, and gives rise to various physical phenomena. Every enquiry undertaken for the purpose of determining that precise factor to which alone the changes wrought on the living organism are due must first take cognisance of the several effects produced on the tissues 
by the very different physical agents which go to make up the sum of these phenomena.

On the surface of and within a vacum-tube, in action, we find:

I. Heat;

2. Ozone;

3. Cathode-rays;

4. Ultra-riolet rays;

5. Rays composed of material particles;

6. Roentgen-rays;

7. Spark- and brush-discharges from the high-tension electricity accumulated on the surface of the tube;

8. Electric or electro-dynamic wares;

9. Rays of unknown character.

With a knowledge of these facts, the author in 1896 made the experiments described on page 230 . In these, by suitable arrangements, he absolutely excluded the action of other physical agencies and was able to ascribe a definite influence to the Roentgen-rays themselves. Hc stated in effect that it is to the Roentgen-rays alone that we must look for the cause of the changes in the skin ${ }^{1}$ ) The possibility of heat, ozone, or ultraviolet rays having anything whaterer to do with his results was precluded by the condition of his experiments.

The assumption that other factors are accountable for the tissue-changes is, moreover, disapproved by other circumstances.

T'esla's ozone theory ${ }^{2}$ ) could not explain the deep-lying tissue-changes which Gilchrist ${ }^{3}$ ) and Kaposi ${ }^{4}$ ) had observed taking place in the nerves, nerve-sheaths, periosteum and bones of the extremities, and which $\|^{\circ}$. Reid" ") had seen in the back of a patient whose chest had alone been exposed to the rays "). Still less comprehensible by this theory is that fundamental

1) Wiener med. Woch., 1897, No. 10.

2) Public Opinion. Vol. XXI, No. 24. quoted by (iilchrist.

3) Johns Hopkins IIopp. Bulledin, Falmary, 1807.

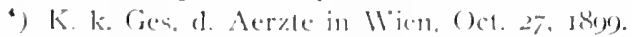

${ }^{\circ)}$ lirit. Med. Journal, Isefs. Vol. II.

-) Luuted by Müller. 
peculiarity of Roentgen-radiation-its cumulative action. It is a widely known fact that the changes in the skin after Roentgenradiation do not immediately follow on the exposure, but appear many days later. Even then they are announced by alteration in the deeper structures of the skin (shewn by falling of the hair, pigment changes, subjective symptoms, etc.), while the surface of the skin itself often remains throughout unaltered in appearance and function.

Borcles, ${ }^{1}$ ) Stenbeck, $\left.{ }^{2}\right)$ and Elliot ${ }^{3}$ ) ascribed the changes in the skin to the action of ultra-violet rays coming from the Roentgen-tube. There can be no question that ultra-violet rays are contained to some extent in the phosphorescent light excited by the cathode-rays on the tube-wall. But we must not forget that those ultra-violet rays have their origin within the tube; in order to be effective they must, therefore, first penetrate the glass wall of that tube. Now ordinary glass absorbs these rays to a great extent; only certain kinds (crown-glass and light phosphate-crown-glass) permit their passage to any degree ${ }^{4}$ ).

It is, therefore, very questionable if many ultra-violet rays reach the outside of the tube at all. Even were this the case, the author's investigations (p. 23 I) and the observations of M. Möller "i) would seem to exclude the possibility of their exerting any real action in Roentgen-radiation.

Moller observed that a woman who was being treated by X-rays for lupus of the face shewed signs of reaction also (pigmentation, etc.) on the chest, which was covered by a red bodice and a black dress. Molle'r believes that this phenomenon, which the author also has repeatedly observed, completely disproves the assumption that ultra-violet rays have anything to do with the reaction, since they would certainly have been absorbed in this case by the woman's clothing ${ }^{6}$ ).

1) The British Journal of Dermatology, July, I897.

) Quoted by Möller.

$\left.{ }^{3}\right)$ Journal of Cutaneous and Gen.-L'rin. Dis., Feb. I897.

$\left.{ }^{4}\right)$ For the properties of different kinds of glass, see Eder \& Valcnta, Denkichr. d. math.-natur. classe der K. akademie d. Wiss., May 4. 1894.

5) Einfluss des Lichtes auf die Hant. Billiotheca Medica. Stuttgart, rgoo.

") Thomson (Boston Med. \& Surg. Journal. Dec. 3. 1896) describes an experiment which also disproves the effect of ultra-violet rays. 
The author's experiments with regard to the possible action of the phosphorescent light gare negative results. The idea of any real effect proceding from the heat-rays of the racuumtube may be dismissed as improbable. The heat crolved, especially with the slow rate of intermption of the primary current usual in Roentgen-therapy, can be readily prored to be so insignificant that it can have no real physiological effect. This idea is further confirmed by the author's experiment with the aluminium screen (p. 23I).

These three factors, therefore-ozone, ultra-violet rays, and heat-rays - can obviously have nothing to do with the physiological effects of Roentgen-radiation. There is, perhaps, more to be said for the remaining factors above mentioned, though there are many objections to be urged against nearly all of these.

Against the cathode-ray theory of Gilchrist, $\left.{ }^{1}\right)$ Ames, $\left.{ }^{2}\right)$ and Foveau de Courmelles, ${ }^{3}$ ) it may be urged that though cathode-rays have the power of penetrating very thin layers of aluminium, as Hertz showed, their progress is arrested by even thin layers of other bodies, such as glass. Consequently the glass wall of the vacuum-tube would prevent most, if not all, of these rays from reaching the exterior of the tube ${ }^{4}$ ).

A very interesting theory has been propounded that the skin-changes are due to a direct bombardment with material particles emanating from the electrode. Crookes") believed that when the discharges from a Ruhmkorff coil are passed through a vacuum-tube, small particles are driven off from the cathode. These are either particles of the gas within the tube or are particles of the cathole itself; they may, again, be electrolytic elements of the gas-molecule. It is a matter of common knowledge that the region of a tube opposite the cathode becomes blackened after prolonged use. 'This blackening is due to a deposit of material particles driven off the elec-

1) I. c.

2) Quoted by Gilchrist.

3) Congr. f. Neurolog., Brussels, ISnz.

1) Gractz. I)ie Elektricitiat, Stuttgart, 18ys, p. 272.

8) Quoted by Gracta. 
trode. But against this theory of material particles it must be urged, (I) that it has not yet been proved that the particles penetrate the tube-wall and so arrive at the skin; (2) that no evidence has been as yet forthcoming of the presence of this foreign matter in the skin. Gilchrist examined a specimen of skin after exposure to X-rays, the pigmentation in which was so pronounced that the idea was suggested of there being possibly an invasion of aluminium particles in the tissue. Nevertheless under the microscope no trace of metallic or other foreign bodies could be discovered. Abel made chemical investigations with the same results. Tesla, who supports the theory of bombardment by material particles (1. c.), gives the comforting assurance that it would take at least a century before a sufficient quantity of the foreign matter collected would seriously threaten the health of the person undergoing irradiation.

Any possible action on the part of the tension-electricity accumulated on the surface of the Roentgen-tube (such as was suggested by Destot, ${ }^{1}$ ) Balthasard, $\left.{ }^{2}\right)$ Jankau,,") Fovean de Courmelles, ${ }^{4}$ ) Apostoli, ${ }^{5}$ ) Lester Lenard, ${ }^{6}$ ) and the author ${ }^{7}$ ), seemed excluded by the above-mentioned experiment; the aluminium screen undoubtedly conveyed all the electricity to the ground; moreover, $W$. Reid's ${ }^{8}$ ) observation seemed also to negative the electrical theory. Reid's observation, confirmed later by Kïmmel and Rerillel, ${ }^{9}$ ) shewed that changes occurred not only in the area of skin directly exposed to the rays, but also in a corresponding area of skin on the opposite side of the body, where the rays emerge. This circumstance seemed also to speak against any electric-wave hypothesis; moreover, any theory of this kind could not explain the negative results of the author's experiment with the reversed current (p. 23I).

1) Quoted by Möllcr.

2) Soc. de biologie. July I7, 1897 .

$\left.{ }^{3}\right)$ Photogr. Monatsh. f. Med., 1896, No. 6.

4) Soc. de biologie, July $17,1897$.

$\left.{ }^{5}\right)$ Internat. med. congr., Moscow, IS97.

-) The American X-ray Journal, I898, No. 5.

$\left.{ }^{7}\right)$ Vortr. in der Gesellsch. d. Aerzte in Wien, Jan. 15, I897.

$\left.{ }^{8}\right)$ British Med. Journal, ISgS, Vol. II.

") Quoted by Möller. 
The author's theory that the X-rays themselves are the active factor of Roentgen-therapy was held also by Gocht, ${ }^{1}$ ) Kümmel,"2) Rieder,"3) Albers-Schönberg, ${ }^{+}$) and others.

Against even this view, however, came the following observation: The author treated a woman for hypertrichosis; during the whole course of treatment very hard tubes only were employed, so that at no time could the least trace of Huorescence be seen on the screen. Plainly there were practically no $\mathrm{X}$-rays evolved from the tube. Nevertheless, after a certain time, the hair began to fall. Struck by this fact, the author treated a similar case with the current reversed throughout. Thus the experiment he made in I $\$_{9} 6$ was repeated, with the exception that in this instance (no result being evident by the twelfth day, as was usul with the ordinary arrangement of current) the exposure was prolonged for $S$ or Io days. By this time the hair began to fall. A repetition of this experiment with a third patient yielded the same result. Obriously, therefore, the Roentgen-rays cannot be the sole cause of the changes in the skin.

This riew was confirmed by $H^{r}$ oyzekowskis") experiments on rabbits. In every case where the X-rays alone were employed, electricity and light being excluded as far as possible, no injurious effects were to be seen; when, however, the X-rays were used in conjunction with the other varieties of radiation emanating from the tuhe, physiological effects followed in from 3-I 2 hours.

A further striking fact, which could be noted at any sitting, seemed also to point to electrical energy as bearing some casual -elationship to the physiological effects. When the apparatus is in action the patient and the objects surrounding him become so charged with electricity that sparks can be drawn with the knuckles from any part of his body. The patient has often

1) Fortschr. a. d. Geh. d. Roentgenstr., Vol. I, No. I.

2) Quoted liy Gocht.

3) Nünchener med. Woch., Iso7, No, ro.

‘) Hhid. 1goo, Nos. 9, 10, I I.

") Qunted by Zarubin, Monatsh. f. prakt. Derm., ISgo. Vol. XXVIII. No. 10. 
the sensation of being in a light current of air ("electric wind"); his hairs are attracted towards the tube. Next came detailed acounts from d'Arsonval and Ondin of the therapeutic employment of Tesla's high-frequency currents ${ }^{1}$ ). On comparing the effects of these currents with those of Roentgen-radiation, the author was at once struck by many points of resemblance between the two. Might not spark-discharges from the coil in the case of Roentgen-radiation also play an important part in the physiological action? Since the experiment with the aluminium screen argued against any direct spark-effect, it seemed natural to conclude that the electric vibrations due to the sparkdischarges were accountable for: the physiological effects, and that the intensity of the latter varied with the physical qualities of the electric vibrations.

This idea, however, to which the author gave expression at a meeting of the Wiener dermatologischen Gesellschaft, May Ioth, I $899,{ }^{\circ}$ ) appeared somewhat at variance with the fact that not only the leaden masks in common use, but even masks of cardboard ${ }^{3}$ ) or ordinary paper, ${ }^{4}$ ) were sufficient for protective purposes. Since electric waves penetrate all bodies, with the exception of metals, and, moreover, since they are transmitted in all directions from the sparking-path (just as waves of light are propagated in all directions from the point of origin), their effect would not be confined to the region directly opposite their source, and still less would their progress be arrested by a sheet of paper. Further, doubts arose whether electric vibrations really emanate from a Roentgen-tube. According to $J$. Thma, ${ }^{5}$ ) most probably no electro-magnetic waves arise in this way.

In the endeavour to throw light on this perplexing problem,

$\left.{ }^{1}\right)$ Annales d'électrobiologie, d'électrothérapie et d'électrodiagnostie, Jan. I5, 1898 .

$\left.{ }^{2}\right)$ Wiener med. Presse, I899. No. 31, and Wiener klin. Woch., I899, No. 39

$\left.{ }^{3}\right)$ Itahn and Albcrs-Schönborg, Nünchener med. Woch., March 13, 1900.

4) Stcnbeck, quoted by Möller.

s) Quoted in the author's work "Die physiolog. Wirkungen der Polentladungen hoch-gespannter Inductionströme." Sitzungsb. d. Akad. der Wiss. in Wien, Math.-naturw. C1., Vol. CIX, Section III, p. 594. 
the identity of the active agent in Roentgen-therapy, the author felt bound to reconsider the question of the spark-discharges. Two considerations in particular influenced him in this direction. The first was the question as to whether in his first experiment the aluminium screen had actually "earthed" all the discharges; it seemed at least within the bounds of possibility that more vigorous sparks should have penetrated the screen. He remembered, moreover, that from the kind of tube he was at that time employing (a small vacuum-tube from Stützerbach), often powerful sparks struck the screen, and that on the corresponding area of skin the necrosis made its appearance later. Again, it seemed not impossible that the vacuolisation degeneration discovered by Gassmann in the cells of the intima of the vesselwalls, in cases of Roentgen-ulceration, might be traced to the effects of direct spark-discharges.

It seemed essential, therefore, to study the physiological effects of spark-discharges pure and simple-hence the experiments described on page I 2 I and onwards. Thus the fact was established that direct spark-discharges from a Ruhmkorff's apparatus have a similar influence on the skin and on bacteria to that which has been ascribed to Roentgen-radiation. This led one to the conclusion that electric discharges constitute the essential factor in Roentgen-therapy.

Recently, however, Sträller, $\left.{ }^{1}\right)$ Kienböck, $\left.{ }^{2}\right)$ and Scholli ${ }^{3}$ ) have described a series of experiments tending to show that $\mathrm{X}$-rays themselves possess a physiological action. These investigators shew that irradiation by means of soft tubes, which evolve X-rays copiously, brings about reaction in the same individuals much earlier than irradiation with hard tubes, which give a comparatively small output of X-rays.

Kienböck urged the fact that the skin reaction only appears on the area exposed to the $X$-rays, and that by fixing a leaden screen between the tube and the surface of the skin a white patch (corresponding to the screen) ${ }^{\prime}$ is produced, surrounded

2) Deutsche med. Wochenschr., August, 1000, p. 546 .

${ }^{2}$ Wiener klin. Wochenschr., rooo, No. 50 .

3) L. C. 
by a zone of erythema. The effect on the skin is weakened according as other bodies are interposed between the tube and the body. The same investigator arranged a tube in such a way that the mirror of the anti-cathode lay at right angles to the surface of the skin. Thereby only that portion of skin underlying the fluorescent half of the tube was subjected to $X$-rays; behind the mirror the tube was dark, and from here but few X-rays struck the skin. Reaction only appeared in the first-mentioned area of skin; the border of this reactive zone coincided with the plane of the anti-cathode mirror.

There are sereral objections to be raised against these experiments. In the first place, it is well known that under certain conditions even hard tubes bring about reaction far earlier than was anticipated. Again, it is quite clear that leaden plates not only intercept X-rays, but also spark-discharges. Finally, it must be mentioned that the high-tension electricity is not evenly distributed over the surface of the tube, as we are given to suppose, but has certain peculiarities in this respect.

E. Riecke ${ }^{1}$ ) examined the distribution of free electricity on the surface of a Crookes tube, actuated by an induction-apparatus, by dusting the tube with a mixture of red lead and powdered sulphur. Peculiarly shaped figures appeared on the tube-surface; a ring was seen opposite the cathode, sharply outlined by yellow powder. The ring was situated somewhere about the border of the brightly fluorescing part of the tube-wall. Within the ring alone red spots were seen; these were composed of positively-electrified red-lead powder. The distribution of the red powder was uneven, probably on account of the uneven condition of the cathodesurface.

Metal wires and plates placed within the tube in the path of the cathode-rays caused shadows to appear on the tube-wall; these shadows were also indicated by the disposition of the powder. The shadows were

1) Wiedcm. Ann., Vol. LXIX, ı899. p. 788. 
sharply outlined by streaks of red powder; the shadows themselves were either free from powder or covered in the middle with powdered sulphur.

The author's own experiments confirmed those of Riecke. He observed a striking arrangement of the yellow powder in the form of broad rings opposite the poles of the tube. Moreover, in the brightly fluorescing portion of the tube placed opposite to the skin the accumulation of free electricity was especially marked.

The following experiment seems convincing:

Scholt ${ }^{1}$ ) irradiated a circular patch of skin, the size of one's palm, on a hog's back. The exposure was continued for $3 / 4$ hour, and the patch was divided into 5 segments; 4 of these segments were covered with lead, glass, aluminium and paper respectively, while the fifth was left uncovered. After 30 days the hairs loosened in the uncovered and paper-covered segments; later on, when the same phenomenon began to shew itself also in the aluminium-covered segment, the first-mentioned segments shewed a superficial necrosis. In the glass- and leadcovered regions absolutely no changes were visible. In a later experiment, Scholiz shewed that mercurial plaster also forms an effective screen against irradiation.

In order to demonstrate that the alteration in the tubevacuum, which occurs during every irradiation, has an important bearing on the physiological effect, $S$ cholt $z^{2}$ ) exposed the left flank of a rabbit to a small "hard" tube, which became "soft" after 5 or 6 minutes' use; after that the tube was brought to bear on the right flank. After four irradiations in this manner an alopecia appeared on the right side, while on the left there was only a slight fall of hair.

In order to decide whether Roentgen-rays are effective after they have actually penetrated the tissues, Scholls exposed certain regions on the backs and throats of rabbits and pigs over which he had fastened the aninals' ears. The irradiation was vigorous, and inflammatory reactions were induced not only on

1) Arch. f. Derm. und Syph., Vol, I,IX, Part I.

2) L. C. 
both sides of the ears, but also on the parts boneath the ears. According to Sihollz, the muscles, cartilages, and bones are not affected; necrosis in these structures is generally secondary only to inflammation of the skin.

Scholtz believes that Roentgen-rays exert their effects in the first instance upon the skin. These skin-effects, moreover, do not only occur where the rays enter the body, but may, after the penetration of thin layers of muscle and cartilage, occur at the site of exit. At the same time comparatively thin layers of tissue, which only show faint grey shadows on the fluorescent screen, considerably weaken the effect of the rays.

Scholtz summarises as follows:

I. The greater the output of $\mathrm{X}$-rays from the tube, the greater the effect on the skin.

On this ground, according to Scholtz, the therapeutic usc of soft tubes is advisable. He recommends varying the intensity of treatment by alterations in the strength of current, the tubedistance, and the duration of exposure.

2. The less a given substance allows Roentgen-rays to traverse it, the more does it restrain effective rays from reaching the skin.

Bodies, such as an aluminium screen, which throw only feeble shadows on the fluorescent screen, hold back, nevertheless, a relatively large part of the effective rays. In other words, it is just the weakly-penetrating rays which are especially active on the skin.

From the foregoing experiments it becomes evident that of all the physical factors emanating from an X-ray tube only the Roentgentrays themselves and the discharges of high-tension electricity from the tube-surface can be considered responsible for the physiological effects. Both these agents appear simullaneously while the tube is working, both have demonstrably similar effects, and these effects supplement and accentuate each other.

The latter hypothesis appears borne out by the following experiment:

A serere case of hypertrichosis of both cheeks and the chin was treated by the author as follows: The right 
cheek was exposed for the first 20 minutes to the "quiet" discharges from the special electrole, which has been described earlier. Afterwards the same check was treated with Roentgen-rars for 15 minutes, the tubedistance being $15 \mathrm{~cm}$. The tube was a very soft one, and could be touched without one receiving any perceptible electric discharge. The left cheek was simply exposed to the same Roentgen-tube for I 5 minutes at the same tube-distance. It would be approximately correct.

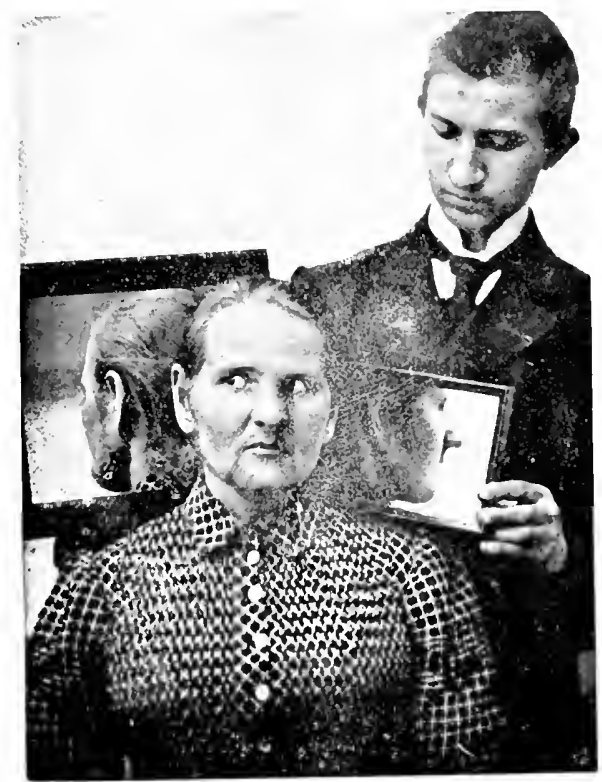

FIG. 86.

therefore, to say that the right check received X-rays plus electric discharges, the left cheek X-rays alone. The experiment was continued daily in this manner, and after the sixth day the right check shewed distinct signs of reaction, the hairs fell, well-marked erythema, etc., followed. The left check shewed as yet no signs of change; this region was, therefore, exposed 8 times further. After this, slight erythemat and pigmentation shewed themselves, and a week later the hairs began to 
fall. The reaction, however, on this side throughout ran a milder course than on the right. The experiment was repeated many times with the same result. Reaction and epilation always followed in a few days after treatment with high-tension electricity and X-rays, and the reaction was always most evident in those places which had been bombarded most vigorously by the discharges (Fig. 86). These regions appeared intensely red. The rest of the skin shewed the usual slight pigmentation and erythema of commencing reaction. The combination of both methods also in cases of lupus ulcerations determined an earlier reaction and hastened the healing process.

As regards the quality of tubes, it is certain that in most cases soft tubes are more powerful and act sooner than hard tubes. At the same time the rays cmanating from hard tubes (including the electric discharges) appear to penetrate more deeply than those from soft tubes.

Holzknecht's opinion is that any deeper action on the part of X-rays is impossible, since the physiologically active rays are absorbed by the skin, and only those rays which are inactive are allowed to penetrate. Against this we need only refer to the fact that animals which have been exposed for a considerable time to Roentgen-radiation show dermatitis on both sides of the body.

In conclusion, we may discuss the mechanism of the physiological process. The action of electric discharges has been already explained (p. I 59). With regard to the X-rays, the assumption that we have here to deal with a pronounced local action is opposed by the so-called tropho-neurotic theory of Ondin and Barthélemy ${ }^{1}$ ). These authorities look upon the reaction as a reflex process arising from the central nervous system through stimulation of the cuticular nerves. This theory may be abandoned in view of the local macro- and microscopical changes already described as occurring in irradiated tissues, bear-

1) Monotsh, f. prakt. Dermatologie, Vol. XXV, I897, p. 4 I7. 
ing in mind, morcover, that there is complete absence of any symptoms on the part of the brain and spinal cord, or of any proof of peripheral nerve lesions ${ }^{1}$ ).

The action of X-rays is doubtless purely local, but as to its precise character there is still much to be learned. Kienböck believes in a chemical action, leading to disturbances of metabolism by which the cells of the tissue are led to react in the form of Roentgen-dermatitis. The action, however, cannot be purely chemical, for most enquiries shew that X-rays have remarkably weak chemical properties").

The action of X-rays on a photographic plate must not be taken as a proof of the direct chemical effect of the rays on bromide of silver. Eder and I'alenta have shewn that X-rays act on silver bromide gelatine, but not on collodion plates; this sufficiently proves that the photographic action of the rays is a highly complicated process, which does not consist merely in the reduction of bromide of silver.

The author believes that through the destruction of tissueelements certain products arise whose absorption leads to constitutional symptoms. This would explain the appearance of fever at the commencement of severe Roentgen-dermatitis, before any excoriation or ulcer affords the opportunity for local infection.

$J_{a n k a u^{3}}$ ) believed that electrolytic analysis of the cclls takes place from irradiation, whereby the tissues are chemically affected and inflammation is provoked.

Kaposi ${ }^{4}$ ) advanced the theory that Roentgen-rays affect the vascular system in the deeper layers of the skin similarly to sunlight, so that first an active and then a passive hyperamia results, which slowly extends to the more superficial vessels.

It is well known that during the phenomenon of osmosis a slight potential-difference arises on both sides of the diaphragm through which the passage of the liquicl particles takes place. H. Bordier ${ }^{5}$ ) affirms that under certain experimental condi-

1) Jutassy, 1. c.

$\left.{ }^{2}\right)$ A. . Hemptinne, Zeitschr. f. physik. (henie, Vet. XXI, 3, 1. 493.

${ }^{3}$ ) Internat. photog. Monatselor. f. Nedicin, Vol. V. No. 1.

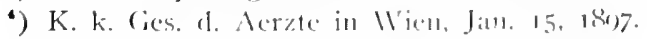

¿) Acad. de Sc. Commot. reml., Vin. (XXVI. 1. 595 
tions Roentgen-radiation retards the process of osmosis. An aluminium plate connected with the earth and introduced between the Roentgen-tube and the osmometer in no way interferes with this retarding process. Consequently Bordier ascribes the retarding influence to the $\mathrm{X}$-rays themselves. Bordier explains this effect by the disturbing influence of $\mathrm{X}$-rays on the electro-capillary phenomenon taking place in the membrane during osmosis. Since many vital processes in the cell depend upon the proper performance of this function of osmosis, Bordier believes that in the retardation of the latter is to be found the cause of many biological and therapeutic effects.

The author believes that the pathological changes in irradiated tissue may be explained by the disturbance of a preexisting electrical equilibrium in the several tissue molecules by the X-rays, which possess the property of discharging electrically-laden bodies. $B$. $W$ aller $^{1}$ ) also shares in this view.

It seems, moreover, not impossible ${ }^{2}$ ) that $X$-rays induce fluorescence in those tissue-elements capable of this phenomenon, and that this process brings about chemical changes, especially in the cells. In this respect those tissues which contain earthy matter avould appear to possess some affinity to the bromide of silver-gelatine, while other tissues, which have no fluorescence capacity, remain unharmed by the rays.

Quite recently, Goldstcin, arguing from the supposition that where Roentgen-rays impinge upon material bodies ultra-violet light of very short wave-length is produced, ascribed to this ultra-violet light the cause of the biological effects produced in the deeper layers of the skin by Roentgen-radiation ${ }^{3}$ ). According to this view, it is difficult to see why the rays should exert their influence mainly on the skin. They penetrate the whole body, and would, therefore, cause the production of

\footnotetext{
1) Fortschr. a. d. Geb. d. Roentgenstrahlen, Vol. I, No. 6, p. 242.

2 The author communicated this view to a meeting of the Vienna Medical Club, Jan. 30, I90I.

$\left.{ }^{3}\right)$ Sitzungsbericht d. k. preuss. Akad. d. Wissenschaften, quoted by Holzknecht, Die Photochem. Grundlagen, etc. Fortschr, a. d. Geb. d. Roentgenstr., Vol, V,
} 
ultra-violet light in the internal organs (the heart, liver, etc., where they are also absorbed) and so bring about changes there, which certain! $y$ is not the case. This difficulty does not arise with the author's hypothesis, for it is well known that the animal tissues do not all possess in the same degree the capacity for Huorescence.

\section{$\S$ 34. Roentgen-ray Dermatitis. ${ }^{1}$}

The pathological changes in the skin which go by the name of "X-ray dermatitis" present the following clinical appearances: At first we have the milder signs of reaction already referred to: turgescence of the skin, pigment changes, palered erythema, loosening of hairs, and subjective phenomena (itching, burning, feeling of tension).

Where the action is stronger the hairs begin to fall in from 3 to 14 days after the last exposure; first the thicker, and then the lanugo-hairs (seldom otherwise). The redness gradually becomes intensified, more and more cyanotic; while a small area of the skin (where the irradiation has been the strongest) soon shows superficial excoriation. The latter, which is accompanied by severe pain (see p. 25I), and often fever, rapidly spreads centrifugally.

Many authors affirm that Roentgen-dermatitis runs a painless course. The author cannot substantiate this from the cases which have come under his notice. These were accompanied by considerable pain, especially when the raw surfaces were exposed to the air. In the case of nævus pigmentosus pilaris, which was mentioned earlier in this work, and which was the occasion of the first experiment in Rocntgen-therapy, the pain was at first trifling. Later on, however, when ulceration shewed itself, the pain became at times very severe, especially at night.

The author has only seen pustular and bullous formations

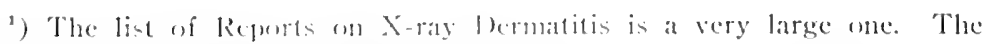
reader is referred to Musnus doller's lemk. 
in the above-cited case, though these are described as constant by several authors (Ehrmain, Kienböck, and others). The excoriations have a yellow-red, smooth, wax-like appearance; they secrete a quantity of thin pus, and are covered by a peculiar (fatty-fibrinous) coating, which is with difficulty removed (this coating does not give a fibrin-reaction: Lion); at their border are seen circular or oblong islands of epithelium which may have bridge-like processes extending to the healthy skin in the neighbourhood. After very powerful irradiation a whitish patch (the "Roentgen-slough") differentiates itself in the middle of the excoriation. This becomes gradually darker and finally separates. The more powerful the irradiation, the sooner these phenomena shew themselves.

Repair sets in as follows :

The slighter signs of reaction which are the result of very weak irradiation soon disappear. Erythema and pigmentation are the first to go; often enough no traces of these are left after three days. Intumescence of the skin persists longer, and to this must be ascribed the excellent appearance of the integument in the earlier period after the suspension of treatment. Weeks or months later, when the skin has returned absolutely to the normal, one may be able to recognise slight wrinkles and atrophic spots-shewing that the irradiation had been a little stronger than was perhaps thought at the time, and that it had consequently left certain permanent changes behind it. Where, however, the strength of the irradiation has been correctly gauged and administered, the excessive glossiness, elasticity, and smoothness of the exposed region disappear, leaving a skin which looks thoroughly healthy and normal.

Six to eight weeks after the commencement of reaction new hairs begin to shew themselves in the smooth and hitherto hairless skin; these hairs soon grow to the ordinary size. It is only after long and intermittent treatment that the hairs remain in a rudimentary condition, being white and small, with superficiallylying roots, so that they can easily be pulled out.

Where very pronounced erythema (with or without excoriation) has been induced, this condition lasts for several days; after this time we find a drying up of the normal secretions, 
thickening of the cuticle, and marked pigmentation. Then, when the swelling has subsided, the skin exfoliates very freely.

In more severe types of Rocntgen dermatitis, with defmite ulceration, the well-pronounced inflammatory phenomena are accompanied by pain and occasionally febrile symptoms for 6-S weeks. After this time the after-effects of the X-rays seem to be completed (note that hair begins to grow again at the expiration of 6 to 8 weeks also).

The X-ray ulcer has intensely red, sharp-cut borders, and heals but slowly towards the centre. The ulcer-secretions have a tendency to dry and form large rupia-like crusts adhering closely to the base of the ulcer; on removing these crusts one sees the characteristic, profusely-discharging Roentgen-ulcer; pain gives way later to a feeling of intense itching. IVhen the ulcer is finally healed (and this may take a year or morc in very severe cases), the scar is smooth, flat, and of good appearance, or it may be sclerodermatous or atrophic. Sometimes telangiectases appear in the scar, either in the form of thick branching vessels or as a reddish "marbling." An ulcer healed in this manner is liable to again break down and take a still longer time in healing. Besides this acute fulminating form of Roentgen-dermatitis, however, we meet with another and milder form. The latter is seen after prolonged irradiation with weak tubes (hard tubes), a long tube-distance, and short exposures. The dermatitis then runs a milder course; the visible signs of reaction are less pronounced than in the preceding form. They have more the appearance of a chronic eczema, such as is seen in laundrywomen-the skin is swollen, dry, boardlike, thickly scaling, and in places excoriated or ulcerated. One often sees, moreover, an atrophic, cigarette-paper-like change in the skin and nails. Sensibility is of course disturbed in these cases where the hands are affected. Cases of this kind, the so-called "chronic radio-dermatitis," may lead to the same cicatricial or atrophic conditions as the acute form.

The author finds himself unable to agree with Oudin's dictum that Roentgen-ulcers have some tendency to become the seat of secondary infection. Antiseptic dressings would in any case negative possibilities of this kind. Still many writers appear to 
have discovered numerous bacteria in their histological examinations of the ulcers.

As before mentioned, Roentgen-irradiation of the hands often brings about changes in the nails. These become dry, fissured, thickened and distorted; the ridges are deepened or become thinner, atrophic, and disappear where the action has been very strong.

Numerous reports are to hand concerning the histological changes in the tissues after irradiation.

Darier ${ }^{1}$ ) noted an extension of the prickle and horn-cell layers, partly due to general hyperplasia of the rete, but more particularly to increase in the number of prickle and horn cells themselves; further, a thickening of the epidermis, great increase of keratohyalin, and degeneration and atrophy of the hairfollicles, hairs, and glands of the skin.

$U n m a^{2}$ ) found slight increase of the nuclei of the papillæ and round the blood-vessels, and copious pigmentation in the upper layers of the cutis, whereas the pigment in the epidermis was not increased. The collagenous layers of the cutis appeared much thickened, swollen and pressed together, so that the intervening lymph-spaces were scarcely visible. They shewed, moreover, a peculiar staining reaction, becoming basophilic. The protoplasmic elements, especially the epithelium of the sweatglands, appeared compressed and very small. The elastic bundles were not seen by ordinary methods of staining, and sections shewed them to have a great tendency to separate from each other and fall away in small fragments.

In Kibbe's case ${ }^{3}$ ) the stratum lucidum was wanting, and keratohyalin, highly coloured, was found in the neighbourhood of the follicles. The cutis was richly cellular, and marked by vascular dilation.

Gilchrist ${ }^{*}$ ) found the horn-layer thickened, copious brown pigmentation in the rete, and dilated vessels in the corium.

Jutassy's investigations have already been quoted (p. 277).

\footnotetext{
1) Monatschr. f. prakt. Dermatol., Vol. XXV, I897, No. 9.

$\left.{ }^{2}\right)$ Deutsche Medicinalzeitung. March i8, i898.

3) New York Med. Journal, Jan, I6, i\$97.

•) Johns Hopkins Hosp. Bulletin, Feb., I897.
} 
Gassmann ${ }^{1}$ ) found the following histological appearances in a small piece of blackened tissue taken from the base of a deep Roentgen-ulcer: The specimen was not necrotic in the ordinary sense, but consisted of different properly characterised and well-staining elements. The main portion was formed of collagenous bundles (white connective-tissue fibres), normal in appearance, and staining readily; their nuclei also stained well with ordinary methods. In places were seen degeneration-forms of peculiar appearance which took the nuclear stain. Between the nuclei, leucocytes and mast-cells were copiously distributed. Elastic bundles were readily shewn with orcein or by Weigert's method.

Microscopic examination of another ulcer ${ }^{2}$ ) shewed newformed granulation-tissue which was in part skinned over; the lesion could in no wise be distinguished from other types of ulcer.

The vessels of the cutis and sub-cuticular region shewed remarkable pathological changes in a Roentgen-ulcer 2 months old; there were seen enlargement and vacuole-degeneration of the intima, loosening of the elastica, vacuolisation and atrophy of the muscularis.

The walls of many vesscls were changed into a swollen mass and completely obliterated. The intima, which was thickened and possessed many, swollen endothelial cells, was in places raised from the underlying layer, so that here and there round spaces were evident in this region. The greater part of the lumen of the vessel was occupied by this twisted, reticular, and vacuole-containing intima. I cong bladder-like nuclei with nucleoli were visible, especially near the lumen, which could be readily recognised as endothelial nuclei. The vacuoles were either apparently quite empty or filled with a fincly-bundled matcrial; here and there, moreover, a nucleus was scen. Only the larger vessels shewed the remains of a much unravelled, unequally thick, and often broken clastica.

The muscularis often presented a honcycombed appearance,

1) Fortschritte, Vol. II, H. 4.

$\left.{ }^{2}\right)$ Ilicil. Vol. II, II. 6 . 
the brown-coloured, smooth, cross-cut muscle fibres with their well-stained normal nuclei being separated from one another by many vacuoles. The muscle-bundles themselves appeared atrophied. (These changes are very similar to those shewn in Fig. 59.)

This vacuolisation degeneration affected both arteries and veins, but was not equally marked throughout the section. The fact that within a vacuole one often found a cell, a muscle-fibre, or a nucleus, made it not improbable, in Gassmann's opinion, that these spaces occupied the site of pre-existing cells. One might suppose that round the cell an exudation had collected which compressed the cell and so made it atrophy.

Besides these vascular changes, Gassmann ${ }^{1}$ ) also observed degeneration of the sub-cutaneous connective tissue, with abnormal staining reactions.

Lion $^{2}$ ) found in his sections changes in the vessel-walls corresponding to those described by Gassmann; he found also vacuolisation in all the layers of the epidermis and cutis; the cell-elements appeared swollen and perforated. He also found copious hæmorrhages.

Scholtz found the following changes in the skin of a hog's back; he excised the specimen for examination 7 days after one hour's irradiation:

"The horn-layer, raised somewhat, contains here and there several nucleated cells. The granular layer is merely indicated; in places it is quite atrophied. The prickle-cell layer is much diminished, and the prickle-cells themselves are greately altered. The latter are swollen, their contour badly defined, their shape in the palisade-layer broader. The protoplasm is diffusely stained with hxmotoxylin, the nucleus is only faintly stained, and the chromatin is seen in small conglomerations and in fragments. The nuclei are in great part swollen, and often indented and vacuolised. Vacuoles are found in the protoplasm, especially in that adjacent to the nuclei.

In nearly every microscopic field are to be seen cells with

$\left.{ }^{2}\right)$ L. C.

$\left.{ }^{2}\right)$ VII Congr. d. deutschen dermatol. Gesellsch., Breslau, Igor. 
two or eren three amitotic nuclei. Mitosis, on the other hand, is not seen, or at least only the commencenent of it. All these signs of degeneration obtain from the palisade-layer to the horn-layer: near the surface the contour of the cells is scarcely more evident and their protoplasm has become almost a homogeneous mass; the nucici are in great part only faintly indicated. 'The cell-changes in the hair root sheaths are quite similar; the loosening and falling of the hair can be readily explained by this cell-degeneration.

The corium is somewhat ordematous; the connective tissue is less easily strained, somewhat swollen and homogencous. Unna's "basophile" reaction is not obtainable with this tissue. The elastic fibres are preserved. The connective tissue cells have distinct, more or less diffusely stained protoplasm, are swollen and often peculiarly shaped. The cells of the sweat-glands shew similar mild degenerative changes, proliferating in places and projecting into the lumen of the ducts. In the media and intima of the larger vessels also are seen mild forms of cellular degeneration, quite similar to that of the other cells. The cells of the intima are swollen, pressing towards the lumen; in places they are manifestly proliferating, becoming loosened and tending to mingle with the blood-stream.

In sections taken from a more powerfully irradiated skin the cellular degeneration was more pronounced, extending to the nucleus as well as to the body of the cell; besides this the signs of inflammatory reaction were seen: marked rascular dilatation, serous effusion into the tissues, conglomeration of leucocytes, and free migration of white blood-corpuscles. Where a high degree of cell-degeneration is attained from strong irradiation the leucocytes collect in masses round the dying cells and so aid in their complete destruction. Noreover, many "highly-charged" mast-cells are visible.

The cells of the intima and media of the blood-ressels shew changes similar to those described by Gassmann.

Round the hatrs, where the rete extends towards the cutis as the root-sheath, quite similar degencrative processes are found among the cell-clements, also inllammatory signs. By these 
means the coverings of the hair are completely destroyed, masses of leucocytes taking their place.

In the severest grades of reaction microscopic examination reveals the appearance of a "Roentgen-slough." The rete malphigii and the horn-layer are completely wanting; in their place is seen a zone of thickly accumulated and mostly well-formed polynuclear leucocytes. The pus-cells are copious, and are sur-

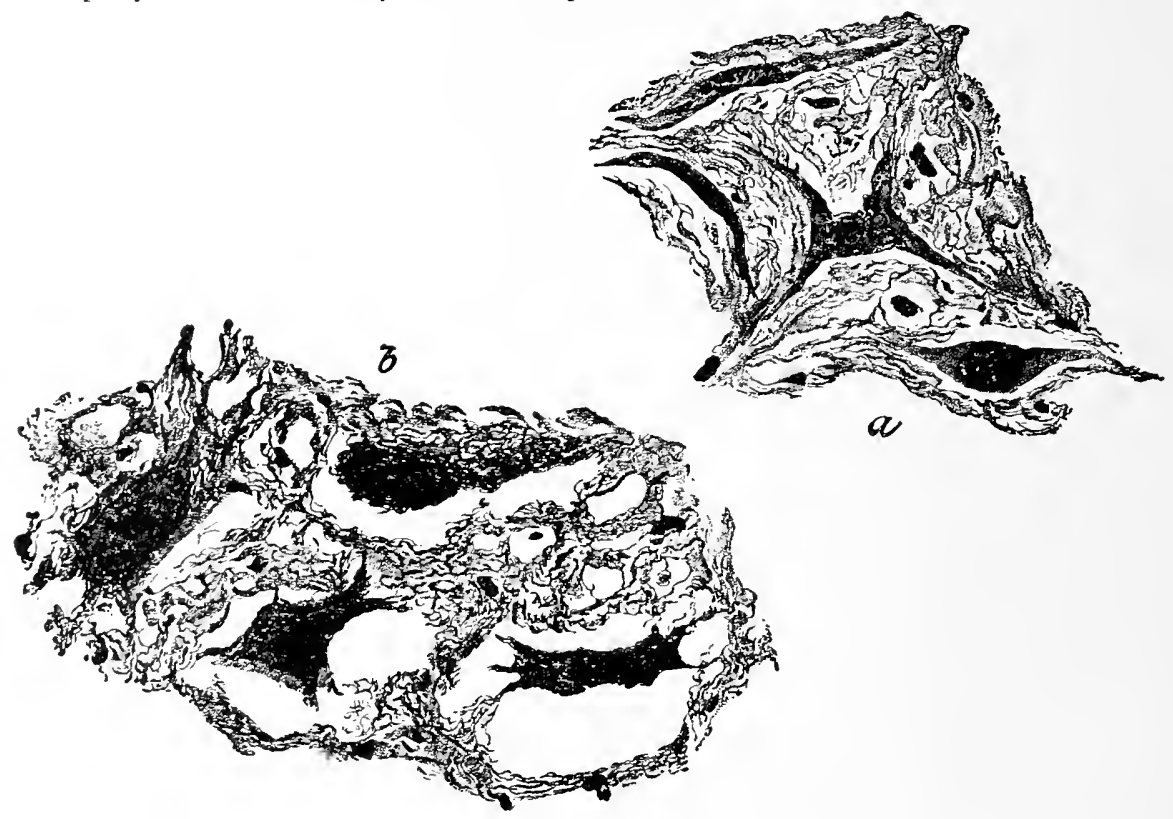

FIG. 87.--(a) Degeneraterl swollen connective-tissue cells-"vacuolised connective tissue." (b) Connective-tissue cells still more altered-giant-cell forms. (From II'. Scholt " "Ueber den Einfluss der Roentgenstrahlen auf die Haut in gesundem und kranken Zustande." Archiv f. Dermatol. 11. Syph., Vol, LIX, Part III.)

rounded by fine thread-like masses. These could be shewn by II'cigert's method of staining to form in places a fine network between the pus-cells. Over this zone of leucocytes lies a thin layer composed of broken-up pus corpuscles, nuclear remains, detritus, and largely of masses of bacilli and cocci.

Towards the cutis the zone of lencocytes either presents a sharply-defined border, or is seen penetrating the connective 
tissue. The papillary body has its outline comparatively well preserved for the greater part, or is at least recognisable. The connective tissue is soaked with serous exudation, swollen, in parts loosened into small bundles. The elastic network is preserved; on the other hand, the connective-tissuc cells shew marked degeneration: the protoplasm is swollen, diffusely stained by hxmotoxylin, containing one and often sereral bladder-like nuclei of peculiar shape. The small ressels are much dilated, gorged with blood, and suriounded by leucocytes. In the zone of leucocytes and in the cutis hemorthages are seen of varying size. In the case of severe Roentgen-ulcers the ressels of the cutis and sub-cutis are in places completely obliterated, the connective tissue vacuolised throughout, the connective-tissue cells forming a kind of giant-cell. (See Fig. 87.)"

Scholtz studied the process of healing of superficial Roentgen-ulcers in several specimens taken from the human skin.

"The infiltration in the corium diminishes; the connectivetissue cells and their nuclei become normal again, and where the papillary body has been destroyed it becomes replaced by fine, well-staining connective-tissue fibres which lie parallel to the surface of the skin. The epithelium slowly spreads from the borders of the ulcer and soon sends processes towarts the still somewhat adematous connective tissuc, or covers over the papillary bodies which may be still retained. In the latter event complete restoration of the normal configuration of the skin is effected, in which only the destroyed follicles are wanting.

Not only does the connective tissue in the healed area remain for some time soft and wanting in strength, but the epithelial cells also shew signs of marked disturbance for a while.

The rete remains celematous for some time, the pricklecells swollen, the nuclei bloated and badly-staining, the prickle, and especially the granular layer, is often mach widened, and the keratohyalin collected in lumps and particles in the cells. In the horn-hyer are found nucleated cells in which the horning process is incomplete. Moreover, in cases where the reaction has not reached the stage or excoriation or ulceration, but merely a well-marked dematitis has shown itself, sereral weeks after 
the irradiation has been discontinued and all inflammatory phenomena have disappeared similar enlargement and changes in the prickle-cell and granular layers are evident.

It is quite comprehensible that under these conditions the newly formed Roentgen-scar and powerfully irradiated areas of skin should be very sensitive to caustic applications or to fresh exposure to the tube. Weak dressings of pyrogallol ointment may readily cause fresh ulceration of the whole, and these new ulcers may be very slow in healing."

The destruction of the cell-elements and especially of the connective-tissue cells is probably the cause of the tedious repair of Roentgen-ulcers, since, as is well known, scar-formation proceeds from the connective-tissue cells.

The above-described histological changes afford a ready explanation of the falling of the hair after Roentgen-radiation. The rays soon take effect on the prickle-cell layer and bring about degenerative changes in its cell-clements; it naturally follows that the cells of the hair-sheaths, in which the cells of the rete form the outer layer in the depth of the cutis, are soon affected, and may be completely destroyed. This only happens where a certain degree of intensity in the irradiation has been attained, whether by one single powerful exposure or by the accumulated effects of several weaker exposures; moreover, it may occur without the rest of the tissues being especially affected.

Salomon ${ }^{1}$ ) described pronounced changes in the elastic ibres in a case of lupus treated by X-rays. These fibres were quite normal in the deeper layers of the cutis; more superficially, however, "innumerable small, very fine fibrils were seen, which could be demonstrated by the Unna-Tänzer or IV'eigert methods. These were slightly twisted, often wary, and sometimes lay parallel to the surface, sometimes vertically. They increased in numbers towards the surface and reached to the small-celled infiltration below the epidermis, without actually coming into contact with the epiclermis itself. The same appearance was manifest in the thickened adventitia of the blood-

\footnotetext{
1) Archiv. i. Dermat. u. Syph., Vul. LX, Part II.
} 
vessels, but here the fibres lay always parallel to the vesselwall." Clearly these were very young elastic fibres.

Zehmann ${ }^{1}$ ) examined hairs which had been caused to fall by Roentgen-radiation. In most hairs the portion of the shaft nearest to the hair-root was considerably thinner, and instead of the usual bulbous appearance scen in the root itself was a thin, pointed remmant. In other cases, where the hair-shaft retained its usual calibre, in place of the bulb a stumpy, conical end was seen. Often the bulb shewed a slight swelling at the lower end. In a few cases hairs were found having wellmarked bulbs with a peculiar brush-like condition. In view of the three first-named conditions, Zehmann considers that we have to deal in these cases with a rapidly developing atrophy of the hair-root, reminding one in some ways of the condition obtaining in alopecia areata.

The prognosis of Roentgen-dermatitis depends upon the following factors: the intensity of the irradiation, the extent and depth of the pathological changes, the locality affected. The sooner a powerful reaction makes its appearance, the more severely does it run its course, and the worse are the prospects of early cure. As a rule, dermatitis and ulceration shew a tendency towards improvement after about two months, by which time in ordinary cases the after-effects of the irradiation have exhausted themselves, and fresh hairs begin to make their appearance. Of course the rapidity of healing depends largely upon the size of the ulcer. The extent of the scarring, telangiectasis, etc., which may follow, depend, moreover, upon the intensity of the dermatitis and the extent to which the tissues have been transformed. These after-conditions, therefore, are controlled by carlier factors-the intensity of the treatment and the reaction. The appearance of a white patch on a larger, red, shallow excoriation is a bad prognostic sign; this indicates later ulceration. Ulcers lying over superficial bony prominences (the chin, vertebral spines, etc.) are especially obstinate.

The treatment of these affections is not so ineffective as is generally supposed. In the mildest case, marked only by sub-

1) Freund. Wiener merl. Wockenschr.. 1897, No, 10. 
jective symptoms, the application of a dusting powder is quite sufficient. If erythema or excoriation be present, tepid dressings of boracic lotion, a $15 \%$ boracic lanolin, or diachylon ointment, are useful. Oudin recommends peroxide of hydrogen dressings.

Cold applications of liquor Burowii, which are so often recommended, are directly harmful.

The author repeatedly observed in several cases of hypertrichosis under treatment by $\mathrm{X}$-rays during a cold winter season that when the patients reached the stage of reaction the skin, under the influence of the extreme cold, became darkly cyanotic, so that the patients and their friends became not a little alarmed. This discolouration, and a strong burning sensation which accompanied it, disappeared in a few days. Perhaps the condition is to be referred to the relaxing effect of the cold upon the blood-vessels.

The application of warmth to the affected regions is always acceptable to the patient, whether in the form of hot fomentations, poultices, or otherwise. The author recently treated, in conjunction with Prof. Elrmann, two cases of deep Roentgenulceration which had resulted from exposure to soft tubes by a Viennese practitioner; radiant heat from a powerful incandescent lamp ( I oo candle power) was employed, with daily half-hour sittings. This not only relieved the subjective symptoms, such as intense itching, but rapidly healed the sores. The good results attained in these two cases would appear to speak well for the method ${ }^{1}$ ). Very obstinate ulcerations should be thoroughly and deeply excised and a plastic operation afterwards performed.

Apostoli and Oudin have seen good results from the employment of spark-discharges.

The pigmentation left after irracliation disappears as a rule spontaneously in a short time. Török and Sclein hasten its departure by the shaling action of naphthol ointment.

$\left.{ }^{1}\right)$ The author heard afterwards that $B$ ur had achieved good results in the treatment of Rocntgen-ulcers with red light. Referred to by Oudin at the II Congr. internat. d’Electrologie et de Radiologic, I Sept. Igoz. 


$$
\text { IV. }
$$

BECQUEREL-RAYS. 



\section{BECQUEREL-RAYS.}

\$3. This is the name given to a physical phenomenon, strikingly similar in its effects to the $\mathrm{X}$-rays, which was discovered by $H$. Becquerel in 1896 . Whilst, however, the X-rays can only be produced by the aid of rather complicated apparatus, in which electrical processes take place, the Becquerel-rays require no physical instrument at all. They are emitted by certain chemical elements procured usually from uranium pitchblend, and, so far as is at present known, no external action on the substances is required to bring about their emission. Neither directly nor indirectly is any electrical apparatus needed for the production of these rays, and the radiation, for some reason still unexplained, goes on, undiminished, without any assignable external cause.

Becquerel found that not only uranium salts, but also metallic uranium possesses radio-activity. Mme. Slodkorwska Curie proved that when the bismuth is eliminated from natural uranium pitch-blend, traces are found of a substance which gives out Becquerel-rays of great intensity. This substance M. and Mme. Curie called poloninum. A second radio-active constituent of pitch-blend is found combined with barium; this is called radium. Debierne discovered yet another substance with radioactive properties in pitch-blend; this he called actinium. Thorium, too, and its combinations have been found to be radioactive. Afanasjew and Crookes discovered that all minerals containing radium and thorium are more or less radio-active, even when extremely minute quantities of these elements are present. According to Elsler, many, indeed, perhaps all, bodies present on the surface of the earth emit Becquerel-rays. The radiation of polonium, of the radio-active lead discovered by K. A. Hofmamu and E. Stranss, and of other radio-active substances, falls off markedly in course of time, but sulphate of lead is said to regain the power under the influence of cathode- 
rays; the elementary nature of these substances is a matter of doubt, and it is surmised that they owe their activity to induced radiation or to incorporated traces of radium or actinium, which permanently retain their activity. Becquerel, for instance, found that a non-active body brought near to an active body and exposed for some days to irradiation from the latter, itself becomes for some time radio-active.

Elster and Geite! have lately proved that in the atmosphere too, particularly where (as in enclosed spaces) it is seldom renewed, some radio-active substance is present, which diffuses electricity in the atmosphere.

It is exceedingly difficult to procure these radioactive preparations. Herr Giesel told the author that from soo grammes of raw material he gained only 0.3 gram of radio-active substance. Hence these preparations are very costly. Fon Lengyel lately succeeded in obtaining synthetically radio-active substances (sulphate, chloride and carbonate of barium).

A distinctive mark of radio-active salts is their self-luminosity. They phosphoresce unintermittenly, without any preceding illumination, by means of their own rays, which proceed from the depths of their own substance. Giesel found this quality specially marked in active bromide of barium, freed from water. The luminosity of radio-active substances disappears when they are warmed and re-appears as they become cold.

Becquerel-rays blacken a photographic plate; like the cathode-rays (Becquerel, Goldstein), they produce certain aftereffects of colour on rock-salt, chloride of potassium and fluorite; and, like the $\mathrm{X}$-rays, they colour glass violet (Villari, Berthelot). M. and Mme. Curie, Berthelot and Becquerel found other chemical effects of Becquerel-rays. Thus, they impart ozone to the air, and darken barium platino-cyanide, and, in presence of oxalic acid, reduce corrosive sublimate to calomel. Further points of similarity between the cathode-, Becquerel-, and X-rays were proved. Thus, P. Bary found that all the chemical compounds which are made luminous by the $\mathrm{X}$-rays become Dhosphorescent also through the others. According to Him- 
stedt, both kinds of rays lessen the resistance of selenium-cell; the property of ozonizing the air, too, is common to both cathode- and Becquerel-rays. The best known peculiarity of these rays is their power of passing through dark opaque bodics (metals, too, with the exception of lead), and then acting on a photographic plate or a fluorescent screen. Becquerel-rays, however, differentiate less than the Roentgen-rays. A metal object enclosed in an opaque covering is recognisable on the luminous screen and on the photographic plate, but not so the bones of the hand.

With suitable experimental conditions the Becquerel-rays have a similar electric action to the short-waved light rays; thus they impart a certain electrical conductivity to gas permeated by them ${ }^{1}$ ).

Geitel holds that air in a normal condition has some slight conductivity, which is raised by the merest trace of a radio-active substance. When radio-active substances have been kept in any room the walls for a long time after radiate radium rays (induced radiation) and ionize the air, so that, for experiments on the normal conductivity of the air, it is necessary to work in places where it is certain that no radio-active substances have been stored.

The rays of radio-active substances are capable of discharging a charged electroscope; the brush and spark-discharge of an induction-apparatus between a sphere (as anode) and a disc (as cathode) is converted into a glow-discharge. Becquerelrays possess this peculiarity to such an extraordinary degree that it may be used to prove the presence of very feeble rays, and of the rays of those bodies which preserve their radio-activity for a short time only. Under the influence of Becquerelrays the delaying of the process of spark-discharge (discovered by Jaumann) is suspended

Giesel, v. Schweidler, and St. Me'yer regarded the deflectivity of these rays in the magnetic field as a negative-electricity

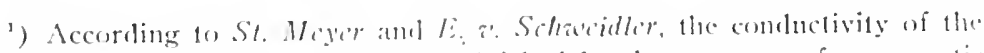
air irradiated by becefuerel rags in diminisled by the presence of a magretic field. 
morement. This behaviour in the magnetic field points to the deflectible rays carrying with them negative electrical discharges. In the electrostatic field, too, as is seen from Becquerel's and Dorn's experiments, they are movable. It has been concluded from this behaviour, and from other qualities in harmony with $i t,{ }^{1}$ ) that the rays proceeding from the radio-active substances are physically identical with cathode-rays, and that they owe their origin to the emanation from the radio-active bodies of very minute particles.

The rays proceeding from radio-active bodies are not homogeneous, but are of several kinds, quite distinct in character, which vary in their power of penetration ( $W$ alter $)$, in their behaviour in the magnetic field ( $P$. and $S$. Curie, Becquerel, Villard, Giesel, St. Meyer, and others), and in their physiological action on the eye and the skin (Aschkinass, and the writer). It has been shown that besides the rays which undergo distinct deflection in the magnetic field and have great penetrating power, whose co-efficient of absorption diminishes as the thickness of layer increases, radium, uranium, and Giesel's polonium emit other rays very similar to the $\mathrm{X}$-rays. These rays, like $\mathrm{X}$-rays, are not affected by a magnetic field, and have far less penetrating power than those in the first group; their co-efficient of absorption increases with the thickness of the layer. Dorn discovered the remarkable circumstance that rays not magnetically deflectible are converted almost wholly into magnetically deflectible rays by passing through paper, and partially so by passing through aluminium.

The wave-length of the Becquerel-rays has not yet been determined; it is surmised that it is far less than that of the Roentgen-rays (calculated at o.00I4 micron). Their velocity has been calculated by Kaufmann at about $250,000 \mathrm{~km}$. per second. This is not very different from the velocity of light $(3 \mathrm{IO}, 000 \mathrm{~km}$.$\left.) , and that of cathode-rays (280,000 \mathrm{~km} .)^{2}\right)$.

$\left.{ }^{1}\right)$ For instance, they evoke, like cathode-rays, the thermo-luminosity of fluorspar, which previous heating has destroyed.

$\left.{ }^{2}\right)$ Nany of the facts given here are taken from Dr. J. Elster's comprehensive references in Dr. J. M. Edcr's Jahrbücher für Photographie u. Reproduktionstechnik (for 1900, I90I, 1902), to which the reader is referred for fuller information. 
$\$ 36$. As has been already mentioned, certain biological effects of Becquerel-rays have become known to us. We are acquainted mainly with their influence on the skin and the eye, and on bacteria.

According to Henri Becquerel, the germinating power of seeds is destroyed by prolonged irradiation.

Two Italian investigators were the first to report an action of the rays of radio-active substances on micro-organisms. Pacinotti and Porcelli ${ }^{1}$ ) found that various germs could be killed within 3 to 24 hours by the rays emitted by a freshly obtained powder of metallic uranium, which had been exposed to sunlight and then cooled down to a temperature of $44-55$ degrees in a receptacle from which the air was excluded. The microscope, it was averred, showed distinct changes in these germs, which could only be ascribed to chemical action on their protoplasm.

Experiments were made in this way on staphylococci and streptococci, proteus, and cholera germs, also on the bacilli of tuberculosis, diphtheria, and typhus. Five $\mathrm{ccm}$. of a very poisonous broth culture of streptococcus were injected under the skin of both ears of a rabbit, and one ear was exposed to the action of the uranium rays. No inflammation at all resulted in the irradiated ear, whilst in the other ear the local affection took its ordinary course.

The present writer in the beginning of 1900 made some experiments with a view to testing the possible bactericidal power of Becquerel-rays ${ }^{2}$ ). In his investigations he used the salts of metallic uranium, not uranium itself. M. and Mme. Curie had observed ${ }^{3}$ ) that both natural pitch-blend and the bismuth and barium compounds obtained from it are considerably more active than metallic uranium itself. Of the two substances (radium and polonium), three preparations of radium were at the author's disposal. The first was a few grains of the original preparation of M. and Mme. Curie of Paris, which

$\left.{ }^{1}\right)$ Gazetta degli Ospedali, ref. to in Wiener med. Blätter, I899. No. I, p. 15 .

2) Die physiologischen Wirkungen der Iolentladungen, etc., l. c.

-) Compt. rend., 180,8, CXXVII, 11]. 175, 1215. 
Dr. St. Meyer of Vienna most kindly lent him for purposes of experiment. The two other radio-active substances $A$ and $B$ came from the laboratory de Haën at List, near Hanover, where they had been prepared according to the directions of Herr A. de Haën. $A$ differs from $B$ in being self-luminous to a greater degree; $B$, on the other hand, stimulates fluorescence in the barium-platinum-cyanide screen much more than $A$ does. Several grains of both $A$ and $B$, thanks to the kindness of Hofrath Prof. Dr. J. M. Eder, director of the Imperial Graphischen Lehr-u-Versuchsanstalt, were procured and set aside for experiment. In the first place Dr. St. Meyer established the fact that both $A$ and $B$ had the same electric qualities as the Curic preparation. As the substances are extremely hygroscopic and lose something of their strength on contact with the air, each of the three preparations was first wrapped in a small covering of parchment paper, and this again enclosed in an envelope of fairly thick aluminium foil, any gaps being closed with a cement impervious to air and moisture. These covers of paper and aluminium apparently offered no obstacle to the passage of the rays, as could be easily seen from tests with the barium-platinumcyanide screen. They were marked on the outside $A, B$ and $C$.

On the agar plates $(a, b$ and $c)$ diffused cultures of staphylococcus pyogenes aureus were made; on the centre of each dish a piece of sterilized paper was spread, and on these again the substances $A, B$ and $C$ were laid. They were left, each on its respective dish, for 3 hours, and at the end of that time both they and the papers were removed and the dishes put in the incubator.

After 24 hours all three cultures showed a quite even growth, without any interruption of luxuriantly developing colonies.

The experiment was repeated, with the modification that the irradiation was left to work for 3 days instead of 3 hours.

The result was again a wholly negative one. The subject of the next experiment was a micro-organism of less resisting power, viz., the typhus bacillus. The experiment was arranged like the preceding one. 
In this case, too, even after 3 days' action of the rays on the cultures, there was not the slightest evidence of any influence on the growth.

These experiments, undertaken for the purpose of showing any possibly existing power of the Becquerel rays to kill bacteria, already developed and capable of life, were carried on by the author in precisely similar fashion to the methods he adopted in his experiments on spark-discharges.

These experiments too yielded a negative result, and justify us in the conclusion that Becquerel-rays of the quality and intensity available for enquiry are not in any way capable of checking the development and life of bacteria.

With regard to the action of Becquerel-rays on the skin, observed by Giesel, M. and Nime. Curie, and others, when the preparations were used under other experimental conditions, the author surmised ${ }^{1}$ ) that radium preparations emit various classes of rays, of which some are more biologically active than others.

This surmise is confirmed by the result of experiments reported by Aschkinass and Caspari. These writers made experiments on the bactericidal propertics of Becquerel-rays with a very powerfully radio-active preparation of bariumradium-bromide, investigating first the action of the non-absorbable rays which had passed through aluminium foil.

Results were absolutely negative; the bacteria developed under the action of these rays just as they did without irradiation. But when the absorbable Becquerel-rays were examined, the result was positive; the casily absorbable rays checked the development of the bacteria on an agar plate, the organism growing luxuriantly in the part on which no rays fell. Aschkinass and Caspari proved by special control experiments that bacterial development was checked, not by the air changed (ionized) by the Becquerel-rays, nor by the bromine freed from the bromide, but solely by the easily absorbable rays falling on the bacteria. Where these rays had to pass through a thicker

') Nalurforscherversammlung, IIamburg, Igor. 
stratum of air before they reached the agar plate no effect was seen; the effective rays had been absorbed by the air ${ }^{1}$ ).

Strebel, too, reported ${ }^{2}$ ) experiments with positive results; according to him bactericidal action is sometimes present, sometimes not.

Quite lately the author was again in a position to make some experiments, with a radium preparation made by Herr Saubermann of Berlin. This time he did not use an aluminium covering, but enclosed the preparation in parchment paper only; in spite of this the result was negative, after the bacteria culture had been' exposed as close as possible to the rays for 3 days. This preparation too, therefore, emitted rays too weak to act on the bacteria.

The writer, simultaneously with these experiments on bacteria, made the first experimental investigation of the action of Becquerel-rays on the skin.

The 3 aluminium envelopes were enclosed side by side in a gutta-percha paper, and this was made adhesive with chloroform and applied to the outer aspect of the upper arm. The whole was bound closely to the arm with a bandage. For 3 days the little packet was left on the same spot. At the end of this time it was removed, when the skin did indeed show some redness, but so slight that it seemed to the author more probable that this had come from the rubbing and the irritation of the gutta-percha paper than from any action of the rays.

Grouven too made experiments on himself with a radioactive preparation. This was bound on the arm for $\mathrm{I} 2$ and 36 hours; after 6 weeks there was still no sign of reaction ${ }^{3}$ ).

On the other hand, $W$ alkhoff $^{4}$ ) observed an action of the Becquerel-rays on the human skin exactly corresponding to that of the X-rays,

1) Pfïger's Arch. f. Physiologie, 1901, Vol. LXXXVI, pp. 603 ff.

2) 73. Versammlung der Naturforscher und Aerzte in Hamburg.

3) Versamml. der Naturforscher und Aerzte in Hamburg.

") Phot. Rundschau, XIV, p. I89. 
On February I9th, 1901, Dr. Giesel told the author: "W'alkhoff laid on his arm for 20 minutes on two occasions $0.2 \mathrm{grm}$. of a preparation of mine in a double celluloid capsule. Alter I 4 days the skin became violently inflamed. The spot was still reddish brown after six months, and is even yet distinguishable. By mistake I applied to myself for two hours $0.3 \mathrm{grm}$. of a more powerfully active preparation. At first there was only faint redness, but after 2 to 3 weeks violent dermatitis set in, followed by blistering and destruction of the epidermis as after a burn. At this time the exactly circumscribed spot looked so bad that I put myself under medical treatment (Dr. Stermlhal), fearing that the destruction of tissue might extend deeper. Two or three days after that the place began to heal, and in two days more the skin had formed all over it; only a roughness was left for some time. The place now, after about three months, is faintly indicated by the absence of hairs.

"I was the first to find that radium reacts also on the living plant leaf after contact for a few hours; the chlorophyll disappears after some days, and months later the spot is still recognisable by the autumnalyellow colouring of the whole leaf and the brownish spots on the surface.

"Even the best preparations have absolutely no effect on bacteria, as $I^{\prime}$ alkhoff has proved in the Munich Physiological Institute.

"To-day a medical man, who wanted to experiment on the eye with one of my preparations, assured me that he had not been able to observe the slightest bactericidal properties on the part of radio-active bodies."

Dr. A. Stermlhal, who had treated Dr. Giesel for this dermatitis, reported at the Breslau Congress of Dermatologists ${ }^{1}$ ) that in the site of the inflammation a smooth, white, somewhat depressed scar was to be seen, with more colour round its bor-

1) Verhandlungshericht, 1) 480. 
ders. The spot was absolutely hairless, whilst hairs grew all round about it on the arm.

M. P. Curie made several interesting experiments, based on those of Giesel on himself, with a preparation the activity of which was about 9,00o times as powerful as that of uranium.

Curie gave the author the following particulars of the experiments: August Ist, I90I. "I allowed the preparation to act on my arm for ıo hours. It was separated from the skin by a thin layer of gutta-percha, and held firmly in place by a bandage, for it is necessary that the substances should be very close to the skin and be only separated from it by very thin media. The action under such experimental conditions was more powerful than I desired, for even now, 4 months after the irradiation, the wound is not yet healed.

"Stronger preparations-and we possess some I,O00,000 times more powerful than uranium-act from a distance and through glass, and an exposure of Io minutes suffices to produce inflammation of the skin. The reaction sometimes does not show itself until 19 and even 30 days after irradiation."

Becquerel put into a glass tube $\mathrm{I} \mathrm{cm}$. long and $3 \mathrm{~mm}$. in diameter a few decigrams of radium-barium chloride, which has considerable radio-activity, surpassing that of metallic uranium about 800,000 times. The glass tube was hermetically closed, wrapped in paper, and put in a little cardboard box. On the 3 rd and $4^{\text {th }}$ of April this box was repeatedly put into the pocket of the waistcoat worn by the experimenter. The latter kept it in his pocket for perhaps 6 hours in all. On the $13^{\text {th }}$ of April he noticed that the rays, which had traversed the glass tube, the wrapping paper, the cardboard of the box and the clothes, had produced on the skin of the body a red oblong patch, $6 \mathrm{~cm}$. long and $4 \mathrm{~cm}$. broad. On the 24th of April the skin peeled, and the most severely affected part began to suppurate and discharge fragments of necrotic tissue. The wound did not heal 
until 49 days after the rays had acted. Thirty-four days after the experiment dermatitis of a less violent nature showed itself also in a second part, corresponding to the other end of the waistcoat pocket, to which the glass tube had probably got shifted for a time. Mme. Curie, who had carried a few centigrams of the same radio-active substance in a glass tube from one place tw another, had similar inflammation on her hand, though the glass tube was enclosed in a case (which had very thin sides). P. Curic and Becquerel further report') that in the course of their experiments, beside the abovementioned lesions, they suffered from peeling of the skin of the hands, and at the tips of the fingers, with which they had carried the little tubes, from very painful spots, which caused trouble for some time.

Aschkinass notes ${ }^{2}$ ) the interesting fact that the power of the Becquerel-rays is not lessened when the preparations are allowed to act on the skin packed in aluminium capsules.

Giesel discovered ") that the Becquerel-rays act on the eye. If a radium preparation is wrapped in light-proof paper and brought in the dark near to the closed eye an intense sensation of light is felt. 'This is strongest when the preparation is laid on the lid, but it is still very clearly perceptible when the hand is interposed or the preparation brought near the temporal region. According to Giesel, this phenomenon is probably due to some phosphorescence in the interior of the eye.

Giesel's results have been substantiated by numerous other investigators also. M. Maier ${ }^{4}$ ) reported radium rays as not acting on the normal eye, but this theory is contradicted by the observations of all the other investigators.

F. Himstedt and $I ! \cdot 1$. Nagel have subjected to closer examination Giesel's discovery of the light-sensation produced

1) Acad. de Sc., June 131h, 190т.

2) 73. Versammlung deutscher Naturforscher und Aerzte in Iamburg.

s) Physik. Zeitschr., I899. No. 3, 1. 43.

4) Bleiblätter, etc., 24, 1900, P. 1344. 
on the eye by Becquerel-rays ${ }^{1}$ ). They confirm his experience only in the case of an eye adapted to the dark, just as Roentgen-rays and ultra-violet light can only be perceived by a completely rested eye. It was not possible to determine whether the Becquerel-rays act directly on the light-perceiving organs of the retina, rods or cones, as they produce fluorescence (which acts as a diffused light source on the eye) in the transparent media of the eye, the lens and the vitreous humour. Himstedt and $\mathrm{Nagel}$ proved a stimulative action of ultra-violet and Roentgen-rays on the frog's eye.

F. Himstedt reports that it is surprising how quickly the eye tires of this sensation of light. If two perfectly similar little bags of light-proof paper are prepared, the one filled with radium, the other with a corresponding quantity of sand, and if these are placed alternately on the eyes, it is impossible, after about 30 repetitions of the experiment, for any one to tell on which eye the radium and on which the sand is placed.

Blind persons who have lost their sight through opacity of the cornea or of the lens, have, as might be expected, a sensation of light under the action of the Becquerel-rays.

Grummach noticed phenomena of irritation in the retina when working with radium. For some hours after he felt a glimmering sensation in his eye.

Dr. Javal ${ }^{2}$ ) together with $M$. Curie made several experiments on blind persons with a very powerful radium salt; this was enclosed in a glass tube, which again was kept in an opaque box of cardboard. The two blind persons first examined saw nothing; the radium gave them no sensation of light. One of them had lost his sight through atrophy of the visual nerves, the other through glaucoma. Javal and Curie pursued their investigations further in the Blind Institute in Paris. One blind person, a boy who had lost his sight through displacement of the retina, had still some slight capacity for receiving light impressions. Under the influence of the rays from the radium his whole ficld of vision was lighted up, and again, even when

1) Physik. Zeitschr., г90т, II. Jahrg. p. 362.

$\left.{ }^{2}\right)$ Physikal. 7eitschr., 1900, I, P. 476. 
he covered his eye with both hands, he perceived light. From this experiment Dr. Javal draws the conclusion that if it were possible to restore the transparency of the cornea the boy might recover his sight to a great extent.

Little is known as yet as to other physiological action of Becquerel-rays.

E. Aschkinass and $W^{\top}$. Caspari ${ }^{1}$ ) were unable even after several hours' exposure to Roentgen- and Becquerel-rays to discover that they had any effect on the consumption of oxygen by muscle preparations made from frogs.

$\$ 37$. H. Strebel made therapeutic experiments with radium substances in lupus. According to his reports, ${ }^{2}$ ) reaction took place in so far that the nodules became distinctly paler and the tissues softer. On another occasion he reports ${ }^{3}$ ) having produced an ulcer in a lupus patient.

Danlos and Bloch cured two cases of lupus erythematosus by the application for 24 to 63 hours of two preparations with an activity of 5,000 and 5,200 respectively ${ }^{4}$ ). Further, Danlos treated ${ }^{5}$ ) four cases of lupus vulgaris with two radium preparations with an activity of 2,500 and 19,000 . The period of application varied from 24 to 36 hours. The treatment answered excellently. The scars were smooth, soft and superficial. With weaker preparations too (activity of $\mathrm{I}, \mathrm{OoO}$ to I,80o) Danlos gained, as he reports, favourable results for the time, but recurrence soon took place. Ianlos considers the stronger preparations to be the more suitable for therapeutic use, as the weaker ones have to be applied for a very long time before they produce any effect, and this inevitably causes ulceration. Such complications may, he believes, be avoided by using the stronger preparations, which require less time.

According to Danlos, the course of radium reaction is as follows: The first redness shows itself, then after 6 to 20 days

1) 73. Versammlung 1. Naturforscher t1. Aeræte in IIamburg, Igor.

$\left.{ }^{2}\right)$ VII Congress of the German Dermatological Soc., Breslau, Transactions, p. 488 .

$\left.{ }^{3}\right)$ 73. Versammlung (1. Naturforscher t. Acrate, Hamburg, 1901.

4) Soc. d. dermatolog. et d. syph., Nov. $71 \mathrm{~h}, 190 \mathrm{t}$.

s) Ibid. July $3 \mathrm{rd}, 1902$. 
the epidermis becomes whitish, macerated, and peels off. Often a bulla appears, which bursts and leaves an ulcer. The ulcer discharges freely, and is deeper or more superficial and more or less painful according to the intensity of the ray's action. The pains, as in Roentgen-dermatitis, are often more pronounced by night. Danlos recommends radium in lupus vulgaris and erythematosus, hypertrichosis, superficial malignant disease and nævus.

Hallopeau and Gadaud ${ }^{1}$ ) treated lupus verrucosus of the hand with radium. The preparation had an activity of 19,0oo. It was applied from 72 to 120 hours. After this there was a change of colour, and in 15 days an ulcer appeared with yellowish base and regular borders; the ulcer was the seat of shooting pains. At the same time the hand became very stiff. Other parts which were only exposed for 24 hours appeared markedly improved.

Oudin proposed that in order to allow only the X-rays from radium preparations to act the substance should be encased in aluminium, which absorbs cathode-rays better than X-rays. He believes he has proved by experiments on guinea-pigs that various parts of the body react differently to radium rays.

From the little that we know as yet of the biological action of the Becquerel-rays we may assume that they are in this respect. very similar to the Roentgen-rays. Like the latter, they produce on the skin-earlier or later, according to the intensity of the irradiation-a kind of dermatitis, which besides other peculiarities (epilation) is specially marked by its slow course and the sluggish character of the lesions induced. Their action, too, on the eye is analogous to that of the Roentgen-rays. With regard to the bactericidal action of the rays of radio-active substances opinions are still divided. It would seem that only one special kind of rays is effective (i. $c$., those easily absorbed), and that only under special conditions and when very powerful preparations are used.

We require further experience before we can determine how far these rays are of practical therapeutic value. For the pres-

') Soc. 1. dermatulog. et d. syph., Nov. 7 th, tgor. 
ent they possess only scientific interest, partly because of the difficulty of making radio-active preparations and their enormous price, but partly also because few preparations are actinic to the same extent, and it is hence impossible to give directions for the strength and duration of treatment which shall be universally applicable. 

V.

TREATMENT WITH HEAT AND LIGHT RAYS. (PHOTOTHERAPY.) 



\section{TREATMENT IVITH HEAT AND LIGHT RAYS. (PHOTOTHERAPY.)}

\section{Elements of Photophysics.}

$\$ 3$. Light is the agency which renders bodies visible. There are two hypotheses concerning its nature. Niaton held that luminous bodies emit a very fine matter, which produces in the eye the sensation of light. This hypothesis is called the e'mission theory. According to the wave or undulation theory, formulated by Huyghens at the end of the I th century, light consists in an undulatory movement of ether, an exceedingly elastic, imponderable medium which pervades the whole universe, penetrating even between the molecules of material bodies. Waves proceeding from the source of light reach the eye and irritate the visual nerves of the retina, thereby producing in the brain the sensation of light. This theory enables us to explain readily most optical phenomena.

To Thomas Young we owe the fundamental discovery that the law of interference is applicable to light as well as sound. He applied the wave-theory successfully to explain the colours

$\left.{ }^{1}\right)$ Text-books and references: J. M. Eder, Ansführl. Handbuch der Photographic, Ialle, I891.-J. M. Eder, Jahrbücher f. Plontographie und Reproductionsverf. I. bis. 16 Jahrg., IIalle a. S.- II. H. Vogel. Handlusch der Photographic, Berlin, 1894. - Mïller-l'ouillet, Lehrlueh der Physik, Vol. II, 1. - II. Kayscr, Lehrbuch d. Physik, Stutgart, 19oo. - I: Kömer, Lehrbuch d. Physik, Vienna, 1897 - E. Riecke, Lehrls, d. Experimentalphysik, Leipzig, $1896 .-F$. Frankenlä̈lser, Das Licht als Kraft, Rerlin, 1902. - Nicls R. Finsch, Ueher die Anwendung won concentrirten Lichtstrahlen in der Medicin, Leipzig, 1S99. - Finsen, Dic Bulenung der chem. Strahlen des Lichtes, Leipzig, 1899. - Masnus Mueller, Der Einfluss des Lichtes anf die Haut, Bibliotl. med., Stutgart, 1900. - Cobhurd. Dic lleilkraft des Lichtes, Leipzig, 1898. - O. Lassar, Ceder die neueren Nethoden der Lupusbehand-

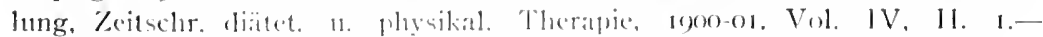
II. Strebel, Die Verwendung dece lichtes in der Therapie, Munich, 1902.J. Raum, Der gegenwärlig Stind un-wer Kontnisse neder den Einfluss des Lichtes etc., Zeitschr. f. Iygiene. Vul. VI. p. 312 etc. - II. Rieder, Handbuch d. physikal. 11. diatet. Therapic, I'art I, Vol. II. 
on certain kinds of thin plates and ridged surfaces. All his experiments in interference were explicable on the assumption that light is a wave-movement; the idea that light consists of flying particles explained nothing. Young discovered also the difference between light waves and sound waves, viz.: that in sound waves the air vibrates in the direction of propagation (longitudinal air-vibrations), whereas in light waves the ether particles move vertically to the direction of propagation (transverse ether-vibrations).

Just as there are high and low notes, so there are varying rates of ether-vibration. Violet light, for instance, makes nearly twice as many vibrations as red. The waves of red light are therefore nearly twice as long as the waves of violet light, for in the atmospheric ether all are propagated with equal velocity. The vibrations of ether particles may proceed along straight lines vertical to the ray of light, and in so doing may either lie all on one plane throughout the ray-this is rectilinear polarisation-or they may move in turn in all directions, as in the case of ordinary light. When the vibrations proceed along circular or elliptic courses, circular or elliptic polarisation takes place. The surface of a luminous, like that of a resonant, body may produce waves of varying size; hence daylight or composite light-like complexity of sound-consists of light of all possible colours and directions of polarisation. The simplest light is that which shews only one cclour, or which consists of waves of equal length and is polarised in straight lines.

Of late a theory has been brought forward which is founded largely on the work of Maxwell and Hertz and on our gradually increasing knowledge of the correlation of electricity, magnetism, and light. This theory regards ether-movements as the common cause of all three classes of phenomena. Scientists have inclined lately more and more to the assumption that luminosity is brought about not by the movements of atoms themselves, but by the movements with and upon them of their electrical charges, electrons.

$\$ 39$. Bodies which are in themselves sources of light, $i . e$, which become visible through their own power, are called selfluminous bodies. 
Amongst terrestrial bodies those which glow and burn are self-luminous.

As long as they are at a fairly low temperature (less than $400^{\circ}$ ) they send out rays of great wave-length (heat-rays); with increase of temperature the wavelength lessens. The heating power of light-rays lessens in the spectrum towards the riolet end. As the temperature becomes higher, the constant movement of the molecules composing the bodies becomes more violent. The atoms of which the molecules again consist begin to vibrate when the latter collide, and the vibration is communicated to the ether surrounding the atoms. As the molecular movement grows in intensity the clashing of the molecules becomes morc violent, and thereby quicker vibrations of the atoms and the ether are induced.

Again there are in nature (in the vegetable, animal and mineral kingdoms) bodies which are self-luminous in the dark.

Amongst self-luminous bodies we must in the first place include the sun and the fixed stars; of terrestrial substances there are, firstly, those which glow by reason of their high temperature (hot or molten metals, glowing carbon in illuminating gas, in electric lamps, etc.); secondly, luminous organisms $(e . g \cdot$, those on rotten wood, fireflies, the infusoria which produce marine phosphorescence, certain jelly-fish, etc.). The source of light in the latter class is as yet by no means accounted for.

Non-luminous bodies may be classiffed as transparent, translucent, and opaque, according as they allow more or less free passage to the ether waves striking them, or as they absorb them entirely. There are no absolutely transparent bodies. Thick layers of even the most transparent bodies appear slightly coloured, a sign that part of the white light falling on them has been absorbed. The space through which light passes is called (whether it contain solid matter or not) the medium. A very small luminous body or a very small luminous surface is called a "point" of light. 
$\S 40$. A straight line along which light is propogated is called a ray of light. Objects may be discerned through straight tubes, not through curved ones.

Light is propagaled in straight lines in all directions.

The extension of light in straight lines may be observed where sun rays fall into a dusty room. The formation of shadows, too, is only explicable on the hypothesis of the rectilinear extension of light.

I. The term "light ray" must be conceived of as a purely geometric definition, as the straight connecting line which we imagine as drawn from a point of light to some illuminated point. A light ray has no physical existence at all. A number of light rays are called a bundle of rays.

The phenomenon of the reception of light by one point from another luminous point in the same medium, in spite of the breaking of the straight connecting line between the two points by an opaque body, is called "deflection of light." The velocity of propagation of light was first estimated by astronomical means. It may be taken as being 299,300 kilom. a second.

$\S 4 \mathrm{I}$. If light is thrown on a sheet of paper from, say, a candle flame, the paper is illuminated less brightly in proportion as it is removed further from the source of light; or, the distance from the source of light remaining the same, as the angle of inclination at which the light rays strike the paper becomes less.

Light rays being projected along straight lines, it follows that the light effect spreads over spherical surfaces, of constantly increasing diameter, the superficial area being proportional to the squares of the diameters; hence the intensity of illumination of a given surface by rays striking it perpendicularly diminishes in proportion to the square of its increasing distance from the source of light.

The strength of light varies inversely as the square of the distance.

If the distance be doubled, the strength of the light is diminished to one-fourth; if the distance be increased threefold, the strength of light is one-ninth, and so on. Thus, if the distance 
be multiplied by four, the strength of the source of light must be multiplied by sixteen in order to gain an equally powerful illumination.

A surface is illuminated most powerfully when the effective light rays strike it at right angles. If the illumination is oblique, fewer rays fall on the same plane; some of the rays, too, are longer, and hence feebler in light as they strike the plane.

Whilst therefore the brightness of a self-luminous body depends on the intensity of the light in each single point and on the size of the illuminating plane, the brightness of illuminated bodies depends further on their distance from the source of light and on their position in relation to it.

The strength of illumination of a plane surface by parallel light rays is proportional to the cosine of the angle of incidence of the rays.

The brightness of a body, i. e., the amount of light which it reflects, is further dependent on its colour and its albedo.

The albedo of a body is that numerical value which gives the proportion of white light falling on a body compared with that which is reflected from it.

$\$ 42$. It is not possible, by the eye alone, to estimate with even approximate accuracy the relative brightness of two illuminating planes, or of the various points $(e \cdot g .$, in a gas flame) where the intensity varies. The aid of photoneters has therefore to be called in; these make it possible to estimate with certainty the relative intensity of two illuminating bodies.

The strength of light is denoted in normal candle-power. In Austria and Germany the nomal parafin candle $(N K)$ (20 millimetres in diameter and with a Aame 50 millimetres in height) was formerly often used in denoting the intensity of a light source. It is generally known as the German unit. In the last few years the more exact // ef ner-Alleneck normal lamp, burning amyl-acetate, has becn more and more used as the light-unit.

In Fngland the light unit is a certain sperm candle (standard canclle), in France a certain oil lamp (bec carcel). The unit of measurement for the illuminating power of an illuminated plane is the metre candle ( $1 \mathrm{M}$. K.), i.e., the illuminating 
power produced by I normal candle at a distance of I metre, where the light rays strike perpendicularly. Photometers serve to measure the optical intensity of a source of light, $i$. e., to compare its strength with that of a normal candle.

In Rumford's shadow-photometer two shadows of an opaque stick are cast on a white plane, the one by the source of light to be measured $(L)$, the other by the normal candle $(l)$; one source of light remaining at the same distance $(d)$, the other is moved on (up to $D$ ) until the two shadows appear equally dark. Then $L: l=D^{2}: d^{2}$.

$$
\therefore L=\left(\frac{D}{d}\right)^{2} \cdot l
$$

In L. IF cber's photometer light is thrown on 2 opalescent glasses, on one from a constant flame, on the other from first one and then the other source of light. A completely reflecting prism brings the images of the glasses side by side. By regulating the distances equality of brightness is produced.

The principle of Bunsen's grease spot photometer is that a grease spot on a piece of white paper appears dark when light is thrown on it from a source of light in front, and light when it is lighted up from behind. If light is thrown on the paper from the back and the front at once, the grease spot will look alternately dark and light, according as the back or the front light is the stronger. If the one light is kept stationery and the other brought nearer or further, a point will soon be found at which the grease spot will vanish, $i$. e., become invisible, because it will then look as bright as the surrounding paper.

Here, as in the case of the Rumford photometer, the equation holds good: $L: l=D^{2}: d^{2}$.

These photometers can only determine the intensity of the effect of the light on our eye. But this is essentially different from the intensity of the chemical effect of the light. No direct 
conclusion can therefore be drawn with regard to the chemical effect of various sources of light from their brightness as determined by the photometer. For testing the chemical intensity of a source of light recourse is had to photographic photometers or actinometers (i. c., semsilometers), which are based on the chemical action of light on substances sensitive to its effect. Sensitiveness of various substances ( $c$. g., silver salts) to the chemically effective rays varies according to their colour sensitiveness.

The photometer of Bunsen and Roscoe is based on the fact that chlorine and hydrogen combine to form hydrochloric acid, slowly in diffused and explosively in direct sunlight. The amount of hydrochloric acid which is formed in a given time and dissolves in the water serves as a measure of comparison for the various sources of light.

The Eder photometer is based on the decomposition of a mixture of bi-chloride of mercury with neutral ammonium oxalate. The intensity of the effective light is estimated according to the amount of sub-chloride of mercury precipitated. Eider proved experimentally that about $90 \%$ of the precipitate of chloride of mercury was the work of the ultra-violet rays, only ro\% being precipitated by the rest of the spectrum.

Further, there are actinometers made from sensitised papers. These are constructed on one or other of two principles. Either exposure is made until the paper assumes a certain normal colour (photometers on Bunsen's and Roscoe's principle), or exposure is made with a scale of regularly diminishing strength of light (as, c. g., with superimposed strips of transparent paper), and observations are taken of the progress of the photographic effect ${ }^{1}$ ).

A. Larsen with his chloride of silver paper photo. meter measures the blackening of the paper as the light passes through.

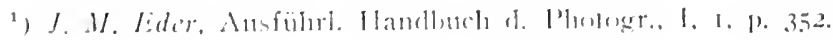


While photometry by means of the ordinary photographic papers is used to measure the intensity of the blue-violet and ultra-violet rays alone, it is possible, by adding certain colouring matter to the bromide of silver, to render the normal papers sensitive to other classes of rays. This was shown by Andresen with rhodamin paper, which is sensitive to yellow light; the bromide of silver indeed still remains very sensitive to blueviolet, but this is counteracted by the use of yellow filters (anramin).

A. W'ingen's is a normal colour photometer constructed on the same principle. J. M. Eder proved the colour-sensitiveness of Andresen's rhodamin-bromide of silver paper, and of rhodamin-chloride of silver and other papers by a series of experiments, from which it appeared that such photometer papers are affected by different zones of the spectrum, according as they are exposed to a more or less strong light. When shutting out (by yellow light filters) the blue and violet rays, which become effective with longer exposures, it is necessary to take into account the concentration and quality of the filtering colour ${ }^{1}$ ).

Electric photometers are based either on a peculiar property of selenium, which is a better conductor of electricity in the light than in the dark, or on the electric currents resulting from the chemical action of light on chloride of silver plates.

A. Larsen constructed an actinoscope for ultra-violet rays, based on the fact that ultra-violet rays are favourable to the formation of electric sparks. The spark of a Ruhmkorff coil jumps across a longer spark-gap if the negative electrode be irradiated by ultra-violet rays.

The mechanical effects of light and its power of producing phosphorescence have been utilised in the construction of photometric apparatus (radiometers, I'arnecke's phosphorescencephotometer), but such apparatus have been but little used in practice.

$\left.{ }^{1}\right)$ Wiener klin. Wochenschr., I902, No. 29. 
$\$+3$. The theory of the refection of light is called katoptrics.

If a light ray falls on an opaque body the light is thereby prevented from passing along a straight line in the same direction.

In such a case part of the light is thrown back or reflected. If the reflecting surface is smooth the resulting effect on the light is termed reflection; if the surface is more or less rough dispersion or diffusion results.

Mirrors may be plane, or they may have curved surfaces. If the curve is outwards the mirror is coneex; if the mirror is like part of a sphere, polished on the inside, it is concuie. The angle at which a light ray falls on a mirror is called the angle of incidence; the angle at which the ray is reflected from the mirror is called the angle of reflection. incidence.

The angle of reflection of light is equal to the angle of

Light rays falling on a concarc mirror, spherically curved, so as to pass through the centre of the sphere of which the mirror forms a part, are called axis rays. The spherical centre of the mirror surface is called the centre of curvature, and the straight line passing through this point and the curve-centre of the mirror itself is the optical axis of the mirror.

An axis ray is reflected directly back.

Rays paralla to the axis of the concare mirror ( $c$. g., the sun rays, coming from an infinite distance) are reflected in such a manner that all pass through the burning-point or focus. The focus is therefore the gathering-point of all the rays that strike the mirror parallel with the axis.

The focus of a concaie mirror lie's on the main axis, midway between the mirror centre and the sphere centre.

As the source of light approaches the mirror, so that its rays are no longer parallel to each other, the focus recedes further and further, even to infinity, when the source of light is in the focus. The reflected rays are then parallel. They become divergent when the source of light is brought between the focus and the surface of the mirror.

Important practical use has been made of this power of lierht dispersion for liant-projectors. Mangin in his 
apparatus uses spherical concave mirrors, consisting of weak concaro-convex lenses with a silver coating. Schuckerl's glass parabolic mirrors, being in one piece, have the adrantage over Mangin's of being free from chromatic and spherical aberrations (see 52), and of absorbing little light; further, they allow of the choice of a suitable focal distance. An arc-light is used. The carbons lie horizontally; the crater-formation on the positive carbon thus produced enables the source of light to be more fully utilised at a medium focal distance from the mirror.

The laws governing the origin of the various images cast by mirrors will not be treated of here, as they have no bearing on our present subject.

44 . The theory of light refraction is termed dioptrics.

We have seen above that opaque bodies do not allow of the rectilinear extension of light, but reflect it. The direction of light rays is altered further by transparent bodies, $i$. $e$., by those which allow a large part of the light to pass through.

An oblique ray, passing from onc transparent body into another which transmits light at a different rate changes its direction, i. e., is refracted.

Refraction is governed by the following laws:

I. The incident and the refracted rays are on opposite sides of the axis of incidence and lie in one plane-the plane of incidence.

2. The quotient of the sine of the angle of incidence and the sine of the angle of refraction equals the ratio of the velocity of light in the two media, and is called the quotient of refraction, or "refraction" index.

Rays impinging at right angles on the dividing surface of two transparent bodies are not refracted.

The refraction index depends on the rate of vibration of the light ray, i. e., its colours.

The refraction of light by means of polished glasses is frequently turned to practical account. Glasses with polished spherical convex surfaces are called lenses, or convex lenses; they are thicker in the middle than at the edges. Glasses which are 
hollowed out or concave (hence thicker at the edges than at the centre) are called hollow or concave lenses.

The optical axis of a lens is the straight line passing through the curve centre of its two surfaces, or-if one surface be plane -through the one curve centre, falling perpendicularly on the plane surface. Rays striking a concave lens parallel with the axis are dispersed after refraction. The axial rays passing through the centre of the lens are not refracted.

Objects looked at through a concave lens appear smaller and nearer.

Sun rays passing through a lens convex on both sides are so refracted as to converge as one point of light at a certain distance from the lens. A piece of paper held at this converging point, the focus, becomes heated and funally burns. The convex lens acts as a burning glass.

Light rays impinging on a convex lens parallel with the axis are so refracted as all to pass through the focus.

The distance of the focus from the centre of the lens is called the focal distance.

Rays passing through the focus and impinging on a convex lens are, after refraction, parallel with the axis of the lens. The axial rays pass through without refraction.

All objects within the focal distance appear, when seen through a convex lens, larger and more distant. The image of an object outside the focal distance appears reversed.

The effect of a lens depends on its diameter and its curve, as well as on the refracting power of its substance.

$\$ 45$. Parallel rays striking a large spherical concave mirror, or a spherical lens close to the edge, do not converge in one focus after reflection, but spread over a wider zone, whose axis is the focal line proper. The effect of this spherical aberration is to blur the image. It happens especially with thick lenses, and is due to the disturbing influence of the prisms of which one may imagine a lens composed. I enses are further subject to chromatic aberation. They break up white light into its component parts, as does a prism. Hence if a bundle of rays is thrown on a convex lens parallel to the optical axis, the violet rays, being refracted at a sharper angle, intersect each other 
again behind the lens at a shorter distance from it than the other rays; the red rays intersect at the greatest distance. At whatever point the image is caught, only one colour is sharp; the others are all blurred.

Parabolic mirrors, on account of the geometrical properties of the parabola, have no focal plane, but only one single converging point for all parallel rays. Hence parabolic mirrors are used in preference to concave as light projectors.

$\$ 46$. In most cases we have to deal with both reflection and refraction of light; that is to say, the whole of the impinging light is not reflected, but part of it finds its way into the second medium. In other words, reflection is associated with loss of light. The amount of loss depends on the nature of the media and the direction of the rays.

Part of the refracted light, again, does not pass through the second medium, but is used up in it, being converted into other forms of energy (heat, chemical energy, etc.).

Photometric measurements prove that the sum of the strength of the light reflected by a body and that of the light passing through it is less than that of the impinging light rays.

This phenomenon is termed light absorption. An absolutely transparent (diaphanous) body would absorb no light at all, but such a body is as non-existent as an absolutely opaque (adiaphanous) one, which would reflect all the light (total reflection excluded).

Even gold and other metals are transparent if sufficiently thin. A whole class of bodies ( $e . g$. , coloured glasses) absorbs only certain light colours; another (fluorescent bodies) changes the colour of the impinging light.

Transparent bodies become translucent by the incorporation of foreign particles, from which the light is diffusely reflected. Opalescent glass, blood, milk, etc., are examples of such media.

According to the law of the conservation of energy, the force of absorbed light is not lost, but is converted into other forms of energy of equal value. A change takes place in the condition of the light-absorbing substance, and, on the other hand, those rays which are absorbed are the only effective ones. Hence 
light which has passed through a substance and left behind in it some of its component parts is sensibly weakened.

$\$ 47$. A light ray passing through a prism is diverted from its original direction and resolved into its component colours. A colour series (spectrum) appears of red, orange, yellow, green, blue, violet; the red appearing least, and the violet most divergent. The yellow and orange of the sun spectrum are about twice as bright as the green, 30 times as bright as the blue and the red, and nearly 200 times as bright as the violet. The colours in the spectrum are neither of equal size nor sharply defined; they merge insensibly one into the other.

White light then is composite. Dispersion is the resolving of a composite light into its component coloured parts; each of these, in the case of white light, is simple or homogeneous. By means of a lens the colours of the spectrum may be reunited into white light.

Colours of bodies are not identical with the colours of the spectrum, being dependent on the light striking them. A body absorbs certain coloured components of the light and reflects or permits the passage of the rest. It is in the one case opaquely, in the other, transparently, coloured. The colours of bodies have thus no actual existence, but are mere products of light.

A transparent body is transparently colourless if it allows all the parts of the impinging light to pass through equally; it is, e. $g$., transparently blue if it absorbs all but the blue rays.

In a blue solution of copper sulphate the red and yellow rays chiefly are absorbed; the green and violet ones are allowed to pass, but less freely than the blue; hence the blue colour.

Yellow colouring solutions allow of the free passage of the yellow rays, and less freely the red and the green; they absorb the blue and violet entirely.

An opaque body is white when it reflects strongly and equally all the component parts of white light, and black when it absorbs them.

It is interesting to note that colourless bodies which are equally transparent to light rays may vary very much in the degree in which they suffer the so-called 
chemical rays to pass. For instance, "double-spathsoda" absorbs chemical rays less than crown glass and flint glass; rock-crystal and rock-salt absorb them least of all.

Red light penetrates bodies far more readily than blue. If lamp-light, which has been passed through a thick sheet of paper, is subjected to spectrum analysis, it will be found that nearly all the blue light has been absorbed and that only the red and yellow remain. With a thicker absorbing layer, red alone passes through. The same holds good of gases and vapours, e.g., the atmosphere (as may be seen from the red colour of stars, and especially also of the sun, as they near the horizon).

The sharper the angle at which the light is refracted, the more readily will it be absorbed.

According to the wave theory of light, each of the colours making up sunlight has its own rate of vibration. Red has the lowest rate (about 400 billion vibrations per second) ; the vibration rate increases with the other colours of the spectrum accordingly as they approach the violet end, while violet itself has the highest rate of vibration (about 8 oo billions per second).

A ray of white sunlight thus comprises vibrations ranging from 400 to 800 billions per second.

The various light-waves of a composite light ray are diffracted variously in a prism. The red light, having the lowest rate of vibration, is least diffracted, or retarded; the violet, having the highest rate of vibration, suffers most diffraction and retardation. Hence the varying refractive indices of the different rays of the spectrum.

The cause of this retardation of the smaller light-waves was for a long time not understood, and that it could not explain this phenomenon was felt to be a flaw in the wave theory.

Cauchy lent fresh support to the theory by establishing the connection between the velocity and the length of a wave, whenever the molecules of a body are in such a position that the distance between them bears a distinct relation to the wave-length. Different colours have different wave-lengths, decreasing in the spectrum from red to violet. 
Hence the colour rays towards the violet end of the spectrum are known as rays of lesser wave-length, or more highly refrangible rays; those towards the red end as rays of longer wave-length, or less highly refrangible rays.

Various methods, which we will not enter upon here, have been used to gauge the wave-length of the different kinds of light. Fraunhofer first determined the length of the visible rays, Cormu, Esselbach, and Eisenlohr that of the ultra-violet, Abney, that of the ultra-red spectrum.

To Angstroem we owe the more accurate measurements, and his unit $(A E)$ is now used to denote wave-lengths:

$$
\text { I } A E=\frac{\mathrm{r}}{10,000,000} \mathrm{~mm} \text {. }
$$

Light of shorter wave-length has a correspondingly shorter duration of vibration, but a higher rate.

The density of the ether particles amongst the molecules is greater in solid and fluid bodies than in gases; hence, light of shorter wave-length, on passing from the air, is more slowly propagated in solids and fluids than light of greater wavelength. Hence, too, it is more highly refracted.

The solar spectrum has at either end of its apparent limits further rays; beyond the red, the so-called infra-red or ultra-red rays of greater wave-lengths and lesser rates of vibration (less than 400 billions); and beyond the violet, the so-called ultraviolet, of shorter wave-length and higher (more than soo billions) rates of vibration.

The principle that a light ray is refracted more highly in proportion to the shortness of its wave-length applies also to the invisible ultra-red and ultra-violet rays.

Spectroscopes are used for the closer examination of the spectrum.

The white light emitted by growing solid and fluid bodies gives an unbroken spectrum.

If, however, sodium chloride is vaporized in the flame of a Bunsen burner, only two bright yellow lines appear as the spectrum of the sodium vapour.

Lithium vapour gives a red and yellow line, an do also salts of barium, calcium and strontium. Livery metal when valporized 
shows its own special line in the spectrum; hence the presence of certain lines in the spectrum of a flame points conclusively to the presence of the corresponding body in the flame. (Spectrum Analysis.) Gases, too, give such spectra when examined in Geissler tubes and illuminated by an electric current.

If the light of a bright flame be passed through sodium vapour, the spectrum will show two dark lines at the spot where sodium vapour alone would give two bright yellow ones. Following Kirchhoff, we call this phenomenon "reversal of the spectrum." If the light be passed through a coloured glass plate, or a glass vessel filled with a coloured solution, the lines of the colours which have been absorbed on the passage of the light through the coloured body vanish from the spectrum, and parts of the spectrum appear dark; thus we have an absorption spectrum.

This filtering or decomposing of white light has long been turned to practical account in various graphic crafts.

In a self-luminous body, emitting only light of one colour, $i$. e., of one wave-length, the particles all vibrate at the same rate. Vibrations of the same length striking the body set the particles in motion, like a body resounding in unison with another body giving out the same tone. The energy which makes the particles vibrate lessens the energy of the impinging ether vibrations, and weakens the light as it passes through; but the vibrating body at the same time gains in energy. This energy, however, is now radiated in all directions, not only in that of the impinging vibrations, where it is less than before.

This is the explanation of Kirchhoff's law: that a body, which, when glowing, emits only light of a given wave-length, absorbs an impinging light ray of the same wave-length. If it emits light of varying wave-length, it for the same reason absorbs all corresponding light rays. The ratio between the cmissive and the absorptive powers for rays of a like wavelength is, as Kirchhoff has proved, the same for all bodies at the same temperature. (Eiscnlohr.)

What becomes of the light that has been absorbed?

I. Absorbed light is usually transformed into heat. The 
impinging visible rays are then emitted by the heated body as dark heat rays of greater ware-length. Conversely a body played on by dark heat rays may be heated till it shines. ( Tyndall terms this calorescence.)

2. Absorbed light often causes the immediate emission of new light rays of a different colour (this is fluorescence). In such cases the bodies are, as it were, self-huminous throughout the period of their illumination; the colour of the light they emit is different both from that of the impinging light and from that peculiar to the body itself. The colours of bodies are caused by reflected rays, fluorescent colours by absorbed rays.

3. Absorbed light may also cause a more or less continuous emission of new light rays of different colours. This phenomenon, known as phosphorescence, is seen in sulphide of calcium, sulphide of strontium, etc ${ }^{1}$ ).

4. The light may produce chemical effects, notably decomposition. Photography is basect on such effects.

5. Electric phenomena may in many cases be caused by the impinging ether vibrations.

6. The light may, under certain conditions, produce mechanical results. (Crookes' radiometer.)

Emission spectra may be unbroken, in lines, or in bands; absorption spectra show only lines or bands. One end only of

$\left.{ }^{1}\right)$ Becquercl proved with the aid of the phosphoroscone that all solid fluorescent bodies are also phosphorescent, if only for a very short time. Hence he concluded that fluorescence and phosphorescence are in their essence the same, the difference lying mercly in their duration.

Filuorescence is chietly caused by light of short wate-length; the wares in fluoresent light may be longer, but are never shorter, than those in the exciting light.

Luminescence, acording to F. II icdemann, is luminosity produced hy external causes without any corresponding rise of temperature. He distinguishes several kinds, according to the exciting cause:

NAME.

Photo luminescence

Electro " "

Chemico "

Thermo

Trilio

Cryctal
Exciting; Cause.

Irratiation

Flectric dicharge

Chemical processes

Slight heating

Friction

Crystallization 
the spectrum may be absorbed ( $e . g$., alum absorbs ultra-red, glass ultra-riolet), or both.

Fraunhofir observed innumerable fine black lines (known after him as "Frannhofer lines") in the solar spectrum; some of the most important of these are shown in Fig. 88 . They were named by him after the letters of the alphabet.

Kirchholf explained their appearance by assuming that there are certain gases present in the gaseous envelope of the sun which would, if not interfered with, produce bright lines where dark ones are shown in the spectrum; that is to say, that the light irradiated from the white-hot sun suffers on its course loss of certain shades of colour through absorption. Accordingly

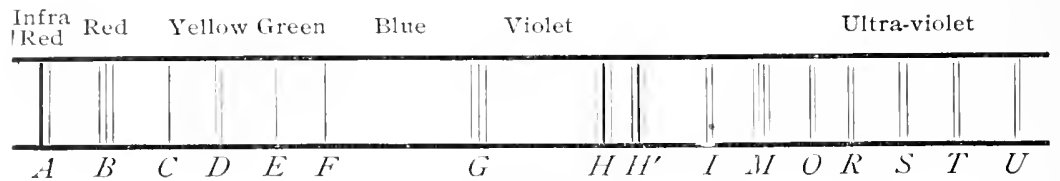

Fig. 88.-Solar Spectrum.

the solar spectrum is an absorption spectrum, and the Fraunhofer lines will show which gases are contained in the sphere of light.

Spectrum analysis, by enabling us to examine the chemical composition of distant bodies, especially of the heavenly bodies, has led to most important results. Further, by its intense sensitiveness to the slightest trace of an element, it has led to the discovery of a whole range of new elements.

The careful measurements of Kirchlioff, Thalén, Huggin, Dewar, Abney, Eder, I'alenta, Exner, Kayser, etc., have determined the wave-length of the light emitted by various elements. Thus:

$N_{\text {al }}$ shows a bright yellow double line, the so-called $D$ line. $(\lambda=5896.16$ and 5890.19$)$.

Li shows red lines (6708 and 6104).

Ba emits green light; its main lines are: 6497,6 I 42, 5828,5536 , etc.

Lines $/$ to $H$ in the spectrum are the most readily recognisable. The eye is most sensitive to the light between $D$ and 
$E$, that is, part of the yellow; from that point towards either end the light is less and less visible, until the red rays beyond $d$ and the ultra-riolet beyond $I /$ are hardly distinguishable.

Our visual organs then are limited in range, like our organs of hearing (we camnot hear sound wares of less than I 1 mm.). $D^{\prime}$ drsonial thus explains the insensibility to his currents.

It does not, therefore, follow that the light rays of either greater or less wave-length have less intensity of vibration. As a matter of fact, as II erscluel showed, the heat of sun rays increases towards the red and goes on increasing in the dark space beyond.

$\$ 48$. By the aid of an intensely sensitive instrument (the bolometer, based on the principle that a wire offers increased electric resistance with increase of temperature) which registers heat-differences to the millionth part of a Celsius degree, Langley discorered a heat spectrum, almost 20 times as long as the visible spectrum, extending beyond the red. In this heat spectrum there are about 700 "lines of cold" (reminding one of the Fraunhofer dark lines), where the bolometer marks no heat.

The wave-length of the heat rays that have been examined ranges from $0.7 \mu$ to $5.2 \mu\left(\mu=\frac{1}{1000} \mathrm{~mm}\right)$.

The heat spectrum, as exammed by the bolometer, shows a constant change of temperiture, at longer and shorter intervals. The spectrum shows lines corresponding to the sudden fall and rise of the temperature; the broader bands of lower temperature may be regarded as absorption bands. Langley's further investigations, at a height of 4,000 metres on Mount Whitney, proved that a great deal of heat is absorhed by the atmosphere; he was able at the same time to determine the amount of solar heat reaching our atmosphere.

This amount is so great that, if the rays struck vertically, it woukl thaw in one minute an ice crust of nearly 3 centimetres. The thickness of the atmosphere which the sun's rays have to penetrate varies at different times of the day (it is much less at noon than at sunset); hence the spectral vary correspondingly. Rays of short ware-length suffer most absorption. At noon the heat maximum is at orange, in the evening towards red. The 
character of the heat spectrum varies also at different seasons of the year.

Further investigation of the heat spectrum may, in Langley's opinion, have important results for meteorology. Both the quality (i. e., the rate of vibration of emitted rays) and the intensity of heat radiation from bodies are dependent on their temperature, their chemical nature, and the character of their surface.

Of the heat rays striking a body part are reflected, part penetrate the body. A diathermanous body is one which allows the penetrating rays to pass through; an athermanous body is one which absorbs them wholly or in part.

Since glass does not allow of the passage of rays of long

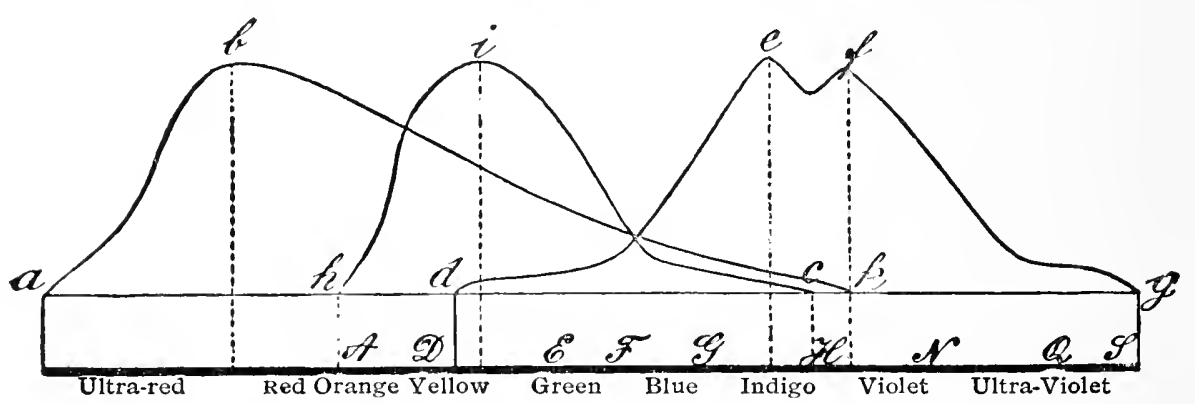

FIG. 89.-From A. Lainer's Lectures on photograph. opt., Vienna, I890, p. Io7.

wave-length, i. e., of heat rays, prisms of rock-salt or fluorspar must be used in these investigations.

$\$ 49$. Rays in the almost invisible part of the spectrum from $I I$ to $R$ act chemically on many light-sensitive preparations. Bromide of silver is affected at $F$, iodide of silver at $G$. On the whole, chemical action is strongest at $H$, and lessens in either direction. (On Fig. 89 , line $a, b, c$ gives the curve of thermal action, line $d, e, f, g$ the curve of the chemical action; line $h, i, k$ gives the light eurve, with its maximum at yellow.) This is true only of the solar spectrum; the spectrum of each light source varies. The spectrum of Drumond's limelight and that of the electric arc-light extend much further towards the ultra-violet end than the solar spectrum, because of the great absorption by the atmosphere of the short-waved sun rays. 
(The amount of ultra-red rays in sunlight depends on the amount of vapour and carbonic acid in the atmosphere.) Still more ultra-violet is shown in the spectrum of the sparks of an induction-coil; most of all in the spectra of aluminium, cobalt, iron or Eder's alloy (lead, zinc, cadmium). These are line spectra, showing innumerable lines, especially in the ultra-violet. The spectrum of iron, $e$. $g .$, has about 5000 lines.

Amongst the media which have been so far cxamined, rock-crystal, white fluorspar, and calkspar have no appreciable absorptive power for ultra-violet; rock-salt, ice, alkali-sulphates, -carbonates, -arseniates, and -borates, alkaline earths, soluble compounds of fluorides, chlorides and bromides of the alkalies and alum have very little. Ultra-violet rays pass without appreciable diminution through the flame of a candle and through pure hydrogen (Schumann).

Organic acids and their compounds, and especially many benzol derivations (Hartley), have great absorptive power. Glass, mica, gypsum, gelatine, and many animal and vegetable liquids absorb ultra-violet largely (de Chardonnel) ${ }^{1}$ (thus, $c$.. ., watery vapour absorbs all waves of less length than $307 \mu \mu$ ). Fluid albumen (i.ce, a $12 \%$ solution) absorbs, according to $G$. P. Drossbach, all waves of less than $320 \mu \mu$, and, when much diluted, all of less than $300 \mu \mu . \Lambda 1 \%$ solution of pepton-gelatin is permeable in the same degree as an equivalent solution of albumen.

Heavy flint glass and all kinds of dense lead-containing glass are most absorbent, crown glass and barytflint-glass less so.

Cornu proved that a quart\% prism gives a solar spectrum stretching beyond $R$ in the ultra-violet; a calkspar prism gives the spectrum to beyond $P$, light flintglass towards $N$ in the ultri-riolet, whilst heavy flint glass stops short of even all the visible violet.

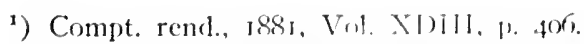

$\left.{ }^{2}\right)$ Deutsche med. Werch., November z, byor. 
Schumann's experiments showed that a glass plate O.I $25 \mathrm{~mm}$. in thickness absorbs a third of the extreme ultra-violet rays of the magnesium spark (more than $\lambda=277)^{1}$ )

Ultra-violet rays are refracted, $i . c$, gatherea, by quartz, rock-salt, or fluorspar lenses.

Ultra-violet rays, like the short-waved rays of the visible spectrum, can produce fluorescence. Cathode- and Becquerelrays make the air light-conductive and convert its oxygen into ozone, and ultra-violet rays of short wave-length $(\lambda=0.000$ I 4 - o.000 I9 mm.) have the same effect.

Her $\approx$ found that irradiation with ultra-violet light does away with the oscillatory character of the discharge from an apparatus emitting electric waves. We may imagine the layer of gas between the two ends of the conductor as becoming conductive through the splitting up of the electrically neutral molecules by the impinging corpuscles ${ }^{2}$ ). Following up this discovery, Hallwachs found that negatively charged metal surfaces are discharged by ultra-violet rays. Lenard ${ }^{3}$ ) discovered that the negatively charged "corpuscles" of a metal surface irradiated by ultra-violet light oscillate so violently in sympathy that they are thrown off from the surface at a great rate, showing all the characteristics of the ordinary cathode-rays produced by an electric discharge. The negative electric charge of a body is, thus, under the influence of ultra-violet rays, transmuted into cathode-rays, which pass off into space, at, however, a noticeably lower rate than light rays.

Side by side with the normal dispersion of light there is, exceptionally, an abnormal dispersion, when the

1) J. M. Edcr's Ausfuehr. Handb. d. Plotog., I, I, pp. 2г3. 283.

") See Lampa, Ueber Stromunterbrechung mit besonderer Berücksichtigung des Wchncltschen Unterbrechers, Sitzungsber. d. Kaiserl. Akademie d. Wissensch., Vienna, I90r.

s) Annalen d. Physik, I900, Vol. III, p. 29S.-Drudc's Ann., Vol. I, I900, p. 486.-Sitzungsber. d. Kaiserl. Akad. d. Wissensch., math.-naturw., Cl, Octuber 19, 1899. 
sequence of colours and lines in the spectrum is wholly different from that of normal dispersion. When a fuchsin alcoholic solution is used, as the prism violet is least diffracted, then red, then yellow, green and bluegreen are quite absorbed.

The position, too, of the spectrum lines may be changed.

Humphreys and Mohler found that when the source of light burns under pressure the lines are always shifted slightly towards the red.

Further, P. Zeemann discovered that when the light source is brought under strong magnetic influence the spectrum lines show peculiar changes: accoraing to the point of riew, they appear either doubled or trebled, with fixed ratios of polarisation.

$\$ 50$. The term interference of $h g h t$ is applied to the phenomena resulting from the mutual effect of light-waves meeting each other.

The interfering waves strengthen or weaken each other in proportion to the variance in their rate of progression. Hence with homogeneous light a series of alternate light and dark bands, with white light, a series of spectra, is obtained.

The colours of thin plates, due to interference, enable us to calculate the wave-length of the light used.

$\$ 5$. Deflection or diffraction of light results when light is passed through a narrow aperture or past a thin rod; the light is then transmitted slightly on one side, thereby giving rise to interference phenomena.

If homogeneous light be used a bright band of the same colour as the light will be seen, with alternate bright and dark bands on either side. If white light be used, a white band will appear, with colour bands to right and left of it. A grating is a series of narrow parallel apertures, made either with fine wires stretched at equal distances across (wire grating), or by scratching parallel lines on a soot-blackened glass plate, or on an ordinary glass plate with a diamond (glass grating). If white light, admitted through an aperture, is passed through : grating, and the image is projected on a sereen, brilliant spectra 
are seen on either side of the white centre, the direct image of the aperture.

In the spectrum nearest to the centre the colours are clear, and the red, yellow, green, and blue bands are of approximately equal breadth. The first spectrum is the clearest and brightest, the second is broader, the following ones are blurred. The same phenomena are observed when the light is reflected from a scratched surface. The reflection gratings most in use now are metal mirrors with very numerous fine scratch-marks ( 700 to the millimetre).

The metal is polished and concave, so as to serve at the same time as a mirror, giving back the images of the aperture without a lens. These are concave gratings.

The diffraction spectrum obtained from this apparatus has not only the advantage of being uninfluenced by absorption (an important point in investigations of ultra-violet, which is so much absorbed by glass prisms), but it has also the further advantage over the refraction spectrum of giving a deflection of the rays proportional to their wave-length. The refraction spectrum gives a quite disproportionate deflection of the more refrangible rays, so that the blue end is much broader than the red.

The identity of heat rays and ether vibrations is proved by the observation or similar interference phenomena in the visible and in the ultra-red (.$e$. , heat) and the ultra-violet rays.

$\$ 52$. Electro-magnetic vibrations of any wave-length are capable of affecting chemical processes. K. Schanm ${ }^{1}$ ) showed that under the influence of electro-magnetic vibrations:

I. Processes are accelerated which go on in the dark also (crystallisation, formation of $\mathrm{HCl}$ from $\mathrm{H}_{2}+\mathrm{Cl}_{2}$ ).

2. Processes are brought about which require light (decomposition of silver halogenide into silver and sub-haloid); many of these are reversed again in the dark (phototropy).

1) Sitzgsher. d. Gesellsch. z. Befoerderg. d. Naturwissensch. zu Marburg, No. 9. July, igor. 
Processes influenced or caused by light may be due to either exothermal (i. e., heat-producing) or endothermal (i. e., heatabsorbing) reaction.

It was formerly held that the red end of the spectrum had special thermal propertics; the centre, where yellow, orange and green seem brightest to the eye, especially optical; and the violet especially chemical.

It is a fact that violet, blue, and eren green light has a preponderating effect on all preparations used in photography, and these rays are called (following W'ollaston) the chemically effective or actinic rays.

Yet recent investigations have proved that no colour is absolutely ineffective chemically; the outermost red and eren ultrared affects silver salts; indeed, red light affects many substances more powerfully than violet.

Again, according to recent investigations, heat is no peculiar characteristic of the less refrangible red and ultra-red rays, but is distributed over all parts of the spectrum.

Thus all the light rays of the spectrum may be chemically effective and be absorbed by sensitive preparations.

These chemical effects are as follows:

I. Under certain conditions light produces molecular changes, resulting in allotropic modifications, or isomeric combinations. Thus, yellow phosphorus is transformed by heat or light into red.

2. Light has the power of setting up processes of chemical combination.

Equal volumes of chlorine and hydrogen will not combine in the dark, but will combine explosively in the light to form $\mathrm{HCl}$.

Light very often farours oxidation (cf. bleaching, the fading of many-coloured materials in light).

3. I.ight causes chemical decomposition. The most notable instance of this is with chlorophyll, the green colouring matter in plants. In sunlight chlorophyll decomposes the carbonic-acid gas of the air, absorbing the carbon, which plants need for growth, and giving out anain the oxyen needed in the air. 
This action of chlorophyll takes place only in the light, and depends on absorption, mainly of the red rays, as is shown by the absorption spectrum.

Photographic processes are of the same nature, depending mostly on the reduction of metallic salts, usually haloid compounds of silver.

Each of the spectrum colours has both an oxidising and a reducing effect; but red light affects metallic compounds for the most part by oxidation, violet light by reduction. Organic compounds are usually most powerfully oxidised by violet light, and pigments by the special light which they absorb.

Photographic preparations $(A g B r, A g C l$, etc. $)$ are decomposed not only by the light they absorb of themselves, but also by the light they absorb by reason of the admixture of certain substances (optical sensitisers).

These substances (chemical sensitisers) favour decomposition by light by binding chemically the constituents (bromine, iodine, oxygen, etc.) eliminated by light.

Artificial light-sources, being very unequal in actinic rays, do not affect the photographic plate in proportion to their visible optical strength. According to Eder's table, e. g., both the magnesium and the electric arc-light have more than 20 times as much actinic effect as lamplight of apparent brilliancy.

The effect of the coloured rays depends on the intensity and the nature of the light and its duration, and on the nature and molecular condition of the illuminated body. Each wavelength ( $i$. e., color) has its own chemical effect. No ray is effective unless it is absorbed. The course of photochemical reaction may be influenced by rise of temperature, pressure, and electricity.

\section{Physiological Effects of Light.}

\$53. By the "physiological effects of light" are usually understood:

I. Its physical effect on matter, on the elementary particles of which the tissues are composed. Such physical and chemical processes are various, and may reveal themselves as optical, 
chemical and heat effects. We know that light may produce fluorescence, phosphorescence, electric phenomena and other physical processes.

2. Its effect on the vital functions. Under the influence of light living tissues may pass from a state of passivity to one of activity, and change of form, of energy, of matter may be induced. In this sense, light works like other physiological irritants, under certain conditions awakening and strengthening elementary forms of life, under others weakening or annihilating them.

These two main effects of light stand in causal relation to each other.

According to Loeb ${ }^{1}$ ) light is not merely an irritant in the physiological sense: it actually infuses the organism with energy. This energy is transmuted into other forms, for the most part of a chemical nature; morphological differences, even chemical, require an organ.

In general the physiological effects of light are great in proportion to the intensity and duration of the energy at work. In many cases, however, the shorter the process, the more powerful is the effect of intense light. Analogies to this may be found in the effect of sudden fluctuations of temperature or of electric currents.

\section{The Effect of Lighl on Planls").}

\$4. Plants, more than any other living organism, need light. Without it they cannot take from the air the indispensable carbonic acid, nor, by means of chlorophyll, assimilate it, giving out the freed oxygen and retaining the carbon in new combinations, such as sugar, gum, starch, cellulose, allumen:").

There are two theories as to the way light works. Most

1) Pflüger's Archis, tsor, Vol. T.Xiti.

2) From C. Mueller and II. Potoni, Botanik, Berlin, 189.3, p. 26o.

3) It can be chemically proved that the amomul of carlonic aciol taken from the air by leaves in sumlight is preciscly the same ats the amomt of oxygen given off. Without sunlight leaves add to the anomint of $C()_{2}$ in the air. 
physiologists assume a direct photochemical effect on the chlorophyll, an effect dependent on the intensity and the quality of the light.

For each plant there is a certain degree of light intensity most helpful to its assimilative process; certain plants (shadeloving plants, marine algx growing at a great depth) flourish best in a dim light, others (light-loving plants) prefer sunny places.

The quality of the light needed for the "chlorophyll function" coincides with that demanded for certain physical and chemical processes. The photochemical effect of light is usually ascribed to the indigo and violet rays (i.e., the more refrangible and short-waved rays).

In the decomposition of carbonic acid by chlorophyll, it is just the red and yellow rays (i.e., the less refrangible) which are effective.

The chlorophyll pigment can only be found in light; plants and plant-parts grown in the dark have no chlorophyll, but are pale yellow in colour (etiolation). But artificial light sources, such as the flame of candles, gas, oil lamps and the ray of the electric arc-light, may take the place of sunlight here; they all have the yellow rays needed for chlorophyll. The quantity of light need not be large. Wiesner proved that sprouting plants will grow dark green in a light which barely suffices for the reading of large print.

The chlorophyll-producing property of light was determined by means of Peletier's bell-jars, vessels filled with coloured solutions to allow the passage of the desired rays alone.

Pringsheim's theory of chlorophyll is opposed to the more widely accepted view of direct photochemical effect. He holds that the chlorophyll pigment, by absorbing the blue, violet and ultraviolet rays, without being itself decomposed, acts as a kind of light screen, lessening the degree of respiration (i. $c$., the oxidation connected with the elimination of $\mathrm{CO}_{2}$ ) and increasing proportionally the assimilative processes, especially the collecting of carbon and giving off of oxygen, going on within the plasma of the chlorophyll body.

There are always changes of energy corresponding with the 
changes of matter brought about in plants by light ${ }^{1}$ ); they are often more noticeable than the changes of matter. Transformation of energy in plants shows itself in morement, of which two great groups may be distinguished, rrow/h-mocement and irritability-movement. In the latter are comprised all the processes in which a change of position of the irritated part is brought about by any physical reaction.

Light is needed for many growth phenomena, but not for all. It is unnecessary for germination, for the growth of roots and many blossoms, as well as for the flourishing of many endophytic (living in the body of plants) and endozoic (living in the body of animals) living parasites. In general light has a retarding effect on growth, both as regards rate and extent (this holds good also of organs which are above ground). This explains the varying rate of growth at different hours of the day, a rate which is not for a time immediately influenced by artificial exclusion of light. The growth of stems and leares is on the whole least towards evening, most towards or in the morning.

$\operatorname{Van}$ Tieghem $^{\circ}$ ) showed that all light rays except the red and ultra-red retard and lessen the growth of plants, and that the effect is most pronounced in the rays that are most refracted in the spectrum. This phenomenon may be, conditionally, regarded as a paralysing effect of the more refrangible rays. According to Flammarion." ) the colour, too, of flowers is influenced by different light rays, so that, $c$. g., very various shades of the lilac may be obtained under variously coloured light. But the effect is greatest on the scent. Thus, strawberries grown under red glass had a wonderful aroma, and crassula flowers, which are nearly scentless in ordinary sunlight, emitted a delicate fragrance, like that of bananas, under the influence of red light.

1) Argustus Ii aller proved that light develops clectromotive energy in the assimilating leaf, more ly the bright red rays, equecially loose aboothed

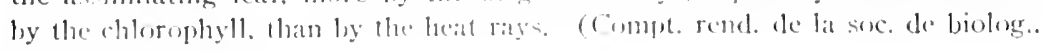
1900. 1.11. 1. 10 (1,3.)

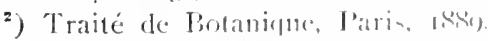

$\left.{ }^{3}\right)$ Gebhard, Die Heilkraft des Lichtes, Leiprig, 18ys. 1). 14. 
The way in which light affects extent of growth is shown most clearly by the fact that plants grown in the dark have abnormally long internodes and leaf stems, but no leaf surface. All green leaf surfaces are furthered in their growth by light through the effect of light on the assimilation of carbonic acid.

We must carefully distinguish between the heat effect and the chemical effect of light (thermal and photo-chemical effect).

Amongst specific growth movements due to light may be noted certain forms of mulation and heliolropism. In so far as light in these cases acts as the stimulus, these movenients may also be regarded as irritation phenomena.

Nutation is the term applied to the automatic movements made once or repeatedly within a certain given period by certain growing parts of a plant.

A special class of these periodic nutation-movements are the sleep-movements (nyctitropic nutations) made by the green leaves of certain plants towards sundown. In the daytime the leaflets are spread open in such a position that the light rays impinge vertically; at night they close up and fold themselves either upwards or downwards, according to their kind, against the common stem.

Heliotropism is the faculty possessed by many parts of plants of turning towards or away from the direction of the strongest light.

Stems and leaf stalks are usually positively heliotropic, $i . e$., they grow towards the light source in the direction of the light rays.

Roots and rhizomes are almost all negatively heliotropic, i. e., they turn away from the light source.

Green leaf surfaces show transverse or dia-heliotropism, $i$. $e$., they turn themselves at right angles to the direction of the light.

Parts of plants removed from their normal position make curvel heliotropic movements.

Heliotropic properties are dependent on the degree of brightness of the light. In a very glaring light organs usually 
positively heliotropic may become negatively so. Heliotropic curvature is conditioned by the direction of the incident light.

We may regard as movements of irritability purely many of those locomotor mevements which light frequently induces in plants.

Many unprotected plasmic bodies, such as the swarm spores of many algx, are capable of independent movement by means of waving cilia. The direction of the morement depends partly on temperature-effects and partly on the action of the incident light.

Light is the cause of change of place with the microscopic inhabitants of the ocean, ponds and lakes, as with the larger animals; they are attracted by it, and in sunlight rise from the depths to the surface. Very often they are present there in such large numbers that the water loses its natural clearness, transparency, and colourlessness, and appears dull-green, bluish, brown, or red. This phenomenon is termed the "flowering of the water." The rising of the water plants to the surface is due, in part, however, to their lessenced specific gravity through the production of oxygen, and, amongst the threads of the alga, or in the higher forms, in the air passages or cavities. Those reproductive cells of the algxe (swarm spores, zoospores), which, like the infusoria, are capable of independent morement by means of waving cilia, show heliotropic movement, $i . \iota$., they move as far as possible in a straight line towards the source of light. A few kinds are negatively heliotropic and are repelled by light. The novements of swarm spores are connected with a turning on the longitudinal axis of their body; whether to right or left depends again on the light rays. The more quickly vibrating blue rays alone influence the direction of the movement (heliotropic action); red rays, like darkness, do not affect it at all.

The creeping or amoboid movements of the plasmodia of myxomycetes, as of flowers of tan, are dependent on light: these bodies are negatively heliotropic, working themselves slowly along on their base, away from the bright spots into the shade.

On this plasma-mosement are possibly dependent the move- 
ments of chlorophyll bodies, which bear some relation to the greater or less intensity of the light.

Portions of the green leaves of phanerogams, mosses and the prothallia of ferns which have been kept continuously in deep shade, assume a darker shade on account of slow changes of position in the chlorophyll corpuscles in the protoplasm. These corpuscles under the influence of light, especially of the short-waved rays, collect mainly in the cell-surfaces turned towards the surface of the leaf; in the dark they collect mainly along the side-walls of the cells, at right angles to the cellsurface. It is uncertain whether we have here a direct influence of light on the protoplasm, or an indirect, induced possibly by primary change in the chlorophyll corpuscles.

Borodin distinguished between day-position and night-position. Stahl divides the position of the (for the most part) flat chlorophyll corpuscles with regard to light incidence into "surface-position" and "profile-position." In all cases the position of chlorophyll corpuscles is governed by the following general rule:

With a medium degree of brightness the chlorophyll corpuscles turn so as to present the broadest possible surface to the light rays. With a minimum of brightness (i. e., darkness) and a maximum of brightness (e.g., direct sunlight) they turn their narrow edge, $i$. e., the least possible surface, to the light.

Locomotor movement is the property of the chlorophyll bodies in all assimilating tissues.

Corresponding to the general rule for locomotor movement is the form-change of chlorophyll corpuscies, depending on degree of brightness. They are flattest in the most favourable light. They are capable of contraction. Hence a green plant may take on a lighter or darker green shade according to the degree of light (just as a chameleon changes its colour).

The protoplasmic current in plant-cells seems under ordinary conditions to be independent of light, though it is conditioned, as may be proved, by temperature, the amount of water contained, or presence of oxygen, and is frequently only recognis- 
able under the microscope after being accelerated by a mechanical stimulus.

According to our present knowledge, the current in the cells goes on unhindered by the cxclusion of light. But E. Josing ${ }^{1}$ ) proved that under changed external conditions the protoplasmic current may after all be radically influenced by light. These changed external conditions he brought about in two ways: first, by subjecting objects with freely flowing protoplasms to the effect of weak solutions of ether or chloroform; and, secondly, by withdrawing, by the aid of suitable agents, the carbonic acid from the surrounding air. Uinder such external conditions the protoplasmic current ceased to flow when the light was excluded, and resumed its course on its re-adnission.

Pringsheim ${ }^{2}$ ) was the first to furnish data as to the injurious effect of light on plants. Fle $m m$ agreed with him in finding no changes specially characteristic of these effects. Rigidity, formation of nodes, concretion of plasma, granulations (especially in the cell-nucleus) occur, but without being specially marked and characteristic. The disorganisation caused by illumination differs from "death by heat" in that no such intensive corporeal movements are ever produced as in the case of sudden change of temperature. For the rest "light intensity raised to its maximum tends directly towards precipitation in the plasma and towards its rigidity." Vacuolisation does not occur; contraction occurs only in the case of death supervening.

\section{The Effect of Light on Bacteria.}

\$5. In I 877 Downes and Bluml") first drew attention to the fact that diffused, and, still more direct, sunlight has the power of killing putrefaction bacteria, that the heat rays play no part here, and that the blue, violet and ultra-riolet rays take the

1) Jahrb. d. wimencels. Jobanik, Igor, Val. XXYT.

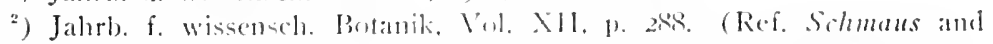

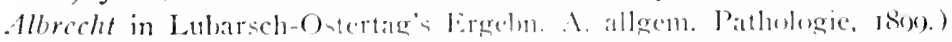

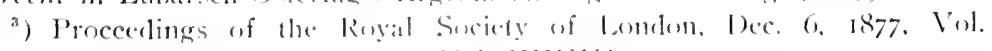

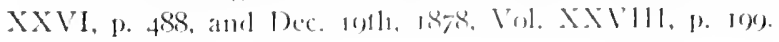


most active share in the effect, though the red and orange rays are not wholly inactive. They showed that the effect is the same whether the bacteria be damp or thoroughly dried; further, that the presence of oxygen is necessary, and that the manner in which the light works in these experiments is not to be sought in a modification of the nutritive basis. They took into account also the possibility that the products of metabolism in the bacteria may be influenced by light. These data, disputed at first by $T y n d a l l,{ }^{1}$ ) were soon confirmed on all sides, and a large number of fresh facts were added to our knowledge of the subject. Whilst Downes and Blunt made their experiments with any bacterial mixture of decomposing liquids to hand, the later investigators used pure cultures. Later on, too, physical conditions were taken more into account, and the arrangements for the experiments made more exact by the use of light sources of varying intensity, and of light filters.

'The fact that light has a very great power of destroying bacteria has now been established. This has been deduced from the experiments of Fatigati, ${ }^{2}$ ) Arloing, ${ }^{3}$ ) Duclaux, ${ }^{4}$ ) Liibbert, ${ }^{5}$ ) Janowski, ${ }^{6}$ ) Santori, ${ }^{7}$ ) Raspe, ${ }^{8}$ ) Geissler, ${ }^{9}$ ) Kolliar, ${ }^{10}$ ) Dandrieu, ${ }^{11}$ ) Chmiliewsky, ${ }^{12}$ ) Gaillard, ${ }^{13}$ ) Marshall Ward, ${ }^{14}$ ) Ledond-Ledard, ${ }^{15}$ ) Pansini, ${ }^{16}$ ) d'Arsonval and Charrin, ${ }^{17}$ )

$\left.{ }^{1}\right)$ Nature, Sept. I5th, i88i, Vol. XXIV, p. 466.

${ }^{2}$ ) Compt. rend., I879, Vol. LXXXIX, p. 959.

${ }^{3}$ ) Ibid. Vol. C, p. 378 , and Vol. CI. p. 5II.

4) Ibid. I 885 .

5) Ref. in Raum, Zeitschr. f. Hyg., Vol. VI.

c) Centralbl. f. Bakteriologie, Vol. VIII, p. I67.

$\left.{ }^{7}\right)$ Boll. della Accad. med. d'igiene, Roma, Vol. XVI, p. 386.

${ }^{8}$ ) Einfluss des Sonnenlichtes auf Mikroben. Dissertation, Schwerin, 1891.

$\left.{ }^{9}\right)$ Centralbl. f. Bakt., Vol. XI, p. I6I.

${ }^{10}$ ) Ibid. Vol. XII, p. 836 .

${ }^{11)}$ Annales d'Hygiène, I888, p. 448.

12) Wratsch, I892, No. 20.

${ }^{13}$ ) Thèse de Lyon, p. 396.

14) Proceedings of the Royal Soc. of London, Vol. LII, p. 393, and Vol. LIII, p. 23.

$\left.{ }^{15}\right)$ Arch. de médec. exp. etc., Ser. I, Vol. V, p. 779.

${ }^{16}$ ) Riv. d'igiene, I889.

${ }^{17}$ ) Arch. de physiologie norm. et patholog., Vol. VI, p. 335. 


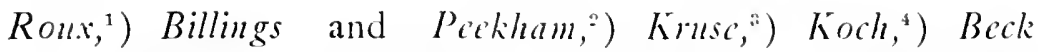
and Schultz,") Dicudonné,") Buchner,") ¿. Esmarch,") Giunti, $\left.{ }^{\prime}\right)$ Martinand, $\left.{ }^{10}\right)$ Momont, ${ }^{11}$ ) I'ittlin, $\left.{ }^{1 "}\right)$ Richardson, ${ }^{13}$ ) Schickhardt ${ }^{14}$ ) and Ruhcmann $\left.{ }^{15}\right)$. Most pathogenic bacteria are injured in their development and growth or are annihilated by light. This was proved by $\mathrm{I}^{\mathrm{T}}$ ard and $B i c$ in the case also of moulds and yeast-cells. The various kinds of bacteria are differently affected (A.sel L. L.ars'n "i")) whilst, e. $g$., the bacilli of typhus, diphtheria, plague and splenic fever have comparatively little resisting power, tubercle bacilli and staphylococci offer greater resistance. Some kinds are, indeed, said to be directly favoured in their growth by light; this was observed in bacterium photometricum (Engelmann), in certain yeast and mould fungi (Gaillard), and in a coccus cultivated from faeces by Schenk ${ }^{i T}$ ).

Different degrees of resistance are offered by the spores and by the bacilli of splenic fever. The former were killed by Arloing in 2 hours' direct sun-heat, whilst he needed 26-30 hours for the killing of the bacillus.

A degree of illumination which is insufficient for the complete checking of development may yet in some cases prove harmful to the formation of pigment. But in the

1) Ann. de linstit. Pasteur, I887.

2) Centralbl. f. Bakt., Vol. X1X. p. 244.

${ }^{3}$ Z Zeitschr. f. Hygiene, I895. 322.

4) Ueber bakteriologische Forschung. Berlin (Hirschwald), I8jo.

5) Zeitschr. f. Hygiene, Vol. XXIII.

-) Arbeiten aus dem Kaiserl. Gesundheitsante. Vol. IX.

${ }^{7}$ Centralbl. f. Bakt., Vol. XI, p. F\&I, Vol. XII, 1. 2!7, and Arch. f. Hygiene, Vol. XVII.

") Zeitschr. f. Hygiene, Vol. XVI.

") Stalt. sper. agrar. jtal., Vol. XVIII.

1") Compt. rencl. Acac. d. Sc., Vol. CXIII.

") Annales de l'instit. Pastenr. Isoz.

1") Wiencr klin. Wochenschr., Bof.

1:) Transact. of the Chem. Soc., 1en,3.

${ }^{16)}$ Fricdrcich's Blätter f. gericht. .Mel. 1893. p. 405.

$\left.{ }^{23}\right)$ Zeitschr. f. diat. und plyys. Therapie, Vol. IV.

15) Nittheilungen aus Finsen's med. Lichtinctitut, I, p. So.

1i) Koch's Jahresb. weluer die Fortachr. in der Lechre von den Gährungsorganismen, 1893,1 1. 5.3 . 
case of other bacteria again, as, $e$. g., micrococcus ochroleucus, light is a necessary condition for this. Side by side with the checking of the development of bacteria, illumination causes a lessening of the virulence of these microorganisms. A raising of the temperature makes the bacteria die more quickly (Geissler, Bang), but that is not necessary to the pirocess. Direct sunlight has a more powerful action than diffused daylight, but it is weaker than simple or concentrated arc-light. Arc-light with metal electrodes (Bang) and the light of the electric spark (Strebel) have a far stronger bactericidal action.

Dicudonné observed that bacteria were killed in half an hour by direct sunlight, in 6 hours by diffused daylight, in 8 hours by electric arc-light of 900 normal candle-power, and after 1 i hours by the electric incandescent light. The bactericidal action of sunlight naturally varies with its intensity at different seasons of the year.

Amongst the different spectrum rays the red and the green are neutral, or even, according to some observers, favourable to the growth of bacteria; the more refrangible blue, violet and ultra-violet rays, on the other hand, have distinct bactericidal properties. As to how the light acts, we assume (I) that it acts directly on the plasma of the bacteria themselves; (2) that it is at the same time, by producing photochemical change, indirectly injurious to the nutritive basis.

Krise found that by subjecting sterile nutritive bases to light complex chemical bodies (peptones) were formed, which checked development.

Richardson proved that in fresh urine under the influence of direct illumination peroxide of hydrogen is formed, which is decomposed by the bacteria, the latter being killed by the liberated oxygen. Diendonn' showed that in water, too, through the action of the chemical light rays peroxide of hydrogen is formed, most freely in the upper layers. This compound is strongly antiseptic. Under illumination, when oxygen was excluded, Dieudonné, and also Tizzoni and Cattani, ${ }^{1}$ ) found

$\left.{ }^{1}\right)$ Arch. f. exper. Pathologie nnd Pharmakologie, Vol. XXVIII, p. 59. 
the bactericidal action very largely diminished, which may be explained by the fact that peroxide of hydrogen could no longer be formed.

The injurious action of light on bacteria, howerer, is not due alone to the fact that it renders the nutritive basis unsuited to their growth, but to the direct injury it inflicts on the protoplasm. IV ard and Krmse proved that dried spores away from all nutritive material are also killed by sunlight. The

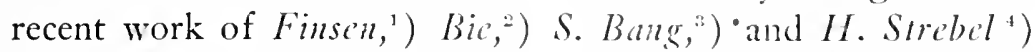
is interesting, not alone because it show's the more powerfully bactericidal action of light sources with preponderating shortwaved rays (concentrated sunlight and arc-light, Finsen; arclight with metal electrodes, Bang and Strebel; electric spark, Strebel $^{5}$ ), but also because in their ingenious experimental appli-

1) Ueber die Anwendung der concentrirten chemischen Lichtstrahlen in der Medicin, Lcipzig. I 899.

$\left.{ }^{2}\right)$ Mittheilungen aus Finsen's med. Lichtinstitut. I.

s) Ibid. III.

-) Deutsche med. Wochenschr., igor, No. 5-6.

5) G. O. Drossbach has heen led by his experiments to differ from others; he is extremcly sceptical as to the parasiticirlal action of light, and regards it cspecially as impossible that bacteria can be affected by the nltra-violet rays at a distance of even ${ }^{1} \frac{1}{6} \mathrm{~mm}$. below the surface of the skin. His experiments on the influence of chemical (ultra-volet) rays on the growth of bacteria yielded negative results. The inoculated nutritive gelatin was divided in two Petri-dishes which were exposed to iron arc-liglat (rooo Watl) at a distance of at most to cm. from the are, the one directly, the other through glass, four times a day for two days (for ro minules at a time) - i. e., for so minutes altogether. The illumination had perforce to be intermittent, as the gelatine melted away when light was applied for a longer time at once. The colonies developed in the same way on both gelatine plates, the mould fungi on the third and the bacteria on the seventio most vigorously. In another case a gelatine plate was subjected to direet rays six times for so long a time as to melt the gelatine. After seven days the colonies had developed normally. Evidently then the effect in checking develumment is very slight, whether the rays pass through glass or through air only. Light wates shorter than those given by sunlight are hence of no value in this respect. If we take furlher into account the slight light strength (as meatured by the bolometer) of the iron arc-light as compred with the callum atre-light or with smlight, the firstnamed may be practically distegareded. In general, the microlscidal action of our artificial someces of light is much less than is often assumed. P'robubly it does not crist at all when the micro-organions are in a really favourable nutritive soil. Pathogenic bacteria, which in any case grow badly in ont artificial culture media, may perbigs when in the latler le affected, if 
ances we have a guarantee for the utmost possible exactitude in estimating, on the one hand, the bactericidal energy of a source of light, and, on the other, the light-resisting powers of a micro-organism. It appeared that concentrated sunlight checked the growth of bacteria after one minute, and caused death in 5 to 7 . Concentrated electric arc-light checks growth of bacteria after 4-5 minutes, and kills them in 15 to 20 . Both the arc-light from metal electrodes and the electric spark kill micro-organisms after a few seconds ( 5 to 40 ).

$V$. Bie, by his experiments, was able to prove exactly with which parts of the spectrum the bactericidal action of light is essentially connected, and how great is the difference between the action of the different parts.

Bie made his experiments with the bacillus prodigiosus and the light of an arc-lamp of 35 ampères and 44-46 volts (about 6 ,ooo NK) ; the light was concentrated by a Finsen apparatus and fell vertically on to the culture. As light filter he used vessels with planoparallel glass walls, enclosing a layer of fluid $3 \mathrm{~cm}$. in thickness. As absorbing media he used:

I. A fresh $I \%$ sulphuric acid solution of quinine with a few drops of sulphuric acid, which allows the passage of all the rays but the ultra-violet;

2. A $5 \%$ solution of sulphate of nickel, which was similar in action;

3. A $1 \frac{1}{2} \%$ solution of chromate of potassium, letting through red to green, inclusive;

4. A $1 \frac{1}{2} \%$ solution of bichromate of potassium, letting through red to yellow, inclusive;

5. A $\frac{1}{7} \%$ solution of fuchsin, letting through red alone.

To determine the light of intensity, Bie compared the degree of blackening of spots produced during given periods of illumination on aristo paper.

The outcome of these expcriments was that all the spectrum

therefore curative action has been proved, it can only be referred to the heat conditioned by light-absorption." (Deutsche med. Wochenschr. Nov. 2I, 190I, No. 47.) 
rays (except the ultra-red, which were not examined), in an increasing ratio from red onwards, check bacterial development. The action increases with the ratio of refrangibility, and is especially marked in the blue, violet, and ultri-violet spectrum. Red, orange, yellow and green rays also act injuriously on bacterial growth, but only after prolonged illumination. Pure red light even did not produce the faintest perceptihle retardation of growth until after an hour and a half's exposure.

The experiments of Sophus Bang, Finsen's most competent colleague, are specially remarkable, the care with which they were conducted making them a molel for all liter investigations of the kind. Bang took into account all the conditions to be noted with regard to light-action, the strength of light used, the distance of the object from the ray-source, the kind of rays passing the filters and the amount passing through, the percentage of light penetrating to the bacteria (taking into account the absorption and refraction of the light through the containing ressel and the culture medium). The experiments were conducted so as to ensure that the light-ray should meet with as few obstacles as possible on its passage from the light source to the object; the reflecting planes were as few and as simple, and the absorption and refraction as slight, as possible; an even temperature was maintained, and measures were adopted for varying the strength of the light according to the gradation desired.

Bang used for these experiments an apparatus in which the bacteria culture was spread out for examination in the thimnest possible layer, $e$.. ., as a suspended drop on a thin quartz plate. This quarts plate is used as the lid of a "moist chamber," this chamber again is fixed in a box filled with water of an even temperature, kept constantly flowing by a paldle-wheel. The light is admitted through a quart, window in the side of the box; its intensity and direction of incidence are exactly estimated.

In this manner, Bang came to the conclusion that under the influence of light at a distance of $28 \mathrm{~cm}$. from an electric are light of 35 amperes and 5 " wolts, at an angle of 45 to the axis 
of the carbon, after part of the heat rays have been kept back by a layer of water ( $25 \mathrm{~mm}$. in thickness) between quartz plates, and at a temperature of $30^{\circ}$, a 3 hours' prodigiosus broth-culture in a pendant drop is sterilised in about one minute, a io to 15 hours' culture in 3 to 5 minutes. The light acts more quickly at $45^{\circ}$ than at $30^{\circ}$, sterilising a 3 hours' culture in about half a minute."

Thus, then, the older the culture, the greater its power of resistance to light action; and further, with increase of temperature comes increase of bactericidal light-action.

The bactericidal effects of sunlight, according to Bucheur, Schickhardt, Diendonné, Uffelmann, von Pettenkofer, Pransnit: and others, probably plays an important part in nature in the "self-cleansing" of rivers. River water, contaminated by. sewage, etc., after having flowed for some distance, becomes again, so far as bacteria are concerned, what it was before the introduction of the contaminating matter. No doubt, however, this is partly the result of the great dilution of the foul matter, the deposit of floating particles as sediment and the absorption or decomposition of substances by living creatures (plants or animals). Wittlin showed that street dust is disinfected in a high degree by exposure to direct sunlight. Von Esmarch tested the bactericidal action of direct sunlight on germ-containing clothes, bedclothes, etc., and found that the action is confined to the uppermost layers of the objects and does not penetrate at all to the interior.

It has also been maintained that the receptivity of an organism to living bacteria and to bacterial poisons is lessened by exposure to light (Kondratjew, $\left.{ }^{1}\right)$ (Gebhard, $\left.{ }^{2}\right)$ (Jousset $\left.{ }^{3}\right)$ ). According to $\left.B \times d e r,{ }^{4}\right)$ however, who investigated this point minutely, this is open to question.

Besides its action, in checking the development and growth of bacteria, we know further that light influences their move-

1) Quoted by Dworetzky, Zeitschr. f. diactetische u. phys. Therapie, Vol. V, bk. 3 .

$\left.{ }^{2}\right)$ Die Heilkraft des Lichtes, Leipzig, I898.

$\left.{ }^{3}\right)$ La Sémaine médicale, 1900, 45.

4) Arbeiten aus dem kaiserl. Gesundheitsamts, I900, Vol. XVII, bk. I. 
ments. According to $/$ ' $^{\prime}$ inogradsky, ${ }^{1}$ ) and Beijerinck, $\left.{ }^{2}\right)$ sulphur-bacteria and the chromogenic bacteria always collect at the lightest spot; they are thus positively phototaxic.

\section{The Effect of Light on Higher Organisms. (Animals, including Man.)}

$\S 56$. In animals, as in plants, light exercises a stimulating influence on the functions of tissue-clements and organs; the :nergy infused into the body by light is transmuted into stimulus, quickening and heightening all the vital processes. As a stimulus, light either influences directly the protoplasm of the irradiated cells, or it brings about by indirect means (through the sense-organs and nerves) certain functions on the part of given organs.

It has long been recognised as a fact that the development of many animals is dependent on light; without light development proceeds slowly or is suspended altogether. William Edwards ${ }^{3}$ ) observed that frog-spawn in an opaque glass died, whilst spawn in a transparent glass became duly developed. The development of tadpoles then proceeds more slowly in the dark. Schnetzler's ${ }^{4}$ ) experiments proved that white light is more favourable than green to such development. E. Young ${ }^{5}$ ) showed that violet light helps on the development of the embryo or rana, salmo and lymenea, whilst darkness or other parts of the spectrum hinder or disturb it. Beclard ") found that flies' eggs develop more quickly under blue and violet glass than under red, yellow, green or white. Guarinoni ${ }^{\top}$ ) believes his experiments to prove that violet light acts favourably on silk-

1) Zur Morphologie und Physiolngie der Schwefellakterien.

2) Centralblatt f. liakteriologic, Vol. XIV, 1) 8.4.

${ }^{3}$ ) De loinfuence des agents pluysigues sur la vie. Paris. 182.4.

1) Archives des sce pllysicgues et naturelles, 1874. Vol. L.l.

") Compt. rend., Vol. I.XXXVII.

") Compt. rend. 1858 .

7) Quoted by Eder, J. M., Ueber die chems. Wirkungen 1. farl. Lichtes, Vicuna, 1879 . 
worms. Godnew ${ }^{1}$ ) noticed that maggots are much more quickly developed in pieces of meat exposed to the light than in meat kept in the dark. Loeb ${ }^{2}$ ) made a study of the action of light on polypi and found that growth is not affected equally by all the rays, but that only the more refrangible (i.e., blue) rays further growth, whilst the red ones have the same effect as darkness. Light exercises a powerful influence also on the growth of the higher animals at a later stage. $P \ddot{o} e y^{3}$ ) believes he has proved that sucking pigs and calves do better in violet light than in white (?). Young rabbits do not get on at all in the dark. Every farmer knows that it is a universal rule that beasts flourish better in bright, sunny sheds than in dark ones. And the healthy development of young children, as is well known, depends in large measure on light.

Not only the general growth, however, is affected by varying supply of light, but also the development of individual organs and parts of organs. It is a well-known fact that a large number of pigment-containing cells are found in the light than the dark (ephelids). Berthold ${ }^{4}$ ) and Finsen ${ }^{5}$ ) have shown that epidermal growth (as of nails and hair) is favoured by light and hindered by darkness. Both patients and nurses in Finsen's clinic acquired a thicker growth of hair on those parts which were exposed repeatedly and for a long time to the powerful electric ray. It has been proved that with amphibia and fishes limbs that have been broken off grew again more rapidly in the light than in the dark.

The stimulating effects of light on transformation of form and transformation of energy, especially on the phenomena of movement, have been proved by many interesting observations.

The arrangement of the protoplasm of amœboid cells '(amoboe, rhizopods, infusoria) is noticeably changed by exposure for any length of time to light or darkness. The plasmodia of æthalium, which in the dark have crawled to the sur-

) Kasan'sche Dissert., I8S2.

Pfueger's Archiv, Vol. LXIII.

Compt. rend., i87r, Vol. LXXiII.

") Muller's Archiv. f. Anatomic und Physiologie, I850, p. I58.

") Withleilgn. ans Finsen's med. Liehtinst., Vol. I, p. IIS. 
face of the tan withdraw again to the depths in a bright light. In the light they develop short, compressed projections; in the dark long, thin, narrow processes. Here then light seems to act in the same way as artificial irritants.

It is well known that change of light causes changes of form in the contractile pigment-cells, in the skin of many fishes, amphibia, and reptiles, thereby producing change of colour in the animals. Under a bright light, $e$. $g$., the black pigmentcells of the frog's skin, which in the dark have wide-spreading ramifications, contract gradually into little balls, making the skin appear lighter. Under sudden illumination pelonyxa palustris contracts in a few seconds into a ball, after the granulestreaming has ceased. If the light is maintained, only weak, slow movements are again observable. When the darkness is dissipated, by the gradual coming on of daylight, there is no irritant effect $\left(\right.$ Engelinann $\left.{ }^{1}\right)$. To Engelmann we owe also the discovery of bacterium photo-metricum, which is extraordinarily sensitive to light stimuli. So long as it is exposed to light, it propels itself swiftly about in the drop of water by the aid of the scourge-like thread which is found at the ends of each bacterial body. When it is brought into the dark the movement of the thread gradually ceases and the bacterium remains motionless, to be stimulated again, howerer, to fresh movement under renewed light-action. By means of a spectrum apparatus Engelmann was able to fix on the orange and ultra-red rays, as the specially active factors in this bacterial movement ${ }^{2}$ ). Light has also a very powerful influence, particularly in regard to the direction, on the ciliary movement of the green swarmspores of the lower plants and flagellates.

According to Uskoff, the ciliary movement of the epithelium of the osophagus is equally swift in red and in violet light, but it is suspended if red light is substituted for previously acting violet.

Even among the ciliary infusoria, which are not generally sensitive to light, isolated specimens are foind whose ciliary

1) Archiv. f. d. ges. P'hycinlugie, XIX, p. r, and Handhuch der l'hysiulogic, Vol. 1, 1. 320.

") I'fluciger's Archir., Vol. XXX. 
movements are stimulated by light. $\operatorname{Verworn}^{1}$ ) observed that pleuronema chrysalis, which when undisturbed lies motionless in the water, makes violent movements when suddenly exposed to light, but only after a period of latent irritation (one to two seconds), not at once when the light falls on it. Verworn proved that this leaping movement is not the result of possible heat-action of the light, but is due in largest measure to the action of the blue and violet rays.

It is true the same effect can be produced with intense (concentrated) heat-rays.

Uskoff ${ }^{2}$ ) made a study of the behaviour of the protoplasm of blood cells. The white corpuscles of frog's blood showed more and longer processes in red light than in violet; further they were in the former spread out, for the most part, in the form of hardly visible discs. According to Hermann, ${ }^{3}$ ) on the other hand, leucocytes are not sensitive to light, whilst red corpuscles show distinct changes of shape. Finsen observed ${ }^{4}$ ) that the red corpuscles in tadpoles' blood changed shape under the influence of sunlight; they contracted and became rounder.

According to Auerbach, light acts as a powerful inducer of contractions on the protoplasm of frog spawn. Finsen, ${ }^{5}$ ) too, noticed in his experiments with frog-spawn and salamander germs, that light possesses in a high degree the power of inducing movements of the germ, and that this power is present to a quite special extent in the blue-violet rays. Daphnia pulex shows with increasing strength of light increased precision and swiftness of motion.

Red light is most favourable to the movements of amœbœ. Both the violet rays and white light have an impeding effect. (Harrington and Leaming.)

Experiments on the influence of light rays on the movements of older animals have given similar results.

${ }^{1}$ ) Quoted from I'cravorn, Allgemein. Physiologie u. psycho-physiolog. Protistenstudien, Jena, I8S9.

$\left.{ }^{2}\right)$ Centralbl. f. d. med. Wissensch., 1879, No. 25.

3) Quted in Strebcl, p. 6.

4) Ueber die Bedentung d. chem. Strahlen, Leipzig, 1879.

$\left.{ }^{4}\right)$ Centralbl. f. d. Wissensch., 1870, No. 23. 
To this class belongs the action of light in directing the movements of the more highly organised animals; it is not always easy, however, to determine the extent of this because of the large part played also by the organs of sense and the nervous system. The experiments of $1.0\left(b^{1}\right)$ and of Parkir and Burmett") have shown that, just as with plants, so also with many animals, light has an intluence on the direction of their movements. Many sightless animals eren show signs of heliotropism.

Light often exercises over animal, as well as regetable organisms, the same kind of irresistibly compelling power that a magnet does on iron; they are forced to turn towards or away from the source of light.

Thus a gnat is always attracted by an artificial light, in whose flame it will singe its wings and lose its life. Blind frogs always turn their heads towards the source of light and place themselves so that its rays may fall symmetrically on both sides of their bodies $\left(V^{\prime}\right.$ wedens $\left.\left.{ }^{3}\right)\right)$. If put in a box, of which one-half is illuminated, the other dark, they will, according to Bert, ${ }^{4}$ ) always try to get to the bright part. It is well known, however, that whilst some animals love the light and always make for sunlight, there are others which dread light and live in the dark.

Many animals, $c . g$. , ants and bees, are able to distinguish colour. Earwigs, wood-lice, carabidde (Finse"ns)), even eyeless creatures, like the earthworm $\left(G r a b r^{* 1}\right)$ ), and blind ones, like the triton, are peculiarly sensitive to the short-waved (blue: violet) rays of light, taking shelter in darkness or the longerwaved (red) rays.

Other creatures again, $c . g$., butterflies, prefer the blue and violet ray's.

1) Der Heliotropismus der Thiere. (te., Würzhurg. s\&on.

2) Wirkung auf Ilamarion, Amer. Journal of lhysiol., IV, S, P. 27.3.

3) Bull. de l'Acarl. des Sc., à Púlethlourge, 1879.

4) Revue Scientif., 1878, 42.

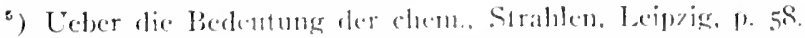

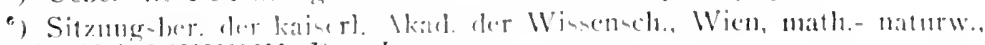
CI. I88. Vol. I.XXXVII, T'art I, 1, 201. 
Cohen and Strassburger proved that in general the shortwaved rays have a stonger phototaxic effect than the longwaved, which, if not present in a too high degree of intensity, have the same action as complete darkness.

The course of phototaxic phenomena, however, often depends on other circumstances. Light affects paramœcium bursaria only when the supply of oxygen is insufficient; with sufficient oxygen the light stimulus is ineffective.

With larva of polygordius, phototaxic phenomena appear only if the temperature is raised at the same time.

Many animals react chiefly to fluctuations in the intensity of the light.

With reference to the action of light on given tissue-elements, a close study has been made of its effect on muscles.

We do not at present know that light has any influence on the movements of striped muscular fibre.

Researches have been made into the action of light in stimulating unstriped muscular fibre by Fr. Arnold, Reinhardt, Budge, Brown-Séquard and Heinrich Mueller ${ }^{1}$ ) and Steinach ${ }^{2}$ ). The incidence of light (without heat) causes the iris to contract in the extracted eye of amphibia or fishes, that is, independently of the central nerve-system, and this though the retina may have been removed and the eye extracted for a long time ( 6 days from an eel in winter!).

Brown-Séquard regards this phenomenon as direct muscleirritation by light. Harless, ${ }^{3}$ ) with human corpses, too observed for a period of 30 hours after death distinct contraction of the pupil of the eye exposed to light, as compared with that of the closed eye.

De Parville (quoted by Buedingen) claims to have proved the red end of the spectrum to be nerve-irritating, and the reverse end (green, blue and violet) to be nerve-soothing. Buedingen, however, was unable to confirm this.

Th. Buedingen $\left.{ }^{4}\right)$ in order to solve the question whether light

$\left.{ }^{1}\right)$ Würzburger naturw. Zeitschr., I861, II, p. I33.

2) Pfliiger's Arch., Vol. LII.

$\left.{ }^{3}\right)$ Abhdign. d. bayr. Akad., is 48, V, p. 490.

4) Zeitschr. f. diaet. und physik. Therapie, Vol. VI, bk. 5, p. 272. 
acts on muscle directly, or through the motor nerves, on a nervemuscle preparation, and further, whether reflex morements can be set up by irradiation of the skin, made experiments with animals whose cerebrum had been removed and the nerve connection between brain and spinal cord severed. These experiments showed that light, as applied in the form of sudden transition from darkness to a blue or red light in concentrated form, had no direct influence on the nerve-muscle preparations made from frogs, and further, that it was not able to modify contractions caused by other stimuli. Neither did the experiments, as to the possible influence of concentrated red and blue arc-light, on the reflex irritability of animals whose cerebra had been removed show the slightest trace of such action. Buedingen concludes that the reflex irritability of the spinal cord is not influenced by light rays falling on the skin.

Besides the muscle-cells, there is a series of other cells which react to the stimulus of powerful light-irradiation. This is proved by the phenomena of erythema solare, dermatitis photoelectrica, xeroderma pigmentosum, hydro, and summer eruptions, pellagra and the various pigment anomalies, due to the influence of light.

Light produces obvious effects on the skin of the higher animals. According to $U$ nna, $\left.{ }^{1}\right)$ the skin becomes coarser and harder in parts exposed to the light, because under the influence of light protoplasm becomes reduced to keratine. Moeller ${ }^{2}$ ) was able to prove, experimentally and microscopically (p.+32), that light sets up hyperplasia of the epidermis and an abnormal horning-process.

The changes in the skin, known as erythema or eczema solare s. photoelectricum, consisting in the diffused production of redness, small blisters and pustules, which may in severe cases lead to leuco-sero-fibrinous inflammation of the whole skin-covering, are regarded as disturbances of the circulation. IVe cannot for the present discuss the question whether these phases of hyperamia and the conditions which they induce,

1) Monatsh. f. prakt. Dermatologie, 1\$85, IV, P. 284.

2) Der Einfluss des Lichtes aul die Haut. Biblioth, med., Stutgart, I900, P. 18. 
which arise also in other organs under strong light, $e . g$., the conjunctiva, the nose, gills (in proteus, Rusconi, ${ }^{1}$ ) are to be regarded as due to action on the vascular nerves, or as being induced by primary injury (degeneration) of the tissue cells. It has certainly been proved, by various histological experiments (Ogneff, Moeller, Glebowssky) (see later) that light produces local pathological changes in the body-cells which are subjected to powerful rays.

It is well known that it is often difficult to get workmen in factories where the work is carried on in strong arc-light, because of the injuries caused by light (Maklakow).

The phenomena of this reaction on the skin are as follows: the skin becomes a bright to copper red, swells, and is burning and painful. These signs are masked in consequence of proliferative processes in the horny layer. After more powerful light action, larger or smaller blisters are formed, with ecchymosis, and even more or less deep-seated necrosis of the tissues. Very marked signs of ill-health may thus be produced. After a few days the skin becomes less red, and increasing pigmentation ensues; the swelling goes down, the blisters dry up, the epidermis peels off, at first in larger flakes, as after scarlet-fever, and later in small scales. Makalkow ${ }^{2}$ ) and H'idmark ${ }^{3}$ ) drew attention to an important fact, in connection with this reaction, viz., that the changes in the skin do not appear at once, immes diately after the action of the light, being in this respect a con. trast to changes brought about by heat-rays, which are visible at once, but die away quickly.

Light erythema, on the other hand, as well as the Roentgen reaction, appears only after being latent for a longer or shorter time; the period of its duration, in which it reaches its height, is prolonged in proportion to the intensity of the light action, and it dies away slowly with desquamation and absorption of piginent.

Maklakow tried to determine the period of latency after the

$\left.{ }^{1}\right)$ Cit. hei Raum, p. 338 .

2) Archives d'ophthalmologie, i889. Vol. IX, p. 97.

$\left.{ }^{3}\right)$ Hygiea, Festband, No. 3.,I889. - Beiträge zur Ophthalmologie, Leipzig, 188 I, P. $43^{8}$. 
use of a powerful arc-light. The effect of an irradiation, lasting only $5_{5}$ seconds, was not seen till after ro hours. A part of the skin, subjected to light action for one minute, showed distinct circumscribed hyperasthesia after half an hour, redness showing itself only after 2.4 hours. Regions of the skin exposed to the light for 3 minutes became red in I I minutes, and parts irradiated for 5 minutes showed erythema, after 3 minutes. These results were confirmed by Finsen ${ }^{1}$ ) and Moel$\left.l e r^{2}\right)$ after accurate experimental research.

If now we compare these facts, with the effects produced on the skin by heat-rays and Roentgen-rays, we may deduce the following laws as to the speed with which the reaction shows itself and the length of its duration:

I. The duration of the period of latency is in inserse ratio to the rave-length of the active-rays; in like manner the effect lasts longer in proportion as the wave-length of the active rays liecomes shorter.

2. The greater the intensity of the light, the earlier does reaction show itself and the longer does it last. If the intensity is less, reaction shows itself later and lasts for a shorter time.

Finsen and Mocller proved by experiment a peculiarity of light reaction precisely corresponding to that observed by the author with Roentgen-ray reaction."). Skin, which has been exposed to powerful influence from chemical (blue, nltra-ciolet) rays, retains for a long time (months and years after the first light-erythema has disappeared) a peculiar predisposition to react remarkably quickly' (by reddening) to mechanical, chemical and thermal stimuli-and also to internal influ'nces (psychical stimulus, itc.).

The relation of the skin pigment to light is very interesting.

We have above $(p .410)$ already referred to the part played by light, in causing colour-change in animals. There are in the skin of rery many animals (also in other organs, $e . g$. , in the iris of amphibia and fishes) pigment-cells or chromato-

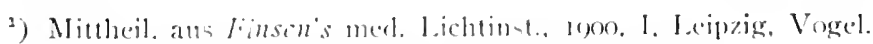

2) Der Einflueseder Lichter anf die llant, Stultgart.

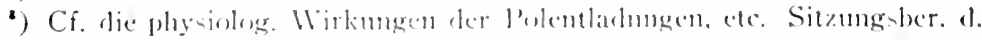
kaiserl. Akatl. d. Wisen.ch. math.-naturw. Cl., lid. CIX, Mbth. III, p. G4. 
phores, the protoplasm of which, on being irritated by light, extends or withdraws processes, thereby distributing the pigment over a larger or smaller space. In many parts (e.g., the iris) these chromatophores may also be regarded as pigmented, unstriped muscle-fibres, or as cells to the periphery, of which muscle-fibres attach themselves, which react to light stimulus by contraction.

Bruecke, who has made the closest study of colour-change, in the chameleon, showed that the movement of the chromato. phores is dependent on the central nervous-system. Darkness acts on the skin of this animal as a stimulus, whilst daylight, even sunshine, reduces the pigment-cells to a passive state. When these creatures are brought out into the sunlight they become dark (projecting the elongations of their pigment-cells to the surface of the body); when they are taken into the dark they become pale in colour (the creature drawing back the dark elongations of its pigment-cells, so that they are covered by the light-coloured pigment in the upper layers of the cuticle).

It is clear, from the varying behaviour of chromato. phores under irritation (strychnine-poisoning, rubbing with turpentine, strokes of a magnetic electromotor) and under paralysation (by cutting through the mainnerves) that we are right in taking the condition in which the cells extend their processes as being the passive condition of rest, and the condition in which they draw in the processes as the active condition of irritability.

The action of light on the skin is local; for according to Bruecke if a band of tin-foil is put round a chameleon and the creature placed in the sun, there will be a light-coloured strip under the tin-foil, whilst the rest of the body will be dark. Bruecke proved that this is really light action, and not the action of radiant heat. P. Bert ${ }^{1}$ ) found that it is the blue constituents of light, and not the red and yellow, which affect the chromatophores and to which we must ascribe the effects discussed.

$\left.{ }^{1}\right)$ Hoppe-Seyler. Physiol. Chemie, I881, p. 25. 
As with the chameleon, so with other animals, it has been proved that the protoplasmic movement of the chromatophores caused by light takes place through the central nervous system. The experiments of Elhrinann, who observed in frogs the direct passage of nerve filaments into the pigment cells, make it probable that the chromatophores are connected with the central nerve organ.

Turbots, which had had the sympathetic nerve cut through, became dark in that part of the body whose nerves had their origin behind the incision.

Blinded fishes become dark in colour through the spreading out of the pigment cells.

Bruccke surmises that there is a reflex action starting from the visual nerve, and that a stimulus of the optic nerve passes on to the central organ, a stimulus which causes the chromatophores to contract; so that when this stimulus is wanting, they permanently cover a larger space ${ }^{1}$ ). According to I' $^{\prime}$ ittich ${ }^{2}$ ) and E. Du Bois-Reymond, ${ }^{3}$ ) the colour of frogs and the sheaffish is also dependent on light, these creatures being black in the dark and turning light-coloured again under the influence of light.

Exner drew attention to the movements of pigment in the eyes of insects as a result of light.

The behaviour of these creatures with regard to light becomes intelligible when we consider that pigment, as we shall see directly, is a natural protection against the action of light. Creatures avail themselves of this protection in proportion as the light-irritation becomes more or less unpleasant to them.

We must distinguish this passing and occasional reaction of the chromatophores to light irritation from the more or less persisting pigmentation, observable in both men and animals in regions exposed to light. According to Ehrmann, where the sun's rays fall on a skin in which melanoblasts are present (i.e., cells which produce melanin, a melanotic brown pigment,

1) Quoted from Brueder, Vorles. neluer Physiologic, 1895. Vol. I.

2) Mucllir's Archiv, 1.5.54.

3) Enterench, zur Naturlehre des Menschen u, der Thiere, von Molescott, $1858, \mathrm{Bd} . \mathrm{V}$. 
formed. only by cells, not in the interspaces) melanin is developed there from the more abundant nutriment received, and under the stimulus to the cells of the light rays. Thence results the sepia-brown colouring of the skin, lasting sometimes for years.

There is a different reason for the deep but evanescent brown of the skin often to be seen, $e . g$. ., in tourists after a glacier excursion, as the remains of erythema solare s. photoelectricum after a single but intense exposure to light. In this case the strong light produces marked hyperæmia of the skin; bloodplasm, in which hæmoglobin is dissolved, finds its way out freely through the walls of the capillaries. In a short time the hæmoglobin is deposited in the interstices of the tissues as golden-yellow hæmosiderin, causing the brownish-yellow colour of the skin, which only disappears in a few weeks after this blood pigment has been reabsorbed. ${ }^{1}$ ) The pigment may also be developed from the red corpuscles directly; they may pass by diapedesis out of the walls of the blood vessels and shrivel up into pigment corpuscles.

As already observed, pigment is the skin's protective organ against light rays. One evidence of this is that races of men exposed always to strong insolation, are, like animals, darker in colour at those parts of the body exposed to the light. IVed$\operatorname{din} g^{2}$ ) observed the remarkable fact that cattle and sheep fed on buckwheat broke out in blisters, if they were light in colour or were exposed to sunlight. Beasts kept in the dark remained healthy. Parti-coloured beasts showed sickness only in the light parts, the dark parts remaining unchanged. WVedding smeared part of one cow with tar; the eruption appeared only on the untarred parts of the skin.

Last summer the author came, by chance, on a corroborating instance. A dark-complexioned man, who had for many years had vitiligo patches on the body and face, after a long walk over the Grossglockner glacier, developed violent inflammation (erythema) in the

$\left.{ }^{1}\right)$ S. Ehrmann, Wiener med. Wochenschr., 1901, No. 30.

$\left.{ }^{2}\right)$ Verhandlungen der Berliner Gesellschaft für Anthropologie, I888, p. 57. 
neighbourhood of the white patches on the face, but in these regions alone. The rest of the skin was quite unaffected.

Finsen proved, by a very interesting experiment, that acquired pigmentation may also have protective power against the injurious action of light rays.

Finsen painted a ring round his arm with black paint and then exposed the arm for 3 hours to very strong sunlight, after which the paint was removed. The skin after a few hours seemed quite white and normal, showing only some redness at the edges of the belt of paint. A few hours later violent erythema was developed in the exposed part, but in the painted belt the skin was white as before. The boundary line between the affected and the normal parts of the skin was extraordinarily sharp and showed precisely the same small irregularities as the edges of the black belt. After the erythema had passed off the skin was a good deal discoloured. Now Fins'n again exposed the arm to sunlight, without biackening any part; the result was precisely the reverse, the white band was attacked by erythema, the neighbouring parts remained unchanged.

Light erythema and light pigmentation are due to the ultra violet rays. Charcot ${ }^{1}$ ) already surmised that it is the so-called chemical rays that produce erythema photoelectricum. I' $e^{\prime} i l^{\prime}$, ) too, showed as early as ${ } S S 7$ that the chemical rays of sunlight produce a peculiar affection of the skin, consisting in swelling of the face and a red cruption, like nettlerash. He recommended the wearing of a red veil to absorb the chemical rays, and this simple remedy acted rery well. $\left.U m n a,{ }^{3}\right)$ too, and $C$. Berliner, ${ }^{4}$ ) also Vollers $^{5}$ ) pointed to the chemical (violet and ultra-violet) light-ray's as the cause of erythema solare, of xeroderma pigmentosum, and of Hutchinson's summer-eruption, and

1) Compt. rend. Soc. linlog., 1,850. 1. 6.3.

2) Vierteljaluresschr. f. I)erm. 11. Syph. Jahrg., I897. p. 277.

3) Monatsh. f. praktische J hematorlogie, IS45. Vol. IV. 1) 277.

4) Ibicl. isio, Vol. XI, Nos, 10, I1.

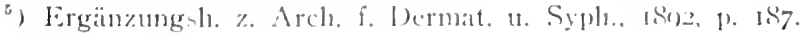


recommended treatment with curucuma-yellow masks, veils or windows, to be used persistently for weeks and months.

Bowles relates (Monatsh. f. pr. Dermatologie, I 894, Vol. XVIII, p. I6) that an officer in India, who suffered much from the sun, protected himself by having his clothes and sun helmet lined with yellow.

Bouchard, Widmark, Hammer, Finsen, Moeller and Maklakow have all made direct experiments to test which part of the spectrum is really injurious. Bonchard ${ }^{1}$ ) used coloured glasses and found violet to act more strongly than the rest.

Widmark ${ }^{2}$ ) investigated the cause of erythema photoelectricum. He used the light of a powerful electric arc lamp, concentrated firstly by a lens of rock crystal; secondly a glass plate was introduced between the irradiated object and the lightcollecting apparatus, to absorb the ultra-violet rays. Further, for another series of experiments, in order to make parallel the diverging rays from the source of light and to cool them down, Widmark used a metal tube, closed at one end by a rock-crystal lens, at the other by a plain sheet of glass with a hole in it, into which a disc of rock-crystal was cemented. The interior of the tube was filled with water, kept constantly in circulation.

Widmark's experiments showed that it was the rays which had passed through the rock-crystal and in which ultra-violet predominated which produced erythema. The heat rays and bright rays played only a subordinate part.

To distinguish the action of light rays from that of heat rays Hammer $^{3}$ ) used a gas lamp with an Argand burner and a ruby glass chimney. Thus the ultra-violet rays and all the light rays, except the red ones, were almost entirely excluded. The upper arm, on which one spot was marked off by adhesive plaster and brown paper, was then exposed and held at about to to I $5 \mathrm{~cm}$. distance from the flame. A fairly intense heat was felt. The experiment lasted an.hour and a half. After 25 minutes the skin showed distinct redness and was warm to the touch. At

1) Compt. rend. Soc. Biolog., I 877 .

2) Hygiea, I889, Festband, No. 3. Verhandlungen d. biolog. Vereins in Stockholm, I889, 20.-Skandin. Archiv. f. Physiologie, I889, I.

$\left.{ }^{3}\right)$ Ueber den Einfluss des Lichtes auf die Haut, Stuttgart, I89r. 
the end of the experiment the skin was moderately reddened and showed a few lighter patches within the red part. Then white patches, during the next few hours, cncroached more and more on the red, and in 24 hours the skin was again normal.

It was further noted that the part covered by the plaster, but not that which was covered loosely by the brown paper, was of the same spcckled red colour as the exposed skin.

In an experiment in which two adjacent strips of skin were irradiated evenly by the electric light, but one of them further exposed to the light of a gas flame with a ruby glass chimney, there was at first more intense reddening of the latter strip and of the parts near it covered by plaster, but later on both the exposed strips showed a quite cven degrec of crythema.

These experiments show quite clearly the difference between the action of heat and of the chemical ultra-violet rays on the skin. Both produce redness, but with this great difference, viz., that redness of the skin, caused by heat, appears very quickly and disappears as quickly, unless the heat has been such as to make albumen fluid. Redness of the skin, caused by the ultraviolet rays, on the other hand, shows itself for the most part some hours later, is very persistent and is followed by desquamation and by pigmentation.

In Maklakow's opinion, ${ }^{1}$ ) the effect of an are-light on the skin is not thermal, but chemical and very like that produced by insolation. Yellow and red light, which have no chemical rays, have also no effect on the skin. I cess powerful sources of light produce no marked effect on the skin, but lead to discolouration, if applied for a long time continuously. Long exposures or very intense light set up necrotic changes: the skin becomes gangrenous as a result of the chemical light action.

Finsen $^{2}$ ) exposed certain parts of the skin to all the rays of the spectrum, other parts to certain rays only. Ior this purpose he frxed on his arm a rock-crystal disc and a row of variously coloured pieces of glass, and painted on the arm a few letters and stripes. All these articles and colours were so arranged on

1) I.. C.

2) Mituheilungen ans Jimsen's merl. I.ichltinstitut, I, l). 9. 
the arm that the skin surrounding each figure was exposed to the full influence of all the light rays; the arm was exposed for Io minutes to the light of an 80 ampère lamp at a distance of $75 \mathrm{~cm}$. The skin was slightly reddened at once; after 2 hours the redness had increased, after 4 hours it had increased still more, but only in the parts which had not been covered. After I 2 hours in all the skin was quite dark red, hot and sensitive. Wherever the skin had been covered during the experiment with paint, it was now quite white and normal, standing out sharply against the red surroundings; the two letters were very clear, white on a red ground. Where the fire pieces of glass had been there were five white streaks of the irregular shape of the glass, and the skin at these places was throughout quite normal. On the other hand, at the part where the rock-crystal had been the skin was quite the same colour, and quite as hot and sensitive as at the parts which had not been covered at all.

Thus this experiment again showed that light erythema is caused by the ultra-violet rays. In a second experiment Finsen collected the arc-light through a quartz lens and held variously coloured bits of glass and also rock-crystals in front of it. At the same time means were taken to pass the light through a layer of cold water, and the irradiated spot was played on with a stream of cold water. Reaction of the skin resulted at the three spots where the light acted through rock-crystal, clear glass, and blue glass, but not the slightest reaction appeared with the other colours. From which we may infer that not only the ultra-violet, but also the visible chemical rays have the power of producing erythema photoelectricum.

A question of great interest to determine is, how deep does the light, and especially the so-called chemically active rays, penetrate into the tissue? Since, as has been shown, a peculiar influence on the phenomena, appearing after insolation, has been ascribed to the ultra-violet rays in particular, it seemed to the author important to test how far the latter assumption is justified. Attention was directed not as to whether the ultraviolet rays did as a matter of fact produce the effects here discussed, but as to whether these rays were capable of having any effect on those structures situated deep in the corium (capillary 
vessels, ends of nerres, chromatophores, etc), from which various unhealthy conditions of the skin have their orimin. The outermost ultra-violet rays, being for the most absorbable by various transparent media, $e . g$. , glass, $\left.{ }^{1}\right)$ it was a matter of interest to find out whether the opaque layers of epidermis prevent to any sensible extent the passage of these rays. The reply to this question has a further practical interest, at the present time, when, owing to the farourable results reported by Finsen on the light treatment of lupus, so much attention is being given to the irradiation of parasitic affections with so-called chemical rays. Quite recently, indeed, Strebel has been substituting for Finsen's treatment with electric arc-light irradiation with the ultra-violet light of the induction-spark, and he claims to have destroyed bacterial cultures far more quickly with this than with the arc-light. Under these circumstances it is specially important to determine the transparency of the epidermis for ultra-violet rays. For if the ultra-riolet rays are indeed so powerful in their action, and if in practice they are actually able to bring their action to bear on the deeper-lying layers of the skin, $i . e$., if they are not intercepted by absorption in the surface tissues, then it would surely be well to use as little as possible the visible constituents of the spectrum, and to make use only of such sources of light as emit the greatest proportion of ultra-violet rays.

In the author's experiments in this direction he wished, therefore, not mercly to test whether the chemical and ultraviolet rays are able to penetrate to the lower layers of the skin, but to determine as exactly as possible which part of the ultraviolet spectrum has this peculiar penetrating power.

It has long been known that animal tissues allow of the free passage of light, or at least of certain constituents of it, and before the discovery of Roentgen-rays this transparency of the tissues was largely taken adrantage of to investigate the character and seat of morbid changes within the human body. 'This

1) Sce the treatise by Eder and ralintw. Die Spectren farloloser und ge. factbter Cilaeser. Denkschr. 1. kats. Akad. d. Wissende. Math.-Haturw.. Cl, Vol. LXI. 
transparency is present in various tissues in a varying degree; it depends not only on the density and chemical nature of the layer, but especially on its uniformity, as regards both matter and density. Most substances found in the human body are not equally transparent to all colours; they absorb the rays of one or several colours and allow passage to rays of the other colours.

The transparency of the skin is shown by the simple experiment of holding the hand in front of a strong light, when the finger tips will show the red light through, or of looking at the sun with closed eyes, when a sensation of red light will be felt. The red colour shows that the red rays have penetrated the tissues. The commonly used test for hydrocele also shows in simple fashion that light penetrates the skin of the scrotum. Dessaignes ${ }^{1}$ ) demonstrated the passage of light through the skin by making a diamond sparkle when the finger covering it was illuminated from above. A similar result followed, when the light had to find its way through a white or chamois leather.

An experiment of Gadncff's ${ }^{2}$ ) threw further light on the nature of the rays penetrating the skin. He filled small tubes with chloride of silver, sealed them hermetically, and then with the aid of a trochar, introduced them under the skin of dogs and cats. When the animals had been exposed for some time to the sunlight, the chloride of silver was found to be blackened, whilst with animals in captivity, which had been kept in the dark, it was unchanged. Finsen proved the passage of light through the skin by placing sensitised paper behind the lobe of the ear and irradiating the latter. The paper was blackened, and to a more marked extent when the blood was driven out of the skin by pressure. Darbois ${ }^{3}$ ) showed that a piece of photographic paper introduced into the mouth under the skin of the cheek between two watch glasses was blackened after one minute by light from the Finsen concentrator, directed on the outside of the cheek. Gebhard ${ }^{4}$ ) inbedded a hand so completely in plaster of Paris that only the back of the hand was exposed.

1) Quoted by Gebhard.

2) Quoted by Boubuoff, Arch. f. IIyg., Vol. X. p. 335 .

3) P. Murbois, Traitement du Iupus vulgaire. Thèse de Paris, I90I, p. 80.

4) D: Ileilkraft des Lichtes, Lecipzig, I898, p. I3I. 
A photographic plate had been placed in the hollow of the palm. Then the hand was exposed for 20 minutes to the light of an electric arc-lamp and afterwards (in the dark room) taken off the plate and the latter developed. The plate was blackened, the contours of the hand and fingers being distinctly seen-a sign that light had passed through the hand. Similar experiments were made in Bechterew's laboratory by Solucha ${ }^{1}$ ). 'Tubes filled with strips of silver-bromide-gelatine were inserted under the skin of dogs and the wound sewn up. Light was projected on the parts from an electrical projection-apparatus of io to 20 ampères current strength and $50-65$ volts. After $1 / 2$ minute the bromide of silver was seen to be decomposed. When, on the other hand, the tubes were inserted deep down into the muscle substance of the glutei, the light had no effect. When the tubes were put behind the ear or inside the check of patients, decomposition soon set in; in the former case after half a minute, in the latter after 2 minutes; when placed behind the forc-arm or in the fist the bromide of silver was unchanged after i 5 minutes.

With a current, then, of the above-mentioned strength, light only penetrates through the skin; with a stronger current, say of 25 ampères and I I volts, it penetrates through the whole body, for under such conditions the sensitised gelatine-film was chemically altered, even when it was placed on the opposite side of the body from the part exposed to the light. Thus Solucha placed it at the back of the neck when he exposed the front of the throat, or at the right side of the body when the light came from the left. Kime and Hortaller ${ }^{2}$ ) proved that sun rays, too, may pass through the human thorax and still have sufficient power to produce an image on a photographic plate. Finsen ${ }^{3}$ ) passed concentrated sunlight through the ear of a white rabbit on to a bacteria culture. After $3 /$ hour he was able to observe distinct weakening or killing of the culture.

Finsen also proved with the skeptroscope that a light ray

1) Wissenschaftl. Sitz d. Vereinig. d. Pelershurger Klinik f. Nerven-

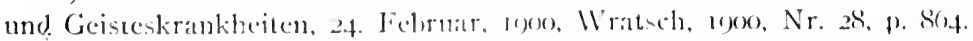

2) Allgem. Photogr.-Zig., 1/m) [1. fle.

3) Cober die Anwendung bul concentrirlen demischen lichtstrablen in der Merlicin, Leipzig, Isen, 1. 31. 
passed through the compressed lobe of a human ear could still be broken up clearly into all the colours of the spectrum.

The quality possessed by the ultra-violet rays of making bodics shine which are capable of fluorescence furnished Strebel ${ }^{1}$ ) with the means of proving the permeability of the human skin. The result of these experiments he gave as follows: "Glass absorbs ultra-violet light powerfully, but epidermis absorbs it rastly more. A plate of horn, about I mm. thick, eclipsed the spectrum from line 4 IO onwards and seemed, therefore, to be very slightly transparent. A human ear compressed between quartz plates absorbed isolated ultra-violet concentrated light completely." In a later communication Strebel reports on the following experinent: "A piece of skin freed from all fat-tissues was fixed between small quartz plates, and, all coloured rays being cxcluded, was irradiated with concentrated ultra-violet light, produced by induction sparks from zinc and aluminium electrodes; the illuminated object was $140 \mathrm{~cm}$. distant from the spark. The fuorescent screen, put up behind the skin, now showed a faint but quite distinct luminescence."

The experiments here given show that not only the optical rays, but also the chemically active ones are able to pass through animal tissue, eren, under certain conditions, through a layer of considerable thickness.

As above stated, the task the author had set himself was to investigate (r) whether the ultra-violet rays can penetrate the epidermis and reach the lower layers of skin, and (2) which part of the ultra-violet spectrum has this peculiar property.

It was only by means of the spectrograph that the task could be solved with accuracy, for even though Strebel's experiments fumished, as it were, the qualitative proof (by the lighting up of the fluorescent screen) of the power of the ultra-violet rays, under given circumstances, to penetrate the skin, still only spectrographic examination of the penetrating light could determine precisely and certainly which constituents of the light possessed this power.

${ }^{1}$ ) Deutsche med. Wochenschr., igor, Nos. 5, 6. 
In the spectroscope commonly used, as is well known, light is decomposed into its constituent parts by means of a glass prism. But glass, like many other transparent media, has the disadrantage of absorbing some parts of the spectrum and ultra-riolet riss in particular. For this reason, in these cxperinents a spectroscope with glass prism was not employed, but a so-called "grating" spectroscope, which produces a refraction-spectrum.

The experiments were undertaken in the photochemical laboratory of the Imperial Graphische Iehr u. Versuchs-Anstalt, in Viemua, under the supervision of Prof. Eduard Ialemta. The author used as material fresh epidermis (1) from burn-blisters, (2) from the bulla of pemphigus vulgaris. Both were carcfully removed with scissors, placed on gliss plates, and with them preserved in the fluid, withdrawn from the blisters by a small pipette during the short distance from the sick-room to the laboratory. (3) Fragments of cpidermis (from animals) which were kept, like the two other preparations, in normal saline solution.

These various membranes were now spread carefully on one quartz plate and covered with another. Then with a strong magnifying glass it was seen that the preparation showed no gaps or tears. The quartz, plates were fastened in front of the opening of the "grating spectroscope." As the source of light the spark was used (intensified by Leyden jars) from a powerful Ruhmkorff coil; the electrodes between which the spark passed were coated with an alloy of lead, zinc and cadmium (Eder's alloy). 'The spectrum of this light source, set up at about fo $\mathrm{cm}$. from the opening, was now photographed, first with, then without, insertion of the preparations. The opening was $0.2 \mathrm{~mm}$. wide; time of illunination, 15 minutes.

The experiments shomed that under these conditions absorption of the ultra-rolet rays begins at the cadmiun line, $\lambda=3260 \mathrm{AE}, i$. e, that this line, under the given conditions, is just recognisable on the film, whilst the light of the more refran- 
gible rays no longer produces blackening, being, therefore, absorbed.

There was no marked difference in the transparency of the three different preparations.

In consequence of this, soe may assume with certainty that, of the blue, riolet and ultra-violet rays, those up to the wavelengtl? of the cadmium line penetrate the epidermis.

The following experiments were made for the purpose of comparing the behaviour of dried epidermis with that of moist, fresh, normal epidermis: Films of almost colourless horn and of horn coloured slightly yellow, 0.5 and $0.56 \mathrm{~mm}$. in thickness, respectively, served as material. They were tested by examination under sunlight with the lattice spectroscope above men. tioned.

The yellowish horn, with an opening of o.I $\mathrm{mm}$. and an illumination of 80 seconds, allowed the ultraviolet light up to the Fraunhofer line $O(\lambda=34+0 A E)$ to pass through; under the same conditions with the colourless horn the ultra-violet rays were effective up to $\mathrm{Q}(\lambda=3287 A E)$.

Whilst, then, colourless, dead epidermis has on the whole the same absorptive power as the living epidermis, the permeability of coloured (pigmented) epidermis was shown to be sensibly less than that of the former.

According to Finsen ( see p. 438 ), the blood circulating in the skin hinders, to a marked extent, the entrance of the chemically active rays. It was for this reason that he constructed his compression apparatus, which also enabled him to lessen considerably the duration of the treatment.

To determine spectroscopically the extent to which the more refrangible rays are absorbed by the blood, a few drops of blood from the finger tips were squeezed on to a quartz film, which was surrounded by a rim of paper $0.17 \mathrm{~mm}$. wide, and covered with another quartz film. The blood completely filled this space of O.I $7 \mathrm{~mm}$. This layer showed a uniformly red colour, without any light space. On examination with a little 
pocket spectroscope, the usual absorption spectrum of blood was seen, with the characteristic absorption band between the Fraunhofer lines $D$ and $E$ in the greenish yellow.

Now, by the aid of a small glass spectroscope, using sunlight, the spectrum was photographed. With an exposure of 5 minutes the image of what had been seen was reproduced.

The absorption began at $F \stackrel{1}{2} G$ and from that point onward, in the direction of ultra-violet, practically no action on the photographic plate was discernible.

The experiments so far described concerned only epidermis and blood. A further experiment was made in order to discover what is the behaviour of living fresh epidermis beneath which blood is circulating.

A frog was put under curari, then two toes of a hind-foot were fastened, with pegs, in such a manner, along the edges of a triangular hole, cut in a sheet of cork, that the web of the foot was stretched tight across the hole. The cork was fixed in front of the opening of the lattice spectroscope. Sunlight was used as the source of light. The opening was $0.15 \mathrm{~mm}$., the exposure, 5 minutes.

It was shown that under these conditions the light from line $H(\lambda=3964 A E)$ onwards was absorbed. When we take into account the thickness of the membrane, the quantity of blood contained in it, etc., it is interesting that so many light rays from the more refrangible part of the spectrum should still be able to pass through.

From all these experiments it follows that a considerable portion of the ultra-riolet rays emitted by various sources of light pierces the epidermis and is able to reach the lower layers of the skin. The amount of these rays corresponds roughly with the third part of the ultra-violet spectrum as at present more exactly known.

Naturally, these results are infiuenced by the intensity of the source of light, the duration of the action and the thickness of the exposed layer. With suitable experimental arrangenents, 
we might, therefore, observe still more favourable conditions of the epidermis as regards permeability.

IVe may here once more refer to the fact, proved by recent photo-chemical research, that chemical action is traceable not only to light rays of short wave length, but to rays of each and every wave length (i.e., colour), according to the nature of the body they fall on. Light falling on a body and being absorbed by it will either produce chemical results (processes of conversion by oxidation), or it will be converted into heat without producing chemical change (absorption phenomena, in the case of substances coloured with pure spectrum colour), or it gives rise to electric phenomena, setting up electric currents or influencing the electric conductibility of the insulated substance. These phenomena seldom have a perfecly simple course, they are usually complicated by various simultaneous processes of similar kind. It is true that most bodies are chiefly affected by the short-lengthed rays, but it is very doubtful, seeing how differently the various animal tissues and fluids are affected by light and how various are their absorption spectra, whether we can group under one designation the action of any one special kind of light on the human organism. We may refer here to the different absorption spectra, given by blood corpuscles and albuminous substances, the former absorbing long waved light from certain parts of the spectrum as well as part of the blue and ultra-violet rays, while the latter absorb mainly the short waved rays.

In any case, as is evident from their absorption spectra, animal organic substances react readily to light. This fact is borne out by the purely empirical observations as to the physiological effects on the organism. Moeller ${ }^{1}$ ) made investigation with the view of discovering what changes of tissue in the skin observable under the microscope corresponded to the various clinical pictures of ordinary sunburn and other more serious light disturbances.

As material he took skin from the head and the ear of rabbits, as well as the skin of his own fore-arm. He used in the 
experiments electric-light arc-lamps of $1200-1$ foo normal candle-power. By rarying the distance between the source of light and the skin, as well as the time of exposure, and by repeated irradiation of the same part of the skin, he obtained various degrees of effect from faint erythema with consequent slight discolouration and pigmentation to more marked changes, such as redness and swelling, formation of resicles, necrosis, etc. By filtering away the heat rays Moeller allowed only the so-called chemically active rays to act on the skin. For his experiments he used I'idmark's apparatus (see p. 422).

Specimens for histological examination were taken from the dermatitis of various degrees thus produced.

The following objects were microscopically examined:

I. Human skin, after slight erythema, photoelectricum harl been produced (object $a$ ).

2. Grayish-toned, thickened, rigicl, but not yet pigmented skin from the head of a rabbit (object b).

3. Skin from the ear of an albinotic rabbit, hyperamic, redematous, dotted with little blisters.

4. The ear of an abbinotic rabbit showing more marked change, swollen on both sides, hyperamic, showing ecchymosis and blisters.

5. A piece of human skin, which had been exposed to the rays and on which a mulberry-shaped, irregular, dark-red hamorthagic blister had formed.

A review of the microscopic changes found in these rarious specimens $\left(a-c^{\prime}\right)$ seems to warrant the following conclusions:

"The first change to show itself in the exposed skin is in the vessels, which become microscopically more or less dilated. In connection with this the epithelium becomes moist throughout, and there is an abnormal formation of horny matter (parakeratosis) of a changed, darker colour. 'The prickle-cell layer of the epidemis and the hormy-layer appear much extended. Within the latter is a deep coloured strip, which consists of horn cells with their muclei ( live go).

Woeller surmises that the skin, which shows microscopically no other change than a yellowish-brown colour, gets its colour 
from this abnormal strip of nucleated cells. With more intense or long-continued action of the irritant exudation supervenes, which is sero-fibrinous or rich in cells; it may often, also, contain red blood corpuscles. The changes are more or less deep seated in proportion to the intensity of the light and the different nature of the exposed skin (whether human or of rabbits, etc.). Nore or less evident derangement of the parts may arise, dependent on the intensity of the exudation; the collagenous tissue begins to swell and become homogeneous, the epithelium swells, becomes relaxed, infiltrated, and raised in builæ.

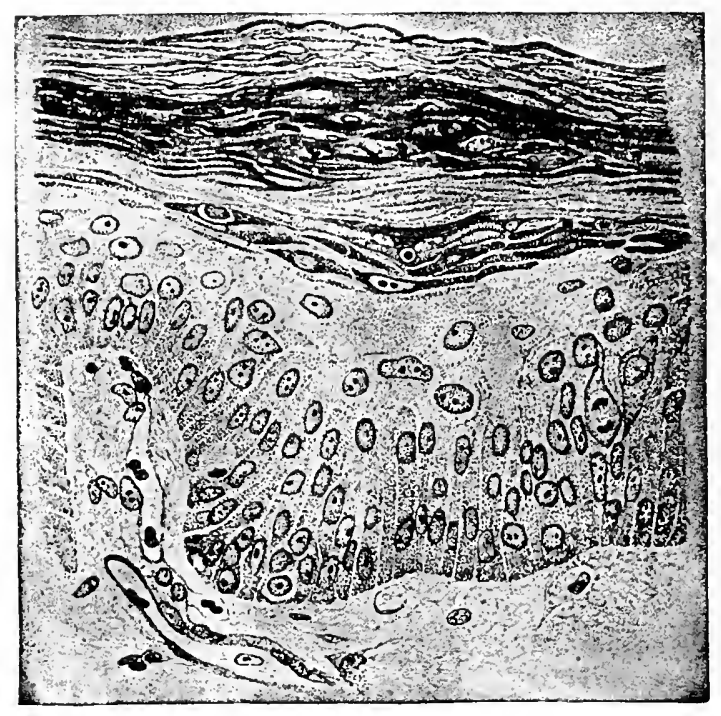

FIG. 90.

The interruption of continuity occurs in various places. With the human being it occurred approximately on the border line between the granular and the horny layers, but this by no means precludes the possibility that on other occasions (other skin and other degree of light intensity) the bullous exudation may arise differently. This would be analagous with the course, e.. . , of pemphigus, ${ }^{1}$ ) where in some cases the blisters appear

1) Jarisch, Zur Anatomie u. Pathogenese der Pemphigusblasen. Arch. f. Derm. u. Syph., i898. Festschrift gewidmet F. J. Pick, Part II, p. 34I. 
between the cutis and the rete, in others between the granular and the horny layer. With more intense light thrombi are formed in the ressels of the cutis."

In Moeller's case the contents of the blister consisted of a fine reticulum, containing numerous red corpuscles and isolated leucocytes. Everywhere close to the surface numerous light, round blisters are to be seen, with a more or less delicatte covering membrane and a light centre (Fig. 9I). In some of the horn-cells, loosened from the covering of the bulla, may be seen very distinctly through the swelling of the cells, a longish

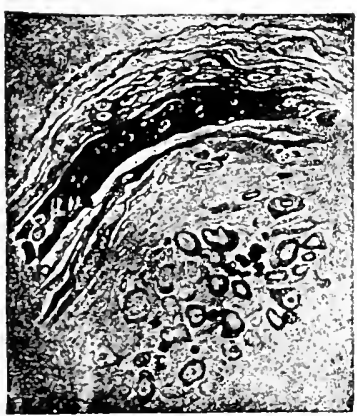

FIG. 9I.

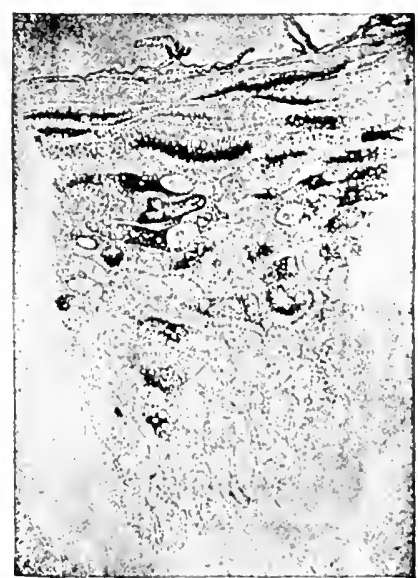

Fiki. 92.

rod-shaped hole in the centre in place of the nucleus. In the remaining prickle-layer, too, which forms the base of the blister, cell changes occur, which vary from a simple swelling to bullous degeneration ( ligg 92 ).

It was interesting to have confirmation of the fact of the deeply-penetrating action of the ultra-violet rays, which was seen very beautifully in the rabbit's ear. For the distmrlances were (achen the light was aery intense) sery maked, also on the recerse side of the carlilase' naty, in consequence, no doubt, of the larger number of vesse!s on that side, they were more noticeable there than on the directly illuminated side. 
In a further series of experiments Moeller proved that heat radiation ( $50^{\circ}$ to $55^{\circ}$ ) acting together with intense ultra-violet irradiation, just the same as heat radiation alone, after the filtering away of the ultra-riolet rays, when directed on the skin of the skull of rabbits, produced more or less intense cerebral disturbances, sometimes even sudden death. The autopsy showed the skin of the head, after intense irradiation of that nature, to be much swollen, and a bloody gelatinous exudation to be present in the subcutaneous tissue. The periosteum, the cranial bones, the dura were discoloured and covered with ecchymoses.

The ressels of the brain surface were much dilated, and it showed numerous, in part confluent, ecchymoses. When, on the other hand, the heat rays were filtered off and the ultra-violet rays alone applied, no central disturbance was observable.

These last experiments show that the action of the ultra-violet rays at any depth is relatively mimportant. No change of tissue was to be seen, even in the spongy subcutaneous tissue, this in direct contrast to the condition after irradiation by heat rays. Both heat rays and ultra-violet rays cause hyperæmia of the cutis, followed in the case of the latter rays by discolouration and hyperplasia of the epidermis, especially of the horny layer, which prevents them from penetrating further into the tissues.

No less important than the changes in the skin are those which light produces in the visual organs. Ogneff ${ }^{1}$ ) asserts that prolonged action of an electric arc-light of high power, with preponderance of violet and ultra-violet rays, produces necrosis in the cells of the cornea in the case of rabbits, pigeons and frogs. In the cells of the comea, necrosis is preceded by amitotic nuclear changes (whilst with brief exposure mitosis results). Of the other parts of the eye, the lens and the vitreous humour are not affected at all, the retina only slightly. Ogneff believed he guarded against any action of temperature in his experiments.

I ight, acting on the retina, bleaches (Kuchne") the red-colouring matter, "rhodopsin," proved by Boll to be present in the

1) Pflucger's Arch., Vol. LXIII, p. 200.

${ }^{2}$ ) Inter-uch. a. d. flyysiol. In-titut Heidelb. Vol. I. u. ff. 
outermost portion of the rods. It is extremely doubtful, however, how far this affects the power of sight.

By means of the local bleaching of the rhodopsin, images of light objects on a dark background $(c . g$., illuminated windows), may, as though by a photographic process, be obtained white on red in the eyes (previously kept in the dark) of either living or dead frogs and rabbits. (In the case of dead animals, the eyes are cut out.) These images may be fixed by means of alum ("optograms").

The pigment granules, when the eye is in the dark, i. $c$., in a state of rest, lie at the back of the pigment cells of the cpithelium, nearest the nucleus; when light falls on the retina, the granules move forward into the ciliary processes, lying between the rods and cones, and the rods and cones themselves draw together and contract. (Boll, Angelucci, Engelmann, r. Genderen, Heger, Perpens and L. Fuchs. $\left.{ }^{1}\right)$ ) A retina that has been kept in the dark changes its electrical condition when light suddenly falls upon it; the electric current which passes normally from the retina to the brain is made stronger (Holmgren). The so-called photo-electrical variations may be here referred to: for the eye accustomed to darkness the maximum of stimulation is found in yellowish green close to the thallium line, whilst the eye adapted to the light reacts most to the ycllow $D$ line of the spectrum (Himstedt and $N a g_{c l}^{2}$ )). Engelmann ${ }^{3}$ ) proved that frogs, from whose eyes light was artificially excluded, reacted with contraction of the interior cones of the retina to irradiation of the skin of the back. This fact proves that the light stimulus reaches the brain by a centripetal course, and is able thence to induce motor phenomena. Buedingen has shown that this reflex action does not take place when the brain is remored, whence we may conclude that this transformation of the stimulus takes place within the brain itself.

According to the researches of Bence Jones, Dupré and Jolm Tyndall, the lens possesses the power of Huoresence in a

2) Lethrb. 1. Augenheitk, I.eiprig mud Wien, $189,3$.

2) Physik. Zcitschr., 19n), Jahug. I1, 1. 362.

2) I'flueger's Arch., 1885, Vol. XXXV. 1, 4ts. 
high degree. "If I bring my eye into a violet ray, I notice a bluish-white glimmer filling the space in front of me. This glimmer comes from the fluorescent light produced in the eye itself. The crystalline hue of the eye, when looked at from without, lights up brightly at the same time ${ }^{1}$ )."

This peculiarity of the lens, which it shares in common with the vitreous body, may perhaps explain why Roentgen and Becquerel rays produce sensations of light with some persons. It is not, however, impossible that electric stimuli of the retina and the risual nerve may have something to do with it.

Of great importance, in connection with the effects of light on the whole human and animal organism, is the influence which it exercises on the blood and the capillary system.

We have already referred to the changes of form caused in the blood vessels by light.

Blood absorbs light in a high degree and in quite a peculiar manner. This is shown by the characteristic absorption spectra in greenish-yellow (Hoppe-Seyler) and in blue-violet obtained by $d^{\prime}$ Arsonval ${ }^{2}$ ) and the author ${ }^{3}$ ). Finsen ${ }^{4}$ ) laid on one side of the lobe of an ear a piece of aristo-paper and directed a pencil of blue-violet rays on to the other side; after the lapse of 5 minutes under these conditions no result was visible on paper. When, howerer, the lobe had been compressed between two glass plates, so as to appear white and bloodless, the paper turned black in 20 seconds. Whence we see that blood, to a considerable extent, prevents the chemical rays from penetrating the tissues.

There is especially one constitutent of blood which uniformly absorbs light, viz., hæmoglobin. Oxyhæmoglobin gives a different absorption spectrum from methæmoglobin. Quincke ${ }^{5}$ ) showed that hæmoglobin gives off its oxygen more quickly in the light than in the dark (the oxyhæmoglobin band

$\left.{ }^{1}\right)$ Das Licht. Sechs Vorlesungen von John Tyndall. Braunschweig, I895, D. I79.

$\left.{ }^{2}\right)$ Arch. de Physiolog., XXII, 2, p. 340.

3) Ci. I. 4.30 .

4) Ueher die Bedeutung der chem. Strahlen des Lichtes, Leipzig, p. 78.

5) Pflueger's Archiv, I894, Vol. LVIT, p. I34. 
in the spectrum vanishes), hence light increases the oxidising power of the blood and, correspondingly, the processes of oxidation in the human body.

IVe must refer ultimately to the action of light on the capillary system, the pigmentation left after exposure to strong light, which comes (cf.p. 4ig) from the accumulation of hæmosiderin in the interstices of the tissues. If this process is widespread, $i$. $c$., if this giving off of blood-colouring matter, in consequence of the exposure of extensive parts of the body to strong light, takes place to a large extent, then it causes a certain impoverishing of the blood in elements and blood-colouring matter, and the body may be stimulated to compensate for this. According to this theory (Lowenthal $\left.{ }^{1}\right)$ ), the influence of light on certain processes of metabolism is intelligible. Graffenber$g e r^{2}$ ) holds that the mass of hxmoglobin contained in the red corpuscles is lessened in the dark; a diminution in the total quantity of blood results from prolonged stay in darkness. Marti, ${ }^{3}$ ) too, by means of experiments with rats, established the fact that deprivation of light lessens the number of red blood corpuscles and to a less extent the amount of hamoglobin, whilst strong and continuous illumination stimulates the formation of erythrocytes and also of hamoglobin ${ }^{4}$ ).

In accordance with the results of these researches are the facts that the women of the far north are much predisposed to amenorrhœa, and are even said to be quite free from menstruation during the long night of winter (Enlenburg,")) whilst at the close of the Polar night the oxyhamoglobin bands in the

1) Deutsche Medicinalzcitung, I800, No. 72.

2) Pflüger's Archiv, I892. Vol. Lili, 1. 238.

$\left.{ }^{3}\right)$ Verl. d. Congr. f. innere Merl., I897.

') P. Borrison', too (cf. Zeitschr. f. phys. n. diät. Ther., Vol. V. p. 237). was anable to prove any influence of either light or darkness either on the number of the red or the white hood corpuscles, or on the formation of hemoglobin. Accoreling to his experiments, the weight of dogs kept in the light increased considerably, after an initial fecline, whilst that of dogs left in darkness showed no increase; the former also possessed a better appetitc.

") Quoted from Strobel, Inje Verwendung des Lichtes in der Therapie, p. 8. 
blood of such persons as were examined showed signs of extension (Holmgren and Gyllenkreut $\left.{ }^{1}\right)$ ).

Whether it is this direct action on the blood or stimulation of the nervous system which again reacts on other vital functions, stimulating them, the fact remains that under the influence of light the tissue-change in men and animals undergoes certain modifications. Several writers have cited interesting observations which favour the second of the two theories.

In the first place we must notice the important results obtained by Quincke, ${ }^{2}$ ) who showed that various tissue-cells (blood, pus, muscle, kidneys, liver, etc.) absorb more oxygen in the light than in the dark. When these substances were mixed with subnitrate of bismuth, the latter was reduced, but only in the light. Severed muscles and nerves, so long as they are not quite dead, eliminate carbonic acid more freely in the light than in the dark (Molescholt and $\left.F_{u b i n i}{ }^{3}\right)$ ).

From these researches we might then conclude that light influences the oxidation of the tissues.

Various experimenters claim to have observed on many occasions that matter-change in living animals and men is thus influenced.

When light is excluded, dogs, hens, pigeons and frogs eliminate less carbonic acid than in the light (Moleschott, ${ }^{4}$ ) Selmi and Piacentini $\left.{ }^{5}\right)$ ) ; the amount of carbonic acid given is in direct ratio to the intensity of the light (Moleschotl) and is not dependent on the freer movement of animals in the light (Chassanorit: $\left.{ }^{6}\right)$ ).

With frogs and toads the blue rays were most effective in increasing the amount of carbonic acid given off $;$ with birds and

1) Quoted from Gebhard and Mocllor.

2) L. c.

$\left.{ }^{3}\right)$ Untersuchnngen zur Naturlehre des Menschen und der Thiere von Moleschott, is8I. Vol. XII, p. 256. - Arehivio di Bozzozero, r879, Vol. III No. I9, P. 23, quoted from Raum.

4) Wiener med. Wochenschr., I885, No. 43.

s) Rendi conti del Reale Instituto Lombard di sc. e. lettre, I87o, Vol. III. Ser. II, p. 5I, ref. Allg. med. Centr. Ztg. i872, p. Sio.

$\left.{ }^{6}\right)$ Ueber d. Einfluss des Lichtes auf die Kolılensäureausscheidung in thisri chen Organismus. Inaug.-Discert. Königsberg, I872. 
rodents the red rays had most influence $\left(\right.$ Pott $\left.\left.^{1}\right)\right)$. Van $P e c h^{2}$ ) found that beetles (Brunchus pisi) take in more oxygen in the light than in the dark. Scharling,") Pettenkofer and $I^{\prime}$ oit, ${ }^{+}$) Fubini and Ronchi ${ }^{5}$ ) proved that human beings give off less carbonic acid in the hours of the night than in the daytime, even with absolute rest. The two last-named writers confined their researches to a single limb (fore-arm and hand).

Brown-Séquard, Pfinger and others hold, however, that the increase of carbonic acid elimination through light is only apparent, being occasioned by the morement and enlargement of the muscle with light causes.

S. Godnew ${ }^{6}$ ) found that persons and animals to whom daylight was accessible excreted more urine, urea, and chlorides than those who stayed long in the dark. S. Daitsch ${ }^{7}$ ) and B. $\operatorname{Kogan}^{\mathrm{s}}$ ) formed the following conclusions from their experiments :

( I) Red light wcakens the processes of both assimilation and dis-assimilation; (2) green light stands lower than white, in regard to the accumulation of nitrogen, as well as to qualitative metamorphoses; destruction changes proceed more vigorously in green light; (3) yellow and violet light induce the maximum of energy in all the rital processes, more complete metamorphosis prevailing under the influence of violet light; (4) darkness causes a diminution in the exchange of nitrogen in the body and, incidentally, a diminution in the daily amount of urine.

$\left.{ }^{2}\right)$ Vergleichende Unters ïber Nengenverhalen d. ausgesch. Kohlensä̈nre. Habilitationsschrift, Jena 1875.

$\left.{ }^{2}\right)$ Amsterdamer Naandliaid vor natuurwet. 1879, p. If6.

3) Ann. de chim. et de pharm., is.4. S. 3. p. 4.88.

4) Bericht der Mïnchener Akarlenie, 10 November, 1866.

${ }^{5}$ Arch. per la sc. med., 18-6. Von. I.

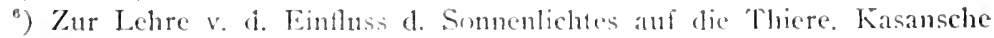
)issert., I882.

$\left.{ }^{7}\right)$ Ueber den Finfluss des weiscen Liehtes nud der verschiedenfarhigen

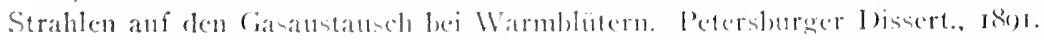

") Ueber den bindlus des weisen (ekektischen) lichtes no der verschiedenfarbigen Strahlen anf die Stickstoffmetamorphose bei Thieren. Petersburger Dissert., I8ry. 
According to Godncw, Gorbazcric ${ }^{1}$ ) Hammond ${ }^{2}$ ) and others, animals gain more weight in light than in darkness.

The influence of light on metabolism may be either direct or indirect; it depends:

I. On the influence of light on the blood and the circulation generally. (See p. 433.)

2. On the influence of light on the tissue elements themselves (cf. p. 4ri ).

3. On its stimulating effect on the nervous system, which produces increased action of the muscles and movement of the body (Locb).

4. On the stimulating effect of light on the organs of sense (the eyes), inducing increased or reflex energy in the body functions.

In connection with this dependence of the processes of matter-change on the accessibility of the sensitive retina to light, Moleschott, Béchard, Selmi and Piacentini, Pott, Pflïger and v. Platten") proved that under the influence of light, through stimulation of the retina, there was marked increase in the elimination of carbonic acid and absorption of oxygen.

Experiments with animals, which have been deprived of not only eyes, but also even of brain and lungs, furnish proof that matter-change may be influenced by reflex action through the skin.

It must, however, be stated, as opposed to the results given above, that papers have been published denying light has any influence at all on matter-change, or assuming only quite a different kind from that shown in the experiments quoted.

According to the observations of Graffenberger ${ }^{4}$ ) and others (Bidder and Schmidt), the metabolism of carbon compounds is lessened in the dark, and more fat is formed and deposited. Hence, animals kept in the dark increase in weight (greese, for fattening, are kept in dark sheds). This retarda-

$\left.{ }^{1}\right)$ Ueler den Einfluss der verschieclenenfarbigen Lichtstrahlen auf die Entwicklung 11. das Wachsthum der Säugethiere. Petersburger Dissert., I883.

") The Sanitarian, I873-74. Vol. I.

2) Arelı. f. d. ges. Physiol., 1875. XI, pp. $263,272$.

4) P’fiuger's Arch., iso2, Vol. LIII. 
tion of matter-change in darkness enables starving animals to live much longer in the dark than in the light (Aducco $\left.{ }^{1}\right)$ ). $B_{i e^{2}}{ }^{2}$ ) does not regard it in any way as proved that light influences the amount of hamoglobin in the blood, or that it promotes the elimination of carbonic acid.

Justus Gauli:") claims to have proved that with frogs in winter time the fatty bodies lying next to the sexual organs disappear in the day time and are formed again at night, and this in the case of blinded frogs as well, which shows that light acts on the fatty bodies through the skin.

It has been assumed by many that light influences the breathing, the temperature of the body and the pulse.

Féré ${ }^{-1}$ ) found in one case that respiration was 19 to the minute in yellow light, 17 in green, and only 15 in red. Under the influence of red light the pulse becomes fuller and slower; in darkness it falls so greatly that the sphygmograph ceases to show oscillation.

J. Godnew,"). too, found that the number of heart beats and the rate of breathing increased noticeably whilst the animals on which he was experimenting were under illumination.

Triwus") made researches under the guidance of IV. v. Bechterew into the influence of coloured lighl on llee pulse of healthy persons. Mosso's plethysmograph was mainly used in the measurements. The subjects were usually kept about two hours in the special coloured room of the clinical hospital. The plethysmograms were taken at the heginning and end of each experiment. In most cases the coloured light caused depression of the pulse, i. e., it became less rapid and less full. Violet is the most depressing colour, and red the least; the action of the other colours correspond with their place in the spectrum, with the exception of yellow, which had no effect, probably because the yellow plates let through nearly all the rest of the

1) Communicazone all' Acald di Torino, quoled in Franlioulä̈user.

2) 20. Congr. f. innere . Neelicin, mon.

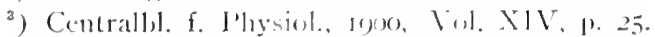

4) begénérescence el criminalité, issos, qunted in Ramm.

5) L. C.

") Wrssensch. Sif

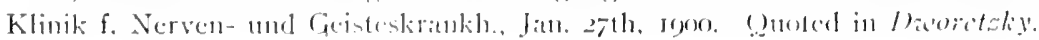


light rays. Triwus surmises that every coloured light ray forming in itself only one part of the energy of the united white stream of light necessary for the physiological nerve-tone, we must look upon coloured light as a peculiar form of light-hunger, which produces a certain minus in the chemistry of the animal body. That is to say, that the effect of any one colour may be explained, not by its own action, but by the absence of that of all the rest of the spectrum.

It is well known that sun baths and light baths affect the pulse and respiration, as well as the temperature, raising all considerably if the exposure is prolonged. In these cases, however, it would seem to be rather the radiating heat than the radiating light which is felt, though the latter may possibly have some share in the result. Raum is inclined to ascribe to light some influence on the daily fluctuations of temperature, conversion of matter and excretion in human organisms, both healthy and sick.

It is evident, from many biological facts already adduced, that light has a powerful influence on the nervous system and the orgains of sense. IVe see it not only in the action on the nervous organs, on consciousness and the mental condition, but also in the many phenomena and modifications of vital functions arising from its indirect action. The various rays of the spectrum have a striking influence on men and beasts. Goethe, in his "Theory of Colours," called attention to the connection between colours and certain emotions. No natural phenomenon affects the mind of man more deeply, no force is more stimulating to his mental powers, than the change from darkness to light or vice rersa. Pon $\approx a$ found by experiments with those mentally affected that blue has a soothing, red an exciting effect.

We know that light gives rise to movements by reflex action, not only by direct action on the tissues of animals. Dogel and Jegorow ${ }^{1}$ ) found that the circulation of the blood, both in men and dogs, was very markedly changed by the action of green

\footnotetext{
1) Qunted from /horelaky's Ref., Zeitschri. f. diät. u. physik. Ther., Vol. V, P. 165 .
} 
light irritating the eye. It was proved that matter change may be affected reflexly by means of the skin and the eye. $P$. Bert ${ }^{1}$ ) found that a chameleon, blinded in one eye, became paler in colour in the whole corresponding side of the body. In the case of other animals ( $\bullet . .$. , the octopus) it can also be proved that light affects the colour of the skin reflexly.

Light has a powerful effect on other nervous organs also. We need only point to the simple and never failing experiment of causing riolent sneezing by light. No doubt this comes through the medium of the nerves, distributed amongst the conjunctiva (trigeminus), for the inclination to snceze caused, or immensely increased, by blinking at the sun, may be at once repressed by closing the eyelids tight, so that the chemicallyirritant rays may be excluded by the lid. But since a good deal of red still comes through the lid (as we perceive when we turn to the sun with closed eyes) we must assume that it is the so-called chemical rays which set up the reflex action of sneezing. Inclination to sneeze, which soon produces actual sneezing, is felt at once when the eyelids are opened a little after a while.

We may ascribe also modifications in the functions of intermal organs to reflex and transferred action of light. Sereral writers (Holsknecht, $\left.{ }^{2}\right)$ Bie $\left.{ }^{3}\right)$ ) have mintained quite recently that we have here only indirect light action, since the so-called chemical rays, which are effectire, are completely absorbed by the organs on the surface of the body.

Holaknecht for this reason regards all light treatment of internal diseases as perfectly useless. I le eren does not hesitate to assert that there is not and cannot be any curative action of rays below the surface, that there neither is nor ever will be any kind of irradiation available for the cure of deep-seated disease, for any irradiation which is effective deep down must at the same time destroy the upper layers of tissue.

The author himself would not pronounce so confident an opinion. We do certainly know that the ultra-riolet rays,

\footnotetext{
1) I., c.

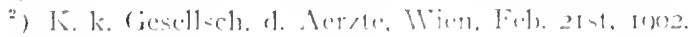

) I. c.
} 
which have decided effect on living tissue, are largely absorbed in the uppermost layers of the skin.

But we know also that the blue and violet-rays (which are likewise very effective physiologically (cf. p. 407), penetrate more deeply than the ultra-violet, and, indeed, if the light is powerful and the duration of exposure prolonged, make their way to relatively deep-lying layers, where, without destroying the superficial layers, they produce powerful effects (e.g., on lupus nodules in the cutis).

It is further known that the penetrating power of rays (into animal tissue) increases in proportion to the wave-length, that, $e$. g., red light pierces relatively large masses of tissue ( $e . g$, the hand) in sufficient quantities to reach greater depths. It is by no means proved that these rays have absolutely no physiological effect; we only know that they do not act on the skin in the same way as the blue, violet and ultra-violet rays. It is, however, just possible that these rays affect other tissues; do we not know that certain constituents of the body ( $e . g$., blood) have a quite specific power of absorption for them? and according to a law of nature, which holds good throughout, every energy that is absorbed must be in some way effective. Neither are the red, yellow and green rays so chemically ineffective as is often assumed. On the contrary, it has been proved that they have a far more powerful effect on certain substances than the rays of shorter wave-length, $c . g$., on dilute solutions of nitroprusside of sodium with sulphide of anmonia, on the brown peroxide of lead produced by oxidation from protoxide of lead under violet light, on green vitriol, on metallic arsenic and arsenious acid, on sulphuretted hydrogen, sulphide of sodium, on cyanine, anci on certain plant pigments, chlorophyll, etc. IVe know from the biology of plants how important these rays are for growth and nutrition. We ought, in the author's opinion, not to pronounce against the possibility of any biological action of these rays until after purely negative results have been yielded by the most careful investigations, with prolonged and intense illumination of the various kinds of tissue with all the ciasses of rays here concerned.

It would seem that such investigations might not be 
entirely fruitless. According to L. Camms, ${ }^{1}$ ) the colouring matter in dog's gall is quickly oxidised, turning green and then losing its colour, in the presence of oxygen by the action of light. The serum of horse's blood, too, becomes first green and then loses colour, when it is kept accessible to oxygen with access of light. Summing up this last chapter we may say that light has the following effects :

I. It has an irritant effect on the skin, producing inflammation.

2. It promotes perspiration.

3. It has a direct effect on the blood and the blood ressels.

4. If large portions of the body are exposed to strong light, it causes a considerable rush of blood to the surface and thereby depletion of the internal organs.

5. It modifies directly or indirectly the transmutation of matter.

6. It incites movement.

7. It exercises influence on the nervous system and the mind.

8. It has parasiticidal powers.

9. Excess of light-stimulus is destructive and paralyzing (causing dermatitis, long-lasting erythema, tendency to recurrence of the same, etc.).

\section{The Therapeutic Lse of Light.}

$\$ 57$. The beneficial therapeutic action of light has been known instinctively, as we may say, for a long time. For example, in China, Japan, Mexico and Iaiti, methods have been followed in medicine which take direct account of the helpful or deleterious power of sunlight; in some cases patients were given sun-baths, in others kept from the light.

According to I. Marcuse, ${ }^{2}$ ) the old (ireeks were accustomed, on grounds of both pleasure and health, to expose themselves (without clothing, and having first anointed themselves) to the sunshine on the flat roofs of their houses. I'stricins and Cicero tell us that the Romans in the same way took sum

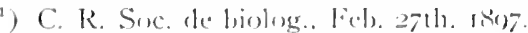

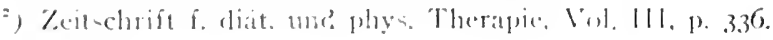


baths, followed often by a cold sponge-bath. In later days they had special outbuildings (Solaria) where the Heliosis was taken. Herodotus made special mention of sun-baths for persons with poor or enfeebled muscles. Antyllus described more precisely the effects of insolation (erythema, profuse perspiration), and gave lengthy descriptions of the action of these sun-baths (loss of fat, lessening of swellings, improvement in cases of dropsy, general bracing, good effect on respiration). The diseases for which he recommended them are: Dropsy, sciatica, affections of the kidneys, elephantiasis, "swellings," abdominal affections, chronic diseases of the bladder, paralysis, uterine diseases and fluor albus.

Herodotus and C. Aurcliun, also Antyllus, recommended light-baths in cases of skin disease.

Heliosis was in those days also the treatment for arthritis, nerve complaints, colic, jaundice, atrophy and constitutional anomalies in children. The sun-bath was used not only generally but locally, $e$. g., as preparative for blistering. Lightbaths and water-baths were often combined (I ittruvius, Pliny).

In the Middle Ages this method of treatment passed into oblivion. Facts, indeed, were known as to both the good and the evil effects of sunshine on the body, but we hear nothing of systematic light treatment before the beginning of the last century. At that time the Jena professor, Löbel, gave a precise account of the indications and contra-indications for the light treatment of sick persons, and described a special apparatus for sun-baths ( $\eta^{\prime \lambda \imath 0} \vartheta \varepsilon^{\prime \prime} \mu \mu^{\prime \prime} s$ ) which was to be used in various diseased conditions. The medical literature of the i $9^{\text {th }}$ century from that time on records a serics of investigations which we must regard as the basis and forerunners of the modern light treatment. In the first rank we must place the researches of the numerous bacteriologists, who have proved the parasiticidal action of light, and have determined to which part of the spectrum this is specially due. Then come the physiological works of Hammer, Widmark and others (mentioned on pp. $420,+22$ ) on the diffcrent action on the skin of the red and the blue end of the spectrum, and Moleschott's on metastasis. We find in the beginnings of chromo-therapy in Ponza, Charpig- 
non, Martin, Veiel, Unna, Berliner, Wolters, and of so-called negative light-therapy in Piorry, Picton, Black, Barlow and Waters. We see attempts to substitute electric light for sunlight (Lahmann), to use concentrated light in the treatment of lupus (Thayer, Otterbein, M $\mathrm{chl}$ ). We find the methods of lightdecomposition (heat and light filters), long used in physics and photo-chemistry, now turned to account in researches into the physiological action of light on the skin (Widmark). Kelogg's works on the effects of electric incandescent baths gave a powerful impetus to the study of light in its bearing on medicine, and mark an important stage in its development.

None, however, has done such work for the furtherance of light-therapy as Finsen (from I 893 onwards). He first made careful experiments of his own, and tested thoroughly those of others, and then, having laid a sound theoretic basis, he constructed the apparatus by which he was able to prove the usefulness of light when applied in its most intense form to malignant growths, such as lupus. Finsen was in the happy position of being able to go on his way unhampered by external circumstances; help came to him from private individuals and from the State, and he was thus enabled to carry his plans to completion and in a comparatively short time to demonstrate forcibly to the medical world, by results with an enormous number of patients, the extraordinary value of the light treatment. We owe it no doubt to this circumstance that lighttherapy has made such strides within a comparatively brief period.

IVe may reasonably hope that the near future will clear up many a doubtful point and widen the range of photo-therapy; for many other workers are busy in the field. Bang, Strebel, Görl, Foveau de Courmelle, Lorte' and Genond have published interesting work on the possible simplifying of the apparatus and the strengthening of its effect; Moeller, Streblel, Glebowsky, the present writer, and others, are secking by theoretic researches to solve certain important problems in light-therapy, and the practical results of light-treatment on most diverse discased conditions are at the present day being studied with the greatest zeal. 
Light may be used for therapeutic purposes either as natural or as artificial light; $i$. $e$., either without being decomposed and collected or as broken up into its component parts and concentrated.

\section{(a) Treatment with Sunlight.}

$\S 58$. The sun is our most natural source of light. Even in our latitudes on bright clear days it is the most powerful light source which we possess.

This may be seen by the following scale (from Picker$\left.\left.\operatorname{ing}{ }^{1}\right)\right)$ :

\begin{tabular}{|c|c|c|c|c|c|}
\hline & \multicolumn{4}{|c|}{$\begin{array}{c}\text { Light-giving Power in the Several } \\
\text { Parts of the Spectrum }\end{array}$} & \multirow{2}{*}{$\begin{array}{c}\text { Total Light } \\
\text { Power } \\
\text { expressed in } \\
\text { Normal } \\
\text { Candles }\end{array}$} \\
\hline & $\stackrel{\mathrm{C}}{\mathrm{Red}}$ & $\stackrel{\mathrm{D}}{\text { Yellow }}$ & $\mathrm{C}^{\prime}$ & $\begin{array}{l}\mathrm{F}_{2}^{1} \mathrm{G} \\
\text { Blue }\end{array}$ & \\
\hline White light..... & 73 & 100 & 101 & I 34 & I \\
\hline Gas lamp....... & $7+$ & I 00 & 103 & I 25 & I 6 \\
\hline Lime light...... & 59 & 100 & I 13 & 285 & 90 \\
\hline Electric arc light. & 61 & 100 & $\mathrm{I} 2 \mathrm{I}$ & 735 & $3^{62}$ \\
\hline Magnesium light. & 50 & I O० & 223 & I, I 29 & $2 I_{5}$ \\
\hline Moonlight ....... & $8_{7}$ & 100 & I 55 & . $3^{6} 3$ & 204 \\
\hline Sunlight........ & 45 & I 00 & 250 & $2,97 \mathrm{I}$ & 70,000 \\
\hline
\end{tabular}

Unfortunately, as regards our use of this source of light, we are dependent not only on weather, but also on other circumstances which affect chemical intensity as well as its optical brightness. As long ago as i 866, Roscoe and Baxendell, following $E d e r^{2}$ ) (from whose standard work we have taken these and the following data), proved that the chemical intensity of light does not by any means coincide with optical brightness. The chemical light-intensity of the sun's rays varies with the time of day and season of the year; that is to say, with the sun's height in the heavens. The maximum intensity is found when the sun is at its highest, i. $c$., at midday. In summer the chemical action both of the sun and of the blue light of the sky is distinctly more powerful than in winter; thus, for instance, at Vienna the

$\left.{ }^{3}\right)$ Ausführl. Handbuch d. Photographie, Vol. I, pp. $320 \mathrm{ff}$.

$\left.{ }^{2}\right)$ From Eder's Ausfïhrl. Handbuch d. Photographie, I, p. 355. 
intensity of the sunlight even at midday in December is only about as great as it is at six o'clock, either morning or evening, in June. The chemical intensity of light is weakened in spring. The optical intensity of sunlight is weakened by about onefourth during its passage through the atmosphere. The chemically active rays lose very much more. Langley found that the following percentage of the various classes of rays passed the atmosphere ${ }^{1}$ :

$\begin{array}{lll}\text { Ultra-violet } & 39 \text { per cent. } \\ \text { Violet } & 42 & \text { ". } \\ \text { Blue } & 48 & \text { " } \\ \text { Greenish blue } & 54 & \text { " } \\ \text { Yellow } & 63 & \text { " } \\ \text { Red } & 70 & \text { " } \\ \text { Infra red } & 76 \text { " " }\end{array}$

According to E. v. Oppolzer,") the atmosphere absorbs about 20 per cent. of the optical rays and about 30 per cent. of the photographic rays. C. Masch") proved that 57.5 per cent. of the sun rays acting on bromide of silver are lost by extinction in the atmosphere.

The extent to which the air absorbs the light is thus considerable; it varies with the amount of rapour, carbonic acid and suspended dust present in the air, also with its movement and temperature. By the atmospheric movement, layers of varying density are formed, and the intensity of the light rises with the temperature.

The chemical intensity of sunshine increases considerably as one rises into the higher regions of the air, $i$. $e$., as the atmospheric pressure becomes less; thus Simony found that the ultraviolet spectrum of sunlight extended much further towards the more strongly refrangible end on the summit of the Pic de Teyde on Teneriffe (3,500 metres high) than in the plain

1) For further information see J. M. Pernter, Meteorolog. Optik. Vienna, Ig02; also J. Hiesner, Studien über das photuchem. Klima. Wiener Akatd.

2) Sitzungslecricht d. K. Akat. d. Wissensch. 107, 1'art II, 1. I 477, 1898.

3) Schriften d. Naturw. Ver. f. Schleswig-Holstin, Vol. XII, Il. 2. 
below. There differences are, however, slight compared with those dependent on geographical latitude.

Corresponding to the course of the sun, which up to the latitude of about 23 passes the zenith twice in the year, there are within these latitudes two maxima of light-stretch, in March and September at the equator, in April and August at lat. $10^{\circ}$. At lat. $20^{\circ}$ there is only one maximum, but that lasts from May to July. The nearer we approach to the higher latitudes the more sharply do we find the curve, which denotes the distribution of light during the year, rising towards the maximum in the summer months. The absolute amount of light given off by the sun to the earth in the course of a day is greatest at the equator and the lower latitudes; it lessens towards the higher latitudes. There is only one irregularity in this respect, $i . e$. , in the highest latitudes of all, where the sun does not set during the whole Arctie summer. (From D. Spitaler ${ }^{1}$ )).

Holetschek estimates the chemical intensity of diffused daylight at mid-day in winter as one-half in the summer, as only one-quarter, of that of direet sunlight. In summer the morning and evening direct sunlight has still about double the ehemieal intensity of diffused daylight, but in winter there is a very slight difference between the two. According to Roscoe and Baxendell, the ratio of the ehemical intensity of direet to that of diffused sunlight is not constant for a given height of the sun in different places, but varies with the transparency and other conditions of the atmosphere.

Naturally, the chemical intensity of daylight is much influenced by the condition of the clouds, $e . g$. , by the depth and density of the layers of cloud and the colouring, shape and position of the clouds relative to the sun. Mist and clouds lessen the ehemical intensity far more than our subjective sensations of light would lead us to believe.

Simple sunlight is used therapeutically in sun-baths and lightand air-baths. Single filtered components of the spectrum are

1) Eder's Jahrl. f. Photogr.. 1888. 2 Jahrg., p. 379. 
used in so-called chromo-therapy. In a concentrated form, either without being decomposed, or after abstraction of certain components (the heat rays), it is used in the treatment of skin diseases.

\section{Sun-Baths.}

$\$ 59$. Patients taking sun-baths ${ }^{1}$ ) are usually placed on rugs or mattresses, with the head raised and protected from the wind, in a verandah entirely open to the south. The head and eyes are protected from the direct rays by dark glasses and suitable shades.

Sun-baths are taken in the height of summer between Io a. m. and 5 p. m.; beginning with at least a quarter of an hour, the time is usually increased to three-quarters, seldom to more than an hour. During the bath the position is changed at intervals, so that the rays may fall on all parts of the body; the same position is maintained until violent perspiration breaks out on the exposed part, but not for longer, or too violent inflammatory reaction might result.

After, and possibly even during the sun-bath, a quick bath (of either the whole body or parts) at $72^{\circ}$ to $82^{\circ}$ is recommended. After this the patient is rubbed down and at the same time massaged, unless there are symptoms which make this not advisable. Many doctors order a dry pack of a quarter of an hour after the sum-bath. When the bathing is done the patient takes exercise.

The sun-baths are sometimes taken every day, but usually only 2 or 3 times a week. Persons in health would find one a week sufficient.

As direct effects of sun-baths, besides the erythemous reaction of the skin (in appearance like a scarlet-fever rash), leading to intense pigmentation and subsequent desquamation, may be mentioned profuse perspiration, rise in temperature to $40^{\circ} \mathrm{C}$. in the case of sensitive persons, nervous disturbances, and with healthy persons a pleasant feeling of refreshment and comfort.

1) For incandescent light baths, sce pp. $360 \mathrm{ff}$. 
The appetite is improved, and the patient feels cheerful and often very sleepy.

Sun-baths are said to be beneficial in disorders of nutrition (obesity, diabetes, gout), in scrofula, rickets, some diseases of the internal organs (asthma, heart-disease), anæmia, cachexy, nervous affections (neuralgia, neurasthenia, sciatica), affections of the skin and mucous membranes.

The beneficial effects of sun-baths are due to their action in promoting perspiration (for the sweat eliminates harmful matter from the body), and also to the stimulation of metabolism and of the nervous system by light, and to the direct action of light on the blood. The violent prolonged hyperæmia of the skin induces a determination of blood from the internal organs to the surface of the body, and thereby the depletion of these organs.

The bactericidal action of sunlight does not count for much here, as the ultra-violet rays (which are most effective in that respect) have little penetrating power. The blue and violet rays, however, penetrate more deeply, and their anti-bacterial action is not to be disregarded.

On the other hand, light promotes the general power of assimilation. The more vigorously this goes on, the greater, according to Liebermeister, will be the vital energy of the body and its power of resistance to everything injurious, especially' to pathogenic micro-organisms. This, as well as the hyperamia induced in limbs exposed to irradiation for a long time ( 2 or 3 hours daily), may explain the cures reported by Cicchansky, ${ }^{1}$ ) Poncet, ${ }^{2}$ ) Perdu and Blanc, ${ }^{3}$ ) in cases of local tuberculosis in joints and bones.

As a substitute for sun-baths in the open air, Rieder in the cold season orders sun-baths within doors-or electric arc-light baths.

$$
\text { Light- and Air-Baths. }
$$

$\$ 60$. In these baths the patients move about in the open air, vith little or no clothing, for a period of from 2 to 6 hours. To

1) Gesellsch. d. Kinderärtze, Moscow, March 7th, 1901.

$\left.{ }^{2}\right)$ Millioz, Thèse de Lyon, 1899.

$\left.{ }^{3}\right)$ Revue intern. de l'électrothérapie, Jan., I900, p. I57. 
keep themselves warm they do some manual work or gymnastics, or have massage. Light- and air-baths are taken morning and evening, with a sun-bath or hot-bath at midday.

According to Rieder, the effective factors with these baths are the thermal stimulus, the increased activity of the skin through the radiation of heat, and the influence of light on metabolism.

Light- and air-baths are used as a general tonic for bracing the nervous system, for treating obesity, in cases of congestion of internal organs, and to stimulate the action of the heart and kidneys. Their effects are similar to those of the "water-cure."

\section{Chromo-Therapy.}

$\S 6 \mathrm{I}$. Coloured, that is filtered, white sun-light in a nonconcentrated state has been hitherto used in the treatment of infectious general diseases (small-pox, measles, scarlatina, erysipelas), nervous afiections and a few skin diseases.

General chromo-therapy is applied by keeping the patient continuously, for some time, in a room to which light is only admitted through suitably coloured glass (red or blue). Chromo-therapy is applied to local affections by wrapping the diseased part of the body closely in suitably coloured stuffs, which.serve as light-filters.

Our experience so far leads to the conclusion that the treatment of localised skin affections with red diffused light leads to good results.

According to Finsen, ${ }^{\text {') }}$ chromo-therapy was first successfully employed in the popular medical practice of past centuries. Thus in China, Japan and Roumania the face and hands of small-pox patients were swathed in red cloths, and graver symptoms thereby averted. The first experiment in chromotherapy on a scientific basis was made in $188_{7}$ by Th. I'ciel, who cured violent persistently recurring eczema solare in the case of a lady by ordering the use of red silk vells."

") Ueber die Pedentung Ier (drem. Strahlen ries Lichtes, etc. Leipzig, ISg9).

$\left.{ }^{2}\right)$ Vicrteljahrschr. f. Iferm. und Syjh.. 1897, 19. I1I3. 
Wolters, $\left.{ }^{1}\right) U_{n n a^{2}}$ ) and Berliner ${ }^{3}$ ) reported similarly good results in 1892 . Instead of the red light filters, Unna and Berliner used yellow (coloured with curcuma) masks, veils and windows.

Quite recently $W$. Winternit ${ }^{4}$ ) has reported similar results in the case of affections not in any way traceable to light action. By covering up with red materials the parts of the body exposed to the sun, he has diminished chronic-hyperæmia of the skin and improved and cured eczema; and by the same means he has brought about distinct improvement in cases of chronic rheumatic affection of the joints, hands and feet.

Acute exanthemata were first treated by chromo-therapy by Niels Finsen ${ }^{5}$ ) in July I 893 . As long ago as 1832 Picton $^{6}$ ) observed that light had an unfavorable effect on the course of small-pox, and Piorry ${ }^{\top}$ ) came to the same conclusion in 1848 . Communications to the same effect are frequent in medical literature. Barlow ${ }^{\mathrm{s}}$ ) and $W$ aters $^{9}$ ) therefore excluded all light from their small-pox patients, and they found that this treatment resulted in the drying up of the pocks without suppuration or scars.

Finsen noticed that persons recovered from small-pox have the deepest and most numerous scars on the face and hands, $i . e$., on those parts most exposed to light. Taking into account his own experiences and those of others, Finsen surmised that the so-called chemical rays, whose injurious effect on the skin had been proved, represent an etiological factor in the suppuration of the lesions. On this assumption he treated his small-pox patients, as Veiel had done before him in the case of eczema solare, with red light, i. e., he kept them in a room from which the chemical rays were excluded. This treatment, according

$\left.{ }^{1}\right)$ Ergänzungsh. z. Arch. f. Derm. u. Syph., 1892, I, p. I87.

$\left.{ }^{2}\right)$ Monatsh. f. prakt. Derm., I885. Vol. IV, p. 277.

s) Ibid. 1890, Vol. XI, Nos. Io and II.

$\left.{ }^{4}\right)$ 22. öffent1. Vers. d. balneol. Geseilsch., Berlin, May 7-I2, Igor.

c) L. c.

$\left.{ }^{6}\right)$ Arch. gén. de méd., XXX, p. 406.

$\left.{ }^{7}\right)$ Traité de médecine pract., T. VII, p. 495.

$\left.{ }^{8}\right)$ The Lancet, I87I, p. I.

9) Ibid. 1871, P. I5I. 
to the reports of Lindholm, Swendsen, Feilberg, Strandgaurd, Benckert, Krohn, Mygind and others, ${ }^{1}$ ) results in the total or partial suppression of suppuration and its concomitant fever, and the absence of scars. Such scars as are left are mostly only superficial, smooth, hyper:emic patches, which clear away later. There is no deep-spreading loss of substance, but at most shallow depressions. As severe ulcers of the skin are avoided there are for the most part no resultant serious general symptoms ( Engel $\left.^{2}\right)$ ).

The results of this treatment in small-pox induced Chatinière, ${ }^{3}$ ) Backmann ${ }^{4}$ ) and Th. Schiiler ${ }^{5}$ ) to try it recently in measles. Here, too, the treatment is said to have been most successful. The hyperpyrexia was lessened and the laryngeal and bronchial symptoms improved. In scarlet fever and erysipelas, too, according to E. Schouli, ${ }^{6}$ ) Festner, $\left.{ }^{7}\right)$ Schiiler and Krukenberg, $\left.{ }^{8}\right)$ good results followed its adoption, the course of the illness being modified in duration and severity.

The question here arises: Are we justified in calling this therapy chromo-therapy? The name might suggest the thought that light of a certain colour has a healing action on these morbid processes.

Such an assumption cannot, we think, be based on the data quoted in the preceding paragraphs. We have seen that it is not only the exclusion of the chemical light rays, that is, of one component of white light, which has good results (Veicl,Finsen); we know that darkness, that is the complete exclusion of light, may act in precisely the same way. This is proved both by the observations of Barlow and IV aiers, and by the fact that persons who have had small-pox are free from scars in the parts which have been covered up, i. $c$., kept in darkness during the

1) Quoted by Finscn.

2) Therapie d. Gegenwart, Igor, No. 3.'

3) La Presse médic., I898, No. 75.

‘) Quoted by Bie, Mittheilungen aus Finson's Lichtinst, II, p. I50.

s) Ibid.

-) Refer. Zeitschr. f. diät, u. phys. Th., Vol. III, p. 612.

") Quoted by Bic, Behandlumg von Masern u. Scharlach mit Ausschl. d. sog. chem. Lichtstrahlen Mithl. a. Finsen's Lichtinst.. II. 1. 1.40.

") München. med. Wochensclır., ıgor, April ist. 
illness. From this point of view we might regard Finsen's redlight treatment as a modification of Barlow's and Waters' darkness-treatment, a form of cure both easier to carry out and more pleasant for the patient. It would not be easy to keep a sick-room permanently and absolutely dark ${ }^{1}$ ).

But it does not follow that the red rays do not play a certain active part in this therapy. We have as yet made no study as to the action of these rays on morbid processes, and it does not seem improbable, when we remember the favourable influence of the allied heat rays, that they too should have beneficial action. This theory would be in accord with Engel's observations; he tells us that the treatment of small-pox patients in a red room has no effect on the eruption in the mucous membrane of the upper air passage, i. e., on parts which are usually in darkness.

Many observations point to the active part played by the less refrangible rays in the various physical phenomena. We know (Cf. p. 446) that on many substances they have a stronger chemical effect than the more refrangible rays. We have evidence, too, of a certain antagonism between the actions of these two classes of rays, of the neutralization, to some extent, by the red rays of certain effects of blue and violet rays.

According to Eder, ${ }^{2}$ ) Herschel, as long as I $830,{ }^{3}$ ) found that red rays have an opposite effect from blue ones on certain photographic papers. Fiseau and Foucault, ${ }^{4}$ ) too, have described very precisely the so-called negative effect of certain light rays. Claudet ${ }^{5}$ ) in I 847 demonstrated that the red and yellow rays of the spectrum check the action of the others (especially blue rays on bromide, iodide, or chloride of silver), or reverse it, if it has already taken place. Later he found ${ }^{6}$ ) that red and yellow light always have a negative or destructive effect on bromo-iodide or bromo-chloride plates, but act on clean

\footnotetext{
$\left.{ }^{1}\right)$ In this sense we should have to look on the exclusion of light, which is insured by dressings, as a factor in the healing of wounds.

") Ausführl. Handb. d. photogr., I, I. p. 262.

3) Biblioth. Univ. de Genève. New Series, Vol. XXIII, p. I85.

4) Compt. rend., Vol. XXIII, p. 679.

$\left.{ }^{5}\right)$ Philosoph. transact., I847. Daguerreian Journ., i851, Vol. I, p. г6r.

") Philosoph. Magaz., Vol. XXXII, p. I99.
} 
iodide of silver plates sometimes negatively and sometimes the same way as blue. As regards the relative action of the single rays, according to Claudet, in order to reverse the action of white light that has acted for the time-unit $\mathrm{I}$, red light requires 50 units, orange $1_{5}$, yellow is. "Waterhouse's ${ }^{1}$ ) investigations showed that each part of the spectrum (from red up to violet) may neutralize a previous action of light on silver bromide.

The importance for our present subject for this kind of action must not, it is evident from these instances, be disregarded.

Yet further experiments with photographic preparations show that the opposed action of the red and the violet light is by no means proved, and that in fact the two ends of the spectrum rather work in concert.

The antagonism in the effect of red and violet rays on phosphorescence is interesting and highly important as bearing on the present question; we shall recur to it later on.

Mental and nervons affections would seem to offer another field for chromo-therapy.

Akopenko, ${ }^{2}$ ) working in Bechterew's laboratory, proved that the colour of the light unquestionably affects the duration of psychical processes, and that the various rays have, in this respect, various effects, according to their position in the spectrum. The nearer we approach the heat rays of the spectrum the more invigorating and quickening become the effects of the colours. The mood of the person under observation is affected: in red light he feels brisk and cheerful, inclined to move and to act. Physical effects are produced; $\iota . g$, at the close of the sitting headache sometimes has disappeared. Yellow light has no special effect on the quickness of physical reaction and on the temperament, being in this respect like daylight. Rays of shorter wave-length have a depressing effect, so that, $c . g$., prolonged stay in a room with green light, though at first pleasant, becomes later oppressive. Under the influence of green light

1) Procecelings of the Royal Sone, London, XXIV, p. I86.

2) Qunted from Inatelaky's Refer. Zeitschr. f. diit. 1. phys. Th., Vol. V. p. 165 . 
psychical processes are retarded: mental quietude results, movement is checked, excitement allayed. This depressing effect is still greater in violet light. The mental attitude becomes melancholy, dreany; after some time headache is felt. The psychical processes are checked and become very slow, whilst the feeling of general depression becomes almost unbearable.

The above coincides with Goethe's observations:

He noted that red and yellow light was bracing, green and blue depressing. Baron Reichenbach's observations were to the same effect.

v. Jaksch, ${ }^{1}$ ) too, emphasizes the soothing, soporific effect of blue light, and he consequently used blue lamp chimneys in his sick wards. On the other hand, G. Olcinikow ${ }^{2}$ ) reports that patients seriously ill, when kept in the red room, suffered from delirium with frightful hallucinations, but these passed away at once on their being carried into a light room.

According to Biné, Féré and Gilles de la Tourette, ${ }^{3}$ ) red light strengthens the nerves. It was noticed in the photographic plate factory of Lumicre Bros., in Lyons, that the workmen in the red rooms were singularly lively over their work, singing, arguing loudly, gesticulating vehemently. When the red panes were replaced by green they became much quieter.

The author's own experience does not quite accord with this. He heard, on enquiry from many professional photographers, that they felt most depressed after spending any length of time in the dark room.

Pon $\approx a^{*}$ ) was the first to try the practical effect of chromotherapy on mental disease. He noticed that persons affected with melancholia, after a short time ( 3 to 24 hours) in the red room, became cheerful and chatty and were ready to take food, whilst the blue room had a quieting effect on maniacs who had already required the straight-waistcoat. The editor of the Inventuo Médica, in Guatemala, is said to have observed the same 20 years before.

20. Congr. f. innere Medicin, 1902.

") Jeshenerdelnik, I900, No. 38 .

3) L'année électr., 1901, p. 368 .

4) Annales Médico-Psychologiques, i876, Vol. XV, Ser. V. 
Up to now blue-diffused sunlight has been little used in photo-therapy. General l'leasanlon claims to have effected complete cure in a case of contusion by thrice-repeated irradiation for half an hour ${ }^{1}$ ). In many districts of the Böhmerwald erysipelas is treated by packing the affected part with blue cloths and carefully keeping off white light. Probably the blue colour of the material that shuts off the light, though insisted on, is of no practical value.

\section{Concentrated Sunlight.}

62. Concentrated sunlight is obtained by using convex lenses or concave mirrors. Long ago isolated cases of lupus were treated (by Thayer, Mohl and a layman mentioned by Otterbein, $\left.{ }^{2}\right)$ ) by means of sunlight concentrated by a burning glass on the affected part, and good results were obtained. Suitable as this simple apparatus is for the intensifying of the light action proper, it has one failing which prevents its general practical use in therapeutics; viz.. with a simple convex lens not only the light, but also the heat rays, are gathered into focus, in which such a high temperature is generated that it is simply impossible to expose living matter to it for any length of time. In order to eliminate the heat rays Finsen availed himself of a method of filtering by means of cold water; which had been often used by physicists, and also in physiological experiments

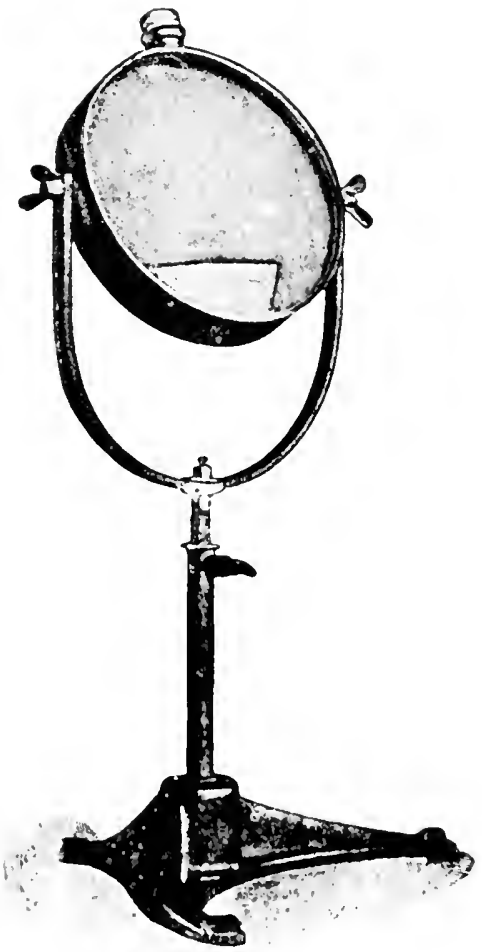

Ficio 9.3.-(Concentrating apparalus for sunlight.-Fimsin.) 
(e.g., by Widmark). His apparatus consists of a plano-convex hollow lens, 20 to $24 \mathrm{~cm}$. in diameter, filled with cold water; this lens is mounted in a forked stand in such a manner that it can be moved in any direction on a vertical and horizontal axis, and can at the same time be raised and lowered (Fig. 93).

With this apparatus all the components of white sunlight are not directed on the diseased spot, for the ultra-violet rays are much absorbed on their passage through the glass. The liquid used in the cold filter has also a great effect on the quality of the light passing through. Distilied water indeed absorbs very little, but the ultra-violet rays are sensibly weakened by the additions of methyl-blue or ammoniacal sulphate of copper which Finsen, who uses only the chemical rays, put in at first to filter away the less refrangible rays.

If a blue filtering fluid of this kind (a solution of double sulphate of copper and ammonia), in a vessel (quartz) allowing the passage of ultra-violet, be introduced in front of the opening of the lattice spectroscope in the path of the light rays from the spark produced between electrodes of Eder alloy, and the spectrum be then photographed, the plate will show, when developed, that - with a layer of 5 per cent. solution of ammoniacopper sulphate, an opening of o. I mm. and an exposure of 3 minutes - the spectrum has been broken off at the air-line $\lambda=3955$. Thus a light filter of that kind, under these conditions, allows blue and violet light to pass, but practically no ultra-violet.

The use of blue light filters has now been given up on account of their weakening of the light.

Quarts, which, according to Stokes, lets more ultra-violet rays through than any other material, should really be used for this optical apparatus, in order to make it as pervious as possible. (According to Hankel, Storer, Eder and Valenta, ordinary glass absorbs very much ultra-violet, hard flint glass and all plumbiferous glass more than crown glass and baryta-flint.) But according to Finsen, glass does let through the relatively weak ultra-violet rays of sunlight, and in any case pieces of 
quartz of the required size would be hard to obtain, and apparatus fitted up with quartz lenses would be very expensive.

For the rest, the favourable results obtained by Finsen with his old apparatus (glass lens and blue light-filters) are proof that it is not so very important that the irradiation should be with pure ultra-violet rays. Lltra-violet rays of low intensity are absorbed by the surface layers of the skin, whilst if raised to higher intensity ( $e . g$. , by the Bang lamp) they soon produce violent inflammation. It would hence appear wiser in treating skin diseases (lupus) not to lay such great stress on the use of the rays of shortest wave-length, but rather to confine oncself to the rays of somewhat greater wave-length. The latter have more penetrating power (vide the blackening of photographic plates by sunlight that has passed through thick parts of the body) and at the same time they possess the desired chemical and therapeutic efficacy (cf. Finsen's results), though not to such an extreme extent as to injure healthy tissues before affecting the morbid growths.

Concave mirrors serve to concentrate greater quantities of light than can be done with lenses. Strebel uses metal reflectors, I metre in diameter, with water-cooling arrangements in front of them.

The method of treatment with concentrated light and its results in lupus vulgaris will be fully dealt with later, in connection with the arc-light treatment, which is based on the same principles.

E. Nesnamow ${ }^{1}$ ) made some interesting experiments with the cure of suppurating processes in the cornea by sunlight. He used a collecting lens similar to Finsen's. Five severe ulcers in the cornea were treated for 2 to 5 minutes daily with blue-violet sunlight, with excellent results.

\section{Treatment aith Irlificial Sources of Light.}

$\S 63$. Sunlight is so little to be relied on that one is driven in

1) Westnik Ophthahmolngii. 100т, Jan. and Fel.; cf. Dzeorctzky, Zeitschr. f. diät. u. phys. Th., Vol. V. Part III. 
photo-therapy to fall back mainly on artificial sources of light. Naturally the attempt is made to bring their intensity as nearly as possible up to that of sunlight, and to heighten as much as may be their illuminating power. Here we must distinguish clearly between the optical brightness of a light, which is a matter of its physiological effect on the eye, and its chemical or photographic illuminating power. Many a light which seems very bright to our eye has little or no effect on photographic plates and other light-sensitive preparations, whilst many pale-blue flames have considerable photo-chemical effect. The illuminating power of the sun is 524 times that of magnesium, but it is only 5 times more powerful chemically. Burning magnesium has as powerful a chemical effect as the sun at $\mathrm{IO}^{\circ}$ altitude with no clouds about, $i$. e., if both light sources have the same apparent superficies. With the sun at $22.4^{\circ}$, its chemical effect is 36 times that of magnesium (Bunsen and Roscoe).

It is desirable for the various purposes of photo-therapy to have light-sources of considerable optical brightness and chemical power. Most kinds of artificial light have very little brightness as compared with sunlight. There are, however, light sources which are extraordinarily powerful, chemically or photographically. Thus the light of burning magnesium is very rich in violet and ultra-violet rays (Schrötter, Bunsen, Roscoe); it is so powerfully actinic that in the fraction of a second 4 to 5 grammes of magnesium powder will develop chemical illuminating power equal to that of a million candles. In the same way there is very much violet and ultra-violet in both the electric arc-light and the light of sulphide of carbon burning in nitrous oxide gas (H. W. Vogel).

These light sources are, as regards their chemical rays, superior to the Drumond lime light, which is very rich in yellow and red rays (Becquerel).

The electric mercury light of the $W^{\prime} a y$ lamp ${ }^{1}$ ) is very rich in blue, violet and ultra-violet rays, being more actinic than the electric light from carbon points (Monckhoven, Bullet. soc. franç., I 87 I, p. 2 I0), but it gives hardly any red rays, differing

$\left.{ }^{1}\right)$ In this a strong electric current is sent through a thin thread of freefalling mercury. 
from the light of the roltaic arc between carbon points; further, it is dangerous on account of the mercurial vapours given of:

This light (II'ay's light) affects photographic bromide and iodide of silver plates almost as powerfully as sunlight, and decomposes iodine salts eren more forcibly. The electric incandescent light from a carbon filament is yellower than the voltaic arc, and much less actinic. The light of burning zinc is rich in blue rays, but, as compared with magnesium, poor in violet.

According to Eder, of all known artificial light sources, magnesium powder gives the most powerful chemical effect relatively with the shortest combustion ${ }^{1}$ ).

Thanks to its richness in the more refrangible rays (blue, violet, ultra-violet), magnesium light is very actinic. Unfortunately, this light burns away with extraordinary rapidity, so that it is not possible to keep it continuously powerful, and, further, it gives off too much smoke to allow it to be used for a prolonged period, as is for the most part necessary in phototherapy. For this reason the electric arc and spark lights, which come next to magnesium light in chemical strength, are mainly employed for photo-therapeutic purposes. Of the other light sources, from which light is emitted by increasing heatenergy of the bodies, only the acetylene light in concentrated form has been so far used in photo-therapy. G. Collecille ${ }^{2}$ ) reports that he has had good results with this in cases of sluggish ulcers. The electric incandescent light is used successfully for all such methods of photo-therapeutic treatment as require mainly rays of greater wave-length.

\section{Treatment with Electric Incandescont Light.}

$\$ 64$. The electric incandescent light has a very similar spectrum to that of petroleum and gas incandescent light; it is poor in violet and blue rays and rich in yellow, red and green. Its chemical (photographic) efficacy is therefore slight, but this may,

1) Eder's Ausführl. Haudb. 1. Photogr.. I, I, PP. 455, 456, 457.

2) Gaz. helid. d. médec. O,t. 5. Isior). 
as well as its brightness, be materially increased by strengthening the current, as is proved by the following table $\left(\right.$ Abney $\left.\left.{ }^{1}\right)\right)$ :

\begin{tabular}{c|c|c}
\hline $\begin{array}{c}\text { Number of Grove } \\
\text { Elements }\end{array}$ & $\begin{array}{c}\text { Illuminating Power } \\
\text { in Normal Candles }\end{array}$ & $\begin{array}{c}\text { Photographic } \\
\text { Effect }\end{array}$ \\
\hline & 0.132 & immensurable \\
12 & 0.26 & 0.35 \\
14 & 1.17 & 1.61 \\
16 & 2.44 & 5.83 \\
18 & 3.84 & 12.84 \\
20 & 6.85 & 36.45 \\
22 & 10.38 & 86.60 \\
24 & & \\
\hline
\end{tabular}

Increasing the strength of the electric current not only affects the optical brightness of the electric incandescent light, but also the amount of its blue and violet rays. Strong currents, however, destroy the incandescent lamp very quickly.

In general 380 incandescent lamps of normal power without reflectors have the same chemical effect as natural light at a distance of one mêtre from the object. When it is a question of producing intense chemical light rays (as in the treatment of lupus) the electric incandescent light is of little value, but it is exceedingly useful where longer-ivaved rays (heat rays, red light) are to be applied.

The treatment with electric incandescent light is either general, in the shape of electric incandescent baths, ${ }^{2}$ ) or local.

The first apparatus for these baths was described by J. H. Kellog in $1894^{3}$ ) and shown at the Chicago Exhibition. The first incandescent baths were introduced into Germany by the Chemist Gebhard; many improvements have since then been made in them. They are arranged sometimes for a sitting, sometimes for a recumbent position of the patient, but are always constructed on the same principle. Sometimes they have arrangements attached by means of which the arc-light and incandescent light can be brought to bear on the patient at once.

\footnotetext{
1) From Eder's Ausführl. Handb. d. Photogr., I, I, p. 463.

$\left.{ }^{2}\right)$ For arc-light baths, cf. p. .383 .

3) American Electrotherapeutic Association, Sept., I894.
} 
Modern incandescent baths are made for connection with the street electric wires. They consist of octagonal boxes, supplied with panes of mirrors, opalescent glass plates, etc., and fitted with a door, fastening on the inside and outside, for the entrance of the patient, and with a movable lid above with a hole for the patient's neck. Along the inner walls to to 60 incandescent lamps, each of 16 normal candle power, are arranged; these lamps can be put in or withdrawn from without, and if necessary from within, by means of several switches (in Ricder's apparatus arranged on one switch-board) in series, along vertical, horizontal or spiral lines. The lamps are protected by lattice work. A thermometer for measuring the inside temperature is fixed on the wall. Recently, too, a window has been put in the wall of the chamber, through which the pulse and the course of the perspiration may be observed. By means of other openings with roll-shutters light may be admitted from an arclight reflector. It is as well to have an electric bell fixed inside the chamber.

The patient is seated naked on a stool in the light box; light from without is completely shut off by a towel round the neck opening. A cold bandage, or ice-cap, is applied to the head.

The temperature of a light-bath should not begin with more than $11 O^{\circ}$ to $122 \mathrm{~F}$; only after the way the patient reacts has been carefully noted may the temperature be raised to $156^{\circ}$ to $167^{\circ}$. The bath should last in all 25 to 30 minutes.

It depends on the special case whether the box should be heated beforehand or not. In the case of an otherwise strong, healthy person, where powerful light-action (e.g., in producing perspiration) is desired, he mily be put into a previously heated chamber. The sudden stimulus of the high temperature in such a case has great effect (similar to the stimulus of the switching on and off of the electric currents). But with weakly, ailing persons this plan must not be adopted; the air in the chamber must be heated gradually after the patient is in the bath. The temperature can be raised by adding to the number of lamps and increasing the strength of the current. During the bath the pulse of the patient must be constantly observed 
and care must be taken for the admission of fresh air. After the light-bath follows a bath, douche, or wet pack.

These baths should not be taken daily, but twice, or at most, three times a week.

The action of the incandescent bath is mainly that of radiating heat. IVe are able by these means to apply heat to the deeper tissues; we have seen ( $p .436$ ) that the heat rays easily penetrate even bones (Moeller), and this it is which constitutes the value of this treatment.

The conducted heat from water, vapour, Russian, Turkish, Irish baths, etc., confines its action to the surface of the body.

Yet the light rays may perhaps, side by side with this action of radiant heat, have certain other effects. The light of these incandescent lamps is indeed poor in so-called chemical rays, and consists mainly of red, yellow and green rays. But, as has repeatedly been mentioned above, it is not impossible that red and yellow rays, too, may have certain biological and therapeutic effects.

The most striking effect of this treatment is its action on the secretion of sweat. Whether this is produced by stimulation of the peripheral nerve-endings or by the raising of the patient's temperature by the radiant heat (Strebel) we cannot decide. In any case the accumulation of heat in the body is obviated through the profuse perspiration.

Kellog ${ }^{1}$ ) observed that the amount of sweat excreted in the electric incandescent bath was twice as much for the same time as in the Turkish bath, whilst the average temperature in the electric light-bath was $S_{\mathrm{I}}^{\circ} \mathrm{F}$, and in the Turkish bath $\mathrm{I}_{4} 0^{\circ}$ to $148^{\circ} \mathrm{F}$. Generally, if all $\left(5^{\circ}\right)$ the lamps of the apparatus are lighted, perspiration begins after 6 to 10 minutes at about $95^{\circ} \mathrm{F}$. If the temperature in the chamber is raised to $140^{\circ}-158^{\circ}$, sweat to the amount of a litre and more may be cxcreted in a short time (a quarter to half an hour).

Below and Aufrecht note the very interesting fact that in the perspiration of persons who years before had been treated. by mercurial inunction traces of mercury were found. Katten-

1) Fortschr. d. Hydrotherapie, Festschr., Wien, Leipzig, I897. 
bracker found 0.26 per cent. of sulphur in the sweat of a glass-blower.

Further effects of incandescent light-bath on the circulation, respiration, metabolism and weight have been observed.

It has been asserted that the action of the heart is unchanged or little affected in the incandescent light chamber. Facts do not bear this out.

$M$. Roth ${ }^{1}$ ) observed that a pulse which had before the lightbath been steady at 72 beats, became rapidly quicker after Io minutes in the chamber, giving $S_{4}$ beats; after I 5 minutes, I04; after 20 minutes, I32. At first it remained fairly steady, but later it grew thin, galloping and irregular.

On the whole we may take it that with the temperature in the light-chamber at about $122^{\circ} \mathrm{F}$. the pulse is quicker each minute by about $\mathrm{I} 5$ to 20 beats.

Strasser ${ }^{2}$ ) and Strebel ${ }^{3}$ ) report to the same effect. These changes in the pulse, however, are often no longer so marked in succeeding baths. Increased blood-pressure, with its consequences (congestion, bleeding at the nose, etc.), has been observed as one of the immediate results of this treatment. In most cases, however, after copious perspiration, when the patient has stayed 20 to 25 minutes in the bath, the bloodpressure subsides again.

Winternit ${ }^{*}$ ) noticed in the case of anxmic persons an increase of hæmoglobin and erythroctes after each light-bath.

Respiration is powerfully affected by the bath. . The breathing often becomes twice as rapid after a short stay (a quarter of an hour) in the chamber, and at the same time more shallow and superficial. The temperature of the patient is raised a little by about 2 F.

Kellog found in the case of three persons under observation, who had previously exhaled on the average 3.60 per cent. of carbonic acid in ten minutes, that the amount given off was by the incandescent bath increased in 5 minutes to 4.10

1) Wr. med. Wochenchir., Ision. No. 19.

2) Encyclop. Jahrib., 1000).

3) L. c.

‘) Blätter f. klin. Hydroth. X. Jahr., 1000, II. 6, p. I44. 
per cent., in 20 minutes to 4.20 per cent., and in 30 minutes, 5. I 3 per cent.

Light-baths seem, according to Roth's investigations on the urine and perspiration, to have no specially marked influence on organic decay in the body. But we may assume that the higher body temperature observable after every light-bath furthers the combustion of fat.

The body-weight shows after each bath a more or less marked decrease, according to the amount of perspiration. If the patient satisfies the violent thirst usually but not always felt by drinking freely, the loss in weight is very soon made up; if, however, he has suitable diet, a comparatively short time suffices to bring down his weight considerably.

Occasionally the light-bath brings on nervous symptoms, such as palpitation of the heart, difficulty in breathing, excitability or languor.

After an ordinary light-bath the skin shows signs of simple though marked hyperæmia. IVith the ordinary type of bath, if its temperature be not raised so as to scorch the skin, the hyperæmia disappears very quickly (like all hyperæmia brought about by radiant heat), without leaving behind any erythema or pigmentation.

All we know as to the action of electric incandescent lightbaths points to their use in all our cases where copious perspiration is desired, with the least possible strain on the heart. The light-bath is distinguished as a convenicnt and efficient source of heat by the fact that its temperature can be exactly regulated. It may safely be raised far higher than is possible in vapour, Turkish, Russian and Irish baths, because most patients, under like conditions, perspire sooner and at a lower temperature in the light-bath than in other baths, and have further only the body and not the head exposed to the heat. Further investigations are necessary before we can determine how far we should take account of the deep-seated action of the penetrating long-waved light rays (red and yellow). Ordinary incandescent light-baths, then, are suitable in cases of faulty assimilation (obesity, diabetes, gout), rheumatic affections of joints and muscles, nerve complaints (sciatica, neuralgia, neurasthenia, 
hysteria), anæmia and chlorosis, chronic poisoning (metallic poisoning, syphilis), chronic exudation and effusions, bronchial asthma and bronchitis, dropsy, ascitis and adema after nephritis, hypertrophic and falty heart, eye complaints (keratitis parcnchymatosa, iridocyclitis, choroiditis), and as a tonic and prophylactic against discase.

According to Strasser, ${ }^{1}$ ) this treatment is especially suited to the hydræmic forms of corpulency, particularly the anamic, pasty-looking type found in young people. For plethoric corpulent patients he prefers packs. According to Strebel, the temperature of the light-bath should never exceed I I $3^{\circ} \mathrm{F}$. for this class of patients.

Incandescent light-baths are usually regarded as not in themselves sufficient treatment for diseases due to faulty assimilation. In combination with suitable diet and medicine they give, however, effectirc help in cases where, owing to heart complications, other methods of inducing perspiration cannot be resorted to. For instance, they are said to be very efficacious in gouty complaints in the joints, arterio-sclerosis, etc. They are, however, not always advisable in all such cases; as Strebel remarks with truth, in gouty complaints a thorough transfusion of the tissues with fluid is desirable, and this wholesale process is checked by the great loss of water through perspiration. Strebel, ${ }^{2}$ ) therefore, does not use baths producing perspiration in such cases, but much prefers protracted sun-baths or arc-light baths, in which light rays and not only heat rays come into play.

The same holds good with rheumatic affections of the joints. In muscular rheumatism, however (lumbago, torticollis, ctc.), incandescent light-baths are excellent.

For the various forms of anxmia, incandescent light-baths are probably relatively less effective than sun-baths. The chemical rays in the latter cause lasting hyperamia and pigmentation, and thereby a removil of blood corpuscles from their ordinary course, thus stimulating the system to replace the blood substance and simultaneously to carry on more vigorously the metabolic processes (I.̈̈rce'nlhal, Strebel). IV cannot here

1) Bläter f. klin. Ifyloulher., rnoo, Nix. 4. 5. p. 0.4.

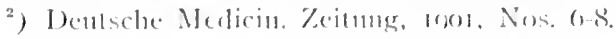


enter into the question of whether the action of light here is helped by the stimulus to the marrow given by the red sun rays penetrating the bone (Strebel).

Incandescent light-baths, producing only temporary hyperxmia of the skin and thereby only temporary depletion of the internal organs, are of less vaiue in these cases where the latter effect is chiefly aimed at, as in cases of congestion of the internal organs or in the venæportæ, difficulty of breathing, valvular heart disease, angina pectoris, etc. In all these cases sun-baths or arc-light baths are more suitable. On the other hand, in dropsy, caused by heart or kidney disease, incandescent lightbaths are a great advance on all previous treatment; they do not, however, much affect the cause of the trouble.

Writers are much divided as to the value of these baths in functional diseases of the nerves. Whilst, for instance, some cannot speak too strongly of their good effects in, $e . g$. , neurasthenia, their soothing influence on general irritability, sleeplessness, singing in the ears, oppression and palpitation of the heart $\left(\right.$ Colombo, $\left.{ }^{1}\right)$ ) others (Strebel) regard a patient suffering from neurasthenia as not a favourable subject for this treatment.

It seems to be much the same with the incandescent lightbath treatment of these nervous conditions as with all the other methods of cure tried for them. One patient is benefited, another is not; much depends on the amount of confidence in the treatment possessed by the patient himself.

Sometimes incandescent light-baths relieve or remove neuralgia and migraine (Strebel, Freund), but by no means always; many cases are quite refractory to the method.

It has been held by many ${ }^{2}$ ) that these baths have a specific influence on tuberculosis, scrofula and other infectious diseases; here bactericidal action of light being assumed.

As we have more than once stated, such an assumption is quite mistaken, for it is well known that incandescent lamps give out very few of the chemically active rays, which are more bactericidal in their action than other light rays, and such chemical

$\left.{ }^{1}\right)$ Revue de Thérapie physique, I90I.

2) Ruhcmann, Apery, Kattcnbracker, Candler, Minin, Clcaves, Below, and others, quoted by Boeder. 
rays as are completely absorbed on their passage through the glass walls of the lamp and the tissues of the body. Obviously then, assuming the light source to be sufficiently rich in actinic rays and sufficiently powerful, we can only consider its bactericidal action as having a very superficial effect.

It may be urged that in the treatment of infectious diseases it is not the actinic rays which matter, but more penetrating longwaved yellow, red and heat rays. Experiments with bacteria cultures show that these rays have no specially pronounced bactericidal properties. These experiments, it is said again, need not be regarded as conclusive; for the rays, by penetrating the body, set up hyperæmia in the infected organs, which acts as a curative.

There is certainly something to be said for this theory, maintained by Strebel amongst others. As a matter of fact, various observers have reported a favourable action of light on infected animals (Kondratiew, De Renzi, Kutschuk, Aufrecht ${ }^{1}$ )). Yet Boeder's ${ }^{2}$ ) experiments show no specific, and specially no specifically bactericidal effect of light rays on infected animals.

Drigalsky") even observed that mice inoculated with splenic fever or other bacteria died more quickly in the lightbath than control-animals, even though they were kept only a short time in the bath. He holds that the very copious perspiration brought on by the heat rays weakens the resisting powers of the body, and calls attention to the danger to many feeble patients, $e$. g., tuberculous persons, from such haphazard treatment.

The whole question calls for more detailed investigation, but so far as our present knowledge goes, we may say that any favourable effect of light treatment on infective diseases of the internal organs must be explained by its indirect action in stimulating metabolism and the action of the heart, and in increasing phagocytosis and perspiration, etc.

Incandescent light-baths are unsuitable in cases of extreme

1) Quoted by Bocder.

2) Arl, atus dem Kaiserl, Fesmolheitsamte, 1900.

$\left.{ }^{3}\right)$ Centralbl. 1. Bakteriolngic, Vol. XXV, II, Nos. 22, 23. 
weakness and arteriosclerosis, for patients with organic heart disease and marked congestive symptoms, in phthisis with night sweats, in hæmorrhagic cases (hæmoptysis, hæmatemesis, apoplexy), and in all cases where no perspiration results from the carlier baths (Ricder, Strebel).

Partial light-baths are a convenient modification of the general incandescent light-bath. For them smaller boxes are used, with reflecting surfaces and incandescent lamps fixed to the sides, and with suitable openings for the insertion of a limb; or else one or more lamps are used, provided with a reflector (Trouvé, Foveau de Courmelles, Laquer).

The apparatus (similar in principle to the above) which the author himself uses for partial light-baths ${ }^{1}$ ) (treatment with radiant heat) consists of two incandescent lamps of roo N. candle-power, each with its own cut-out switch, which are fixed in a parabolic metal reflector. The conducting wires are carried to a rheostat, the lever of which can be so adjusted as to regulate the brightness of the lamps. On its open side the reflector has slots for coloured glass filters or blinds. This apparatus may be either fixed to a stand, or it may be attached to other apparatus. In treating the extremities a drum-shaped box is used, an opening being cut out in its casing corresponding to the exact size of the reflector, which can be fitted in. The drum with its reflector is placed on two supports and fixed with straps; in this position it can be turned so that the rays fall in turn on all parts of the limb under treatment. Asbestos netting is stretched inside the drum at some distance from the reflector to prevent the exposed limb from coming too near the lamps.

Another apparatus for treating the trunk or single parts of it consists of a trough-shaped framework covered with thick heat-retaining material. Curtains of the same material with tapes cover the two ends. The trough has a rectangular opening at the top into which the reflector fits. Below this opening, but attached to two rollers on the outside of the trough, is a thick, close curtain of asbestos, consisting of two nets, one coarse and the other fine-meshed, put one above the other. By draw-

1) This was made by L. Schulmeisler, Vienna, IX. 
ing the one or the other part of the curtain by means of the rollers the irradiation may be varied in strength.

Both these apparatus are fitted with thermometers. The action of such partial light-baths is precisely similar to that of the general incandescent light-baths, but in some respects the former are much supcrior. They are useful for the treatment of bed-ridden patients who cannot be mored to the larger bath. Then they are more suitable for many cases, because a far higher temperature can safely be used with them. A temperature of over $122^{\circ} \mathrm{F}$. is unpleasant in the general bath, but with these apparatus, especially at a second and a third bath, temperatures of $212^{\circ} \mathrm{F}$. and more are borne without inconvenience. When parts only of the body are exposed to the rays, perspiration does not result except at a high temperature (from $194^{\circ} \mathrm{F}$. to $22 \mathrm{I}^{\circ} \mathrm{F}$.), but it is then usually very profuse.

It is interesting to note that irradiation of the extremitics raises the temperature of the whole body. The writer has made repeated observations on this point with patients under treatment for the joints of the hands and feet. Half or threequarters of an hour after the treatment had begun, when the temperature inside the apparatus was about $176^{\circ} \mathrm{F}$., the patients reported themselves as getting comfortably warm over the whole body. At the same time the face became a little flushed, and the temperature, on being again carefully taken, showed a rise of from $3^{\circ}$ to $10 \mathrm{~F}$. The pulse showed on the whole no change; being rarely slightly accelerated. The respiration, too, was unaltered.

The rising temperature may be explained by: I, Heating of the body by heat passing from the apparatus into the tissues; 2, higher temperature of the limb, carricd over the whole body by the heated blood; 3 , sympathetic rise in temperature, through the nervous system. Considering the comparatively rapid rise in temperature and the size of the parts under treatment, together with their slight heat-conducting power, we may probably disregard the first explanation. Which of the other two is the more effective we can hardly for the present determine; possibly each has an equal share in the result.

'The method of this treatment is as follows: If a powerful 
stimulus is to be administered to the affected limb, it is put into an apparatus heated beforehand to about $122^{\circ} \mathrm{F}$.; if, however, a longer irradiation be desired, the current is only turned on after the apparatus is applied to the patient. Various methods may be adopted to make prolonged application of the treatment bearable. The current may be weakened by means of the rheostat when the irradiation becomes unpleasant; or one or both lamps may be switched off for a longer or shorter time; or radiant heat is dispensed with altogether for a time and only the thermometric heat of the box utilised (this is done by simply placing a dry linen compress on that part where the heat is felt unpleasantly, to keep off the direct rays). By these means the treatment may be variously modified. As soon as the desired result, $e . g$., perspiration, is attained, treatment is suspended, the part is thoroughly rubbed down, and either active, passive and resistance movements or massage applied. One should not leave the affected limb in the apparatus after the heat has been turned off and allow it to cool down gradually. The author's experience has proved this procedure to be most harmful to the effect of the treatment.

For the treatment of superficial skin affections, neuralgia, myalgia, etc., we use the simple radiant apparatus with or without coloured filters. We may take the appearance of marked hyperæmia of the skin and possibly slight perspiration as indications for suspending the treatment.

The symptoms indicated for this treatment are precisely the same as those for the general incandescent light-baths. This method, however, may be said to be more universal in its application, inasmuch as it taxes the heart to a much less degree. We have attained excellent results with this method when systematically carried out, both in relieving painful affections of the joints and muscles, and in quickening the re-absorption of serous exudations and effusions into the joints, and in the case of dropsical accumulations. Rheumatic pains are usually immediately reliered. Patients who could hardly stand before the treatment are able to walk without pain after it. But the effect is not lasting; the pains are felt again after one to three hours, and a complete cure is only effected when the treatment 
has been prolonged and combined with suitable medicinal and mechano-therapeutic measures.

It is doubtful whether radiant heat can do much for neuralgia. Patients indeed, in many cases of trigennial neuralgia, sciatica, etc., professed that they felt relief, especially at times from treatment with rays filtered through blue glass. But in the case of other and unhappily of most patients, their suffering was not in the least alleviated by the treatment, and one cannot help thinking that in the successful cases something was due to the effect of suggestion.

On the other hand, it appears that irradation by powerful incandescent lamps has a strikingly good effect on superficial inflammation and suppuration. The author" treated at times with the unfiltered light of an incandescent light apparatus, at other times using a red glass filter (spectroscopically examined), several long-standing cases of acne vulgaris, 2 cases of ulcerating Roentgen-dermatitis, which Prof. Ehrmann had passed over to him, ${ }^{1}$ ) an ulcerating lupus, and a scrofulous abscess which had been left on the neck after ulceration of the lymphatic glands, and a syphilitic ulcer. The disease was checked or lessened in all these cases except the last-named, which was untouched, and the scrofuloderma, which had finally to be treated surgically in spite of improvement at first, because of the ulceration of the neighbouring gland. In the other cases, the inflammatory induration round the acne nodules was lessened, fewer pustules were formed, and the suppuration diminished in the small abscesses, whilst healthy granulation and surface-healing were visibly furthered. The irradiation lasted each time at least half an hour, with the diseased part as close as possible to the light source. In these cases, of course, no other treatment was adopted, the sore places being simply dressed with vaseline after each sitting.

It has been already observed that we are still waiting for a complete explanation of the good results of this treatment. The character of the light source being what it is, we cannot look to any action of chemical rays; we can only take into

$\left.{ }^{1}\right)$ Ges. (1. Aerzte in Wien, licb. 2Ist, I902 
account the rays of greater wave-length, which are produced fairly abundantly, and their power of penetrating the deeper layers of the skin. In this respect, remembering the wellknown good effect of high temperatures on ulcerating processes we may probably assume that the heat rays are beneficial in this way; it may also be that the irritation of the deep-penetrating long-waved rays may stimulate healing and the formation of connective and scar-tissues, just as chemical irritants do when applicd to sluggish ulcers ${ }^{1}$ ) for the stimulation of granulationtissue formation.

The effect may perhaps be made more intense by substituting for the ordinary incandescent lamps the Auer osmium lanıs, which have a stronger illuminating power.

The literature on this subject, especially the Russian, describes a number of results which, even allowing for the enthusiasm of some of the writers, testify to the value of local treatment with electric incandescent light. Thus v. Stcin,") Gatschkowosky,") Minin, $\left.{ }^{4}\right)$ Makawejew, $\left.{ }^{5}\right)$ Kcssler, ${ }^{6}$ ) Turner, $\left.{ }^{7}\right)$ Upensky, ${ }^{8}$ ) and others (all quoted by Divorel $\left.\approx k y,{ }^{9}\right)$ ) speak of its good effect in certain painful affections (lumbago, rheumatism, cephalagia, odontalgia, pleurisy, pains in the chest after influenza, etc.). This treatment was valuable also in the case of exudations (rheumatism, pleurisy, peritonitis, gonorrheal inflammations) and in subcutaneous, subperiosteal and retinal effusions of blood (Tichomi-

$\left.{ }^{1}\right)$ Even in Finsen's are-light treatment, in which recently no blue filters have been used, a curative action of the long-wave rays nay be possible under certain circtmistances. This is not especially the case when the compressing apparatis is not nsed; the blood not being removed by pressure, the actinic short-waved rays are considerably checked in their passage into the tissues, while the long-waved rays pass through comparatively freely.

2) Medizinskoje Obosrenie, i\&go, Vol. XXXIII, p. i 56.

3) Russkaja Nedizina, I892.

2) Wratsch, I899, Nos. 22, 38, 47; 1900, Nos. II, 27.

") Ilid. Igoo, No. 8.

$\left.{ }^{6}\right)$ Il,id. Inoo, No. I4.

$\left.{ }^{7}\right)$ Ibid. Inoo, No. 36 .

$\left.{ }^{8}\right)$ Russky Mediz. Westnik, 1900, No. 19.

") Zeitschr, f. diät. und phys. Thérapie, Vol. V, p. 3. 
row ${ }^{1}$ ) and as a healing factor in skin affections (ulcerations, eczema, lupus, lepra) and in venereal and syphilitic affections.

Minin, Turner and others claim to have cured by irradiation from incandescent lamps ( 6 to 50 candlepower) not only local tuberculosis of the bone, but eren general symptoms of tuberculosis ( $c . g$. , night sweats, etc.), tabes, scurry, sickness. Kaiser," ) too, reported similar results. Possibly, howerer, one may be allowed a little scepticism as to these marvellous results, sceing that they are reported as being obtained with treatment for Io to 15 minutes at a time, repeated every 2 to 3 days, one lamp only being used.

\section{The Electric Arc-Light.}

$\S 65$. The construction of arc-lamps is based on Davy's discovery, in $182 \mathrm{I}$, of the galvanic arc-light. If the pole of a powerful galvanic battery or a dynamo be connected with two carbon rods, a current will be established at once on their contact. If now they are drawn apart to a distance of several millimetres from each other the current will not be broken, but a luminous are will be formed between the carbon points, along which the current passes. At the same time, in consequence of the heat developed, the carbon points will become highly incandescent, hence their use for purposes of illumination. The arc of light is composed of gaseous carbon and of the vapours of metals retained in the carbon, as the spectrum of the arc shows. In lamps with alternating currents the carbons are consumed equally, and hence are approximately equal in brilliancy. giving off, therefore, a nearly equal amount of light on both sides (see Fig. 94) where the length of the radii bounded by the curved lines corresponds with the strength of light radiated in the given directions. In lamps with a continuous current the positive carbon glows more intensely than the negative car-

1) Wratsch, 1900, No. II.

2) K. k. Gesellschaft d. Acrote in Wien, Fel. 7, 1902. 
bons, being consumed about twice as fast (for the resulting light radiation (see Fig. 95). The carbon rods in lamps used for lighting purposes must be kept at a constant distance from each other whilst the lamp is burning, in order that the light

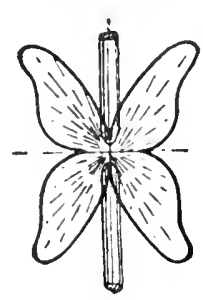

FIG. 94.

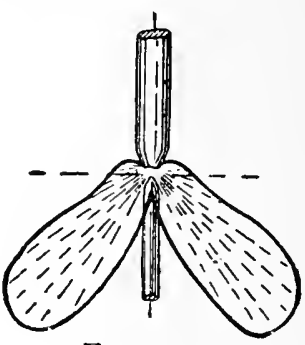

FIG. 95.

point may be kept at the same spot. Generally the carbon points, as they gradually burn away, are adjusted by automatic regulators.

In electric arc-lamps the regulators are almost always worked by the magnetic action set up by the current of the lamp itself. Electric arc-lamps are known as main-current, shunt-wound and differential arc lamps, according to the winding of the coil in the regulator.

Lamps with hand regulators are sometimes used in phototherapy. The light-point is further maintained in an unchanged position by taking the rate of combustion of each of the carbon rods into account when selecting their size. For a continuous current cored carbons are usually taken as the upper or positive ones, homogeneous carbons for the lower or negative ones. As the positive carbon is consumed about twice as quickly as the negative, if both carbons are to burn for the same time, the positive carbon must either be longer or, as is usual, of greater diameter. With an alternating current cored carbons are used both above and below, as they burn away nearly at the same rate. On the whole, thinner rods burn more steadily and give better light than thicker ones, but the latter are often chosen, and are used as thick as possible because of the longer time they last.

The brightness of the electric light, of course, depends considerably on the strength of the current. The following table 
(after $S . \approx$. Gaisberg ${ }^{1}$ )) gives the average illuminating power furnished by open lamps burning without open shades, with currents of different strength and suitable voltage. The table gives further the approximate right diameter of the carbon rods (for rods measuring together foo to $500 \mathrm{~mm}$., in length) if they are to burn fairly evenly.

\begin{tabular}{r|c|c|c|c|c|c|c|c|}
\hline \multicolumn{2}{|c|}{ Continums Current } \\
\hline
\end{tabular}

The roltage given in the above table is deviated from in certain kinds of lamps for which a different material is taken for the carbon rods and a smaller light arc.

Graetz assumes for his average that an arc-lamp with continuous current gives light of about 100 normal candle-power for each amperre consumed.

Palaz gives the following table ") for various strength of current. (See next page.)

The voltage necessary for establishing the are is dependent, with both continuous and alternating current, on the length of the arc, the kind of carbon used, the strength of the current and (with alternating current only) the form of the curve. For continuous current, with current strength of 6 to 14 amperes and

1) Ta-chenluch fïr .Nonteure éleklriucher Belenchtungsanlagen. Mïnchen, Letprig. 1901. 1). 102.

*) R. Ruhlmann, Cirmelzugge der Cileichetrontecknik, Lejpzig, 1901, p. 59 


\begin{tabular}{c|c|c}
\hline $\begin{array}{c}\text { Strength of } \\
\text { Current in } \\
\text { Amperes }\end{array}$ & $\begin{array}{c}\text { Average Spherical } \\
\text { Strength of Light } \\
\text { in Decimal } \\
\text { Candles }\end{array}$ & $\begin{array}{c}\text { Consumption of } \\
\text { Watts for an } \\
\text { Average Spherical } \\
\text { Decimal Candie }\end{array}$ \\
\cline { 2 - 3 }+ & 302 & 0.66 \\
6 & +70 & 0.64 \\
$S$ & 650 & 0.62 \\
10 & 8.40 & 0.60 \\
12 & $1,0.42$ & 0.58 \\
14 & 1,255 & 0.56 \\
I6 & 1,477 & 0.54 \\
20 & 1,960 & 0.51 \\
30 & 3,360 & 0.45 \\
\hline
\end{tabular}

length of arc, 2 to $4 \mathrm{~mm}$. (the more usual numbers), the terminal voltage would be 40 to 50 volts ${ }^{1}$ ).

The illuminating power of the lamp depends, the strength of current remaining the same, in large measure on the length of the light arc. If the distance between the carbon points is too great the light flickers, becomes unsteady, and often goes out with a loud noise. The length of arc must always be proportional to the strength of the current, the number of ampères which pass through the lamp. Graetz puts the right length of arc for lamps supplied with 5 to 6 ampères current at I to $2 \mathrm{~mm}$., for $S$ to 10 ampères at $3 \mathrm{~mm}$., and for powerful lamps with 20 or more ampères strength at 4 to $5 \mathrm{~mm}$. The arc-light may be examined through smoked glass, or superimposed red and green glass.

As is evident from Fig. 95, with a continuous current lamp the measurable strength of light varies very considerably according to the direction in which the light falls on the eye or the photometre. The greatest illuminating power is radiated at an angle of $40^{\circ}$ to $60^{\circ}$ below the horizontal.

In $H$. $V^{\prime}$. Vogel's ${ }^{2}$ ) experiments with a Siemens' lamp, at a nearly horizontal position $\left(\mathrm{I}^{\circ}\right.$, with a current of $\delta 3 / 4$ to

1) J. Herzog and C. P. Feldmann, Handb. d. élektr. Beleuchtung, Berlin and Nünchen, I898, p. I7.

2) Das licht im Dienste der Plbotographie, Berlin, I894, p. I2.3. 
9 amperes and the use of nearly I horse-power), the light was 394 amyl candle-power; at 25,2 below the horizontal $81 / 2$ to $S .34$ ampères gave $1043 \cdot 7+$ candle-power.

Thus, if the illuminating power of an electric lamp of a certain strength of current be giren, it is necessary to indicate also the direction of the radiation.

Lamps with alternating current give approximately the same amount of light above and below. A reflector is usually placed immediately above the are to utilise the light radiated upwards, and in this case the brightness of the alternating lamp is cqual to that of a lamp with continuous current; as regards the actinic rays, however, there is considerable difference between the two lamps. Alternating lamps are seldom used with a less than 4 ampères current, because of their slight illuminating power.

The brightness of the different parts of the spectrum varies quantitatively (cf. scale on p. 450 ) in the electric arc-light as in sunlight. This scale shows that the blue rays preponderate greatly over the red and yellow ones in electric light.

Abney ${ }^{1}$ ) showed that as the rotary morement of the dynamo-electric machine ${ }^{2}$ ) increased in speed, not only does the light as a whole become brighter, but the intensity of the more highly refrangible rays is increased disproportionately to that of the less refrangible rays.

This has been confirmed recently by Absolon Larsen's reports ${ }^{3}$ ).

After direct sunlight and magnesium light, no light is so effective chemically as an electric arc-light. The light of powerful arc-lamps acts on photographic bromide or iodide of silver plates almost as powerfully as sunlight, and decomposes iodine salts even more energetically ${ }^{4}$ ). The following figures of

1) Eder's ansfiibrliches Hatulluth, I, 1, 1). 466 .

2) On which the strength of current or alectromotor puwer dependscf. 1). 90 .

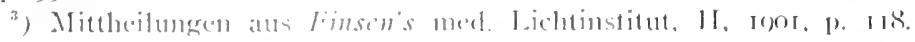

") S. Leeds, Chem, Newn, Vol, XLII, 1. 147. Lider's IIandbuch, Vol, I, 1. 360. 
$E d c r^{\prime} s^{1}$ ) give its strength chemically as compared with that of sunlight.

Photographs of drawings, taken for purposes of comparison, were exposed: 6 minutes in electric arc-light of $\mathrm{I}, 800$ candlepower at a distance of half a metre, a white reflector being used; $2 \mathrm{~T} / 2$ minutes in diffused daylight; 40 seconds in direct sunlight.

Prof. Iogel gives another table with reference to the photographing of paintings. He arranges the different kinds of light, as regards their efficiency for photographic purposes, as follows:

I. Sunlight is 8 to 14 times more powerful than diffused daylight with a clear sky;

2. Electric arc-light (6 lamps of 7,000 candle-power altogether, distance $I \frac{1}{2}$ metres, with white reflector) is 4 times as powerful as daylight in November with a clear sky;

3. Then comes clear sky with white clouds;

4. Blue sky;

5. Dull sky (light often only one-tenth as powerful as white, cloudless sky).

We need hardly point out how fundamentally important for the various forms of phototherapy are these thorough photometric investigations of Eder, Vogel, and others.

Starting from the assumption that the curative results of arc-light treatment are due mainly to the chemical, and particularly to the ultra-violet, rays, attempts have recently been made to construct sources of light which should produce these rays in special abundance. This end was attained by taking as electrode-material, instead of carbon, metals and substances not readily fusible (lime, silicic acid, zircon, thorium, magnesium compounds). Finsen hollowed out the positive electrode and filled it with a mixture of graphite and calcinea lime. Strebel, too, at the Dermatological Congress in Breslau in $1901,{ }^{2}$ ) showed useful apparatus for photo-therapeutic purposes. Great

$\left.{ }^{1}\right)$ Photogr. Mitth., Vol. XX, p. 39, quoted from Eder's Handbuch, p. 465.

2) According to Drossbach, Secchi used iron electrode arc-lamps as long ago as 1873 . 
difficulty was found at first in using pure metals for electrodes, because the metal at the ends of the electrodes is apt to melt and drip, forming a sort of bridge between the electrodes and extinguishing the light. This difficulty was met by Sophus Bang's iron-electrode lamp with water-cooling arrangement, ${ }^{1}$; invented in 1901 (Fig. 96). This lamp is very handy and cheap, and requires only a weak current to produce a noticeable chemical effect. The principle of its arrangement is that the conducting wires $H H$ are carried to two electrode holders $F F$, fastened to the springs $K K$; changeable thimble-shaped iron electrodes $E E$ are screwed on to $F F$. The electrodes are hollow, and connected by thin pipes $G$, running along the inside of the tube that serves as handle, with the water apparatus. The lamp is lighted by pressing the button $A$ to let the electrodes touch and then releasing the button at once. It is blown out like any ordinary lamp. By the screw $B$ the distance between the electrodes can be altered and the voltage kept sufficiently constant. If the lamp burns with 8 ampères and 40 volts an iron electrode lasts on the average four to six hours.

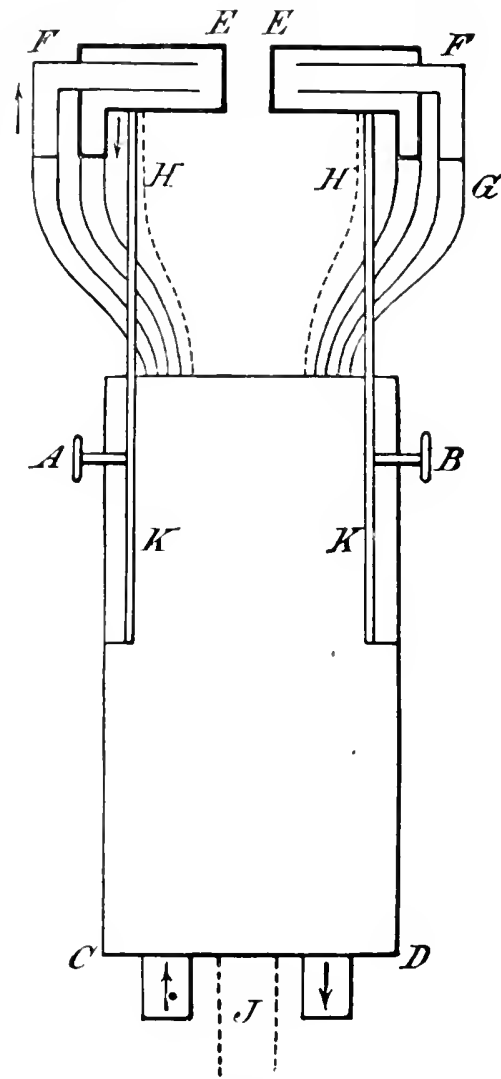

Fis. 96.-Iron-electrode lamp, by Dr.

S. Bang. (From Zeitschr., etc.) The lamp is provided with one of finsen's quartz compression apparatus; this is fixed on the tube $I B C D$ in such a way as to be easily removable, either for cleaning or for being exchanged

1) According to Bang. 11. Sirmens ats far back as 1879 used metal electrodes cooled ly water for are-lanims. 
for pressure apparatus of difierent shape and size. When using this apparatus, one has to bear in mind that if the water-cooling apparatus is not effective little drops of molten iron may form on the electrodes. These drops must be removed with a little

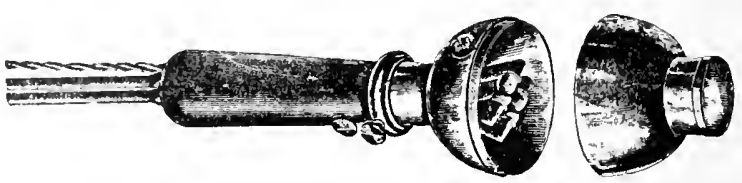

FIG. 97--Iron arc-lamp, by Reiniger, etc.

wooden stick or they may fall down and damage the compressor or hurt the patient. The Kjeldsen arc-lamp "Dermo" is constructed on the same lines. André and Broca, Chatin, too, use arc-lamps having a carbon with metal "core" for positive electrode.

Reiniger, Gebbert \& Schall's iron arc-lamps (Figs. 97 and 98) have a convenient improvement on the Bang lamp. In an

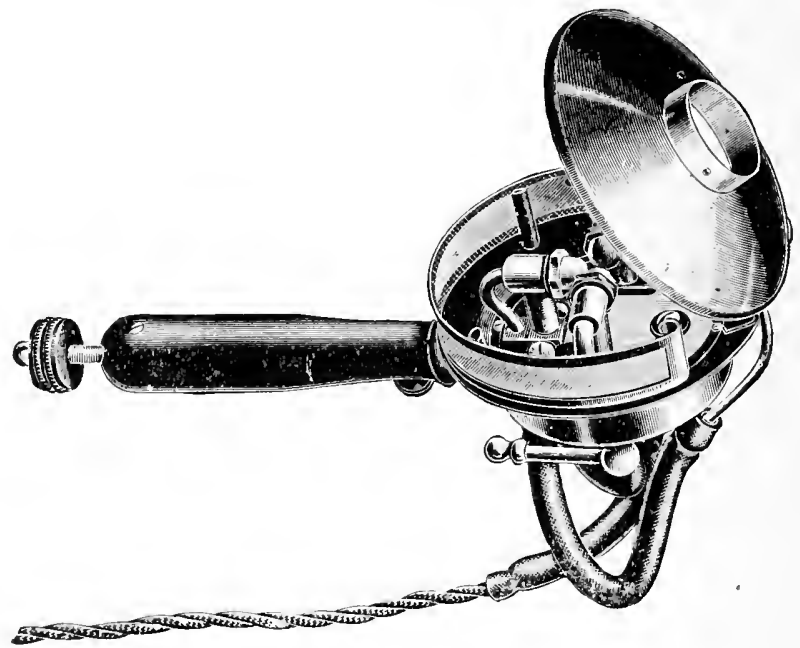

FIG. 98.-Iron arc-lamp by Reiniger, Gebbert \& Schall.

arrangement similar to Bang's, two light arcs are used, put one behind the other. This not only increases greatly the illuminating power of the apparatus but also works the lamp much more economically, for almost the entire energy of the total 
voltage of 1 I o volts is converted into two light arcs of 45 volts each, whilst in lamps with only one arc of 45 volts more than half the energy used is expended to no purpose in orercoming resistance. The greater intensity of the light enables one to diminish the duration of the sitting.

E. Rasch ${ }^{1}$ ) lately described an arc-lamp in which oxides of the metallic earths, here called "electrolyte elcctrodes," are used as rods. To produce the are these rods have to be made conductive by heating, which is done by means of an auxiliary arc between carbon electrodes, arranged close to the others. Arclamps of this kind are said to be highly efficacious, more so, according to the inventor, than any other arc-light; the light is white.

The new arc-lamp of Bremer ${ }^{2}$ ) is constructed on another plan. The poles contain a mixture of carbon and fluor-spar; it is not necessary to heat them. The usual arrangement of the rods one above the other is departed from in this lamp; they are arranged side by side at an angle with each other; the arc of light directed horizontally betwcen the carbon points is turned downwards by the current, so that it spreads out like a fan. This lamp, too, is highly efficacious; its light looks yellow.

Wr. Vedding's") experiments with two Bremer continuous-current lamps, of 12 and 60 amperes, with 44 and 60 volts respectively, the positive carbons of which were made of a calcium compound, show that the smaller lamp had a maximum illuminating power of 6,400 candles, that the illuminating power was constant below the angles $45^{\circ}$ to $90^{\circ}$ to the horizontal, and only then decreased towards the horizontal to I, Ooo candles. The scond powerful arc-lamp had 4 arc-lights arranged on the occasion of the measurcment in two rows. This lamp gave its maximum of $8,3,000$ candles helow $37^{\circ}$. The experiment showed further that with this new lamp three times as much light was obtained as in other

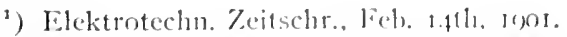

2) Elektrotechn. Zeitschr.. April dth, Iyor.

$\left.{ }^{3}\right)$ Ibid. I900, part 27.
} 
arc-lamps with the same cxpenditure of energy. It shewed advantages over the older systems even when alternating currents were used.

In both Rasch's and Bremer's lamp, no doubt, side by side with the effect of the glowing electrode tips, a considerable part is played by the gaseous metal compounds glowing in the arc. The noxious vapours given off by these lamps may probably stand in the way of their being used for photo-therapeutic purposes.

The arc-light in a non-concentrated form is used for electric light-baths, in a concentrated form for the treatment of localised skin complaints.

\section{The Therapentic Use of Non-concentrated Arc-light.}

$\S 66$. Arc-light baths form a quite distinct class of electric light baths. Whilst in the incandescent light-baths the long-waved rays (heat rays, reddish yellow) come chiefly into play, in the arc-light baths the whole body is exposed mainly to the chemical light rays. The heating action of this light is quite subordinate here to its chemical action. Superior to them both is the natural bath in sunlight, where both the heat rays and the actinic rays act powerfully on the body. It is necessary, therefore, to take account of the special characteristics of the various light-baths when judging which symptoms point to any particular method.

Arc-light baths are taken either in the open or in a chamber. Finsen ${ }^{1}$ ) administers them in the open as follows: Two huge arc-lamps, of I oo ampères each, hang in the middle of a circular room a few metres from the ground. Compartments are formed round the room by a number of radial partitions, and in them are inclined couches, turned towards the light, on which the patient lies naked. The temperature in these light-baths is so low that artificial heat has to be applied to keep the patient from feeling cold, and yet the chemical action on the skin is as powerful as in strong sunlight. They produce a pleasant, slightly prickly, and feebly warming sensation in the skin. Some persons after only ten minutes show distinct erythema, whilst

$\left.{ }^{1}\right)$ Uebur d. Bedeutg. d. chem. Lichstr., p. 7 I. 
others are able to stay in the baths for hours without more than very slight erythema appearing.

The arc-light "enclosed baths" of Kellog ${ }^{1}$ ) are quadrangular boxes in which the patient sits with his head outside, as in the incandescent light-baths. A powerful arc-lamp is fitted in each corner, capable of being turned both upwards and downwards, so that the rays can be directed on the patient in any position. Arrangements are made for the introduction of coloured glass filters to obtain at will either red light or blue light, according as the heat rays or chemical rays are to te excluded.

Strebel ${ }^{2}$ ) brings forward the well-founded objection to the use of arc-lamps for enclosed bath, that in order to produce a chemical effect about equal in degree to that of diffused daylight they develop such tremendous heat in the box that the patient can only stay in the bath for a limited time, however good the ventilation may be.

The use of reflectors to intensify the light has been the objection that burn-blisters are liable to be formed on those parts of the skin on which the concentrated cone of light and heat falls. Another drawback to this use of electric arc-light is the formation of noxious gaseous products (acetylene and other compounds of carbon and hydrogen), which are not only absorbed by the skin, but also, escaping at the neck opening, may penetrate the patient's air-passages (Strebel).

From these considerations it is evident that the Finsen method is the more suitable for electric arc-light baths. The chemical effect of this arc-light treatment might be considerably heightened by the use of parabolic reflectors, which would reflect the otherwise wasted light which is radiated upwards. The irradiation thus utilized has considerably more actinic power than concentrated light, without the disadvantages we have mentioned. According to $I I$. $I V$. Vogel, ${ }^{3}$ ) a Scluckert reflector (in which not only the parabolic reflector but also a

1) Blätter f. Klin. Hydrotherapic, X. Jahrg., P. I4.

$\left.{ }^{2}\right)$ Die Verwendung des Lichtes in der Therapie, München, 1002, p. 20.

$\left.{ }^{3}\right)$ H. W. Vogel, Las Licht in Dienste der Photographice, Berlin s894, p. 123. 
system of cylindrical lenses are used, with the result that the reflected rays are even divergent) with 60 ampères strength of current produced a black photographic reproduction in I 5 seconds, whilst daylight in November required $2 \frac{1}{2}$ to 3 minutes ${ }^{1}$ ).

It is necessary with every kind of arc-light treatment to bear in mind the specifically physiological effect of the so-called chemical light rays (the peculiar and lasting irritation of the skin [Finsen, Möller], the action on the blood [Finsen and others, cf. p. 412], also the as yet uncertain influence on the nervous system) and to judge accordingly the symptoms pointing to its use.

Arc-light baths are often combined with incandescent light by introducing incandescent lamps on the sides of the bathing box; by this means the effects of the two kinds of light are united in one apparatus.

We have as yet no precise, full and suggestive accounts of the therapeutic results of this light-bath method, but we may assume that light by strengthening the organism and furthering the process of phagocytosis in the elimination of microorganisms and toxins will give us a remedy against many diseases.

Metal electrode lamps have been recommended for the treatment of circumscribed skin affections. They are used similarly to the light concentrating apparatus.

\section{Treatment with Concentrated Arc-light.}

$\$ 67$. The idea of using concentrated electric arc-light in the treatment of skin diseases was brought into practice by Niels Finsen. His apparatus in the first place makes the divergent rays from the arc parallel (just in the same way as did the appliance used by $W$ idmark in his experiments). These parallel

$\left.{ }^{1}\right)$ Strehel in his recent interesting work, "Dic Verwendung des Lichtes in der Therapic" (München, Seitz. u. Schauer, I902), states that he has been led by the same conclusion of which Foz'an do Courmelles, the present writer, and many other colleagues, arrived quite independently, to use refracted arclight in place of a sun-bath in his practice, and has done so with good results. 
rays Finsen collects again into a cone, the apex of which falls on the part of the skin to be treated (Fig. 99).

In physical and medical laboratories precisely similar apparatus has long been in use which made the divergent rays from the electric arc first parallel and then again convergent, and filtered the heat rays through layers of water. Thus, in the

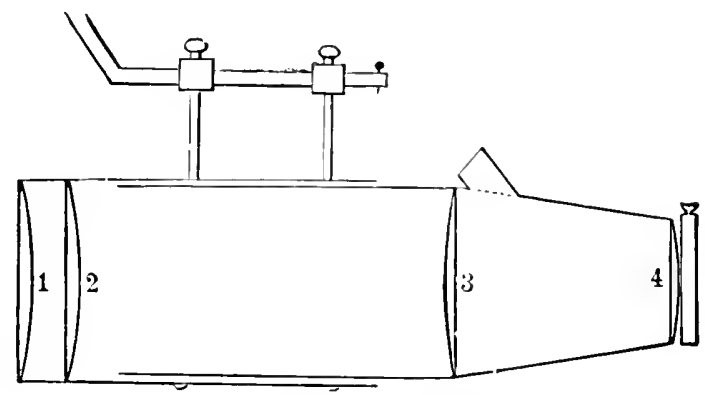

FIG. 99.-Finsen's light apparatus.

Vienna Institute for experimental pathology (Prof. Paltauf, and formerly Prof. Stricker), an apparatus constructed precisely like Finsen's has been used for many years to project light.

Finsen uses as his light source an arc-lamp of 80 ampères with continuous current only; he computes its light intensity at 40,000 candles.

The roltage of powerful continuous current lamps of this kind is at the terminals of the lamp hardly more than half the roltage of the ordinary current in the street. If, therefore, the lamp were to be attached directly to a supply of 110 and more volts over $50 \%$ of the electric energy would be lost in the resistance coils ${ }^{1}$ ). The setting up of two lamps of this kind, one behind the other, is in practice inadvisable on account of their unstealy action. Neither does the supply allow of such strong currents being taken from

3) Naturally a supply of this kind wothld, on acenunt of the large amotunt of energy combuncel. Be much dearer than a current of only the required voltage. 
it, on account of the strain involved on single cables and the resulting fluctuations in voltage for other neighbouring consumers connected with the same street cable. For this reason, when such powerful arc-lamps are used with a street-voltage of more than 65 volts, a transformer has to be employed to transmute driving voltage of the electrical supply to the required lamp roltage, including the steadying resistance.

Alternating cuirrent lamps of the same great intensity of light act unsteadily, and it is difficult to concentrate the light with them; hence for these purposes alternating current lamps are not used.

The connecting apparatus (Fig. 99) consists of two metal cylinders telescoped one in the other, each containing two planoconvex lenses. The two parts of the apparatus may be separated by means of a rack-and-pinion movement. The various lenses are in Finsen's apparatus so proportioned as to size that lenses $\mathrm{I}$ and 2 have together a focal distance of $\mathrm{I} 2 \mathrm{~cm}$., lenses 3 and 4 together a focal distance of $\mathrm{Io} \mathrm{cm}$.

The two lenses, I and 2, nearest to the source of light collect the diverging rays of the arc-lamp into a bundle of parallel rays. Lenses 3 and 4 make these parallel rays converge on the surface to be irradiated. Between lenses 3 and $I$ is a water chamber ${ }^{1}$ ) for the purpose of absorbing the heat rays. As the water in this chamber becomes heated when the lamp is in use, means are taken to keep it constantly replaced by cool water. This is done by a system of tubes (one part bringing the cold, the other carrying off the heated water) in connection with the water supply.

At first Finsen used glass lenses and distilled water, and fixed light filters consisting of glasses filled with a solution of sulphate of copper at the end of the aparatus turned towards the patient. Now, in order to lessen the risk of absorption for the ultra-violet rays, no colour filters are used, and instead of

1) According to Reyn's suggestion, the water chamber is now fixed between the lenses turned towards the lamp. so that they may be cooled and their constant cracking avoided (Quoted A. Huber. Der heutige Stand der Finsen-Therapie, Wiener Med. Wochenschr., I902, No. $20 \mathrm{ff.}$ ) 
the strongly absorbing glass lenses only quartz lenses. A considerable part, however, of the effective energy radiated from the arc of light is still lost on passing through the layer of undisturbed fluid, and the unavoidable use of several lenses weakens the force of the rays which are allowed to pass.

Four of these collecting apparatus are usually arranged round the arc-lamp (Fig. IOO), which is either suspended from

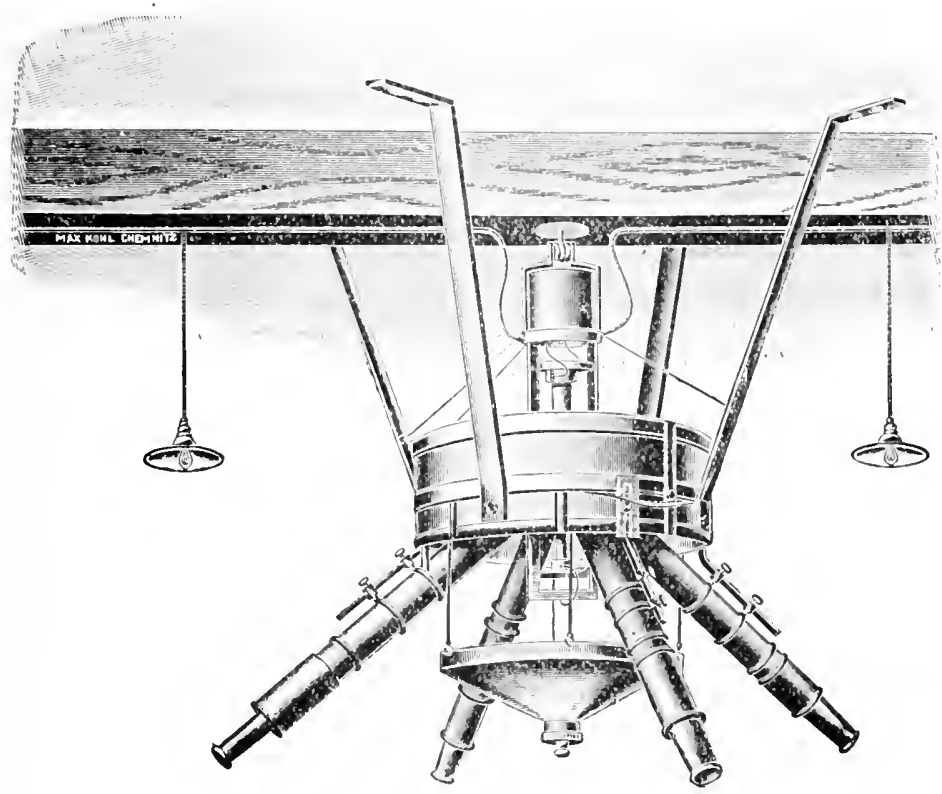

Fiti, 100.-Finsenis apparatus.

the ceiling or mounted on a high iron stand. The spaces between the single concentrators are filled with asbestos plates, so that the operator and the attendants may not be inconvenienced by the strong light. By means of this arrangement four patients can be treated at once and the cxpensue light be fully utilised.

The light from this concentrating apparatus not being eren yet sufficiently cool to be borne without discomfort for any length of time on the skin. Finsell hit upon a very useful plan. A compressor, consistme of two quarty discs fixed in a brass ring, is pressed on the irradiated spot; through this apparatus a constant circulation of cold water is maintained by means of 
a suitable system of tubing ${ }^{1}$ ). This arrangement does away with what is left of the heat, and by the continuous pressure on the irradiated skin it serves the further important purpose of making the skin aniemic. This, as Finsen has shown (cf. p. 426), enables the actinic rays to penetrate far more deeply.

This pressure apparatus is fixed to the patient's body with bandages, or may more conveniently be held on with the hand.

Beurmann drives the blood out of the irradiated parts by applications of adrenalin ( $\mathrm{I}: \mathrm{IOOO}$ ), which constricts the vessels "). But the author's experiments go to show that adrenalin only acts thus when first applied; the effect soon lessens.

As has been said, Finsen replaced by quartz lenses the glass ones, by which ultra-violet is so strongly absorbed. But that had the further disadvantage of necessitating the use of lenses of small diameter $(7-8 \mathrm{~cm}$.) only, for quartz lenses of greater diameter are hard to get and very costly. The light from the voltaic arc, however, can be utilised to a comparatively slight extent with small lenses. In this respect metal reflectors act better. Foreall de Courmelles, Trouvé ${ }^{3}$ ) Kime, ${ }^{4}$ ) J. G. Miiller, ${ }^{5}$ ) Strebel and others, therefore, proposed to have apparatus with mirrors instead of lenses. Strebel described the construction of a concentration apparatus in which the rays are concentrated by metal mirrors of magualia, an alloy of aluminium and magnesium. These mirrors, according to $V$. Schllmann, ${ }^{6}$ ) reflect not only the visible spectrum but also the ultrarioket rays satisfactorily. The light falls on four magnalia concave mirrors ${ }^{7}$ ) mounted in a metal frame at an angle of

$\left.{ }^{1}\right)$ In the accompanying figure the water-supply of the compressor is represented, for simplification of the drawing, as coming from the cooling water of the concentrating apparatus. In practice, however, each of the two apparatus has its own two pipes to the water-supply.

2) Soc. de dermatologie et de syph., July 3rd, 1902.

3) See below.

4) Merlical Record, Oct. I3th, Ig0o.

s) VIl. Dermat. Congr., Breslat, igor.

") I., Mach and $V$. Schumenn, Ein nettes Spiegelmetall. Sitzungsber. d. kais. Nkat. 1. Missensch., Math.-naturw. C1., 1899.

$\left.{ }^{7}\right)$ Other melals tou thight lic suitalsle for such reflectors, c. g., stecl and the Brandes-Schimemann alloy $(4 \% \mathrm{Cu}+26 \% \mathrm{Ni}+24 \% \mathrm{Sn}+8 \% \mathrm{Fe}$ 
$45^{\circ}$ round the arc-light. They project their light through a cooling apparatus fitted with plano-parallel plates or suitably curved lenses. To protect the polish of the mirror from the destructive effect of the ozone, sometimes provision is made for a stream of water to run orer the mirror-surface, ${ }^{1}$ ) or the metal is cooled from the back (Fig. IOI).

The author is not aware if such apparatus has actually been made, or how it acts in practice.

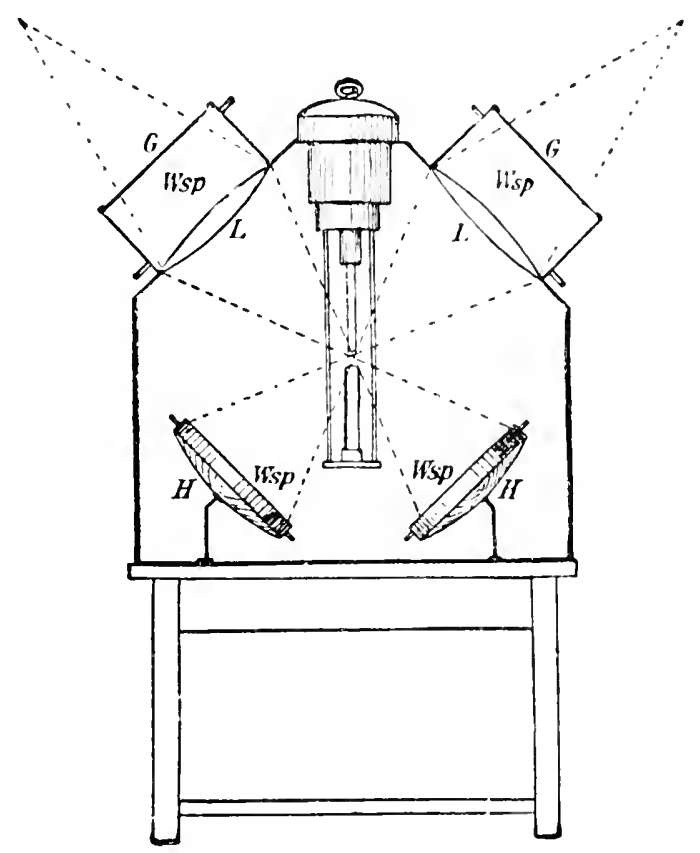

Fici. 10I.-Strocl's Light Apparatus.

According to a fundamental law of optics (cf. p. 372), the intensity of light varies inverscly with the square of the distance Hence we should expect to heighten the effect of

$+\mathrm{I} \% \mathrm{Sb}$ ). the latter of which, according to Glatzol (Physik. \%eitschr., I900, Vol. II, p. I76), lakes a very good polish and resists the action of the air. Both are very well arlapterl for photographic purposes. Silver mirrors reflect $92 \%$ of the visible spectrum, the Brandes-Schuncmann alloy only $50 \%$ : but the latter reflects ultra-riolet mere than other metals.

') Which probatbly. howerer, destroy the wha-violet. 
irradiation by bringing the source of light as close as possible to the object to be irradiated. (The law holds good, indeed, only for non-concentrated light; with concentrated light the distance is not of such great importance.) This law is observed by the metal electrode lamps already described, as well as by the apparatus designed by Fovean de Courmelles and Lortet and Genond. Being designed with due heed to this principle, these apparatus have the advantage of not requiring so extraordinarily powerful a source of light, or so strong a current, as Finsen's concentrating apparatus; and, further, the absorbing media are in
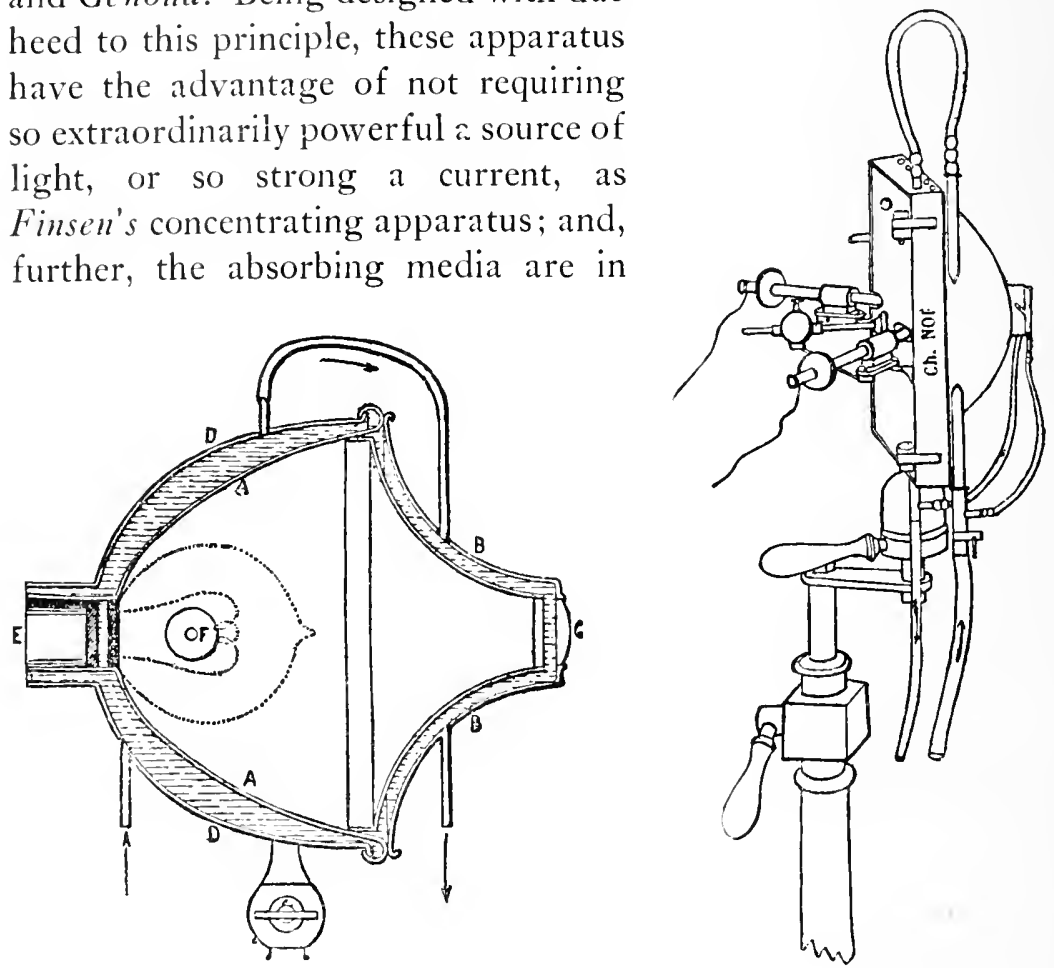

Figs, I02. I03.-Apparatus by Troucé-Fon'cau de Courmelles.

them reduced proportionately in size. Consequently the action with these apparatus is very intense, and the time of exposure can be considerably diminished.

Fovecul de Courmelles and Trouvés apparatus consists of a parabolic mirror, in the focus of which is the arc-light of a lamp of 10 to 22 ampères (Figs. $\mathrm{IO}_{2}$ and $\mathrm{IO}_{3}$ ). Suitable filters are fixed at the opening of the reflector, according as light rays or heat rays or both are desired. The heat filter consists of two 
quartz discs, 2 to $S \mathrm{~mm}$. apart, between which a layer of cold water circulates. These two discs may be of various shapes and sizes, and are applied directly on the part to be irradiated, acting as a pressure apparatus at the same time ${ }^{1}$ ).

At a meeting of the Soc. Franç. de Dermatolog. et Syph. on May ist, 1902, Du Castel reported that he had found this apparatus to act admirably in the treatment of lupus.

Du Castel gained strikingly good results with the Fovean-Trowié apparatus in the case of two patients, giving 65 and 40 one-hour sittings respectively, with a current of 6 amperes. He believes that the length of treatment each time is an essential factor in the cure, and never now has a sitting of less than an hour.

With Lortet and Ge'nond's apparatus, Fig. IO4 (L'Indépendance médicale, March 27th, I90I), a constant arc-light

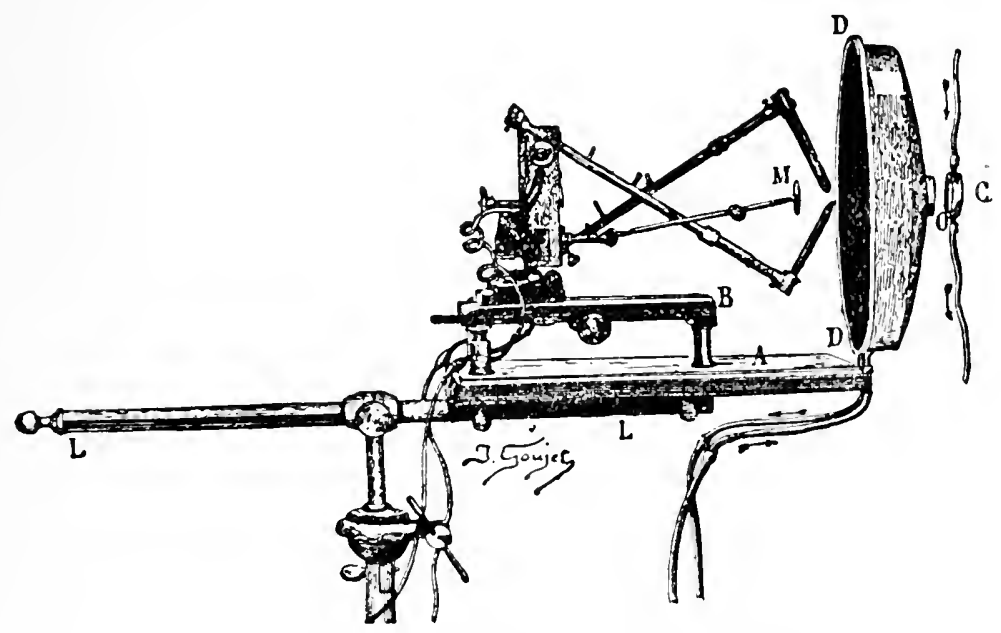

Fig. I0.4-Apparatus by Lortet and Gonoud.

is produced between two carbon points which form so great an angle that the crater of the positive carbon radiates the greater part of the light in the shape of a cone, whose avis passes through the centre of the opening $O$. This opening

1) According to Fon'éau de Commelles (Bulletin office de ha Soc. med. dés Practiciens, 11 Anm. Jume 15. Irot, No. 6). (i. Troure hals from I893 used parabolic mirrors to strengthen the theraluetule action of light. 
forms the centre of a curved metal light-screen $D D$, between the double walls of which cold water is kept constantly circulating.

A small mirror $M$ prevents any light from being radiated backwards, so that almost all falls in front.

A system of screws and slides enables the arc to be regulated and advanced more or less near to the opening. When the apparatus is working the carbons are brought to within $\mathrm{I}$ or $2 \mathrm{~cm}$. of the opening. In front of this is a chamber consisting of two rock crystal discs set in a metal ring; in this chamber a stream of water circulates. This chamber constitutes the compressor. Experience shows that the arc-light may be approached to within 3 to $4 \mathrm{~cm}$. of the chamber without the latter being heated. The heat rays in this apparatus are well absorbed. The size of the part irradiated may be as much as $6 \mathrm{~cm}$. The lamp is said to require not more than Io to $\mathrm{I} 2$ ampères. The time of illumination for therapeutic purposes is given as io to I 5 minutes ${ }^{1}$ ).

The most important use of all these apparatus is for Finsen's treatment of lupus vulgaris. This is applied as follows: Each patient has his own attendant whose business it is to direct the light constantly on the diseased spot. The patient lies on a suitable couch with his head raised, or sits in an armchair with a head-rest. (The patient would hardly be able to keep immovable for a long time, say two hours, in a sitting position.) His eyes are protected by a cloth, the attendant's by darkened glasses. Any scabs or crusts there may be are removed, having been previously softened, with forceps, and the part is washed with a weak antiseptic solution ( $3 \%$ boracic) and dried. Now the light cone is directed on to the site of disease, and care is taken that the axis of the light cone is perpendicular to the area illuminated; the part to be treated is not placed directly at the focus, but a little in front of it so that not one point but a small circle is illuminated. This,

1) Fovcau dc Courmclles, however, asserts (L'année électr., 1902, p. 354) that with this apparatus a supply current of 20 to 25 amp. and sittings of one-quarter to half an hour proved necessary. The unshaded light, too, in the apparatus might be troublesome to the operator. 
moreover, helps to prevent the skin from being heated. A circular area having a diameter of $2 \mathrm{~cm}$. may be treated at one sitting. The surrounding parts are covered with wadding or yellow paper.

Now a suitably shaped compressor ${ }^{1}$ ) is placed on the diseased spot and held there firmly with equable pressure. The duration of the sitting varies according to the quality and intensity of the light used. Finsin recommends a two hours' exposure with his concentrator and a lamp of 30 ampères; with lamps of So ampères the time may be cut down to an hour, or an hour and a quarter. With these powerful lamps, Finsen, after only i 3 to 20 minutes' continuous illumination, effected a definite cure of lupus nodules the size of peas. The designers of the newer kinds of apparatus (Fozcan, Lortet, Bang, Strebel) give the necessary time of exposure for their instruments, on account of the greater light intensity - that is, the greater proportion of ultra-violet rays-as Io to 20 minutes. The treatment consists of one sitting a day, except in cases where the morbid growth is very extensive, when two a day are given.

The patient suffers no pain during the irradiation, except perhaps when the pressure is applied to ulcerated or bony parts, e. g., near the nose.

After the treatment symptoms of erythema solare appear, the irradiated part becomes a little red, the redness very soon increases markedly, and at the same time slight swelling shows itself and burning pains are felt, the skin becomes softer and unevennesses disappear. Often 24 to $4 S$ hours later a large blister forms, filled with serous fluid; this dries awaly in 6 or 8 days to small readily-removable scabs. There is never any loss of substance below the blister.

When the blister has exfoliated, considerable redness is left, which only passes away after months.

To prevent infection of the blisters a dressing is applied of boracic lotion or zinc ointment. After a week or fortnight, when the reaction has disappeared and the scabs have fallen off,

$\left.{ }^{3}\right)$ Fir the skin of the cheek, which yictels realily to pressure, the compresents ated atre comsex, for the forehead concave, and for the temples plane. (Finsen, Sihmidt. Berliner klin. Wochenedur., Igot, No. 32.) 
the same place may be irradiated again, later even more than once; in fact, this is necessary if the therapeutic effect is to be lasting. When one spot seems to have been sufficiently treated a neighbouring spot is treated in the same manner. In this way the treatment proceeds from spot to spot, until the whole part attacked has been exposed to the influence of the light. The treatment should begin at the periphery of the lupus region, and the light must be so directed that in each area of illumination a piece of the apparently healthy skin in the immediate neighbourhood is also included.

After the sitting the spot is marked round with a pencil and so indicated; then the dressing is applied. The compressor, having been cleansed with ether, alcohol, and a solution of carbolic, must be steeped for an hour in carbolic and then placed on the side filled with distilled water. The rock-crystal lenses of the concentrators are cleaned once or twice a week with ordinary water and afterwards rubbed with cork; those nearest to the carbon points are further brushed down thoroughly after each sitting and covered with flannel caps to prevent too rapid cooling and consequent cracking ( $S$ chmidt).

When a patch of lupus has been sufficiently treated the nodules and the raised edges of the lesions become flattened; where formerly confluent nodules formed continuous lupous infiltrations appear now isolated nodules with strips of healthy skin between. By degrees these isolated nodules also disappear; the light reaches not only the superficial but also the deeper lying ones. Ulcers grow less both in surface extent and in depth, and cicatrise. The redness of the skin gives place to normal colouring. The cosmetic result as regards the appearance of the scars is excellent. The scars are smooth and soft, the losses of substance are slight and only such as arose from the morbid processes, not from the treatment, which does not affect the normal tissues.

Gaston, Bandonin and Chatin report hypertrophic and keloid scars, as well as smooth scars, in several cases healed by means of the Lortet-Genoud apparatus ${ }^{1}$ ).

$\left.{ }^{2}\right)$ Soc. de dermatolog., April I, I902. 
Finsen's treatment, beginning as it properly does at the periphery and taking in always the neighbouring healthy skin, has the effect at once of checking the spread of destructive lupous processes. The action of the light on lupus is not only an immediate, but also a lasting one, going on even after the treatment has stopped. Suspicious spots have often been known to resume a healthy appearance during the course of several months after treatment with the rays has been suspended. Finsen concluded from this that the tubercle bacilli are killed by the light in a much shorter time than is required for the slow process of transforming the diseased reddish-brown tissue into healthy skin of a normal colour.

It seems to the author doubtful whether this bactericidal power of light is really the essential curative force in this treatment. He would rather, with $S$. Bang, $\left.{ }^{1}\right)$ G. J. Miiller, ${ }^{2}$ ) Glebowsky, Serapin, Sack (cf. below) and H.E. Schmidt, ${ }^{3}$ ) lay stress on the action of the light in producing inflammation. Light seems to him to act as an irritant penetrating, like the Roentgen-rays, very deeply. Its action goes decper than that of many chemical irritants, $e . g$., pyrogallic acid, resorcin and lactic acid, the effect of which is weakened by the albumen compounds soon formed. The light irritant stimulates the granulation tissue, which is usually but little inclined to change into connective tissue, to the formation of connective tissue and cicatrices. Probably the extraordinarily powerful illumination acts on the diseased tissues, which are specially sensitive to light and less able to resist its action, as a hyper-irritant, and this, as is well known, kills the cells. By this means the morbid tissues are destroyed and prepared for reabsorption.

There is a further circumstance which may make for the favourable action of light, viz.. the artificial and lasting hyperamia produced by it in the diseased area. We know that in the treatment of various tuberculous processes hyper:emia has been aimed at again and again, and that good therapeutic

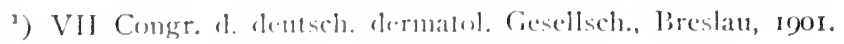

2) Ilisil.

${ }^{3}$ Berlin. Kin. Wixhemachr., 1gon, No, 32. 
results have thereby been gained. We need mention here only Bier's treatment of tuberculous affections of the joints, with which in this respect we may compare the action of the old Koch tuberculin (Neisser, Scholt $\approx$ ), and that of the penetrating chemical caustics, pyrogallol, resorcin (Ehrmann), and lactic acid (Max Joseph).

As the result of this hyperæmia the chemical products of the bacteria are said to be accumulated, a circumstance which is inimical to the bacteria themselves; Bier holds further that hyperamia promotes the formation of connective and scar tissues ${ }^{1}$ ).

Light, in the author's opinion, acts in the case of all such affections exactly in accordance with the action which all physiological irritants, following a universal law, exercise: it acts partly as a stimulus and partly as a strong irritant, paralysing and destroying. He recognises in these rays, as in the Roentgen-rays, just those kinds of irritants which have a specific action and are of special therapeutic value:

I. Because of the specific quality of the rays;

2. Because of their power of penetrating deeply.

The latter quality is specially important in treating deeplylying granulomata, for the other remedies, such as copper sulphate, camphor, etc., which have been used for ages to stimulate torpid granulations, ulcers, and wounds to the formation of connective tissue and scars, have only a superficial action.

Glebowsky made a histological study of the process of

1) Since the compressor drives away the blood from the diseased spot during the treatment, and thus tends to prevent the hyperamia aimed at, $E$. Lang's theory as to the pressure of the capsule being an essential curative factor seems to the author unfounded. His assertion (Wiener dermatolog. Gesellschaft and IV Internat. Congr. f. Dermatologic und Syph., Aug. Igoo, Compt. rend., p. I7I) that he cured hupus by pressure with the lens alone is quite unique and has not been confirmed. On the contrary, G. J. Milller reports (VII Congr. der deutschen dermatolog. Gesellschaft, Breslau, Verhandlungenslocr, p. 471) that he found no result from glass pressure tried experimentally for weeks for as much as eight hours at a time, but that a distinct effect was noticeable from the action of light alone on sensitive spots without pressure. Bang and Lesser (ibid.) have observed the same. 
healing in cases of lupus of the skin under the influence of light treatment, and arrived at the following conclusions ${ }^{1}$ ):

Pieces of skin removel for examination $2+$ hours after irradiation showed the vessels dilated and the surrounding parts infiltrated with nigrating leucocytes. Besides this, it was seen that the interstices of the connective tissue were somewhat wider, and that there was slight vacuolisation degeneration which was clearly marked in the giant-cells.

In pieces removed after $+\$$ hours the same phenomena were much more marked, and there was,

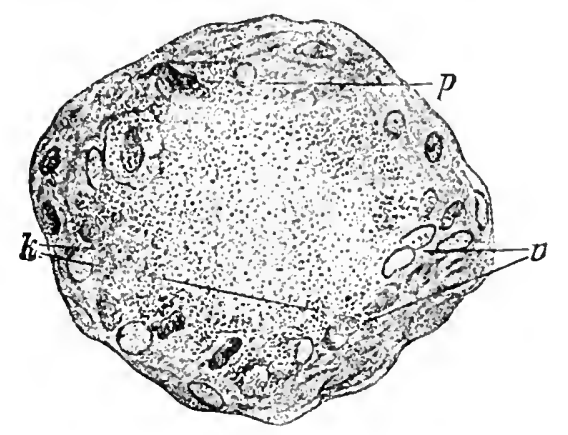

FIG. I05-Giant-cell from a case of lupus two days after Finson treatnient. (Gleboasky-Serapin, die Verinderungen im Lupusgranulom unter der Einwirkung des concentrirten Bogenlichtes nach der Finsen'schen Methode. Verhandl. d. 1). dermat. Gesell. VII Congr. p, prknosis; $v$, racuolised nuclei; $k$, fat.)

further, fatty degeneration of the protoplasm and necrobiotic changes in the nuclei of the granuloma-cells, especially in the giant-cells (pyknosis and chromatolysis, Fig. 105). After repeated sittings these destructive appearances in the giant-cells increased, and finally the cells disappeared entirely. This happened on an average after + to 5 irradiations. The processes of degeneration in the epithelioid elements were much less marked as compared with those in the other granuloma

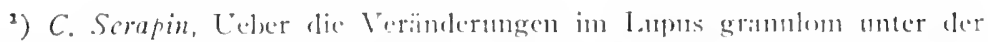
Einwirkung des concentrirten Pagenlichtes, ete. Vll Comgr. d. dentsch. de:matolog. Gesellsch. Verliandlengsucriche, p. 500 ff. 
elements (a point of difference from the changes in the lupus growth after treatment with the Roentgen-rays, achen such varieties of effect have not been observed, (cf. p. $2 S_{7} \mathrm{ff}$ ); but Glebowsky and Serapin noticed in them changes of a purely progressive character, e. g., elongations of the nuclei and the cell-bodies and elements of a spindle shape were met with, which seemed at times to pass into fibres. The lymphoid elements of the lupus, too, showed during the acute reaction rather pronounced fatty degeneration, and later on oval and spindle-shaped cells were observed amongst them. As the process of reaction advanced and died away the cell elements in the granuloma disappeared more and more, beginning with the upper layers of the corium, and the connective tissue became more and more conspicuous (Fig. I06). Only at the end of the treatment were the numerous blood vessels contained in the granuloma quite obliterated.

According to $d$. Sack's investigations on lupus, ulcus rodens, and nævus vasculosus planus ${ }^{1}$ ) the blood-vessels are the first point affected by the light. The endothelium lining their walls swells and grows rapidly and endarteritis results, with final obliteration of the vessels. The retrogressive changes in the character of the cells which show themselves, and are in part of a necrobiotic nature, are purely elective, attacking only certain less persistent elements of the disease tissue, whilst the other elements both within and without the morbid growth are stimulated to activity.

Since at each sitting only one small spot can be treated, and as this has to be treated again more than once, the whole process of cure cannot but be of long duration; precisely how long depends on the intensity and extent of the disease. Small isolated lesions can be cured in a few sittings, but large growths may need to be treated for a year or more, and tax severely the patience of both doctor and patient. In order to shorten

1) Mïnchen. med. Wochenschr., July 8, I902. 
the period of treatment, very extensively affected parts which are much infiltrated and darkly pigmented are first treated with pyrogallol ointment, and especially deep-seated nodules may be destroyed by the cautery.

In the light treatment of lupus vulgaris, just as in the $\mathrm{X}$-ray treatment, it is well to make a break with the treatment after a powerful reaction, so that it may be seen, when the acute

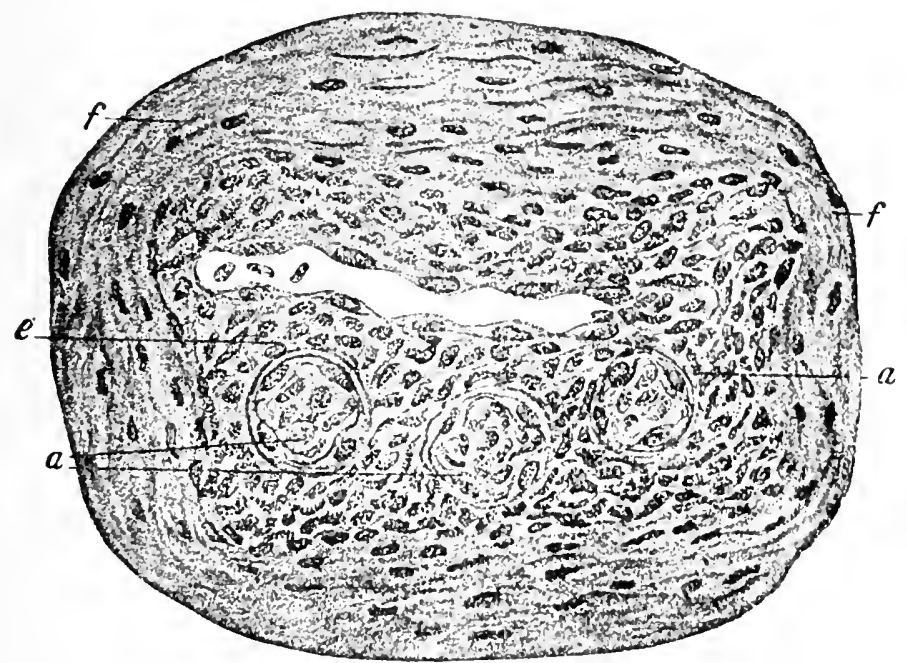

F.g. I06.-A Lupus nodule after four sittings. (From Glibousky-Serapin 1. c.) (a) Blood vessels with hyperplasia of the endothelium. (c) Grantlation elements between the epithelioid cells. (f) Connective tissue.

effects have died away, whether it is advisable to continue the treatment.

According to Forchlammer ${ }^{1}$ ) generally after some lapse of time, when the reaction has died away and the whole part is again in a nermal condition, there are remains of the disease left in the shape of isolated deep-seated nodules. It is, therefore, necessary to watch the case carefully and to give, where necessary, short supplementary treatment once or several times, as the case may be.

In the case of not ton extensive and inveterate lupus growths

1) VII Congress d. deutsch, dermatol. Gesellscli, Breslan, igor. 
of not more than Io years' standing the average duration of the first treatment may be taken as 3 to 4 months. Cases of greater extent and longer standing are likely to require longer treatment, and complete cure is in such cases more uncertain, in great measure on account of the changes brought about in the lupus tissues in the course of the disease, and by the treatment previously tried for it. These changes are of the nature of fibrous scar-tissue, intense brown pigmentation, and considerable infiltration (Forchiammer), and they all render it more difficult for the light to penetrate the tissues.

Both Finsen and his fellow-workers lay special stress on the importance of keeping the patients under observation for a long time after treatment.

Although the method gives the most favourable results (according to Forchliammer, $85 \%$ of cures), there is yet a small number of cases ( 2 to $3 \%$ ) in which it fails. Then there are the cases in which the disease attacks the mucous membrane, but even when it is localised at the body orifices, the gums, the palate or the tongue, it may be treated by this method (Lebon, ${ }^{1}$ ) Török and $\left.S_{c h e i n}{ }^{2}\right)$ ). With a considerable number of patients there are relapses. Forchhammer reports that this is especially the case with patients who neglect to come up for inspection after the end of the treatment, or with cases where there is extensive inflammation of the mucous membrane, or, finally, with patients who are attacked very soon after treatment by severe and debilitating disease, e. $g$., erysipelas or influenza.

Broc ${ }^{3}$ ) mentions one case in which irradiation produced always the most violent eczema and oedema, so that it could not be adopted.

According to M. Morris and E. Dore, ${ }^{4}$ ) cases of lupus are unsuited to the Finsen treatment, which are accompanied by much scarring and pigmentation and great vascularisation and thickening of the skin, and those which occur in certain regions

\footnotetext{
') La Photothérapie, Paris, Société d'éditions scientifiques, Igor.

$\left.{ }^{2}\right)$ L. C.

$\left.{ }^{3}\right)$ IV Intern. Congr, de Dermatologie. Paris, Igoo.

4) Lirit. Nerl. Journ., Feb. 9, IgOI.
} 
(eyes, mouth, nostrils). Leredde and Paulier recommend preparatory treatment with scarification and cauterisation in cases where previous remedial efforts have produced sclerosis ${ }^{1}$ ).

There are a few drawbacks to set against the adrantages of this method, its efficacy, its elective action on lupus whilst preserving all sound tissuc, its painlessness, its excellent cosmetic results, its freedom from unpleasant and unexpected after-effects. The method demands a large plant, which is costly' both to buy and to maintain in operation (on account of the powerful current required); it necessitates a trained staff of attendants, and by its tedious course it makes great demands on the patience of both doctor and patient, and requires from both considerable powers of physical endurance. Comparisons have been drawn between the light treatment and the Roentgen-ray treatment, and the latter method has been termed the more simple, quick and cheap. Many (Kiimmel and others) have, therefore, recommended that isolated lupus patches should be treated by the Finsen method, and larger areas of disease with the Roentgen-rays. Objectively considered, the Roentgen treatment is certainly simpler; the tube needs only to be directed rightly on the patient, and, provided he remain still, he can be then left to himself and there is no need of specially trained attendants. The duration of the single sittings is much shorter than with the Finsen treatment, and the Roentgen-ray treatment is just as painless; the area which it is possible to treat each time with the X-rays is much larger than with the Finsen treatment, and the appearance after treatment is quite as good. But on the other hand we must consider that even though the Roenlgen treatment does not require so efficient a staff of attendants it presupposes in the operator great experience and practice, and clemands from him, not indecel the same physical endurance, but the capacity of judging correctly and measuring the intensity of irradiation, the suitable time of exposure, etc., whilst with the Finsen method no such nicetics have to be taken into aceount.

Further, in many cases, from the Roenlgen treatment there

1) Soc. de 1)ermatologg., April 1, 1002. 
can only be assurance of definite results if violent and painful dermatitis, persisting to the end of the cure, be accepted as part of the process; and its results are by no means always so evident as with the rival method.

There is probably not much difference between the two methods as regards either their value in lupus of the mucous membrane or the total length of time required for a cure. With each method the main treatment needs to be followed by several periods of supplementary treatment. We may say indeed that the advantages of the physical treatment of lupus are not to be sought in any shortening of the time of treatment (which is generally not less than that required by any of the previous methods), but in the fact that they are conservative and almost painless and give excellent cosmetic results.

These considerations point to the $X$-ray treatment as being suitable in cases where, for reasons not connected with the disease itself, the light treatment cannot be used, and on the other hand a suitable Roentgen apparatus is available.

Finsen's method and its results have received recognition on all hands. Numbers of medical men have travelled to Copenhagen to see and study the light treatment on the spot. The present writer is one of those who have had an opportunity of inspecting the arrangements of the Finsen Institute, and he can only express admiration of the excellent way the whole thing is planned, the high scientific attainments of the heads of departments (Bang, Forchliammer, Ryn [Larsen], Bie) and the zeal and enthusiasm with which they work to further the good cause.

Finsen's statements have already been amply confirmed by the most eminent authorities. Lassar, Lesser, Jadassohn, Sabonrand, Petersen, Malcolm Morris, Leredde and Pautier, Ehlers, Burgsdorf, Macke'nsie, Dore, Sequeira and many others report similar experiences.

Treatment with concentrated sunlight is carried out in just the same way as with the arc-light apparatus; only care has. to be taken to keep the stand of the concentrating lens constantly in a right position with regard to the sun.

As has already been mentioned, various simplifications of 
the Finsen apparatus have been designed (Fovean-Tronvé, Lortet and Genond), and quite satisfactory results are said to have been attained with short exposures ( 15 to 30 minutes: Gaston, Baudonin, Chatin, Focen de Courmelles, ${ }^{1}$ ) Du Castel, $\left.{ }^{2}\right)$ Lebon,,$^{2}$ ) Gastou, Baudouin and Chatin $\left.{ }^{3}\right)$ ).

The new iron electrode lamps do not seem to be so suitable as the Finsen apparatus for the treatment of lupus vulgaris.

S. Bang ${ }^{4}$ ) himself writes that "the Finsen apparatus have maintained their supremacy unrivalled in the treatment of deep-seated affections such as lupus vulgaris; for these we should advise against the iron light." Strebel, too, says ") : "As the light from the electric spark and from the iron electrode lamp can only act on the supcrficial skin hecause of its preponderance of ultra-violet, which is absorbed in the epidermis to a great extent, the earlier Finsen method still maintains its position; the deap-seated lupus nodules must be treated with light rich in colour rays, which alone can penetrate deeply."

Bang would have his apparatus used in cases where with simple and cheap means a powerful reaction of the skin and a superficial bactericidal action is required. Kromayer, Liese, Below, Kattenlracker and Schiff claim to have had good results with the Kjeldsen lamp in cases of lupus vulgaris and erythematosus, alopecia areata, acne rosacea, eczema, syphilitic ulcers, condylomata, favus, and ulcerating hxmorrhoids.

\section{Alopecia Areata.}

Since this affection is believed by many dermatologists to be of a parasitic nature, and since light kills bacteria and stimulates the growth of hair whilst producing local inflammation of the skin, ${ }^{6}$ ) O. Jersild ${ }^{7}$ ) suggested the application of the light treatment to alopecia areata.

1) Le mérlicin, 1902, Nr. 7. Pruxclles.

2) L. C.

${ }^{3}$ ) Soc. de dermatolugie, $A_{1}$. 1, 1002 .

•) Deutsche med. Wochensclir., Ig02, No, 2.

s) L. C., P. 62.

-) The methods of treament nsually tried am also at producing hyperaemia by means of chemical, mechanical (facpuet), and electric irritants (Ehrmann, Bordire).

$\left.{ }^{5}\right)$ Annales de dermatolugie, 1899, 1.20. 
His method is much the same as that indicated by Finsen for cases of lupus vulgaris.

The concentrated light used is from very powerful light sources. The hair is cut off round the patches in a zone of one to two centimetres.

The illumination starts at the periphery in the healthy part and advances gradually towards the centre. One or two sittings of an hour and a quarter are given each day. Jersild thinks it is not necessary to use a compressor on the morbid spots; the skin is cooled down now and again by moistening with cold water. If carried out rightly the treatment is painless.

Each diseased spot is irradiated only once. The duration of the treatment depends naturally on the extent to which the disease has spread.

The immediate result of the treatment is to stop at once the further falling off of the hair at the spots treated $\left(\right.$ Spiegler, $\left.{ }^{1}\right)$ Jersild $\left.{ }^{2}\right)$ ).

After a longer or shorter time (at the earliest after eleven days-Jersild) lanugo appears on the bald patches, and this gradually becomes pigmented and thicker.

The more recent the origin of the disease, the better are the prospects of cure; this method of treatment is of no use in cases of universal alopecia areata of many years' standing.

The results, as reported by Jcrsild, are very encouraging.

According to Forchllammer's statistics, 30 cases out of 49 were cured in Finsen's Institute. Saiouraud's experience was less happy $\left.{ }^{3}\right)$. He reports that no specially good results were attained in active cases of alopecia areata by this method, but that it was successful with more chronic cases, limited in extent. Sabourand, too, attributes the effect of the light treatment in alopecia to the local congestion it produces ${ }^{*}$ ).

1) VII Congr. d. deutschen dermatol. Gesellseh., Breslau, p. 469.

2) Nitheilungen aus Finsch's med. Lichtinstit., I, p. II3.

$\left.{ }^{3}\right)$ In a letter kindly sent to the author recently, the distinguished French investigator writes: "J'ai expérimenté pendant six mois la photothérapie de Finsen dans la pelade. Et j'ai obtenu des résultats médiocres, beaneoup plus médineres que l'Ecole de Copenhagne me semblait les annoncer dans le traitment de cette maladie."

4) Quoted by II. Lebon, La Photothérapie, Paris, I9or, p. 22. 


\section{Lupus Eryhemalosus.}

Finsen, Bang, Forchlammer, ${ }^{1}$ ) Leredde,") Petersen,;) Sabourand,,$^{+}$G. J. Milller ${ }^{5}$ ) and others have tried treating this affection with the concentrated arc-light. It acts quite satisfactorily in fresh cases, but with cases of longer standing, and especially if the lupus erythematosus is generalised, it often has no effect. Still, according to Leredde, there have been cases which could not be effectively treated by other methods where the light treatment has brought about distinct improvement. Leredde strongly recommends treating the plaques beforehand with high-frequency currents. Sabourand combines the light treatment with scarification and galvanopuncture.

J. M. H. Macleod") treated 5 cases of lupus erythematosus by the Finsen method; two were decidedly improved, two showed no change, and one showed obvious change for the worse.

\section{Epilhelioma.}

Finsen, Petersen, Burgesdorf and others have seen distinct improvement from the light treatment with superficial cpitheliomata in the early stages. Sequeira prefers treatment with the Roentgen-rays for this disease.

\section{Nerus rascularis.}

According to Forchlammer, the light treatment reduces materially the deep red colour of this disease, and in some cases the affection was entirely cured. Petersen observed with one case, in which the nærus extended from the forehead to the eyelid, that there was improvement not only in the part of the

') Congrès pour l'étude de la tuberculose, Paris, 1898, and Dermatolog. Congress, Breslau, igor.

2) Bulletin généra! de thérapeutique.

${ }^{3}$ ) VII Congr. d. deutschen dermatolog. Gesellsch., $190 \mathrm{I}$.

4) L. c.

s) L. c.

a) Brit. Med. Assoc, meeting at Manchester, July 30, 1 yoz. 
forehead treated, but also in the part of the nævus on the upper lid, which of course could not be irradiated.

\section{Other Skin Diseases.}

Treatment with intense light irradiation has been tried with a variety of other skin diseases (acne vulgaris, Finsen; furunculosis, Strebel, Barbensi; acne rosacea, Finsen, Strebel; rhinophyma, Leredde; psoriasis, G. J. Mïller, Strebel, Barbensi; sycosis parasitaria and non-parasitaria, Finsen, G. J. Müller; biskra-button, Petersen; favus, Finsen); the experience gained is still limited, the results varying and uncertain. Varicose ulcers, too, septic wounds, fistulæ after operation on bubo, etc., are said by $G$. J. Miiller and others to heal more quickly under light-treatment.

Finsen ${ }^{1}$ ) recommends also concentrated lighttreatment for bacterial affections which are not quite superficial, and where there is the possibility of rendering the tissues partially or entirely anæmic. Such, $e . g$., would be the case with tuberculous affections of the smaller joints and the extremities in the case of small children; here the tissues are very transparent, and might readily have the blood expelled with an Esmarch bandage. G. Hurtado claims to have cured arthritis tuberculosa in the elbow-joints by this means ${ }^{2}$ ).

The author is not aware if there are any further experiences of the kind.

It has been already mentioned (cf. p. 32 I) that sun-baths have, under certain conditions, proved efficacious with such complaints.

\section{Venereal Discases.}

\section{G. Barbensi and Strebel ${ }^{3}$ ) treated primary syphilitic}

$\left.{ }^{1}\right)$ Ueber d. Anwendung von conc. chem. Lichtstrahlen, Leipzig, I899. p. รo.

$\left.{ }^{2}\right)$ Revista Ibero-Americ. de C. Med., IgoI, No. I2.

$\left.{ }^{3}\right)$ Revista critica di Clinica Medic., quoted in Foveau de Courmolles, L'année électr., 1902, p. 392. 
chancre, gummata, and soft chancre with concentrated light and irradiation with ultra-riolet rays (from iron electrodes or spark-light); the local specific lesion in the case of primary chancre is said to heal quickly under this treatment, but secondary eruptions are not thereby prevented. The light-treatment would, therefore, have to be combined with mercurial treatment, or iodide treatment in the later stages. According to Strebel, with venereal ulcers two or three irradiations suffice to heal the sore within a few days. There is no evidence of distinct influence on glandular swellings.

\section{The Therapeutic Use of Other Sources of Light.}

$\$ 68$. In the method treated of in the foregoing chapter the voltaic arc is the source of the light used for irradiation. In Strebel's method the light of the condensed spark from an induced current of high tension is used. It has long been known that this light is very rich in ultra-violet rays, and experts in photography and spectrography have for many years made use in their spectroscopic and photo-spectrographic experiments of the induction spark between suitable metal alloys, strengthened by Leyden jars ${ }^{1}$ ).

In view of experiments in other quarters which had established the fact that ultra-violet rays have a powerful bactericidal effect, it was to be presumed that light irradiation from the induction spark would have a specially powerful effect in this direction. Marshall $\mathrm{V}$ ard ${ }^{2}$ ) and Strebel ${ }^{3}$ ) have furnished the practical proof of this. Strebel showed that the spark of an induction coil at a sparking-distance of $20 \mathrm{~cm}$. kills all kinds of microbes at 70 to $140 \mathrm{~cm}$. distance in a few minutes, and, if the objects are brought to the source of light, within a space

1) Rood (Fortschr. der Physik, 1864, p. 257), Berthclot (Ann. Chim. Phys. (7) 19. p. 150), Tommasi (Beil). Ammal. Plyys. Chem., I886, P. 427), E. H. Cooks (Phiios, Nag., 1899, Ser. 5, Vol. XXXVII, p. 40), and others have shown that the silent. dark electric effuria of influence machines and Ruhmkorff induction crils also contain many ultra-violet rays, and have the same actinic action on photographic preparations as light rays.

2) Proc. of the Roy. Snc. of I.oncton, 1894. Vol. I.IV, p. $472 \mathrm{ff}$.

$\left.{ }^{3}\right)$ Deutsche med. Wochenschr., 1901, Nos. 5, 6. 
of time which compares well with any results gained with the arc-light. The effect on the skin of rays from the induction spark is precisely the same as that of those from the voltaic arc; they, too, produce erythema and pigmentation.

The biological action of the induction light is in large part the action of ultra-violet rays. Rays of greater wave-length are present to so small an extent that any possible action on their part would be quite subordinate.

Further investigations have yet to determine what part in the known and still unknown action of this irradiation is played by the "electric waves" originating in the spark.

One reason in favour of using spark-light is that with it, as with metal electrode arc-lamps, the source of light can be brought quite close to the object, and the effect of the light thus increased. Bearing this in mind, and also the circumstance that the light source proper does not require such careful guarding and regulating as the apparatus before mentioned, and that it can be packed in small compass, the Strebel spark-light apparatus would seem to be specially suitable for use in cases where it is desired to bring the action of the ultra-violet rays to bear on the body cavities.

The Strebel instrument for producing this light consists of a small spark-induction coil, or it may be a large one, but in any case only a small spark-gap is used. A Leyden jar (or a battery of such jars) is inserted parallel with the spark-gap, $i$. $e$, one pole joined to the inner, the other to the outer, foil.

The so-called capsule apparatus for irradiating the external skin consists of a short ebony tube, $6 \mathrm{~cm}$. broad, which is closed on one side by a quartz lens, on the other by a concave mirror of magnalium metal. The conducting wires pass through the wall of this apparatus and terminate in one or more pairs of aluminium electrodes, which stand opposite to each other, a short distance apart, in front of the concave mirror (Fig. I07).

Gorl $\left.{ }^{1}\right)$ inserted insulated aluminium balls one behind the other in the shape of an "S" between the electrodes. The spark flashes from ball to ball along the "S."

$\left.{ }^{1}\right)$ München ned. Wochenschr., 190I, No. 19. 
Air is forced into the capsule through an opening by means of a small bellows, and this both prevents the electrodes from becoming heated and drives out the ozone and metallic vapour formed by the spark ${ }^{1}$ ). The apparatus serves as both light source and compressor, and is pressed by the patient himself against the part of skin to be treated.

For the irradiation of the cavities of the body Strebel constructed similar instruments, shaped to fit the parts to be treated, like catheters, etc. The instruments are intended on insertion to have the effect of causing local anæmia through pressure and the stretching of the mucous membrane.

The idea of this apparatus may be good, but the way in which it is carriec out, i. e., by glass tubes, etc., into which insulated wires pass, makes it of doubtful practical value. Glass is used as material for the apparatus, without regard to the fact that glass is a powerful absorbent of ultra-violet, and that, therefore, very few of the ultra-violet rays of the induction spark traverse it.

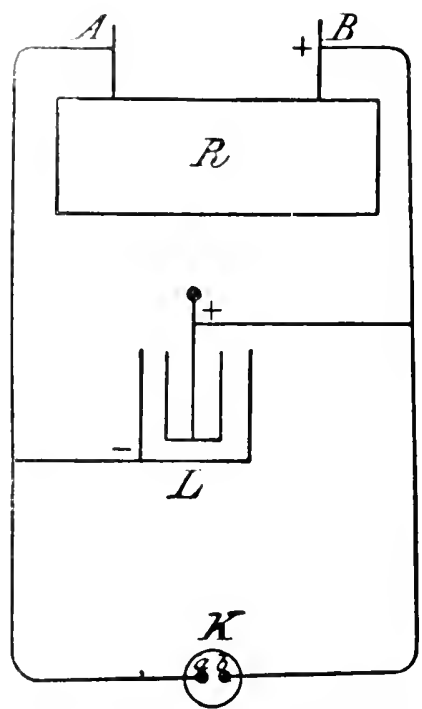

FIG. 107.-Strebel Instrument.

It is true Strebel directed that quartz windows should be put in the instruments opposite the sparks, but so far as we are aware this is never done.

With glass apparatus of this kind, it would only be after very long irradiation that any tiological or therapeutic action whatever could be expected.

The introduction of a glass catheter into the human urethra is not without its danger. We may recall that not long ago a case was mentioned where a glass sound 
broken off in the urethra, which could only be removed by operation.

Strebel proposed as another form of spark-light the rays given out by the opening spark of a Wagner hammer with an induction apparatus. Strebel showed that this light, when the interrupting-spark is produced by aluminium contacts, is very rich in colour rays, though not to so marked an extent as the voltaic arc-light, and is even richer than the latter in ultra-violet. Cooling arrangements have to be provided, as this light is very hot.

Both the writer ${ }^{1}$ ) and Strebel ${ }^{2}$ ) have pointed to the possible therapeutic utilisation of the ultra-violet rays in the electric brush-light. Strebel constructed a small condenser, which projects these electrical discharges in a circular plane. S. Leduc, too, gave directions for a suitable apparatus ${ }^{3}$ ). His condenser is insulated in a capsule with a quartz end, and can be used as a compressor. Five to ten of such apparatus can be inserted in one current and several patients thus treated at the same time.

Irradiation for a quarter of an hour to an hour each time, every one to three days, is applied with this apparatus, according to the length of time before the action shows itself. According to Strebel, the treatment proceeds rapidly with this capsule apparatus, as a patch $5 \mathrm{~cm}$. across may be at once irradiated.

We have so far only Strebel's report of results from this method. He claims to have had very good results: Lupus vulgaris (distinct improvement after several irradiations of three-quarters of an hour); venereal ulcers (healing after several irradiations of half an hour); obstinate psoriasis ( 8 sittings of an hour and a half each); a plaque of herpes tonsurans as large as a 5 mark piece (2 I irradiations of half an hour); sycosis ( 12 sittings of 25 to 30 minutes-simultaneous epilation) ; eczema madidans (two sittings); ulcus cruris (healing

1) Die physiolog. Wirkungen der Polentl. Sitzungsber. d. k. Akad. d. Wissensch. in Wien, Math. naturw. Classe, Vol. CIX, Part III, I900, p. 652.

2) L. c.

3) Quoted in L'année électrique, I90I, p. 389. 
after 9 to 20 sittings), and alopecia areata ( 8 sittings). This treatment is said to have been markedly successful with diseases of the mucous membrane, arresting the discharge and causing the disease to disappear (Huor albus blenorrh. irradiation for 20 minutes every fourth day, combined with salt water douches; chronic metritis- Io intrauterine irradiations; gonorrhoa, in the male- 5 or 6 sittings of 15 minutes each; otorrhœa-Io sittings of 15 minutes; venereal warts-3 sittings).

Strebel himself allows that this treatment may possibly produce violent irritation of the mucous membrane (inflammatory swelling, pain on micturition, etc.).

Even when carried out for a very long time the treatment of lupus vulgaris with electric brush-light does not produce completely satisfactory results. Of this the author has convinced himself with his own electrode, which (cf. plate Fig. 7) gives out brush-light very freely. He could, indeed, perceive distinct improvement ${ }^{1}$ ) with lupus ulcers, and a visible tendency to skin over; but constantly, a fter a longer or shorter time, there was a relapse.

With the forms of light-treatment so far described the sources of light used were bodics which were made to shine, $i$. $e$., to give out light rays, by their heat energy being raised to the point at which light irradiation begins. As we (p. 385) have seen, besides this there are other kinds of light development: luminescence, which are produced by external agency, indeed, but with no corresponding rise in temperature. The literature of medicine in the last few years describes various attempts to turn this kind of light to therapeutic account. IVe might include the induction spark amongst light of this order.

The light produced by electricity in the rarefied atmosphere of Geissler tubes is, in spitc of its apparent feebleness, good actinically, having a considerable propoition of ultra-violet rays, as has been proved by the researches of Norley," $) H$. W. Vogel,

1) Dic Verwendung der Spannungselektricitä, etc. Referat f. d. VIII Congr. d. deutschen dermatologe. Gesellsch., Berlin, 1901. Verhandlungsber., p. 71 .

2) Photograph. Mithleilungen, 187, Vol. VIII, p. I02. 
Caprion, $\left.{ }^{1}\right)$ and others. This light is cold. Strebel availed himself of the photo-chemical property of glow light by constructing instruments for the irradiation of the cavities of the body, in which helium was used as the gaseous medium. He reports the light from this apparatus as being strongly bactericidal, penetrating the tissues well. He uses this light chiefly for the treatment of affections of the mucous membranes, in similar manner to the light of the induction spark.

Certain physiological and therapeutic effects have been reported of another group of luminous phenomena: fluorescence and phosphorescence.

By fluorescence we understand a peculiar self-luminosity of certain bodies which is evoked by light rays and lasts only as long as the irradiation.

Fluorescence is a peculiar effect of absorbed light.

Fluorescent light contains, as a rule, only wave-lengths which are not smaller than those of the light producing it. When, $e . g$., we examine the fluorescence of quinine solutions we see that the action which produces the fluorescence is peculiar mainly to the short-waved light. But in this fluorescent light there are also kinds of light of greater wave-length than that of any component of the light producing the fluorescence; there are, however, none of shorter wave-length. The law that fluorescent light cannot contain light of less wave-length than the exciting light does not apply to other substances, $e . g$., eosin, Huorescin, and naphthaline red.

The quality of producing fluorescence is, as we have seen, common to those rays which have the most marked physiological effect (the ultra-violet, Roentgen- and Becquerel-rays). This is remarkable for the reason in particular that these rays, according to our theories, are not closely related. It seems natural to look for the common source of the physiological action in their power of producing fluorescence; that this is so seems proven: ( I) by the capacity of the animal tissues for fluorescence, proved by various investigators, and (2) by the

$\left.{ }^{1}\right)$ Photographed Spectra, London, 1877. 
peculiar biological effect of fluorescent light, as shown by Tappeiner and Raab.

H. v. Tappeiner ${ }^{1}$ ) induced O. Raab to test the action on infusoria of the Huorescent light obtained by the illumination of phenylacridin. Paramocicum caudatum in a suspended drop in a damp chamber was the subject of this experiment. It was seen that paramocia, in an acridin solution ( $\mathrm{I}$ in 20,000), died in sunlight in 6 minutes, in diffused daylight in about 60 minutes; but if kept in the dark they were alive after 6,000 minutes ( I oo hours). An eosin culture, mixed in an eosin solution ( $\mathrm{I}$ in 800 ), arranged in the green part of the spectrum of a powerful electric arc-light, broken up by a quartz prism, showed after two to four hours' exposure all degrees of injury, even to death. The other parts of the spectrum (which induced the fluorescence of eosin but little) had no effect on the culture. When a paramecia culture in an acridin solution ( $\mathrm{i}$ in 20,000) was arranged so that all the light reaching it had to pass through a 4 to $5 \mathrm{~cm}$. layer of a concentrated acridin solution ( $\mathrm{I}$ in 500 ) the light no longer took effect; the paramocia were healthy after a week, even when exposed to sunlight. But when a quinine solution was taken as screen the light acted as usual, obviously because now the screen absorbed only the inactive ultra-violet rays, and not the violet rays which produce fluorescence in acridin. This last experiment proves at the same time that it is not the fluorescent light which is harmful, but the process of fluorescence stimulation itself. $\approx$. Tappeiner sums up the results of his experiments as follows: Light becomes highly injurious to paramecia in the presence of acridin, phenylacridin, eosin, and quinine, in solutions in which these substances in themselves (in the dark) are hardly, if at all, poisonous. 'This action of light is closely connected with the fluorescent quality of the substances named. The injurious power, however, lies not in the fluorescent light produced, but in the process of its production.

$O$. Raab surmises that we have here a conversion of the

') Münchener med. Wrochencehr., rgoo, No. 1, P. 5, and Zeitschrift f. Binlogie, Vol. XXXIX. 
energy of the light rays into chemical energy, analogous to that of chlorophyll, which also is a highly fluorescent body. There is only this difference, that this conversion is the cause of death to the paramocia, whereas to plants it is the condition of continued life.

v. Tappeiner holds that this kind of light action comes into play with those animal organs and fluids (skin, retina, blood and lymph serum) which have the capacity for fluorescence. He surmises, too, that the cause of the skin inflammation noticed by $\left.V^{\prime} e d d i n g^{1}\right)$ in beasts fed on buckwheat lies in the fact that substances get into the body which are capable of fluorescence.

A species of luminosity very closely allied to fluorescence is phosphorescence (according to Becquerel, fluorescence is only phosphorescence of very short duration). A large number of bodies, under the influence of powerful illumination, especially from light sources which emit a large amount of ultra-violet rays, radiate a soft light precisely similar to that of fluorescence. In phosphorescence, however, the radiation persists for an appreciable, often even for a considerable, time after the illumination has ceased, whilst fluorescent substances shine only during its continuance.

A considerable number of natural and artificial substances have this quality of phosphorescence to an evident extent: among natural phosphorescent bodies ("light-absorbers" and "light-magnets"), we may mention diamonds, calcareous spar, and chlorophane. Artificial phosphorescent bodies include the sulphates of the alkali earths, which are obtained by heating sulphur with limestone, barytes, or strontium salts. Sulphide of calcium, which has a violet phosphorescence, is the best and most brightly phosphorescent substance known up to now. This substance is called after its discoverer "Balmain's luminous colour."

The colours of phosphorescent light are not only dependent on the chemical composition of the substances which emit, but they are to a large extent dependent on their physical nature

$\left.{ }^{1}\right)$ Cf. p. 332 . 
and their temperature. The intensity of phosphorescent light is increased by heating. Like fluorescent light, phosphorescent light consists of rays of greater wave-length than the exciting light; there is much in favour of the assumption that in phosphorescent light energy is given off which has been taken from the absorbed light of the exciting light source. We have as yet, unfortunately, no well thought out theory, free from all objections, as to the process of transforming the light movement of the exciting light into that of the phosphorescent light (A. Lampa). The light of the best and most brightly shining phosphorescent substances excited by daylight is comparatively weak; according to Eder, ${ }^{1}$ ) when in immediate contact with a bromide of silver gelatine plate, it acts about as powerfully as the light of one normal candle at $50 \mathrm{~cm}$. distance. Phosphorescent light has always a far wcaker effect than the light which excited the phosphorescence.

Seebeck and Becquerel discovered the remarkable fact that the yellow and red rays counteract the action of the violet rays, extinguishing, or at any rate, considerably weakening, the brightness produced by them.

Whilst fluorescent plates are used to render visible the ultra-violet spectrum (this spectrum producing visible fluorescent rays on the regions whereon it falls), phosphorescent plates are used, according to Becquerel, to make ultra-red visible. If, $e . g$., one brings into the dark a plate covered with Balmain's luminous colour, which has been exposed to daylight and is therefore luminous, the luminosity will disappear where ultra-red rays fall and a negative picture of the spectrum will be given.

It is, perhaps, permissible to connect this remarkable fact with certain effects of the red end of the spectrum on animal tissues; all the more since it is proved that certain tissues are capable of fluorescence, and that the process of inducing fluorescence produces changes in the tissue.

Rays of phosphorescent light give in general a continuous

1) Ausführl. Ilandls. d. Photogr., I, 1, p. $46 \mathrm{r}$. 
spectrum extending into the blue. The colour of the radiated light is independent of the colour of the exciting rays, $i$. e., a certain luminous substance gives off always the same light, be the exciting light blue or violet or white.

The duration of phosphorescence after illumination varies with different bodies. There is no connection between the intensity of the phosphorescent light and the duration of the luminosity.

As may be seen from the tables in Eder's "Recepte und Tabellen für Photographie und Reproductionstechnik," page 72; the photographic effect (brightness) of blue phosphorescent sulphide of calcium diminishes rapidly after the light impression has been received. If we take the brightness immediately after illumination as 100 , after $\mathrm{I} S$ seconds it will be 56.5 ; after 45 seconds, 25.1 ; after I minute 35 seconds, I4. I, etc. The luminous power of phosphorescent colours, too, lessens quickly after the light impression, more rapidly in the first few seconds after insolation than later.

After the discovery of the X-rays, H. Becquerel, ${ }^{1}$ ) and simultaneously with him a number of other physicists, expressed the opinion that phosphorescent bodies under suitable experimental conditions may be made to emit dark rays capable of acting on the photographic plate after passing through opaque bodies, and, in consequence, to exercise the same kind of action as Roentgen-rays ${ }^{2}$ ).

This induced the author to make some experiments with a view to testing the possible biological effect of phosphorescent light.

Herr Hofrath Eder placed at the author's disposal for this purpose a standard light plate. This consists of a layer, enclosed within two glass plates, of the so-called Balmain luminous colour, i. e., of a mixture of sulphur compounds of calcium, barium and strontium, which emit an extraordinarily powerful and long-lasting phosphorescent light. (The duration of the brightness is only relatively long-cf. above.)

1) Fourth edition, Knapp, Halle, I896.

2) Compt. rend., I896, p. 420. 
To make the phosphorescent plate shine, $21 / 2 \mathrm{~cm}$. of magnesium ribbon was burned and the flame moved to and fro as close as possible to the surface of the glass. The plate then shone out with a very beautiful intense blue light in a slightly darkened room. As the luminosity of the phosphorescent substance lessens rapidly, the illumination with magnesium light had to be repeated every three minutes. A longer picce of magnesium ribbon was not burned, as the brightness gained by $2 \mathrm{I} / 2 \mathrm{~cm}$. could not be increased.

This luminous layer was brought às close as possible to a diffuse culture of staphylococcus pyogenes aureus on agar in a Pérri dish, a very shallow dish being chosen for the experiment, in which a threefold thick nutritive medium was poured.

A sheet of non-transparent paper, in which a cross had been cut out, was inserted between the culture and the phosphorescent plate. This was done to make it possible to distinguish between the growth of the irradiated and the non-irradiated parts.

For three hours the experiment was continued, the phosphorescent plate being illumined every three minutes with a piece of magnesium ribbon $2 \frac{1}{2} \mathrm{~cm}$. in length, and then laid again on the open dish. During the illumination with magnesium light the culture was, of course, put away in a dark place, so as to be kept unaffected by any action of the powerful chemical rays of the magnesium light. After 3 hours the culture was placed in the incubator.

The next day the nutritive medium was covered with a thickly packed eren growth, which showed no trace of variety of development.

The experiment was repeated in the same way for 6 hours but gave the same negative result, as did also a typhus culture with like treatment.

Phosphorescent light, therefore, of the intensity arailable for these experiments, has no influence on baclerial growth.

Quite recently $C$. Rolh has employed the light radiated by 
phosphorescent bodies in various affections of the cavities and passages of the body, and has, according to his accounts, obtained favourable results in cases of chronic nasal catarrh. Any result of this kind would appear from the above described experiments to be in no wise due, as Roth seems inclined to believe (Zeitschr. f. angew. Chemie), to a deleterious action of phosphorescent light on bacteria.

Looking back on the results of investigation thus far in the realm of phototherapy, we see that, thanks to the work of photo-chemists and physicists and thanks to the initiative of numerous medical men, we already possess considerable knowledge of the peculiar qualities and effects of light, and are in a position to turn this force of nature to practical therapeutic account. There remain, however, many great gaps in our knowledge which cannot be filled up without further hard work. Especially does it seem advisable in future, side by side with the close observation of all the circumstances under which the more carefully studied highly refrangible short-waved rays come into action (e.g., besides the chemical activity also the brightness of the light, the quantitative determination of the absorbed rays, etc.), to study also the manner in which the less refrangible long-waved rays act $(c \cdot g$., those that penetrate more deeply into the tissues), as well as the way in which various tissues and organs react to illumination with rays from this region of the spectrum. 


\section{APPENDIX TO THE TREATMENT BY X-RAYS}

In the endeavour to place the "dosage" of this treatment on a more exact basis, scveral writers have suggested auxiliary apparatus for denoting the amount of the radiant cnergy employed (the intensity of the X-rays). As has been pointed out (p. 245), Beclère, Gaston, Oudin and others recommend the spintemeter and radiochromometer. We have observed that neither of these instruments appears to be quite adequate, in the case of the spintemeter for the following reasons: The spintemeter is designed to give the spark equivalent to the degree of hardness of the tube. But no account is taken of the fact that various induction coils produce varying electric energies, which come into action in equal-sized sparking-distances. Consequently a Roentgen-tube of a certain vacuum, inserted in the equally large spark-gaps of induction coils of different construction will give rise to varying phenomena. If, $c . g$., we insert a vacuum tube, which in a roltohm coil of $25 \mathrm{~cm}$. sparking-range corresponded to a spark-gap of $71 / 2 \mathrm{~cm}$. into the spark-path of a Dessaucr apparatus (which, as is well known, gives more powerful secondary currents), we should have with the Dessaner apparatus a whole sheaf of sparks at a distance $(8 \mathrm{~cm}$.) across which, with the first apparatus, no spark could flash. The data given of the spintemeter hold good, therefore, for the apparatus of the experimenter concerned alone, not necessarily for other apparatus. In order to make the results of the individual apparatus available for all it would be necessary to give also the construction of the apparatus, and thereby make it possible to calculate the given values in terms of other apparatus - in any case a matter of some difficulty.

It is well known that the $X$-rays produce certain colours in many substances (Villard, Holzknechl). Holzknechl determines the amount of $\mathrm{X}$-rays absorbed by a certain salt (the 
composition of which is not given) by comparing the colour resulting in this body from irradiation with a standard scale of shades. From the intensity of the colouring, that is, from the corresponding number in the scale of comparison $(H)$ conclusions may be drawn, according to Holzknecht, as to the amount of X-rays absorbed, and, therefore, also as to the probable physiological effect of the same amount of rays.

In using the chromo-radioneter, the apparatus is placed on the part of the exposed surface of skin which is most adjacent to the tube-focus. The tube is either kept working at one sitting till the desired darkening effect is reached (this is determined by comparison with the standard scalc), or, where the treatment is to be extended over several sittings, the apparatus may be packed away in some non-transparent receptacle until the next sitting. If the sittings occupy more than 5 days, the irradiation may be pushed a little beyond the regulation "dose," since the skin recovers somewhat between the sittings. 


\section{N D E X}

A

Aberration, 378,379 .

Absorption of light, 380 .

Absorption of ultra-violet rays 388 .

Absorption-spectrum, 384 .

Accumulators, 27.

Accumulators, capacity of, 29.

Accumulators, charging of, 216.

Accumulators, discharging of, 218 .

Accumulators, power of, 29.

Acetylene-light, 475 .

Acne pilaris, 267.

Acne vulgaris, 237,300 .

Acne rosacea, 300.

Actinic rays, 293.

Actinium, 35I.

Actinometer, 375 .

Adrenalin, 494.

After treatment, 235, 278 .

Aigrette, II5.

Albedo, 373 .

Alopecia areata, 237, 272, 509.

Alopecia, persistent, 299.

Alopecia, pityroides, 275 .

Alternating currents, 48 .

Alternating currents of high frequency, 88.

- Alternating current machines, 49.

Ampère. 22, 32.

Ampèremeter, 39 .

Ampère-hour, 32, 46 .

Amplitude, 51.

Analgesia, 166.

Anæsthesia, I04.

Angle of incidence, 377 .

Angle of reflection. 377 .

Angström's unit, 383 .

Anode, 25, 188.

Anode-rays, 191, 229.

Anti-cathode, 202.

Anti-cathode, over-heating of, 202.

Arc-liglits. 43.

Arc-light lamps, 470, 486.

Are-light baths, 488 .

Armature, 50.

Athermanous borlies. 388 .

Augmenting lndex, 14.

Aureola, 117.

Auromin, 376 .

Auto-conduction, roo.
B

Bacteria, influence of Becquerel rays on. 355 .

Bacteria, elfect of electricity 011, 140.

Bacteria, effect of high-frepuency currents on, 126.

Bacteria. effect of light on, 401 .

Bacteria, effect of $\mathrm{X}$-rays on, 305,312

Balmain's luminous paint, 520 .

Bariun!, 351.

Battery, electric, 15.

Battery, galvanic, 24 .

Battery connection, 34 .

Battery poles. 24.

Biliary calculi, 304.

Bismuth, 351 .

Bipolar treatment, IOI.

Blepharitis, 27I.

Boloneter, 387.

Breaks. See Interrupters.

Bremer lamp, 487.

Brush-discharge, 1 I 6 .

Brush, faradic, 103.

Butterfly patch, 298.

\section{$\mathrm{C}$}

Cathode, 25, 188 .

Cithode rays, $159,187,23 \mathrm{I}$.

Cathode rays, nature of, I88.

Callus, effect of X-rays, 305 .

Calorescence, 385 .

Capacity, 7,12

Capacity of accumulators, 29, 32 .

Carcinoma, 294. 302 .

Cascarle-battery, 15 .

Channel rays, 191.

Charging, electrical. 5 .

Chemical action of light, 393.

Chemical action of X-rays, 194.

Clacnical action of Beconerel-rays, 352.

Chloropliyl, influence of Beccuerel-rays on, 350.

Chlorephy]-function, 396.

Chondros salfoma, 303.

Chromorationeter. 525.

Commutator, 4 \%.

Compressors, 4(1)3.

Concave-grating. spectrosenpic, 392.

Concentrating apparatus for are-light, 495. 
Concentrating apparatus for sunlight. Electricity, bound, I2. 508.

Condensation, Ioo.

Condenser, I2, 57.

Conductors, 3, I9, 20.

Constant-current machines, 49.

Corpuscles, $\mathrm{x}, 390$.

Coulomb, 32.

Coulomb's law, 6.

Crookes' radiating matter, I\&g.

Cimulative effect of $\mathrm{X}$-rays, $24 \mathrm{I}$.

Current closed, 32.

Current collector, 50.

Current interrupter, 64 .

Curve, chemical, 388.

Curve, height of, 388 .

Curve, thermal, 388 .

\section{$\mathrm{D}$}

D'Arsonvalisation, Ioo.

D'Arsonval's apparatus, 93, 95.

Dark cathode space, I88.

Decade resisting principle, 508 .

Deflection, 391.

Degeneration, vacuolisation, I54, 342, 503.

Density, electric, 6.

Depigmentation, 24i.

Dermatitis from Becquerel-rays, 359.

Dermatitis from high-frequency currents, $16 \mathrm{I}$.

Dermatitis from $\mathrm{X}$-rays, 337.

Dermatitis from $\mathrm{X}$-rays, prognosis of, 347.

Dermatitis from X-rays, treatment of, 347.

Dermatitis papillaris capillitii, 229, 237, 270.

Dermo lamp, 486.

Diabetes, I67.

Diathermanous bodies, 388.

Dieletric, I3.

Dieletric, constant, I3.

Diffraction, 39I.

Dioptric, 378 .

Discharge point, 6.

Discharge, caterpillar, I20.

Discharge, silent, I2O, $5 \mathrm{I} 3$.

Discharge, oscillating, I6.

Disconnector, 6o.

Dispersion of light, $28 \mathrm{I}, 377$.

Dispersion of light, abnormal, 390.

Distance, influence of, 235 .

Distance effects, xiii.

Drossel-tubes, 2 I 5.

Dynamos, 37.

Dyne, 7 .

Electricity, negative, 3 .

Electricity, positive, 3 .

Electricity, friction, 3 .

Electricity, static, I2.

Electricity, voltaic, I9.

Electric current, 8.

Electric current, magnetic effect of, 45 .

Electric equilibrium, I2.

Electrical oscillation, 87.

Electric spark, 13.

Electric meters, 46.

Electric vibrations, physiological effects of, I63.

Electro-dynamometer, 40.

Electrolysis, 25.

Electrolyte, 25 .

Electro-magnet, 49.

Electro magnetic theory of light, 87 .

Electro-motor, 52.

Electro-motive power, 9, 19, 22, 33.

Electrode for monopolar coil-discharges, I 49.

Electrons, xi, 26.

Electrostatic discharge, I 6 .

Electroscope, 6.

Electro-therapy, I62.

Elements, galvanic, 2I, 24 .

Elephantiasis, I77, 237, 301.

Energy, electrical, 7.

Epidermis, absorption of light by, 430 .

Epithelioma, 237. 39I, 5I I.

Erythema or Eczema solare, 415.

Erysipelas, chromotherapy in, 457.

Erythema from X-Rays, 254.

Etiolation, 396.

Exposure, influence of length of, 235. 245.

Extra current, 54.

Eye, effect of Becquerel-rays on the, 36I.

Eye, effect of light upon the, 436 .

Eye, effect of magnetic field on the, 184 . 3 IO.

Eye, effect of X-rays on the, 308.

\section{F}

Faradic brush, IO3.

Farad, I2.

Faradisation, 99, II4, 275.

Favus, 237, 263.

Field, electric, 8.

Field, magnetic, 7 .

Filtration of light, 384 .

Fissura ani, I79.

Fluorescence, I88, 385, 518 .

Fluorescent-screen, 202.

Focus-tubes, 202.

Folliculitis barbæe, 266, 267.

Foucault's currents, 56.

Foucault's interrupter, 68.

Focus of lenses, 379. 
Franklinisation, 114, 162, I72.

Franklin's plate, I4. 90.

Fraunhofer's lines, 386.

Furunculosis, 300.

\section{G}

Galvanic current, 2 I.

Galvanic current, heat and light effects of, $4 \mathrm{I}$.

Galvanization, 163.

Galvanism. I9.

Galvanometer, 38 .

Geissler tubes, 89 .

Generator, 52.

Gramme’s ring, 50.

Grating, 39I.

Growth movement, 397.

\section{$\mathrm{H}$}

Hair, loosening of, by X-rays, 253.

Hair, falling, causc of, 346 .

Heat rays, ix, 325,387 .

Heliotropism, 398 .

Henoque's method, 107.

Hertzian rays, 88.

Hertzian waves, 197.

High-frequency currents, 85 .

Hirschmann's electrolytic interrupter, 77.

Histological investigations, 152.

Hot wire instruments, $4 \mathrm{I}$.

Hyperesthesia, I04.

Hypertrichosis, 237, 276.

Hyper-ultra-violet rays, $x, 364$.

Hysteresis, 45.

\section{I}

Impeding-resistance, 64 .

Impotence, I73.

Incandescent light, 42.

Incandescent light baths, 466 .

Induced current, 48 .

Induction, 47.

Induction, electro, 52 .

Induction, electro-static, I 2.

Induction-spark, microbicidal action of, $5 \mathrm{I} 3$.

\section{Influence, 7 .}

Ions, 26.

Ionisation, 196.

Intensity, chemical, of sunlight, $45 \mathrm{I}$.

Intensity, chemical, of artificial lightsources. 464.

Intensity, chemical, of electric current, $22,32$.

Intensity, chemical, of X-rays, determination of, $240,245,247,525$.

Intensity, chemical, of X-rays, influence of, 235,245 .
Interference of light. 391.

Interruption, influence of rate of, 247 .

Interrupters, 57.

Interrupters, electrolytic, 59, 7\$, 75, 77. 79. 80 .

Insulators, 4 .

Invisible discharge. I17.

Irritability movement, 397.

Iron arc-lamp, 405, 485, 486, 509.

\section{$\mathrm{J}$}

Jar residue, $\mathbf{1 5}$.

Joule's law, 4I.

\section{$\mathrm{K}$}

Katoptrik, 377.

Keratitis, light treatment of, 464 .

Kohl's electrolytic interrupter, 75 .

\section{$\mathrm{L}$}

Latent period after light-treatment, 4I7.

Latent period after X-ray treatment, 3I 2.

Lead, radio-active, 352 .

Lead fuses, $4 \mathrm{I}$.

Lenard rays, I90.

Lenses, 378.

Lenz's law, 56.

Lepra, 237, 294.

Levy's interrupter, 70 .

Leyden-jars, 15 .

Lichen ruber planns, 298.

Light cathode band, I88.

Light-power of the sum, 450.

Light, 360 .

Light, stimulating action of, 409, 4 I5.

Light, destructive action of, 397.

Light-filters, 407.

Light and air-baths, 454 .

Light-sources, 470 .

Light-strength, 372 .

Light-rays, 372 .

Light, electro-mangetic theory of, 370 .

light, emission theory, 3(n).

Light, nurlulatory theory. 369 .

1.ight. deflection of. 391.

Liglit, refraction of, 37 s.

Lines of force, magnetic, 8 .

L.mminescence, 517 .

Lupus erythematosus, 177, 237, 298. 364. $51 \mathrm{I}$.

Lupus vulgaris, 236, 240, 28!, 364, 498.

\section{MI}

Nachines, electrical, 17 .

Magnetic field, ploysinlogical effect of, 120.

Masks, 260.

Nagnetic iuduction, fis. 5.3 .

Massige, electrical, 177.

Measles, chromutherapy in, 457. 
Measurement of electric current, 38 .

Measurement, technical, 32.

Mechanical concussion, I61.

Mercury interrupter, 66, 7I.

Metabolism, influence of ligh-frequency currents on. Io6.

Metabolism, influence of light on, 439 .

Metabolism, influence of $\mathrm{X}$-rays on, 305.

Meters, 46 .

Moist chamber, 407.

Monopolar coil discharge, I43.

Monopolar voltaic current, I8I.

Morton's currents, 85 .

Mucous membranes, 174 .

Multiple-phase currents, $5 \mathrm{I}$.

Mycosis fungoides, 237, 294.

\section{$\mathrm{N}$}

Nævus flammeus, 237, 301, 364 .

Nrevus pigmentosus pilosus, 229.

Nevus vascularis, with light-treatment, 5 II.

Nails, changes in, after X-rays, 340.

Neef's hammer, 65 .

Nervous system, influence of d'Arsonvalisation on, Io9.

Nervous system, treatment of, 172.

Nerves, influence of $\mathrm{X}$-rays on, 305.

Neuralgia, 303.

Normal candle, 373 .

Normal candle lamp, 373 .

Nutation, 398.

Obesity, I67.

Ohm, 30, 34.

Ohm's law, 33.

Opening-spark, 57.

Optical axis, 377 .

Optogram. 437.

Osmosis, 215 .

Osteoperiostitis, 305 .

Ozone, 325.

\section{$\mathrm{P}$}

Parallel connection, 37.

Peletier's jars, 396.

Pemphigus foliacetss, 298.

Penetrator, 203.

Period, 57.

Peritonitis, tubercular, 304.

Permeating electricity, I82.

Phase, 5I.

Phosphorescence, 188, 385, 520.

Photometer, 374 .

Phototaxis. 478 .

Pigment changes from X-rays, 252.

Pigmentation after light treatment, 420.

Pitchlblend, 35I.

Plate, collecting, 12.
Plate, condensing, 13 .

Plate interrupter, 8I.

Platinum interrupter, 79.

Platinum, deposit on Roentgen-tubes, 202.

Plant seeds and X-rays, 320.

Polarisation of current, 26.

Polonium, 35I.

Pole-finding, 217.

Potential, 6, 8, 10.

Potential-difference. 9.

Primary coil, 52.

Protozoa, action of X-rays on, 320.

Prurigo, 298.

Psoriasis, 237, 296.

Quantity, 7, I2.

\section{Q}

\section{$\mathrm{R}$}

Radiochrometer, 206, 245.

Radium, 351.

Radium-rays, 190.

Ramification of current, 36.

Rays, short-waved, 383 .

Red-room, 456.

Reflex-excitability, influenced by X-rays, 305.

Reflection of light, 377 .

Refraction, 378 .

Resistance, 30 .

Resistance, ballast, 3 I.

Resistance, specific conducting, 30.

Resistance, unit of, 33 .

Resistance, external, 34 .

Resistance, internal, 34 .

Resonator, Hertz's, 92.

Resonator, Oudin's. 95.

Resonator, bipolar, Rochefort's, 96 .

Rheostats, 30.

Rheostat lever, 3 I.

Rheotome, 57.

Rheumatism, 304.

Roentgen-apparatus, installation of, 219 .

Roentgen-apparatus, depreciation of, after use, 227.

Roentgen-rays, I87.

Roentgen therapy, 229.

Rotary current, 5I.

Ruhmkorff coil, 57 .

\section{$\mathrm{S}$}

Sarcoma, 294. 302, 303.

Scarlatina, chromotherapy in, 457 .

Schulmeister's interrupter, 69.

Sclerodermia after X-rays. 279.

Sclerosis, with light-treatment. 507, 512.

Scrofuloderma, 290.

Secondary coil, $27,5^{2}$. 
Seeds, germinating power of, 355 .

Selenimm, 376 .

Self-induction, 54 .

Self-induction, co-efficient, 55 .

Self-potential, 55 .

Sensitometer, 375.

Shimmering liglit, I 7 .

Short current, 27.

Short-circuiting of coils, 227.

Shunt circuit, 38 .

Siemens' double-T-magnet, 49.

Siemens' dynamo, 49.

Siemens' interrupter, 8o.

Simon-interrupter, 79.

Sinusoidal-currents, 104 .

Skiameter, 206.

Skin, atrophy of after X-rays, 278 .

Skin diseases. 174.

Sleep-movements, 398 .

Solenoid, 45.

Sound waves, 200.

Spark-discharge, stimulating action of. I2I.

Spark-discharge, delay of, 353.

Spark-gap, 64.

Spark inductor, 56.

Spectroscope, 383 .

Spectrum, 38I, 386.

Spectrum, cold-lines in, 387 .

Spintemeter, 345. 525.

Staphylococcus, 3 I 6.

Subjective phenomena after X-ray irradiation, 255.

Sun-baths, 453.

Sulphate of lead, 351 .

Sycosis, 237, 266.

Sycosis parasitaria, 271 .

\section{$\mathrm{T}$}

Telangiectasis after X-rays, 279.

Tension, terminal, 37.

Tension, surface, 7 .

Tension connection, 15 .

Tesla-currents, 89 .

Therapeutical application of high-frequency currents, 165.

Thermo-clectric current, 44.

Thermo-pile, 217.

Thorium, 35 !.

Thrombosis, zor.

Transformer, Tesla's, 88.

Transparency of tisslles, 425 .

Trancverse vibration, 197.

Trichotylosic, 262, 271 .

Tropho-neurotic theory, 334.

Tuberculirles, 280 .

Tubercular ulcers, zyo.

Tuberculosis of bone, healed under Xrays, 304
Tuberculosis with light-treatment, 45., $5+2$.

Tuberculosis of lings, treatment by $\mathrm{X}$ rays, 305.

Tuberculosis treated by high-frequency currents, 170 .

Tubes, Roentgen, degree of hardness of, 205.

Turbine, mercury interrupter, 69, 72, 73.

\section{$\mathrm{U}$}

Ulcus perforans, 291, 295.

Ulens, rodens, 291.

Ulerythema sycosiforme, 267.

Ultra-red rays, 383

Ultra-violet rays, 325,383 .

Ultra-violet rays, discharging properties of, 195, 390 .

Ultra-violet light, 190.

Unit of resistance, 33 .

Unit of electro-motive force, 33 .

Unit of current capacity, 33 .

Unit of light, 373 .

Uranium salts, $35 \mathrm{I}$.

Urticaria pigmentosa, 301 .

\section{V}

Vacuolisation, 155.

Vaciulm. 205.

Vacuum penetrator. 203.

Vacuum, Müller-Uri, 203.

Vacumm, Voltohm, 204.

Vacumm, MI. Levy, 204.

Vacuum, Dessaner, 204.

Vacuum, with water-cooling, 204.

Vacuum-tubes, 201.

Vacuum-tubes, adjustable, 208.

Vacuum-tubes, regulation of, 208.

Vacuum-tubes, arrangement of, 224.

Vacunm-tubes, importance of condition of, 242.

Variola, cliromotherapy in, 457 .

Verruce, 30I.

Visceral compiications after X-rays, 307 .

Vitiligo, 420.

Volt, 9, 33.

Volta inditction, 52.

Voltuleter, 39.

Voltholn-tubes, 204.

Wehnelt's interrupter. 59.

Wheatstone's bridge, 37 .

Whirl currents, 56.

Wind, electric, 7,113 .

Work of electric current, 22, 33. 
X

X-ray, stimulating effect of, 320 .

$\mathrm{X}$-rays, elective action of, 284 .

X-rays, the nature of, 196 .

$\mathrm{X}$-rays, penetrating power of, 187.

X-rays, physical properties of, I88.

$\mathrm{X}$-rays action on fluorescing bodies, 187 I9I.

X-rays action on photographic plates, I87, 192.
$\mathrm{X}$-rays action with varying distance, I92, 196.

$\mathrm{X}$-rays, chemical action of, 194 .

$\mathrm{X}$-ray apparatus installation, $2 \mathrm{I} 5$.

$\mathrm{X}$-ray method of treatment, 243 .

Z

Zeemann's phenomenon, 39r. 


\section{LIST OF AUTHORS}

Abney, 385, 386, 466, Baudet, I78.

$483 . \quad$ Baudlonin, 500, 509.

Abraham, P. S., 300. Baxendell, 450, 452.

Aducco, 443 .

Afanasjew, 351 .

Akopenko, 459.

Bazy, 304.

Beard. II 4 .

Beauregard, 3 I3.

Albers-Schönberg. 77, Béchard, 442.

$205,248,259,266,269$. Bechderews, 427, 44j. $282,286,289,296,327$.

Ames, 325.

André, 486,

Andresen. 376 .

Angelucci, 437.

Anthony, IV. A., I87.

Apery, 472.

Apostoli, 107. 140, 166, $168,326.348$.

Arago, I 19.

Arloing, 402.

Arnold, 4I4.

\begin{tabular}{l|l} 
Aron, 46. & Benedikt, 166, 248, 280. \\
A ronstam, N. H. 200. & Benkert, 457,
\end{tabular} 459 .

Beck, 3I3. 403.

Beck, C., 302.

Beclard, 409.

Béclère, 245. 525.

Becquerel, 44. 190, 35 r, $361,385,464,520$.

Bédard, 30.4.

Beer. B.. I84. 310.

Behrend, 29, 252, 279.

Beijerinck, 409.

Bclow, 468, 472, 509.

Benedikt, I66, $248,280$.

d'Arsonval, 79, 90-95, Benoist, 206, 245, 257.

I00, I04-II3, I2I, I25. Berg, Otto, I9I, 231.

132. 140, 167, 22.3. 310, Bergman, 248. 328, 387, 402, 438. Bergonié, 304.

Aschkinass, xy, 199, 319, | Berliner, 42I, 449, 456. 354.

Auerbach, 412.

Aufrecht, 468, 473.

Ausset, 304.

Axenfeld, 308, 322.

Backman, 457.

Baedeker. 104. I08-1 10, 167,173 .

Bagge, Ivar, 290.

Balmain, 520 .

Balthasard, 326.

Balzer, 279.

Berlioz, io6, 313 .

liernard, 245.

Bert, 4I3, 4IS. 445.

Berthelot, I65, 352, 513 :

Berthold, 4ro.

Bertin, 308.

Berton, 313.

Besnier, 267.

Beurmann, 494.

Bidder, $4+2$.

Bie, $405,443,445,457$, 508.

Bier, 502.

Bang, 404-407, 449, 463, 485. 499, 50I, 508, $51 \mathrm{I}$.

Banister, 239.

Barbensi, 5 I2.

Bardet, 309.

Barlow, 449, 456.

Billings, 403.

IBiné, 460 .

Bisserié, II2, I74-I77. I $80,248$.

Black, 449.

Jlaikice, 3I 3 .

Barthélemy, 174. 248, Blaise, 313. $256,279,281,307,334$.

Bary, 352 .

Basch, I I0, I14.

Batelli, 200.
Boczar, 250, 266.

Bceder, 408, 472.

Boesè, 28.

Boisseau du Rocher, I73.

Bois-Reymond, 419.

Boll, 437.

Bollaan, 174.

Bolleau, 292.

Bolton, I 40.

Boltzmann, 197.

Bonetti, 95.

Bonome, III, 140, 313 .

Bordier, I74, 335, 509 .

Bordin, 400.

Borrisow, 439.

Bouchacourt, L., 85, 115.

Bouchard, I66, 422.

Bowles, 324. 422.

Brahams, Pl.., I83.

Brandes, 309.

Bremer, 48I.

Broca, 239. 486.

Brooke, A. G., 294.

Brocq, 174-177, 506.

Brown-Séquard, $44 \mathrm{I}$.

4I4,

Brücke, 4IS.

Buchner, 403, 408.

Budge, 4I4.

Büdinger, Th., 4I4. 437.

Büttner, I87.

Bukofsky, 266.

Bum, I67.

Bumsen, 24, 375, 464.

Burci, iqo.

Burgsdorf, 508, 511.

Burnett. 413.

Caffarena, i 10.

Cailletet, 118.

Calderone, 265.

Caldwell, 79.

Cimp de la, 294.

Campenon, 305.

Camus, L., 447.

Candler, 472.

Capranica. 305.

Capriati, 164.

Caprioni, 518.

Carmichael, 195.

Carré, 17. 
Carvalho, I09, II4.

Caspari, W., xv, 3I9. 357.

Cattani, 404.

Cauchy, 382 .

Chabaud, I93, 215.

Chabry, 174.

Chalupecky, 309, 31 I.

Chamberlain, 292.

Chanteloube, 304.

Chatzky, I64.

Charcot, 44 .

Chardonnet de, 389 .

Charpignon, 448 .

Charrin, I IO, I40, 402.

Chassanowitz, 440.

Chatin, 486, 500, 509.

Chatinière, 457 .

Chaves, Br., $27 \mathrm{I}$.

Chmiliewsky, 402.

Ciechansky, 454.

Cohen, 4I4.

Cohn, T., 3, 108, I10, I40, I68, 173.

Clark, 33, 289, 302.

Claudet, $45^{8}$.

Cleaves, 472.

Colardeau, 207.

Colleville, 295, 465.

Colombo, I8I, 472.

Conrad, 238.

Corrado, 158.

Cornu, 383. 389 .

Coudres, Th. des, I99.

Cooks, E. H., 5I3.

Crookes, I89, 197, 208, $35 \mathrm{I}$.

Cruet, 167.

Curie, 35I, 36I.

Daitsch, J., 44I.

Dandrieu, 402.

Daniel, J., 24, 238.

Danielewsky, B. J., I64.

Danlos, 363.

Darbois, 426.

Darieix, 309.

Darier, 307, 310, 340.

Davenport, I6I.

Davy, 479.

Denoyés, I07.

Debierne, 351.

Deprez, 65.

Descamps, 304.

Despeignes, 302 .

Dessaignes, 426.

Dessauer, F., 29, 63-66, $204,215,245,250,525$.

Destot, II5, I4I, 304.

Dewar, 386.

Didsbury, I67.
Dieudonné, 403-408.

Dobereiner, IIS.

Dogel, 444.

Donath, B., 34, I87, 200, 221.

Dore, E., 506, 508.

Dorn, 354.

Dort, van, 290.

Doumer, 85, IO4, I IO, I68, I80.

Doutrelepont, 288 .

Downes, 402.

Drigalsky, 473 .

Drossbach, G. P., 389, 405,484 .

Drummond, 388 .

Dubard, II5, I4I, 304.

Dubois, III.

Ducastel, 290, 304, 497, 509.

Duclatux, 402 .

Ducretet, E., 95, 208.

Dumstrey, 280.

Dupré, 437.

Dworetzky, 408, 443, $459,463,478$.

Ebert, H., I88, I90.

Eder, J. M., I87, I92, $205,230,324,335,356$, $369,375,386,394,425$, $450,458,462,465,483$. 521 .

Edwards, W., 409.

Ehlers, 508.

Ehrmann. S., i76, 237, $243,253,270,275,280$, $294,338,348,419,477$, 502, 509.

Egau, P. R., 290 .

Einthofen, I05.

Eisenlohr, 383.

Eijkman, P. H., 302.

Eliot, 324.

Elster, 35I.

Engel, 457.

Engelmann, 161, 403 , 4 II, 437.

Ernecke, 65.

Escherich, 304.

Esselbach, 383.

Esmarch, v., 403, 408, Gatschowsky, 478. 5I2.

Eulenburg, 439.

Exner, F., I9.

Exner, S., xiii., 386, 4 I9.

Faraday, 25, 48, 92, 105 , I 13 , I 16.

Fatigati, 402.
Faure, 27.

Feddersen, 16.

Feilberg, 457.

Feldman, 482.

Feré, Ch., $184,443,460$.

Ferguson, 293.

Festner, 457

Finsen, U., 25, 48, 92, $105,275,284,369,405$, $4 \mathrm{IO}, 4 \mathrm{I} 7,42 \mathrm{I}, 426,43 \mathrm{O}$, $438,449,455,458,463$, $478,483,488,492,498$, 50I, 506, 509, 512.

Fiorentini, 3I3, 316.

Fizeau, 57, 458 .

Flammarion, 397.

Flemming, 176 .

Forchhammer, 505, 508, 5 IO.

Forster, 239, 24I, 312.

Foucault, 47, 56, 66, 68, 458.

Foveau de Courmelles, 85, I69, I86, 239, 245, 290, 310, 325, 327, 449, 474, 490, 496, 509 .

Frankenhäuser, F., 369.

Franktin, 90, 94, II9.

Frantzius, 313.

Fraunhofer, 383, 386.

Friedenthal, I40.

Frister, 230.

Froscani, I 40.

Fubini, 440.

Fuchs, E., 437.

Fuchs, S., 3Io.

Gadean, 279, 364.

Gadneff, 426 .

Gaiffe, I2I.

Gaillard, 403.

Gaisberg, S., v., 48I.

Gaudil, I7I.

Garbaso, 200.

Gassiol, I88.

Gassmann, xiv., I55, 206, $243,247,252,269,287$, 289,341 .

Gastou, I 74, 250, 257, 266, 269, 306, 500, 509, 525 .

Gatti, 3 Io.

Gaugain, I I7, I I9.

Gaulé, J., 443.

Gautier, I06, I78, 300.

Gebhard, 309, 369, 408, 426, $440,466$.

Geissler, 89, I88, 402, 517.

Geitel, 352. 
Genderen, 437.

Genoud. 3I3, 315. 449. 496, 509.

Geyser, 289.

Giesel, 352-359.

Giesson, 152 .

Gilchrist, 323. 340.

Gilmann, Joln G., 302.

Gintl. 198.

Giunti, 403.

Glatzel. 495.

Glebowsky, xiii. $4 \mathrm{I} 6,449,501,504$.

Gocht, I87, 252. 281, 289, 327.

Godnew, 4IO. 44I, 443.

Goethe, 444, 460 .

Görl, 449, 514.

Goldstein, xv., II4. 189. $336,352$.

Golgi, 159 .

Gorbazewicz, 442.

Gottstein, 140.

Graber, 413.

Graetz, L., 38 I, 48 I.

Graf, Ch., 183 .

Graffenberger, $439,+42$.

Greiner and Friedrichs, 207.

Grenee, 183.

Gross, 313 .

Grouven, 243, 250, 263, $269,280,287,289,358$.

Grunmach, 3, 280, 289 . $295,303,313,362$.

Guarinoni, 409.

Guelcher, 28, 44.

Guichard, 309. 313 .

Guillaume, ro7.

Guilloz, Th., 108, I 68 .

Gundelach, 207, 213, 245. 316.

Gyllenkreutz, 440.

Hagen, 28.

Hahn, 237, 248, 266, 269. $280,286,296$.

Hall-Edwards. 250, 280 .

Haller, III.

Hallopeau, 279, 364.

Hallwachs, 389 .

Hammer, 422, 448 .

Hammond, $4+2$.

Hankel, 462.

Harless, 414.

Harringtom, 412 .

Harris, IIs.

Hartley, 380 .

IIavas, 250 . 28 r.

Hegger, 4.37

Helmbolt\%, xi.
Hemptinne, A. v., 194.

Henocque, 107.

Hermann, 184. 421.

Herschel, $387,+58$.

Hertz, H., x, 87, 92, 189 . 199. $370,390$.

Ilertzog, 482 .

Hess, C., I57.

Himmel, 286, 289, 3 I I.

Himstedt, F., I 20, 352, $36 \mathrm{I}, 437$.

Hirschmann, IV., 72, 77. $212,245$.

Hittorf, IV., I89, 213, 230.

Hoilge, 157.

I Iofmann, K. A., $35 \mathrm{I}$.

Holetschek, 452.

Holland, 290.

Holmgren, 4.37, 440.

Holzkneclit. G., 190. 237, 273, 313. 319, 334, 445, 525.

Hoorweg, L.. 105.

Hoppe-Seyler, 4 is, 438 .

Hortatler, 427.

HIuber, A.. 492.

Hueter, 282.

Huggin, 386.

Humphreys, $39 \mathrm{I}$.

Hurtado, (i.. 512.

Huyghens. 369 .

Hummel, 41 .

Ishewsky, ${ }^{8} 83$.

Jackson, 202.

Jacquet, I77. 509.

Jackissohn, 290, 508.

Jaksch, $\therefore$. 460.

Jankall. 326, 335.

Janowski. 402.

Jagnot, 177.

Jatumann, xr, 353.

Javal. 362.

Jegorow, 444.

Jellinck, S., 158, I60, 165.

Jency. 200.

Jersild, O.. 509.

Johnuon, 20)2, 302.

Johnston. Jannes C., 279.

Joseph. Il.. xiv, $24,3,32$ I.

Joscph, Max, 502 .

Jones. 13., 24)0, 4.37.

Josing. E... for.

Joll-4et. 408.

Jutitsy, 2.37. 248, 260 , tallis: 107.

$277,281,299,295,300$, Lacatille, 107.

340 .
Kahane, Max, 171, 173.

Kaiser, G., 178, 230, 250, 259. 479 .

Kalischer, 187.

Kaposi, 236, 250, 268, $270,299,323,335$.

Kattenbracker, 468, 472 , 509.

Kaufmann, x, xiii, 196, 199, 206, 354.

Kayser, xv, 3, 369, 386.

Kellog, 449, 466, 468, 489.

Kelvin, Lord, 102, 197.

Kessler, 478.

Kibbe, 340 .

Kienböck, 205, 237, 242, $248,257,272,275,28 \mathrm{I}$, $305,312,319,329,338$.

Kime, 427. 494.

Kindler, $169 . .174$.

King, E. E., 238 .

Kirchhoff, G., 86, 87, 38.3. 386.

Kiribuchi, 157.

Kirmisson, 290, 304.

Kistiakowsky, xiv.

Kjeldsen, 486. 509.

Klemm, I56, 161, 402.

Klemperer, 1 to.

Knox, 290.

Koch, 40.3. 502.

Körner, F., 3, 369.

Kogan, B., 4tI.

Koht, Max, 99, I21, 223, 250.

Kohlrausch, 20.

Kolle, 238.

Kondratiew, 408, 473.

Kopp. 229.

Kotliar, 402.

Korybut-1)askiewicz, 157

Kreill, A., 3io.

Kromayer, 509 .

Krohn, 457.

Krüger, 140.

Krukenberg, 457.

Krisse, 402, to5.

Kiilune, 156,436 .

Kïmmel, 237, 282, 289, $327,507$.

Kurella, H., 85. 96, 106 , IC4.

Kurlliaum, F., 204.

Kutschuck, 47.3 .

Kumitzky, I8.3.

Lalbarseli-Ostertag, 140.

Libman11, 449. 
Lamberts, 157. . $\quad$ Mach, L., 494.

Lampa, A. V., ix, x, 3, Macintyre, 238. 39I, 52I. Mackenzie, 408.

Lancashire, G. H., 269, Macleod, M. H., 5I I. 28I, 285, 290, 293. Nagini, I57.

Lancaster, 304, 305.

Lang, E., 502.

Langley, $387,45 \mathrm{I}$.

Lapinski, 290.

Laquer, 474.

Laquerrière, I40.

Larsen, A. L., 376, 403, $483,508$.

Lassar, O., 369, 508.

Laulanie, IIO.

Lazat, I06.

Leaming, 4I 2 .

Lebon, 506, 509, 510.

Lecercle, 306.

Leclanché, 24.

Lecher, E., xv, 3,87, I20.

Leeds, 483 .

Ledond-Ledard, 403.

Leduc, St., 85, I I0, 5 I6.

Lemström. I65.

Lenard, Ph., I89, 19I, I99, 390.

Lengyel, v., 352.

Lenz, 49, 56 .

Leonard, Lester, 210, 326.

Leredde, I77, 507, 5II.

Leroy, L., 72.

Lesser, 502, 508.

Lewandowsky, II4.

Levack, J. B., 293.

Levy, Max, 63, 70, 7I, $74,79,204,2 \mathrm{I} 2,215$.

Levy-Dorn, 280.

Leyden, I5, 513.

Liebermeister, 454.

Liese, 509.

Lindholm, 457.

Lion, V., 257, 266, 269, $27 \mathrm{I}, 282,289,300,338$, 342.

Llaberia, 300.

Lodge, I97.

Loeb, 395, 4IO, 4I3, 442

Locbel, 448.

Löwentlal, 439, 47 I.

Loewy, A., I08, i I0, 168.

Londe, A., 72, I87, 20I, 203.

Lopriore, 320 .

Lortet, 313, 3I5, 449, 496, 509.

Luebbert, 402.

Lugaro, 158.

Luraschi, 313.

L'Orosi, I4I.
Maier, M., 36 I.

Majorana, I95.

Makawejew, 478 .

Maklakow, 4I6, 423.

Maldiney, 320 .

Mann, I 58.

Mangin, 378 .

Maragliano, V., I05.

Marangoni, 193.

Marcuse, J., 447.

Marcuse, W., 229, 238.

Marmier, I4I.

Marti, 439.

Martin, 449.

Martinaud, 403.

Martre, I07.

Mascart, II8.

Masch, C., $45 \mathrm{I}$.

Masson, II9.

Maudityl, I 44.

Maxwell, Clark, 87, 92, 370.

Mehl, 449, 46r.

Meidinger, 24.

Mendelsohn, I40.

Meril, 292, 302.

Merk, L., 250.

Méry, P., 305.

Meyer, St., 352.

Michelson, 200.

Mies, J., 239.

Minck, 313.

Minich, K., 277.

Minin, $472,478$.

Mizuno, T., 58.

Mohler, 391.

Möller, M., I87, 29I, $324,369,415,419,432$, $440,449,468,490$.

Moleschott, 4I9, 440, 448.

Momont, 403 .

Monckhoven, 464 .

Mongour, 304.

Monseaux, 279.

Norgan, 293.

Morris, M., 506.

Norrow, P. A., 290.

Morton, 85, 293.

Mosso, 443 .

Moutier, I09, I68.

Mülısam, 313, 316.

Mïller, I22, 187.

Müller, C. H. F., 208, 395.

Müiller, E. K., I82.
Müller, G. J., 250, 3Iо, 494, 501, 502, 51I.

Müller, H., 4I4.

Müller-Pouillet, 3, 369.

Mïller-Uri, R., 203.

Muraoka, ix, 200.

Mygind, 457.

Nagel, W. A., 36r, 487.

Narkiewicz-Jodko, I8I.

Neisser, 283, 288, 502.

Neef, 65, 67, II9, 220.

Nesnamow, E., 463.

Neumann, I. V., 85, I76, $266,274,285,289,300$.

Newman, 289.

Newton, 370 .

Nicolou, 257, 270.

Niels, 369 .

Nikolski, I72.

Nissell, I59.

Nobele, J. de, 290.

Norley, 5 I7.

Novak, V., I92.

Ogneff, 4I6, 436.

Oleinikow, G., 460.

Oppolzer, E. v., 45I.

Ortt, 200.

Ott, I49.

Otterbein, 449, 46I.

Oudin, 85, 92-IO4, I IOII3, I32, I67, I74, 223, $248,256,28 \mathrm{I}, 307,31 \mathrm{I}$, $328,334,339,348,364$, 525 .

Pacinotti, 355.

Palaz, 48I.

Paltauf, 49I.

Pansini, 402.

Parker, 4I3.

Parville, de, 4I4.

Pautier, 457.

Payne, 296.

Pearsons, I74.

Pease, D. H., I40.

Pech, van, 44I.

Peekham, 403.

Peletier, 396.

Perdu, 454.

Perntner, 45I.

Perpens, 437.

Perrin, I96, 199.

Petersen, v., 508, 5I I.

Petri, I29, I35.

Pettenkofer, v., 408, 44I.

Pfaundler, 3 .

Pfeffer, I56.

Pflüger, 105. I 57, I 84 , I88, 436. 
Phisalix, I Io.

Piacentini, 440, 442.

Pick, Ph. J., 253, 434.

Pickering, 450 .

Picton, 449, 456.

Piorry, 449, 456.

Planté, 27.

Platten, v., 442.

Pleasanton, 461.

Plücker, I84, I88.

Poey, 4 Io.

Poggendorff, 63, I84.

Pokitonoff, 237, 300.

Pollak, 28, 8I.

Poncet. 448, 454.

Potonié, 395 .

Ponza, 444, 460 .

Porcelli, 355 .

Porter, 205, 208.

Pott, 313, 44I.

Pollitzer, J., $27 \mathrm{I}$.

Prausnitz, 408.

Pringsheim, 396, 401.

Prochownik, I 40 .

Provazek, S., xiv, 243, 321.

Pscheidl, IV., 3.

Pugh, 293.

Puluj, I98, 213. 215.

Purkinje. 3Io.

Pusey, 187, 250, 281, 289. 293.

Queen \& Co., 208.

Quénisset, 302, 306.

Querton, L., I08.

Quincke, 440.

Raab, O., 5 I9.

Radiguet, 59, 95.

Radman, G. H., 290.

Radzikowsky, I64.

Rasch, E., 487.

Raspe, 402.

Raum, J., 369, 416, 440, 443, 461 .

Realé, 108.

Regnier, I67.

Reichenbach, Baron, 184, 460.

Reid, 323, 326.

Reinhardt, 414.

Reiniger, 3I, 7I, 85, 250, 486.

Rémond, A., 85, II5.

Rendu, 304.

Renzi, de, 108, 473.

Reyn, 4 I9.

Revillet, 326 .

Richardson, 403, 404.

Ricliarz, 73.
Richer, P., 239.

Ridolfi, II 9.

Riecke, E., 330, 360.

Ricder, 174, 3I3, 327, $369,454,467,473$.

Riehl, 238.

Riess, I 19.

Righi, 195.

Rineliart, J. F., 293.

Rivière, 17I, 306, 313.

Rochefort. O., 96-98.

Rockwell. I I4.

Rodari, I82.

Rodet, 308 .

Röntgen, I91, 196, 201, 507.

Roiti, 196.

Rona, 289.

Ronchi. 44I.

Rood. 5 I3.

Roscoe, 45o, 464.

Roth, C., 523 .

Roth, M., 469.

Ronillies, 304.

Ronvière, I07.

Roux, I57, 403.

Rubens, $x$.

Rubenstein, 286.

Rühlmann, II9.

Ruhemann, 403, 472, 481 .

Rulumer, 57.

Rulumkorff, 79 .

Rirotte, 245.

Rusconi, $\& 16$.

Ryn, 508.

Sabouraud, 274, 508.

Sabrazés, 2I3, 306.

Sack, A., 50I, 504.

Sagnac, I95.

Sainton, 304.

Salomon, 279, 346.

Salmonsen - Wertheim, 59.

Sambuc, 3т3.

Santori, 402.

Scharling, 44I.

Schandinn, xiv, 320.

Schanm, K., 392.

Schein, 250, 254, 257, 264, 301, 348, 506.

Schell, 289.

Schenk, 403.

Schenkel, 206, 247, 252, $260,289$.

Schickharelt, 403, 408.

Schiff, E., 175, 178, 230, $237,240,250,266,27 \mathrm{I}$, $275,280,289,293,509$.

Schmirl, Ch., 250, 289.

Schmidt, G. B., I90, 230. Stembo, 179, 303.

Schnetzler, 409.
Schmidt, H. E., 442, 499, 501.

Scholtz, 237, 248, 252, $257,263,269,282,288$, 294, 312, 316, 329, 342, 502.

Schouli, E., 457.

Schrötter, v., 464.

Schuckert, 378, 489 .

Scliüler, Th., 457.

Schiirmayer, 259.

Schulmeister, L., 69, 149, 474.

Schumann, V., 389, 494.

Schultz, 242, 3I 3, 403.

Scliweidler, E. v., 353.

Secchi, 484.

Sederholm, 280, 289, 295.

Secbeck, 52I.

Seifert \& Co., 223.

Seguy, G., 207, 302, 306.

Sehrwald, I93.

Sella, 195, 238, 252.

Selmi, 440, 442.

Sequeira, 29I, 508, 511.

Serapin, 501, 503.

Sliarpe, 28o, 289, 295, 300.

Sholefield, 289.

Siemens, IV., 49, I2I, 485.

Sigaud de la Fond, I14, 223, 250.

Simon. 79, 80, 8I.

Simony, 451.

Sinapius. 304.

Sjögren, 237, 280, 289, 290, 295.

Smirnow, I40.

Smith, 289, 293.

Sokolow, 303.

Solucha, 427.

Sonnenburg, 289.

Sorel, 237, 30I.

Soret, 30I.

Sormani. 3I3.

Southgate, Leigh, 304.

Späth, I 40.

Spasski, N., roo.

Spiegler, 266, 269, 500.

Spicler, 313.

Spilker, II4, I40.

Spitaler, 452.

Squance, J. C., 290.

Stalil, 400 .

Starke, H., 195.

Startin, J., 28I.

Stcin, v., 478.

Steinach, 414. 
Stenbeck, 237, 289, 290.

Sternthal, 359.

Stokes, 462.

Stoney, xi, I99.

Storer, 462.

Strandgaard, 457.

Strätter, 242, 329.

Strassburger, 4I4.

Strasser, 469, $47 \mathrm{I}$.

Strauss, 177, 35I.

Strebel, 358, 363, 369, $404,425,428,439,449$, $463,468,469,47 \mathrm{I}, 473$, 489, 499, 509, 5I2, 5 I 7.

Stricker, 49I.

Sudnik, R., I7I, I79.

Sule, I92.

Swendson, 457.

Tänger, 346 .

Tappeiner, H. v., 5 I9.

Tarkhanoff, 306.

Taylor, 293, 300.

Tesla, N., 86, 88, 89, 90, I08, I 20.

Thalen, 323, 328, 386.

Thayer, 449, 46I.

Thielée, I78.

Thomson, J. J., x, xi, 86, I I8, 195, I99, 324 .

Thompson, E. P., 47, I87, 284 .

Thouvenin, 320 .

Thurnwald, 290.

Tichomirow, 478.

Tieghem van, 397 .

Tizzoni, 404.

Török, 250, 254, 257, 264, 301, 348, 506.

Tolomei, I4I.

Tommasi, 5 I 3 .

Tourette, Gilles de, 460 .

Tripet, 107.

Triwus, 443.

Trouvé, 473, 494, 496.

Tschdanow, I79.

Tudor, 28.

Tuma, J., 328.
Turner, 478.

Tyndall, 385, 402, 437.

Uffelmann, 408.

Ullmann, K., 28I, 300, 408.

Upensky, 478.

Uskoff, 4I 2 .

Unna, P. G., I52, I 55 , 259, 340, 4I5, 42I, 449, 456.

Valenta, E. V., I42, I92, $205,324,335,425,429$, 462.

Valenza, 158.

Vas, I 57.

Vedding, W., 487.

Veiel, 449, 454, 456.

Verworn, 156, 412.

Vial, I40.

Vieira, 250, 257.

Vietti, G., I08.

Vigouroux, II 4.

Villard, P., I94, 245, 354. 525.

Villari, 196, 352.

Vinaj, G. S., Io8.

Viola, iा

Vogel, H. W., 369, 482, $489,517$.

Voigt, 302.

Voit, 44I.

Voller, 77, I89, I92.

Volta, I9.

Vosmaer, 200.

Wade, 3 I 3.

Wagner, 5 I 6 .

Walker, Norman, 266, 290.

Wallentin, J. G., 3, I8.

Walkhoff, 358 .

Waller, A., 397.

Walsh, 307.

Walter, B., 3, 54, 60, 77, I I6, I33, I92, 200, 205. 210, $214.336,354,456$.

Ward, Marshall, 402, $405,513$.
Warnecke, 376.

Wassilief, I72.

Waterhouse, 459.

Waters, 449, 456.

Watson \& jons, 203.

Weber, 40.

Wedding, 420, 520 .

Wehnelt, A., 59-62, 73$79,94,100$, I20, 19I, 200, 212, 217, 221, 247.

Weichselbaum, A. V., II 3, I 22 .

Weigert, I52, 288, 34I.

Weldor, 302.

Widmark, 4I6, 422, 433, $448,462,490$.

Wiechert, E., I99.

Wiedemann, E., 3, I 6 , I I9, I88, 385 .

Wien, W., I99.

Wiesner, 396, 45I.

Wild, 203, 2I4, 3II.

Wilke, 217.

Wilkinson, I I4.

Williams, Chisholm, I75, 293.

Wimshurst, I8, I20.

Winkelmann, 196.

Winkler, F., 85.

Winogradsky, 408.

Winternitz, W., 456, 469.

Wittich, 4I9.

Wittlin, 3I3, 403, 408.

Wolters, 421, 449.

Wolff, M., 313.

Wollaston, 393 .

Wood, N., 280, 300.

Woyzekowsky, 327.

Wwedensky, 4I3.

Young, 370, 409.

Zarubin, 187.

Zechmeister, 250, 27I.

Zeemann, I99, 23I.

Zehmann, 347, 39I.

Zeit, 3 I9.

Ziemssen, v., 237, 266 289, 295. 


\title{
NOTES
}

\author{
( ) $N$
}

\section{INSTRUMENTATION}

(Published in connection with the linglish Translation of RAIIO-THERAPY, by LE(OPOI.I) FRLUNI)

BY

Clarexce A. Wratiht, F.R.C.S.lE.), F.F.P.s.(i.

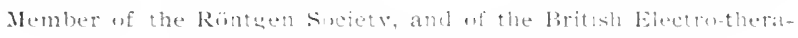

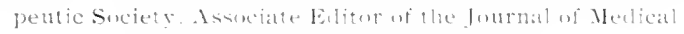

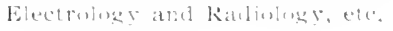

\section{! I, I, L S R A T E D}
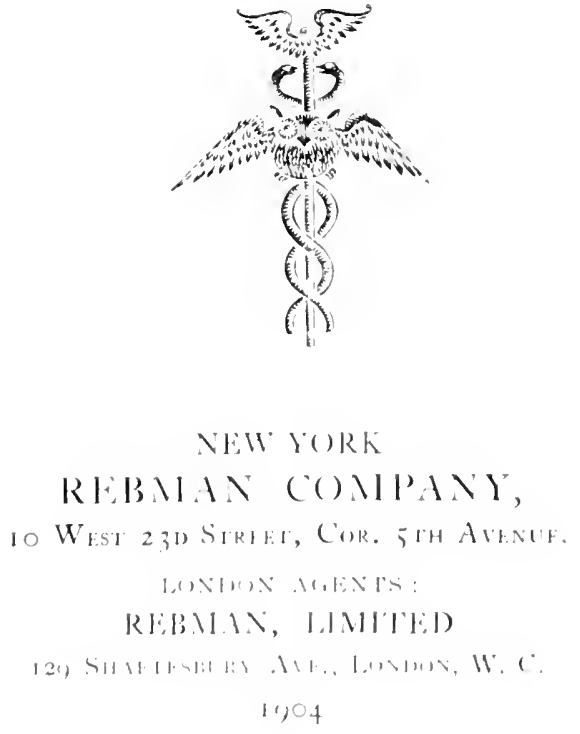



\section{NOTES ON INSTRUMENTATION}

SOURCE OF ELECTRIC ENERGI. As the chuice of an ilectromotor or source of electrical energy is a matter of primary importance, it were well to preface our remarks on the more recent advances in instrumentation by a short preliminary survey of this subject.

The question first to be decided by the reader, is the form of treatment that he is desirous of adopting. As far as Photothirafy goes, he must rentember that treatment by the Finsen Arc Lamp requires a continuons current of about 80 ampères at 50 volts. A Finscn-Reyn Lamp, in working oft the same current, consumes 20 amperes at 55 volts. Lamps of the Lortet-Genoud type on a continuous current supply require only one half this amomt of current. but they consume an additional 6 amperes when working off an alternating one. The little Dermo lamp of Dr. Bang takes a continunus current of about 60 volts and 5 to 10 ampères. The more powerful and efficacions Strebel Lamp consumes 7 ampères at 50 to roo volts on a continuous or alternating main. It is evident therefore that the form of apparatus to be nsed for Phototherapy must have a considerable influence on our selection of a sontec of electrical energy.

Again, for Radiography and Radiotherapy, we notice that we require a current of very high electro-motive force ( 50 to 500 thousand rolts), but of small ampèrage ( $\mathrm{I} .6$ to $\mathrm{IO} \mathrm{m} / \mathrm{a}$, according to the degree of vacunm it the focus tube). We can obtain the necessary current direct from a static matchine, or indirectly by means of some form of Induction-Transformer. which, when actioned by a current of low voltage, will produce in its secondary circuit one of the required tension. Inasmuch as portability, or the amoum of floor space available, is a question of moment, Spark Coils will be found by far the most suitable form of apparatus for our purposes, more especially as they can also be turned to account for High Frequency Treatment. For coils yielding a spark of 12 to 16 inches in air-the size most suitable for general use-a current of 4 to 12 amperes at 16 to 80 volts will be required, according to the form of interrupter cmployed.

It is obvious, therefore, that the source of electricity best suited for practising all these three branches of Physico-therapentics must be one which can furnish us with a current of 60 to 80 rolts at 16 to 20 amperes. Th. problem, therefore, resolves itself into a consideration of the forms of electrical energy capable of furnishing the necessary amount of current. The restrictions imposed by environment and the conditions of life, under which the practitioner must work, to a large extent determine his choice of a source of electrical encrgy.

In towns, where a current from the commercial main is avilable, he is naturally anxious to use it. In order to do so it is necesiary for him te ascertain the nature of the current alpplied, whether comtiminu or alter nating; its voltage, and in the care of an alternating current, its frenumey as well.

CONTINEOES CIRRENTS-In working off the commercial main,

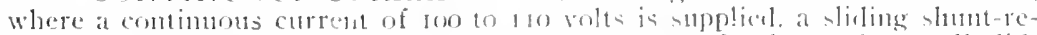
sistance or thenatat, with crank w vary the number of rults, and a small slif-

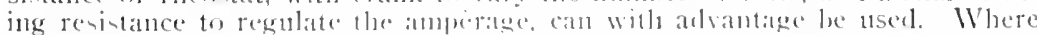

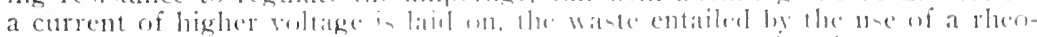

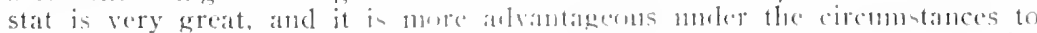

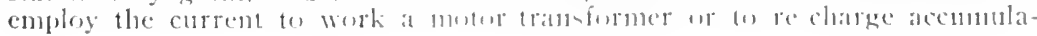


land. admirable as stationary storage batteries. For up-country stations, like India, where labonr is cheap. the hand dynano shown in Fig. 2 is to be recommended to charge accmmulators with. Where gas, oil or petroleum is available as a motive power, a dymano worked by a 1/2 H. P. motor can be employed.

CONTINLOLS CLRRENT ISXAMO-In working off an alternating street current. or from comnercial mains supplying a continnous current at very high voltages (200 to 300 volts). a rotary converter is an aclvantage. The initial ontlay is heary, lut it sives the necelless llittering away of

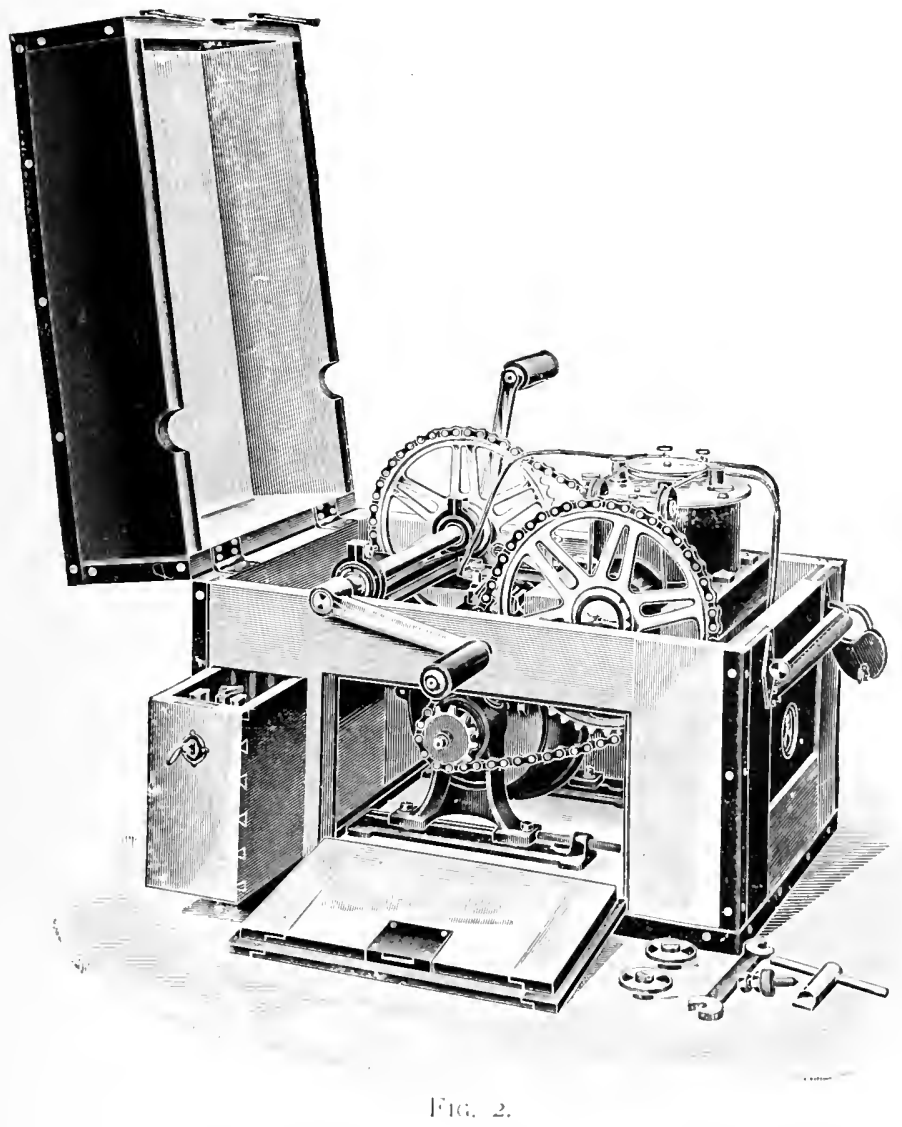

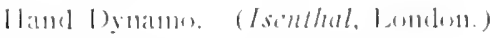

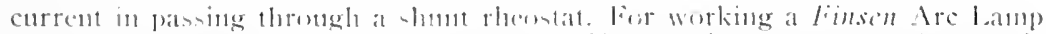

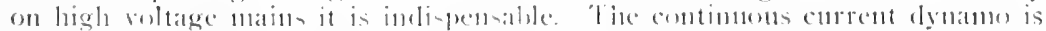

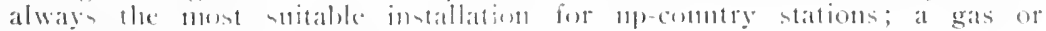
petrolentul engine formi-lrige the nutive power.

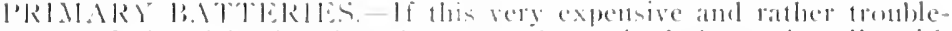

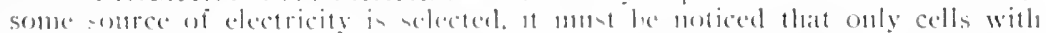

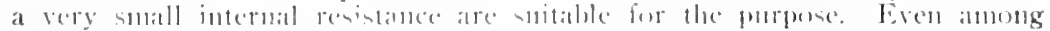

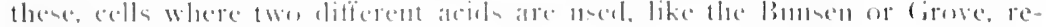

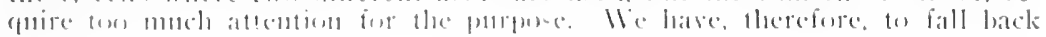




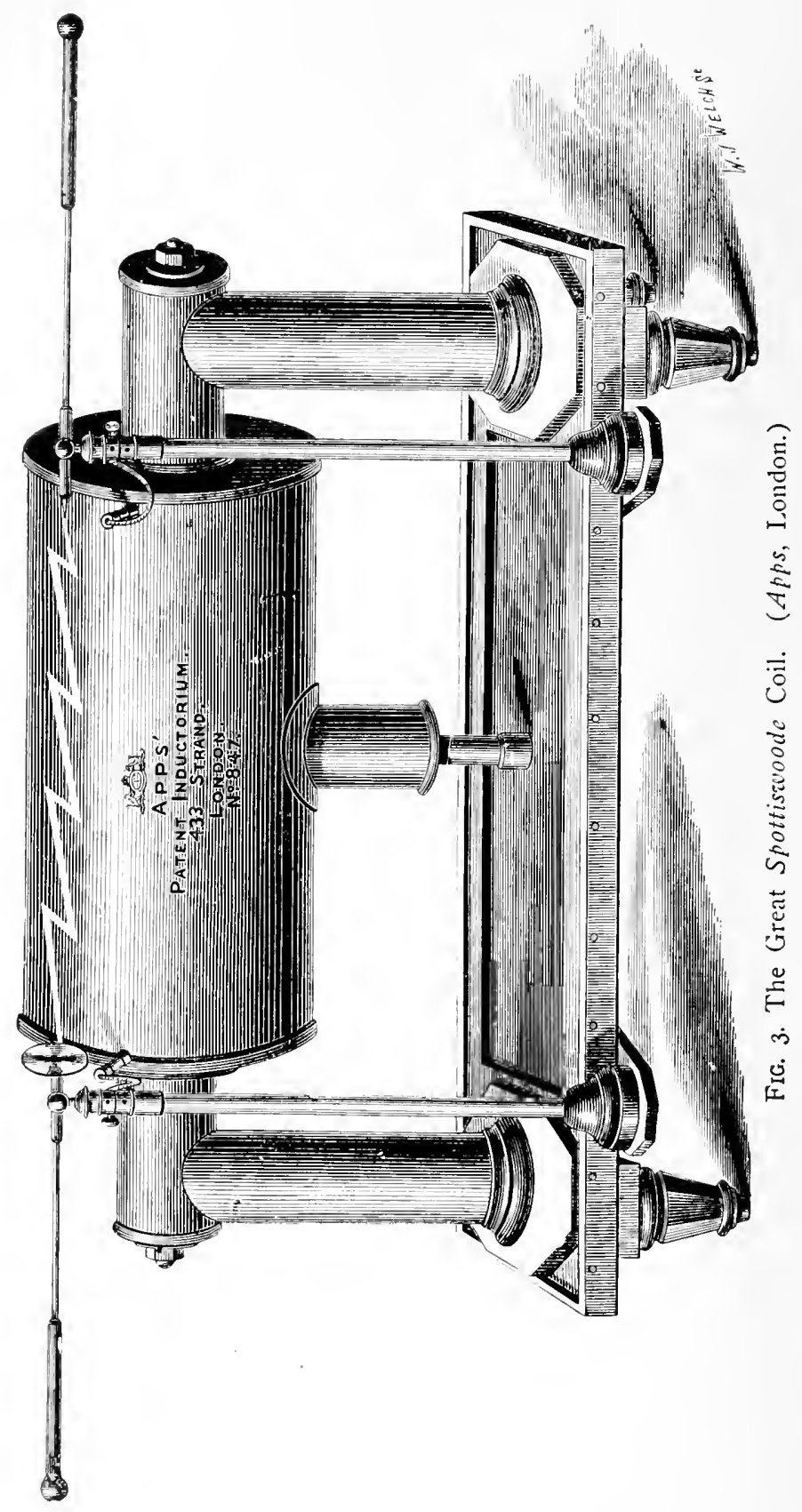


on the Biblormate cells, whose chict objection is their want of constancy. To obviate this difficulty, care must be taken that the two electrodes are suifficiently far apart to allow of the circulation of a wide stream of acid between them, and also that they be of the largest dimensions possible. With the zinc plates well amalgamated. These precantions taken, the only objection to them is that of expense, which is decidedly greater than that of electricity derived from any other source, currents of high voltage (100 volts) inclusire.

Having so far dealt with the somres of electricity, we may next consider Induction Coils and the accessories tending to their proper working, care being taken to point out the factors upon which their efficiency depends, and any drawbacks attending their use.

INDUCTION COILS.-Introduced by Masson and improved by Rulmkorti. they attained to a very high degree of excellence in the Spottiswoode Coil (Fig. 3), which in Isis excited the admiration of the scientific world. This coil was furmished with two interchangeable primaries. in one of which the layers were subdivided and could, by a novel mechanical arrangement, be connected either in series or parallet. The secondary of the coil consisted of 280 miles of wire (with a total resistance of I 0.200 uhms), wound in four sections, the two outer being of wire of somewhat larger sectional area. Each section was wound in that discs, of abont 200 layers. The total number of tums in the secondary was 3+1.850. Strange to say, the condenser of this coil was not larger than that usually fitted to coils of much smaller dimensions. It consisted of 125 sheets of tinfoil (IS x SI inches) separated by a double layer of varnished paper about one-hundredth of an inch thick. The coil, with 5-quart Groc'c cells. gave a spark of 28 inches of air; with Io similar cells one of 35 inches: and with 30 such cells sparks ranging from $3 \pi / 2$ to 42 inches. It is evident, therefore, that cren as early as the sear I877, the principles molerlying the manufacture of large coils were well understood in England.

Althongh passable Rodiographic work has been done in the field with coils of smaller size, those mont suitable for the purpose should yielel a spark of Io to 16 inches in air. In the selection of coils one has first to concider their efficiency, the value of the materials and devices used in their contruction, and lastly, the arrangements that exjet to fit them to the varying conditions of focus tube vacumm, and of the intermpter employed.

EFFICIENCY.-The true criterion of efficioney is the amomt of electrical energy that can be transformed. This quantity is the product of two factors; the electro-motive iorce and the amperage of the derived current. The former determines the spark length of the steondary circuit; the latter can roughly be ganged by the number. hrightness and thickne-s of the sparks passing between the knobs of the discharger: so that with coils giving sparks of the same length we may say that the one which profluces the greatest number of fat. bright sparks. jis the lest. In deciding this point. it is important to remember that the a'olume of sparls of matrinmm lingth and intonsity must be obtuined with a relatialy hish mumler of intrivuptions (soo or more) per minute.

CONSTRLCTION OF COIIS. (Figs. a am 5.) The utility of a spark-coil for heavy discharges depend upun two falctors. namely, the perfection of its insulation and the quality of the materials msed in its construction.

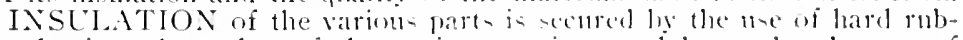
ber or elonite tubes: that of the variss sections and layers by the we of hard paraffin wax, or better still, ly emplowing a semi-solicl viscid hycluocaslonn similar to that used as an in-ulitur in the kochefort lansformer. The insulation of the wires of the primary and secomlary is hest carried ont by nsing only the best domble silk-eoated wire in the comstrmetion of these parts.

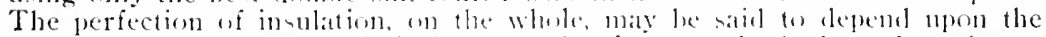

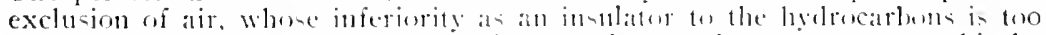

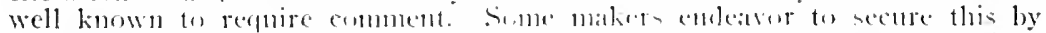
prolonged immersom of the wires in melled was, reme wing lhe exeess in wind-

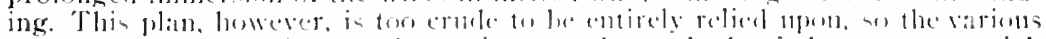
parts, when wommel hase to be igate treated lo it hath of the same material.

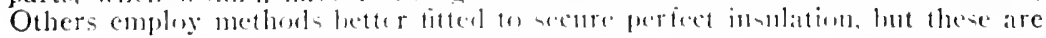


carefully guarded as trade secrets. The use of cotton for coating the conducting wires is in every way to be condemned. It is a pity to saerifice efficiency for the sake of a slight reduction in the price.

THE QUALITY OF MATERIALS.-All materials used in the manufacture of Heavy Discharge Induction Coils should be of the best quality. Soft, well-annealed iron is best suited to minimize loss of power by hysteresis. Lamination or division of the metal core in a direction parallel to the

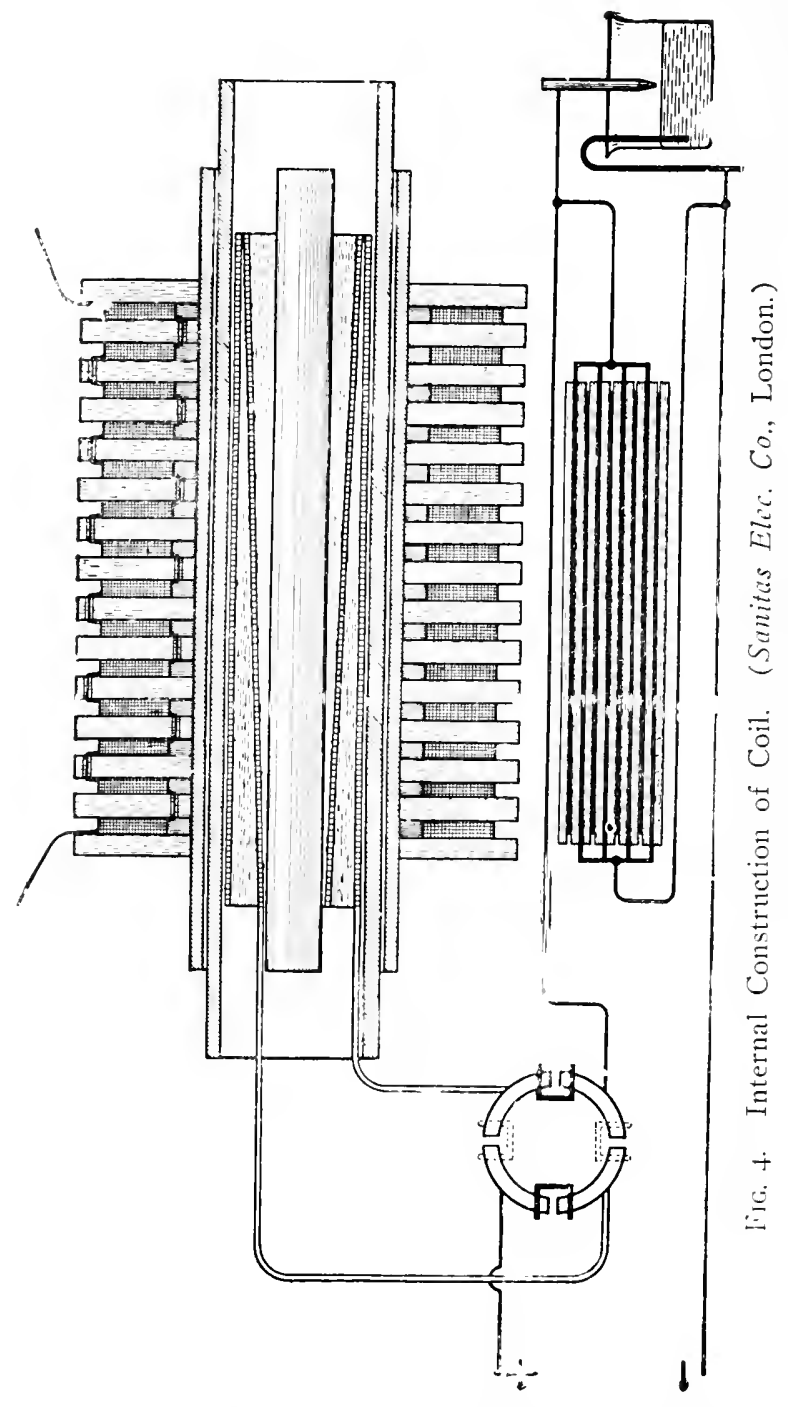

How of magnetion. conpled with insulation of the adjacent plates or wires with thin paper, varnish or paraffin wax, will reduce the Eddy currents to an almost negligible anmunt. For this reason, it is nstral to make nse of the best anmealed tranformer inon wire for the cylindrical core, on which the primary 
is wound: instration again-t Liddy current being secured by thorulngl impregnation with paration wax and sparking between the core and primaty by binding round with tapes smblarly treated. The wire emploged for the

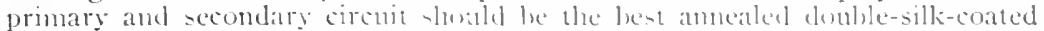

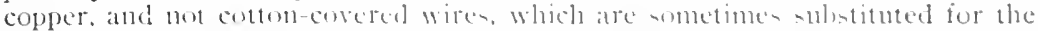
sake of cheapmess. Fach laver of the primatry should be imsulated from those adjacent to it by sheets of pratfined paper, and the primary at a whele further insulated ly immerion in patrafin wax. It is for the same purpose arefully separated from the secondary ly a stout tule we elwnite. Which is cluned in at both ends by dises of the sime material. Is the stratin on the dielectric. due to the difference of potential hetween the contiguns turns. in diminished by sectional winding. the -uld-division of the secondary intu sections, each only at fraction of an inch in thickness, is a print upon which stess should he hatel

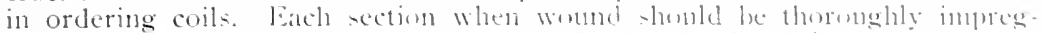
nated by soaking in hest paralin wax and further insulated from the two ardjacent sections by a thin diec of elenite or sleeves of well-paratfined papers.

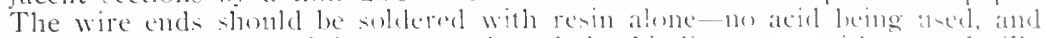
the insulation of the joint strengthened. ly bineing over whin waxed ille.

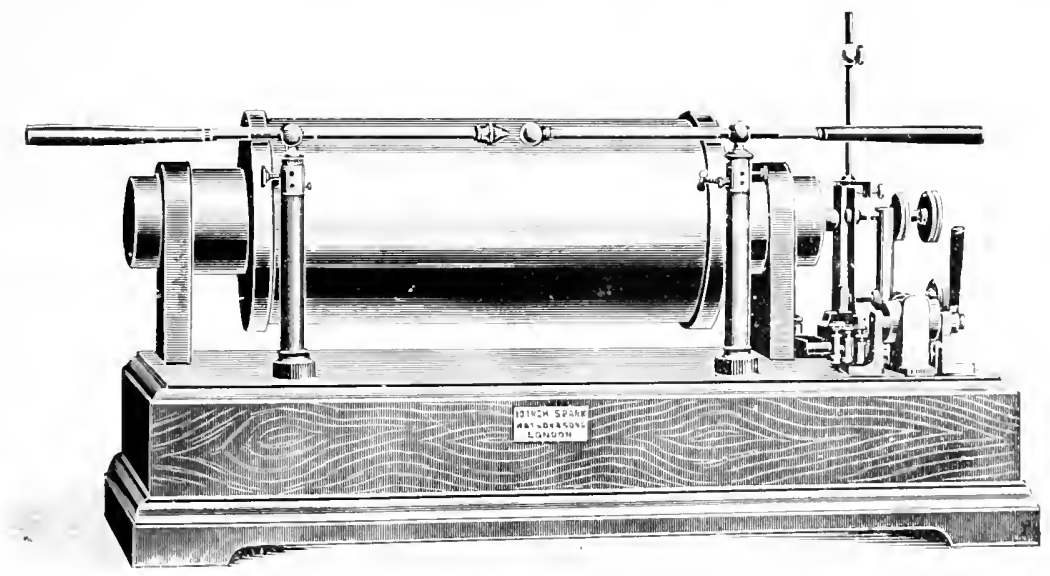

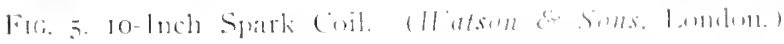

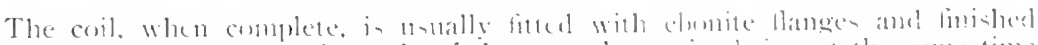

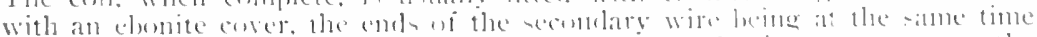

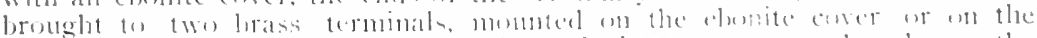

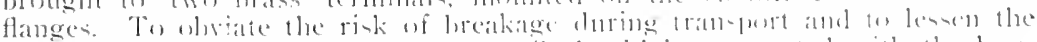

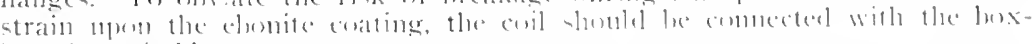
base ly - mitalble : npport

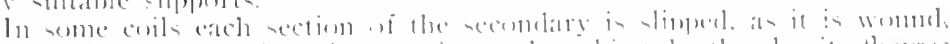

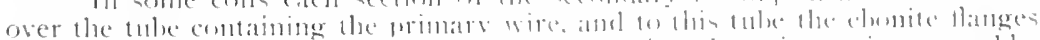

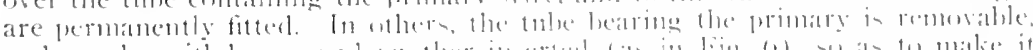

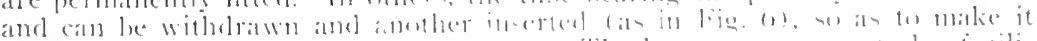

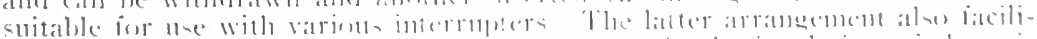

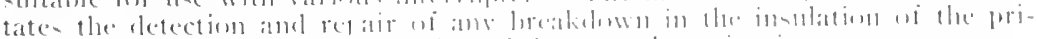

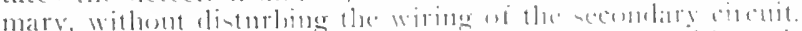

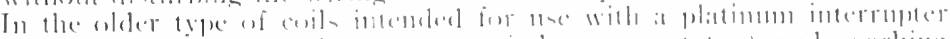

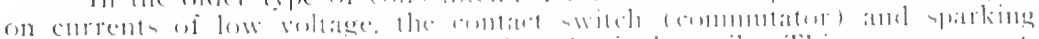

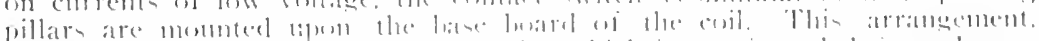

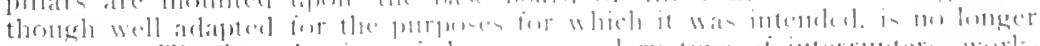

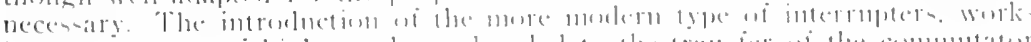

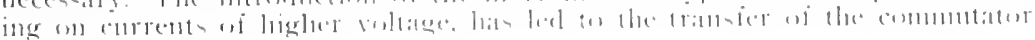




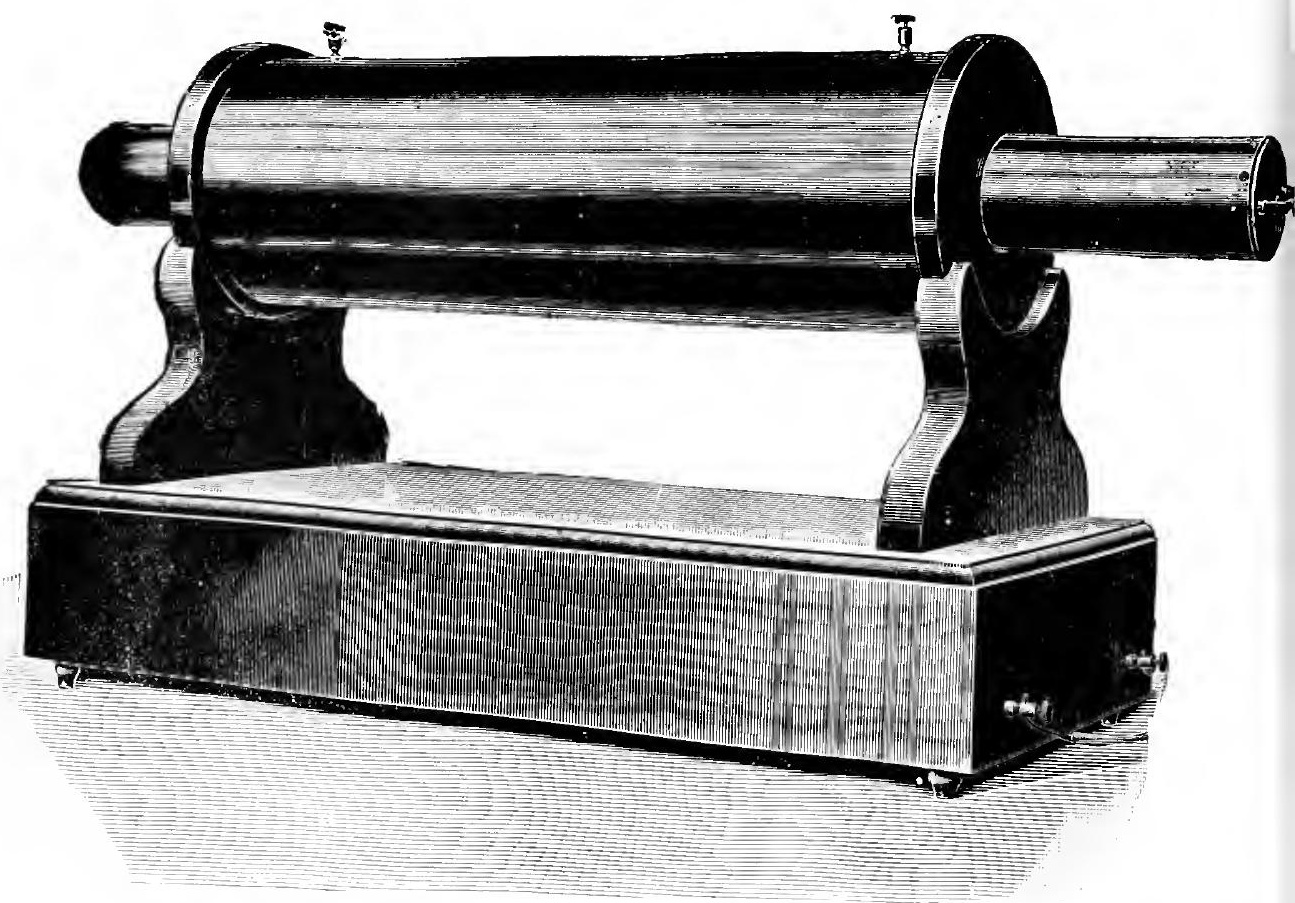

Fis. 6. Large Coil. with Condenser. (/schthal, London.)
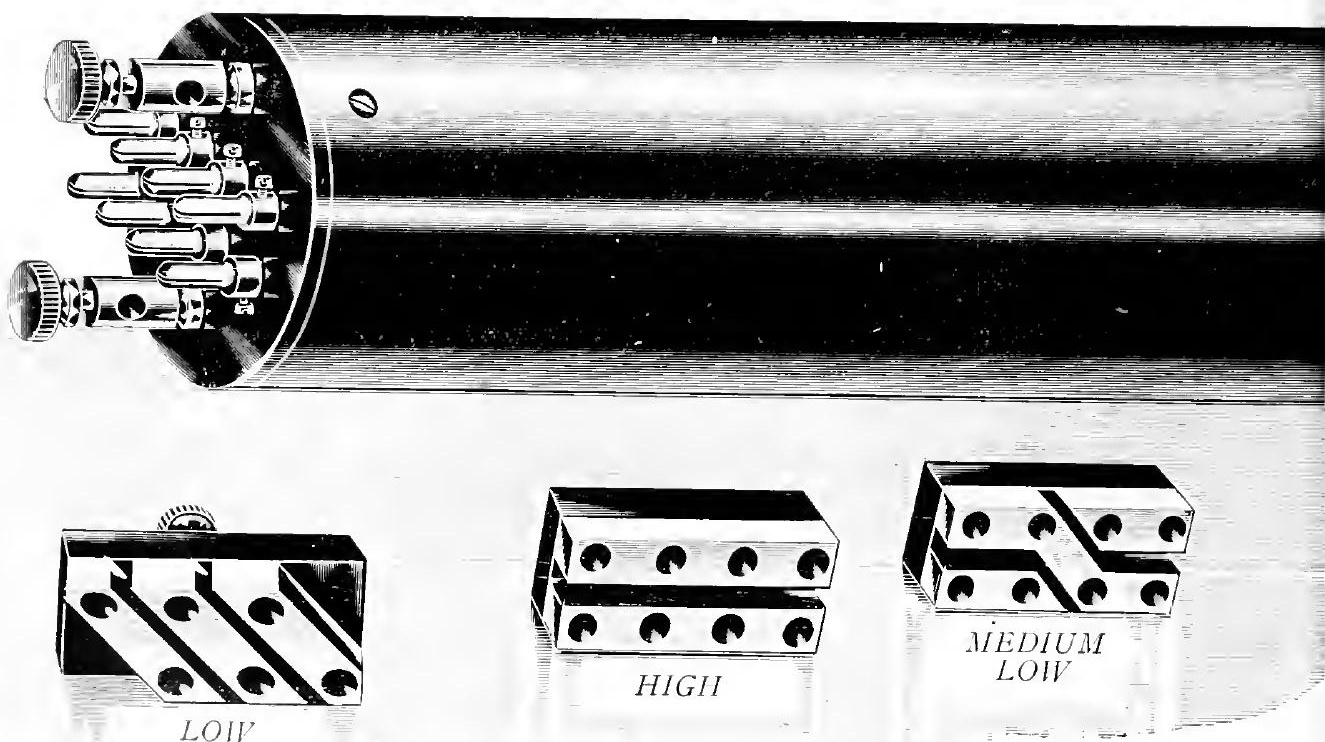

Fir. 7. Invided Primary. (Sanitas Elcotl. Co., London.) 
it. a moment later, through the primary circuit, it hastens the demagnetization of the core, and thereby increases the E. $\mathrm{H}$. F. of the induced direct current in the secondary circuit. The size and capacity of the condenser are usually proportioned to the maximum length of the spark, but some coils are fitted with a subdivided condenser and a mechanical device whereby each or all of its subdivisions can be thrown in or out of the circuit. This allows of an ex tensive degree of regulation of the size and intensity of sparks obtained from the secondary terminals.

The best types of coils possess both these regulations.

ACCESSORIES. The two most important accessories for convenience in working coils are the commutator and the leat fuse, the latter being in most coils comspicuous by its absence. The communtators most commonly used with the spark coil are the Rhumkorff, the de IVallerille, the Sicmens-Halske, the Caster. In working with molern types of interrupters the commutator is best mominted with the wher atecessories on the wall switchboard or upon a table distributor.

As much of the effieiency of spark coils depends on the nature of the arrangentent for automatically interrupting the flow of the current in the primary circuit. it will he wise to consider them at some length.

INTERRLPTERS.--The chief type of interrupters in use at present are:

(a) The Viloratory Interrupter.

(b) The Rocking Nercury Interrupter.

(c) The Nercury Notor Intermpter $\begin{aligned} & \text { Rotary Type } \\ & \text { Jipue Type }\end{aligned}$

(d) The Rotary Break i Film Truse

(c) The Electrolytic Interrupter, Film Type

(f) The Jet Brakk.

IIBRATORY INTERRLPTERS.-The older type of spring platinum Interrupter is rapidly falling into disuse, the fow remaining representatives of this class. like the "Tril" Break (shown in Fig. 9), maintain their popularity for small coils by reaton of the fire adjustment which, by allowing the core to lecome nearly saturated befure lireaking the contact, induces a higher E. M. F., in the secondary circuit. The difference in the electromotive force which can be thts obtained is very great. while at the same time the wear on the contact-studs, by the sparking set np at "break" is redteed to a minimum. It must. however, be remembered that the heat so engendered, even if minimized. is not withont it effects num the contact spring, the untempering of which is mainly responsille for the increase of sparking. To obviate this diffeulty. the resiliency of the spring is now alolished and the suddenness of interruption at the point of maximum saturation promoted by a simple mechanical device. A light piece of flat netal, balanced on its edge, is substituted for the movable contact, and is maintained in position, while the current is passing, by spiral spring, whose tension can be easily regulated. The adju-turent for contact duration is thereby increased and the interruptions can be timed to always occur at the phase of maximum induction.

The maximmin voltage that can legitimately be employed with a platintm lireak, should not exceed 36 volts. For coils yielding sparks of is inches and over. every form of platinum interrupter is unsuitable, as the contact surfaces are too rapidly destroyed by the powerful sparking which takes place. In working with these some form of mercury break or more modern interrupters should be employed.

THE ROCKING MERCLRY INTERRUPTER is fast becoming an object of antiguarian interest. The lest model of this group is the "Interrupteur oscillant à movement rectiligne" of $M$. Rochefort. In it the movement of the dipper is perfectly perpendicular to the surface of the mercury, as may be sathered from concentric rings that form upon the surface of the isolating fluid. It can furnish any number of interruptions up to r.200 per minute. The rapidity of oscillation is regulated by an adjustable weight. The amount of current comstumed in working it is very small. 
Another electromagnetic interrubler of the divper type ts dat of .I. Radiguet. In it both the fixed and noring comblets are mate of enper.

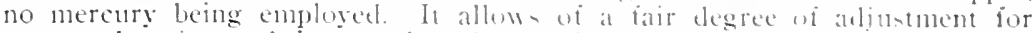
contact duration and for speed. and is ly fat the best dectromagnetic interrupter with which I an anguanted.

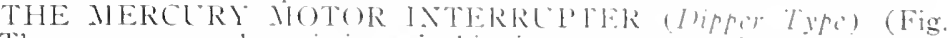

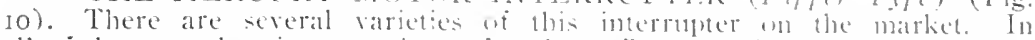
all of them an electric motor is emplosed u elfect the dip and withdratwal of

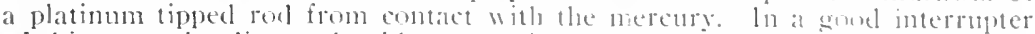

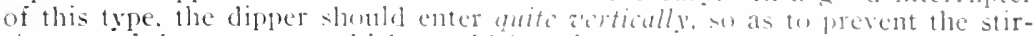
ring wp of the mereury. Which westel inter fere with the aceuratey of the interruptions. Most forms of reif patimg broks allow some degree of regulation

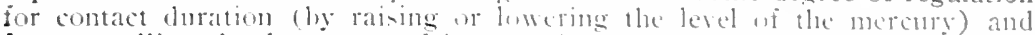
for controlling the frepuency of intermption (hy regulatiog the -peed wi the

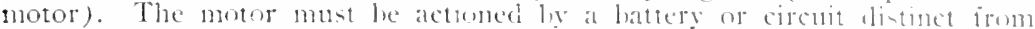
that of the coil. Interruptions ranging from soo to 1,200 per minute can be

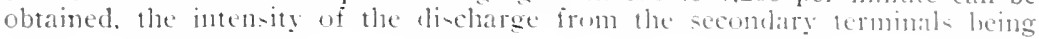

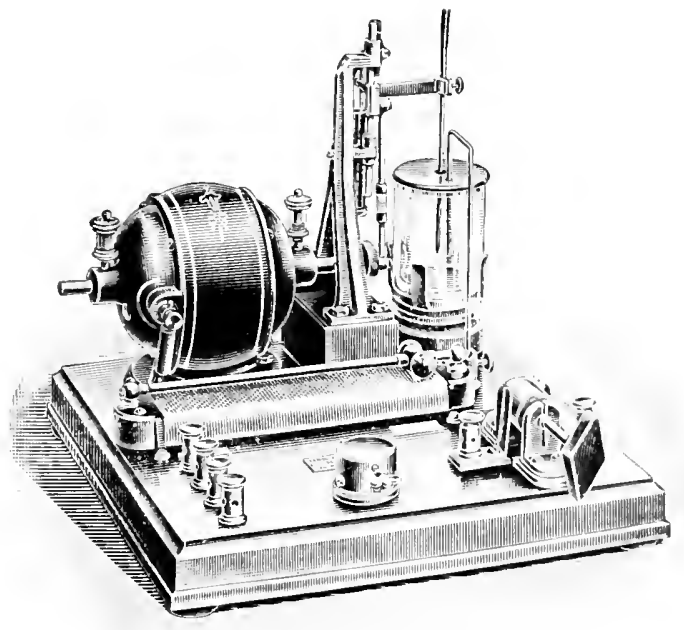

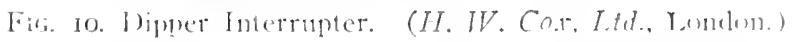

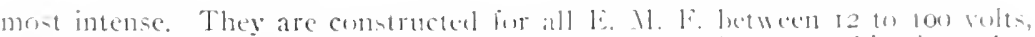
and are almost moiscless in action: but are open to the same oljections that can be levelled against all furms of meremy break. The mereury alter a time requires cleaning: for the intensity of the spark in the entate spate tends to

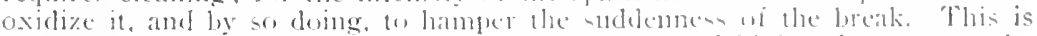
more especially the case when run with a emrent wi hish roltage upon the primary of the coil.

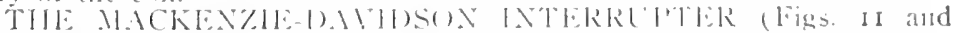

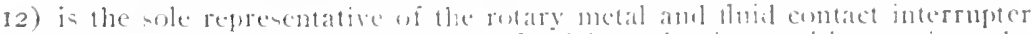

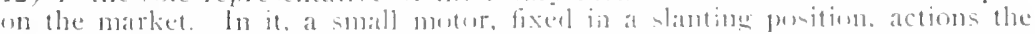

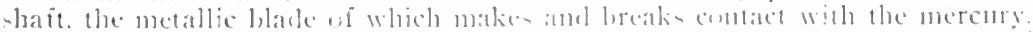

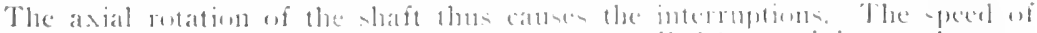

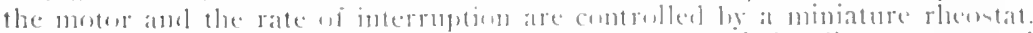

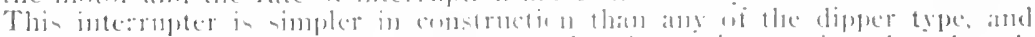

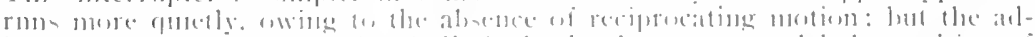

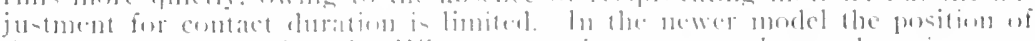

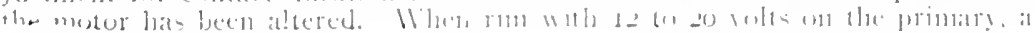


comparatively small number of sparks are elicited, with a higher speed and a suitable resistance in the circuit, a higher E.M.F. (up to Ioo volts) can be employed without breaking down the coil. Where a very high number of

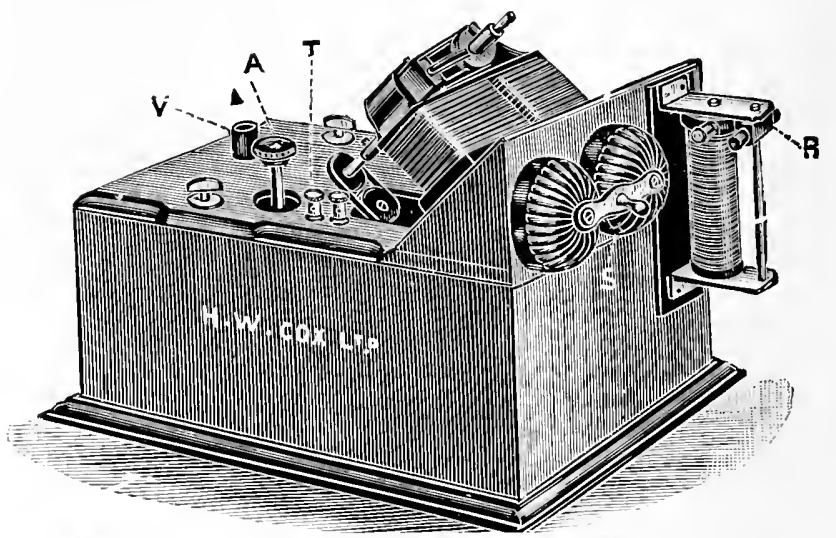

FiG. I I. Old Mackenzie-Davidson Interrupter. (H. W. Cox, Ltd., London.)

interruptions must be obtained, the mercury motor breaks of this type yield place to the rotary interrupters.

ROTARY BREAKS.-In this class of interrupters, the separation of the contacts is effected by the movement of a revolving disc or drum, actioned by an electric motor. The tendency to spark at break is diminished by submersion of the contacts and drum in petroleum or alcohol. The two chief types of this break are

The "Film" Type and the "Brush Sector Contact Break." The Film type consists of a rotary drum fitted with copper segments (with their apices directed downwards) and one or more contact brushes, which press firmly against its curved surface. To insure perfect electrical contact and minimize friction, a film of mercury (pumped from a cell in the bottom of the jar) is spread between the two contact surfaces, which along with the drum are

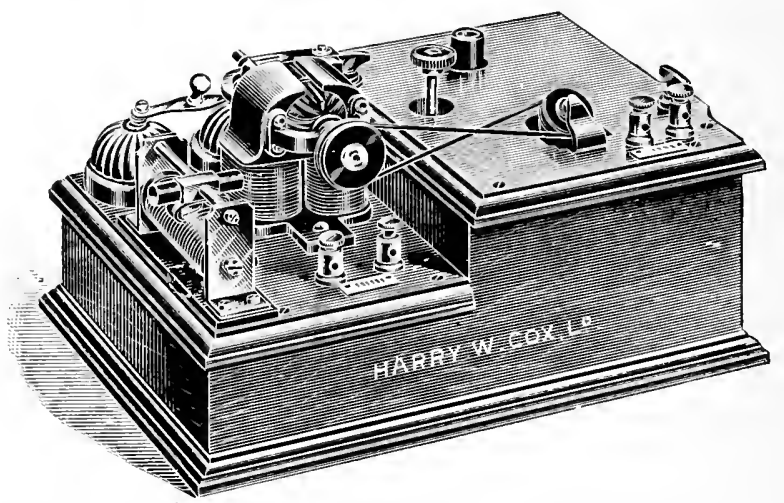

FIG. I2. New Mackenzie-Davidson Interrupter. (H. WV. Cox, Ltd., London.)

immersed in paraffin oil. By sliding the rod bearing the brush in a direction parallel with that of the axis of the drum and by regulating the speed of the motor, the relative period of contact and interruption, as well as its frequency, 
can be altered at will. Every intermpter of this type which is actioned by a separate motor requires to be started before the current is switcled on to the primary of the coil, so as to prevent the damage that the rush of an excessive, heary current might produce. An interrupter of this class is shown in Fig. I3.

THE BRUSH SECTOR CONTACT BRE.MK.-In all types of this interrupter, the circuit is made and broken by two contact brushes pressing against a segmented revolving copper cylinder. The absence of mercury and the consequent cleansing it entails is an advantage. It is, moreover, perfectly silent in action. There is. however, some wear on the bruslues, duc to this steady and continuous pressure against the rotating copper sectors. The drum is actioned by an electric motor. The circuit remains closed as long as the two systems of brushes press against the same segment of the cylinder. The rapidity of interruption. Which varies from 800 to 1.200 per minute, is

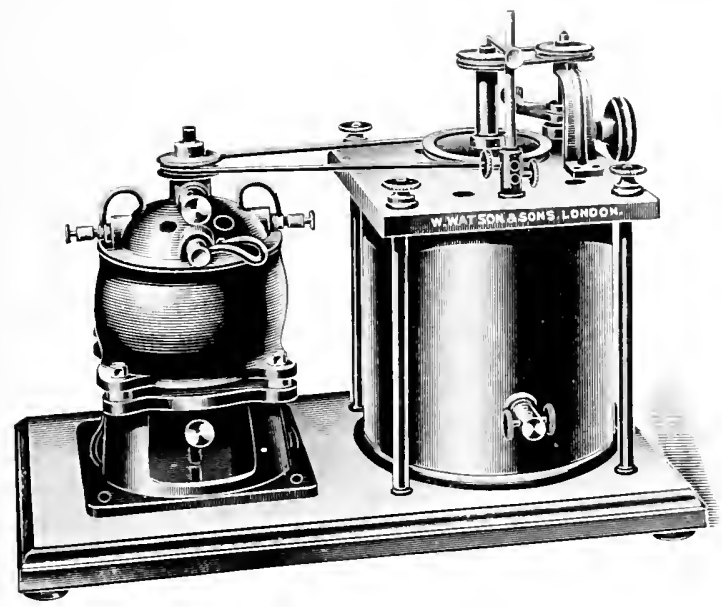

FIG. I3. The "Fram" Break.

controlled in part by regulating the speed of the motor, and in part by an adjustment of the movable brush.

ELECTROLITIC INTERRLPTERS.-In this ype of interrupters advantage is taken of the intermptions, caused by the increase of current density generating sufficient heat to vaporize the clectrolytic fhid and thus produce an insulating sheath about the surface of the anode or at the aperture of communication between the inner and onter jars of the Caldatell liquid interrupter. The two chici sub-groups, into which this class is subdivded are (I) The Vehnelt or "Film," and the Simon-Calda'll or "Liquid" interrupters.

The "FlL.W" type (Fig. I4). In this type the interruptions are canced by the formation of a film of non-conducting vapor or gas about the active electrode, which is the anode. As the break in the circuit is instantancous and complete, no confenner is necded. The apparatus has heen said to work with a minimum E. M. F. of 16 to 24 volts: but the best resulte are obuanerl with a current of 50 to 120 volts. The number of interruptions raries with the voltage of the current and the area of the exposed platimum surface, hut is always very high. It is important. therefore, that both these factors should be easily controlled, the former by a rheostall, the latter by varying the length and sectional area of the platinum dip exposed. As the eifects are in a measure proportionate to the size of the wire amplinged, interrupters, with thee or more wires of different gauges, alle an alvamlage. With a voluge of to to roo volts, it answers admirably fur shert rums, but if used comtinuously for 
half an hour or more, the acirl grows hot tntil it at length stops the working. To diminish fatigne caused by the excessive generation of heat in the electrolyte, a leaden pipe, through which cold water is kept circulating, sometimes forms the passive electrode. In other forms of this apparatus, the container is made very large. Some makers seek to minimize the production of heat

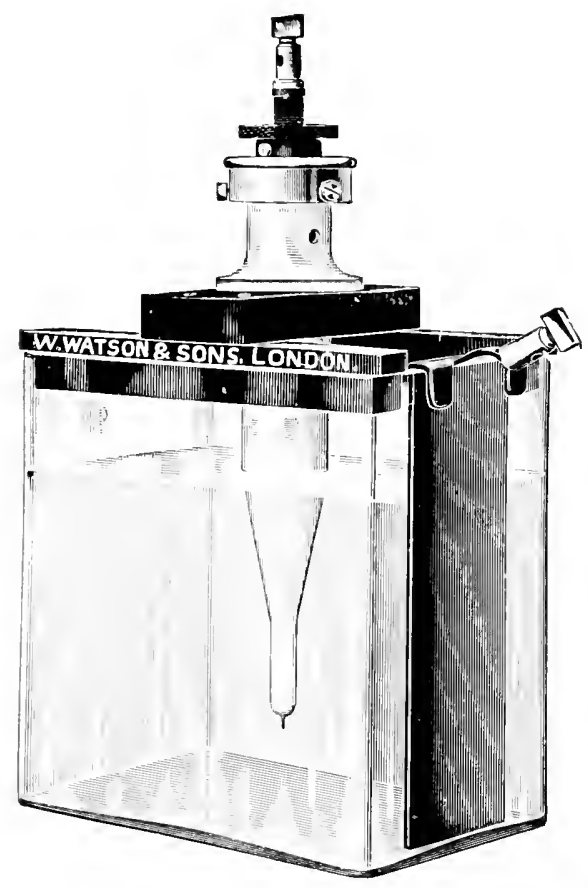

FIG. I4. Electrolytic Film. (I'atson \& Son, London. )

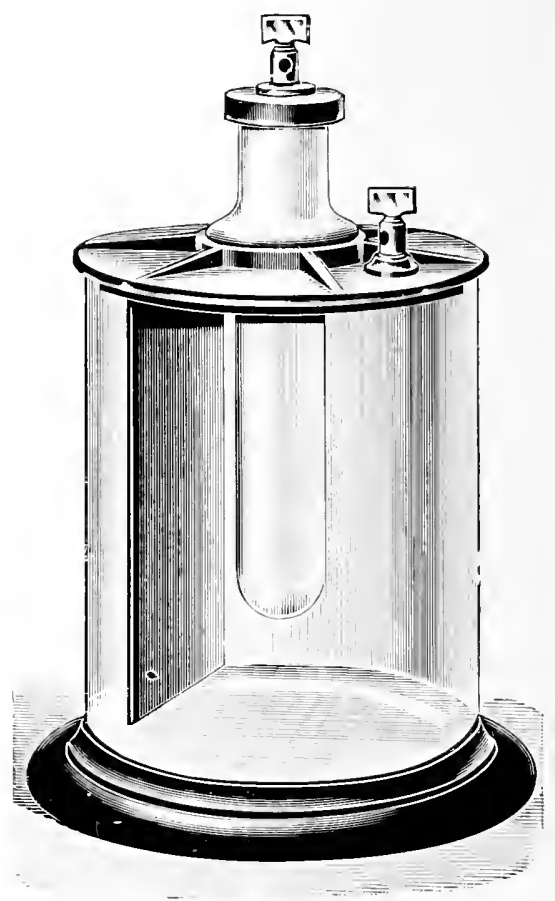

FIG. I5. Stmon Interrupter. (Sanitas Electrical Co., London.)

by enclosing the break in a large container through which cold water is kept circulating. The substitution of the sulphates of magnesia and of potash alum for the acidulated solution also tends to retard the generation of heat. Interrupters of this type require but little attention and are easily kept in order, but the loud humming noise they make while working is a great drawback to their use.

TIIE LIQUID ELECTROLYTIC INTERRUPTER (Fig. I5).--This was simultaneous and independently described by /)r. Simon and Mr. Caldwell, in the year $\mathrm{I} 899$. In it the interruptions are brought about by the evaporation of the electrolyte at the aperttre connceting the two chambers. The frequency of interruption is proportionate to the strcngtlo of the current, the size of the aperture and to some extent also to the inductance of the circuit. The size of the aperture is altered by the movement of a pointed rod of nonconductive material. thus allowing some control to be exercised over the frequency of the interruptions. The liability of the inner tube to be damaged by the tnerual expansion of the glass of which it was made led to the substitution for it of perforated porcelain dises. Which now form the septum between the two containers.

The very considerable heating of the electrolyte during the working of the break, reguires some of the varions methorls mentioned in dealing with "Film" Interrupters to be adopted. 
THE JET INTERKL'P LER.-In this intermpter a jet of meremry convey the current, while a revolying drum carrying two or more metallic segments (according to the voltage of current) makes the circuit by intersecting this stream. The breali is instantaneons and complete. The duration of contact can be varied by rasing or lewering the contact plate relatively to the jet. The number of intermptions is easily controlled and can be varied from soo to 12.000 per minute. This hreak vields a thicker spark of greater intensity than that obtaned by any other method. It is silent in action. and can be aldapted to any E. M. F. from 1210250 volts.

A small rheostat controls the speed of the motor, while a large one is ustrally interposed between the eoil and the mains. This is the only interrupter that antomatically switches off the current traversing the primary cirenit. In the II odal Intermpter (Fig. 16), llse mercury pumped irom the reservoir is forced throngh a number of small apertures arranged axially, insteat of a single one. The contaet-duration is varied by altering their number by means of a regulating screw.

APPARATLS FOR LSE ITTH ALTERNATING CURRENTSTo obtain a pulsating or uni-directonal currout in the secondary eirenit of an Induction Coil, when a commercial alternating current is the only source of energy avalable, it is advisalble to adop one of the following expedients:

(a) Convert the alternating current into a contimous current by means of Hotor-Ginerators or Electrolytic Rectificts.

(b) Convert the altemating enrent into an intormittent current by suppresing one phase by means of an Electrolytic break, a Synchromized Platimum or Rotary Intermpter.

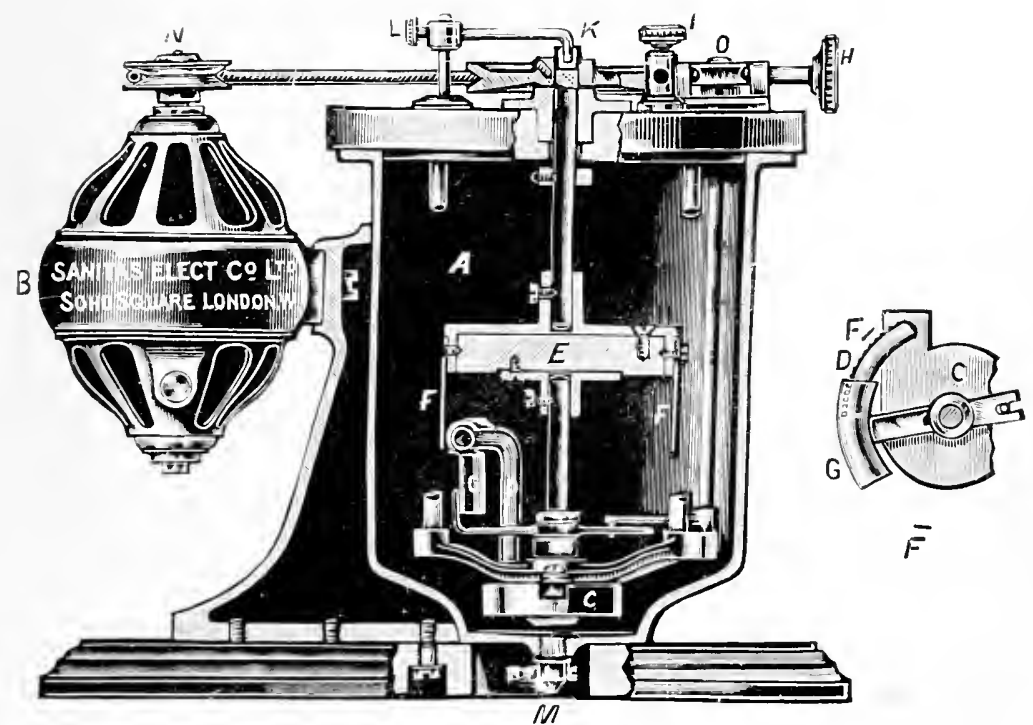

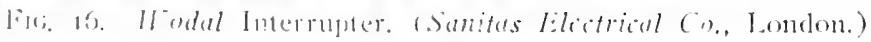

MOTOR GENERATORS (Fig. 17).- This is the most perfect and ecomonical way of working with commereal alternatting currents. A steady

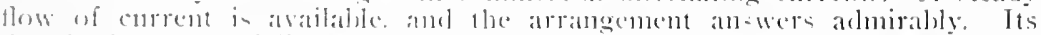
drawhack are at follows: The intial cost of the inctallation is great and the intrentetion of machinery, which, moles carefully arected, in the base-

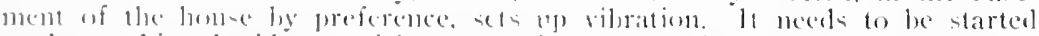
at the maldine, lesiden reguiring a certaton ammunt of attention for regulation etc. Hotor Transformes, whe of real hervice. Homld have a scenndary 
output of not less than 300 watts. A rheostat for starting and controlling the speed of the alternating motor is indispensable.

ELECTROLYTIC RECTIFERS.-The principle underlying their action is the great resistance (dne to polarization) that the current has to encounter when the almminimm electrode is the cathode. In its simplest form-

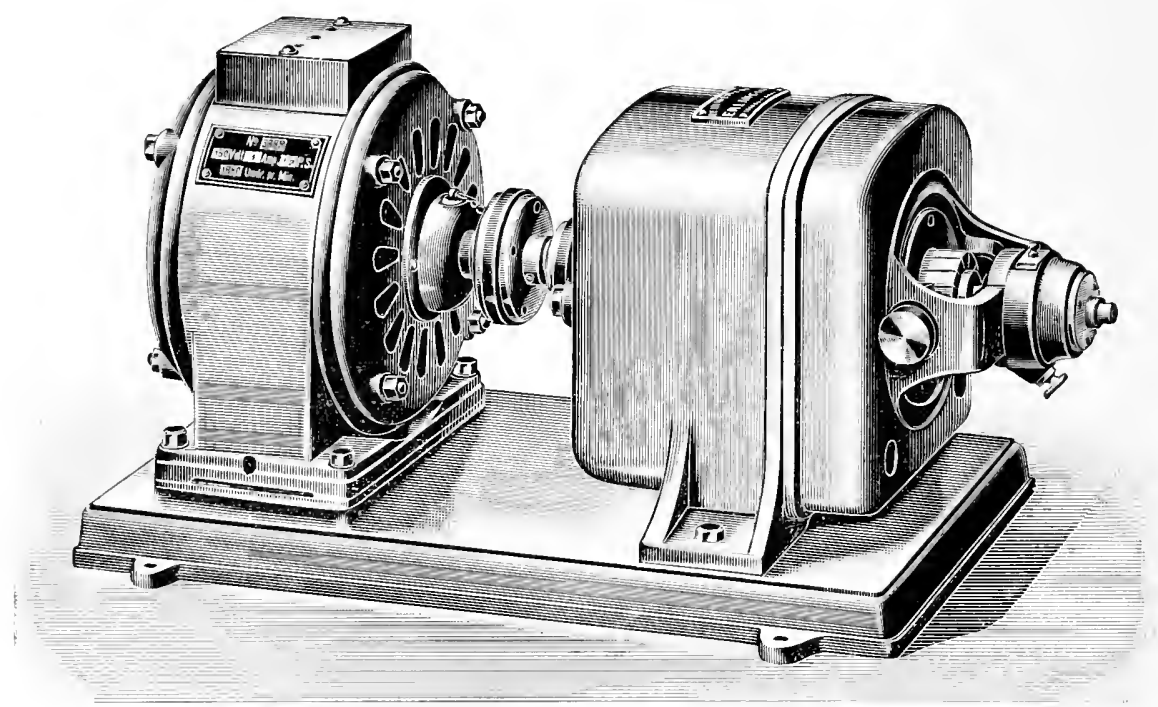

FIG. I7. Notor Generator. (Schall, London.)

the Graet Aluminimm Carbon Cell-the aluminitum is the smaller and active electrode, which polarizes rapidly and offers a high resistance to the passage of the current when it is the cathode; lut freely allows the passage of the current when it is the anode. The resistance of each cell is calculated to be sufficient to stop a current of 22 volts from passing. Its efficiency is about 35 per cent. less than that of the supply circuit. The cells in working grow hot, and when a certain temperature is reached polarization ceases. Special precautions have, therefore, to be taken to prevent this. The cells are sometimes placed in a zinc container, through whicl a stream of cold water is kept circulating; but the nse of hollow lead pipes (with cold water circulation) as the large passiz' electrode is to be preferred. A form of electrolytic rectifier, which of late years has attracted much attention, both on acconnt of the simplicity of its constrnction and its higl efficiency, is the Nodon Valve (Fig. I8). In it, the active electrode is a slender rod, made of an alloy of aluminimm and zinc: while the passive electrocle is the iron container. The electrolyte is a solution of phosphate of aluminitm, which is deposited as a thin film upon the active electrode, when it is the cathode. This is sufficient to prevent the pascage of any current below 22 volts in this direction. When the aluminium is the anode, the current flows freely. The cells heat up slowly when working. When they have been lying idle for some time, a small resistance between them and the mains is needed to start polarization. The number of cells (connected in series) which should be inserted into the prinary circuit depends on the voltage employed. The chici advantages of electrolytic rectifiers are:

(a) Complete independence of the periodicity of the commercial current, or of any change in the same.

(b) The entire absence of all noise and motion.

(c) The small amomint of attention required.

(d) Their high efficiency $(65 \%)$ and low cost as compared with motor generators. 
Sometimes the starting-up process is unduly prolonged or quite impossible. This shows that the cells require to be cleaned. Apart from the heat engendered by fatigne is that due to defects in the aluminium alloy, the boiling over so caused being often productive of a short circuit.

ELECTROLITIC BREAKS.-Both the "Film" and "Liquid" types are available for use on alternating mains. They act by suppressing one phase of the current. In the IVEHNELT tvpe. the current only passes when the platinum is the anode. The chief objection to their use with alternating currents is a great waste of platinum. They are also very liable to fatigue with prolonged working. The current is, moreover, but imperfectly converted into a uni-directional one, mnless the primary of the coil is specially wound to allow of a variation of its self-induction. When the CALDWELL type is used, the smaller lead electrole is replaced by one made of aluminium; but even then it is, at its best. only a poor substitute for the more efficient electrolytic rectifiers. which it seeks to replace.

In the SINCHRONIZED PLATINUM BREAK the vibration of a bar-magnet, whose movements are synchronized with one phase of the commercial current, gives rise to the interruptions. The objections to its use are that it not only wastes one phase of the current, but also is only available for use witls coils of small or meditum size. It is, hesides, rather noisy, requires

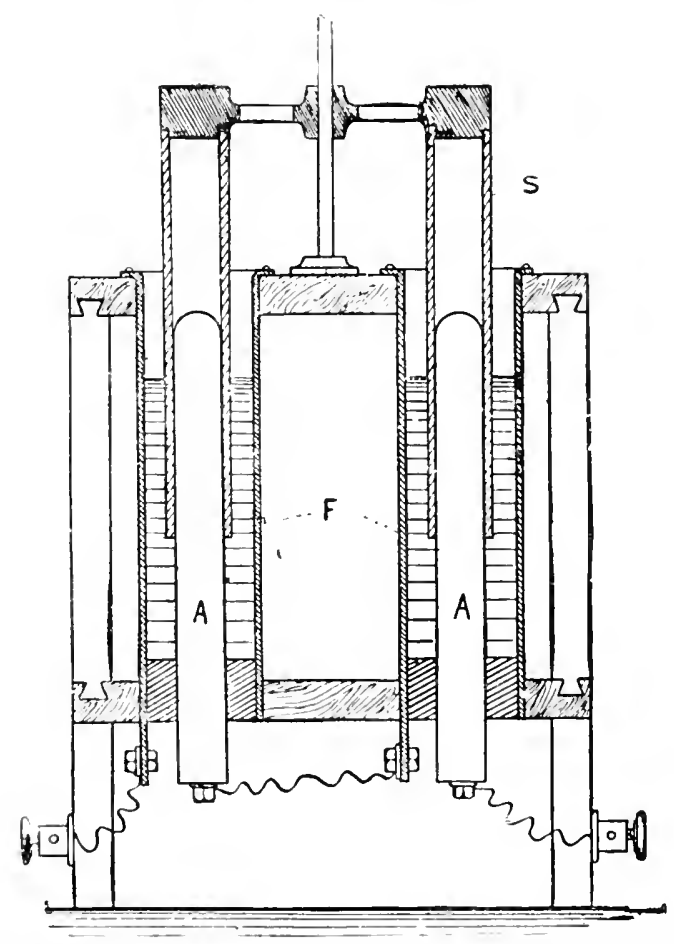

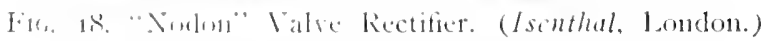

much delicate arlju-tment and frapuent attention, as well as presenting all the other disallantages of the ordinary platinum interrupter.

The idea underlying the construction of THE SYNCHRONIZED ROTARY BRlo.K (Fig , 11) and 20), is to interrupt the current once in either phase and at the moment of maximum intensity. As the circuit is open 
for one phase only of the current, the flow is intermittent. The rapidity of the interruptions is determined by the periodicity of the main and is capable of no variation. The conditions under which these interrupters attain their maximum of efficiency is where the interrupter-spindle and that of the synchronizing motor are firmly coupled together without any elastic or slipping couplings. Jet Interuptirs and Turbinia Brouls, when so coupled logether, give the best results. The motor has to be started by a flywheel driven by hand-power until the correct speed is arrived at. It has further to be adapted to the dynamo which feeds it. If the dymamo runs mooth and maintains a constant frequency, the results are excellent; but this cannot always be secured. Another objection to its use is its great cost.

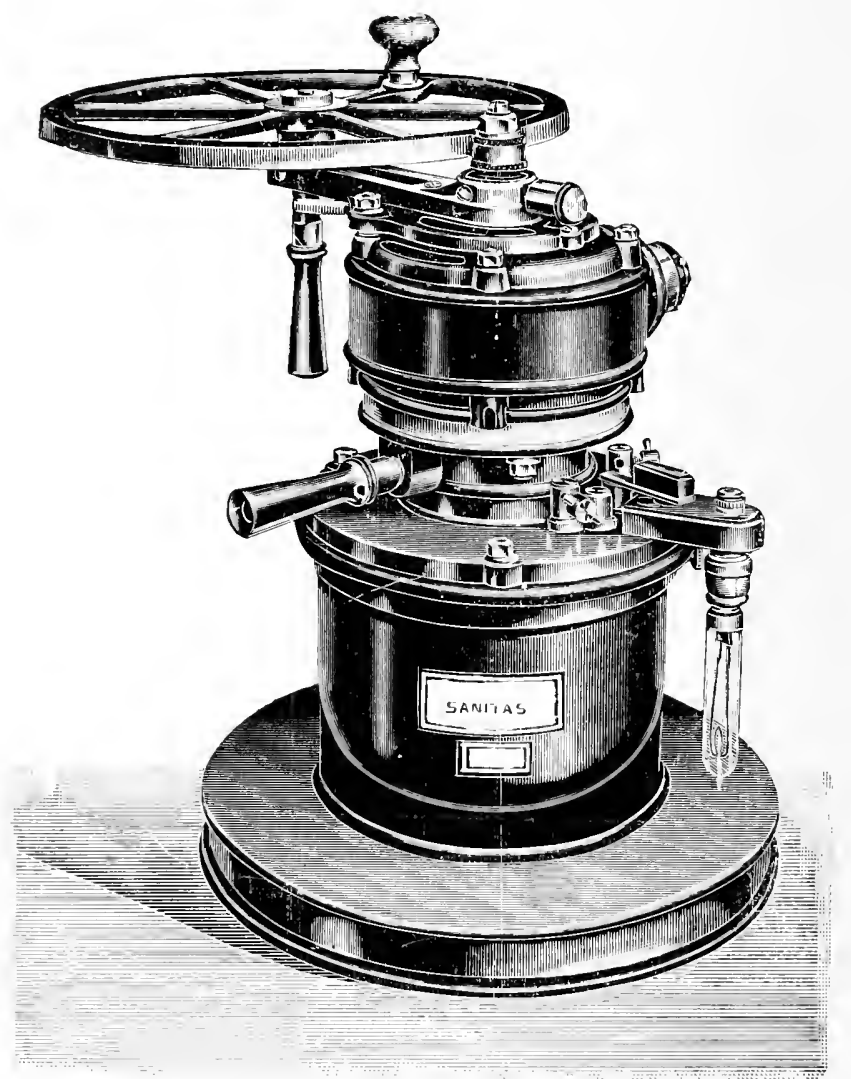

FIG. Ig. Synchronized Turbine Interripter. (Sanitus Flatrical Co., London.)

STATIC MACHINES (Figs. 2I, 22 and 23) are the only dircot sonrce of uni-directional currents of high potential and small amperage, like that required to ofrerate a focus tulye: ind as -uch is better fitted for this purpose than to charge the condenser of a lligl Fromincy Apparatus. Althongh generally said to derive its efficiency from Frictional Electricity, this popular belief is a very imperfect statement of the truth-at least in the case of modern lnfucho Machines, which derive the major part of their charge from Elcctro-Static Induction. For the sulutle influence, which one electrified body has on all other conductors brought into proximity with it, has more 
to do with their efficiency than the cnergy derived from friction. The three principal types of Influenci lathing in common use are the HOLTZ. the VOSS, and the WIMSILRST.

In the fl imshurst machine. buth the circular dises, which are about 1/4 incl apart, are made to revolve in opposite directions; in the Holta machine, on the other hand. one plate-the linger-is permancntly fixed, and has the other revolving much choser o it. In the earlier models the revolving plates were made of glass. whore liability to hreak when made to revolve at a high speed is so great that in sufficient suphly of current. steady and ample enough for all purposes. contl only be attained by the costly multiplication of gigantic plates. whose we sze increased their liability to fracture. The substitution of ebonite and compressed micat plates is an improrement in the right direction, as the increase in speed make un for the diminished surface-

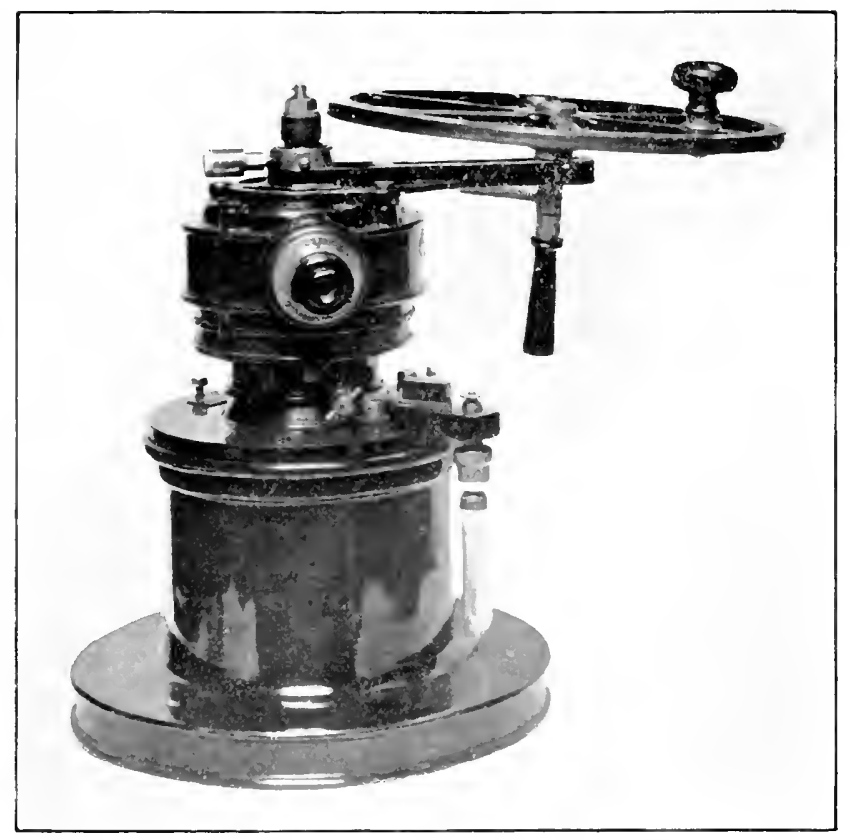

Fis, 20. Synchronized Turline Interrupter. (Ischthal, London.)

capacity of the dises. The saving in thoor space thus olutained is an inportant

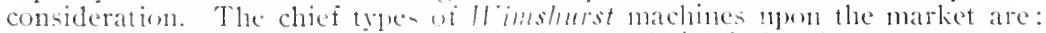

( I) Nachines with multi-sector plates and simple brushes.

(2) Nacluine with multiple brinher and ho sector, and machines of the mixed type, which combine the aluantages of both systems-auto-excitation, with increaned sutput. The only portable machine of the llimshurst

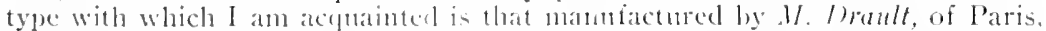

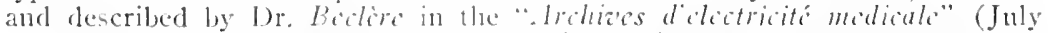
I5th, Igoo). It was eeprecially intended for radiographic work, weighs only 32 kilus, and pack away along with all aceessorien into a catse meanuring 82 by 87 by $53 \mathrm{~cm}$.

ADVANTAGIS ANI) IISAIM INTAGES OF TIE STATIC MACHINE- The simplicity of it com-truction, the absence of delicate and complicaterl apparatus, the independenee of the operater of local contingencies and 
circumstances, the minimum of attention required to maintain it in good working order, and the almost perfectly continuous current it produces are among its chief advantages. The low amperage. which unfits it to charge the condensers of a High Firequency machine, the liability of its strfface to ac-

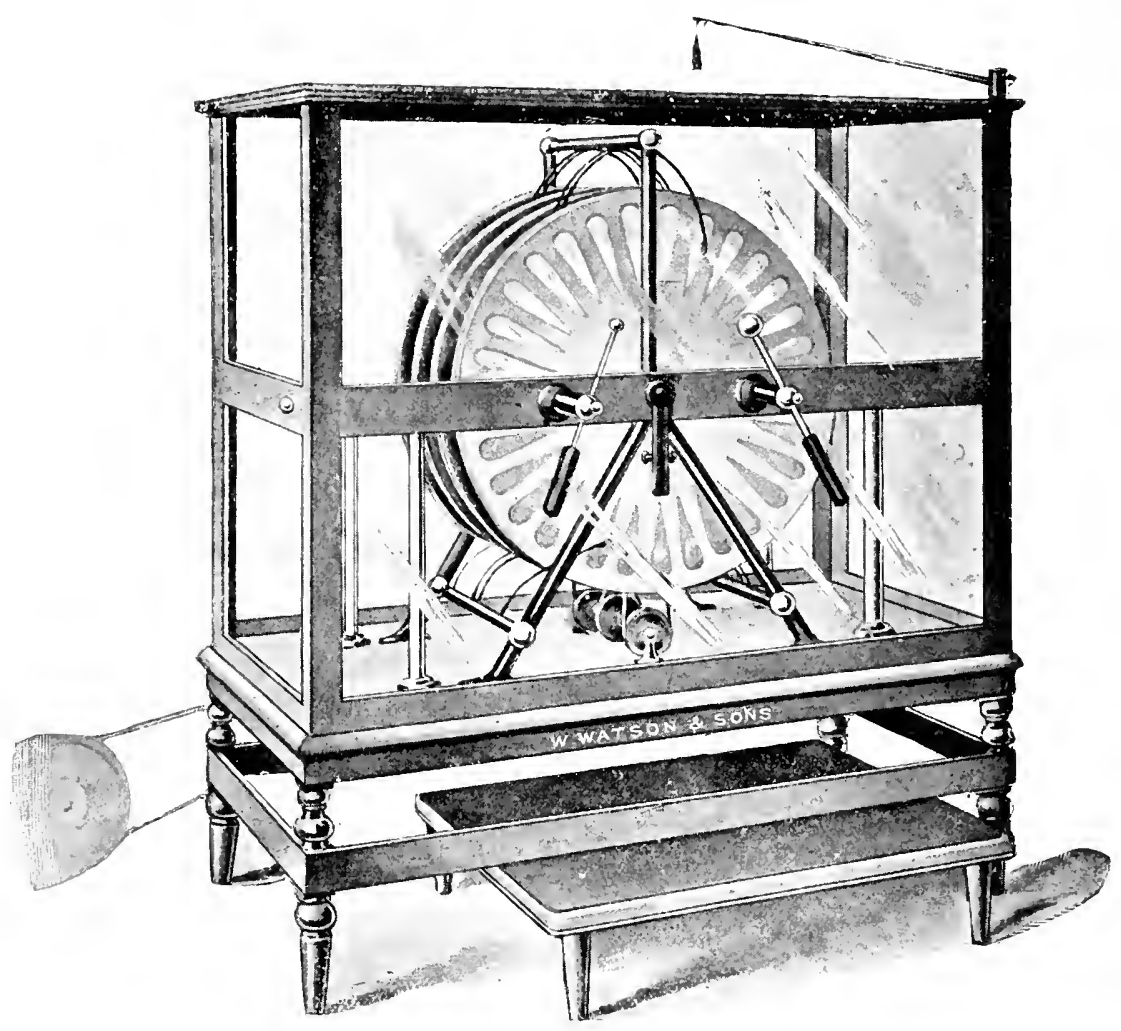

FIG. 2I. Static Filn Machine.

cumulate damp, and of the metallic sectors to oxidation, the erratic reversal in its polarity (especially when used for radiography), and its liability to electrical leakage and set up brush discharges, compled with its large size and high initial cost, are among its chief disadvantages. It is better fitted for fluoroscopic examination than for radiographic work, the most probable reason being that a high potential difference in the electrical charges on its armatures is more easily maintained with a high resistance focus tube in the circuit than by the use of one of lower vacumm-resistance. To obviate the difficulty of using low-resistance tubes, Drs. IFilliams and Rollins have introduced into the circuit between one or other of the terminals of the tube and the positive or negative leads, a multiple spark-gap-a device which allows the radiation of the tube to be very largely modified so as to give hard or soft screenic effects. Before attaching the leads to the terminals of the tube, the polarity of the current should be ascertained by watching the character of the sparks that pass between the knobs of the dischargers; these converge to a point at the positive, and spread themselves over a wider area at the negative pole. 


\section{APPARATLS EMPLOYED IX IIBRATORY ELECTRISATION.}

In noticing the apparatus nsed in the treatment of diseases by currents of high frequency, we can for purposes of classification divide then into four chief groups:

(a) Apparatus employed in gencrating the oscillatory currents.

(b) Apparatus emploted in rasing the tension of the currents of high frequency:

(i) Apparatus whose nee is peculiar to the individual methods of application.

(d) Instruments for measuring the amperage of the currents, etc.

(a) APPARATUS EAPLOYED IN GENERATING THE OSCILLATORY CLRREXTS. - Allongh Tesla has, by running an alternator with $38+$ poles at a very higl speed (3.000 revolutions per minute), been able to obtain a current with a frequency of 10,000 periods per second, this mechanjcal process has toot been generally adopted; the oscillatory discharge of condensers, on the contrary, is the method most commonly employed. The period of vibration of the currents so ubtaned is measured by hundred-millionths of a second. The number and arrangement of the condensers vary somewhat in different types of apparatus, but the principle underlying their application is the same in all.

In the Carre of Tishe Transformers only one condenser is employed.

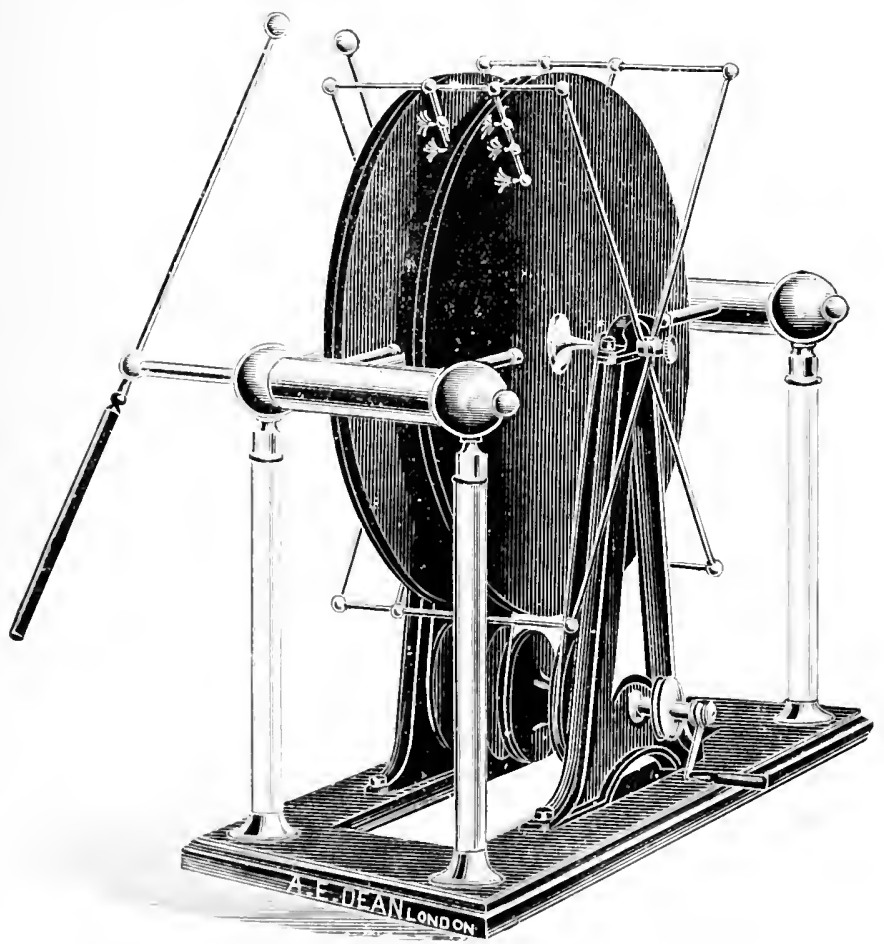

lin. 22. Migh-Speed Secturless Static Machine.

Its armatures are connected with the secondary terminals of a high intensity spark-coil on the one hand, and with the discharging pillars of an adjustable spark-gap on the other; while the snlenoid of high frequency, which consists of 12 to 20 turns of stout copper wire, forms part of the circuit of discharge 
between the external armature and the pillar of the spark-gap with which it is connected.

The Tereira Trunsformer (Fig. 2t) is in many respects the same, with the exception that in it a hatery of Leydon jars $(2$ to + in number and connecterl in series) is sulstituted for the single condenser of which both Carré and Tesla make use.

In the D'Arsonial Transformer (Fig. 25), and all modifications of this apparatns, two condensers are employed. The internal armature of each is connected with one of the secondary terminals of the induction coil and with the discharging pillars of the spark-ga1): while the external armatures are

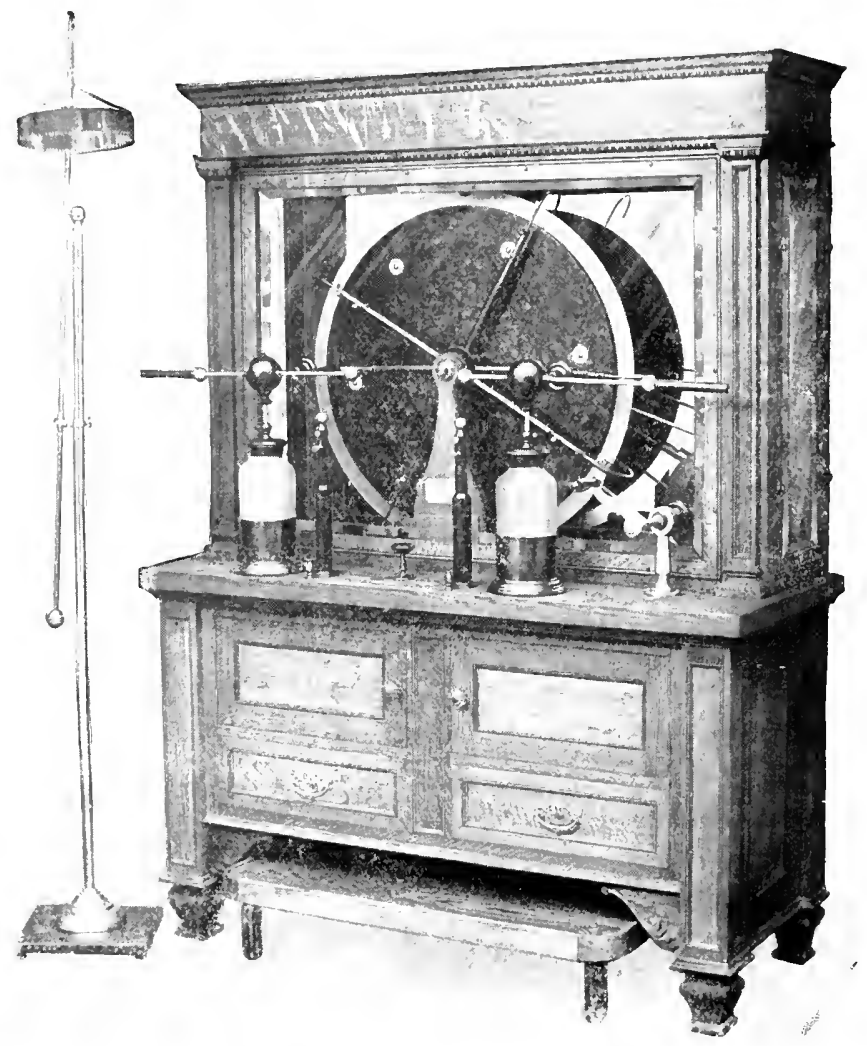

Firi. 23. Static Machine. (Smith if Ilade, Lomdon.)

united by the small solenoid of high irequency formed of 15 to 20 spirals of thick copper wire.

The apparatus of $O^{\prime}$ Farril \& Lebailly (Fig. 26) is a modification of the 1) Arsonteal Transformer in which the solenoid of high frequency is replaced by a duplex resonator, with which the external arnatures are connecterl.

An apparatus somewlat smilar is the Rochefort nudel (Fig. 27), for lise with two condensers. In it similar bipolar effects are ohtained by the use of two ordinary resonators, the free extremities of their lowest pirals being directly comnected with one antother. 
The apparatus, however. which .I. Rochetort most strongly recommends is that represented in Fig. 2s. Here four condensers, divided into two batteries. are emploverl. The internal anmatures of these are connected with the terminals of the secomblary of his transformer, which serves all the
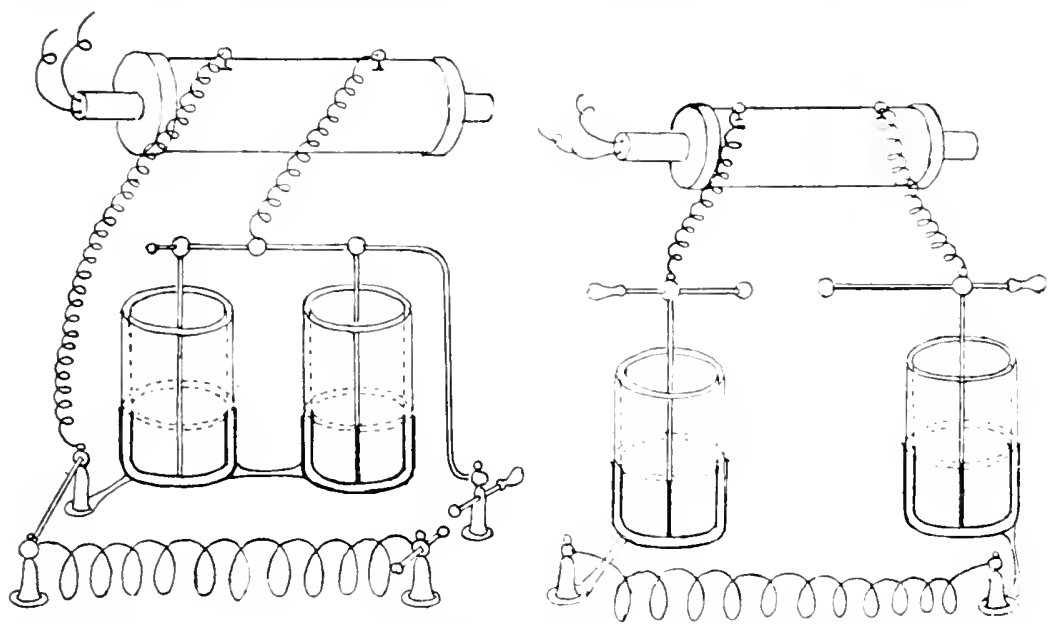

Fig. 24. The Tiacina Tramsormer. Fus. 25. The D'. Irsonial Transformer.

purposes of a spatk-cuil. int with greater efficincy. The external armatures are comnected with the two resmatters after the mannes shown in Fig. 27; the ure of the snall whenuid of high frequency is entirely dispensed with.

In all these transformer the cipacity of the condensers employed is proportioned to the self-intuction and resistince of the circuit, so as to main-

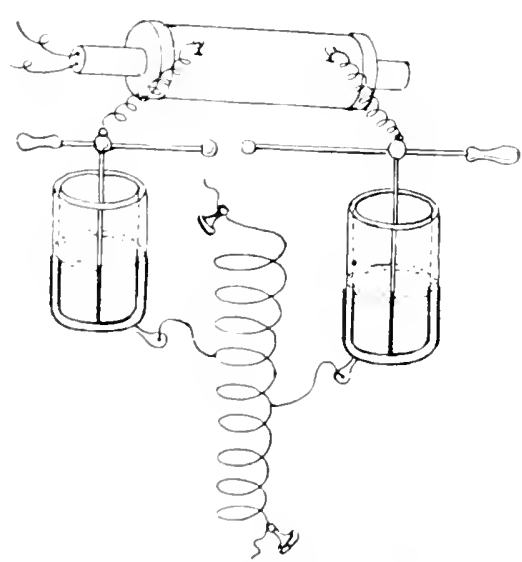

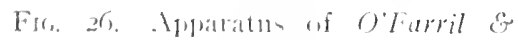
l.iluilly:

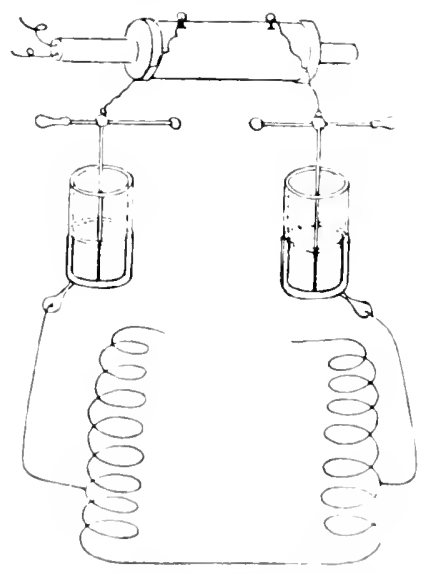

lini. 27. Roihefort's Model for L'se with Twu Conklenters.

tain the weillatory chatracter of the sark dinchatges, which ate the source

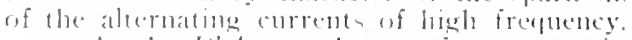

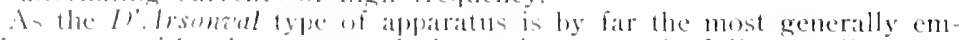
ploged. we can with advantage -tmely its various parts in fuller detail. 
CONDENSERS.-There are two types of condensers used by different makers in the construction of this transformer. The one is the Leyden jar, the other the plate condenser. The former are chiefly employed by instrument makers both in Germany and England; the use of the latter is at present confined to France. The chief advantage claimed for the plate-condenser is that the dielectric can be more easily obtained of a uniform thickness, so that no undue electrical stress is brought to bear on any point of its coated surface. This lessens the liability of the glass to be pierced by a disruptive discharge passing between its two coats. By stubmersion in a bath of highflash petroleum oil, these plate-condensers are rendered less liable to become oxydised, and so preserve their coats intact for longer periods than Leyden jars, which are not insulated in the same way. On the other hand, it has to be noticed that the inductive influence of the charge imparted to the internal armature of the Leyden jars is increased by their configuration, while at the same time the capacity of the jars can be more readily varied so as to alter the frequency of the oscillatory discharges passing between the discharging balls of the spark-gap.

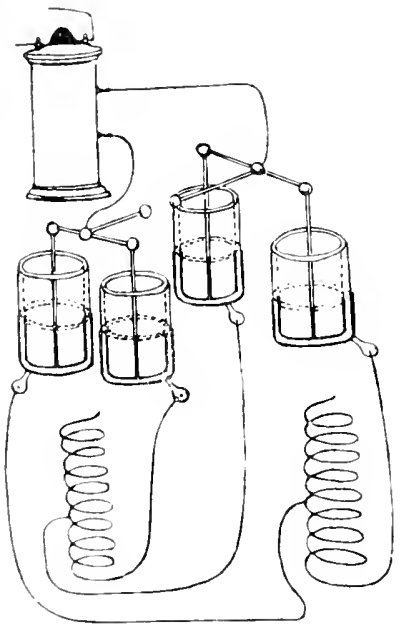

FIG, 28. Rochefort's Model for Use with Four Condensers.

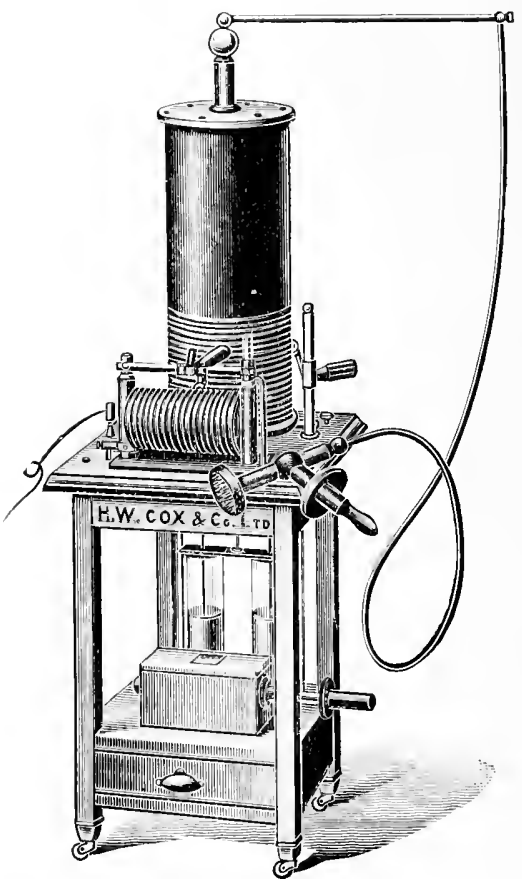

FiJg. 29. ITigh-Frequency Apparatus, with Square Spark-Box.

The Detonator or Spark-box (Fig. 29). - This useful accessory has been given various shapes by different makers, who have endeavored by various expedients to correct the several drawbacks attending its use. The passage of an oscillatory discharge between the knobs of the discharger gives rise to a loud reverberating cackling noise. which is nowise conducive to quieting the alarm of nervous patients. In the attempt to muffle the sound by enclosing the discharging knobs in a glass or earthenware sparkbox, it was noticed that nitrous compounds were generated, which, by uniting with the moisture of the enclosed air, formed nitric acid. The deposit of this conducting fluid on the inner surface of the detonator increased the tendency 
to areing. In its simplest form the spark-box consists of a cubical earthenware or glass box with rulcanite lid. As this does not entirely suppress the disagreeable sounds, it was considered advisable to further enclose it in a feltlined wooden box. which, on account of its efficiency, is well named the silent spark-bor. It figures in the apparatus shown in Fig. 30 . As the fre-

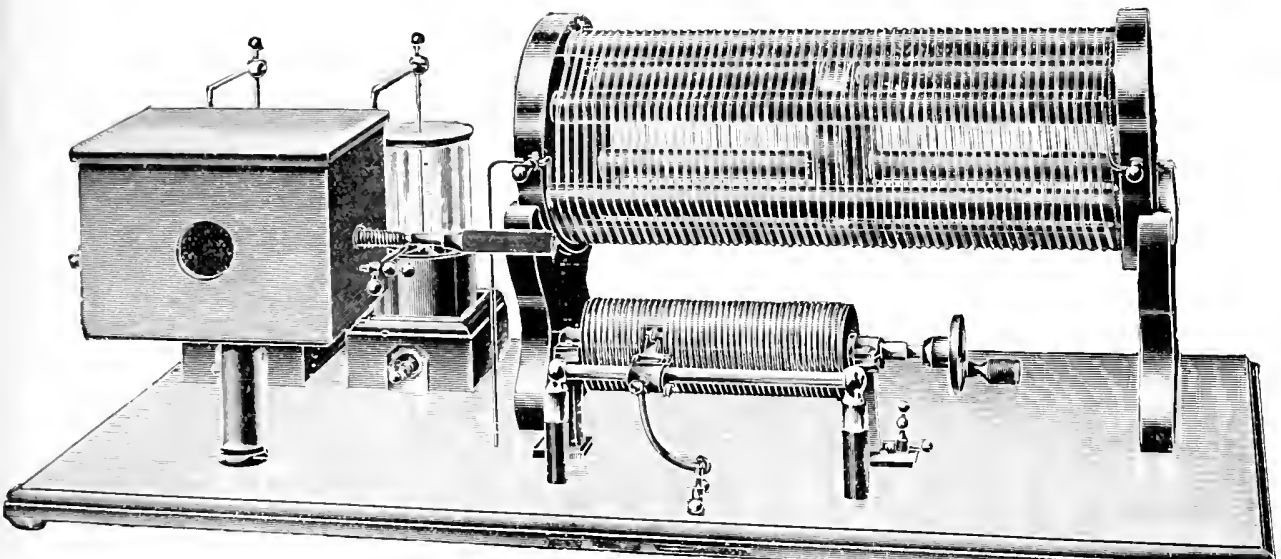

FiG. 30. New Nodel H. F. Apparatus, with Rotary Solenoid and Silent Spark-Box. (Iscuthal, London.)

quency of oscillation of the alternating discliarge depends upon the resistance of the air-gap between the knobs of the discharger, it was considered advisable to enclose it in a transparent cylindrical glass tube, fitted with vulcanite (felt-lined) caps, as in Fig. 3I, through which the distance between the balls could be accurately gauged without opening the lid of the spark-box; but as the same object can be more accurately effected by means of the graduated

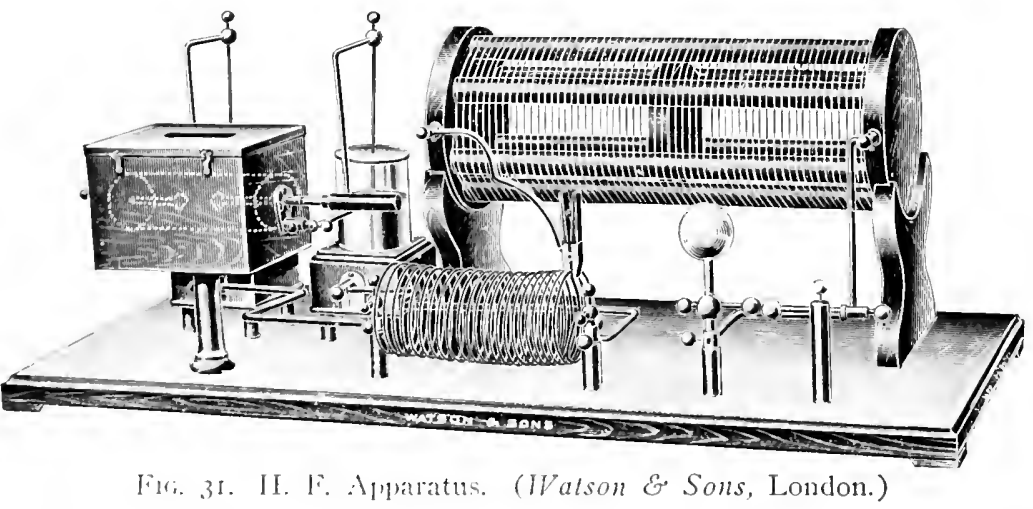

slicling-rot of the Silent Spark-Bow there is no real advantage to be gained by the use of glass. The liability of the spark discharge to arc and so destroy the box has been met by the use of a magnalium detonator, which effectually prevents the deposit of moisture upor these surfaces. The movable arm of the detrnator is, in the apparatus of some makers, spirally grooved or 
threaded, to prevent any unintentional alteration of the distance between the knobs of the discharger, and to allow a finer graduation in its adjustment.

The Small Solcnoid of High Frequency. so ntanted from its intimate association with the armat!res of the condensers, consists of 12 to 20 turns of coarse copper or mangano-copper wire, connected through the supporting pillars with the external coats of the condensers. As the amount of current passing into the body of the patient in the bipolar method of direct applica-

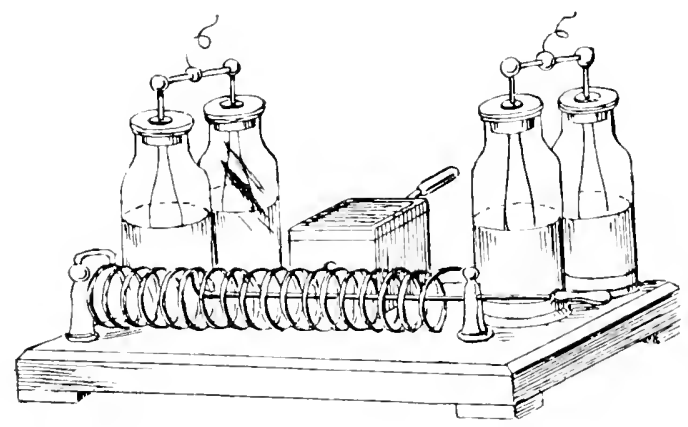

Fic. 32. Contact Rod Regulator.

tion and also in bipolar condensation depends on the self-induction of this circuit, the method employed to vary the nmmler of spiral turns introduced into it is a matter of paramount impurtance. The five clief methods of control are as follows:

The Sliding Contact Rod Regulator (Fig. 32).-This consists of a metallic rod pernanently connected with one end of the small solenoid and fitted with an insulating handle By sliding along a groove in the supporting

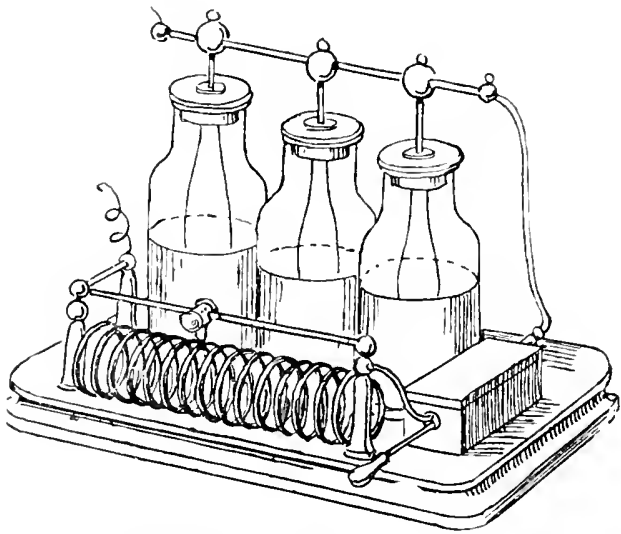

Fiti. 3.3. Movable Contact-Point Regulator.

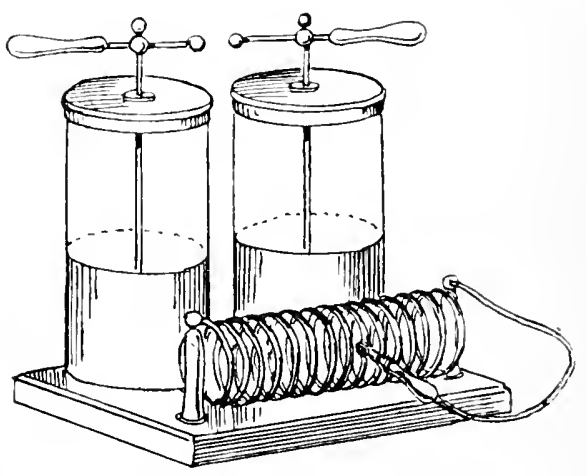

Fic. 34. Adjustable Spring Catch.

pillar it can touch any desired spiral, and by so doing throw out of the circuit nne or more turns of the solenoid. In some models it passes into the interior of the sotenoid: in others it touches the spirals externally. The tension of the current in the shunt circuit diminishes as the rod is pushed home, and increases as it is withdrawn. The brass tube-regulator that works in the interior of the solenoid of the Carré Transformer is a variation of this method of control. This arrangement allows a certain measure of general control, 


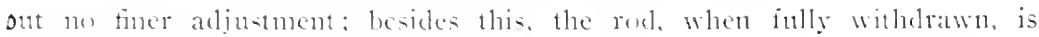
liable to get in the way of the operator.

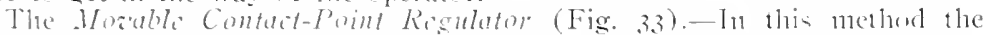

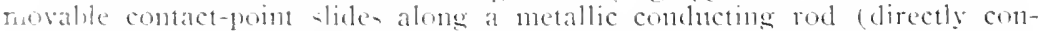
nected with one of the -nuporting pillars of the solenoid. but in-ulated from the other, which aloo helps to supmort it). The free extremity, ly touching the wire of the solenoil. can entilinh contact whth any desired spiral. This allows of an irregular and somewhat conse graduation of the intensity of the current, without any marked interruption of its flux, provided the contact piece be hroad enongh to touch lwo adjacent spirals.

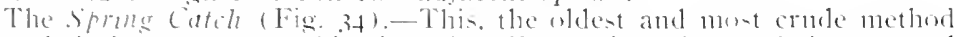

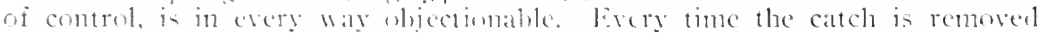

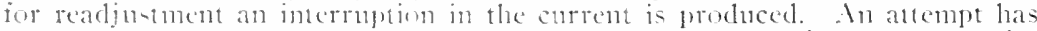
been made a remedy this by connecting the spring-eatch with ome extremity

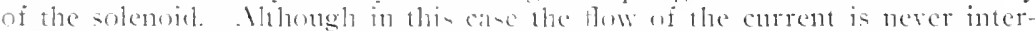
rupter. still the remural oi the catcle from a spiral immediately throws into the direct cirenit exery turn of the solomoid. and as atdenly increases the intensity of the current in the derived circuit.

The Kendeing solenoid.-In thic form of eontrol the wire solenoid

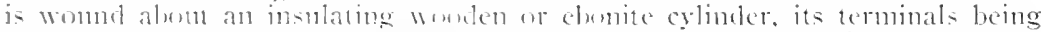

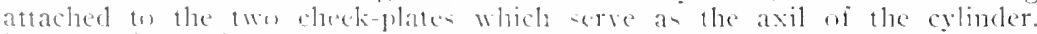

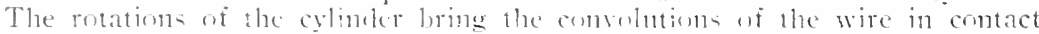

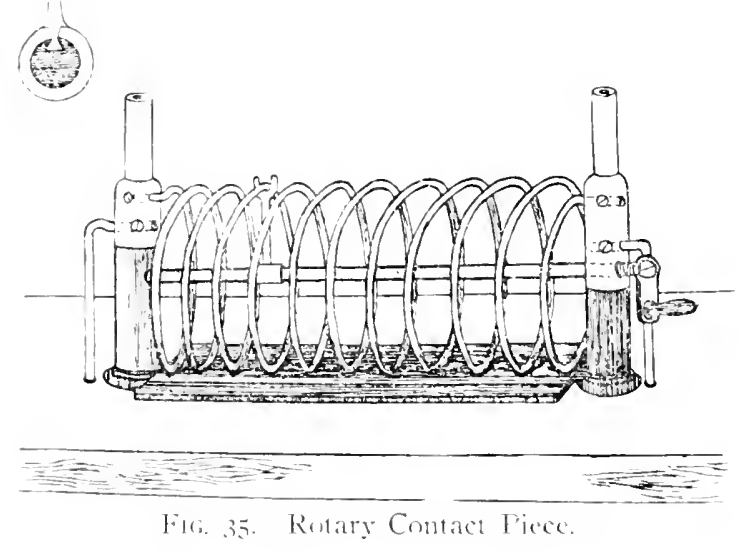

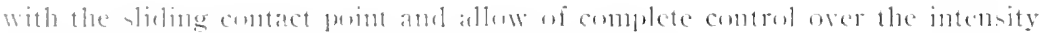

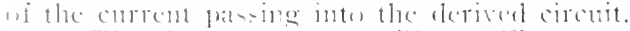

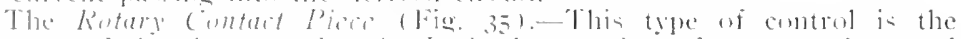

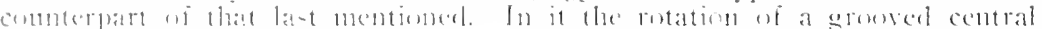

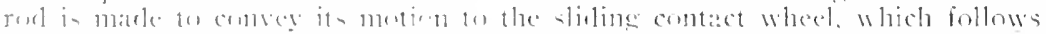

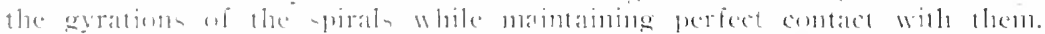

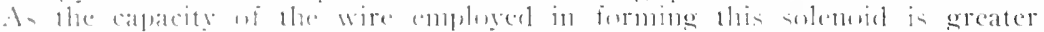

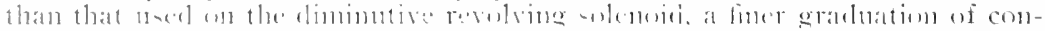

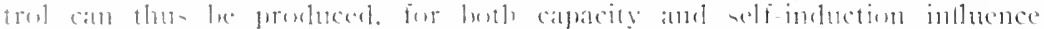

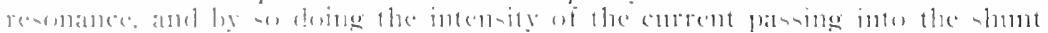

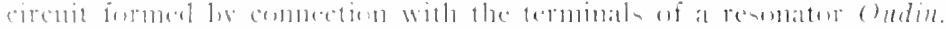

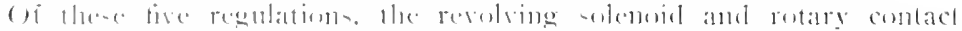

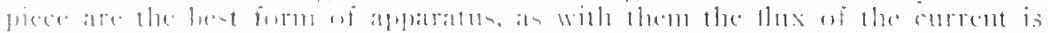

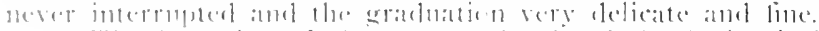

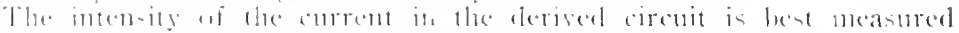

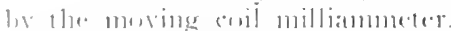

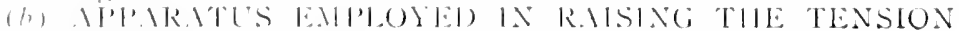

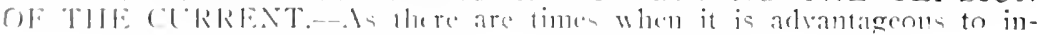


crease the tension of the currents employed in vibratory electrisation, several different kinds of apparatus have been constructed for the purpose. The chief of these are:

(I) The Tesla Coil.

(2) The Oudin Resonator.

(3) The D'Arsonval Bipolar Coil of High Tension.

(4) The Spirals of Guilleminot.

(5) The Cones of Reus.

(I) The Tesla Coil, the first in order of time of the apparatus devised for raising the tension of the current, was invented by Tesla in the year I89r.

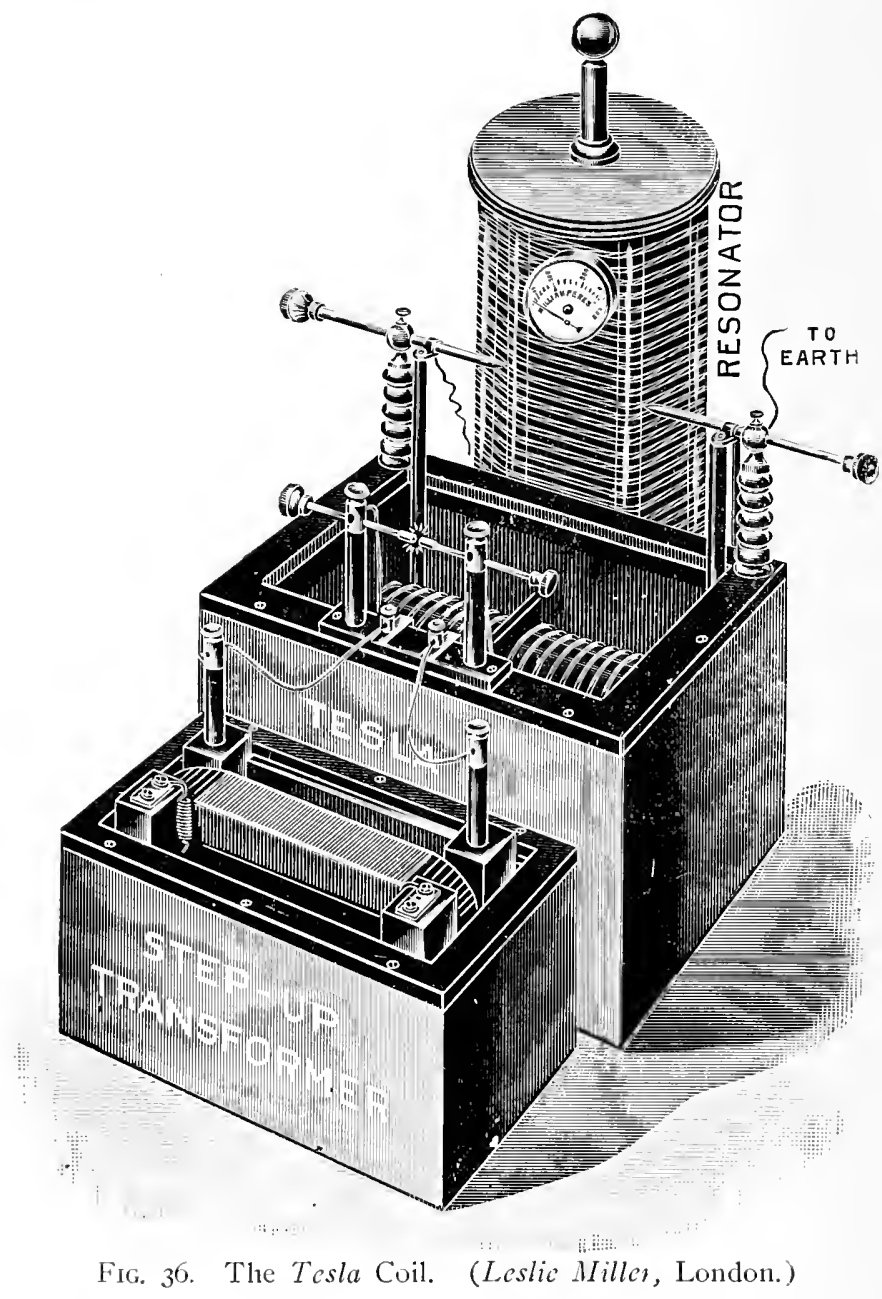

It essentially consists of an inner and an outer coil, with the former of which a condenser and spark-gap are connected in series. Both coils and the condenser are usually submerged in an insulating container filled with oil. The primary or inner coil consists of a few turns of stout copper wire, 
While the secondaty or onter is a single laser of many tums of fine copper wire, wound romd the primary, from which it is carefully insulated. The apparatus can be ied by either a spark-coil or step-up transformer. It yields a plentiful supply of sparks of any desired length, but the noise made when working is deatening.

(2) The Oudin Risonator.-Alhough Hirlz had previonsly emploged the plenomena of resonance in his experiments, it is to Dr. Oudin that the resonator owes its introductioni into electrotherapentics. He first, in I\$82. made use of resonance clitects to elevate the tension of currents of high frequency. The apparatus devised by him consists of a large solenoid of uninsulated copper wire, of medimm thickness, wound spirally about a vertical cylinder of well-paraffined wood. The total length of the wire employed varies from 45 to oo metres, and its diameter from 2.5 to 3 millimetres. It makes 50 or more turns abont a wooden cylinder to to 50 centimetres in lyeight and 30 centimetres in diameter; while the distance between the spirals is about 8 millinetres.

As forcid resonance is more powerful in its effects than fre resonance. the resonator was at tirst attached to one pole of the solenoid of high frequency; the other pole remained free or was comnected to eartli. This arrangenent, however, was soon modified. It was fomnd that better elfects could be produced by connecting both poles will the resonator. A subsequent modification led to the solcnoid of high frequency being suppressed and the resonator directly comected with the external armatures of the condensers. The lowest spiral of the resonator was then mited to one armature, while a spiral four to seven turns above it was connected with the other armature by an adjustable clip. The resonator was thus dirided into two colenoids-a lower and an upper. The lower, or prinary, solenoid, of small size, consisted of the few turns of wre intercepted between the points of attachment of the two cords leading to the external armatures. In it circulated currents of both high and medium frequency, those of high frequency alone being free to pass into the upper solenoid; for the currents of lower frequency were clo-e-circuited by it. The secondary or upper solenoid was longer, and was travered by currents of high frepuency alone; these, by reason of the selfinduction of the circuit. attained an enormons tension. When the three esicntial magnitudes-canacity, self-induction and resistanceof the two solenoids were by trial proportioned to one another. the upper spirals of the resomator and its free teminal were seen lathed in a lively brush discharge, like to that produced by a Tesla coil or inthence machine. This efflure is made use of for therapentical purposes, by connecting the free teminal of the resonatir or one of its nper spirals with the wire leading to the electrode.

Althongh the calihe of the wire, which forms the resonator, does not appear in materially interfere with the production of these resonance effect, yet it has, according to () udin, a considerable intluence on the plysical character of the effluve and spark. A line wire yiclds a spark, long, thin, sinuous and searcely painful, with an efluwe as porty nourished: while with a wire of larger dimensions. loth efthe and spark gain in forec. This depends on the capacity of the wire rather than it self-induction, for every increase of calpacity remlers the spark more vigomons and painful.

The manner in which comnection was established between the poles of the prinatry solenoid of the resomator and the extermal armatures of the condensers next attracter attention. It was recognised that the nature of the arrangement that exint for this purpose largely influenced the adiustment of the two solenoride and the regnlation wi the elfects produced. The crmele

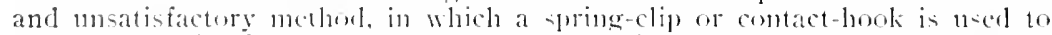
connect the wire from the external armat me with the desired piral soon gave place to sthers which allewed mere perfect regulation and adjustment of be made witlont in any way interrupting tae circuit. Ducretst and Bonnetti inventerl an alplaratus in which a gromed contact-slip is mate on revolve albout a fixed resrmator, and then cetablish contalet with the spirals; while Radiguet causef the revomatur itedf to rotate before a statiomary contact-point. Both these methorls allow of at more perfect gratuation of effects and an eacier 
adjustment of the solenoids to each other, without any interruption of contact.

The resonators above described, however, are only suitable for producing unipolar effects. If double efluvation is to be practised, the apparatus of Lcbailly $\& O^{\prime}$ farril or that of $M$. Rochifort must be resorted to.

In the former the primary solenoid is centrally situated, the external armatures being connected with two intermediate spirals. This creates two secondary solenoids, which are situated one on either side of the primary, and allows of bipolar applications to be made by connecting the wires leading to the electrodes with the free outer terminals of these two solenoids.

Although the apparatus is an improvement on the unipolar resonator of Ondin, still it is not always easy to equalise the current density at the two poles, and for this reason that of $M$. Roclucfort is to be preferred.

In the Rorhefort model (aide Fig. 28) four Leyden jars are employed. These are dirided into two hatteries. comnected hy their internal armatures with the secundary terminal of an induction tran-former or spark-coil. The cxternal armatmes are connected with two separate resonators, those of each battery leing connected, the one with the lower terminal of one resonator, the other with the uppermost spiral of the primary solenoid of the other resomator. When carcfully adjusted, the density of the current traversing each of the cecondary solenoids is ermalised; and being of opposite sign,

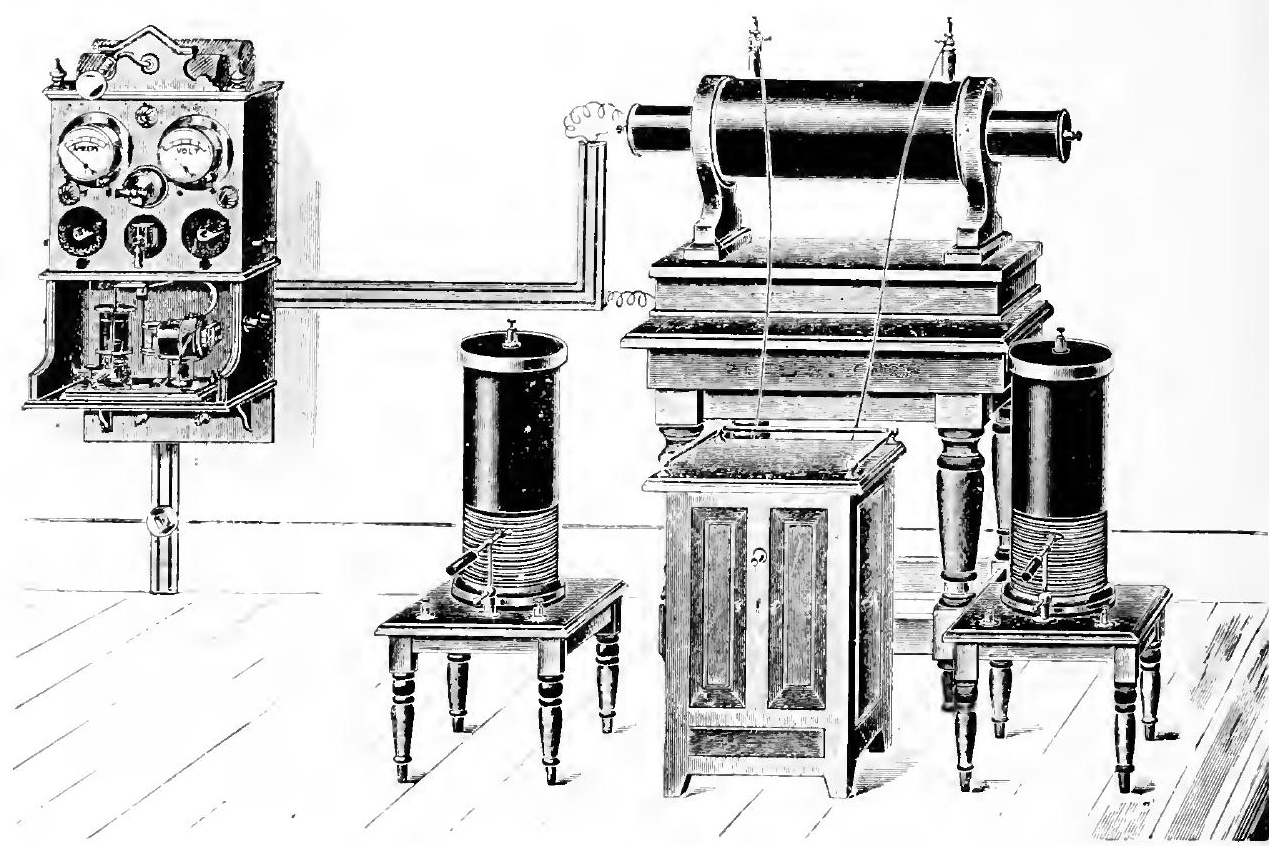

Fin. 37. Donble Model Resonator. (I)ian, london.)

(minl)le effluation ean easily be practiced and the effects produced readily controlled.

The position of the resonator in the aplyatus of different makers calls for a few remarks. In some apparatus, as in Fig. 37. the solenoid stands 11pright on the cabinet enclosing the condenser and spark-gap: in others, as in Fig. 38 , it is fermanenty connected with the comlensers; in others, again, 
the resonator is horizontally placed so as to allow its inclusion in a cabinet; and lastly, it may be inverted and fixed by its lase to the bottom of the baseboard of the high frequency table, as in Fig. 39. As there is no special adrantage gained by any of these peculiar arrangements beyond the mere matter of aring space, these eccentricities call only for a passing notice. lluch more important, however, is the arrangement for graduation. The

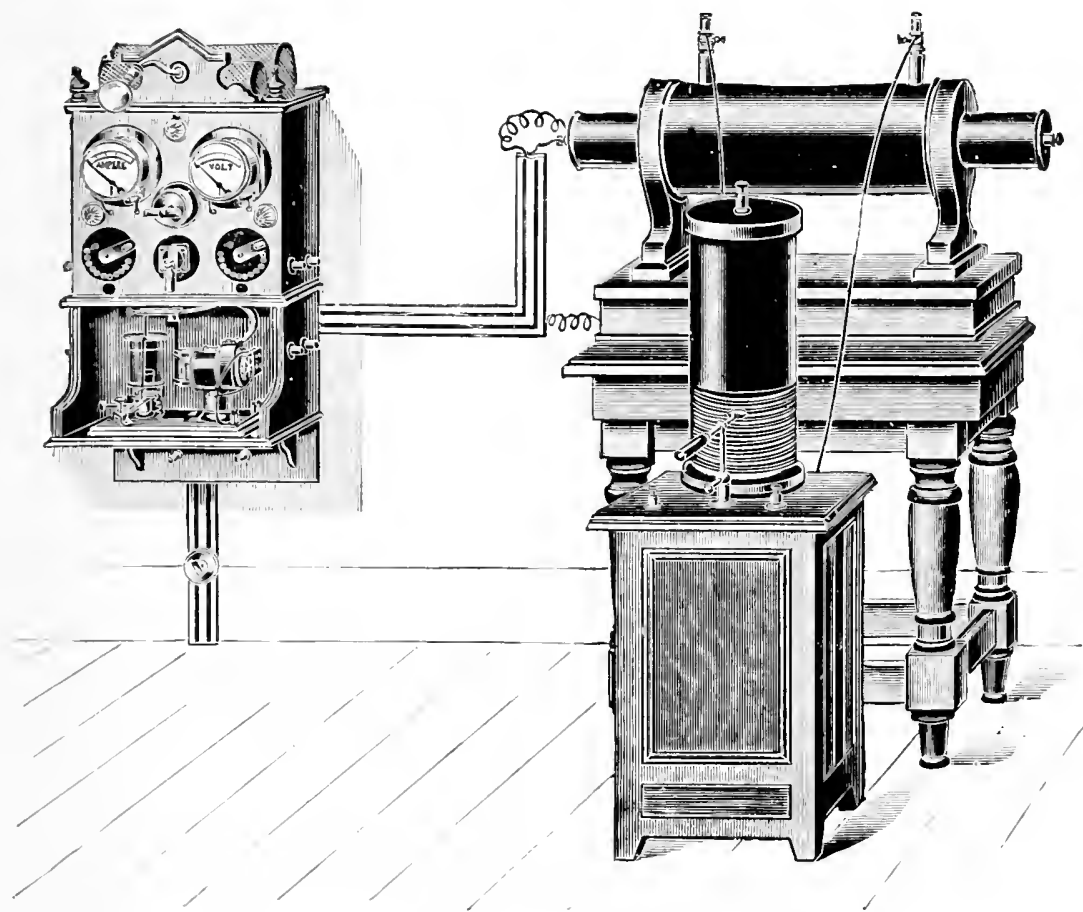

Fic. 38. Single Nudel Resonator. (Dean, London.)

crude method of regulation by the contact clip must only be mentioned to be condemned. The regulation by means of a revolving cylinder, running on rubberer tires, whose movements can le graduated by the movement of a handle, is the one most to be commended. Not less worthy of mention is the model shown in Fig. 4o. Here adjustment is effected by revolving the solcnoid by meanc of the insulating spokes fixed to the disc at its base.

D'ARSONVAL'S BIPOLAR COIL OF HIGII TENSION.-This apjaratus is marle up of an inner and an onter coil. withont any direct communication between them. The inner or secondary coil is formed of a single laver of medium-sized copper wire, making many turns about a central insulating cylinder. The currents derived from its terminals are employed in double effluvation. The outer or inducing coil rests on two insulating supports. It consists uf four turns of thick copper wire, wound parallel, and at some distance from the inner coil. lis terminals are connected with the external armatures of the Ievden jars. This primary coil can be moved to and fro abont the secundary. When centrally situated, the currents derived from the sceondary terminals are of equal density and of opposite sign; but as it is made to approacl one or other extremity, this proportion becomes altered, so that the density at one pole is increatsed while that of the other diminishes. 
This allows of either bipolar or unipolar effects being produced, or effuva. tion to be practised with currents of unequal density.

GUILLEMINOT'S SPIRALS.-In resonators formed of wire wound into a helix or solenoid, the energy of the electrostatic field developed by the inducing spirals is only to a very limited extent utilised in the production of induced currents. For the form of the resonator of Oudin does not permit the powerful oscillatory field. created in the vicinity of the spirals, to be employed in generating bipolar currents. Dr. Guilleminot has, however, by changing the form of the resonator from a helix to a spiral been able to utilise the energy of the field in producing bipolar effects. The results of his experiments were published in 190I. The resonator devised by him consists of a spiral of 15 to 20 spires of copper wire held in position by insulating cords which radiate from a central nave to the periphery of the circular frame work which supports it. The innernost spire has a diameter of 20 to 30 centimetres. The distance between the snccessive spires is about $18 \mathrm{~mm}$. centrally, but somewhat increases towards the periphery. The central extremity

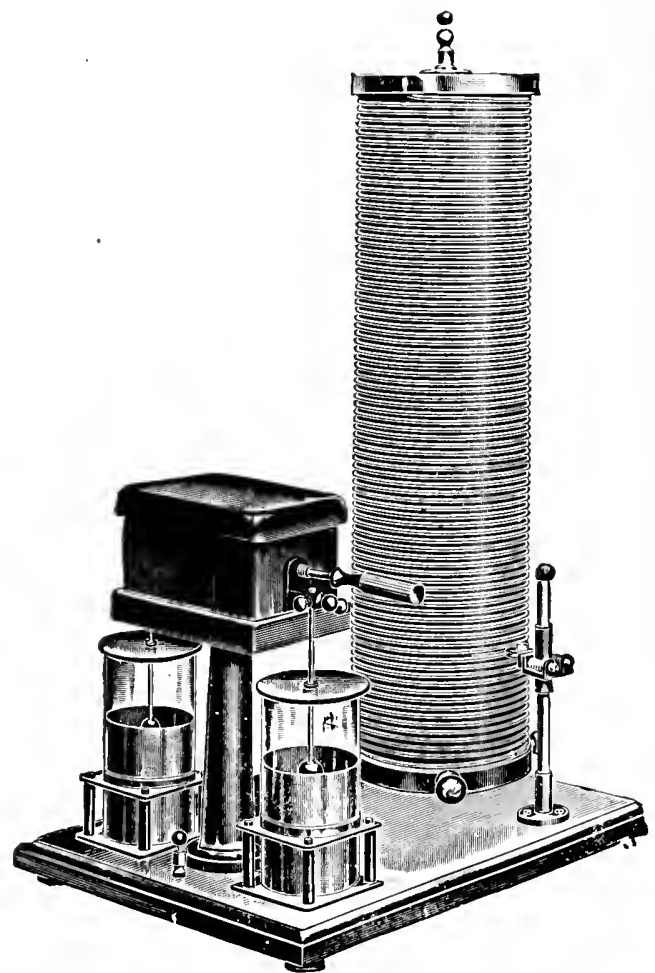

FIg. 39. Small H. F. Apparatus, without Solenoid. (Isenthal, London.)

of the spiral is connected with the nave, which provides a socket, into which the spider electrode of Truchot can be fixed, or with which any other electrode can be attached directly or by means of a tlexible cord. The onter end of the spiral is connected with the binding screw fixed in the supporting frame.

Such a spiral can be influenced by currents of high frequency derived from the external armatures of the condensers. The eflluve, however, will differ accorling to the mode of connecting the armatures with the wires of the piral. For the central effluve obtained by infliencing the outer spirals 
is more solit and tractable than that induced externally by influencing the central spires. Bipolar effects can also beduced by placing one or more of the intermediate spirals in circuit with the onter coats of the leyden jars.

IIth a 16 -inch spark coil and a prinary current of 6 to 8 amperes, the maximum effect is obtaned by connecting the ambtures with a single spire.

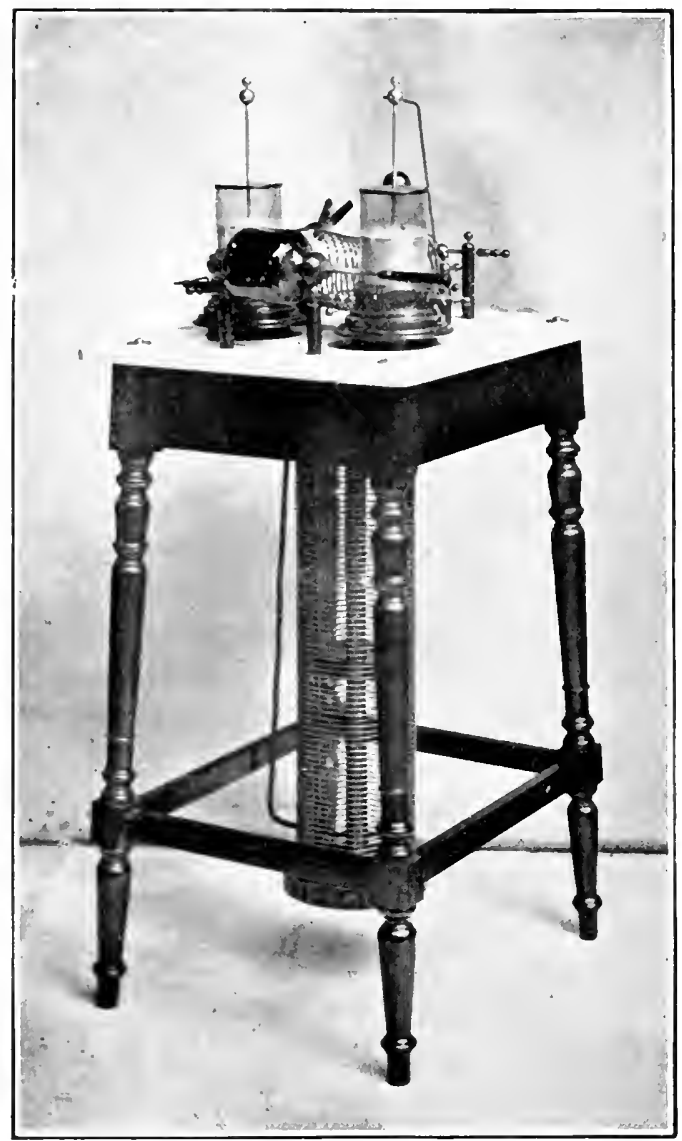

Fib. fo. H. F.. with Solenoid under Table.

This is the condition mo-t favourable to the study of the phenomena of high frepuency for the action of the inducing spirals on one another is thereby eliminaterl.

lis studying (irom centre to periphery) the length of the sparks. which tend to pass between the spires, it is fomme that they are leant at the centre and increase up to the union of the $3+$ internal with the 4 external spires, after which they rapritly diminish in size. Thee results are comstant, what ever be the intensity of the prinary current or kind of spiral emploned.

The influence of two prirals ont eate ofher is equally interesting.

The subject can le bent studied in comsidering separately-

(a) The relative inlluence of an atetive and it pasive spiral aproached to each other. 
(b) The interrelation of two active spirals which do not reciprocally influence each other. other.

(c) The reaction between two active spirals which mutually affect each

(a) The relative influence of an active and a passive spiral approached to each other. If the surfaces of the two spirals, brought in relation to each other, be parallel and their centres in the same horizontal line, the influence of the spirals on each other varies according to the direction of their winding.

If the two spirals wind in a direction contrary to each other (Fig. 42), the brush discharge which issues from the central spire of the active spiral is diminished on both sides of it. At the same time an intense effluvation is induced about the central spire of the other. The closer the spirals are brought to each other, the more beautiful and intense do the interpolar effects become, a free exchange of effluve taking place between them.

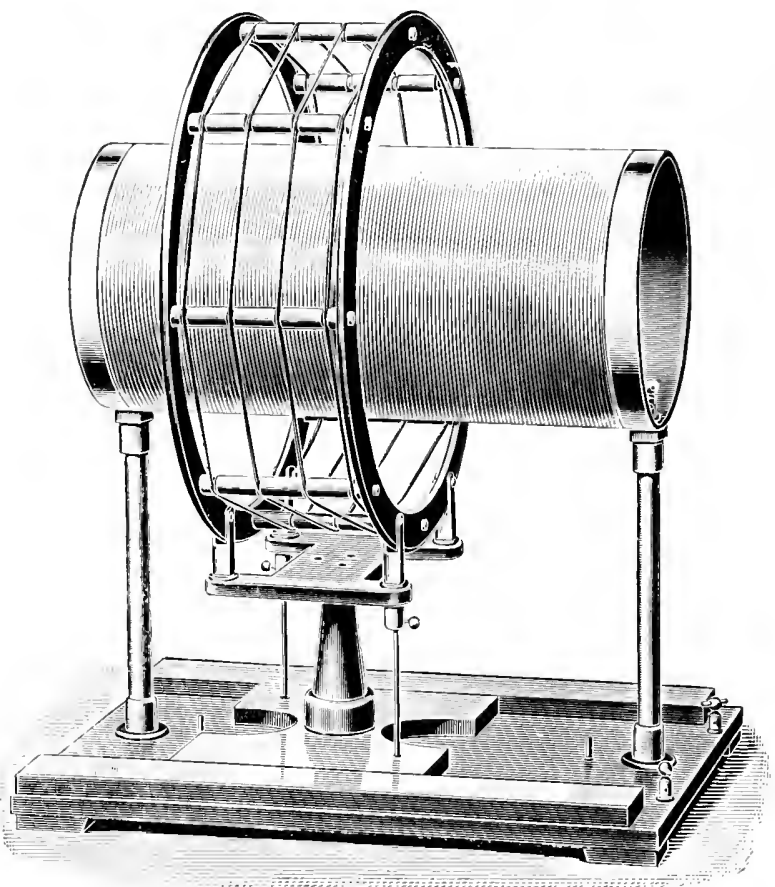

FIg. 4I. D'Arsonval Bipolar Coil, High Tension. (Schall, London.)

These interpolar effects are abolished and effluvation ceases when the central ends of the two spirals are joined by a conducting wire. The connection to earth of the central spire of the passive spiral has but little influence on the effluve derived from the active spiral.

When the two spirals wind in the same direction (Fig. 43) very different effects are produced. The brush discharge about the central spire of the active spiral is but little modified by its proximity to the passive spiral. It is only when the distance between them is less than 20 to 30 centimetres that it becomes asymetrical, being almost absent on the side facing the passive spiral and very vigorous on the side turned away from it. It presents the appearance of being blown away by the intensity of the charge induced in the passive spiral, which, however, is very meagre when compared to the free effluvation 
observed in the preceding cane. It is needlese to say that no interspiral phenomena are produced nor dow effluvation cease when the inner extremities of the wo spiral are compled together. Connection to earth of the

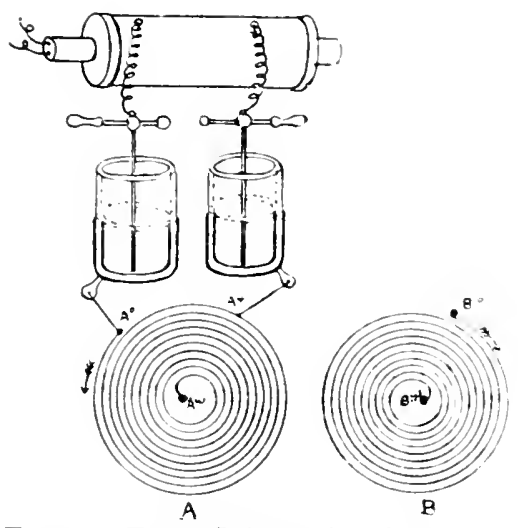

FIG. 42. Two Spirals in Contrary Directions.

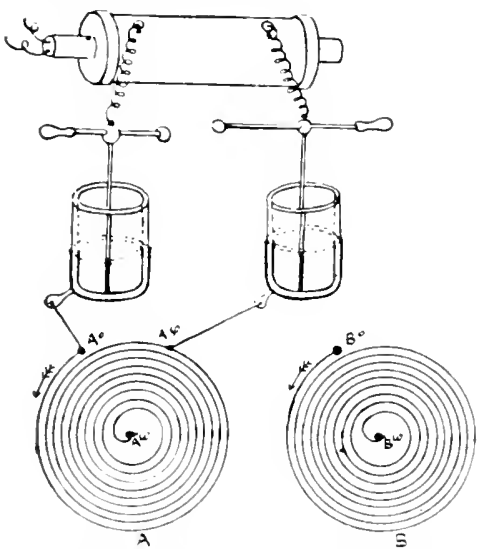

Fui. t.3. Two Spirals in Same Direction.

central extremity of the passive spiral not only re-establishes the symmetry of the effure of the active spiral, but also diminishes it.

(b) Effects obtained with tiio actiac spirals which do not reciprocally influence one anoth'r. For purposes of demonstration, let it be supposed that $A^{\circ}$ and $B^{-}$are the peripheral extrenities of the spirals, $A$ and $B$ respectively, and to and $B$ o points in their first spires, which mark the end of the inducing circuit and the connmencenent of the induced, whilst $\mathrm{L}^{1}$ and $\mathrm{L}^{2}$ are two batteries of Leyden jars. whose external armatures are connected with the poles of the inducing spires. The effects observed will differ according to the method adopter in compling the armatures of the condensers with the coils of the sprirals, and also with the direction of winding of the spirals.
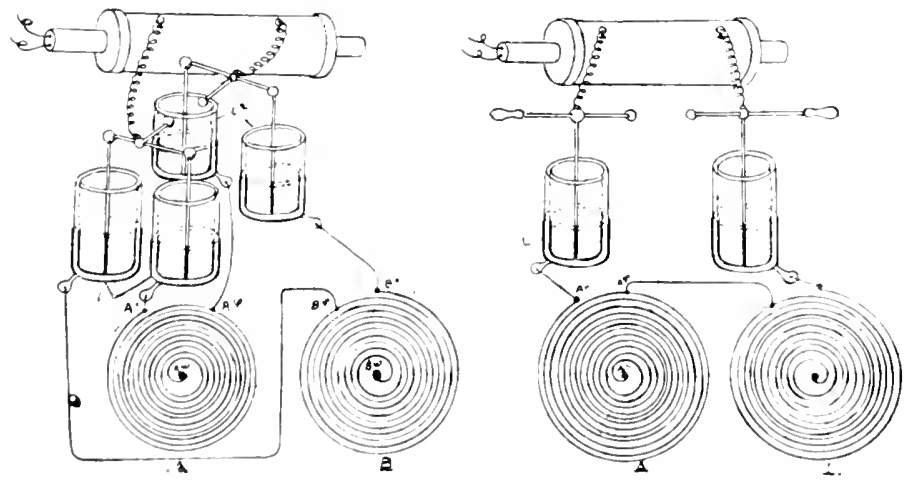

Firs. 4. Active. mit Inllencing One Another.

Case I. Where the flow oi the current is homologous and centripetal in loth spirale. - Here the direction of winding of the two spirals is the same (ligig. 4t). When munted in quantity, the current derived from $\mathrm{L}^{1}$ flow from $A$ to $A$ in the spiral $A$. and from $B^{\circ}$ to $B \phi$ in the spiral $B$, the 
points $\mathrm{A} \phi$ and $\mathrm{B} \phi$ being connected with the battery of condensers at $\mathrm{L}^{2}$. When the spirals are monnt d in tension, the discharge circuit of the inducing current is $\mathrm{L}^{1}, \mathrm{~A}^{\circ} . \mathrm{A} \phi, \mathrm{B}^{\circ}, \mathrm{B} \phi, \mathrm{L}^{2}$. The effects of this mode of accouplement are: (I) The effluvation, which takes place between the central spires of A $\omega$ and $\mathrm{E} \omega$, is not abolished by comnecting their inner extremities; on the contrary, the conductor by which connection is established itself becomes the centre of a lively brush discharge; (2) Connection to earth of the central end of one spiral tends to increase the effluve of the other, and when the spirals are mounted in tension this effluve attains to the length that it would possess had this spiral alone been placed in the circuit, being somewhat less dense, but more soft and tractable.

Case II. Where the current flows centripetally in the one spiral and centrifugally in the other (Fig. 45).-To bring abont this result the spirals must wind in contrary directions. They may be mounted either in quantity or in tellsion.

When mounted in quantity the inducing current derived from $\mathrm{L}^{1}$ flows centripetally from $A^{\circ}$ to $A \phi$ in the spiral $A$ and centrifugally from $B \phi$ to $B^{\circ}$ in the spiral B. When momnted in tension. the circuit of discharge of the inducing carrent is $\mathrm{L}^{1} \mathrm{~A}^{\circ}$ 的 $\mathrm{B} \phi \mathrm{B}^{\circ} \mathrm{L}^{2}$. The effects observed in this case are opposite to those noticed in the preceding. Effluvation ceases when the
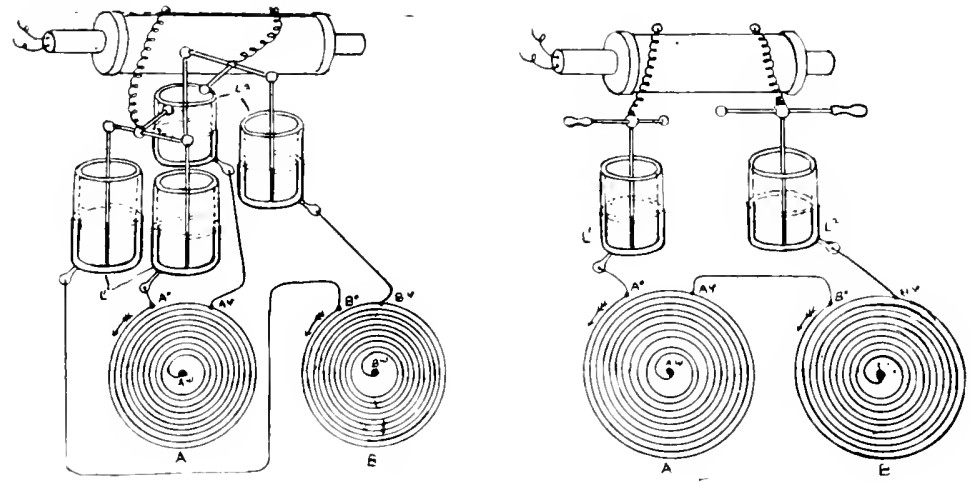

FIg. 45. Bipolar.

central ends of the two spirals are mited; connection with earth of one of the central spires tends to lessen the effluve of the other.

From a consideration of the preceding cases it will appear that the spirals receive a charge which will be either similar or opposite according to the mode of couplage.

The charge is similar-

(I) When the spirals are momted in quantity, so that $\mathrm{L}^{1} \mathrm{~A}^{\circ} \mathrm{A} \phi \mathrm{L}^{2}$ and $\mathrm{L}^{1} \mathrm{~B}^{\circ} \mathrm{B} \phi \mathrm{L}^{*}$ are the 1 wo inducing circuits connecting the condensers $L^{1}$ and $L^{2}$.

(2) When they are mounted in tension, so that the inducing current traverses the circuit $\mathrm{L}^{1} \mathrm{~A}^{\circ} \mathrm{A} \phi \mathrm{B}^{\circ} \mathrm{B} \phi \mathrm{L}^{2}$.

(3) And judging by analogy of effect it will be so when a passive spiral is influenced by an! active spiral, wound in the same direction as itself.

On the other liand, a difference of charge is observed-

(I) When the spirals are mounted in quantity. so that $\mathrm{L}^{1} \mathrm{~A}^{\circ} \mathrm{A} \phi \mathrm{L}^{2}$ and $\mathrm{L}^{1} \mathrm{~B} \phi \mathrm{B}^{\circ}$ and $\mathrm{L}^{2}$ are the circuits of the inducing currents circulating in the spirals $\mathrm{A}$ and $\mathrm{B}$.

(2) When mounted in tension, so that $\mathrm{L}^{1} \mathrm{~A}^{\circ} \mathrm{A} \phi \mathrm{B} \phi \mathrm{B}^{\circ} \mathrm{L}^{2}$ is the circuit of discharge between the condenser $\mathrm{L}^{1}$ and $\mathrm{L}^{2}$.

(3) Judging by similarity of effects, when the active spiral is wound in a direction contrary to that of the passive spiral, with which it is in relation. 


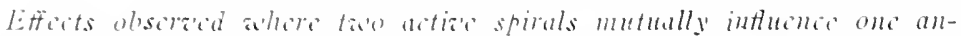
other.- ts the proximity of the spirals lo cach other and their mode of

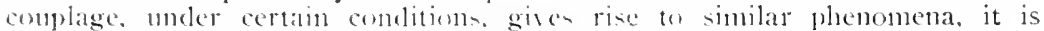
criclem that the effects which acerte from a combination of both will be proportionately increased. For intanee, if the two prirals $A$ and $B$, wound in

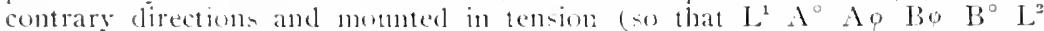
forms the discharge circuit of the inducing current). be approached to each other. libe mont brilliant intermblat effeen manifest themselves, and a lively brush discharge takes place letween the central phires of $A$ and $B$. These interpolar effects are abolished and efthration ceases when the inner extrem-

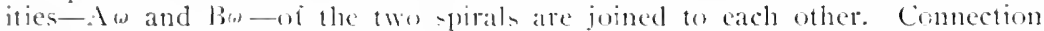
with earth of the entral yire of bo has very lithe intluence on the eftluve of $1 \omega$

But if the two spirali I and li be wound in the same direction and momuted in temsion (so that $\mathrm{I}^{\mathrm{I}} \mathrm{A}^{\circ} \mathrm{A}, \mathrm{B}^{\circ} \mathrm{B} \% \mathrm{~L}$ is the circuit of discharge of the inducing carrent), no interpolat effects will be observed when the spirals are apptoalehed to each other, nor will the comnection of the central

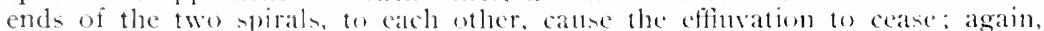
connection with eath of the eantral end of ome spiral increases the effluve of the other.

If a nentral capacity-say, the hamel or boly of a patient-les introduced between two spirals, compled so as to problice interpolar effects, it is at once deluged by the free effluve from both, but will spirats in which the How of the current is homolergows the opposite effect is produced, i.t. an effluve isstles from the calpacity to impinge on their spires.

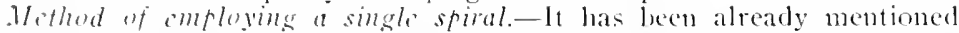
that minolat or hijular effects can le produced with a single spiral according to the monle of complage. Lnipolar effinvation. homever, is the result it is best fited to probluce. It can le employed to administer a ceplatic donche. The spiral is llen attacheel of the ceiling at a emvenient height above the head of the patient. Who cither sits or stailds leneath it. lts ontermost spite is inHuencerl by a carrent of high frepuency. derived from the extermal armatures of the leyden jats. At the same time, in the entral socket, which forms the hul, of the spiral, is fised the spider clectrole of Truchot. A single spiral can also he used with an other electrole either directly attached to the central sockel or commected with it ly al comblucing cord.

Mode of utilising ta's spirals in aibratery tratment.-To derive the full bencit of the powerful electrostic ficld leveloped between the spirals the patient mul be placel letween them. They may or may not be titted with special clectrodes, alcourling to the requirements of the case. To pro duce bipolar effects, the spirals must he so arranged that the direction of winding in the whe is oppusite to that in the other. This canses the inducing current to circulate centripetally in the one, while it Hows contrifugally in the

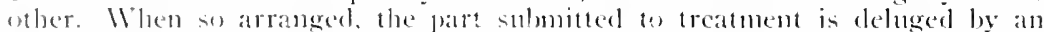
efluse. which proecels from luth. The effect produced can le regulated by mosing fhe spral; cloner to each wher or further apart. For honopular effects, the wires of the spirats must wind in the same direction: an effluve

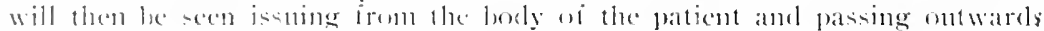

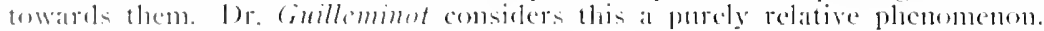
and explatins it in lle following waty

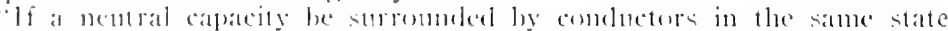

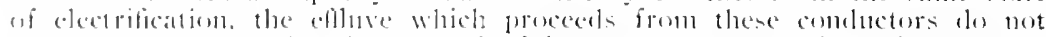

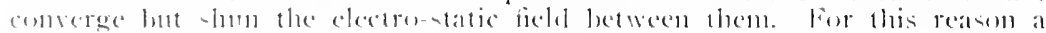

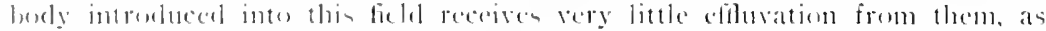

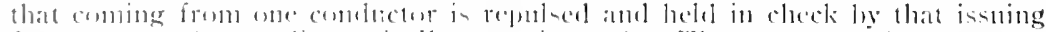

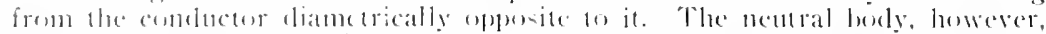

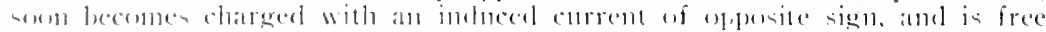

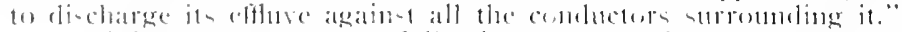

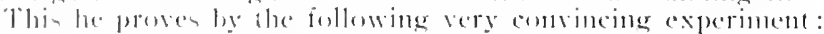

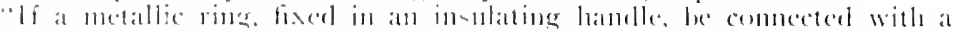

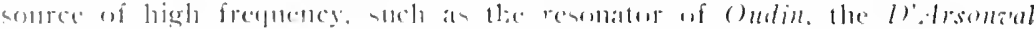

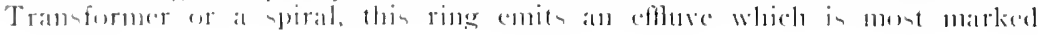


eccentrically; for the rays passing centripetally are checked and opposed by those proceeding from the part diametrically opposite. If a finger be brought in external relation $\|$ ith the ring. it receives an effluve from it, but emits very little. If, on the other hand, it be introduced into the ring centrally, it will be seen to emit a brisk brush discharge, but to receive next to nothing."

In summing up, it may be said:

( I) A capacity both receives and emits an effluve when placed in the vicinity of a conductor charged to a very high potential. This is the case where one spiral alone is employed.

(2) The effluve that issues from a neutral capacity, placed between two conductors, which mutually influence each other, is minimised and lost in

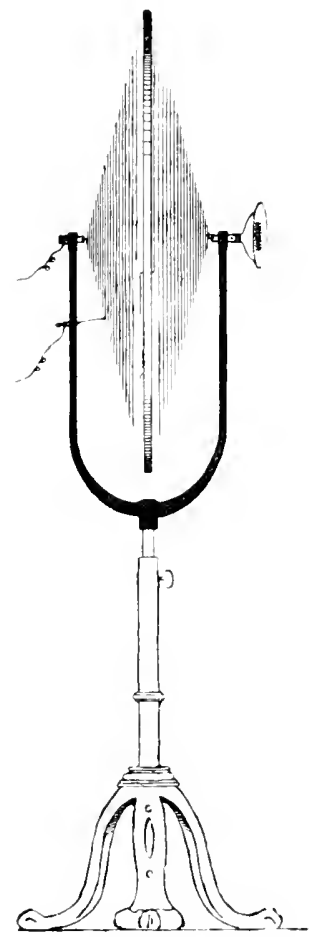

Fut th. Reus Cones.

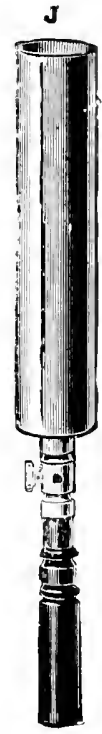

FIG. 4\%. Hand Electrode. (lican, London.)

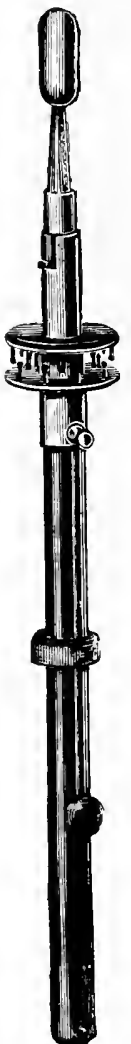

FIG. $f^{?}$. Rectal

Electurelc.

the deluge of effluve, which it receives from these conductors. This is the case of bipolar effluvation with a capacity introduced between the spirals.

(3) The effluve from a capacity introduced between two conductors emiting a homopolar effuvation is increased while that of the conductors themselves is minimised by the electro-static field intervening between them. This is the case with spirals mounted so as to produce homopolar effects.

Besides this, a patient can also be submitted to the influence of one spiral, and the efiluve of the other, by connecting him with the one by a handelectrorle and placing him in the vicinity of the other, the spirals being meanwhile arranged either to produce homopolar or bipolar effects. 
Rins' Cones (Fig. 46). - This apparatus occupies an immediate place between the that spiral of Dr. Guilleminot and the longitudinally coiled resonator of Dr. Oudih. It essentially consists of a biconvex lenticular disc (33 inches in diameter) formed of ebonite or some other good insulating substance, upon which a layer of wire (No. 9. B. W. G.) is coiled so as to form two cones. united by their bases. The wire makes 3 I spiral turns on each side of the disc. As the peripheral extremity of the two cones are continuous with each other, and the direction of their winding contrary, the inductive effects of the spiral is always a maximum. When in use, the central extremity of one cone is commected with the external armature of a Leyden jar. while the corresponding armature of the other condenser is connected by an adjustable contact clip with one of its intermediate spirals, so as to obtain a maximtm of output on the free central terminal. to which the electrode is attached. The apparatus is available for use as a ceplatic

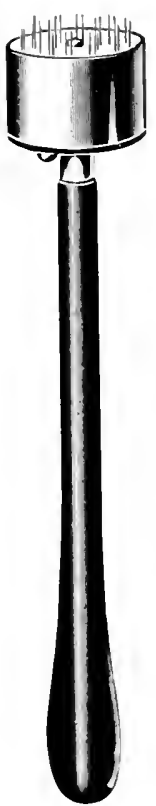

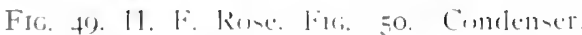
(Sanitas Electl. (Isinthal, Lomdon.) (o., L.onrlin1, )
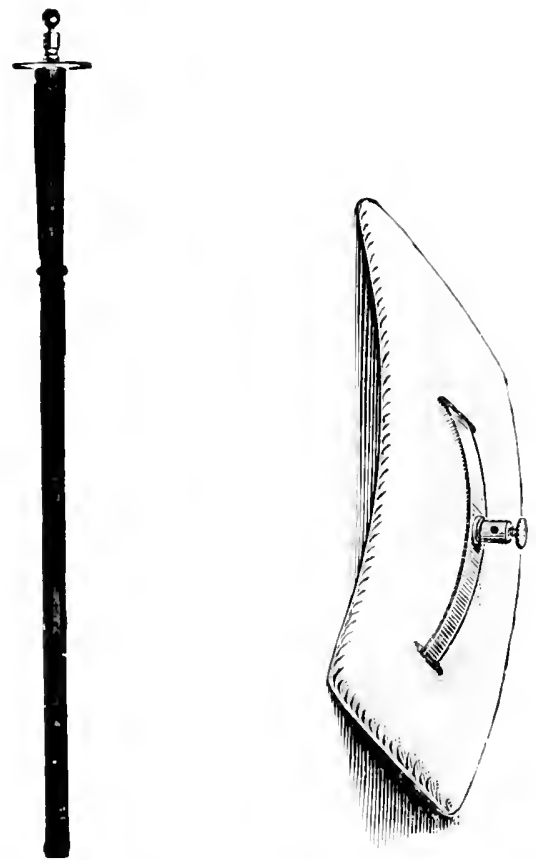

lili. 1 1) 1) Elece trode. (Silhall. L.minton. )

douche or for unipolar applications, but two cones are required for double effluvation and bipolar application.

Ipparatus l'eculiur to Indisidual Mothods of Application.-These may be be-t consirlered in reference to the methods to which they apply. The four recognised metherle of application are : Jerivation, Elftuvation, Conden-ation and . Intoconduetion.

For treatument by the Stabile method wi lerivation we require handelectrodes (Fig. 47), luth for mipmlar and hipular applications; or flat dise electrorles (fige. 51). Fon rectal ine we reduire the electrodes shown in Fig. fo. For labile applications we emples cither the glolulat. the

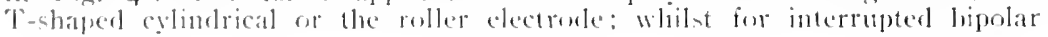


applications we use various sized lrushes for generai purposes, and an Erbmodel electrode for parts where a finer graduation of effect is desired.

In EFFLUVATION we make use of a rose-effluve, of condenser electrodes, and of vacuum and water electrodes.

THE ROSE OR EFFLUVER (Fig. 49) essentially consists of a flat disc of metal studded with concentric row of blunt pointed pegs. In the improved models all the metal part of the electrode (except the projecting tips of each peg) is heavily insulated.

THE CONDENSER ELECTRODE (Fig. 50) is formed of a metal or graphite carbon conducting rod enclosed in an insulating sheath of glass or ebonite. They constitute with the integument of the part, to which they are applied, a condenser. The delicate violet coloured sparks thrown ont by these electrodes, and al:o by the vacuntm and water-electrodes, are termed "Eucomides," to distinguish them from the more painful "Sparl" discharges and the gentle "Effluve" from the Rose or Effluver.

IACLU.H ELECTRODES are made of vulcanite or glass, and exhausted to various degrees of vacuum. The discharge proceeds from a small pin, placed centrally in the attached extremity of the electrode. When the exhanstion is high, X-ray effects are said to he produced.

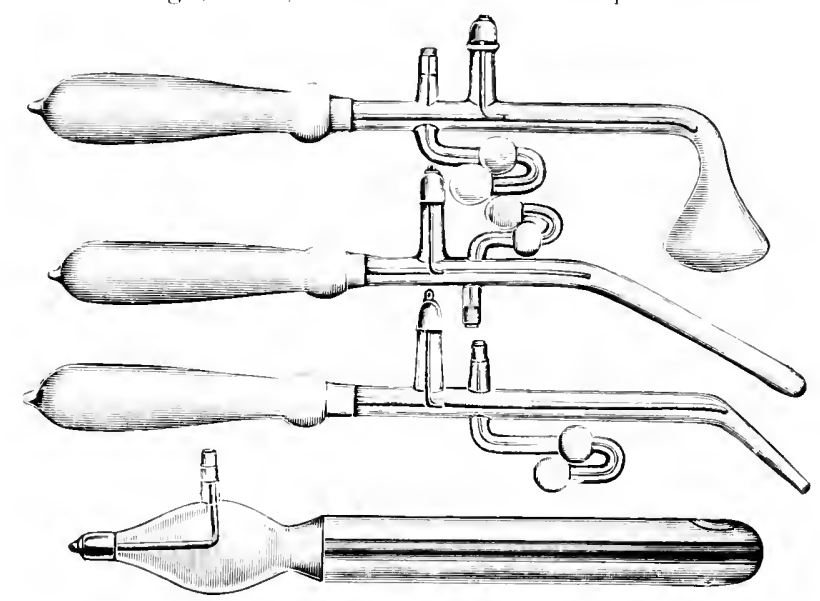

FIG. 52. Fluid Electrode. (Schall, London.)

FLCID ELECTRODES (Fig. 52).--These are formed of glass or ebonite, with the interspace between the sheath and central pin filled with a slight acidulated saline solution. It is not mnusual to convert a vacuum electrode in which the vacuum is clamaged into a water electrode, so as to save expense of having it exlansted.

For treatment ly Condensation we require a couch condenser for general condensation, and various shaped splint condensers for more localised applications.

THE COUCH CONDENSER (Fig. 53).--It has been given various forms by different makers. In some it is a massive. lumbersome article of furniture better suited to the spacions receiving-roms of a general hospital than to the private affices of the consultant or general practitioner; in others its dimensions have been whitted down to a size that renders its nse more awkward than efficacious. For consulting roms in which the amount of available space is limiterl, the lent-wool conel shown in Fig. 5.3 is most suitable. It is at once strong, useful and elegant. and does away with the multitucle of unsemly expedients that have to le resorted to so as to render the chair-eonclenser effective It, moreover, allows the patient to rest in an easy, reclining position, the advantages of whel are too well known by the 
experienced operator. With the exception of the scat, it in nowise differs in construction from those usually met with in furniture stores. The seat is a stout metallic sheet, carefully fitted to the body of the couch, and coated on

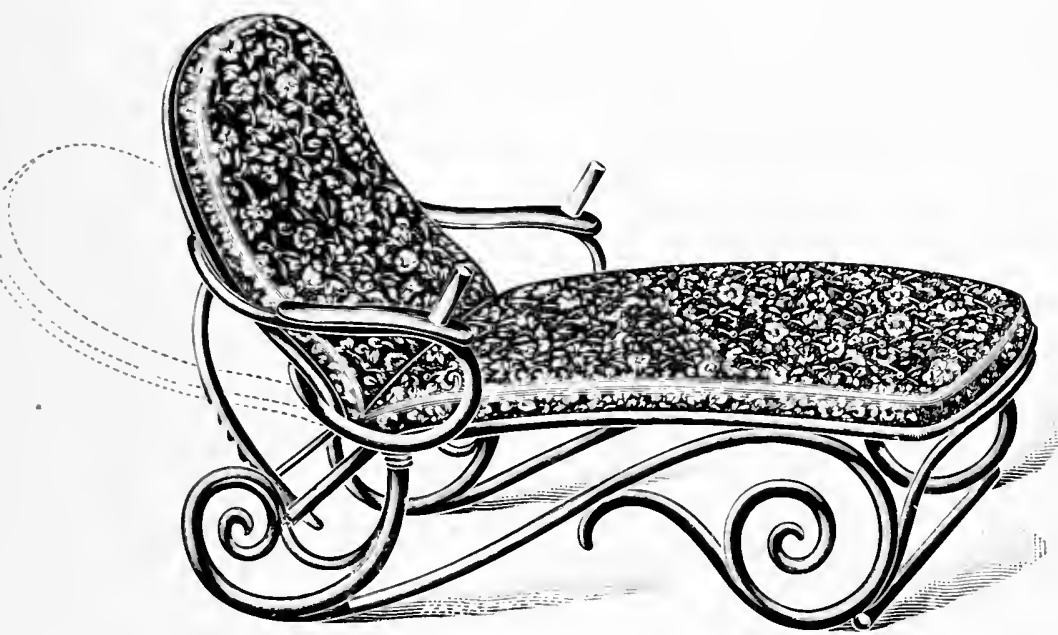

Fig. 53. High-Frequency Cunch. (II. II. Co.r, London.)

both sides with a layer of some insulating material. At the head the sheet somewhat protrudes to allow of the attaclment of the binding-screws, which connect it with an apparatus of high frequency. It is usually made of tin. copper, lead or zinc. and forms that armature of the conch-condenser which
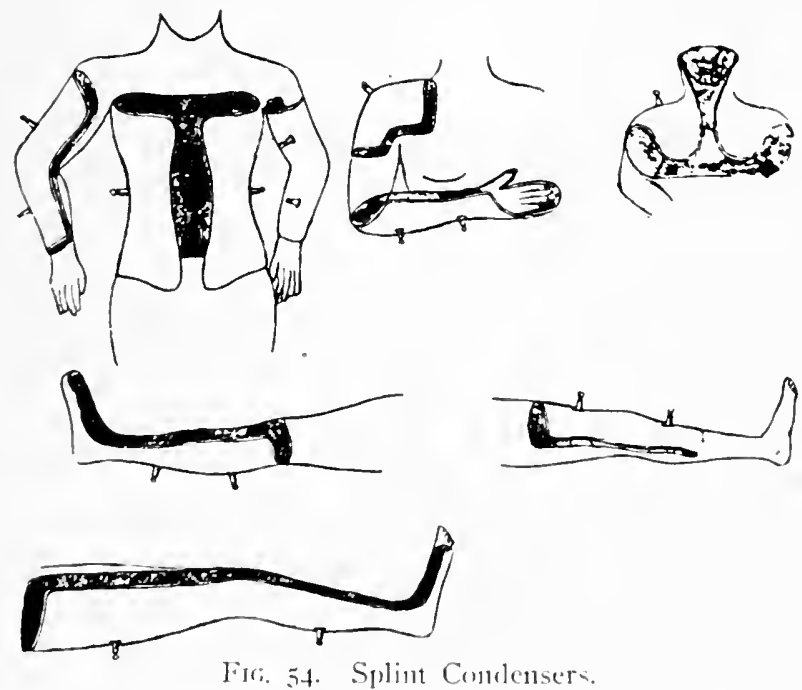

is always active. The insulating subutance employerl ats dielectrics are varions. Some maker une a horseluar-pardeded cusbion to cover the metallic sheet; but its dielectric capacity is very limited, both on accomt of the 
nature of the substance of which it is made and the thickness of the layer required to secure perfect insulation. Reus favours a composite material, composed of mica, shellac, wool fibre and well-pulped paper, which is carefully prepared and rolled in sheets. In appearance, it much resembles papier-mâché, but is more durable and easily applied, not so liable to crack, and takes a better polish. Another material equally useful is coarse fibrous felt, impregnated with shellac and then compressed. Guttapercha and

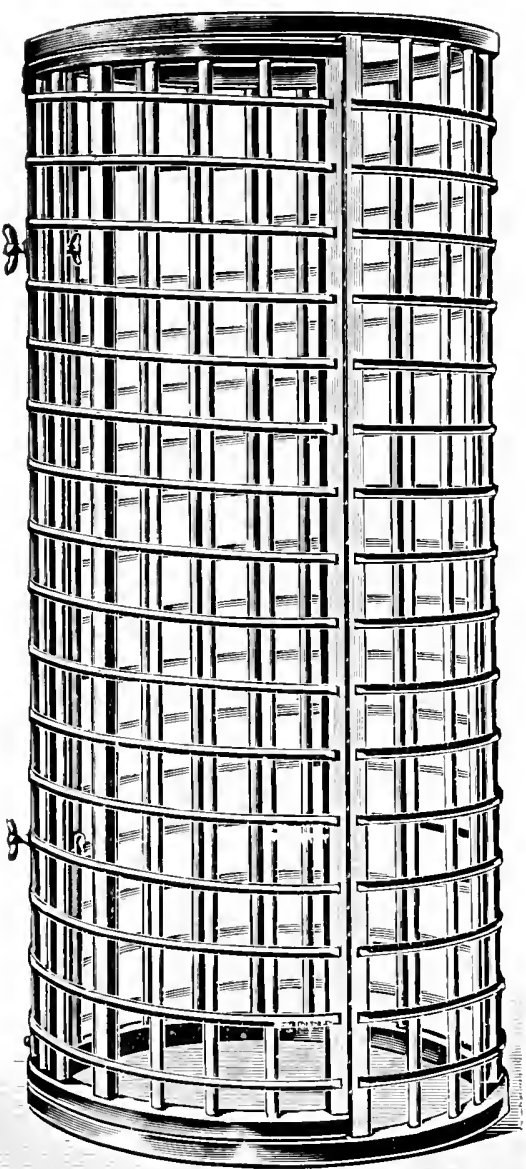

FIG. 55. Upright Cage. (Sanitas Elec trical Co., London.)

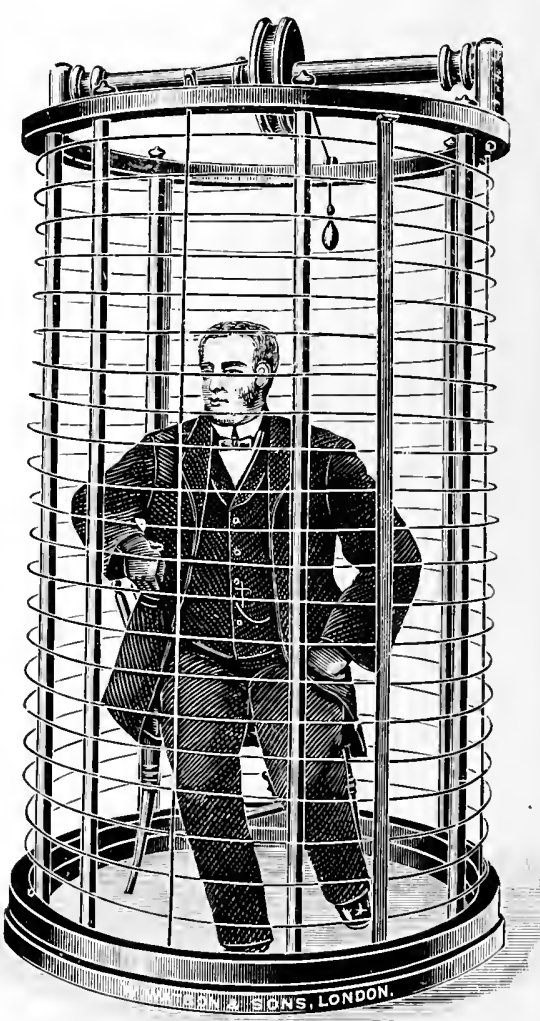

Fig. 56. Upright Cage. (Watson \& Sons, London.)

ebonite have been similarly employed. All of these are good dielectrics, not easily pierced by sparks; while the thinness of the coat required much intensifies the inductive effects.

SPLINT CONDENSERS (Fig. 54) are made of aluminium, sheet lead or wire-gatuze and encased except at the points where the connections are made in a close-fitting layer of the composite material already described. The 
patient, to whom the splints are applied, is placed on an insulated coucl and connected by means of a disc or hand electrode with one pole of the small solenoid. while the wire from the opposite pole is attracted to the terminal binding screw on the splint, which, of course, has to be carefully adapted to the contour of the part to which it is applied. In treatment by general autoconduction the cage of antoconduction is a necessity, while in local antoconduction the smaller solenoids which are shown in Fig. 55 are employed.

THE SOLENOID OR CAGE OF AUTOCONDUCTION is a large helix. 7 feet long by 36 to 48 inches in diameter, formed of 18 to 20 turns of stout copper wire ( 15 to $20 \mathrm{~mm}$.) ribbon or tubing. Its spirals are held in position by suitable insulating supports. which may either be cords and tapes of silk or of specially prepared thax, or pillars and bars of ebonite or well-paraffinated wood. As the solenoid is intended for the reception of the body of the patient. it must be sufficiently large and spacious not only to accommodate

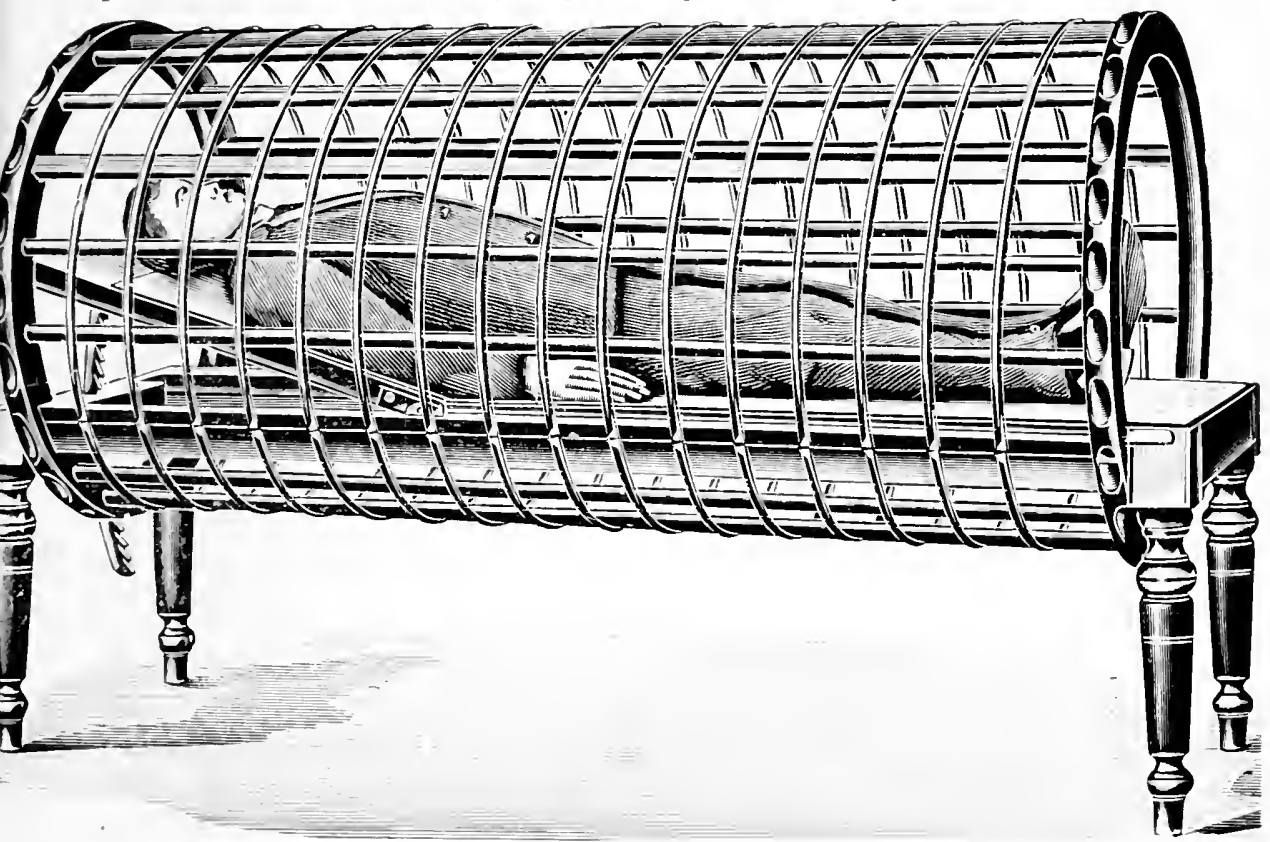

Fig. 57. IIorizontal Cage. (Sanitas Electrical Co., London.)

it. but also to allow of easy access and enclosure. Those of the dimensions above mentioned have leen found to well serve the purpose. There are two chief types of solenoids in general use, the one intended for use in the vertical, the other in the horizontal position. The arrangement of the wirespirals and their number differs in the apparatus of different makers. In some forms of the large upright solenoid the wires are permanently fixed to the woorlen framework of the cage, a loor being provided to admit the patient; in others the wire coils are suspended by silken tapes or cords, and can le drawn up like a venetian blind to alford access. The break in the continuity in the wire of the spirals in the first case causes a certain amount of yarking and interruption in the flow of the current, and is for this reacom not so advantageously cmployed as the other, which, when not in use. can be frawn up to the ceiling so as to place more space at the disposal of the aperator. In the horizontal model the wires are cither fixed about the 
insulating couch or else they can be drawn into position after the patient has been placed upon it by a cord and pulley arrangement. Although both types of solenoids can be used in treatment by Apostoli's method, still the vertical model (Fig 56) is to be preferred, more especially in dealing with the hypersensitive, the infirm and the bedridden. It must, however, be remembered that whatever may be the form of solenoid adopted, its terminals require to be connected with a source of currents of high frequency. This can be effected by connecting them with the two poles of the solenoid of high frequency; but they are more often directly united to the external armatures of the condensers in the apparatus of production, thus deriving a current of greater potential. The cage is also usually provided with an induction ring with an incandescent lamp or Geissler tube, whereby the intensity of the current induced in the body of the patient can be roughly estimated.

The apparatus in use for local antoconduction (Fig. 58 ) is similar in construction but snaller in dintensions than the wire-frame horizontal cage employed in general autoconduction. In the majority of cases a single limb or region is all that is introduced into these solenoids, but the limit can be

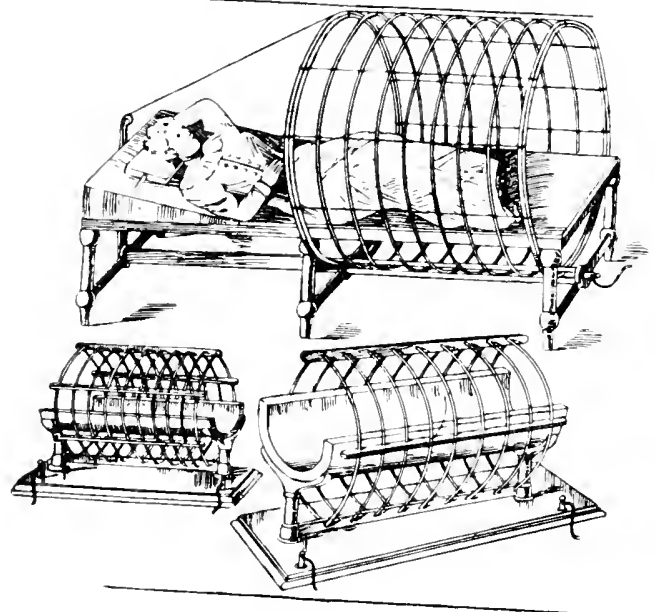

FIG. 58. Local Autoinduction Cage.

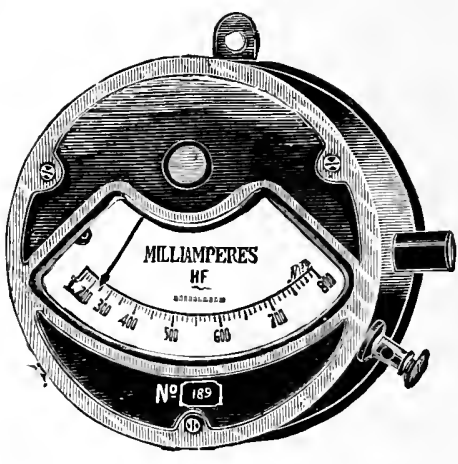

FIG. 59. Milliampère-meter.

extended to embrace the entire trunk below the shoulder girdle. It is actioned by connecting its terminals with the poles of the small solenoid of high frequency or the two terminals of a bipolar resonator, by varying the self-induction of whose spirals a graduation of effect can be produced. In extended local autoconduction the patient is placed upon an insulating couch, about which the cage is fixed so as to leave the head and shoulders outside the sphere of direct electro-magnetic influence. In purely local autoconduction the limb is centrally introduced into one of the various smaller solenoids and rests upon the insulating splint condenser in its interior. It can be connected with a pole of the solenoid of antoconduction, as in the combined method of Apostoli, or remain free from contact, as in the simpler method of D'Arsonval instruments for measuring the intensity of the currents passing into the body of the paticnt. Besides the voltmeter and ammeter, which registers the amount of current passing into the prinary of the induction coil, it is at all times necessary to know the intensity of the current passing into the body of the patient. This can be measured by the universal gatvanometer of Gaiffe. a dead beat milliampere-meter of the $D^{\prime}$ Arsonzal type (Fig. 59), in which the elongation of a fine metallic wire (nade of an alloy of platinum and 
iridium) canses the passage of a current through to infinence the movenent of the light pointer which then indicates the intensity of the current passing throngh the eirenit. This instrument, to be of real service, should register as far as up to $700 \mathrm{~m}$ a.

APPAR.ITLS FOR N-R.AY WORK-The foctus-tube is the most essential part of an X-ray outtit. Withont endeavonring to trace in detai? the evolution of these from the printitive firisslir and Crooks tube of laboratory fame to the latest and most improved mode? now on the market.

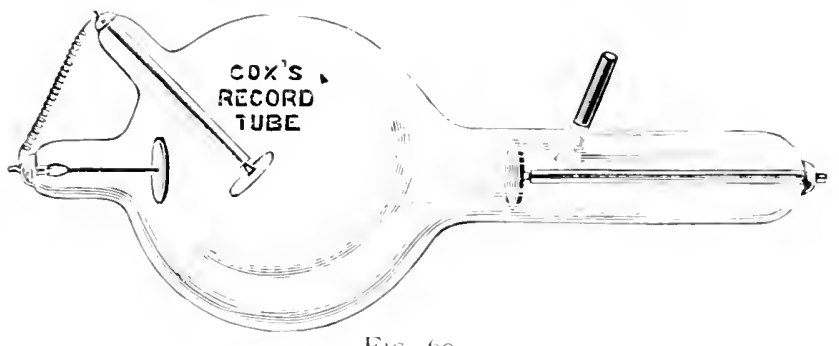

Fic. 00.

it may be said that as cathodic tubes were never specially designed for X-ray work, there was always a considerable amount of diffusion of the cathodic stream with the inevitable result-hat defintim at short distances from the tube. The first real step towards improvenent in instrumentation was the use of the focus tube. in which the stream of cathodic rays are brought to a focns at some part of the surface of the anode. By thus limiting the size of the area from which the $\mathcal{X}$-rays proceed, a better definition of shadows cast by objects held close to the tulse could be obtained.

The next important step formard in the development of these tubes was the introduction of a target-the anti-cathode of Professor Sitionus $P$.

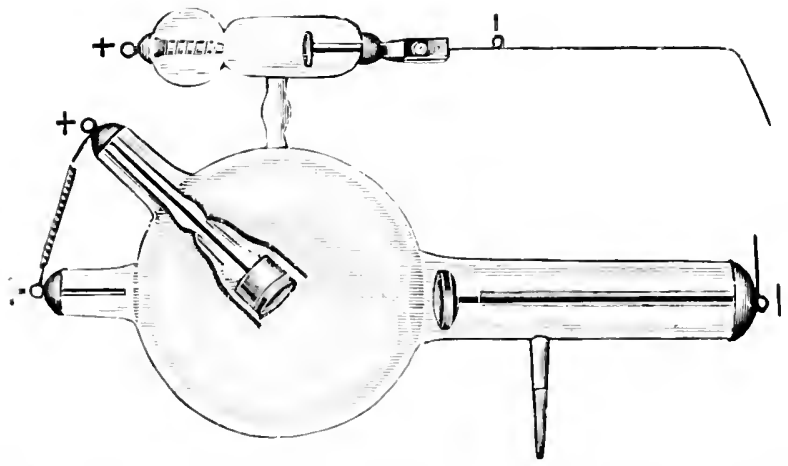

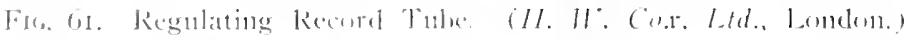

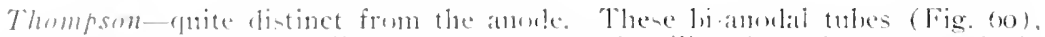

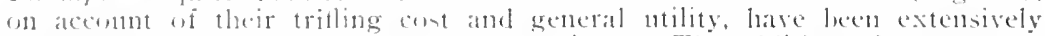
userd, buth in this country and on the continent. The additinn of a regentera-

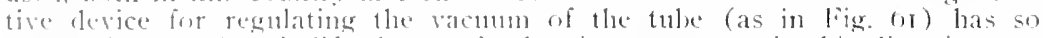
greatly increated their life that no further improvenents in this direction can le effecterl. The gratlual rine wi valumble resintance of at tule with continned

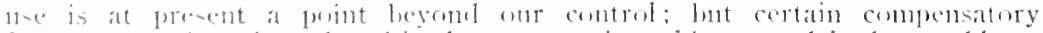
features have lecen introfuced in the comstruction of latter models that enable us to renre at greater alegree of constancy. The tubes provided with these 
devices are known as "regenerative tubes," in contradistinction to the "ordinary" tubes generally made use of.

REGENERATIVE TUBES.-The vacum of a hermetically sealed tube may be altered by one of the following devices:

(I) By the application of heat. A tube whose resistance has risen beyond the working limit may temporarily be restored by warming the glass from the outside by means of a spirit lamp, sand-bath or Bunsen burner; this can also be effected by forcing a powerful electric current through the tube till the anti-cathode grows incandescent. Althongh this method is applicable to all tubes, the "Double-Bulb" tube (shown in Fig. 62) allows

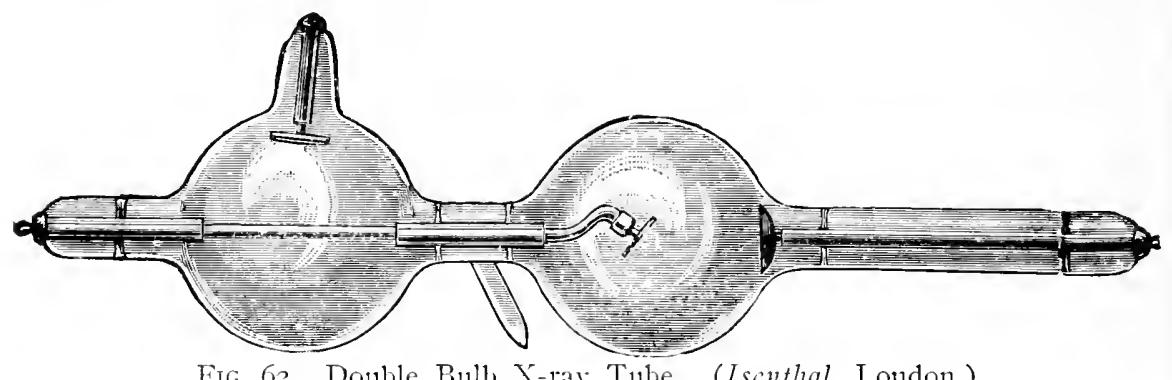

Fig. 62. Double Bulb X-ray Tube. (Iscuthal, London.)

of this method of regeneration to be used to the best advantage. The most prominent characteristic of this tube is the addition behind the working bulb of a regenerating chamber, the heating of which by the passage of the electrical discharge allows the condensed gas which clings to its inner wall to be driven into the main tube, and thus lower its resistance.

(2) By the introduction of substances which absorb gases at ordinary temperatures and yicld them up when heated. The substances more commonly employed are mica discs. sodium and potassium hydrate, palladium, permanganate of potash and charcoal. They are generally placed in a small

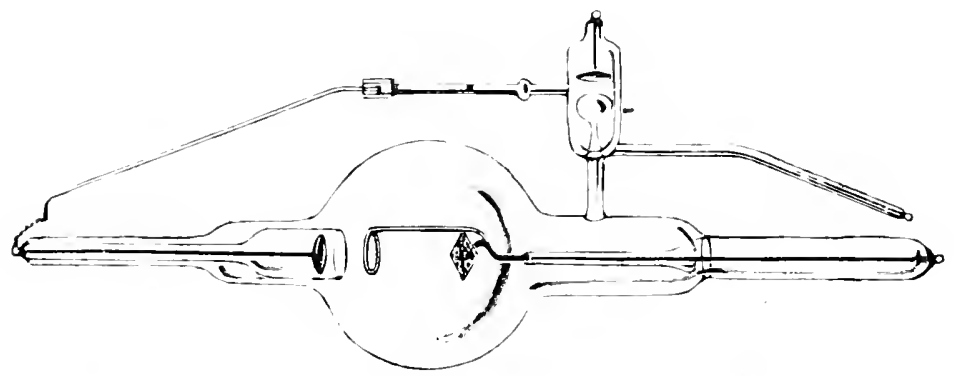

FIG. 63. Chemical Substance Regenerator. (Dian, London.)

auxiliary chamber, by the careful heating of which the resistance of the main bulb can be gradually lowered to any required degree. An arrangement which allows the necessary heating to be done automatically by means of an alternative discharge circuit with an adjustable spark-gap is shown in Fig. 63. When the vacuum in the main tube rises beyond the desired limit, the current, selecting the path of least resistance, passes through the alternative circuit and heats the chemical in the auxiliary bulb until sufficient vapour has been driven into the main chamber to lower its resistance to the required extent. It is evident, therefore, that the resistance in the vacuum chamber will depend on the length of air-gap employed.

The great disadvantage of all automatic regulators in which an alterna- 
tive path of discharge is employed is that, whilst the regulatory sparking is going on in the by-eircuit, there is no discharge through the niain clamber. The anti-cathode. therefore, has time to owol and ocelude non it suface some of the released raponr, which, when the current reverts to the main circuit and again heats the larget, may be given off so rapidly as to render the vactutun too low.

MECHANIC $\backslash L$ REGENERATION.-Screral attempts have been made to vary the penetration value of the rays by altering the distance between the cathode and its target but as the required manipulative dexterity their use entails can only be acquired by long practice, and after the sacrifice of a great number of tubes, one seldom sees any of these regenerative tubes used by any but the inventors themselves.

ELECTROSTATIC REGENER.ITION.-Is the charge gencrated by electrostatic induction upon the outer surface of a focus-tube when in the has a considerable effect upon the discharge passing through it, attempts have been made to regenerate the tulx and lower its resistance by drawing off this onter charge by a commection with an carthed wire or with the inner surface of the tube. This has been effected by covering the neck of the tube in the plane of the cathode alge witli a ring of copper wire or with strips of tinfoil and comnectung it ly an adju-table spark-gap with earth or the wire leading to the cathode. As the risk of perforation is to some extent increased hy the facilitice offered for the pasage of the sparls romel the

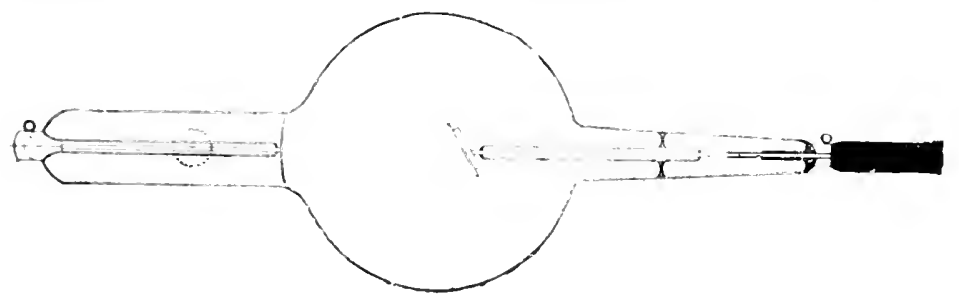

Fic. O4. Onnusis Tubes. (1)an, Lomlon,)

tube, Belinger has snggested the wise of emi-comdnctors-a wouden cylinder moistened with glycerine for the matallic conting. Althongh this method of regeneration is rery promising, there is still a vast amount of room for improvenent.

BI OSMIOSIS. - Amuther and the most promising method of regulating the vacutm of a tube repents nom the fact that certain metals. more especially platmum, when heated to redness. beconne pervions to hydrogen. If a cloned tule of platisum be sealed into the anxiliary chamber of a focus tule, it will, when cold, be perfectly air-tight, but when heated to redness in the flame of a spiritamp. the hydrogen from the flame parses into the bull, and thes lowere its resistance. As there is no linit to the supply of hyelengen that can thus les passed into the tube, its superiority to other methorl in obvious. The anmealing of the platinum, howerer. requires the utmont cate 10 present the prosilility of leakage. This type of regenerator is largely cmploged. and a tule with this regulation is shown in Fig. 64. In some make if tule, facilities are also afforded for the removal of any

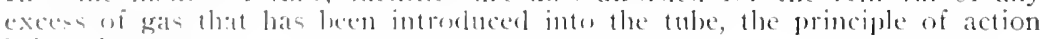
being the same.

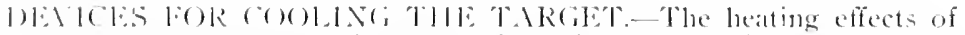
the catherlic -tream now the anti-cathrele placed in the exat foem when used with very - frong exciting entrent is very great and it is always arlvantageous

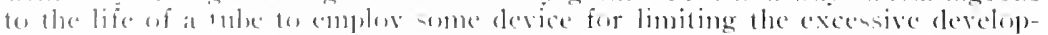
ment of heat. The smplest of these is the wse of heary dincharge targets.

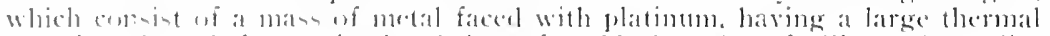

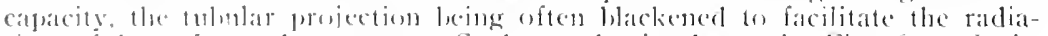

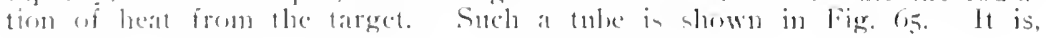


however, more advantageous to employ in conjunction with these a small porcelain disc, which is inserted between the sheet of platinum and the tubular shaft. This arrangement answers admirably, and the life of the tube is much prolonged by its use. The "contrast" tube, shown in Fig. 66, pos-

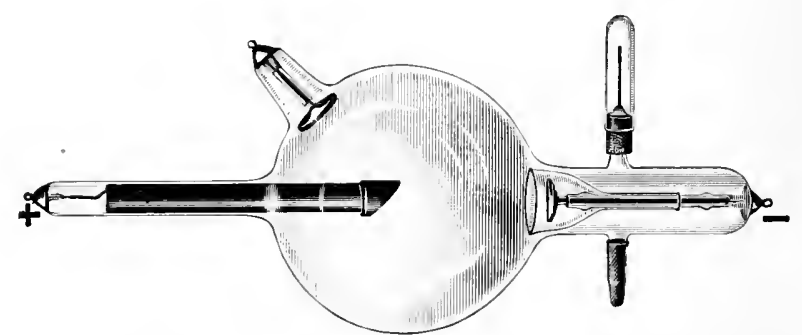

FIG. 65. Heavy Anode Regulating Tube. (Schall, London.)

sesses all these advantages. Another method of obviating the difficulty is by using water-cooled tubes, either by means of a water circulation or by making the anti-cathode hollow and filling it with a quantity of water, with a provision for the escape of the steam generated in working. The detrimental effects of the closing spark upon focus tubes when currents of high voltage are employed on the primary of the coil has lately been remedied by the introduction of "valve" tubes, which are inserted in series with the tube. The additional outlay this involves is comterbalanced by the saving effected in the increased length of life of the focus tubes.

FLUOROSCOPES AND FLLORLSCENT SCREENS.-The only two of the many salts that Ruoresce under the action of the X-rays that are

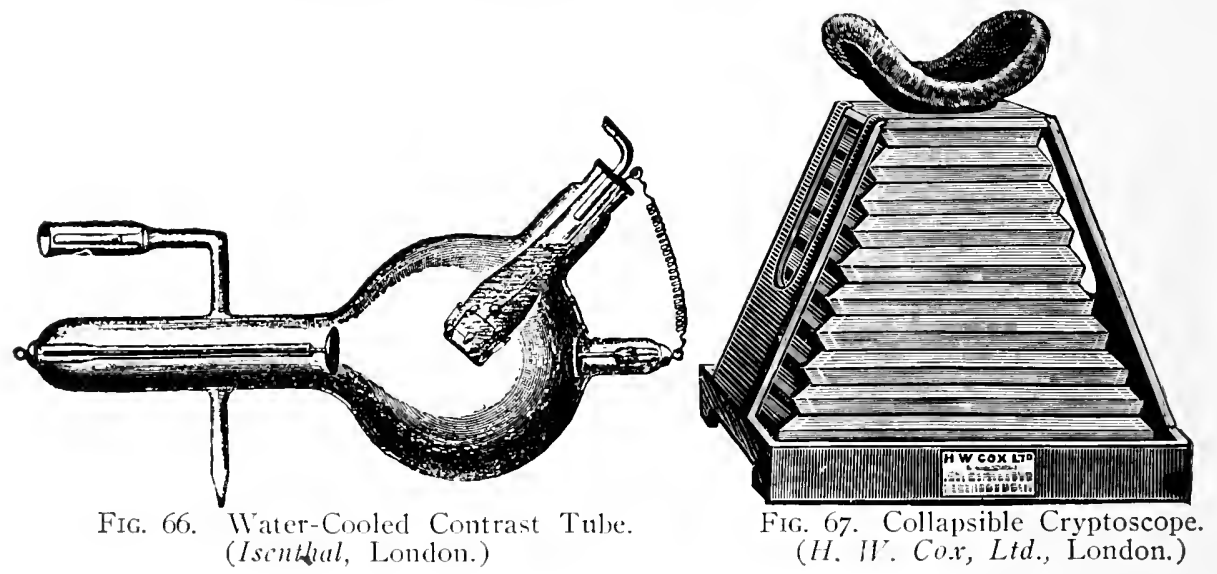

employed in the manufacture of fluorescent screens are Barinm platinocyanide and Calcium tungstate. The fluorescence of the former is a brilliant yellowish green; that of the latter is bluish-white and not so intense; but on account of its colour, it possesses a greater photo-chemical activity, and is therefore used as an intensifying screen for radingraphic work. In the screens of English makes the harinii crystals are larger and more thickly spread; in thore manufactured on the Continent the coating is more evenly spread.

A cryptoscope is a close-fitting hox. intended to shut out all extraneous light from the screen. It is sometimes fitted with accordion folds (Fig. 67), 
which allow the distance between the eve and screen to be adjusted; but the advantage does not compensate for the increased cost.

INTENSIFYING SCREEIS.-The fogged appearance of negatives obtained by radiographic exposures, more especially of those through the thicker parts of the bedy, is diee, according to I'illard, to the fluorescence of the surrounding air. Of all the various attempts to prevent this by

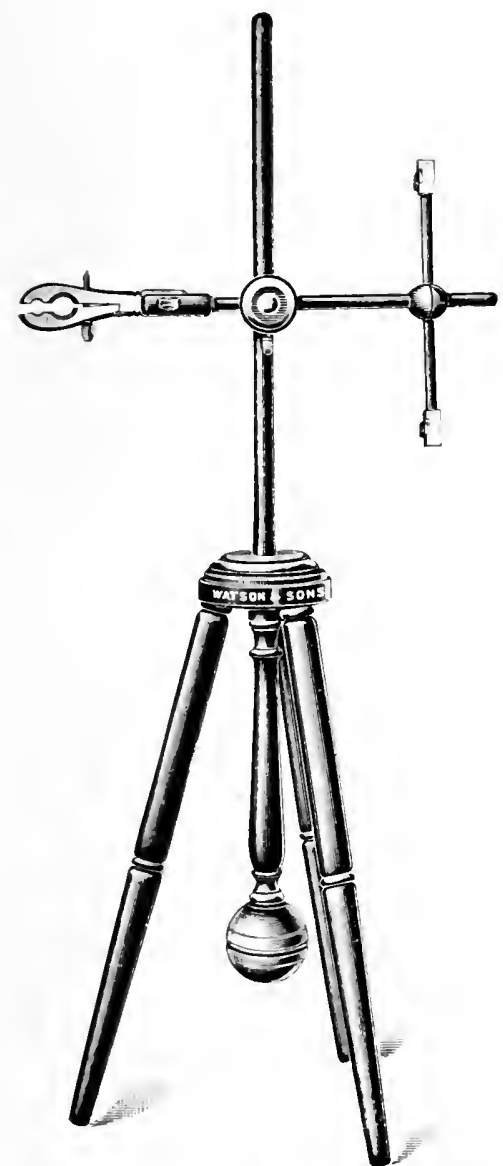

Fig. 68. Tube Holkler. " ith Ball Weight.

(I'atson \& Sons, London.)

Fic. 6o. Bunsen stand Tube Holder. (H. IV. Cox, Ltd., London.)

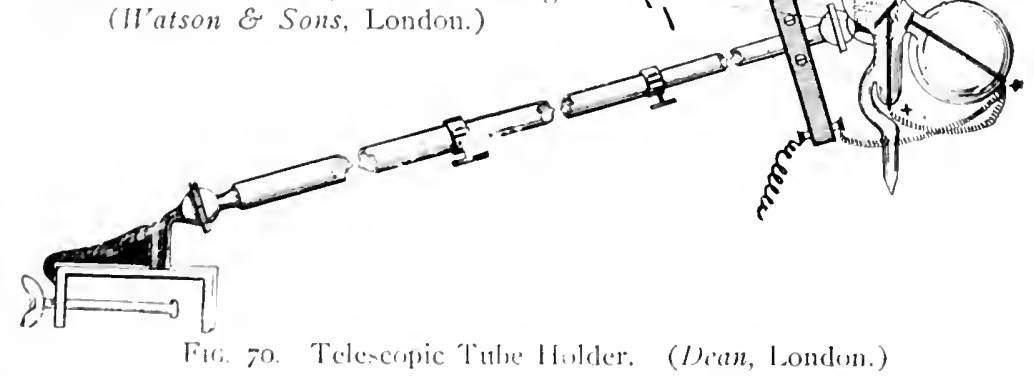


accelerating and intensifying the effects of the rays upon the sensitized film, the only method that has hitherto been attended with any measure of success is the use of certain substances which fluoresce when excited by the X-rays and act upon the sensitized film like ordinary light. Owing to the granular state of the fluorescent screen, the resulting radiograms somewhat lack definition, so that minor details, especially of the smaller osseous structures, are effaced. If a platino-cyanide of barium screen be employed for this purpose, the photo-films must first be colour-sensitized by treatment with a .or\% solution of erythrosine and drying in a perfectly anactinic chamber. In taking radiograms by this method, the intensifying screen is placed immediately above the film or plate, so that the rays must pass through it before they can act upon the plate.

The substitution of amorphous calcium tungstate for the granular platino-cyanide in the preparation of the screen has allowed better skiagraphs to be obtained with ordinary dry nlates. In using such a screen the coated surface is placed in contact with the emulsion of the plate or film, which is placed nearest to the object to be radiographed. By combining the two methods, Mar Lery has been able to greatly intensify the effects and proportionately shorten the duration of exposure. He employs a plate or film, coated on both surfaces with a sensitive emulsion, and placed between two intensifying screens. As contact with these gives double density to the negative the time of exposme is materially shortened.

TUBE HOLDERS (Figs. 68, 69 and 70).-Although for experimental work the ordinary Bunsen retort holder with heavily weighted base allows of the tube being fixed in any desired position, still, for clinical purposes, we require a stand which will enable us to fix the tube at any height from the floor, and allow it to project far enough from its support to be brought over the operating table or conch. For this purpose a stand with a heavily weighted base and firmly.clamped projecting arm with swivel and ball joint is necessary. The projecting arm, which carries the tube and the connecting wires is always made of wood or ebonite, to prevent any spark from the terminals, in passing into the metal, perforating the tube.

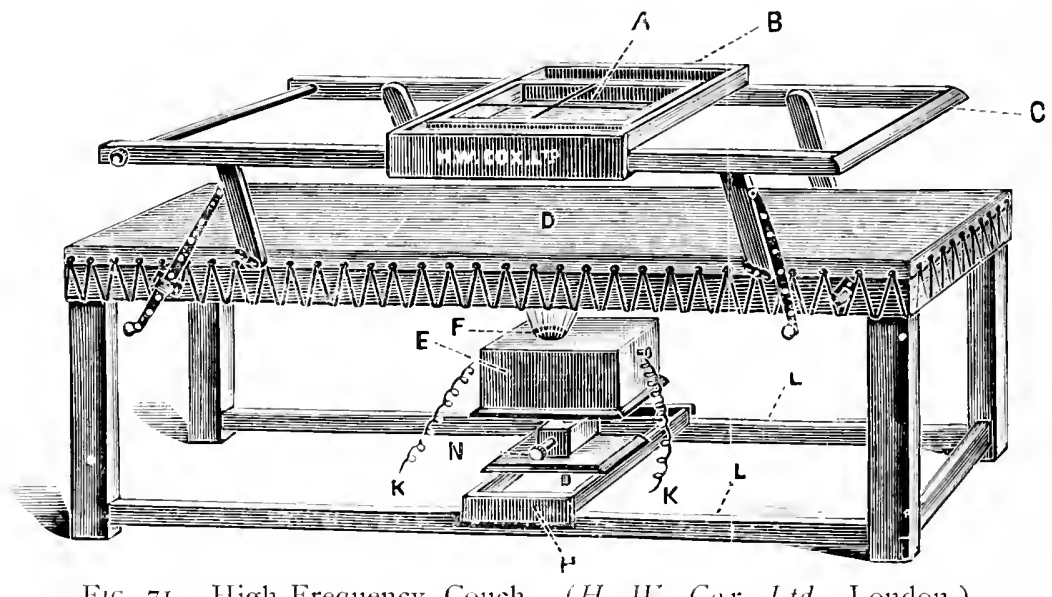

Fıg. 7r. High-Frequency Conch. (H. H. Co.r, Ltd., London.)

For use with overhead coils, and in offices where the amount of floor space is limited, the "WALL BRACKET" type of tube-holder will be found very convenient; while for portable installations a telescopic tube-holder is almost indispensable.

COUCHES (Figs. $7 \mathrm{I}$ and 72 ).-Whenerer radiograms have to be taken of a patient in the recumbent position, a radiographic couch will prove a very 
noful accessory. It esentially conlists of a strong wooden franework. over the top of which a theet uf canvas, thin leather or other material radioscopically transparent in tretched. By placing the tube below the conch, the patient can ea-ily be examined with the fluorescent screen. In taking radiograms. a photugraphic plate is placed below the screen. and the tube bronght as close to the hudy as is considered advisable. Conches fitted

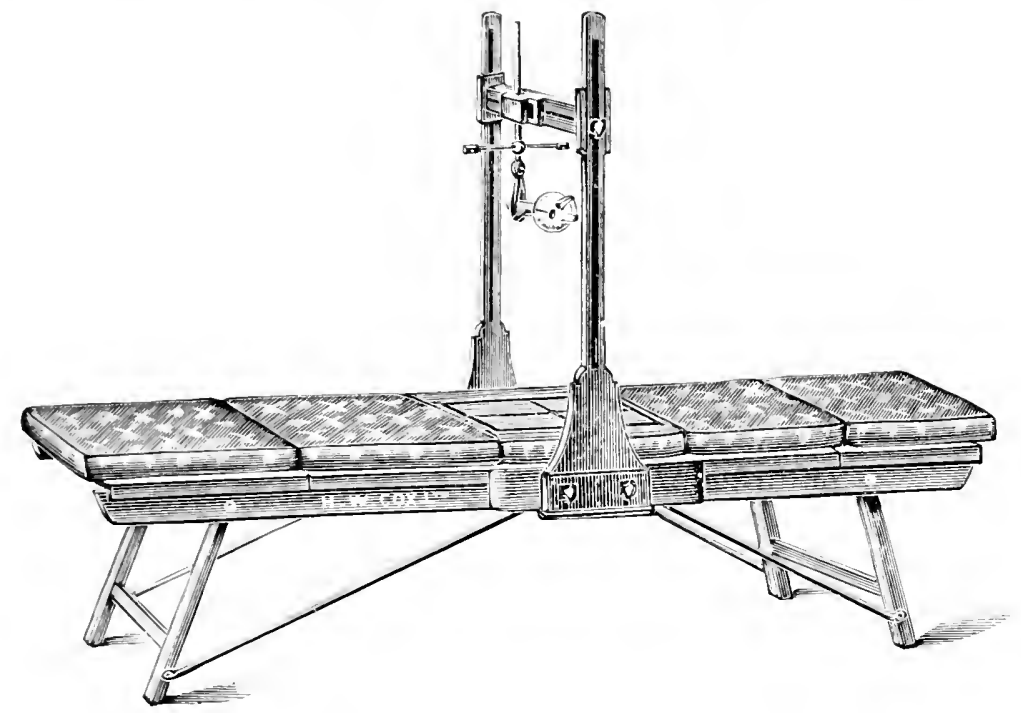

FIG. 72. Nackenzie Davithon Couch. (H. M'. Corr, Ltd., Lomdon.)

with a plate-bolder which can lee brouglat in contact with the part to be radiographed allows the radiographer to operate to the best advantage; as once the object is fixed with the fluoroscopic screen, no change in the position of the patient or tube is necessary to obtain? a good skiagraph.

Sume couches are especially designed for localisation and stereoscopic radiography. In these the tube holder and plate-rest (although adjustable) are perminently connected with each other in order to maintain a certain fixed relation between the tube and object. The plate-holder is so constructed that the plates may be changed without disturbing the patient or causing him to alter his position, and the holder possesses cross and orientation wires. while both tulse and plate-holder move simultaneonisy so as to bring the latter in relation to the particular part of the body to be radiographed. STEREOSCOPIC R.ADIOGR.IPHY (Jigs. 73, 7t and 75).Drs. Hedley, Hackenzie Davidson. Imbert and Bertin-Sams have shown that two radiogrants taken with ttbes $2^{\mathrm{T}}+$ inches apart, when snitably monnted ancl viewer in a sterenscope, will give an object in proper relief. Radiographic stereograms, however, differ from ordinary stereograms in as much as it in immaterial which picture is monnted on the right or left-hand side of the stereoscope, as the change only produces a difference of aspect (i.e. gives the anterior or puterin view). In order to see objects in stereoscopic relief on the fluorencent acreen. two tubes must be placed with their target 25 inches aprart, on that the distance between them is coplat to the

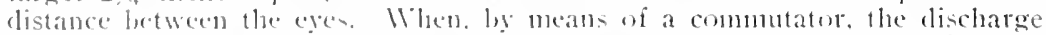
from an Induction Coil is alternately directed into one or other of these. whilst the oferator lonking through two openings in a sereen fitted with a disc sector. Which allow the eye to he illuminated synchrononily with the alternations of the discharge in the two tubes, the object viewed ceases to 
appear as a flat shadow and stands out as an inage which conveys the sense of actuality and relief. It is, of course. essential to the success of these experiments that the two tubes should have the same penetration, so as to excite the same degree of fluorescence in the screen. The trouble is to find two such tubes, and although the difficulty has been partially solved by the use of two bulbs with a medium of communication between them. it is not always possible in working with these to prevent the active tube sparking across to the inert, and this confuse the shadows. We are therefore restricted to the use of soft tubes of comparatively little penetration, which correspondingly diminishes the fluoroscopic shadow effects. It must, however, be admitted that stereoscopic radiography promises, when development is sufficiently advanced, to be the easiest exact method of localisation.

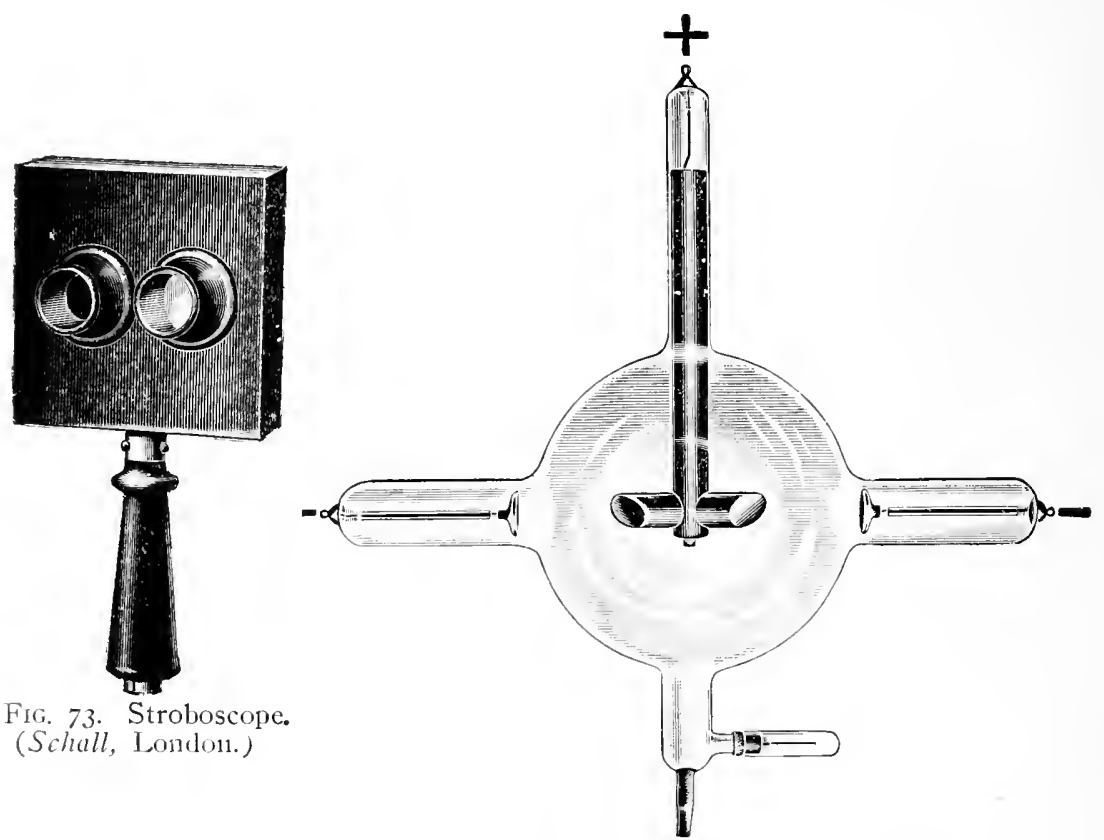

Fig. 74. Stroboscope, Louble Tulue. (Schall, London.)

DEVICES FOR RESTRICTING THE EFFECTS OF THE RAYS TO PARTICULAR AREAS.--The two chicf devices employed for this purpose are ADJUSTABLE DIAPHRAGMS and TUBE BOXES.

Adjustable Diaphragms. These consist of a skeleton frame, to which can be fitted, according to the needs of the moment, metallic diapliragms of sheet lead, or of some other equally opaque metal, with a central aperture of the required size. This framework is usually permanently connected with the tube-holder. Among later models we notice diaphragms in which the size of the aperture can be increased or dininished by removing or affixing ringslips, and others in which this is effected by arlintment of the iris diaphragm.

Tube Boxes (Fig. 76). - These are made of wood and lined with several layers of lead-foil similar to that used for lining tea-chests. The interior is insulated against sparking by a thick padding of felt. This arrangement practically prevents the rays from passing out except through a circular aperture, 3 inches in diameter, made in one ide of the box. The size of the aperture can be further reduced by means of diapluragms of heavy sheetlead. In order to prevent sparking from the lead plate a diaphragm of plate- 
glass is aloo inserted. In come molets, instead of using lead-foil, the interior of the box is coated with several layers of lead-paint. The size of the box

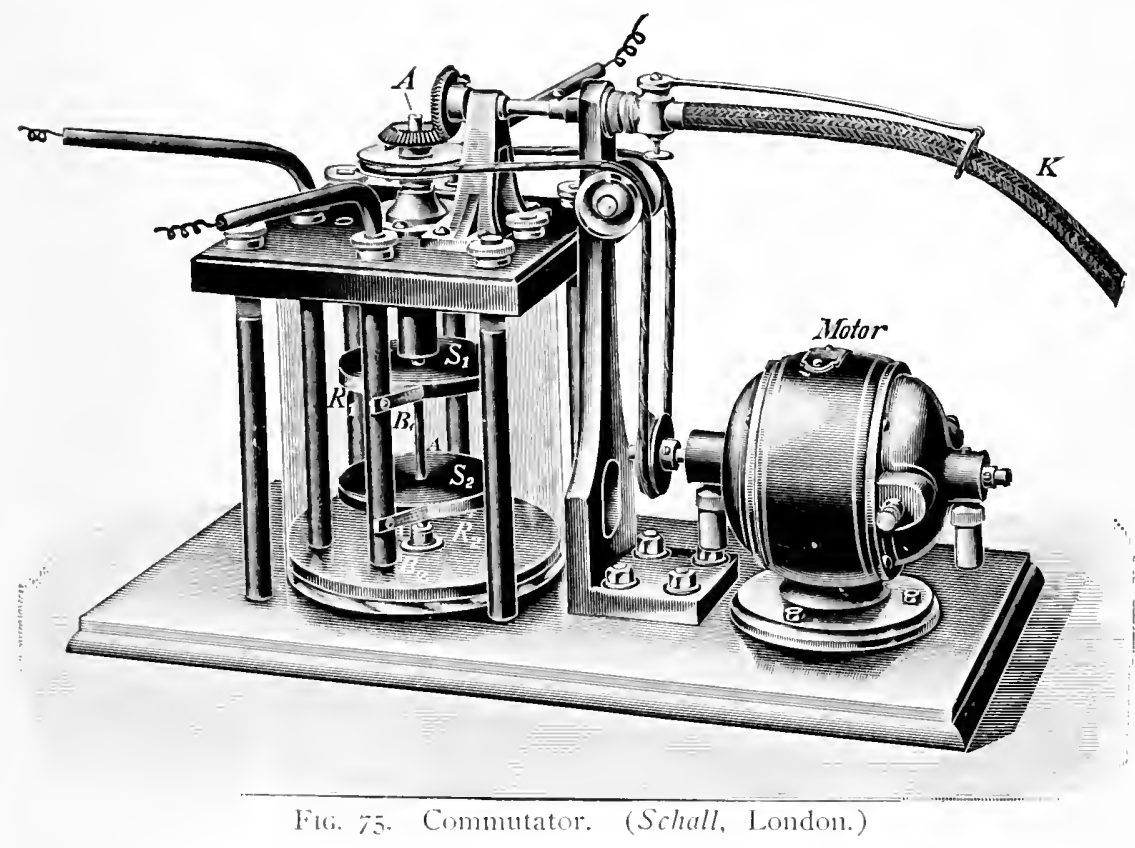

varies in the models of different makers. some being umecessarily large and cumbersone, while others are too small to secure perfect insulation. Attempts

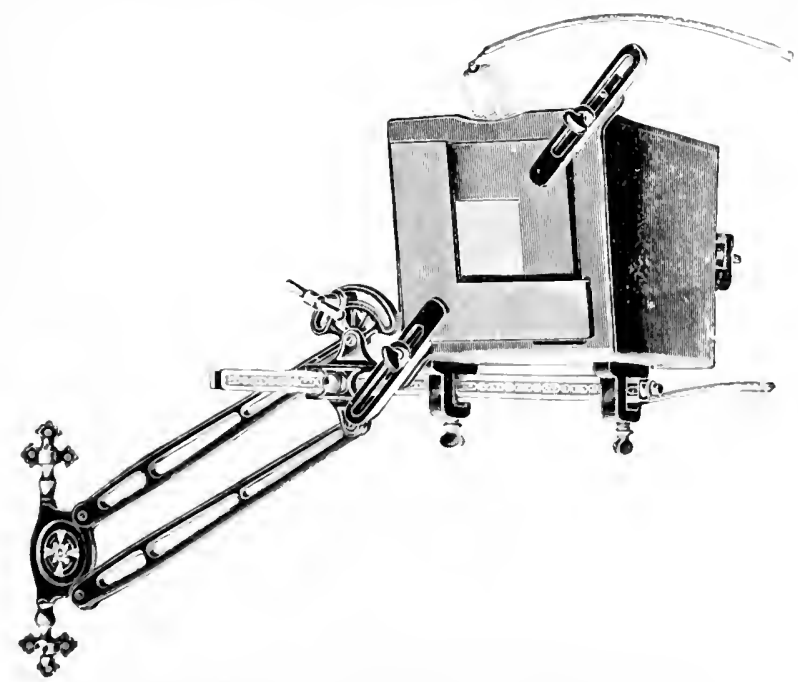

Fir. 76. Liracket Tinle Bux. (Schall, I ondo:1, ) 
have also been made by mechanical means to allow the position of the box being altered; but for use with the exploration couch the simpler models are the most efficient. These, when used for therapentic applications to the face, require to be mounted on a pedestal or attached to a wall-bracket.

MASKS. - These protections are made of either lead plates and covered with flannel or paraffined paper, or they are made of gauze or guttapercha, moulded while warm to the shape of the part and covered with tinfoil. The edge of the holes cut into them being covered over with a layer of paraffinated or shellacked paper. In making exposures in the mouth, pharynx or ragina, a speculum of block tin can easily be improvised. The use of adhesive plaster and of ointments made of bismuth and zinc is only advisable when it is impossible by any other means to protect the tissues against burns.

PROTECTIVE SCREENS.-These, in accordance with Elihu Thomson's directions, are made of aliminimm and earthed. They appear to absorb

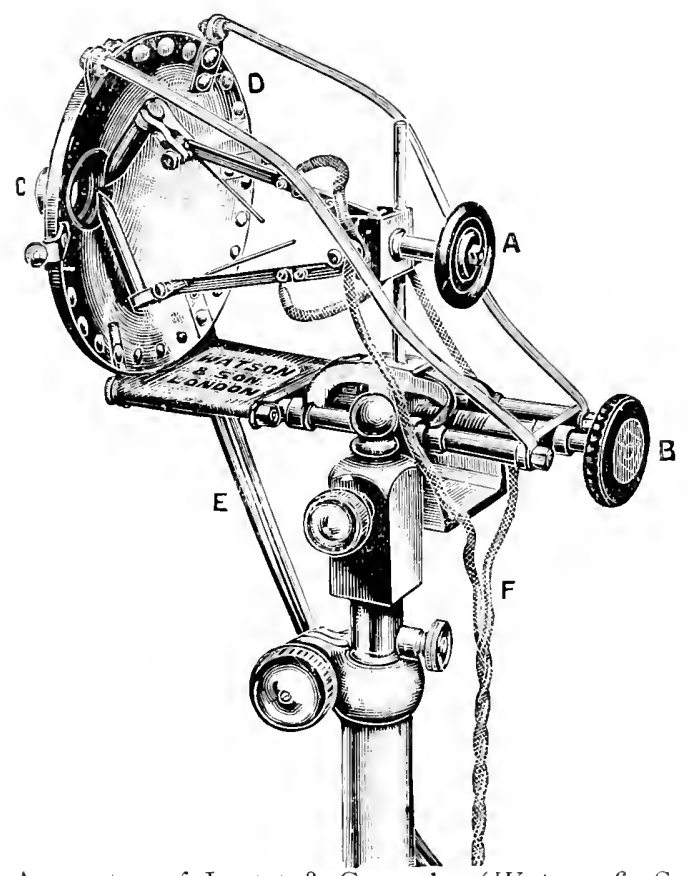

FIg. 77. The Apparatus of Lortet \& Genoud. (IVatson \& Sons, London.)

the softest rays, which exert the greatest influence upon the skin (i.e., setting up a dermatitis) without affecting the more penetrative radiations.

APPARATUS USED IN TREATMENT BY CONCENTRATED CHEMICAL LIGHT.-In order to intensify the bactericidal effect of the solar rays and render them therapeutically effective, lenses and mirrors, which concentrate the rays and bring them to the definite focus are used. The apparatus which is the more often employed is a hollow bi-convex lens, filled with a solution of methylene blue or ammoniated sulphate of copper, so as to ocelude the greater number of heat-rays.

The amount of sunlight available for use in northern latitudes being very limited, it has been thought necessary to supply the deficiency by having recourse to artificial illumination. The light best fitted for this purpose is the voltaic arc, for that given out by incandescent lamps contains too few chemical rays. 
As the rays from an are lamp are divergent, special apparatus employed must be provided for rendering them parallel before pasing through the hollow lens which focuses them upon the part to be treated. The apparatus cmployed for this purpoie consists of two cylindrical telescopic tubes, fitted with plano-convex lenses for rendering the rays parallel (the space between them being nsually provided with a water-circulation); and the hollow bi-convex lens already described for focusing the rays on the part. This extension piece of the Finsen Lamp is mounted upon the netallic support of the lamp. In order to render bloodless the regions submitted to the action of the light. a compressor apparatus, which essentially consists of a slightly convex plate of glass set in a metallic frame, is employed. This is held in position by elastic bands or ly being pressed firmly over the part to be treated.

THE FINSEN ARC-LAMP consints of an arc lamp of 60 to so ampères. in which the positive carbon is placed above the negative, so that the rays, in passing from the crater, are projected downward and outward. In
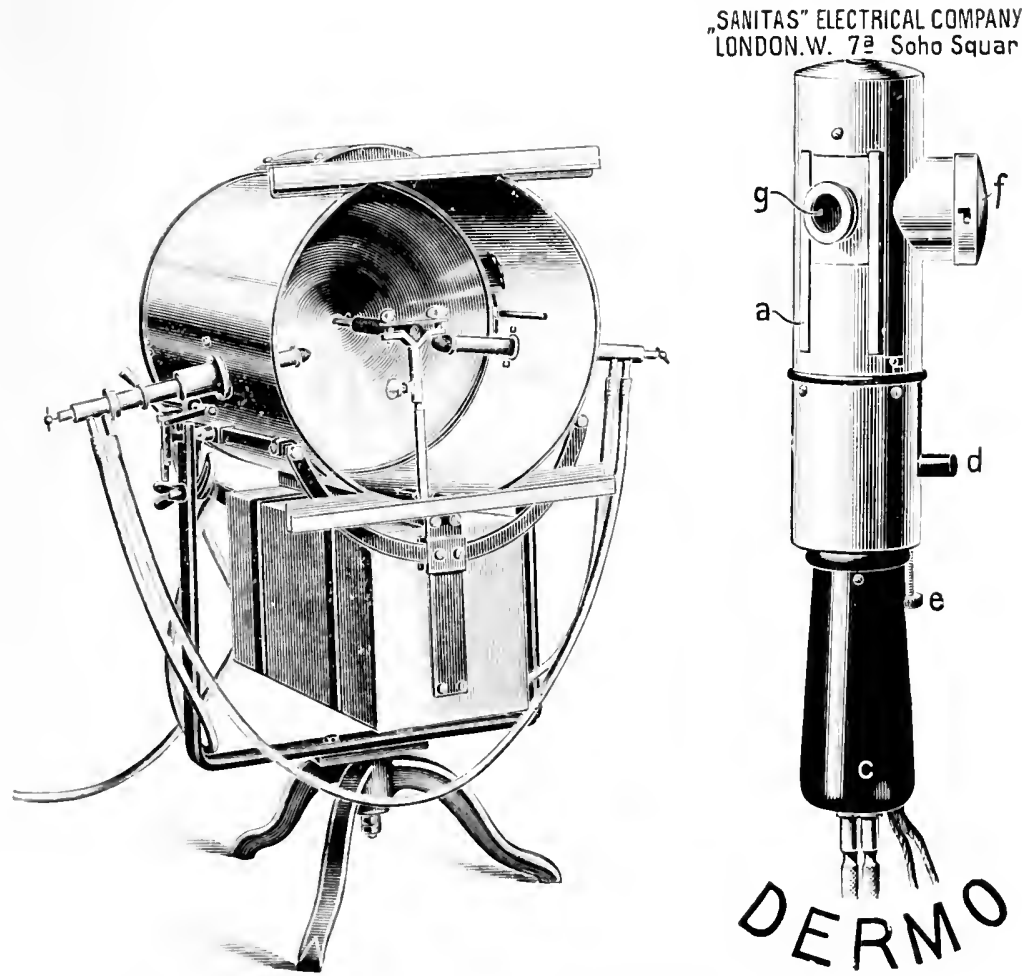

Fig. -8. Dermu Lamps. (Sunitas Electrical Co. Landon.)

the axis of the rays issung from the luminous point are four copper telescopic tulses of the kind already described, the lenses of which are made of rock-crystal so as not to intereept the chemical rays, each one being provided with a water circulation for conling the rays as they pass out.

THE APPARATUS OF LORTET AND GENOUD (Fig. 77).-In it the are-light employed is fecbler than in the Finsen, and consumes only io to 20 amperes. The carbons are inclines towards one another. It also 
possesses a water circulation. The distal end of the screen is fitted with a compressor lens identical with that of the Finsen lamp. As the arc is brought quite close to the patient, the amount of light wasted is greatly diminished; so that a much larger area of skin can be efficiently treated at each sitting whose duration is at the same time considerably reduced.

THE APPARATUS OF DR. SCHALL.-This was intended to allow operators working off the alternating commercial mains to enjoy the same advantages as those using a continuous current. This apparatus can also be used on the continuous current supply. In working off a continuous current the crater-like depression formed in the negative carbon prevents diffusion of the luminous rays; but in use on alternating current mains both carbons are pointed, so that the light is dispersed equally in all directions. To prevent loss from this dispersion, Dr. Schall has introduced a reflector that can withstand the very high temperature of the arc. It consists of a disc of fine clay, into whose mass some oxide of magnesium has been incorporated. One side of the disc is hollowed out so as to form a cylindrical cavity, in which the points of the carbons protrude. A refractor that can be adjusted by means of a milled screw regulates the distance between the two carbon points, while the intercalation of a third carbon between these establishes the arc. It consumes a current of 8 to 10 amperes at 50 to 60 volts.

THE DERMO LAMP OF DR. BANG.-As the amount of ultra-violet rays depends on the chemical composition of the substance of which the electrodes are composed. several attempts have been made to turn this factor to

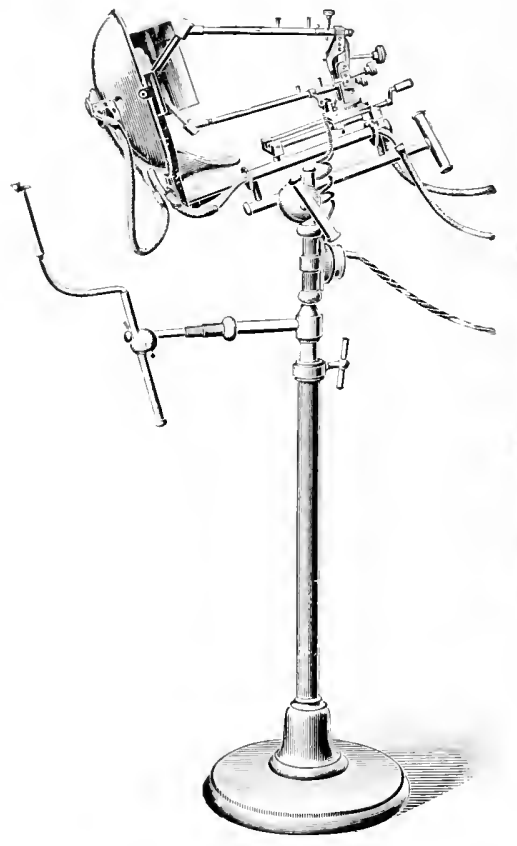

FIG. So. Triplet Lamp. (Sanitas Elcctl. Co., London.)

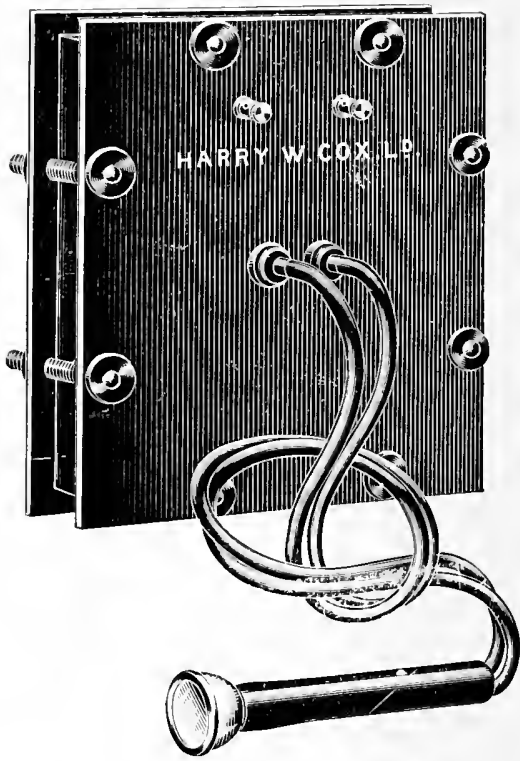

FIG. Si. Spark Lamp. (H. IV. Cor, Ltd., London.)

account. Strcbl has experimented with electrodes of magnesium oxide, aluminium oxide and zircone. He has also made use of aluminium electrodes.

Righi has demonstrated that the electric arc passing between a positive electrode of zinc and a negative electrode of carbon produces purer chemical 
rays than the ordinary are: but it was Bung. the director of the Finsen Institute. who first succeeded in constructing a lamp with metallic electrodes suitable for phototherapy. This lamp is intended to yield a maximum of chemical rays, with a minimum production of heat: by using iron for the electrodes, and reducing their hear resistance by a water circulation. For large lamps the whole of the electrode except the tip is cooled by being plunged in a container filled with water. This lamp is only sulable for the treatment of superficial cutaneous alfections. as the rays possess no powers of deep penetration, but expend their whole energy on the cuticle.

THE APPARATUS OF BROCHA \& CIIATIN-It resembles the Bang Lamp in the use of metallic electrodes, but differ from it in possesing no refrigeration apparatus. It consumes much more current. The electrodes

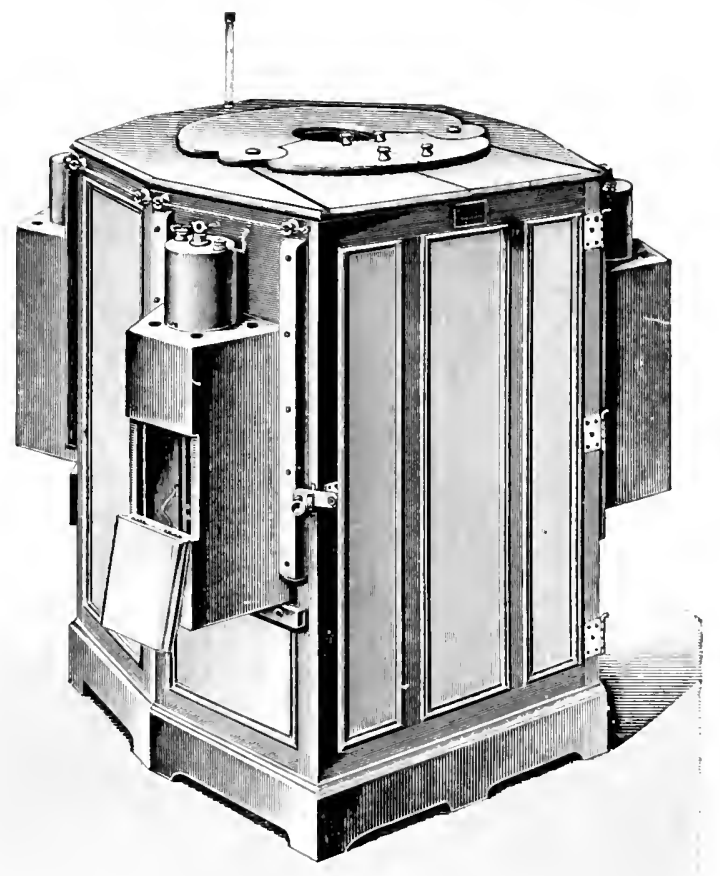

Frt. 82. light bath, with Scarch-light. (Schall, London.)

consss of a positive electrode of carbm with an fron core and an ordinary carbon negative electrode. It is littcl with an automatic regulator and a metal screen for protecting the operator from the chemical effects of the light. It has four telescopic tubes, three of which are intended for triating patients, the fourth for viewing the carlons. It combumen 15 to 20 amprires. ()wing to the ab-ence of a water-conling arrangement. one has, in using it, in rely on very energetic compression (while keeping the patient 3 or 4 inches irom the are) to prevent hurns.

TIE FINSEN REYN LAMU,-This apparatus alus combines the

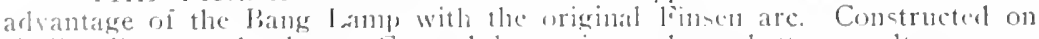
similar lines to the Lertet-Genomel lamp. it porduces lecter results. as one

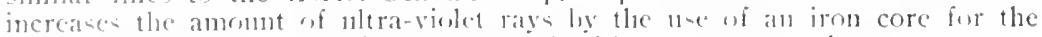

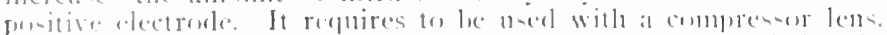

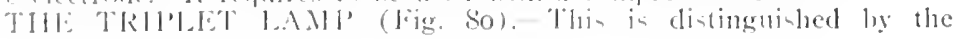


following features: It allows of carbon and iron electrodes to be used either separately or together, so as to produce a mixed light; so that it is equally applicable for the treatment of stperficial and deep skin affections.

THE STREBEL LAMP.-This is fitted with two positive carbon
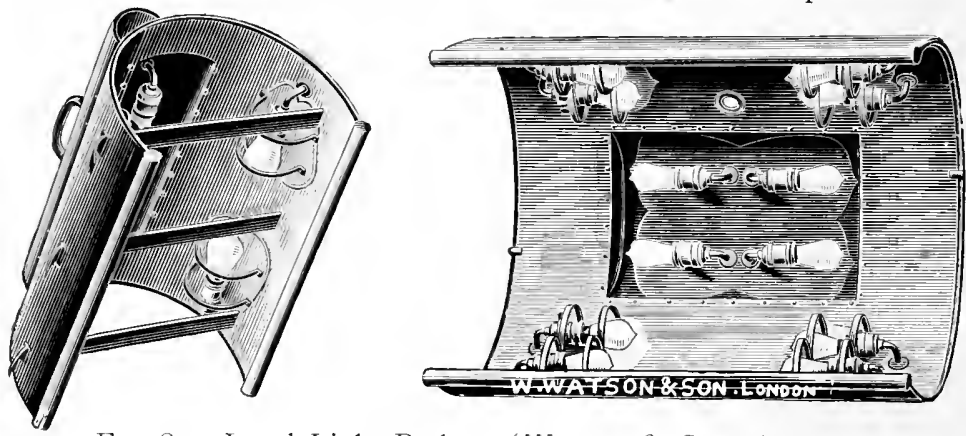

Fig. 83. Local Light Baths. (Tratsun \& Sons, London.)

electrodes with iron cores, and two ordinary carbon negatives, and is provided with a reflector of magnalitum, a rock-crystal lens and a water-cooling arrangement. It can be used for both deep and superficial diseases of the cutis.

THE SPARK LAMP (Fig. 8I).-This apparatus makes use of the

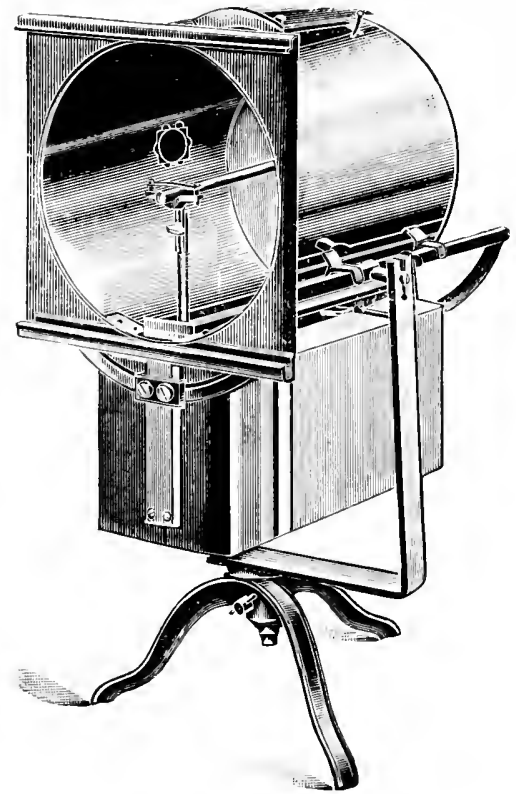

Fig. 84. Search Light. (Schall, London.)

oscillatory discharge of a condenser as a source of ultra-violet radiations. The electrodes are of metal enclosed in a circular ebonite case. which is fronted by a compression lens of quartz.

LIGHT BATHS (Figs. 82 and 83 ).-The use of light baths for the 
treatment of diseascs bas of recent rears attracted some attention. Their therapentical effects differ according to the sonrec of light-rays. A bath fitted with incandescent lamps produces leating effects alone: while those supplied with arc-lamps in addition suplly the more chemically potent ultraviolet radiations. A bath of this nature is shown in Fig. 82. In this model the window is provided to allow the light from a powerful search-light to play upon the body of the patient. To prevent the skin being scorched. the rays are made to pass throngh a filtering screen of spectroscopically tested blue glass. cut into narrow strips to allow them to expand under the influence of the heat withont breaking.

THE KAISER LIGHT TREAT MENT (Fig. 84).--1)r. G. Kaiser of Tienna has found that the blue and riolet rays of the spectrum produce only a slight inflammation, and in consequence of this both circulation and metabolism are stimulated. The apparatus nsed consists of a juwerful search-
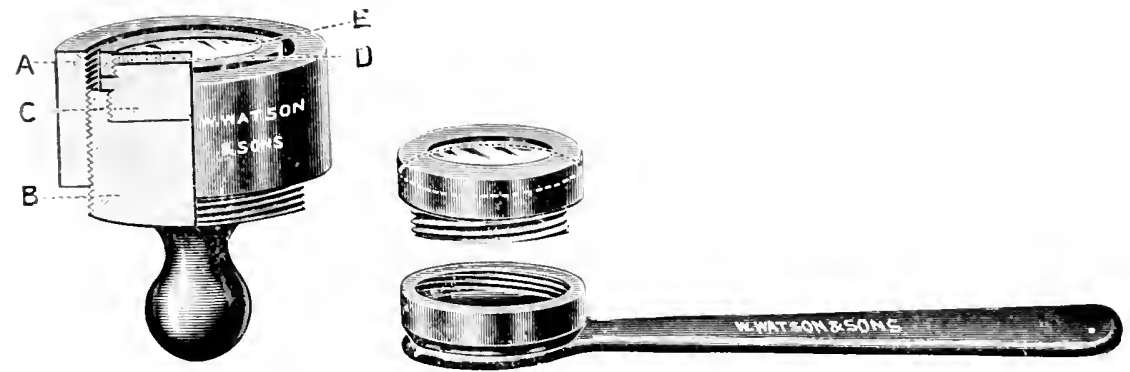

Fus. 85 and 80. Radimu Applicators. (II'atson \& Sons, London.)

light with magnainum reflector, a bhe light-filter of -pectroscopically tested glass cut into narrow strips to prevent fracture from unequal expansion, and a hollow lens filled with a solution of methyline bhe to which a small quantity of alum has been adder. The light consumes a current of 16 ampères at 55 volts.

RADILM THERAPY (Figs. 85 and 86).-In the experimental applications of radium to diseared structures, the metallic salt is enclosed in a container like that hown in the accompanying woodent (Fig. 85). The second applicator is intencled for making applications to the tomgue (Fig. 86). 



\section{ISENTHAL \& CO.}

85 Mortimer Street, London, W.

The most PERFECT and EFFICIENT

\section{INSTALLATIONS}

FOR

\section{Radiography, High Frequency, Electrotherapy, Sismotherapy, Light Baths, Radiotherapy.}

ELECTRICALLY HEATED STERILIZERS

and other Medical Appliances and every kind of Electrical Instrument or Apparaltus used by up-to-date Physicians and Surgeons.

LOWEST PRICES AND

GREATEST SELECTION.

Write for our NEW LIST, the most complete ever issued.

We alle not bound 10 one system but can offer you the choice of all the best makes.

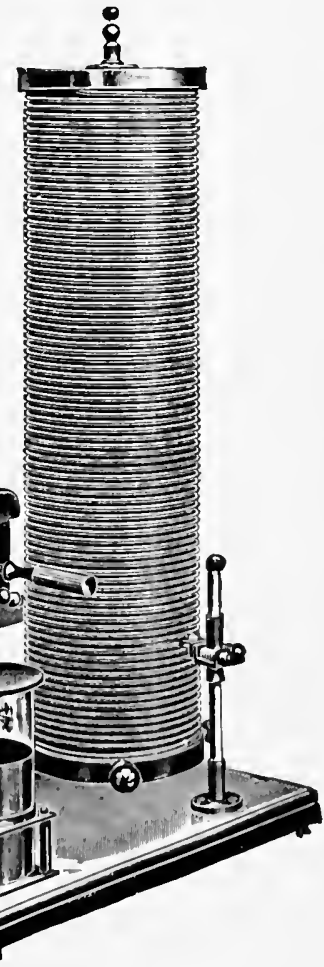




\section{ELECTR0-THERAPEUTICS.}

W. WATSON \& SONS,

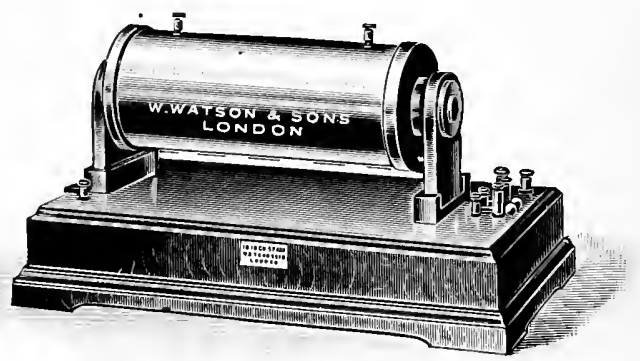

WATSON'S HEAVY DISCHARGE INDUCTION COILS

For X-Rays, Lupas, Cancer, etc.

WATSON'S HIGH-FREQUENCY APPARATUS

For Neurasthenia, Rheumatism, etc.

WATSON'S SPARK LAMP, LIGHT BATHS AND FINSEN TYPE LAMP

For Lupus, Rodent Ulcer, Psoriasis, etc.

I'rile for Ncw Illustrated Price list and full particulars. Post Frce on Application.

WATSON'S PRACTITIONER'S OUTFIT consisting of 6 inch Coil and

FOR "Viil" Contact Break, Accumulator, Rheostat, Tube-holder, 2 Tubes and Fluorescent Screen, complete in travelling case, $\mathbf{E 2 9 . 1 4 . 6}$

RADIOGRAPHY WATSON'S HOSPITAL OUTFIT consisting of 10 inch Spark Coil and

"Vril" Contact Break, Accumulator, Tubes, Tube-holder, Rheostat and Fluorescent Screen

$£ 42.16 .6$

Tubes from $18 / 6$ each. Special Tubes for Electrolytic Breaks, 50/

FOR HIGH-

COMPLETE SETS OF HIGH-FREQUENCY APPARATUS only

$£ 10.10 .0$

FPEOUNNCY COMPLETE SETS OF HIGH-FREQUENCY APPARATUS, including Coil, Break,
etc from CURRENT,

LARGE CAGE SOLENOID for Autoinduction, as illustrated . $15 \mathrm{~S}$

ELECTRODES, from . . 7/6

Full Instructions given with each Se1.

LIGHT BATHS for ams and limbs $£ 5.10 .0$ FOR LIGHT THERAPHY.

\section{LIGHT BATH for trunk}

£9.0.0

SPARK LAMP for Ultra-Violet Light . . $\$ 3.0 .0$

FINSEN LAMP . . . $\quad \mathbf{\$ 2 0 . 0 . 0}$

Estimales, Price Lists, Suggestions, post free on afplicalion ESTABLISHED 1837.

W. WATSON \& SONS, majesty's Government, 313 High Holborn, London, W. C.

6, Forrest Road, Edinburgh Depots: $: \begin{aligned} & 78, \text { Swanston Street, Melbourne, Australia } \\ & \text { W. W. Scott \& C0., 78, Sauchiehall Street, Glasgow }\end{aligned}$ 


\section{THE DOWSING \\ Radiant heat and Cight Creatment}

For Rheumatism, Gout, Arthritis, Lumbago, Sciatica, Sprain, Stiff and Painful Joints, and many Disorders of the Digestive Organs.

The Dowsing Radiant Heat and light Tieatnent is a new and stecessul method of applying light and teat Rays of great intensity ho the limbs and pants of the hody:

This system of treatment dilfers thom all ro-called hot-at applications, and it is

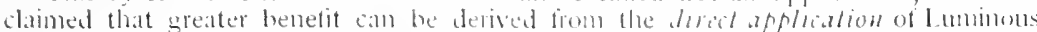
Heat Rays, which in the Dowsing ststem ate Jhom diect upon the hody. The physiological ation of the Dow sing Bath show the following phenomena:-

(1) Very marked redness in the skin.

(2) Very abundant skin perspiration and the elimination of considerable quantites of carbonc acid by the lungs.

(3) Acceleration of the pulthe and increase of $9 \mathrm{~cm}$ perature of the subject: these 1 wo phenoment are less marked in proportion than in luarkish baths.

(4) lncrease of the volume of solid materhals of the urine and urea, and less unc acid.

(5) Greater actwity of the functions of general nutriton, and the elimmation of orgatic oxidathon products.

(6) Penetrating power of the heat rays much more than that of ubscure heat
(7) Parsicular excitation of the skin by the chemical rays and on the oilieg part of pronuanced bactericidal prieperticts.

(4) specit arton of the Luminous Rays on the red globules of the blood, the number of which incleates the oxygenatum.

Ratiant Luminous lleat is the healing vehicte, par excellence, for gout and rheumatsum, contusions. spuans. etc. : as regards arthritis, very bat cases have been cured by communed baths.

I.IN.III. among general affections, such as

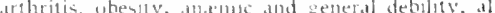
are happily intluenced the the action of Lumbous

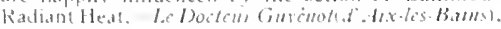

The following are some opinions of teading English Medical Men :-

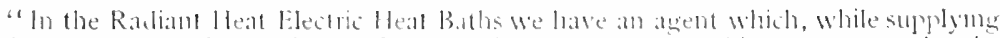

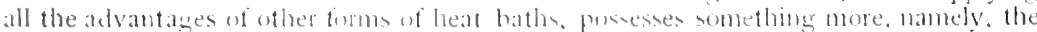

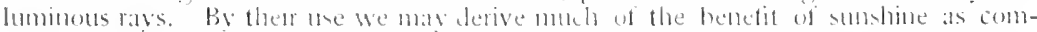
pared to that of other modes of heating."

"If one conviders for a moment the remakahle effects which can he produced by sun baths, it is forcel uron the mind that in the luminoms tols there ane probably

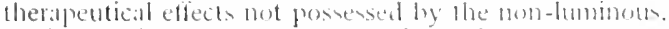

"I consider the ideat source of heat for steh a purpose is a luminnus one."

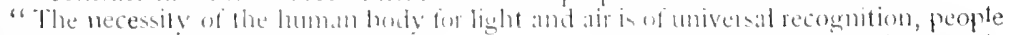
becone andenic when deprived of light. properties in radiant heat."

Hoce are some spectal thetapentic

The Dowsing Apparatus is fully protected by a number of important patents, and the Radiant Heat Treatment is regristered. The apparatus is supplied only for use by lledical Hen, or Institutions. Hospitals, Nursing llomes, fe., Hmber their care. We have no agents, and the apparatus is not sold; the object being to prevent it falling into unqualified bands.

The Profession is warned atgainst copies and infringemenrs offered as similar to the Dowsing System, the apparatus so utate having no tharapcotic value whatever:

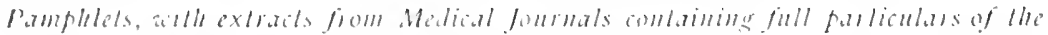

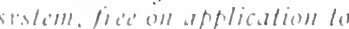

\section{THE DOWSING RADIANT HEAT CO., LTD.}

\section{Registered office: 24 BUUD(BI:RON', CANNON SIRIET, E.C.}

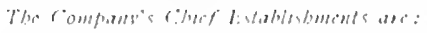

LONDON, 28 York Place, Baker Street, W. BOURNEMOUTH, Glen Fern Gardens
BATH, $50 \times$ ford Row.

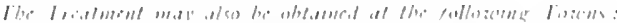

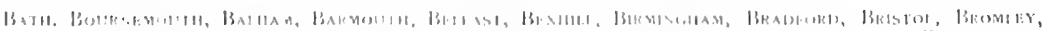

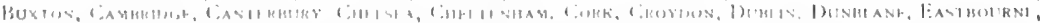

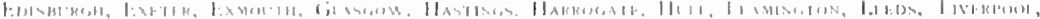

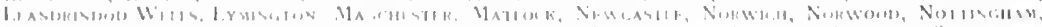

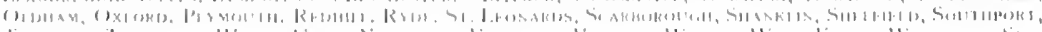

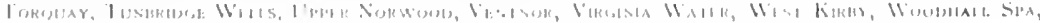
Y'ARMUII, YORK. 


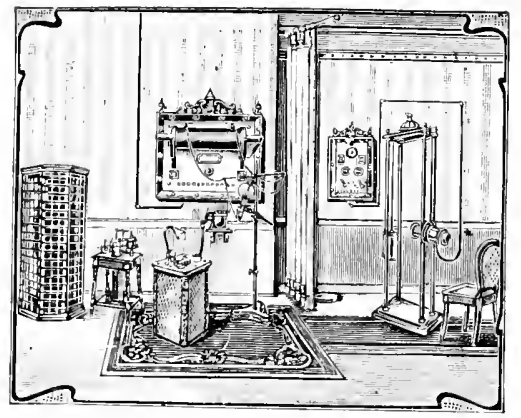

Installation of $\mathrm{X}$-Ray and High-Frequency Apparatus, showing Switch-table from which everything is controlled, and apparatus for Magneto Theraphy.

Total price about $£ 150$.

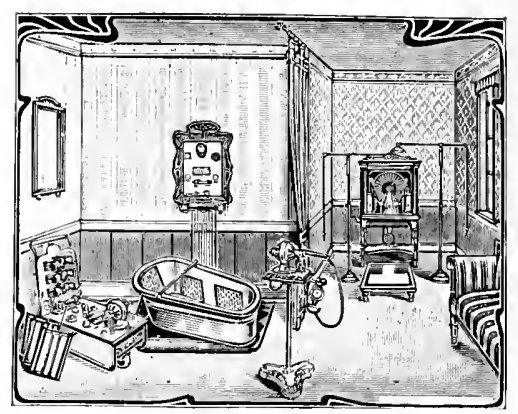

Installation for Sinusoidal by Dr. Smith. Galvanic and Faradic Water Baths. Static Machine and Vibrator for Massage.

Total price about $£ 100$.

\section{The Saniltas} Electrical Co., Ltd.

7a SOHO SQUARE LONDON, W. MANUFAC?URERS OF

Complete Installations of apparalus for all modern melhods of treatment, by Light, Heat, Electricity and Physical Exercise, elc.

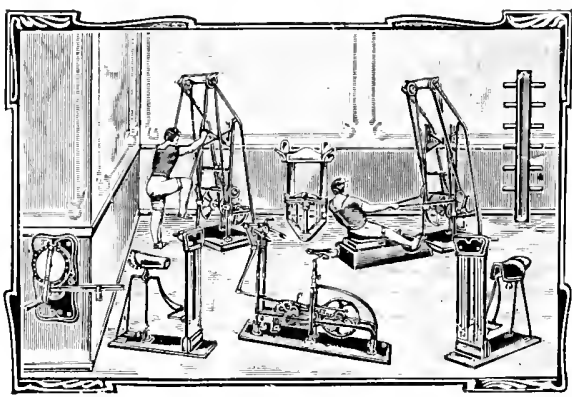

The Sanitas Electrical Co., Ltd.

7a SOHO SQUARE LONDON, W.

The whole of our appliances may be inspected in working order in our Show room.

Special Catalogues and actallet information on appliation.

Instaliàtion of Apparatus for Physical Exercise. "Sano" tor Rowing, Hill Climbing, Pulling, etc., etc. "Velotrot" for the motion of Cycling and Horse-riding (combined or otherwise) with the atlendant highly beneficial vibration of the whole body; and four other practical appliances for different Total price about $£ 55.15 .0$

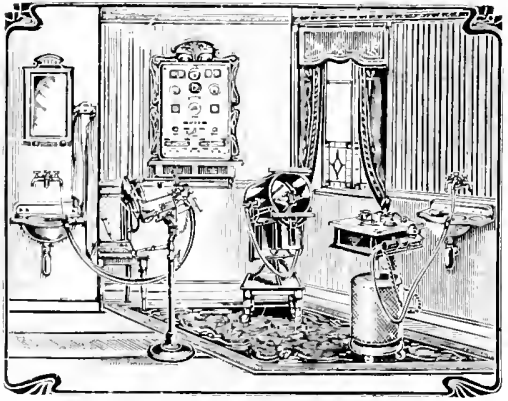

Installation for the treatment of Skin Disease: by the carbon and iron light of the "Dermo" and "Triplet" lamps and "Combined" Projector. Also Switchboard tiur Cautery, Galvanisation, Faradisatton, Electrolysis, Cataphorests, etc.

Total price about $£ 150.0 .0$

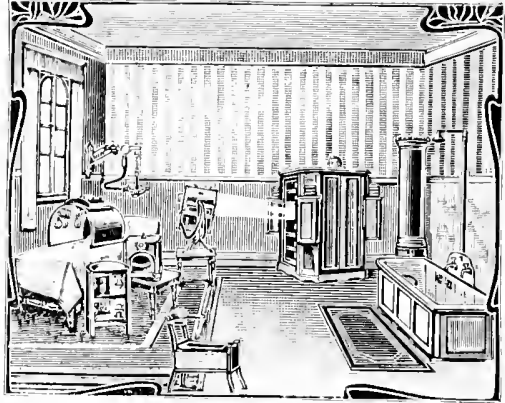

Installation for Light Bath treatment by "Combined" double I ight Bath with Arc and Incandescent l.amps, and with Projector for additional illumination from outside of actual seat of complaint. Portable light Baths for local treatment of Trunk or Lime and bidet for treatment of female diseases by light.

Total price about $£ 125.0 .0$ 


\section{IMIORTANT ANNOL NCEMENT}

JACOBI A Portfolio of Dermochromes. By l'befentul

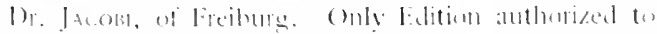

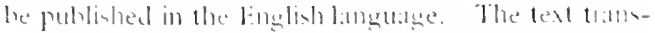

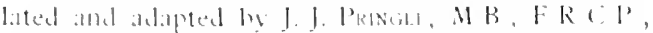

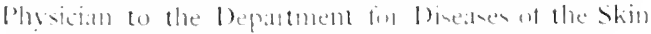

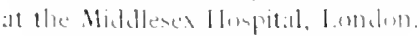

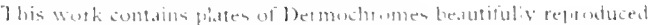

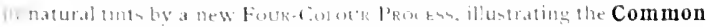
Diseases of the Skin and Venereal Affections which the ciener.al

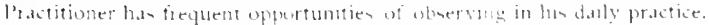
fach plate is acompanted by a page or mote of explatatory text contath

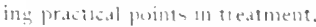

The work. complete in ino vilumes. is publishes at the vely

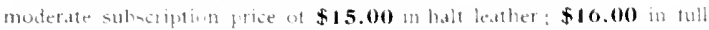
flexible leather with silt edges, thus bringing it within teath of all

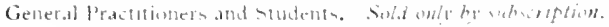

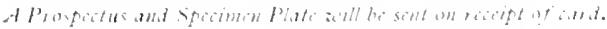

"Charm of noseliy . . accurate telinemions. . . faith-

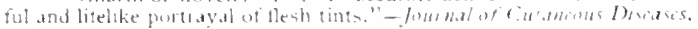

"llapplent results we have seen - . Prificult lo magime a

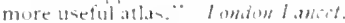

"Remarliable plates. . Nothing comblesced the stitl with

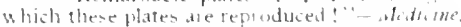

"surpasing in every genect anything that has peviously bllen

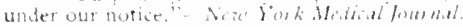

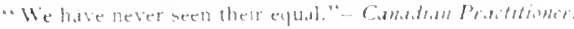

"Two much pratse canmot be besti.wed woun them."

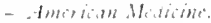

b. Finest gates ever seen in any wation skin diseasen."

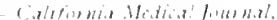

WIILIAMS-High Frequency Currents in the Treat-

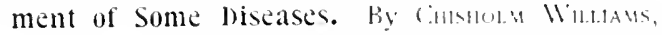
FR.C.S, Edin, ahe Electortherapention w the

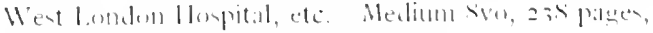
with 75 illustiations. Price, $\$ 2.75$.

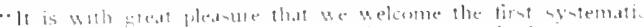

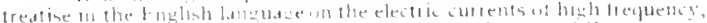

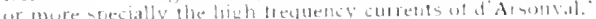

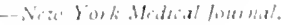

Nill YuRk

$I() N 1)(N$

RLBMAN COMIPANY

RIBMAN LIMITLI)

11) Mtsk 2311 S1k1

120 SHAH II SBUR: AVI. 


\section{WAITE \& BARTLETT MFG. CO.}

217 East 23d Street, New York, U. S. A.

\section{HIGH-GRADE ELECTRO-MEIICAL APPARATUS}

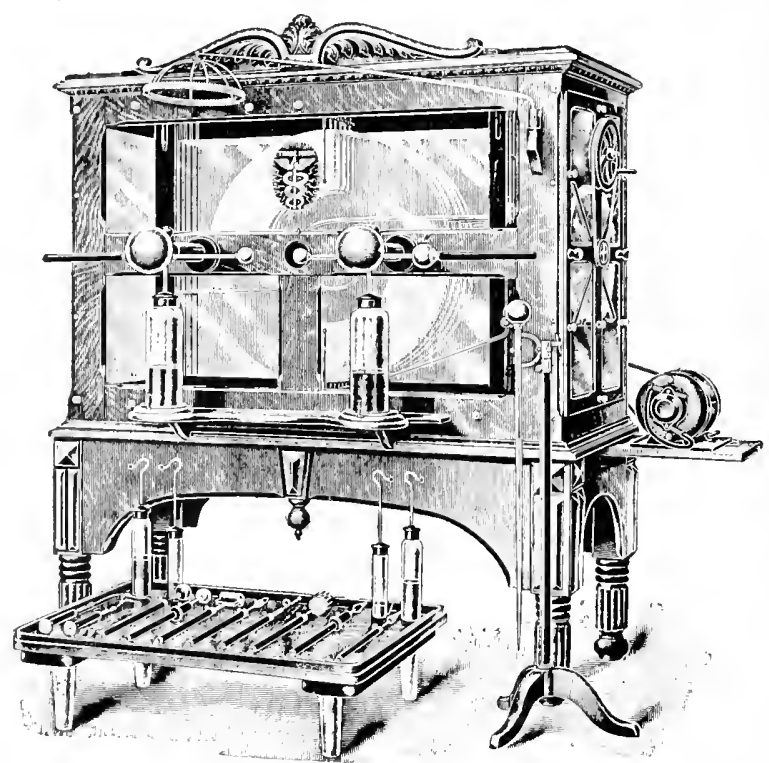

Static Machine

$X=$ Ray Coil

Piffard's Higli=Frequency Apparatus

Piffard's`Utra=Violet Ray Lamp

Piffard's Hand Arc Lamp

Galvanic and Faradic Switch Boards for Commercial Curreuts, Alternating or Direct

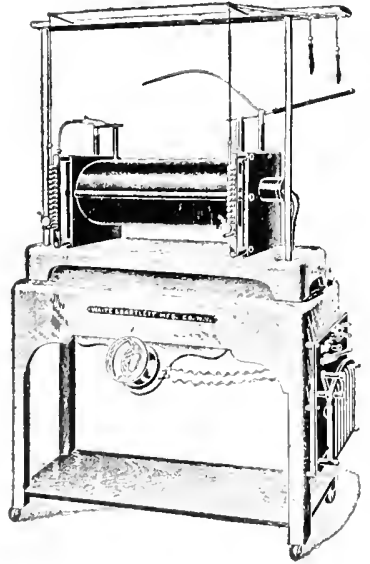

SEND FOR CATALOGUE
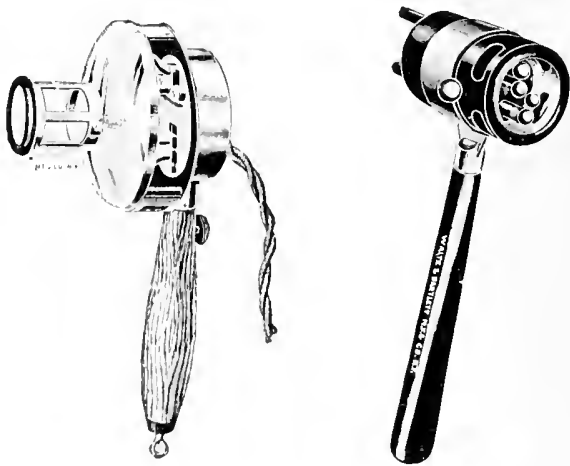


\section{$\mathbf{S}$

for continuous-current with variable self-induction for any tension up to 250 volts.

SWITCHING AND MEASURING TABLES whth cased-in W'ehnelt-interrupter.

SWITCH-B0ARDS for use with intermpters having several contact pins or rotary interrupters.

ROENTGEN-INSTALLATIONS for Military home and tield hospital. (The Roentgen-installations in all the hospitals of Saxony and a great number of the Prussian hospitals are supplied from my works)

ALL APPARATUS and installations for Radio-therapy
NEW! Intensity shades and protective ypectacles fitted with lead glass; a preventive against the had effects of the Roentgen rays.

ACCESSORY apparatus for all purposes.

HARDNESS-MEASURE for detemining the hardness of tubes.

CHROMORADIOMETER for losage in the $X$-Raly treatment of lupus.

RADIUM-BROMIDE chemically pure, giving : tounding results. Fnclosed in capsules. Prices on application.

STATIC-MACHINES formedical purposes, etc. Ilighest efficiency at molerate prices.

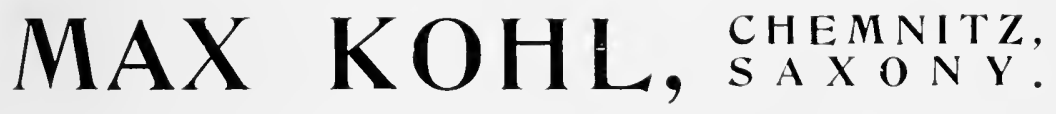

Estimates, Directions for Use, Refirilies. eti., sellt fiee oul aptication.

NOTICE! orders can be execuled either direct or through the Agency of Messrs. Isenthal \& Co., 85 Mortimer Street, London, W.

\section{ELECTRICAL INSTRUMENTS} FOR Surgieal and Medieal Purposes, Motors, X Rays, Light Baths.

New Catalogue of over 200 pages will be sent free K. SCHALL, on application to35, Great Marylebone Street, LONDON, W. 
The First and ONLY ORIGINAL Journal ever published on X-Rays!

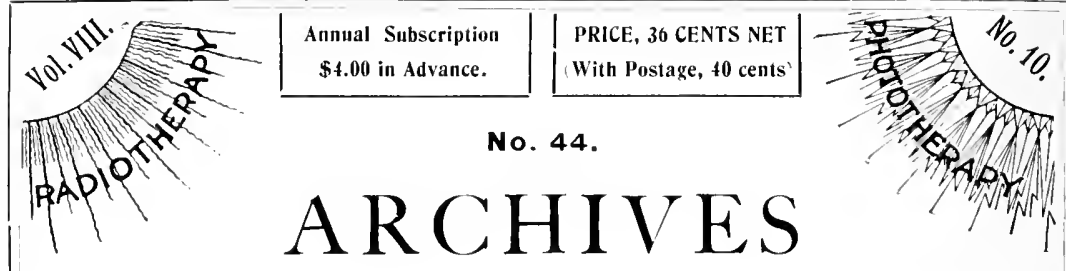

$-\mathrm{OF}-$

\section{THE ROENTGEN RAY}

AND ALLIEI) PHENOMENA

(Formerly Archives of SKIAgraphy)

PUB L IS H E D B Y

REBMAN COMPANY, Io, West 23rd St., NEW YORK

REBMAN, LIMITED, I29 Shaftesbury Ave., London, W. C.

\section{CONTENTS.}

EDITORIAL:

Sir Oliver LODge's l.f.ctures 1 :0

ORIGINAI. ARTICIE:

Lectures to Medical Practi-

TIONFRS UN PHYSICS API'LIER

to Menicine. By Sir Oliver

l.ODGE, F.R.S.

LECTURE 1. ON RaYS IN Gentral . . $1 \neq 0$

ITEMS OF INTEREST:

Treatment of Cancer . 104

X-Ray Treatment ol Cancek

OI THE UIERUS.
THE ROENTGEN SOCIETY PAgE MEETING, NOVEMBER ; , 1903. By Mr. Leslie Miller:

IMPROVEMENTS IN INDUCTION Colls and High-Frequency RESONATORS . . 165

High-Frequency Apparatus . 109

THE ROENTGEN SOCIETY

MEETING, DECEMBER 3, 1003. 173

THE ROENTGEN SOCIETY

MEEIINC;, JANUARY 7, 1004.

The Relflations of Radum. By DR. Geokge B. Batten . 173

D)ESCRIPTIONS OF PLATES . 182

$$
\text { PLA T F. S. }
$$

PLATE CIXXXVII.

RaDUMGRAM (F HANI), SHOWING INJECTED Arteries. By A. G. FryetT, F.R.M.S.

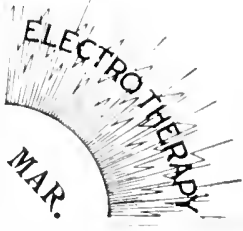

(Melbolirne, Australia)

I'LATE CLXXXVII.

Stereoscorlc Rambographs of Elbow JoInts, Aktfries Injected. BY A. G. Fiktet, F.R.M.S. (Melbourne, Ausiralia)

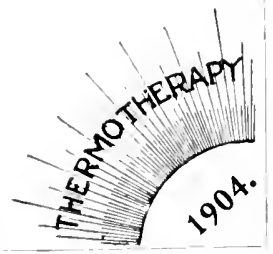

Size of Journal: Royal 4to, or $12 \frac{1}{2} \times 10$ inches. 


\section{John J. Griffin \& Sons, LIMITED.}

Makers of

\section{SCIENTIFIC APPARATUS.}

\section{STATIC MACHINES}

in

MAHOGANY CASES FOR

HAND OR MOTOR DRIVE.
WIMSHURST MACHINES FOR $X=R A Y$ WORK.

INDUCTION COLLS, MEDICAL. COILS, EI.ECTRODES.

$X=R A Y$ TUBES, THERAPEUTIC TUBES, RADIUM.

$20-26$ Sardinia Street,

LINCOLN'S INN FIELDS, LONDON, W. C.

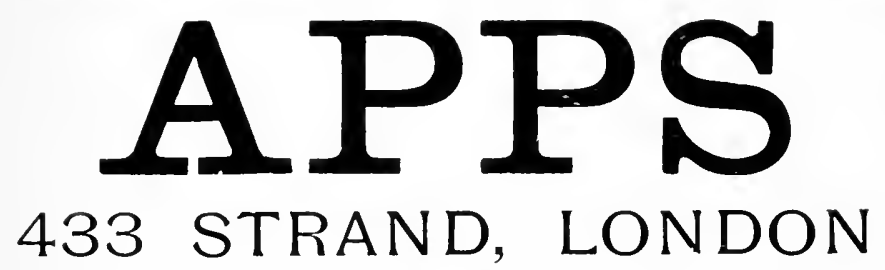

MANUfaCtURER of Ophical, Mallemalical and philosophical InstruW ments 10 the Gewerument bepartments. Apparatus for X-Ray Work, ete. INDUCTION COILS, APPS'S PATENTED INSTRUMENTS, small and large sizes, very highest efficiency and durability. All instruments guarantecd. All simes 1 in. to $30 \mathrm{in}$. stuckel. FOUR FEET SPARK COILS to order.

SPECIAL PATTERNS will be matle to arder, induling pointless Sec. fions and Winding. Flat, atso Crnical, my 1 s6h Patterns, foro ohms Resistance, ro-in. Covils w) orter.

IMPROVED MERCURY BREAK, $6^{6} 6^{\prime}$. This can be fllted to any Induction Coil.

HOSPITAL X-RAY SETS, complete in every detail. from f25 cach. The "Model" see of X-Ray apparatus atopled for South Africa during the late war, with bitteriec, Steam Motur, elc.

HIGH-FREQUENCY APPARATUS of the latest patterns.

A SPECIAL SERIES OF COILS wiboul Comtart bicakers, ele., specially mented in at phan but very durable manned, about two-thinds of cataloguc prices.

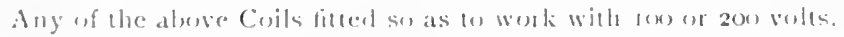

PRICES PEK RETURN OF POST. 


\section{$X$-RAY AND \\ HIGH FREQUENGY APPARATUS.}

As supplied by us to H.M. Government, Middlesex Hospital, King's Coliege Hospital, The General Hospital, Birmingham, The Cancer

Hospital, Fulham; and the Leading Hospitals and $X=R a y$

Workers throughout the World.

We are the Actual Manufacturers, and Guarantee all our Coils against Breaking Down.

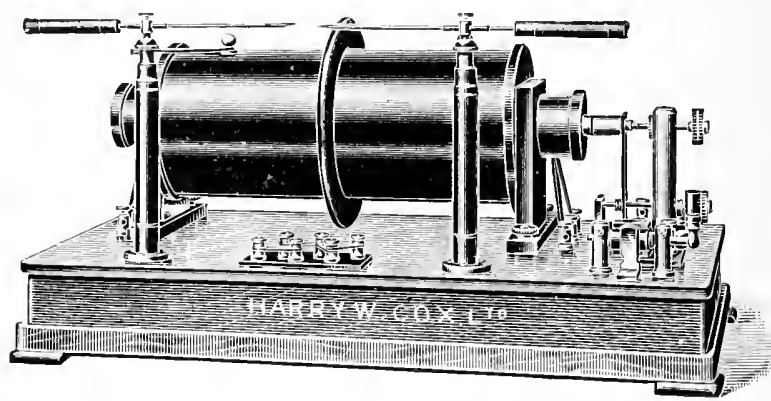

Extract from a letter from a lange Medical Institution, referring to Coil and complete Accessories:-

"You will, I ans sure, be ghad to know that the apparatus you fixed up for us gives every satisfaction; a great deal of work has been done with it."

\section{COX'S PORTABLE COMPLETE OUTFIT}

as supplied to H.M. Admiralty, War Office, the new Royal Yacht, and in use by a

large number of Hospitals and General Pactitioners in all parts of the Work.

HIGH FREQUENCY APPARATUS.

N.B.-OUI INDUCTION COILS and APPARATUS are not only less costly than other makers', but give results unexcelled by any of them.

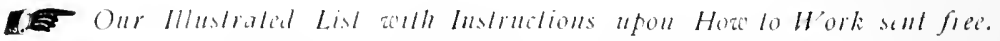

Lessons given in X=RAY and HIGH FREQUENCY WORK.

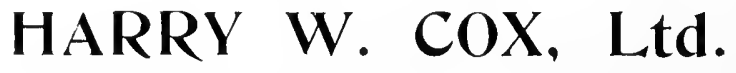

9, 10 and 11, CURSITOR ST., CHANCERY LANE, LONDON. 






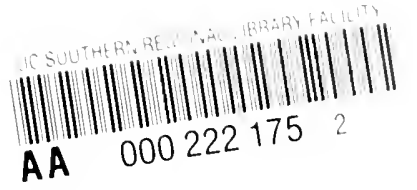


